MÉMOIRES DE LA SMF 88

\title{
THE WAVE DIFFRACTED \\ BY A WEDGE WITH \\ MIXED BOUNDARY CONDITIONS
}

Olivier Lafitte

Société Mathématique de France

Publié avec le concours du Centre National de la Recherche Scientifique 
O. Lafitte

Commissariat à l'Énergie Atomique-DAM., DCSA/MLS,

Centre d'Études de Bruyères-le-Chatel, 91680 Bruyères-le-Chatel.

Massachusetts Institute of Technology, Math. Department, Cambridge, MA 02 139,

USA.

DM2S/DIR., Centre d'études de Saclay, 91191 Gif-sur-Yvette Cedex.

2000 Mathematics Subject Classification. - Primary 35L05, 35A21; Secondary 35Q60, 78A25.

Key words and phrases. - Diffraction, electromagnetic waves, wedge, impedance.

This work has been supported in part by the contract 93-1187/A000 of the Délégation Générale pour l'Armement (D.R.E.T).

The author completed this work while he was at the C.M.L.A., ENS Cachan.

He revised this work while in CMAT, École Polytechnique, 91128 Palaiseau Cedex.

The author wants to thank R.B. Melrose and the Massachusetts Institute of Technology for the year he spent in 1993-1994, when he started the present study. 
À Christine, Marine et Geoffrey 



\title{
THE WAVE DIFFRACTED BY A WEDGE WITH MIXED BOUNDARY CONDITIONS
}

\author{
Olivier Lafitte
}

\begin{abstract}
We study the diffraction of a conormal wave by a curved wedge in $\mathbb{R}^{2}$, each face + or - of the wedge being characterized by a mixed boundary condition of impedance type $\partial_{n} u+z^{ \pm}(x) \partial_{t} u=0$. We reduce the problem to a system on the two traces of the diffracted wave on each face of the wedge. The principal matricial symbol of this system is the matrix of the "straightened" system obtained with the tangent diedra and with the boundary condition $\partial_{n} u+z^{ \pm}(0) \partial_{t} u=0$.
\end{abstract}

\section{Résumé (L'onde diffusée par une arête avec conditions au bord mixtes)}

Nous étudions la diffusion d'une onde conormale analytique par une arête (ou un dièdre) à faces courbes, muni de conditions de type impédance sur chaque face, de la forme $\partial_{n} u+z^{ \pm}(x) \partial_{t} u=0$. Nous ramenons ce problème à l'étude du système sur les traces et les dérivées normales sur chaque face. Ce système a pour terme principal le système obtenu en remplaçant chaque face par la face tangente et les conditions au bord par $\partial_{n} u+z^{ \pm}(0) \partial_{t} u=0$ et nous montrons que le système principal est inversible. 



\section{CONTENTS}

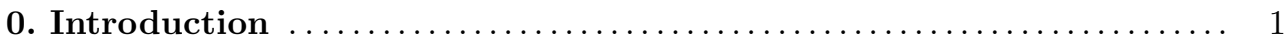

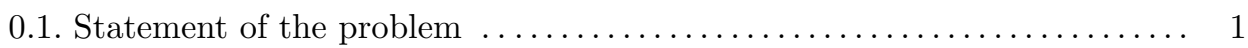

0.2 . Rigorous asymptotic behavior of the solution $\ldots \ldots \ldots \ldots \ldots \ldots \ldots \ldots$

1. Existence, uniqueness, and regularity of the solution $\ldots \ldots \ldots \ldots \ldots 13$

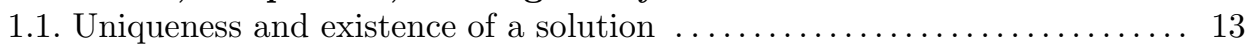

1.2. The application to the system of Maxwell equations $\ldots \ldots \ldots \ldots \ldots \ldots \ldots$

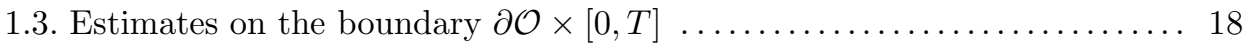

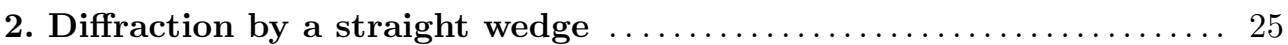

2.1. Existence of the solution of an elliptic problem $\ldots \ldots \ldots \ldots \ldots \ldots \ldots \ldots$

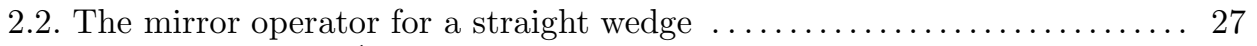

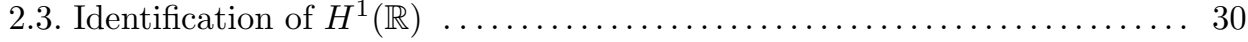

2.4. Expression of the boundary operators using the Mellin transform . . . . . 32

2.5. The fundamental analytic system for the traces $\ldots \ldots \ldots \ldots \ldots \ldots \ldots \ldots 35$

2.6. The invertibility in $L \times L$ of the analytic system $\ldots \ldots \ldots \ldots \ldots \ldots \ldots$

3. Microlocal propagation of singularities for the mixed problem . . . 43

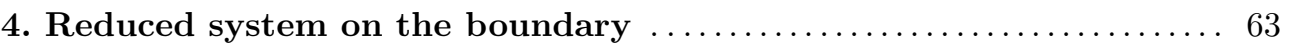

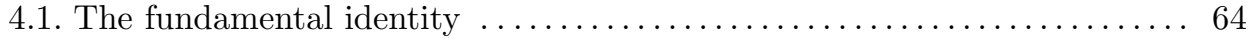

4.2. Expression of the Neumann to Neumann operators $\ldots \ldots \ldots \ldots \ldots \ldots \ldots 66$

4.3. The Dirichlet to Neumann operators ......................... 67

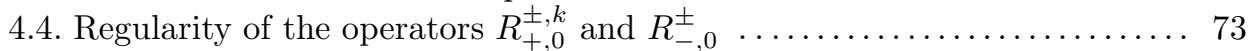

4.5. Asymptotic expansions of the operators $K_{ \pm}^{+, k}$ and $R_{ \pm, 0}^{+, k} \ldots \ldots \ldots \ldots \ldots 78$

4.6. System of equations and microlocalisation of the sources $\ldots \ldots \ldots \ldots \ldots 9$

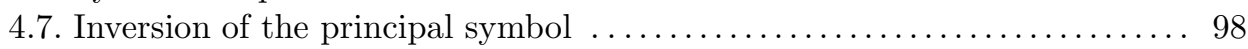




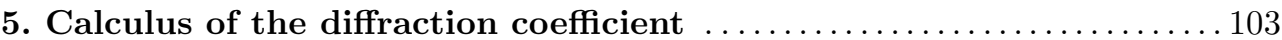

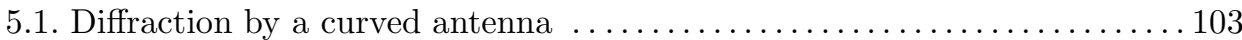

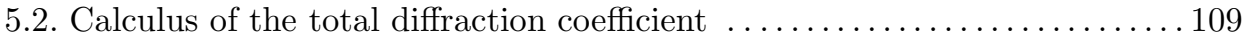

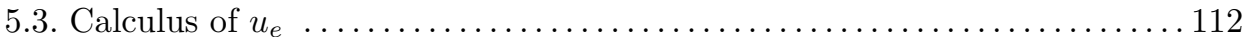

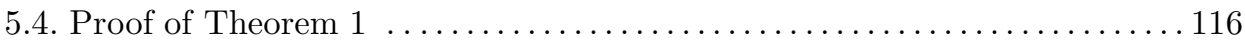

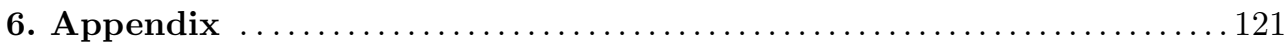

6.1. Properties of the Helmholtz equations around corners . . . . . . . . . . . 121

6.2. Calculus of the distributions on the boundary $\ldots \ldots \ldots \ldots \ldots \ldots \ldots \ldots \ldots$

6.3. Asymptotic expansions of the DTN operators (from Gérard and Lebeau)

Bibliography 


\section{CHAPTER 0}

\section{INTRODUCTION}

\subsection{Statement of the problem}

Let $F=\left\{(x, y) \in \mathbb{R}^{2}, x \geqslant 0, b(x) \leqslant y \leqslant a(x)\right\}$ be a wedge in $\mathbb{R}^{2}$, the curves $y=a(x)$ and $y=b(x)$ are called in this paper the faces of the wedge. We assume $a(0)=b(0)=0, a(x)>0>b(x)$ for $x>0$ :

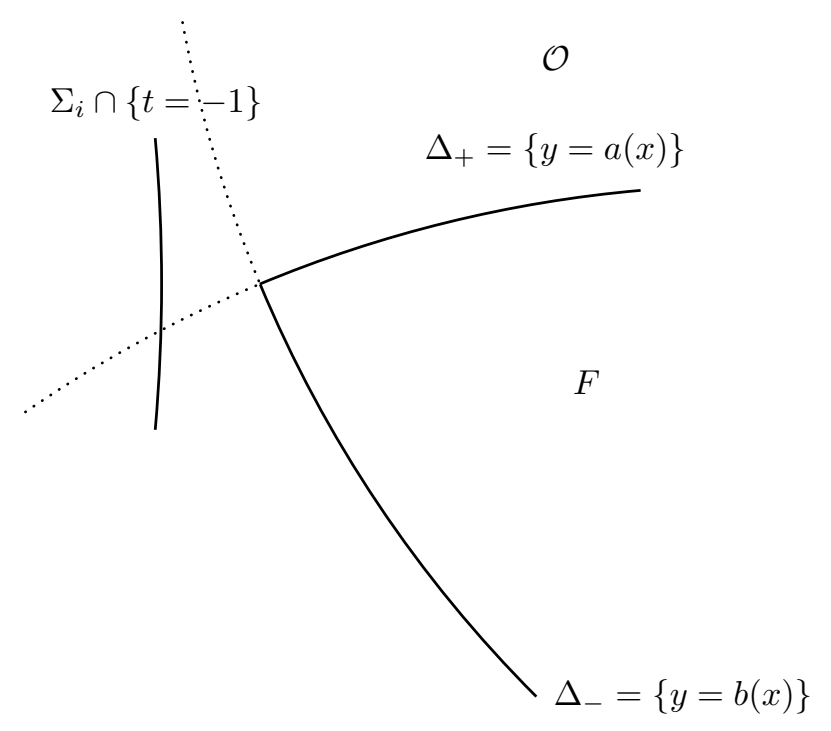

Figure 0.1 . Wedge $F$, incident wave front set $\Sigma_{i}$

The space domain is $\mathcal{O}=\mathbb{R}^{2}-F$. We denote the faces of the wedge by $\Delta_{+}$ and $\Delta_{-}$. With the notation $\widetilde{\Delta}_{+}=\{(x, y, t), y=a(x), t \in \mathbb{R}, x \in \mathbb{R}\}$ and $\widetilde{\Delta}_{-}=$ $\{(x, y, t), y=b(x), t \in \mathbb{R}, x \in \mathbb{R}\}$, we verify that $\Delta_{ \pm}=\widetilde{\Delta}_{ \pm} \cap\{x>0\}$. The functions 
$a$ and $b$ are assumed to be analytic functions on $\mathbb{R}$. We define the exterior domain $\Omega=\mathcal{O} \times \mathbb{R}_{t}$. It will be convenient to consider that $\partial \mathcal{O}=\partial \mathcal{O}_{+} \cup \partial \mathcal{O}_{-} \cup\{(0,0)\}$. In all the sequel, $p$ will denote the projection from $\mathbb{R}^{2} \times \mathbb{R}_{t}$ to $\mathbb{R}^{2}$.

The problem of diffraction by a wedge with Dirichlet or Neumann boundary conditions has been studied by other authors before (starting with Poincaré [34], [35] and Sommerfeld [40], then Garnir [19], Bernard [3], [5], [7], [8], Kaminetzki-Keller [23], Bouche-Molinet [9], [30], Cessenat [12], Assous-Ciarlet [1]). However, these authors considered a wedge with straight faces or a wedge whose faces are circular arcs (Bernard [5]). The generalization to a curved wedge with analytic faces was done for a Dirichlet boundary condition by Gérard and Lebeau [20]. Other authors studied related problems: Kondrat'ev [24] considered more general boundary conditions and a cone, as well as Eskin [17, 18] or Bernard [7]. Grisvard [21], Azaiez-Dauge [2], Assous-Ciarlet-Sonnendrucker [1] studied elliptic problems outside polyhedra.

The results of Gérard and Lebeau were used by Burq [11] to obtain a control result with open sets with corners. A generalization of the propagation result to a wedge in $\mathbb{R}^{d}$ is due to Lebeau $[\mathbf{2 9}]$.

We generalize in this paper the results of $[\mathbf{2 0}]$ for more general boundary conditions. We assume that each face of the wedge is characterized by an impedance boundary condition, that we describe below by equation (6).

Let us consider an incident wave $u_{i}(x, y, t) \in H_{\text {loc }}^{1}\left(\mathbb{R}^{2} \times \mathbb{R}_{t}\right)$, solution of the wave equation $\left(\Delta-\partial_{t^{2}}^{2}\right) u_{i}(x, y, t)=0$. We assume that $u_{i}$ is conormal analytic to a surface $\Sigma_{i}$ such that $\Sigma_{i} \cap\{t<0\} \subset \Omega, \Sigma_{i} \cap\{t=0\} \cap \partial \Omega=\{(0,0,0)\}$ and $u_{i}(x, y,-\delta)$ is supported on the side of $p\left(\Sigma_{i} \cap\{t=-\delta\}\right) \subset \mathbb{R}^{2}$ which does not contain $F$ (see Figure 0.1). The wave $u_{i}$ is the generalization of a plane wave ${ }^{(1)}$. This wave can be written, in a neighborhood of $t=x=y=0$

$$
u_{i}(x, y, t)=\frac{1}{2 \pi} \int_{-\infty}^{+\infty} e^{i \omega\left(t-\theta_{i}(x, y)\right)} \sigma_{i}(x, y, \omega) \mathrm{d} \omega+a(x, y, t)
$$

where the function $a$ is analytic in the neighborhood of $(0,0,0)$ and $\theta_{i}(0,0)=0$, $\nabla \theta_{i}(0,0)=(1,0)$. The symbol $\sigma_{i}$ is analytic and satisfies

$$
\sup _{s \geqslant 0} \int_{-\infty}^{+\infty}\left(1+|\tau|^{2}\right)\left|\sigma_{i}(x, y, \tau-i s)\right|^{2} \mathrm{~d} \tau<+\infty .
$$

Let us define the impedance boundary conditions. For this purpose, we define, when they exist, the two traces $\partial_{+}$and $\partial_{-}$, which are the normal derivatives on each face of the wedge (unlike in [20], where the normalization coefficient was not present):

$$
\left\{\begin{array}{l}
\partial_{+} f(x)=\left.\left(1+\left(a^{\prime}(x)\right)^{2}\right)^{-1 / 2}\left(\partial_{y} f-a^{\prime}(x) \partial_{x} f\right)\right|_{y-a(x)=0^{+}} \\
\partial_{-} f(x)=\left.\left(1+\left(b^{\prime}(x)\right)^{2}\right)^{-1 / 2}\left(b^{\prime}(x) \partial_{x} f-\partial_{y} f\right)\right|_{y-b(x)=0^{-}}
\end{array}\right.
$$

\footnotetext{
$\overline{\text { (1) For example, }^{2}}$ when $\sigma_{i}(x, y, \omega)=(1+|\omega|)^{-3}$ and $\theta_{i}(x, y)=x, u_{i}$ is (up to a regularization) the inverse Fourier transform of what is called a plane wave propagating in the $x$ direction.
} 
Notice that these derivatives are the exterior normal derivatives to the wedge $F$; when we consider the usual Green's formula in $\mathcal{O}$, the exterior normal derivatives to $\mathcal{O}$ are $-\partial_{ \pm} u$.

0.1.1. The tangent straight wedge. - If the functions $a(x)$ and $b(x)$ are linear functions, the wedge is an angular sector, which is defined in polar coordinates by $-\tan ^{-1}\left|b^{\prime}(0)\right| \leqslant \theta \leqslant \tan ^{-1} a^{\prime}(0)$, and the exterior normal derivative to the wedge is $r^{-1} \partial_{\theta} u\left(r, \tan ^{-1} a^{\prime}(0)\right)$ on the face $y=a^{\prime}(0) x$ and $-r^{-1} \partial_{\theta} u\left(r, \tan ^{-1} b^{\prime}(0)\right)$ on the face $y=b^{\prime}(0) x$. We shall write $a=a^{\prime}(0)$ and $b=b^{\prime}(0)$ to shorten the notations. The angular sector associated with $\mathcal{O}$ is denoted by $\mathcal{O}_{0}$. We introduce $\Omega_{0}=\mathcal{O}_{0} \times \mathbb{R}_{t}$ to be in accordance with the classical calculus on the diffraction by a wedge (J.M. Bernard $[\mathbf{8}])$. We verify that, when the situation is the one described in the figure below:

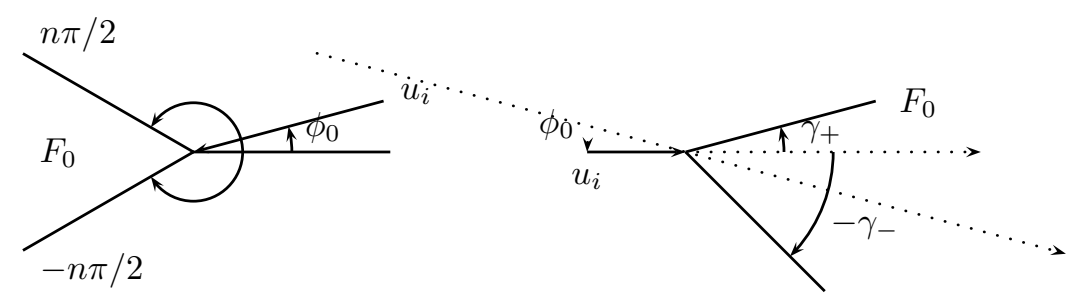

Figure 0.2. Definition of $n$ and $\phi_{0}$

the face $\theta=n \pi / 2$ is the face $y=b^{\prime}(0) x$ of the straight wedge we consider in our hypothesis. The normal derivative $\partial_{-}$on this face is $-r^{-1} \partial_{\theta} u(r, n \pi / 2)$, and the boundary condition written in (4) of $[\mathbf{8}]$ is, on this face

$$
r^{-1} \partial_{\theta} u(r, n \pi / 2)+i k \sin \theta^{+} u(r, n \pi / 2)=0 .
$$

This boundary condition is thus $\partial_{-} u-i k \sin \theta^{+} u=0$. In a same way, the boundary condition on the other face $\phi=-n \pi / 2$ is

$$
r^{-1} \partial_{\theta} u(r,-n \pi / 2)-i k \sin \theta^{-} u(r,-n \pi / 2)=0,
$$

that is $\partial_{+} u-i k \sin \theta^{-} u=0$. Finally, the incident wave written in (1) of [8] is $u_{i}=e^{i k r \cos \left(\phi-\phi_{0}\right)}$. The equation of the wave front associated with this representation, assuming that the projection wave front at $t=-t_{0}$ is away from the wedge, is $t=$ $-r \cos \left(\phi-\phi_{0}\right)$. With this remark, we notice that (according to the Physics notation for the Fourier transform in time of the incident wave) the corresponding incident wave is

$$
U_{i}(r, \phi, t)=\frac{1}{2 \pi} \int_{\mathbb{R}} e^{i k\left(r \cos \left(\phi-\phi_{0}\right)+t\right)} \mathrm{d} k
$$

which implies that the boundary condition in the $(x, y, t)$ system of coordinates is

$$
\partial_{ \pm} u-\left.\sin \theta^{\mp} \partial_{t} u\right|_{\Delta_{ \pm}}=0 .
$$


0.1.2. The boundary conditions for the problem. - The definition of $\partial_{ \pm}$is extended to functions of $\Omega$ (as being the quantity $\vec{n}_{ \pm}(x) \cdot \nabla u(x, y)$ ).

Let $z^{+}(x)$ and $z^{-}(x)$ be two complex functions, defined and analytic on $\mathbb{R}$, satisfying ${ }^{(2)}$

$$
\Re z^{+}(x)>0, \quad \Re z^{-}(x)>0 .
$$

The condition $\Sigma_{i} \cap\{t<0\} \cap \partial \Omega=\varnothing$ implies the relations $\partial_{ \pm} \theta_{i}(0)<0$ (which are fulfilled because $b^{\prime}(0)<0$ and $a^{\prime}(0)>0$ ).

Consider the diffraction of $u_{i}$ by the half space whose boundary is $\widetilde{\Delta}_{ \pm}$and containing the point $(1,0)$. The condition

$$
\partial_{ \pm} \theta_{i}(0)-z^{ \pm}(0) \neq 0
$$

implies that the Dirichlet to Neumann operator $\partial_{ \pm} u-z^{ \pm} \partial_{t} u \rightarrow \partial_{ \pm} u$ is defined, microlocally in a neighborhood of $\left(0,0, \tau,-\tau \partial_{ \pm} \theta_{i}(0)\right) \in T^{*}\left(\widetilde{\Delta}_{ \pm}\right)$. The condition (5) rewrites $\partial_{ \pm} \theta_{i}(x)-z^{ \pm}(x) \neq 0$ at a point $(x, a(x))$ of $\partial \mathcal{O}_{+}=p\left(\Delta_{+}\right)$(respectively $(x, b(x))$ of $\left.\partial \mathcal{O}_{-}=p\left(\Delta_{-}\right)\right)$and it is necessarily fulfilled for any point where $u_{i}$ is an incident wave, that is a point of $p\left(\Delta_{+}\right)$where $\partial_{+} \theta_{i}(x)<0$ or a point of $p\left(\Delta_{-}\right)$where $\partial_{-} \theta_{i}(x)<0$. This is a consequence of $(4)$ and of the inequalities $\partial_{ \pm} \theta_{i}(0)<0$, which ensures that $\Re\left(\partial_{ \pm} \theta_{i}(0)-z^{ \pm}(0)\right)<0$.

Let $u \in H_{\text {loc }}^{1}\left(\mathbb{R}^{2} \times \mathbb{R}_{t}\right)$ be a solution of the wave equation in $\Omega$, equal to $u_{i}$ for all strictly negative $t$ 's, satisfying impedance boundary conditions on each face of the wedge. If it exists, it is the solution of the system (6) (which is the same system as (1.4) of $[\mathbf{2 0}]$ with mixed boundary conditions):

$$
\left\{\begin{array}{l}
\left(\Delta-\partial_{t^{2}}^{2}\right) u=0 \text { in } \Omega \\
\partial_{+} u(x, t)-z^{+}(x) \partial_{t} u(x, a(x), t)=0 \\
\partial_{-} u(x, t)-z^{-}(x) \partial_{t} u(x, b(x), t)=0 \\
\left.u\right|_{t<0}=\left.u_{i}\right|_{t<0} .
\end{array}\right.
$$

To this system, we associate the problem with constant coefficients in the tangent straight wedge $\Omega_{0}$, which is

$$
\left\{\begin{array}{l}
\left(\Delta-\partial_{t^{2}}^{2}\right) u=0 \text { in } \Omega_{0} \\
\partial_{+} u(x, t)-z^{+}(0) \partial_{t} u(x, a x, t)=0 \\
\partial_{-} u(x, t)-z^{-}(0) \partial_{t} u(x, b x, t)=0 \\
\left.u\right|_{t<0}=\left.u_{i}\right|_{t<0} .
\end{array}\right.
$$

In this paper, we prove that the wave $u$, in the neighborhood of $t=x=y=0$, can be written as the sum of two reflected waves (called $u_{e,+}$ and $u_{e,-}$ ) and of a diffracted wave $u_{d}$. The reflected waves $u_{e, \pm}$ are described below and are computed classically.

\footnotetext{
${ }^{(2)}$ Note that the global condition on $\mathbb{R}$ is needed if we intend to construct a solution in $\mathcal{O} \times \mathbb{R}_{t}$, but if we assume the existence of a solution and we want to evaluate the influence of the edge $(x, y)=(0,0)$, this condition is only necessary in a neighborhood of $x=0$, hence at $x=0$ if we are free of the choice of the neighborhood of $t=0$.
} 
The diffracted wave $u_{d}$ is conormal analytic to $\left\{t=\left(x^{2}+y^{2}\right)^{1 / 2}\right\}$, hence there exists a symbol $\sigma_{d}(x, y, \omega)$ such that

$$
u_{d}(x, y, t)=\frac{1}{2 \pi} \int e^{i \omega(t-r)} \sigma_{d}(x, y, \omega) \mathrm{d} \omega
$$

where $\sigma_{d}$ has an asymptotic expansion in inverse powers of $\omega$, of the same type as the one obtained by Gérard and Lebeau in $[\mathbf{2 0}]$ for the diffracted wave associated with a Dirichlet boundary condition. The traces $\left.u\right|_{\Delta_{+}},\left.u\right|_{\Delta_{-}}, \partial_{+} u, \partial_{-} u$ are solution of a pseudodifferential system, which symbol is invertible in an algebra of pseudodifferential operators. This solution is computed explicitly by the inversion of the pseudodifferential system. We then prove the validity of the asymptotic expansion of the solution. The leading order term of the solution of (6) is the leading order term of the solution of the problem (7). This leading order term is computed $([\mathbf{8}],[\mathbf{9}],[\mathbf{3}])$ using Sommerfeld-Maliuzhinetz inversion formula [30], [31], [40].

The main difficulty which arises here is the fact that we have to control the solution at the corner, arising from the fact that the boundary condition is not the Dirichlet boundary condition. Another question one may ask is the problem of the behavior of the trace on the boundary, to be able to compute the Calderòn operators. These questions make the generalization of the techniques of Gérard and Lebeau not straightforward, as we shall see along this text.

\subsection{Rigorous asymptotic behavior of the solution}

We will see in Section 2.1 the conditions (29) such that the problem (7) is a well posed problem. At each point $\left(x_{0}, y_{0}, t_{0}, \xi_{0}, \eta_{0}, \tau_{0}\right)$ such that $x_{0} y_{0} \neq 0$, the condition (4) implies that the problem is a microlocally well posed problem, and the condition (29) implies that the harmonic problem obtained after Fourier transform in time of the diffraction problem is well posed for all $\omega \in \mathbb{C}^{-}=\{\omega \in \mathbb{C}, \Im \omega<0\}$.

We denote by $z$ the function

$$
z=z^{+} 1_{\widetilde{\Delta}_{+}}+z^{-} 1_{\widetilde{\Delta}_{-}} .
$$

Kaminetzki and Keller [23], following the works of Sommerfeld [40], found formally that the harmonic wave diffracted by the wedge was characterized by a term $e^{-i \tau r} /(\tau r)^{1 / 2}$.

Gérard and Lebeau [20] proved for Dirichlet boundary conditions that the front $\Sigma_{d}=\left\{t=\left(x^{2}+y^{2}\right)^{1 / 2}\right\}$ is a subset of the wave front set of the solution $u$. We prove the same result for mixed boundary conditions. Both solutions $u_{e}$ and $u_{d}$ contribute to the symbol on this front. A precise description of the principal symbol of $u_{d}$ and of $u_{e}$ is given in Section 5 . 
0.2.1. Description of $u_{e}$. - Let $u_{r,+}$ be the solution of the wave equation obtained by reflection of $u_{i}$ by $\widetilde{\Delta}_{+}$:

$$
\left\{\begin{array}{l}
\left(\Delta-\partial_{t^{2}}^{2}\right) u_{r,+}(x, y, t)=0, \quad y>a(x) \\
\partial_{+}\left(u_{i}-u_{r,+}\right)(x, t)-z^{+}(x) \partial_{t}\left(u_{i}-u_{r,+}\right)(x, a(x), t)=0
\end{array}\right.
$$

and the wave $u_{r,-}$ diffracted by $\widetilde{\Delta}_{-}$:

$$
\left\{\begin{array}{l}
\left(\Delta-\partial_{t^{2}}^{2}\right) u_{r,-}(x, y, t)=0, \quad y<b(x) \\
\partial_{-}\left(u_{i}-u_{r,-}\right)(x, t)-z^{-}(x) \partial_{t}\left(u_{i}-u_{r,-}\right)(x, b(x), t)=0 .
\end{array}\right.
$$

These two functions are (up to analytic functions $a_{ \pm}(x, y, t)$ ) described by integrals analogous to (1)

$$
u_{r, \pm}(x, y, t)=\frac{1}{2 \pi} \int_{-\infty}^{+\infty} e^{i \tau\left(t-\theta_{ \pm}(x, y)\right)} \sigma_{ \pm}^{z}(x, y, \tau) \mathrm{d} \tau+a_{ \pm}(x, y, t) .
$$

Introduce $\theta_{a}(x)=\theta_{i}(x, a(x))$ and $\theta_{b}(x)=\theta_{i}(x, b(x))$. The functions $\theta_{ \pm}$are the solutions of

$$
\begin{aligned}
& \left(\nabla \theta_{+}\right)^{2}=1, \partial_{+}\left(\theta_{+}+\theta_{i}\right)(0,0)=0, \quad \theta_{+}(x, a(x))=\theta_{a}(x), \\
& \left(\nabla \theta_{-}\right)^{2}=1, \partial_{-}\left(\theta_{-}+\theta_{i}\right)(0,0)=0, \quad \theta_{-}(x, b(x))=\theta_{b}(x),
\end{aligned}
$$

following the notations of $[\mathbf{2 0}]$ p. 370 . This allows to introduce the front of each wave:

$$
\Sigma_{+}=\left\{(t, x, y), t=\theta_{+}(x, y)\right\}, \quad \Sigma_{-}=\left\{(t, x, y), t=\theta_{-}(x, y)\right\} .
$$

The symbols $\sigma_{ \pm}^{z}(x, y, \tau)$ are the solutions of the transport equations of [26] associated with the impedance boundary conditions ${ }^{(3)}$ :

$$
\begin{aligned}
& \partial_{x} \theta_{+} \partial_{x} \sigma_{+}^{z}+\partial_{y} \theta_{+} \partial_{y} \sigma_{+}^{z}+\frac{1}{2} \Delta \theta_{+} \sigma_{+}^{z}+(2 i \tau)^{-1} \Delta \sigma_{+}^{z}=0 \\
& \left(z^{+}(x)-\partial_{+} \theta_{i}(x)\right) \sigma_{+}^{z}(x, a(x), \tau)+\frac{\partial_{+} \sigma_{+}^{z}(x)}{i \tau} \\
& =\left(z^{+}(x)+\partial_{+} \theta_{i}(x)\right) \sigma_{i}(x, a(x), \tau)+\frac{\partial_{+} \sigma_{i}(x)}{i \tau}, \\
& \partial_{x} \theta_{-} \partial_{x} \sigma_{-}^{z}+\partial_{y} \theta_{-} \partial_{y} \sigma_{-}^{z}+\frac{1}{2} \Delta \theta_{-} \sigma_{-}^{z}+(2 i \tau)^{-1} \Delta \sigma_{-}^{z}=0 \\
& \left(z^{-}(x)-\partial_{-} \theta_{i}(x)\right) \sigma_{-}^{z}(x, b(x), \tau)+\frac{\partial_{-} \sigma_{-}^{z}(x)}{i \tau} \\
& =\left(z^{-}(x)+\partial_{-} \theta_{i}(x)\right) \sigma_{i}(x, b(x), \tau)+\frac{\partial_{-} \sigma_{i}(x)}{i \tau} .
\end{aligned}
$$

The relation (5) allows us to solve in the neighborhood of $x=y=t=0$ the system of transport equations (14), (15).

We introduce the functions

$$
u_{ \pm}=u_{i}-u_{r, \pm} \in H_{\mathrm{loc}}^{1}\left(\mathbb{R}^{2} \times \mathbb{R}_{t}\right) .
$$

${ }^{(3)}$ We used the relation $\partial_{+} \theta_{+}(x)=-\partial_{+} \theta_{i}(x)$ and $\partial_{-} \theta_{-}(x)=-\partial_{-} \theta_{i}(x)$ for any point of $\Delta_{+}$or $\Delta_{-}$ assumed to be in the lighted region. 
We denote by $\delta_{+}$and $\delta_{-}$the single-layer distributions on the face $\Delta_{+}$and $\Delta_{-}$. We denote also by $\delta_{+}^{\prime}$ and $\delta_{-}^{\prime}$ the double-layer distributions on the face $\Delta_{+}$and $\Delta_{-}$. The canonical representation of such distributions is given through an orthogonal representation of $T_{(x, a(x))} \mathbb{R}^{2}$ and of $T_{(x, b(x))} \mathbb{R}^{2}$. For this purpose, we introduce the local semi-geodesic transformations $h_{+}$and $h_{-}$from $\left[0, \rho_{0}\right] \times\left[0, \rho_{1}\right]$ to the corresponding neighborhood of the boundary:

$$
\begin{aligned}
& h_{+}(x, n)=(x, a(x))+\frac{n}{\left(1+\left(a^{\prime}(x)\right)^{2}\right)^{1 / 2}}\left(-a^{\prime}(x), 1\right) \\
& h_{-}(x, n)=(x, b(x))+\frac{n}{\left(1+\left(b^{\prime}(x)\right)^{2}\right)^{1 / 2}}\left(b^{\prime}(x),-1\right) .
\end{aligned}
$$

The transformation $h_{+}$is a diffeomorphism of a neighborhood of $\left(x_{0}, 0\right)$ into a neighborhood of $\left(x_{0}, a\left(x_{0}\right)\right)$. Its inverse transformation is denoted by $\rho_{+}$. Let $g \in \mathcal{D}^{\prime}(\mathbb{R})$. The distributions $g \otimes \delta_{+}$and $g \otimes \delta_{-}$are defined by the action on the test function $\phi_{1} \otimes \phi_{1} \in C_{0}^{\infty}\left(\mathbb{R}^{2}\right)$ equal to $\phi_{1}(x) \phi_{2}(n)$ :

$$
\left\{\begin{array}{l}
\left\langle g \otimes \delta_{+}, \phi_{1} \otimes \phi_{2}\right\rangle=\left\langle g,\left(1+\left(a^{\prime}(x)\right)^{2}\right)^{1 / 2} \phi_{1}\right\rangle \phi_{2}(0) \\
\left\langle g \otimes \delta_{-}, \phi_{1} \otimes \phi_{2}\right\rangle=\left\langle g,\left(1+\left(b^{\prime}(x)\right)^{2}\right)^{1 / 2} \phi_{1}\right\rangle \phi_{2}(0) .
\end{array}\right.
$$

In a similar way, we define, for $g \in \mathcal{D}^{\prime}(\mathbb{R})$, the distributions on $\mathbb{R}^{2}$ denoted by $g \otimes \delta_{+}^{\prime}$ and $g \otimes \delta_{-}^{\prime}$ through the relations

$$
\begin{aligned}
& \left\langle g \otimes \delta_{+}^{\prime}, \phi_{1} \otimes \phi_{2}\right\rangle=-\left\langle g,\left(1+\left(a^{\prime}(x)\right)^{2}\right)^{1 / 2} \phi_{1}>\partial_{+} \phi_{2}(0)\right. \\
& \left\langle g \otimes \delta_{-}^{\prime}, \phi_{1} \otimes \phi_{2}\right\rangle=-\left\langle g,\left(1+\left(b^{\prime}(x)\right)^{2}\right)^{1 / 2} \phi_{1}\right\rangle \partial_{-} \phi_{2}(0)
\end{aligned}
$$

This way of defining the two boundary distributions is intrinsic. Note that we choose here a way of orienting the boundary; in the case of $y=a(x)=0$, we verify that

$$
\left\langle g \otimes \delta_{+}^{\prime}, \phi(x, y)\right\rangle=-\int_{\mathbb{R}} g(x) \partial_{y} \phi(x, 0) \mathrm{d} x .
$$

Let $u_{e}$ be the solution in $\mathbb{R}^{2} \times \mathbb{R}$ of

$$
\left\{\begin{array}{c}
\left(\Delta-\partial_{t^{2}}^{2}\right) u_{e}=u_{+}(x, a(x), t) 1_{x>0} \otimes \delta_{+}^{\prime}+u_{-}(x, b(x), t) 1_{x>0} \otimes \delta_{-}^{\prime} \\
\quad+\partial_{+} u_{+}(x, t) 1_{x>0} \otimes \delta_{+}+\partial_{-} u_{-}(x, t) 1_{x>0} \otimes \delta_{-} \\
\left.u_{e}\right|_{t<0}=\left.u_{i}\right|_{t<0} .
\end{array}\right.
$$

Introduce the fundamental solution of $\Delta-\partial_{t^{2}}^{2}$ supported in $t \geqslant 0$, denoted by $\left(\Delta-\partial_{t^{2}}^{2}\right)_{+}^{-1}$. We have

$$
\begin{aligned}
u_{e}=u_{i}+\left(\Delta-\partial_{t^{2}}^{2}\right)_{+}^{-1} & {\left[u_{+}(x, a(x), t) 1_{x>0} \otimes \delta_{+}^{\prime}+u_{-}(x, b(x), t) 1_{x>0} \otimes \delta_{-}^{\prime}\right] } \\
+ & \left(\Delta-\partial_{t^{2}}^{2}\right)_{+}^{-1}\left[\partial_{+} u_{+}(x, t) 1_{x>0} \otimes \delta_{+}+\partial_{-} u_{-}(x, t) 1_{x>0} \otimes \delta_{-}\right]
\end{aligned}
$$


0.2.2. Contribution of the jump at the corner. - For the description of the solution and of $u_{e}$, we introduce the contribution of the jump between $u_{+}(0,0, t)$ and $u_{-}(0,0, t), \theta$ being any function identically equal to 1 in a neighborhood of 0 , say $[-1,1]$, and compactly supported, and $R$ being a small real number

$$
u_{0}(x, y, t)=\left(\Delta-\partial_{t^{2}}^{2}\right)_{+}^{-1}\left[\left(u_{+}(0,0, t)-u_{-}(0,0, t)\right) \theta\left(\frac{x}{R}\right) 1_{x \geqslant 0} \delta_{+}^{\prime}\right] .
$$

We shall see later on that the solution $u$ satisfies $\left.u\right|_{[0, T] \times \partial \mathcal{O}} \in L^{2}\left([0, T], H^{1}(\partial \mathcal{O})\right)$, hence this jump is artificial, because it is introduced by the difference $u_{+}(0,0, t)-$ $u_{-}(0,0, t)$.

From the relation (143) of the Appendix, and the regularity results of Lemma 6.5 of the Appendix, we check that $u_{0}$ is a distribution of $L_{\text {loc }}^{2}$ for which we have the jump formula $\left[\left.u_{0}\right|_{\partial \Omega}\right]=\left(u_{+}(0,0, t)-u_{-}(0,0, t)\right) \theta(x / R) 1_{x \geqslant 0}$. The distribution $u_{e}$, given by the system (18), is associated with a trace on the boundary (defined by $\left(\left.u\right|_{\Delta_{+}},\left.u\right|_{\Delta_{-}}\right)$) which is in $H^{1}\left(\Delta_{+}\right) \otimes H^{1}\left(\Delta_{-}\right)$. The distribution $u_{e}-u_{0}$ writes:

$$
\begin{aligned}
u_{e}-u_{0}=\left(\Delta-\partial_{t^{2}}^{2}\right)_{+}^{-1} & {\left[\left(u_{+}(x, a(x), t)-\left(u_{+}(0,0, t)-u_{-}(0,0, t)\right) \theta\left(\frac{x}{R}\right)\right) \delta_{+}^{\prime}\right.} \\
+ & \left.u_{-}(x, b(x), t) \delta_{-}^{\prime}\right]+\left(\Delta-\partial_{t^{2}}^{2}\right)_{+}^{-1}\left[\partial_{+} u_{+} \delta_{+}+\partial_{-} u_{-} \delta_{-}\right]+u_{i} .
\end{aligned}
$$

We consider the distribution on $\mathbb{R}^{2}$ equal to

$\operatorname{tr}(x, t)=\left(u_{+}(x, a(x), t)-\left(u_{+}(0,0, t)-u_{-}(0,0, t)\right) \theta\left(\frac{x}{R}\right)\right) 1_{x \geqslant 0}+u_{-}(-x, b(-x), t) 1_{x<0}$

The restriction of $t r$ on $\mathbb{R}_{+}^{*} \times \mathbb{R}_{t}$ (respectively on $\mathbb{R}_{-}^{*} \times \mathbb{R}_{t}$ ) is a distribution of $L^{2}\left(\mathbb{R}_{t}, H_{\text {loc }}^{1}\left(\mathbb{R}_{+}^{*}\right)\right.$ ) (respectively of $L^{2}\left(\mathbb{R}_{t}, H_{\text {loc }}^{1}\left(\mathbb{R}_{-}^{*}\right)\right)$ ), and $\operatorname{tr}\left(0_{+}, t\right)=\operatorname{tr}\left(0_{-}, t\right)=$ $u_{-}(0,0, t)$. Hence $\operatorname{tr}(x, t)$ is an element of $L^{2}\left(\mathbb{R}_{t}, H^{1}(\mathbb{R})\right)$. We apply the fundamental solution on a Dirichlet trace which is in $H^{1}(\partial \Omega)$ and a Neumann trace in $L^{2}(\mathbb{R})$. It is again a consequence of Lemma 6.5 of the Appendix and of the regularity of $\left(\Delta-\partial_{t^{2}}^{2}\right)_{+}^{-1}$ that $\widetilde{u}_{e}=u_{e}-u_{0}$ is in $H_{\text {loc }}^{1}(\Omega)$.

The distribution $\widetilde{u}_{e}$ is convenient to take into account the behavior at the corner, but will not be convenient to use the 2-microlocal propagation result, because the trace of the distribution $u_{e}-\widetilde{u}_{e}$ is not $V_{ \pm}$-outgoing according to the definition of Gérard and Lebeau $[\mathbf{2 0}]$.

We introduce $\sigma_{ \pm}(x, \tau)$ such that $u_{+}(x, a(x), t)=(1 / 2 \pi) \int \sigma_{+}(x, \tau) e^{i \tau\left(t-\theta_{a}(x)\right)} \mathrm{d} \tau$ (respectively $\left.u_{-}(x, b(x), t)=(1 / 2 \pi) \int \sigma_{-}(x, \tau) e^{i \tau\left(t-\theta_{b}(x)\right)} \mathrm{d} \tau\right)$.

The jump between $u_{+}(0,0, t)$ and $u_{-}(0,0, t)$ cannot be avoided, because in general we have $\sigma_{+}(0, \tau) \neq \sigma_{-}(0, \tau)$. The traces of $u_{+}$and of $u_{-}$used in what follows are computed in Lemma 5.2 (Section 5.3). The analytic wave front set of $u_{e}$ is included in the conormal set to the smooth part of $\Sigma_{d} \cup \Sigma_{+} \cup \Sigma_{-}$(because $u_{e}$ is conormal analytic to the smooth part of $\Sigma_{d} \cup \Sigma_{+} \cup \Sigma_{-}$). The detailed calculus of the symbols is written in Section 5 . 
0.2.3. Statement of the high frequency result. - Let $\left.\gamma_{+} \in\right] 0, \pi / 2[$ and $\left.\gamma_{-} \in\right]-\pi / 2,0[$ be defined by

$$
a^{\prime}(0)=\tan \gamma_{+}, \quad b^{\prime}(0)=\tan \gamma_{-} .
$$

The angle of the wedge $F$ is $\left.\gamma=\gamma_{+}-\gamma_{-} \in\right] 0, \pi[$.

Let $\theta_{ \pm}$be the Brewster angles of the faces, defined for example by J.M. Bernard in $[\mathbf{5}$, p. $322(2)]$ :

$$
\sin \theta_{ \pm}=z^{ \pm}(0){ }^{(4)}
$$

We get that

$$
\sigma_{ \pm}(0, \tau)=\frac{2 \sin \gamma_{ \pm}}{\sin \theta_{ \pm} \pm \sin \gamma_{ \pm}} \sigma_{i}(0,0, \tau)\left(1+O\left(\frac{1}{\tau}\right)\right) .
$$

Let $\Omega^{\varepsilon}=\mathcal{O}^{\varepsilon} \times \mathbb{R}_{t}=\left[\mathbb{R}^{2}-\left(F \cup\left\{(x, y), \tan \left(\gamma_{-}-\varepsilon\right) x \leqslant y \leqslant \tan \left(\gamma_{+}+\varepsilon\right) x\right\}\right)\right] \times \mathbb{R}_{t}$. We introduce the set $\widetilde{\Omega}^{\varepsilon}=\Omega^{\varepsilon} \cap \Omega^{+, \varepsilon} \cap \Omega^{-, \varepsilon}$, where

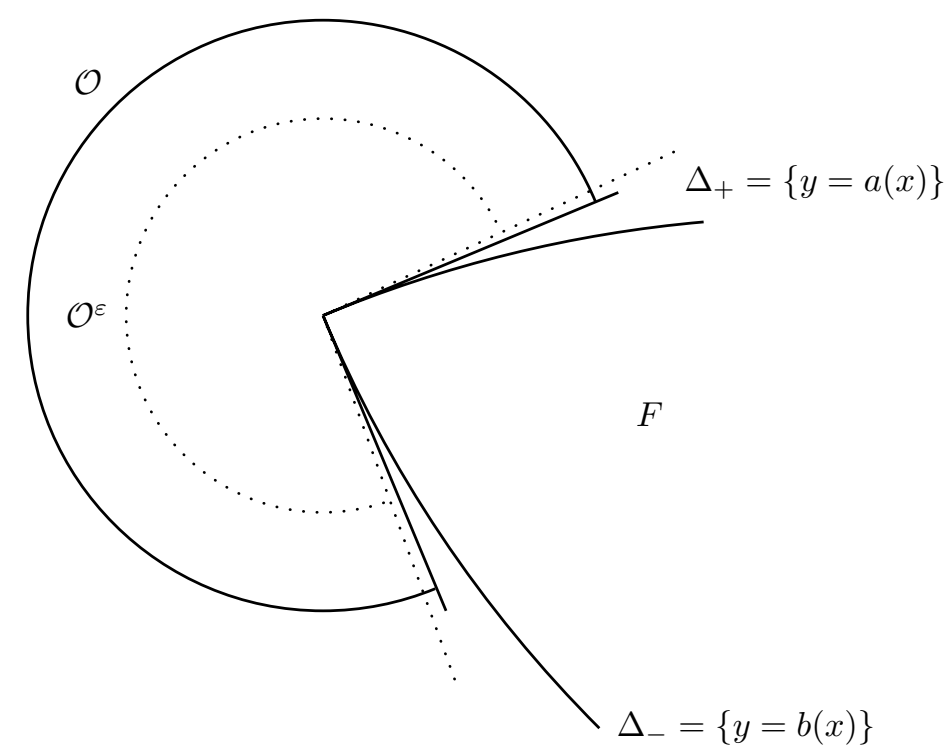

FiguRE 0.3 . Region of validity of the high frequency result

- $\Omega^{ \pm, \varepsilon}=\mathbb{R}^{2} \times \mathbb{R}_{t}$ when $\Im z^{ \pm}(0) \neq 0$

- when $\Im z^{ \pm}(0)=0, \Omega^{ \pm, \varepsilon}$ excludes the sector between the angles $\gamma_{ \pm}-\theta_{ \pm}-\varepsilon$ and $\gamma_{ \pm}-\theta_{ \pm}+\varepsilon$, and the sector $\gamma_{ \pm}+\theta_{ \pm}-\pi-\varepsilon, \gamma_{ \pm}+\theta_{ \pm}-\pi+\varepsilon$

We prove in this paper that the asymptotic expansion of the wave is known in the high frequency regime outside the corner for small times. In other words, we can find

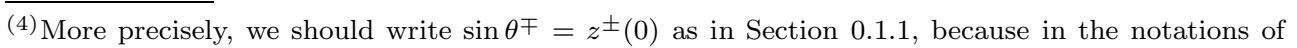
$[8]$, the face - is the face called + here, as was noticed in Section 0.1.1. We drop this notation and adopt the notation (21). 
the analytic wave front set of $u$, and it is the union of the reflected wave front set and of a diffracted wave front set (Corollary 1 ).

A precise description of the symbol of $u$ on each front is available, and this is the aim of the main Theorem:

TheOREM 1. - We assume $\Re z^{+}(0)>0, \Re z^{-}(0)>0$.

- For all $\varepsilon$, there exists $\varepsilon_{1}$ such that, for $0<t<\varepsilon_{1}$ and $(x, y, t) \in \widetilde{\Omega}^{\varepsilon}$

$$
u(x, y, t)=u_{e}(x, y, t)+u_{d}(x, y, t) .
$$

The distribution $u_{d}+u_{0}$ belongs to $H_{\mathrm{loc}}^{3 / 2}\left(\bar{\Omega}^{\varepsilon}\right)$ and is conormal analytic with respect to $t=r$, that is there exists a symbol $\sigma_{d}(x, y, \omega)$ such that

$$
u_{d}(x, y, t)+u_{0}(x, y, t)=\frac{1}{2 \pi} \int_{-\infty}^{+\infty} e^{i \omega(t-r)} \sigma_{d}(x, y, \omega) \mathrm{d} \omega
$$

where the symbol $\sigma_{d}$ satisfies

$$
\sup _{s \geqslant 0} \int_{-\infty}^{+\infty}\left(1+|\tau|^{2}\right)^{3 / 2}\left|\sigma_{d}(x, y, \tau-i s)\right|^{2} \mathrm{~d} \tau<+\infty
$$

- There exists a function $D\left(\theta, \gamma_{+}, \gamma_{-}, \theta_{+}, \theta_{-}\right)$, depending only on $\theta_{+}, \theta_{-}, \gamma_{+}, \gamma_{-}$, and $\theta$ such that the symbol $\sigma_{d}^{0}(x, y, \tau)$

$$
\sigma_{d}^{0}(r \cos \theta, r \sin \theta, \tau)=1_{|\tau| \geqslant 1} \frac{e^{i \pi / 4}}{(2 \pi \tau r)^{1 / 2}} \sigma_{i}(0,0, \tau) D\left(\theta, \gamma_{+}, \gamma_{-}, \theta_{+}, \theta_{-}\right)
$$

satisfies

$$
\sup _{s \geqslant 0} \int_{-\infty}^{+\infty}\left(1+|\tau|^{2}\right)^{5 / 2}\left|\sigma_{d}(x, y, \tau-i s)-\sigma_{d}^{0}(x, y, \tau-i s)\right|^{2} \mathrm{~d} \tau<+\infty .
$$

The wave front set of the solution is thus known:

COROLlary 0.1. - The function $u_{e}$ is conormal analytic to the smooth part of

$$
\Sigma_{i} \cup \Sigma_{r,+} \cup \Sigma_{r,-} \cup \Sigma_{d}
$$

and the function $u_{d}+u_{0} \in H^{3 / 2}\left(\bar{\Omega}^{\varepsilon}\right)$ is conormal analytic to $\Sigma_{d}$.

Using explicit calculations of special functions, J.M. Bernard [5] computed the diffraction coefficient for the diffraction of a plane wave by a straight wedge with constant coefficients mixed boundary conditions. This result gives the coefficient of diffraction $D\left(\theta, \gamma_{+}, \gamma_{-}, \theta_{+}, \theta_{-}\right)$(section 5$)$.

As Gérard and Lebeau did, we transform (6) into a system of two relations on the boundary between distributions constructed from the traces of $u-u_{e}$, and we prove that the leading order term of this system is invertible if and only if the system (7) is a well posed problem (Section 4). We prove that the system (42) (which is the Fourier transform of the system (7) with a Fourier parameter equal to $\tau e^{-i \alpha}$ ) is a well posed system (Proposition 2.1 in Section 4.7). This ends the proof of the validity of 
the computation. We apply the pseudodifferential calculus introduced by Gérard and Lebeau and we deduce the leading term of the wave diffracted by a wedge.

We solved a few technical problems as well as fundamental ones. The key argument which makes this paper work is the fact that $\left.u\right|_{\partial \Omega} \in L^{2}\left(\mathbb{R}_{t}, H^{1}(\partial \mathcal{O})\right)$. This is achieved by proving that the trace of $u$ on each face is in $H^{1}\left(\widetilde{\Delta}_{ \pm}\right)$and that $\left.u\right|_{\partial \mathcal{O}}$ belongs to $H^{1 / 2}(\partial \mathcal{O})$ owing to the boundary condition. The most technical problems were caused by the terms coming from the diffracted solution associated with the distribution $\left(\Delta-\partial_{t^{2}}^{2}\right)_{+}^{-1}\left(g \delta_{\partial \mathcal{O}}^{\prime}\right)$, which normal derivative belongs to $L^{2}(\partial \mathcal{O})$ when $g \in H^{1}(\partial \mathcal{O})$, and is no longer in $L^{2}(\partial \mathcal{O})$ when $g \in H^{1}\left(\partial_{+} \mathcal{O}\right) \otimes H^{1}\left(\partial_{-} \mathcal{O}\right)$ and $\left.g(0)\right|_{\partial_{+} \mathcal{O}} \neq\left. g(0)\right|_{\partial_{-} \mathcal{O}}$. This regularity result is a consequence of a precise calculus of the Dirichlet to Neumann operators. However, the Dirichlet to Neumann operators are more complicated than the "Neumann to Neumann" operators $K_{ \pm}^{+}$and $K_{ \pm}^{-}$introduced by Gérard and Lebeau in [20]. These Dirichlet to Neumann operators are a sum of operators, which have the same type of regularity as $K_{ \pm}^{+}$and $K_{ \pm}^{-}$, acting on $g$ and its tangential derivative. Moreover, according to the jump between $\sigma_{+}(0,0, \tau)$ and $\sigma_{-}(0,0, \tau)$, it has been necessary to include the resolution of a Helmholtz problem with a given jump of the trace of $u$ on $\partial \Omega$.

The second important tool is the generalization to the mixed impedance boundary condition of the 2-microlocal propagation result obtained for Dirichlet boundary condition.

The pseudodifferential system we obtain here is complicated, and cannot be inverted in the same simple way of Gérard and Lebeau; in particular the compatibility condition at the corner has to be taken into account. However, we reduce the problem of existence to the computation of the inverse of an operator where this compatibility condition at the corner is absent. 



\section{CHAPTER 1}

\section{EXISTENCE, UNIQUENESS, AND REGULARITY OF THE SOLUTION}

In this chapter, we prove that the problem of diffraction of a $H_{\text {loc }}^{1}$ conormal analytic wave is a well posed problem, through the Hille-Yosida theorem. We show that, under a physical assumption, the system (6) is a good tool to study the diffraction of an electromagnetic wave by a dielectric piecewise analytic object. Finally, we show that the trace $\left.u\right|_{\partial \Omega}$ of the solution of (6) belongs to $H^{1}(\partial \Omega)$ and that the normal derivative $\partial_{n} u$ belongs to $L^{2}(\partial \Omega)$, which enable us to use the techniques of Gérard and Lebeau.

\subsection{Uniqueness and existence of a solution}

Consider three positive real numbers $T, t_{0}, \delta$, with $t_{0}+\delta<T / 3$, and a function $\theta_{0}, C^{\infty}(\mathbb{R})$, compactly supported, with support in $[-T, T]$, identically equal to 1 in $[-T / 2, T / 2]$. We introduce

$$
\begin{aligned}
& \widetilde{u}_{0}(x, y)=\theta_{0}\left(\sqrt{(x+\delta)^{2}+y^{2}}\right) u_{i}(x, y,-\delta), \\
& \widetilde{u}_{1}(x, y)=\theta_{0}\left(\sqrt{(x+\delta)^{2}+y^{2}}\right) \partial_{t} u_{i}(x, y,-\delta) .
\end{aligned}
$$

We consider the problem

$$
\left\{\begin{array}{l}
\left(\Delta-\partial_{t^{2}}^{2}\right) u=0 \\
\partial_{+} u(x, t)-z^{+}(x) \partial_{t} u(x, a(x), t)=0 \\
\partial_{-} u(x, t)-z^{-}(x) \partial_{t} u(x, b(x), t)=0 \\
u(x, y,-\delta)=\widetilde{u}_{0}(x, y) \\
\partial_{t} u(x, y,-\delta)=\widetilde{u}_{1}(x, y) .
\end{array}\right.
$$

We have the proposition

Proposition 1.0. - Under the conditions (4) and the conditions

$$
\Delta u_{i} \in L_{\mathrm{loc}}^{2}\left(\mathbb{R}^{2} \times \mathbb{R}_{t}\right), \quad \partial_{t} u_{i} \in H_{\mathrm{loc}}^{1}\left(\mathbb{R}^{2} \times \mathbb{R}_{t}\right),
$$

the problem (24) is a Cauchy problem with mixed boundary conditions. It admits an unique solution which belongs to

$$
\mathcal{E}=C\left(\mathbb{R}_{+, t}, H^{2}(\mathcal{O}) \cap H^{1}(\mathcal{O})\right) \cap C^{1}\left(\mathbb{R}_{+, t}, H^{1}(\mathcal{O})\right) \cap C^{2}\left(\mathbb{R}_{+, t}, L^{2}(\mathcal{O})\right) .
$$


This proposition proves that the problem (6) admits an unique solution, if it exists. This does not prove the existence of a solution, because we have not enough regularity conditions on the initial data to ensure the application of the Hille-Yosida theorem. However, if we consider a function $\phi_{\delta / 2}$, regularization in time, compactly supported for $-\delta / 2 \leqslant t \leqslant \delta / 2$, and if we consider the initial data $\widetilde{u}_{\delta}=\theta_{0}\left(\sqrt{(x+\delta)^{2}+y^{2}}\right) \phi_{\delta / 2} \star$ $u_{i}$, the solution for $t \geqslant 0$ of the problem (24) with initial data $\left.u\right|_{t<-\delta}=\left.\widetilde{u}_{\delta}\right|_{t<-\delta}$, which exists because the initial data are regular enough in time, is equal to $\phi_{\delta / 2} \star u$, where $u$ is the solution, if it exists, of (6).

Now consider $u_{i}(x, y,-\delta)-\widetilde{u}_{0}(x, y)$ and $\partial_{t} u_{i}(x, y,-\delta)-\widetilde{u}_{1}(x, y)$. The support of these two distributions is contained in the intersection of the half space whose boundary is $\theta_{i}(x, y)=-\delta$ which does not contain $(0,0)$ and the complementary of the disk of radius $T / 2$ passing through the point $(-\delta, 0)$.

Note that $u_{i}$ is a solution of the d'Alembert equation, thus we know the contribution at infinity. Let us define the distribution

$$
\begin{aligned}
S_{i}(x, y, t) & =u_{i}(x, y, t)\left[\theta_{0}^{\prime \prime}\left(\sqrt{(x+\delta)^{2}+y^{2}}\right)+\frac{\theta_{0}^{\prime}\left(\sqrt{(x+\delta)^{2}+y^{2}}\right)}{\sqrt{(x+\delta)^{2}+y^{2}}}\right] \\
& +2 \theta_{0}^{\prime}\left(\sqrt{(x+\delta)^{2}+y^{2}}\right)\left[\partial_{x} u_{i} \frac{x+\delta}{\sqrt{(x+\delta)^{2}+y^{2}}}+\partial_{y} u_{i} \frac{y}{\sqrt{(x+\delta)^{2}+y^{2}}}\right]
\end{aligned}
$$

which is in $L^{2}\left(\mathbb{R}^{2} \times \mathbb{R}_{t}\right)$, according to the fact that $\theta^{\prime}$ and $\theta^{\prime \prime}$ are compactly supported and $u_{i} \in H_{\text {loc }}^{1}\left(\mathbb{R}^{2} \times \mathbb{R}_{t}\right)$. We introduce the distribution $h$ solution of

$$
\left\{\begin{array}{l}
\left(\Delta-\partial_{t^{2}}^{2}\right) h(x, y, t)=S_{i}(x, y, t) \\
\left.h\right|_{t=-\delta}=0 \\
\left.\partial_{t} h\right|_{t=-\delta}=0
\end{array}\right.
$$

This distribution exists (see Wilcox [41]) because $S_{i}$ belongs to $L^{2}\left(\mathbb{R}^{2} \times \mathbb{R}_{t}\right)$. Hence $w=h+u_{i}\left(1-\theta\left(\sqrt{(x+\delta)^{2}+y^{2}}\right)\right)$ is a distribution in $\left.\left.H_{\mathrm{loc}}^{1}\left(\mathbb{R}^{2} \times\right]-\infty, t_{0}\right]\right)$ solution of

$$
\left\{\begin{array}{l}
\left(\Delta-\partial_{t^{2}}^{2}\right) w=0 \\
w(x, y,-\delta)=u_{i}(x, y,-\delta)\left(1-\theta\left(\sqrt{(x+\delta)^{2}+y^{2}}\right)\right) \\
\partial_{t} w(x, y,-\delta)=\partial_{t} u_{i}(x, y,-\delta)\left(1-\theta\left(\sqrt{(x+\delta)^{2}+y^{2}}\right)\right)
\end{array}\right.
$$

Then $w+v$ is solution of (6) for $t \leqslant t_{0}$. The uniqueness of $w+v$ is not obvious with this method, because $w$ and $v$ depend on the truncation function. However, given a $T$ and a truncation $\theta$, the distributions $w$ and $v$ are unique. The solution of $(6)$ for $t \leqslant t_{0}$ is the superposition of the solution $v$ of (24) and of the solution of a free propagation problem in $\left.\left.\mathbb{R}^{2} \times\right]-\infty, t_{0}\right]$, with initial conditions $v(x, t,-\delta)=$ $u_{i}(x, y,-\delta)-\widetilde{u}_{0}(x, y), \partial_{t} v(x, y,-\delta)=\partial_{t} u_{i}(x, y,-\delta)-\widetilde{u}_{1}(x, y)$. As $t_{0}+\delta<T / 3, v$ is identically 0 in $B(0, T / 3) \times\left[-\delta, t_{0}\right]$ (see Figure 1.1). 


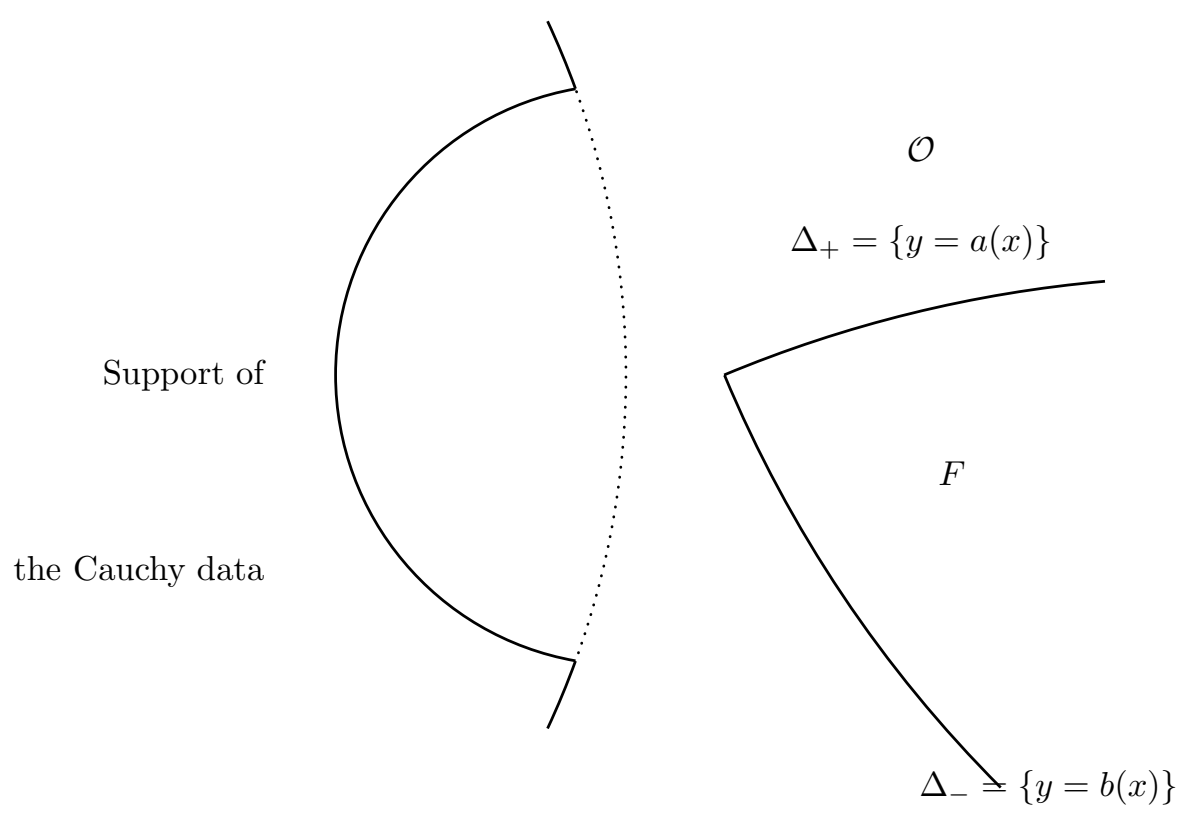

Figure 1.1. Support of the wave $w$

\subsection{The application to the system of Maxwell equations}

The situation described above is adapted for the high frequency scattering of an electromagnetic wave by a wedge in $\mathbb{R}^{3}$, if we assume that the wedge is $F \times \mathbb{R}_{z}$ and that the wave front set of the incident electromagnetic field is the surface

$$
\Sigma_{i}=\left\{t=\theta_{i}(x, y), z \in \mathbb{R}\right\} \subset \mathbb{R}^{4},
$$

and that this incident field, solution of the Maxwell equations in a neighborhood $W$ of $(0,0,0,0)$, is conormal analytic to $\Sigma_{i}$ in $W$, supported in the part of $\mathbb{R}^{4}$ characterized by $t \geqslant \theta_{i}(x, y)$ :

$$
\begin{aligned}
\vec{E}_{i}(x, y, z, t) & =\frac{1}{2 \pi} \int e^{i \tau\left(t-\theta_{i}(x, y)\right)} \vec{e}_{i}(x, y, \tau) \mathrm{d} \tau, \\
\vec{H}_{i}(x, y, z, t) & =\frac{1}{2 \pi} \int e^{i \tau\left(t-\theta_{i}(x, y)\right)} \vec{h}_{i}(x, y, \tau) \mathrm{d} \tau .
\end{aligned}
$$

We omit from now the dependency in $z$. The boundary condition we assume on each face of the wedge is computed using [27], and we make the following physical assumption (which is not true for the case of a wedge filled with dielectric material because the Calderón operator is well defined on $H^{-1 / 2}(\operatorname{div}, \Gamma)$, see Cessenat [13]). 


\section{Hypothesis 1.1}

(H) The boundary condition on $\partial \Omega \times \mathbb{R}_{z}$ is the trace of the boundary condition on each face $\partial \mathcal{O}_{+} \times \mathbb{R}_{t} \times \mathbb{R}_{z}$ and $\partial \mathcal{O}_{-} \times \mathbb{R}_{t} \times \mathbb{R}_{z}$ induced by the incident electromagnetic wave.

The Maxwell equations inside the dielectric write

$$
\left\{\begin{array}{l}
\operatorname{rot} \vec{E}+\mu \partial_{t} \vec{H}=0 \\
\operatorname{rot} \vec{H}-\varepsilon \partial_{t} \vec{E}=0
\end{array}\right.
$$

Let $Z$ be the pseudodifferential matrix operator taking into account the boundary condition. When the time dependency is $e^{-i \tau t}$, recall that the boundary condition induced by a material of dielectric constants $\varepsilon, \mu, \Re(\varepsilon \mu)>0, \Im(\varepsilon \mu)<0$ contained in $\{(x, y, z), y<a(x), x \in \mathbb{R}, z \in \mathbb{R}\}$ is given by the relation

$$
\vec{n}_{+} \wedge \vec{E}=\mathrm{Op}(Z(x, a(x), \eta, \tau))\left[\vec{n}_{+} \wedge\left(\vec{n}_{+} \wedge \vec{H}\right)\right]
$$

where

$$
\vec{n}_{+}=\frac{1}{\left(1+\left(a^{\prime}(x)\right)^{2}\right)^{1 / 2}}\left(-a^{\prime}(x), 1,0\right) \quad \text { and } \quad-\eta=\nabla \theta_{i}(x, a(x))-\partial_{+} \theta_{i}(x) \vec{n}_{+}(x) .
$$

We introduce $\nu_{+}(x)=\left(\varepsilon \mu-\eta^{2}\right)^{1 / 2}, \Im \nu_{+}(x)>0$, that is

$$
\nu_{+}(x)=\left(\varepsilon \mu-1+\left(\partial_{+} \theta_{i}(x)\right)^{2}\right)^{1 / 2} .
$$

In a similar fashion, we define $\nu_{-}(x)=\left(\varepsilon \mu-1+\left(\partial_{-} \theta_{i}(x)\right)^{2}\right)^{1 / 2}$. The eigenvalues of $Z_{0}$, leading order term of $Z$, are $\mu / \nu_{+}(x)$ for the eigenvector $\nabla \theta_{i}(x, a(x))-$ $\partial_{+} \theta_{i}(x) \vec{n}_{+}(x)$ and $\nu_{+}(x) / \varepsilon$ for the eigenvector $\vec{e}_{3}$, because the tangent space to the boundary is spanned by these two vectors and $\vec{e}_{3}=\left(\nabla \theta_{i}(x, a(x))-\partial_{+} \theta_{i}(x) \vec{n}_{+}(x)\right) \wedge \vec{n}_{+}$. The application of $Z$ to a function characterized by a phase function of the form $e^{-i \frac{\omega}{c} \phi(x, z)}$ (which is defined on the boundary) is given by the leading order term of $Z$ computed at the point $\eta=-\nabla \phi$ through the application of the stationary phase lemma.

As we assume the behavior in time of the solution to be characterized by $e^{i \tau t}$, we get $-\nu_{+}(x)$ in the eigenvalues, because we need to have the decay in the obstacle of the form $e^{-i \tau s \nu_{+}(x) l-i \tau \theta_{i}(x, a(x))}$. When $l \rightarrow+\infty$, this factor goes to 0 if $\Re\left(-i \tau s \nu_{+}(x) l\right)<$ 0 , which implies, for $\tau>0$, the choice $s=-1$.

We consider only the leading order term of the boundary condition, and we get

$$
\begin{aligned}
Z_{0}\left[\vec{n}_{+} \wedge\left(\vec{n}_{+} \wedge \vec{H}\right)\right]= & \frac{\mu}{\nu_{+}(x)} \frac{\left(H_{1}+a^{\prime}(x) H_{2}\right)(x, a(x), t)}{\left(1+\left(a^{\prime}(x)\right)^{2}\right)^{1 / 2}}\left(\begin{array}{c}
\frac{1}{\left(1+\left(a^{\prime}(x)\right)^{2}\right)^{1 / 2}} \\
\frac{a^{\prime}(x)}{\left(1+\left(a^{\prime}(x)\right)^{2}\right)^{1 / 2}} \\
0
\end{array}\right) \\
& +\frac{\nu_{+}(x)}{\varepsilon} H_{3}(x, a(x), t)\left(\begin{array}{l}
0 \\
0 \\
1
\end{array}\right) .
\end{aligned}
$$


We deduce from this relation the boundary condition induced by the leading order term:

$$
\begin{gathered}
E_{3}(x, a(x), t)=\frac{\mu}{\nu_{+}(x)} \frac{H_{1}(x, a(x), t)+a^{\prime}(x) H_{2}(x, a(x), t)}{\left(1+\left(a^{\prime}(x)\right)^{2}\right)^{1 / 2}} \\
\frac{a^{\prime}(x) E_{2}(x, a(x), t)+E_{1}(x, a(x), t)}{\left(1+\left(a^{\prime}(x)\right)^{2}\right)^{1 / 2}}=-\frac{\nu_{+}(x)}{\varepsilon} H_{3}(x, a(x), t) .
\end{gathered}
$$

We use the Maxwell equations in the free space

$$
\operatorname{rot} \vec{E}+\mu_{0} \partial_{t} \vec{H}=0, \quad \operatorname{rot} \vec{H}-\varepsilon_{0} \partial_{t} \vec{E}=0
$$

and take the derivative in time of the two boundary conditions to obtain

$$
\begin{aligned}
\partial_{t} E_{3}(x, a(x), t) & =\frac{\mu}{\nu_{+}(x) \mu_{0}} \frac{-\partial_{y} E_{3}(x, a(x), t)+a^{\prime}(x) \partial_{x} E_{3}(x, a(x), t)}{\left(1+\left(a^{\prime}(x)\right)^{2}\right)^{1 / 2}} \\
\partial_{t} H_{3}(x, a(x), t) & =-\frac{\varepsilon_{0}}{\nu_{+}(x) \varepsilon} \frac{-a^{\prime}(x) \partial_{x} H_{3}(x, a(x), t)+\partial_{y} H_{3}(x, a(x), t)}{\left(1+\left(a^{\prime}(x)\right)^{2}\right)^{1 / 2}}
\end{aligned}
$$

which writes

$$
\left\{\begin{array}{l}
\partial_{+} H_{3}(x, a(x), t)+\frac{\nu_{+}(x)}{\mu_{r}} \partial_{t} H_{3}(x, a(x), t)=0 \\
\partial_{+} E_{3}(x, a(x), t)+\frac{\nu_{+}(x)}{\varepsilon_{r}} \partial_{t} E_{3}(x, a(x), t)=0
\end{array}\right.
$$

As $\Im \nu_{+}(x)>0, \Re \nu_{+}(x)<0$, we check that

$$
\frac{\nu_{+}(x)}{\mu_{r}}=\left|\mu_{r}\right|^{-2}\left(\Re \nu_{+} \Re \mu_{r}+\Im \nu_{+} \Im \mu_{r}\right)
$$

We add the usual condition $\Re \mu_{r}>0$ to obtain $\Re\left(\nu_{+}(x) / \mu_{r}\right)<0$. Hence this boundary condition is of the type (6) and satisfies the hypotheses of Theorem 1 . The set up of this section is summarized in

LEMMA 1.1. - The solution of the system of Maxwell equations in $\Omega \times \mathbb{R}_{z}$, assuming that the incident electromagnetic wave is of the form (25) and that the boundary condition is deduced from $(H)$ is given when the solution of

$$
\left\{\begin{array} { l } 
{ ( \Delta _ { x , y } - c ^ { - 2 } \partial _ { t ^ { 2 } } ^ { 2 } ) H _ { 3 } = 0 , \Omega \times \mathbb { R } _ { z } } \\
{ \partial _ { \pm } H _ { 3 } + \frac { \nu _ { \pm } ( x ) } { \mu _ { r } } \partial _ { t } H _ { 3 } = 0 , \Delta _ { + } \times \mathbb { R } _ { z } } \\
{ H _ { 3 } | _ { t < - \delta } = H _ { i , 3 } | _ { t , - \delta } }
\end{array} \quad \left\{\begin{array}{l}
\left(\Delta_{x, y}-c^{-2} \partial_{t^{2}}^{2}\right) E_{3}=0, \Omega \times \mathbb{R}_{z} \\
\partial_{ \pm} E_{3}+\frac{\nu_{ \pm}(x)}{\varepsilon_{r}} \partial_{t} E_{3}=0, \Delta_{+} \times \mathbb{R}_{z} \\
\left.E_{3}\right|_{t<-\delta}=\left.E_{i, 3}\right|_{t,-\delta}
\end{array}\right.\right.
$$

is known. Thus we have

$$
\left\{\begin{array}{l}
\mu_{0} \partial_{t} H_{1}=-\partial_{y} E_{3} \\
\mu_{0} \partial_{t} H_{2}=\partial_{x} E_{3} \\
\varepsilon_{0} \partial_{t} E_{1}=\partial_{y} H_{3} \\
\varepsilon_{0} \partial_{t} E_{2}=-\partial_{x} H_{3}
\end{array}\right.
$$

The divergence free condition is fulfilled as soon as the wave $\left(\vec{E}_{i}, \vec{H}_{i}\right)$ satisfies it (it is a Cauchy condition for a first order system of partial differential equations). 
The Theorem 1 above implies the diffraction result for the system written in Lemma 1.1, which gives the electromagnetic field diffracted by a wedge filled with absorbing dielectric material under the hypothesis 1.1. This gives the proof of the hypothesis used by Bernard in $[4]$.

\subsection{Estimates on the boundary $\partial \mathcal{O} \times[0, T]$}

Consider $u$ the solution, if it exists, of (6). We denote by $\underline{u}=u 1_{\Omega}$ the distribution extending $u$ to $\mathbb{R}^{2} \times \mathbb{R}_{t}$. The function $\underline{u}$ belongs to $L_{\text {loc }}^{2}\left(\mathbb{R}^{2} \times \mathbb{R}_{t}\right)$ (unlike in Gérard and Lebeau, because there is a jump of the trace of $u)$. We define $v_{ \pm}=\left.\left(\partial_{ \pm} u\right)\right|_{x>0}$ as distributions in $\mathcal{D}^{\prime}\left(\Delta_{ \pm}\right)$, because $u$ is a solution of the wave equation and the boundary is non characteristic. We have

\section{Proposition 1.1}

(1) There exists a constant $c$ such that $\operatorname{supp}\left(v_{ \pm}\right) \subset\{(t, x), 0 \leqslant x \leqslant c t\}$.

(2) The distributions $v_{ \pm}$belong to $L^{2}\left(\mathbb{R}_{+} \times[0, T]\right)$.

(3) The trace of the solution on the boundary belong to $H^{1}(\partial \Omega)$.

(4) The jump formula is valid:

$$
\left(\Delta-\partial_{t^{2}}^{2}\right) \underline{u}=\left(v_{+} \delta_{+}+v_{-} \delta_{-}\right)+\left(\left.u\right|_{\widetilde{\Delta}_{+}} 1_{x>0} \delta_{+}^{\prime}+\left.u\right|_{\widetilde{\Delta}_{-}} 1_{x>0} \delta_{-}^{\prime}\right) .
$$

1.3.1. Proof of the $L^{2}$ regularity. - The first item of Proposition 1.1 comes from the fact that the distribution $v_{ \pm}$of $\mathcal{D}^{\prime}\left(\Delta_{ \pm}\right)$satisfies $\operatorname{supp} v_{ \pm} \subset\{t \geqslant 0\}$. As we have finite speed of propagation, the solution is 0 for $\left(x^{2}+y^{2}\right)^{1 / 2}>t$, hence $v_{+}$is supported in $\left(x^{2}+(a(x))^{2}\right)^{1 / 2} \leqslant t$ and $v_{-}$is supported in $\left(x^{2}+(b(x))^{2}\right)^{1 / 2} \leqslant t$. We denote by $c^{-1}=\min \left(\left(1+(a(x) / x)^{2}\right)^{1 / 2},\left(1+(b(x) / x)^{2}\right)^{1 / 2}\right)$ and we get the result.

We introduce a function $\chi$ compactly supported in a subset of $\left.B\left(0, r_{0}\right) \times\right]-T, T$, equal to 1 in a neighborhood of $(0,0)$ and $\rho_{\varepsilon}$ the product of $\chi$ with a time regularization $f_{\varepsilon}(t)$. We introduce

$$
u_{\varepsilon}=\rho_{\varepsilon} u=\chi(x, y, t)\left[f_{\varepsilon} \star u\right](x, y, t)=\chi(x, y, t) \int f_{\varepsilon}\left(t^{\prime}\right) u\left(x, y, t-t^{\prime}\right) \mathrm{d} t^{\prime} .
$$

The distribution $u_{\varepsilon}$ is now in $C_{0}^{\infty}(]-T, T\left[, H^{1}(\mathcal{O})\right)$. As $u \in H_{\mathrm{loc}}^{1}(\bar{\Omega}), u_{\varepsilon} \in H^{1}(\bar{\Omega})$, and we have uniformly in $\varepsilon$ the inequality $\left\|u_{\varepsilon}\right\|_{H^{1}(\bar{\Omega})} \leqslant C$ where $C$ is independent of $\varepsilon$. As $u_{\varepsilon}$ is regular in $t, \partial_{t}^{2}\left(\rho_{\varepsilon} u\right)$ is regular, as well as $\left.\partial_{t}\left(\rho_{\varepsilon} u\right)\right|_{\partial \Omega}$. These distributions belong to $H^{1}\left(\mathbb{R}^{2} \times \mathbb{R}\right)$. Note that the norm in $H^{1}\left(\mathbb{R}^{2} \times \mathbb{R}\right)$ of these distributions can depend on $\varepsilon$.

We check that $u_{\varepsilon}$ satisfies

$$
\Delta u_{\varepsilon}=\left[\Delta, \rho_{\varepsilon}\right] u+\rho_{\varepsilon} \partial_{t^{2}}^{2} u
$$

As we have $\rho_{\varepsilon} \partial_{t^{2}}^{2} u=\chi\left(f_{\varepsilon} \star \partial_{t^{2}}^{2} u\right)=\chi \partial_{t^{2}}^{2}\left(f_{\varepsilon} \star u\right)$, where $f_{\varepsilon} \star u$ belongs to $C_{0}^{\infty}(]-T, T\left[, H_{\text {loc }}^{1}(\mathcal{O})\right)$, we get $\rho_{\varepsilon} \partial_{t^{2}}^{2} u \in H^{1}$. Thus $\Delta u_{\varepsilon} \in L^{2}(\Omega)$. From the results of Grisvard [21] (see Section 6.1.2), we can define in $H^{-1 / 2}(\partial \Omega)$ the normal derivative 
of $u_{\varepsilon}$, because $\Delta u_{\varepsilon} \in L^{2}(\Omega)$ and $u_{\varepsilon} \in H^{1}(\Omega)$. The normal derivative is defined through the equality, for $v \in H^{1}(\Omega)^{(1)}$ :

$$
\left\langle\partial_{n} u_{\varepsilon},\left.v\right|_{\partial \Omega}\right\rangle_{H^{-1 / 2}, H^{1 / 2}}=\left\langle\Delta u_{\varepsilon}, v\right\rangle_{L^{2}(\Omega)}+\left\langle\nabla u_{\varepsilon}, \nabla v\right\rangle_{L^{2}(\Omega)} .
$$

From the results of Dauge and Azaiez [2], and Costabel and Dauge [15], as $\gamma \in] 0, \pi[$, there exists $\varepsilon^{\prime \prime}>0$ such that $u_{\varepsilon} \in H^{\frac{3}{2}+\varepsilon^{\prime \prime}}$ because

$$
u_{\varepsilon} \in L^{2}\left(\mathbb{R}, H^{1+\frac{\pi}{2 \pi-\gamma}-\varepsilon^{\prime}}(\mathcal{O})\right), \quad \forall \varepsilon^{\prime}>0 .
$$

We note that this implies in particular $\partial_{n} u_{\varepsilon} \in L^{2}(\partial \Omega)$ (with the norm possibly depending on $\varepsilon$ ) as well as $\partial_{t} u_{\varepsilon} \in L^{2}(\partial \Omega)$. The aim of the first part of this proof is to show that the norm of $\partial_{n} u_{\varepsilon}$ in $L^{2}(\partial \Omega)$ is independent of $\varepsilon$. For these distributions, we have $\left\langle\partial_{n} u_{\varepsilon},\left.\partial_{t} u_{\varepsilon}\right|_{\partial \Omega}\right\rangle=\left(\partial_{n} u_{\varepsilon},\left.\partial_{t} u_{\varepsilon}\right|_{\partial \Omega}\right)$.

Introduce

$$
a(\varepsilon)=\left(\left(\Delta-\partial_{t^{2}}^{2}\right) u_{\varepsilon}, \partial_{t} u_{\varepsilon}\right)=\left(\left[\Delta-\partial_{t^{2}}^{2}, \rho_{\varepsilon}\right] u, \partial_{t} u_{\varepsilon}\right) .
$$

The operator $\left[\Delta-\partial_{t^{2}}^{2}, \rho_{\varepsilon}\right]$ is of order $2+0-1=1$, bounded uniformly in $\varepsilon$ (because the family of operators of order $0 \rho_{\varepsilon}$ is uniformly bounded in $\varepsilon$ ). Hence $\left\|\left[\Delta-\partial_{t^{2}}^{2}, \rho_{\varepsilon}\right] u\right\|_{L^{2}(\Omega)} \leqslant C_{1}$. We have of course $\left\|\partial_{t} u_{\varepsilon}\right\|_{L^{2}(\Omega)} \leqslant C$. Hence the term $a(\varepsilon)$ is bounded uniformly in $\varepsilon$.

It is also a consequence of (26) that

$$
\left\langle\partial_{n} u_{\varepsilon},\left.v\right|_{\partial \Omega}\right\rangle_{H^{-1 / 2}, H^{1 / 2}}=\left\langle\left[\Delta-\partial_{t^{2}}^{2}, \rho_{\varepsilon}\right] u, v\right\rangle+\left\langle\nabla u_{\varepsilon}, \nabla v\right\rangle-\left\langle\partial_{t} u_{\varepsilon}, \partial_{t} v\right\rangle
$$

which implies the inequality

$$
\left|\left\langle\partial_{n} u_{\varepsilon},\left.v\right|_{\partial \Omega}\right\rangle\right| \leqslant\left(\left\|\left[\Delta-\partial_{t^{2}}^{2}, \rho_{\varepsilon}\right]\right\|_{L^{2}(\Omega)}+\left\|\nabla u_{\varepsilon}\right\|_{L^{2}(\Omega)}+\left\|\partial_{t} u_{\varepsilon}\right\|_{L^{2}(\Omega)}\right)\|v\|_{H^{1}(\Omega)} .
$$

Hence there exists a constant $D$, independent of $\varepsilon$, such that, with a good choice of $v \in H^{1}(\Omega)$, of given trace $\left.v\right|_{\partial \Omega} \in H^{1 / 2}(\partial \Omega)$ (we know from Grisvard [21] that the lifting lemma is valid from $H^{1 / 2}(\partial \Omega)$ to $H^{1}(\Omega)$ for a polyhedral boundary)

$$
\left|\left\langle\partial_{n} u_{\varepsilon},\left.v\right|_{\partial \Omega}\right\rangle\right| \leqslant D\left\|\left.v\right|_{\partial \Omega}\right\|_{H^{1 / 2}(\partial \Omega)} .
$$

The norm of $\partial_{n} u_{\varepsilon}$ in $H^{-1 / 2}(\partial \Omega)$ is bounded independently of $\varepsilon$ by $D$.

Note now that $\left(-\partial_{t^{2}}^{2} u_{\varepsilon}, \partial_{t} u_{\varepsilon}\right)=0$, because $\partial_{t} u_{\varepsilon}$ is in $C_{0}^{\infty}(]-T, T\left[, H^{1}(\mathcal{O})\right)$. We deduce the equality

$$
\begin{aligned}
a(\varepsilon)=\left(\Delta u_{\varepsilon}, \partial_{t} u_{\varepsilon}\right) & =-\left(\nabla u_{\varepsilon}, \nabla \partial_{t} u_{\varepsilon}\right)+\left\langle\partial_{n} u_{\varepsilon},\left.\partial_{t} u_{\varepsilon}\right|_{\partial \Omega}\right\rangle \\
& =-\left(\nabla u_{\varepsilon}, \nabla \partial_{t} u_{\varepsilon}\right)+\left(\partial_{n} u_{\varepsilon},\left.\partial_{t} u_{\varepsilon}\right|_{\partial \Omega}\right)
\end{aligned}
$$

We check that $\int_{\Omega} \nabla u_{\varepsilon} \nabla \partial_{t} u_{\varepsilon} \mathrm{d} x \mathrm{~d} y \mathrm{~d} t=\frac{1}{2} \int_{\mathbb{R}} \mathrm{d} t \partial_{t}\left(\int_{\mathcal{O}}\left|\nabla u_{\varepsilon}\right|^{2} \mathrm{~d} x d y\right)=0$. Hence $\left(\Delta u_{\varepsilon}, \partial_{t} u_{\varepsilon}\right)=\left(\partial_{n} u_{\varepsilon},\left.\partial_{t} u_{\varepsilon}\right|_{\partial \Omega}\right)$.

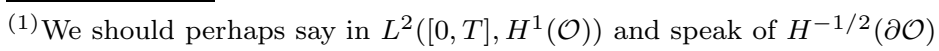


Let $n_{+}(x)=\left(1+\left(a^{\prime}(x)\right)^{2}\right)^{-1 / 2}\left(-a^{\prime}(x), 1\right)$ and $n_{-}(x)=\left(1+\left(b^{\prime}(x)\right)^{2}\right)^{-1 / 2}\left(b^{\prime}(x),-1\right)$. We have the equalities

$$
\begin{array}{ll}
\vec{n}_{+} \cdot \nabla u_{\varepsilon} & =\left.\left(\partial_{+} \chi\right)\left(f_{\varepsilon} \star u\right)\right|_{\Delta_{+}}+\chi f_{\varepsilon} \star \partial_{+} u \\
\vec{n}_{-} \cdot \nabla u_{\varepsilon} & =\left.\left(\partial_{-} \chi\right)\left(f_{\varepsilon} \star u\right)\right|_{\Delta_{-}}+\chi f_{\varepsilon} \star \partial_{-} u \\
\left.\partial_{n} u_{\varepsilon}\right|_{\Delta_{+}} & =\left.\left(\left.\partial_{+} \chi\right|_{\Delta_{+}}-z^{+}(x) \partial_{t} \chi\right)\left(f_{\varepsilon} \star u\right)\right|_{\Delta_{+}}+\left.z^{+}(x) \partial_{t} u_{\varepsilon}\right|_{\Delta_{-}} \\
\partial_{n} u_{\varepsilon} \text { vert }_{\Delta_{-}} & =\left.\left(\left.\partial_{-} \chi\right|_{\Delta_{-}}-z^{-}(x) \partial_{t} \chi\right)\left(f_{\varepsilon} \star u\right)\right|_{\Delta_{-}}+\left.z^{-}(x) \partial_{t} u_{\varepsilon}\right|_{\Delta_{-}}
\end{array}
$$

We then get

$$
\begin{array}{ll}
\left.\partial_{t} u_{\varepsilon}\right|_{\Delta_{+}}=\frac{\partial_{n} u_{\varepsilon}+\left.\alpha_{+}(x, t)\left(f_{\varepsilon} \star u\right)\right|_{\Delta_{+}}}{z^{+}(x)}, & \alpha_{+}(x, t)=\left.\partial_{+} \chi\right|_{\Delta_{+}}-\left.z^{+}(x) \partial_{t} \chi\right|_{\Delta_{+}} \\
\left.\partial_{t} u_{\varepsilon}\right|_{\Delta_{-}}=\frac{\partial_{n} u_{\varepsilon}+\left.\alpha_{-}(x, t)\left(f_{\varepsilon} \star u\right)\right|_{\Delta_{-}}}{z^{-}(x)}, & \alpha_{-}(x, t)=\left.\partial_{-} \chi\right|_{\Delta_{-}}-\left.z^{-}(x) \partial_{t} \chi\right|_{\Delta_{-}}
\end{array}
$$

We use now the relation $a(\varepsilon)=\left(\partial_{n} u_{\varepsilon},\left.\partial_{t} u_{\varepsilon}\right|_{\partial \Omega}\right)$ and $|a(\varepsilon)| \leqslant C_{0}, C_{0}$ independent of $\varepsilon$, to get

$$
\left|\left(\partial_{n} u_{\varepsilon}, \partial_{n} u_{\varepsilon} / z\right)+\left(\partial_{n,+} u_{\varepsilon}, r_{+}\right)+\left(\partial_{n,-} u_{\varepsilon}, r_{-}\right)\right| \leqslant C_{0}
$$

where $r_{ \pm}(x, t)=\left.\frac{\alpha_{ \pm}(x, t)}{z^{ \pm}(x)}\left(f_{\varepsilon} \star u\right)\right|_{\Delta_{ \pm}}$.

As $\chi$ is identically equal to 1 in a neighborhood of $(x, y)=(0,0)$, we verify that the two distributions $r_{+}$and $r_{-}$belong to $H_{0}^{1 / 2}\left(\mathbb{R}_{+} \times \mathbb{R}_{t}\right)$, identically 0 in a neighborhood of the corner, and satisfy $\left\|r_{ \pm}\right\|_{H^{1 / 2}\left(\mathbb{R}_{+} \times \mathbb{R}_{t}\right)} \leqslant C_{ \pm}$independent of $\varepsilon$. Hence the distribution $r=r_{+} 1_{\Delta_{+}}+r_{-} 1_{\Delta_{-}}$is in $H^{1 / 2}(\partial \Omega)$, with norm bounded by $C=\max \left(C_{+}, C_{-}\right)$. This implies that

$$
\left(\partial_{n,+} u_{\varepsilon}, r_{+}\right)+\left(\partial_{n,-} u_{\varepsilon}, r_{-}\right)=\left\langle\partial_{n} u_{\varepsilon}, r\right\rangle_{H^{-1 / 2}, H^{1 / 2}} \leqslant C\left\|\partial_{n} u_{\varepsilon}\right\|_{H^{-1 / 2}(\partial \Omega)} .
$$

We deduce the inequality

$$
\left|\left(\partial_{n} u_{\varepsilon}, \partial_{n} u_{\varepsilon} / z\right)\right| \leqslant C_{0}+C\left\|\partial_{n} u_{\varepsilon}\right\|_{H^{-1 / 2}(\partial \Omega)} \leqslant C_{0}+C D .
$$

For $x$ in $\left[-c t_{0}, c t_{0}\right]$, we have $0<c_{0} \leqslant \Re z \leqslant c_{1}$ thus there exists a constant $D_{0}$, independent of $\varepsilon$, such that

$$
\left\|\partial_{n} u_{\varepsilon}\right\|_{L^{2}(\partial \Omega)} \leqslant D_{0} .
$$

The first part of our regularity result is proven. We prove now that $\partial_{\tan } u \in L^{2}(\partial \Omega)$, where the operator $\partial_{\tan }$ is defined on $\Delta_{+}$by $\left.\left(1+\left(a^{\prime}(x)\right)^{2}\right)^{-1 / 2}\left(\partial_{x}+a^{\prime}(x) \partial_{y}\right)\right|_{y=a(x)}$ (and a similar definition on $\Delta_{-}$). For every real vector field $X$ on $\mathbb{R}^{2}$, we can write $X$ on $\partial \mathcal{O}$, almost everywhere, as $X=(X \cdot \vec{n}) \partial_{n}+(X \cdot \vec{t}) \partial_{\tan }$. The following equality is true for $v \in H^{1}(\Omega)$ (using the representation $\left.X=\alpha_{1}(x, y) \partial_{x}+\alpha_{2}(x, y) \partial_{y}\right)$ :

$$
\begin{aligned}
(v, X v)_{L^{2}(\Omega)}= & \int_{\Omega} v\left[\alpha_{1} \partial_{x} \bar{v}+\alpha_{2} \partial_{y} \bar{v}\right] \mathrm{d} x \mathrm{~d} y \mathrm{~d} t \\
= & -\int_{\Omega}\left[\partial_{x}\left(\alpha_{1} v\right) \bar{v}+\partial_{y}\left(\alpha_{2} v\right) \bar{v}\right] \mathrm{d} x \mathrm{~d} t \mathrm{~d} y \\
& +\int_{\partial \Omega}\left[\left.\alpha_{1}\right|_{\partial \Omega} n_{x}+\left.\alpha_{2}\right|_{\partial \Omega} n_{y}\right]|v|^{2} \mathrm{~d} \sigma \mathrm{d} t
\end{aligned}
$$


hence

$$
\Re(v, X v)_{L^{2}(\Omega)}=-\frac{1}{2} \int_{\Omega} \operatorname{div} X|v|^{2}-\frac{1}{2} \int_{\partial \Omega}(X \cdot n)|v|_{\partial \Omega}^{2} \mathrm{~d} \sigma \mathrm{d} t .
$$

We also verify that $a_{0}(\varepsilon)=\Re\left(\left[\partial_{t^{2}}^{2}-\Delta, \rho_{\varepsilon}\right] u, X u_{\varepsilon}\right)$ is uniformly bounded in $\varepsilon$ because $X u_{\varepsilon}$ is uniformly bounded in $L^{2}(\Omega)$ and the operator $\left[\Delta-\partial_{t^{2}}^{2}, \rho_{\varepsilon}\right]$ is bounded uniformly from $H^{1}(\Omega)$ to $L^{2}(\Omega)$. We have the equalities

$$
\begin{aligned}
\Re\left(\left(\partial_{t^{2}}^{2}-\right.\right. & \left.\Delta) u_{\varepsilon}, X u_{\varepsilon}\right)=a_{0}(\varepsilon) \\
& =-\Re\left(\partial_{t} u_{\varepsilon}, X \partial_{t} u_{\varepsilon}\right)-\Re\left(\Delta u_{\varepsilon}, X u_{\varepsilon}\right) \\
& =-\Re\left(\partial_{t} u_{\varepsilon}, X \partial_{t} u_{\varepsilon}\right)+\Re\left(\nabla u_{\varepsilon}, \nabla X u_{\varepsilon}\right)-\Re\left(\partial_{n} u_{\varepsilon}, X u_{\varepsilon}\right) \\
& =-\Re\left(\partial_{t} u_{\varepsilon}, X \partial_{t} u_{\varepsilon}\right)+\Re\left(\nabla u_{\varepsilon}, X \nabla u_{\varepsilon}\right)+\Re\left(\nabla u_{\varepsilon},[X, \nabla] u_{\varepsilon}\right)-\Re\left(\partial_{n} u_{\varepsilon}, X u_{\varepsilon}\right) .
\end{aligned}
$$

If we introduce

$$
\begin{aligned}
a_{1}(\varepsilon) & =\frac{1}{2} \int_{\Omega} \operatorname{div} X\left|\partial_{t} u_{\varepsilon}\right|^{2} \mathrm{~d} x \mathrm{~d} t \mathrm{~d} y, \\
a_{x, y}(\varepsilon) & =\int_{\Omega} \operatorname{div} X\left|\partial_{x, y} u_{\varepsilon}\right|^{2} \mathrm{~d} x \mathrm{~d} t \mathrm{~d} y, \\
a_{2}(\varepsilon) & =\Re\left(\nabla u_{\varepsilon},[X, \nabla] u_{\varepsilon}\right),
\end{aligned}
$$

these four terms are bounded uniformly in $\varepsilon$. As $u_{\varepsilon}$ is at least in $H^{\frac{3}{2}+\varepsilon^{\prime \prime}}(\mathcal{O})$, the traces of $\partial_{t} u_{\varepsilon}, \partial_{x} u_{\varepsilon}, \partial_{y} u_{\varepsilon}$ on the boundary are well defined, and we get

$$
\begin{aligned}
-\frac{1}{2}\left(\partial_{t} u_{\varepsilon},(X \cdot n) \partial_{t} u_{\varepsilon}\right)+\frac{1}{2}\left(\partial_{x} u_{\varepsilon},(X \cdot n) \partial_{x} u_{\varepsilon}\right)+\frac{1}{2} & \left(\partial_{y} u_{\varepsilon},(X \cdot n) \partial_{y} u_{\varepsilon}\right) \\
& =\left(a-a_{1}-a_{2}-a_{x}-a_{y}\right)(\varepsilon) .
\end{aligned}
$$

We rewrite, on the boundary,

$$
\left(\partial_{x} u_{\varepsilon}\right)^{2}+\left.\left(\partial_{y} u_{\varepsilon}\right)^{2}\right|_{\Delta_{ \pm}}=\left(\partial_{ \pm} u_{\varepsilon}\right)^{2}+\left(\partial_{\tan } u_{\varepsilon}\right)^{2} .
$$

There exists a vector field $X$ and a constant $d_{0} \neq 0$ such that $X \cdot n \geqslant d_{0}^{2}>0$ for almost every point in $\partial \Omega$. We proved previously that $\left\|\partial_{t} u_{\varepsilon}\right\|_{L^{2}(\partial \Omega)}$ is bounded uniformly in $\varepsilon$, hence this leads to

$$
\left(\partial_{\tan } u_{\varepsilon}, X \cdot n \partial_{\tan } u_{\varepsilon}\right)_{L^{2}\left(\Delta_{+}\right)}+\left(\partial_{\tan } u_{\varepsilon}, X \cdot n \partial_{\tan } u_{\varepsilon}\right)_{L^{2}\left(\Delta_{-}\right)} \leqslant C^{\prime}
$$

where $C^{\prime}$ is independent of $\varepsilon$. This implies that $\partial_{\tan } u_{\varepsilon}$ is bounded in $L^{2}\left(\Delta_{ \pm}\right)$, independently of $\varepsilon$. As $\partial_{t} u$ is equal to $\partial_{n} u$ on each face (up to the impedance coefficient), we obtain that $\left.u\right|_{\Delta_{+}} \in H^{1}\left(\Delta_{+}\right)$and $\left.u\right|_{\Delta_{-}} \in H^{1}\left(\Delta_{-}\right)$. As the support of $u$ does not meet the wedge for $t<0$, we verify that $\operatorname{supp}\left(\left.u\right|_{\Delta_{+}}\right) \subset\{t \geqslant 0\}$ and $\operatorname{supp}\left(\left.u\right|_{\Delta_{-}}\right) \subset\{t \geqslant 0\}$. Hence we define, for all $\tau \in \mathbb{C}^{-}$, the partial Fourier transform in time of $\left.u\right|_{\Delta_{+}}$, which is a holomorphic function in $\tau$ with values in $H^{1}\left(\partial \mathcal{O}_{+}\right)$(denoted by $\widehat{u}_{+}(\tau, x)$ ) and $\widehat{u}_{-}(\tau, x)$ in a similar fashion. The distribution $\left.u\right|_{\partial \Omega}$ is supported in $t \geqslant 0$ and belongs to $L^{2}\left(\mathbb{R}, H^{1 / 2}(\partial \mathcal{O})\right)$ (see Simon $[\mathbf{3 7}]$ for this injection), hence its partial Fourier transform in time is holomorphic in $\tau$ on $\mathbb{C}^{-}$with values in $H^{1 / 2}(\mathcal{O})$. From the fact that $\widehat{u}_{ \pm} \in H^{1}\left(\partial \mathcal{O}_{ \pm}\right)$, we deduce the existence of $u_{+}(\tau)=\lim _{x \rightarrow 0} \widehat{u}(x, a(x), \tau)$ and of $u_{-}(\tau)=\lim _{x \rightarrow 0} \widehat{u}(x, b(x), \tau)$. 
The last step here is to use Theorem 1.5.2.3 of [21]: the mapping from $H^{1}(\mathcal{O})$ to $H^{1 / 2}\left(\partial \mathcal{O}_{+}\right) \times H^{1 / 2}\left(\partial \mathcal{O}_{-}\right)$with the additional condition

$$
\int_{0}^{\delta}|u|_{\partial \mathcal{O}_{+}}(x(s))-\left.\left.u\right|_{\partial \mathcal{O}_{-}}(x(-s))\right|^{2} \frac{d s}{s}<+\infty,
$$

where $x(s)(0 \leqslant s \leqslant \delta)$ parameterizes $\partial \mathcal{O}_{+}$and $x(-s)(0 \leqslant s \leqslant \delta)$ parameterizes $\partial \mathcal{O}_{-}$, is continuous. Hence we obtain the necessary relation $u_{+}(\tau)=u_{-}(\tau)$ for all $\tau \in \mathbb{C}^{-}$(equality between holomorphic functions) ${ }^{(2)}$. The distribution $U(s, \tau)=$ $u_{+}(x(s), \tau) 1_{s \geqslant 0}+u_{-}(x(-s)) 1_{s>0}$ is thus, for all $\tau \in \mathbb{C}^{-}$, in $H^{1}(\mathbb{R})$ which means that $\widehat{u}(x, \tau) \in H^{1}(\partial \mathcal{O})$ and is holomorphic in $\tau \in \mathbb{C}^{-}$, because there is no discontinuity at $x=0{ }^{(3)}$.

The distribution $w(x, t)=\left.u\right|_{\Delta_{+}}(x, t) 1_{x>0}+\left.u\right|_{\Delta_{-}}(-x, t) 1_{x<0}$ satisfies $\left.\partial_{t} u\right|_{\partial \Omega}$ equal to $\left(z^{ \pm}\right)^{-1} \partial_{n} u$ on $\Delta_{ \pm}$hence $\partial_{t} w \in L^{2}\left(\mathbb{R} \times \mathbb{R}_{t}\right)$ and $w \in L^{2}\left(\mathbb{R}_{t}, H^{1}(\mathbb{R})\right)$, supported for $t \geqslant 0$. Along with $\left.u\right|_{\partial \Omega} \in L^{2}\left(\mathbb{R}, H^{1 / 2}(\partial \mathcal{O})\right)$, we obtain $\left.u\right|_{\partial \Omega} \in H^{1}(\partial \Omega)$. Additionally, $\left.u\right|_{S} \in L^{2}(\mathbb{R})$ with support in $t \geqslant 0$.

Recall the usual jump formula when $\Omega$ is an open set of regular boundary. The exterior normal to $\Omega$ is called $\vec{n}$. Then

$$
\Delta\left(u 1_{\Omega}\right)=(\Delta u) 1_{\Omega}+\left[\partial_{n} u\right] \delta_{\partial \Omega}+[u] \delta_{\partial \Omega}^{\prime} .
$$

We have to give a precise distribution of $\delta^{\prime}$, which is

$$
\left\langle\left[\partial_{n} u\right] \delta_{\partial \Omega}+[u] \delta_{\partial \Omega}^{\prime}, \phi\right\rangle=\int_{\partial \Omega}\left[\left.\left[\partial_{n} u\right] \phi\right|_{\partial \Omega}-\left.[u](\vec{n} \cdot \nabla \phi)\right|_{\partial \Omega}\right] \mathrm{d} \sigma .
$$

In the case studied here (that is in the neighborhood of any regular point of the boundary), the interior is $\mathcal{O}$ and the wave in it is $u$, the exterior is $F$, the wave in it is 0 , and the exterior normal unit vector to $\mathcal{O}$ is $-\vec{n}_{ \pm}$. Hence $\left[\partial_{n} u\right]=-\vec{n}_{ \pm} \cdot \nabla 0-$ $\left.\left(-\vec{n}_{ \pm} \cdot \nabla u\right)\right]=\partial_{ \pm} u,[u]=[0-u]$, and $\left.(\vec{n} \cdot \nabla \phi)\right|_{\partial \mathcal{O}}=-\partial_{ \pm} u$. Hence the formula $(27)$ leads to $\left(\Omega=\mathcal{O} \times \mathbb{R}_{t}\right)$

$$
\left\langle\Delta\left(u 1_{\Omega}\right), \phi\right\rangle=\int_{\partial \Omega}\left[\left.\partial_{ \pm} u \phi\right|_{\partial \Omega}-\left.u\right|_{\partial \Omega} \partial_{ \pm} \phi\right] \mathrm{d} \sigma .
$$

Hence $\left(\Delta-\partial_{t^{2}}^{2}\right)\left(u 1_{\Omega}\right)-\left.u\right|_{\partial \Omega} \delta_{\partial \Omega}^{\prime}-v_{+} \delta_{\Delta_{+}}-v_{-} \delta_{\Delta_{-}}$, distribution of $H^{-2}(\Omega)$, is supported by $(0,0) \times \mathbb{R}_{+, t}$. It is necessarily of the form $S(t) \delta_{(0,0)}$ with $S \in L^{2}\left(\mathbb{R}_{t}\right)$, supported for $t \geqslant 0$.

Let $\psi$ be any $C^{\infty}(\mathbb{R})$ function, with values in $[0,1]$, identically equal to 1 for $x \leqslant x_{0}$ and 0 for $x \geqslant y_{0}\left(0<x_{0}<y_{0}\right)$. A choice of $\psi$ is done in the next Section. For $\Im \tau<0$,

\footnotetext{
${ }^{(2)}$ Note that, precisely, the equality $u_{+}(t)=u_{-}(t)$ is true in $L^{2}(\mathbb{R})$, and the equality of the Fourier transform is true in $\Im \tau<0$ as usual $L_{\mathrm{loc}}^{\infty}$ functions, because the distributions are supported in $t \geqslant 0$. ${ }^{(3)}$ Note that a function $u$ which is in $H^{1}\left(\mathbb{R}, L^{2}(\mathcal{O})\right)$ is continuous in time, and is well defined in $L^{2}(\mathcal{O})$ for all time $t$. Moreover, as these distributions are in $L^{2}\left(\mathbb{R}, H^{1}(\partial \mathcal{O})\right)$, supported for $t \geqslant 0$, we apply the same remark as above for their partial Fourier transform in time for $\Im \tau<0$. We can thus speak of the value in time of such distributions as well as of the value of the Fourier transform.
} 
we introduce the distributions solution of the Helmholtz equation:

$$
v_{S}(x, y, \tau)=\frac{i}{4} \widehat{S}(\tau) H_{0}^{(1)}(\tau r)
$$

and

we have

$$
\phi_{\widehat{u}(0,0, \tau)}(x, y, \tau)=\left(\Delta+\tau^{2}\right)^{-1}\left[\left.\widehat{u}\right|_{S}(\tau) \psi(|x|) \delta_{\partial \Omega}^{\prime}\right] .
$$

$$
\begin{aligned}
& u 1_{\Omega}-\mathcal{F}^{-1}\left(v_{S}+\phi_{\widehat{u}(0,0, \tau)}\right)(x, y, t) \\
& \quad=\left(\Delta-\partial_{t^{2}}^{2}\right)_{+}^{-1}\left[\left(\left.u\right|_{\partial \Omega}-u(0,0, t) \psi(|x|) \delta_{\partial \Omega}^{\prime}+v_{+} \delta_{\Delta_{+}}+v_{-} \delta_{\Delta_{-}}\right] .\right.
\end{aligned}
$$

The distribution $u 1_{\Omega}$ admits a limit for $(x, y) \in \mathcal{O}$ tending to a point of $\partial \mathcal{O}$ and we proved previously that the trace $\left.u\right|_{\partial \Omega}$ was continuous on the boundary. The distribution $\widehat{\phi}_{u(0,0, t)}(x, y, t)$ is also continuous on the boundary.

From usual results on the boundary outside the corner points, the distribution

$$
\left(\Delta-\partial_{t^{2}}^{2}\right)_{+}^{-1}\left[\left(\left.u\right|_{\partial \Omega}-u(0,0, t) \psi(|x|)\right) \delta_{\partial \Omega}^{\prime}+v_{+} \delta_{\Delta_{+}}+v_{-} \delta_{\Delta_{-}}\right]
$$

is also well defined at the corner. However, owing to the fact that $H_{0}^{(1)}(\tau r)$ is equivalent to $\frac{1}{2 \pi} \log \frac{1}{r}$, the term $\widehat{v}_{S}$ is not continuous on the boundary for $r=0$ if $S \neq 0$. Hence $S=0$ for $\Im \tau<0$. As the distribution $S$ is supported for $t \geqslant 0$, we have $S=0$. This ends the proof of the jump formula. The proof of Proposition 1.1 is complete.

The equality of the fourth item of Proposition 1.1 leads to the following definition, which gives the boundary operators used throughout this paper:

\section{Definition 1.1 (Mirror operators)}

- For $f \in L^{2}\left(\mathbb{R}_{+}\right)$, we define the mirror operators (which are the normal derivatives of the single-layer solutions associated with a density $f$ on the boundary $\Delta_{+}$(for the lower index + ) or on the boundary $\left.\Delta_{-}\right)$:

$$
\begin{aligned}
& K_{ \pm}^{+, k}(f)(x, k)=\psi(x) \partial_{+}\left(\left[\Delta+k^{2}\right]^{-1}\left(1_{x^{\prime} \geqslant 0} f\left(x^{\prime}, k\right) \psi\left(x^{\prime}\right) \delta_{ \pm}\right)\right) \\
& K_{ \pm}^{-, k}(f)(x, k)=\psi(x) \partial_{-}\left(\left[\Delta+k^{2}\right]^{-1}\left(1_{x^{\prime} \geqslant 0} f\left(x^{\prime}, k\right) \psi\left(x^{\prime}\right) \delta_{ \pm}\right)\right)
\end{aligned}
$$

- For $f \in H^{1}\left(\mathbb{R}_{+}\right)$, we define the mirror operators (which are the normal derivatives of the double-layer solutions associated with a density $f$ on the boundary $\Delta_{+}$(for the lower index + ) or on the boundary $\Delta_{-}$)

$$
\begin{aligned}
& R_{ \pm}^{+, k}(f)(x, k)=\psi(x) \partial_{+}\left(\left[\Delta+k^{2}\right]^{-1}\left(1_{x^{\prime} \geqslant 0} f\left(x^{\prime}, k\right) \psi\left(x^{\prime}\right) \delta_{ \pm}^{\prime}\right)\right) \\
& R_{ \pm}^{+, k}(f)(x, k)=\psi(x) \partial_{-}\left(\left[\Delta+k^{2}\right]^{-1}\left(1_{x^{\prime} \geqslant 0} f\left(x^{\prime}, k\right) \psi\left(x^{\prime}\right) \delta_{ \pm}^{\prime}\right)\right) .
\end{aligned}
$$

- Let $U \in H^{1}(\partial \Omega)$. To $U$ is associated an element $f$ of $H^{1}(\mathbb{R})$, which can be split in two elements $f_{+}$and $f_{-}$of $H^{1}\left(\mathbb{R}_{+}\right)$, such that $f_{+}\left(x^{\prime}\right)=f\left(x^{\prime}\right) 1_{x^{\prime} \geqslant 0}$ and $f_{-}\left(x^{\prime}\right)=f\left(-x^{\prime}\right) 1_{x^{\prime} \geqslant 0}$, satisfying the relation $f_{+}(0)=f_{-}(0)$. We define the operators $R^{ \pm, k}$ through

$$
R^{ \pm, k}(f)=R_{+}^{ \pm, k}\left(f_{+}\right)+R_{-}^{ \pm, k}\left(f_{-}\right)
$$


Note that the operators $K_{ \pm}^{+, k}$ and $K_{ \pm}^{-. k}$ are Neumann to Neumann operators (studied by Gérard and Lebeau) and the operators $R_{ \pm}^{+, k}, R_{ \pm}^{-, k}$ are Dirichlet to Neumann operators.

The aim of the sections that follow is to find a pseudodifferential expression for the operators introduced in Definition 1.1 and to compute their asymptotic expansion, in the pseudodifferential calculus written by Gérard and Lebeau in [20]. This will be found in Lemma 4.2 .

In the following section, we consider the operators of Definition 1.1 in the case of a straight wedge; the operators will be denoted by small letters $k, r$ instead of capital letters $K, R$. The upper indices denotes the derivative we choose $\left(\partial_{+}, \partial_{-}\right)$, the lower index denotes the boundary on which the density is defined. 


\section{CHAPTER 2}

\section{DIFFRACTION BY A STRAIGHT WEDGE}

Let $F_{0}=\left\{(x, y) \in \mathbb{R}^{2}, \tan \gamma_{-} x \leqslant y \leqslant \tan \gamma_{+} x, x \geqslant 0\right\}$. Let $\mathcal{O}_{0}=\mathbb{R}^{2}-F_{0}$, $D_{+}=\left\{\left(x, \tan \gamma_{+} x\right) \in \mathbb{R}^{2}, x \geqslant 0\right\}, D_{-}=\left\{\left(x, \tan \gamma_{-} x\right) \in \mathbb{R}^{2}, x \geqslant 0\right\}$. The normal derivatives on $\partial \mathcal{O}_{0}=D_{+} \cup D_{-}$are

$$
\begin{aligned}
& \left.\partial_{n} u\right|_{D_{+}}(x)=-\partial_{+} u(x)=\left(\sin \gamma_{+} \partial_{x} u-\cos \gamma_{+} \partial_{y} u\right)\left(x, \tan \gamma_{+} x\right) \\
& \left.\partial_{n} u\right|_{D_{-}}(x)=-\partial_{-} u(x)=\left(-\sin \gamma_{-} \partial_{x} u+\cos \gamma_{-} \partial_{y} u\right)\left(x, \tan \gamma_{-} x\right) .
\end{aligned}
$$

We verify that

$$
\begin{aligned}
& \bar{F}_{0}=\left\{(r \cos \theta, r \sin \theta), r \in \mathbb{R}_{+}, \theta \in\left[\gamma_{-}, \gamma_{+}\right]\right\} \\
& \mathcal{O}_{0}=\left\{(r \cos \theta, r \sin \theta), r \in \mathbb{R}_{+}^{*}, \theta \in\right] \gamma_{+}, 2 \pi+\gamma_{-}[\} .
\end{aligned}
$$

Using the translation $u(r, \theta) \rightarrow u\left(r, \theta-\gamma_{-}\right)$, we reduce $F_{0}$ to $\mathbb{R}_{+} \times\left[0, \gamma_{+}-\gamma_{-}\right]$, $\gamma_{+}-\gamma_{-}<\pi$ and using the translation $u(r, \theta) \rightarrow u\left(r, \theta-\gamma_{+}\right)$, we see that $\mathcal{O}_{0} \simeq$ $\left.\mathbb{R}_{+}^{*} \times\right] 0,2 \pi-\gamma_{+}+\gamma_{-}\left[, 2 \pi-\gamma_{+}+\gamma_{-}>\pi\right.$.

For simplicity's sake, we shall consider the set $\left.\mathbb{R}_{+}^{*} \times\right] 0, \alpha[$, where $\alpha \in] 0,2 \pi[, \alpha \neq \pi$.

We consider the diffraction problem in $\mathcal{O}_{0}$, the boundary condition on each face of the wedge $\mathcal{O}_{0}$ being $\left.\partial_{n} u\right|_{D_{ \pm}}+\left.\sin \theta_{ \pm} \partial_{t} u\right|_{D_{ \pm}}=0$. In Proposition 2.1, we prove a regularity result under the assumption

$$
\Re \sin \theta_{ \pm}>0, \quad \Im \sin \theta_{+} \times \Im \sin \theta_{-} \geqslant 0 .
$$

\subsection{Existence of the solution of an elliptic problem}

In this Section, we consider $\mathcal{O}=] 0, \beta\left[\times \mathbb{R}_{+}^{*}\right.$ for $0<\beta<2 \pi$. We denote by $E$ the space of distributions $u(r, \theta)$ on $[0, \beta] \times \mathbb{R}_{+}$such that:

$$
E=\left\{u \in C_{\theta}\left([0, \beta] ; H^{1}\left(\mathbb{R}^{+}\right)\right) ; \frac{1}{r} \partial_{\theta} u \in C_{\theta}\left([0, \beta], L^{2}\left(\mathbb{R}^{+}\right)\right) ; u(0,0)=u(0, \beta)\right\} .
$$

For $\chi$ a $C^{\infty}$ function constant in the neighborhood of 0 , we notice (as p. 420 of [20]) that $[\Delta, \chi]$ transforms $E$ into $L^{2}$. Gérard and Lebeau proved in Appendix D of [20], the 
TheOREM 2. - Let $0<\beta<2 \pi$ and $\omega \notin \mathbb{R}$.

(1) The problem $\left(\Delta+\omega^{2}\right) u=0,\left.u\right|_{\partial \mathcal{O}}=f \in H^{1}(\partial \mathcal{O})$ admits an unique solution in $E \cap H^{1}(\mathcal{O})$.

(2) The problem $\left(\Delta+\omega^{2}\right) u=0, \partial_{n} u=g \in L^{2}(\partial \mathcal{O})$ admits an unique solution in $E \cap H^{1}(\mathcal{O})$.

and used the following result, from Grisvard [21]

Lemma 2.1. - Let $0<\beta<2 \pi$ and $\omega \notin \mathbb{R}$.

(1) The problem $\left(\Delta+\omega^{2}\right) u=f \in L^{2}(\mathcal{O}),\left.u\right|_{\partial \mathcal{O}}=0$ admits in $E \cap H^{1}(\mathcal{O})$ an unique solution.

(2) The problem $\left(\Delta+\omega^{2}\right) u=f \in L^{2}(\mathcal{O}), \partial_{n} u=0$ admits in $E \cap H^{1}(\mathcal{O})$ an unique solution.

We easily deduce from these results the existence and uniqueness in $E \cap H^{1}(\mathcal{O})$ of a solution of $\left(\Delta+\omega^{2}\right) u=h, \partial_{n} u=g$ for $h \in L^{2}(\mathcal{O})$ and $g \in L^{2}(\partial \mathcal{O})$.

We prove:

Proposition 2.1. - Let $\omega \in \mathbb{C}-\mathbb{R}$, more precisely $\Re \omega>0$, $\varsigma \omega<0$. Under the condition (29) and the assumptions $g_{ \pm} \in L^{2}\left(\mathbb{R}^{+}\right), h \in L^{2}(\mathcal{O})$, the problem:

$$
\left\{\begin{array}{l}
\left(\Delta+\omega^{2}\right) u=h \\
{\left.\left[i \omega \sin \theta_{-} u+\partial_{n} u\right]\right|_{\theta=0}=g_{-}} \\
{\left.\left[i \omega \sin \theta_{+} u+\partial_{n} u\right]\right|_{\theta=\beta}=g_{+}}
\end{array}\right.
$$

has a unique solution in $E \cap H^{1}$.

We notice that this result is proven if there exists a solution $U$ of the problem (30) which satisfies the relation $\partial_{n} U \in L^{2}(\partial \mathcal{O})$ (that is $r^{-1} \partial_{\theta} U(r, 0) \in L^{2}\left(\mathbb{R}_{+}\right)$and $\left.r^{-1} \partial_{\theta} U(r, \beta) \in L^{2}\left(\mathbb{R}_{+}\right)\right)$. If such a $U$ exists, the problem

$$
\left(\Delta+\omega^{2}\right) V=h, \quad \partial_{n} V=\partial_{n} U
$$

admits an unique solution $V \in E \cap H^{1}(\mathcal{O})$ through Theorem 2 and Lemma 2.1 and the function $w=V-U$ is solution of the problem

$$
\left(\Delta+\omega^{2}\right) w=0, \quad \partial_{n} w=0
$$

which unique solution is 0 (Theorem 2). The function $U$ is thus equal to $V$, hence $U$ is in $E \cap H^{1}(\mathcal{O})$. The Proposition 2.1 is a consequence of

LEMma 2.2. - The problem (30) admits a unique solution in $H^{1}$ for $h \in L^{2}(\mathcal{O})$ and $g_{ \pm} \in L^{2}\left(\mathbb{R}_{+}\right)$. This solution satisfies $\partial_{n} u \in L^{2}(\partial \mathcal{O})$.

The proof of Lemma 2.2 is in the Annex. 


\subsection{The mirror operator for a straight wedge}

Let $\mathbb{C}^{-}=\{k \in \mathbb{C}, \Im k<0\}$. The partial Fourier transform in time of $u(t, x, y) \in$ $\mathcal{D}^{\prime}\left(\mathbb{R} \times \mathbb{R}^{2}\right)$ is denoted by $\widehat{u}(k, x, y)$, and we denote also by $\widehat{u}_{\lambda}(k, x, y)$ the distribution $\widehat{u}(\lambda k, x, y)$.

Form now, we drop the notation $\otimes$ for distributions in $\mathcal{D}^{\prime}\left(\mathbb{R}^{2}\right)$ whenever it is clear that the definition is correct. We define, for $\xi \in \mathbb{R}$ and $k \in \mathbb{C}^{-}$,

$$
\xi_{0}=\left(k^{2}-\xi^{2}\right)^{1 / 2}, \quad \Im \xi_{0}>0 .
$$

Using the notations of Definition 1.1 and the notations for the operators associated with the straight wedge, we have

\section{LEMMA 2.3}

(1) Expression of the single-layer terms (operators computed by Gérard and Lebeau)

- For the regular terms (face + to face + or face - to face -)

$$
\begin{aligned}
& k_{+}^{+, k}(f)(x, k)=\frac{1}{4 \pi \cos \gamma_{+}} \int_{\mathbb{R} \times \mathbb{R}_{+}} \psi(x) \psi\left(x^{\prime}\right) f\left(x^{\prime}\right) e^{i \frac{\left(x-x^{\prime}\right) \xi}{\cos \gamma_{+}}} \mathrm{d} x^{\prime} \mathrm{d} \xi=\frac{1}{2} \psi f(x) \\
& k_{-}^{-, k}(f)(x, k)=\frac{1}{4 \pi \cos \gamma_{-}} \int_{\mathbb{R} \times \mathbb{R}_{+}} \psi(x) \psi\left(x^{\prime}\right) f\left(x^{\prime}\right) e^{i \frac{\left(x-x^{\prime}\right) \xi}{\cos \gamma_{-}}} \mathrm{d} x^{\prime} \mathrm{d} \xi=\frac{1}{2} \psi f(x)
\end{aligned}
$$

- For the singular terms $(\text { face }+ \text { to face }- \text { or face }- \text { to face }+)^{(1)}$

$$
\begin{array}{rl}
1_{x \geqslant 0} k_{-}^{+, k}(f)(x, k)=\frac{1}{4 \pi \cos \gamma_{-}} \iint 1_{x^{\prime} \geqslant 0} 1_{x} \geqslant 0 & f\left(x^{\prime}\right) \psi\left(x^{\prime}\right) \psi(x)\left(\cos \gamma-\frac{\xi}{\xi_{0}} \sin \gamma\right) \\
& \times e^{-i \frac{x^{\prime} \xi}{\cos \gamma} e^{i \frac{x}{\cos \gamma}\left(\cos \gamma \xi+\sin \gamma \xi_{0}\right)} \mathrm{d} x^{\prime} \mathrm{d} \xi} \\
1_{x \geqslant 0} k_{+}^{-, k}(f)(x, k)=\frac{1}{4 \pi \cos \gamma_{+}} \iint 1_{x^{\prime} \geqslant 0} 1_{x} \geqslant 0 & f\left(x^{\prime}\right) \psi\left(x^{\prime}\right) \psi(x)\left(\cos \gamma-\frac{\xi}{\xi_{0}} \sin \gamma\right) \\
& \times e^{-i \frac{x^{\prime} \xi}{\cos \gamma} e^{i \frac{x}{\cos \gamma_{+}}\left(\cos \gamma \xi+\sin \gamma \xi_{0}\right)} \mathrm{d} x^{\prime} \mathrm{d} \xi}
\end{array}
$$

(2) Expression of the double layer operators:

$$
\begin{aligned}
r^{+, k}(g)(x, k) & =\frac{1_{x \geqslant 0} \psi(x)}{4 \pi \cos \gamma_{+}} \int_{\mathbb{R}} i \xi_{0} \mathcal{F}\left(\psi g_{+}\right)\left(\frac{\xi}{\cos \gamma_{+}}\right) e^{i \frac{x \xi}{\cos \gamma_{+}}} \mathrm{d} \xi \\
& -\frac{1_{x \geqslant 0} \psi(x)}{4 \pi \cos \gamma_{-}} \int_{\mathbb{R}} i\left(\cos \gamma \xi_{0}-\sin \gamma \xi\right) \mathcal{F}\left(\psi g_{-}\right)\left(\frac{\xi}{\cos \gamma_{-}}\right) e^{i \frac{x\left(\xi \cos \gamma+\xi_{0} \sin \gamma\right)}{\cos \gamma_{+}}} \mathrm{d} \xi
\end{aligned}
$$

\footnotetext{
(1) Note that these two relations write

$$
1_{x \geqslant 0} k_{-}^{+, k}(f)(x, k)=\frac{\psi(x)}{4 \pi \cos \gamma_{-}} \int e^{i \frac{x}{\cos \gamma_{+}}\left(\xi \cos \gamma+\xi_{0} \sin \gamma\right)}\left(\cos \gamma-\frac{\xi}{\xi_{0}} \sin \gamma\right) \mathcal{F}(\psi f)\left(\frac{\xi}{\cos \gamma_{-}}\right) \mathrm{d} \xi
$$$$
1_{x \geqslant 0} k_{+}^{-, k}(f)(x, k)=\frac{\psi(x)}{4 \pi \cos \gamma_{+}} \int e^{i \frac{x}{\cos \gamma_{-}}\left(\xi \cos \gamma+\xi_{0} \sin \gamma\right)}\left(\cos \gamma-\frac{\xi}{\xi_{0}} \sin \gamma\right) \mathcal{F}(\psi f)\left(\frac{\xi}{\cos \gamma_{+}}\right) \mathrm{d} \xi
$$ 


$$
\begin{array}{r}
r^{-, k}(g)(x, k)=\frac{1_{x \geqslant 0} \psi(x)}{4 \pi \cos \gamma_{+}} \int_{\mathbb{R}} i\left(\cos \gamma \xi_{0}-\sin \gamma \xi\right) \mathcal{F}\left(\psi g_{+}\right)\left(\frac{\xi}{\cos \gamma_{+}}\right) e^{i \frac{x\left(\xi \cos \gamma+\xi_{0} \sin \gamma\right)}{\cos \gamma-}} \mathrm{d} \xi \\
-\frac{1_{x \geqslant 0} \psi(x)}{4 \pi \cos \gamma_{-}} \int_{\mathbb{R}} i \xi_{0} \mathcal{F}\left(\psi g_{-}\right)\left(\frac{\xi}{\cos \gamma_{-}}\right) e^{i \frac{x \xi}{\cos \gamma-}} \mathrm{d} \xi
\end{array}
$$

We identify in these two operators the regular and the singular part. We have the relations

$$
\begin{aligned}
& r_{+}^{+, k}(g)(x, k)=-g_{+}(0) \frac{1}{4 \pi} \int_{\mathbb{R}} \frac{\xi}{\xi_{0}} e^{i \frac{x \xi}{\cos \gamma_{+}}} \mathrm{d} \xi \\
& +\frac{i k^{2} \psi(x)}{4 \pi \cos \gamma_{+}} \int \frac{1}{\xi_{0}} \mathcal{F}\left(\psi g_{+}\right)\left(\frac{\xi}{\cos \gamma_{+}}\right) e^{i \frac{x \xi}{\cos \gamma_{+}}} \mathrm{d} \xi \\
& -\frac{\psi(x)}{4 \pi} \int \frac{\xi}{\xi_{0}} \mathcal{F}\left(\partial_{x^{\prime}}\left(g_{+} \psi\right)\right)\left(\frac{\xi}{\cos \gamma_{+}}\right) e^{i \frac{x \xi}{\cos \gamma_{+}}} \mathrm{d} \xi \\
& r_{-}^{+, k}(g)=g_{-}(0) \frac{\psi(x)}{4 \pi} \int_{\mathbb{R}}\left(\sin \gamma+\frac{\xi}{\xi_{0}} \cos \gamma\right) e^{i \frac{x\left(\xi \cos \gamma+\xi_{0} \sin \gamma\right)}{\cos \gamma_{+}}} \mathrm{d} \xi \\
& -\frac{i k^{2} \psi(x) \cos \gamma}{4 \pi \cos \gamma_{-}} \int_{\mathbb{R}} \frac{1}{\xi_{0}} \mathcal{F}\left(\psi g_{-}\right)\left(\frac{\xi}{\cos \gamma_{-}}\right) e^{i \frac{x\left(\xi \cos \gamma+\xi_{0} \sin \gamma\right)}{\cos \gamma_{+}}} \mathrm{d} \xi \\
& \frac{\psi(x)}{4 \pi} \int_{\mathbb{R}}\left(\sin \gamma+\cos \gamma \frac{\xi}{\xi_{0}}\right) \mathcal{F}\left(\partial_{x^{\prime}}\left(\psi g_{-}\right)\right)\left(\frac{\xi}{\cos \gamma_{-}}\right) e^{i \frac{x\left(\xi \cos \gamma+\xi_{0} \sin \gamma\right)}{\cos \gamma+}} \mathrm{d} \xi \\
& r_{+}^{-, k}(g)=-g_{+}(0) \frac{\psi(x)}{4 \pi} \int_{\mathbb{R}}\left(\sin \gamma+\frac{\xi}{\xi_{0}} \cos \gamma\right) e^{i \frac{x\left(\xi \cos \gamma+\xi_{0} \sin \gamma\right)}{\cos \gamma-}} \mathrm{d} \xi \\
& +\frac{i k^{2} \psi(x) \cos \gamma}{4 \pi \cos \gamma_{+}} \int_{\mathbb{R}} \frac{1}{\xi_{0}} \mathcal{F}\left(\psi g_{+}\right)\left(\frac{\xi}{\cos \gamma_{+}}\right) e^{i \frac{x\left(\xi \cos \gamma+\xi_{0} \sin \gamma\right)}{\cos \gamma_{-}}} \mathrm{d} \xi \\
& -\frac{\psi(x)}{4 \pi} \int_{\mathbb{R}}\left(\sin \gamma+\cos \gamma \frac{\xi}{\xi_{0}}\right) \mathcal{F}\left(\partial_{x^{\prime}}\left(\psi g_{+}\right)\right)\left(\frac{\xi}{\cos \gamma_{+}}\right) e^{i \frac{x\left(\xi \cos \gamma+\xi_{0} \sin \gamma\right)}{\cos \gamma}} \mathrm{d} \xi \\
& r_{-}^{-, k}(g)(x, k)=g_{-}(0) \frac{1}{4 \pi} \int_{\mathbb{R}} \frac{\xi}{\xi_{0}} \cos \gamma_{-} e^{i \frac{x \xi}{\cos \gamma}} \mathrm{d} \xi \\
& -\frac{i k^{2} \psi(x)}{4 \pi \cos \gamma_{-}} \int \frac{1}{\xi_{0}} \mathcal{F}\left(\psi g_{-}\right)\left(\frac{\xi}{\cos \gamma_{-}}\right) e^{i \frac{x \xi}{\cos \gamma_{-}}} \mathrm{d} \xi \\
& +\frac{\psi(x)}{4 \pi} \int \frac{\xi}{\xi_{0}} \mathcal{F}\left(\partial_{x^{\prime}}\left(g_{-} \psi\right)\right)\left(\frac{\xi}{\cos \gamma_{-}}\right) e^{i \frac{x \xi}{\cos \gamma-}} \mathrm{d} \xi
\end{aligned}
$$

Lemma 2.3 is a consequence of Lemma 4.2 for the operators of order 0 and is the result of Proposition 6.2 for the operators of order 1. The proof of these results is written in Section 4.2. The boundary operators associated with the straight wedge are given by the equalities (81), (82), (83), (84) of Lemma 6.5 below (reproduction of the calculus of Gérard and Lebeau) in the case of the operators of type $K$ for $\mu_{+}=a$, $\mu_{-}=b, a(x)=a x, b(x)=b x, a=\tan \gamma_{+}, b=\tan \gamma_{-}$.

The operators $k_{+}^{+, k}$ and $k_{-}^{-, k}$ are (up to the localization $\psi$ ) equal to $\frac{1}{2}$ Id. 
We notice that the distribution $\frac{\psi(x)}{4 \pi} \int_{\mathbb{R}} \frac{\xi}{\xi_{0}} e^{i \frac{x \xi}{\cos \gamma+}} \mathrm{d} \xi$ (and more generally the distributions coefficient of $g_{+}(0)$ and $g_{-}(0)$ for the double-layer operators) are not in $L^{2}(\mathbb{R})$, but belong to $H^{-1}(\mathbb{R})$. Hence it will be useful to consider the operators

$$
\begin{aligned}
& r_{+, 0}^{+, k}(g)(x, k)=+\frac{i k^{2} \psi(x)}{4 \pi \cos \gamma_{+}} \int \frac{1}{\xi_{0}} \mathcal{F}\left(\psi g_{+}\right)\left(\frac{\xi}{\cos \gamma_{+}}\right) e^{i \frac{x \xi}{\cos \gamma_{+}}} \mathrm{d} \xi \\
& \quad-\frac{\psi(x)}{4 \pi} \int \frac{\xi}{\xi_{0}} \mathcal{F}\left(\partial_{x^{\prime}}\left(g_{+} \psi\right)\right)\left(\frac{\xi}{\cos \gamma_{+}}\right) e^{i \frac{x \xi}{\cos \gamma_{+}}} \mathrm{d} \xi \\
& r_{-, 0}^{+, k}(g)=-\frac{i k^{2} \psi(x) \cos \gamma}{4 \pi \cos \gamma-} \int_{\mathbb{R}} \frac{1}{\xi_{0}} \mathcal{F}\left(\psi g_{-}\right)\left(\frac{\xi}{\cos \gamma_{-}}\right) e^{i \frac{x\left(\xi \cos \gamma+\xi_{0} \sin \gamma\right)}{\cos \gamma_{+}}} \mathrm{d} \xi \\
& +\frac{\psi(x) \cos \gamma}{4 \pi} \int_{\mathbb{R}}\left(\sin \gamma+\cos \gamma \frac{\xi}{\xi_{0}}\right) \mathcal{F}\left(\partial_{x^{\prime}}\left(\psi g_{-}\right)\right)\left(\frac{\xi}{\cos \gamma_{-}}\right) e^{i \frac{x\left(\xi \cos \gamma+\xi_{0} \sin \gamma\right)}{\cos \gamma_{+}}} \mathrm{d} \xi \\
& r_{+, 0}^{-, k}(g)=\frac{i k^{2} \psi(x) \cos \gamma}{4 \pi \cos \gamma+} \int_{\mathbb{R}} \frac{1}{\xi_{0}} \mathcal{F}\left(\psi g_{+}\right)\left(\frac{\xi}{\cos \gamma_{+}}\right) e^{i \frac{x\left(\xi \cos \gamma+\xi_{0} \sin \gamma\right)}{\cos \gamma_{-}}} \mathrm{d} \xi \\
& -\frac{\psi(x) \cos \gamma}{4 \pi} \int_{\mathbb{R}}\left(\sin \gamma+\cos \gamma \frac{\xi}{\xi_{0}}\right) \mathcal{F}\left(\partial_{x^{\prime}}\left(\psi g_{+}\right)\right)\left(\frac{\xi}{\cos \gamma_{+}}\right) e^{i \frac{x\left(\xi \cos \gamma+\xi_{0} \sin \gamma\right)}{\cos \gamma_{-}}} \mathrm{d} \xi \\
& r_{-, 0}^{-, k}(g)(x, k)=-\frac{i k^{2} \psi(x)}{4 \pi \cos \gamma} \int \frac{1}{\xi_{0}} \mathcal{F}\left(\psi g_{-}\right)\left(\frac{\xi}{\cos \gamma_{-}}\right) e^{i \frac{x \xi}{\cos \gamma_{-}} \mathrm{d} \xi} \\
& +\frac{\psi(x) \cos \gamma_{+}}{4 \pi} \int \frac{\xi}{\xi_{0}} \mathcal{F}\left(\partial_{x^{\prime}}\left(g_{-} \psi\right)\right)\left(\frac{\xi}{\cos \gamma_{-}}\right) e^{i \frac{x \xi}{\cos \gamma_{-}}} \mathrm{d} \xi
\end{aligned}
$$

proven in Proposition 4.3 to be operators from $H^{1}\left(\mathbb{R}_{+}\right)$to $L^{2}\left(\mathbb{R}_{+}\right)$.

We have the relation, for $k \in \mathbb{C}^{-}$:

$$
\int_{\mathbb{R}}\left(\sin \gamma+\frac{\xi}{\xi_{0}} \cos \gamma\right) e^{i \frac{x}{\cos \gamma_{+}}\left(\xi \cos \gamma+\xi_{0} \sin \gamma\right)} \mathrm{d} \xi=\int_{\mathbb{R}} \frac{\zeta}{\zeta_{0}} e^{i \frac{x \zeta}{\cos \gamma+}} d \zeta .
$$

This is a consequence of the change of variable $\zeta=\xi \cos \gamma+\xi_{0} \sin \gamma$. To prove (32) we must take some care and differentiate between the two cases $\gamma<\pi / 2$ and $\gamma>\pi / 2$. We introduce also $\zeta_{*}=-\xi \sin \gamma+\xi_{0} \cos \gamma$. Simple algebra yields $\left(\zeta_{*}\right)^{2}+\zeta^{2}=k^{2}$, hence $\zeta_{*}$ and $-\zeta_{*}$ are candidates to be equal to the root of positive imaginary part of $k^{2}-\zeta^{2}$. We first deform the contour of integration $\xi \in \mathbb{R}$ to $\xi \in \mathbb{R} e^{i \alpha}$. A simple perturbation argument implies that we can take $k \in \mathbb{R}_{+}$. When $\xi \in \mathbb{R} e^{i \alpha}, \xi \cos \gamma+\xi_{0} \sin \gamma \in \Gamma_{\gamma}$, which is a contour in the complex plane. Note that $\Gamma_{\pi-\gamma}=\Gamma_{\gamma}$ and both contours are described in opposite directions (the contour $0<\gamma<\pi / 2$ is described from $-\infty$ to $+\infty)$.

For $0<\gamma<\pi / 2,\left(k^{2}-\zeta^{2}\right)^{1 / 2}=\zeta_{*}$. As $d \zeta=\mathrm{d} \xi \cos \gamma-\frac{\xi}{\xi_{0}} \mathrm{~d} \xi=\frac{\mathrm{d} \xi}{\xi_{0}} \zeta_{0}$ we obtain

$$
\int_{\mathbb{R} e^{i \alpha}}\left(\sin \gamma+\frac{\xi}{\xi_{0}} \cos \gamma\right) e^{i \frac{x}{\cos \gamma+}\left(\xi \cos \gamma+\xi_{0} \sin \gamma\right)} \mathrm{d} \xi=\int_{\Gamma_{\gamma}} \frac{\zeta}{\xi_{0}} e^{i \frac{x}{\cos \gamma+}} \zeta \frac{\xi_{0}}{\zeta_{0}} d \zeta
$$


The contour $\Gamma_{\gamma}$ is described from $-\infty$ to $+\infty$ (which means that it starts from the branch in $\Re<0$ to end with the branch in $\Re>0$ ), hence, as there is no branching point or pole in the loop with $\left(-\infty e^{i \alpha}, \infty e^{i \alpha}\right)$, we obtain the equality $(32)$.

For $\pi / 2<\gamma<\pi,\left(k^{2}-\zeta^{2}\right)^{1 / 2}=-\zeta_{*}$ and $d \zeta=-\frac{\mathrm{d} \xi}{\xi_{0}} \zeta_{0}$, hence

$$
\int_{\mathbb{R} e^{i \alpha}}\left(\sin \gamma+\frac{\xi}{\xi_{0}} \cos \gamma\right) e^{i \frac{x}{\cos \gamma_{+}}\left(\xi \cos \gamma+\xi_{0} \sin \gamma\right)} \mathrm{d} \xi=-\int_{\Gamma_{\gamma}} \frac{\zeta}{\xi_{0}} e^{i \frac{x}{\cos \gamma+} \zeta \frac{\xi_{0}}{\zeta_{0}}} d \zeta .
$$

The result comes from the fact that $\Gamma_{\gamma}$ is described from $\Re>0$ to $\Re<0$ and is thus replaced by $\left(+\infty e^{i \alpha},-\infty e^{i \alpha}\right)$.

This leads to the equalities

$$
\begin{aligned}
& r^{+, k}(g)=r_{+, 0}^{+, k}\left(g_{+}\right)+r_{-, 0}^{+, k}\left(g_{-}\right), \\
& r^{-, k}(g)=r_{+, 0}^{-, k}\left(g_{+}\right)+r_{-, 0}^{-, k}\left(g_{-}\right) .
\end{aligned}
$$

For the computation of the operators $r$, we consider the Mellin transform of both the restriction of $f \in H^{1}(\mathbb{R})$ to $\mathbb{R}_{+}$and to $\mathbb{R}_{-}$, and of the derivative $\partial_{x} f$ on $\mathbb{R}_{+}$and on $\mathbb{R}_{-}$. We thus need to construct the space of holomorphic functions of $z$ in $\Im z<0$ associated with $H^{1}(\mathbb{R})$. This is the aim of the next paragraph.

\subsection{Identification of $H^{1}(\mathbb{R})$}

Let $L$ be the space of holomorphic functions in $\Re z<0$, satisfying

$$
\sup _{s<0} \int_{-\infty}^{+\infty}|\phi(s+i \xi)|^{2} \mathrm{~d} \xi<\infty .
$$

Let $\phi \in L$. There exists (from Paley-Wiener theorem) a function $\Phi(x) \in L^{2}\left(\mathbb{R}_{+}\right)$such that

$$
\phi(z)=\int_{0}^{\infty} e^{z x} \Phi(x) \mathrm{d} x .
$$

The relation between $\phi$ and $\Phi$ is bijective from $L$ to $L^{2}\left(\mathbb{R}_{+}\right)$.

The generalization of this result to the functions of $H^{1}(\mathbb{R})$ is given by

LEMMA 2.4. - The transformation $\left(\Phi(x) 1_{x \geqslant 0}, \Phi(-x) 1_{x \geqslant 0}\right) \mapsto\left(\phi_{+}, \phi_{-}\right)$is a bijection from $H^{1}(\mathbb{R})$ to the subspace of $L \times L,\left(L^{1}\right)^{2}$, defined through

$$
\left(L^{1}\right)^{2}=\left\{\left(\phi_{+}, \phi_{-}\right) \in L \times L, \exists c_{0} \in \mathbb{C},\left(z \phi_{-}+c_{0}, z \phi_{+}+c_{0}\right) \in L \times L\right\}
$$

Proof. - For $\Phi \in H^{1}(\mathbb{R})$, we define $\Phi_{+}(x)=\Phi(x) 1_{x>0}$ and $\Phi_{-}(x)=\Phi(-x) 1_{x>0}$. Both distributions $\Phi_{+}$and $\Phi_{-}$belong to $L^{2}(\mathbb{R})$ and are supported in $x>0$, hence belong to $H^{1}\left(\mathbb{R}_{+}\right)$.

The transformation

$$
\Phi \in H^{1}(\mathbb{R}) \longmapsto\left(\phi_{+}(z)=\int_{0}^{\infty} \Phi(x) e^{z x} \mathrm{~d} x, \phi_{-}(z)=\int_{0}^{\infty} \Phi(-x) e^{z x} \mathrm{~d} x\right) \in\left(L^{1}\right)^{2}
$$


is a bijection. For $\Phi \in H^{1}(\mathbb{R})$, the definition of $\phi_{+}$and of $\phi_{-}$follows form the equalities

$$
\phi_{+}(z)=\int_{0}^{\infty} e^{z x} \Phi(x) \mathrm{d} x, \quad \phi_{-}(z)=\int_{0}^{\infty} e^{x z} \Phi(-x) \mathrm{d} x .
$$

The fact that $\Phi \in H^{1}(\mathbb{R})$ implies that $\partial_{x} \Phi \in L^{2}(\mathbb{R})$, hence

$$
\widetilde{\phi}_{+}(z)=\int_{0}^{\infty} e^{x z} \partial_{x} \Phi(x) \mathrm{d} x, \quad \widetilde{\phi}_{-}(z)=-\int_{0}^{\infty} e^{z x} \partial_{x} \Phi(-x) \mathrm{d} x
$$

belong to $L$. Using a classical integration by parts, we have

$$
\widetilde{\phi}_{+}(z)=-\Phi(0)-z \phi(z), \quad \widetilde{\phi}_{-}(z)=-\Phi(0)-z \phi_{-}(z) .
$$

There exists a constant $c_{0}$, equal to $\Phi(0)$, such that $c_{0}+z \phi_{+} \in L$ and $c_{0}+z \phi_{-} \in$ $L$, and $\phi_{ \pm} \in L$. Hence $\left(\phi_{-}, \phi_{+}\right) \in\left(L^{1}\right)^{2}$. As $\phi_{+}(-i \xi)=\int_{0}^{\infty} e^{-i x \xi} \Phi(x) \mathrm{d} x$ and $\phi_{-}(-i \xi)=\int_{0}^{\infty} e^{i x \xi} \Phi(-x) \mathrm{d} x$, the function $\Phi$ belongs to $L^{2}(\mathbb{R})$ hence

$$
\widehat{\Phi}(\xi)=\phi_{+}(-i \xi)+\phi_{-}(-i \xi)
$$

(as the functions $\phi_{+}$and $\phi_{-}$belong to $L$, the limit, for $s \rightarrow 0$ of $\phi_{ \pm}(s-i \xi)$ exists in $\left.L^{2}\right)$.

Let us start now from $\left(\phi_{+}, \phi_{-}\right)$element of $\left(L^{1}\right)^{2}$. The $\operatorname{limit}_{\lim _{s \rightarrow 0}}\left(\phi_{ \pm}(s-i \xi)\right)$ exists in $L^{2}(\mathbb{R})$, hence the distribution $\Phi_{ \pm}^{s}$, equal to the inverse Fourier transform of the holomorphic function $\phi_{ \pm}(s-i \xi)$, has a limit, as $s \rightarrow 0$, in $L^{2}\left(\mathbb{R}_{+}\right)$. Let $\widetilde{\Phi}_{+}^{s}(x)$ be the inverse Fourier transform of $(s-i \xi) \phi_{+}(s-i \xi)+c_{0}$. The relation

$$
\int_{\mathbb{R}}\left|(s-i \xi) \phi_{+}(s-i \xi)+c_{0}\right|^{2} \mathrm{~d} \xi \leqslant C
$$

independent of $s$, proves that $\widetilde{\Phi}_{+}^{s}$ admits a limit in $L^{2}\left(\mathbb{R}_{+}\right)$as $s \rightarrow 0$. The relation

$$
\begin{aligned}
\int_{\mathbb{R}}\left[(s+i \xi) \phi_{+}(s+i \xi)+\right. & \left.c_{0}\right] e^{i x \xi-\varepsilon \xi^{2}} \mathrm{~d} \xi \\
& =\left(s+\frac{d}{d x}\right)\left[\int_{\mathbb{R}} \phi_{+}(s+i \xi) e^{i x \xi-\varepsilon \xi^{2}} \mathrm{~d} \xi\right]+c_{0} e^{-x^{2} / 4 \varepsilon}\left(\frac{\pi}{\varepsilon}\right)^{1 / 2}
\end{aligned}
$$

implies that the distribution $\Phi_{+}^{s}$ is solution of the equation

$$
\left(s+\frac{d}{d x}\right) \Phi_{+}^{s}+c_{0} \delta_{0}=\widetilde{\Phi}_{+}^{s}(x) .
$$

Taking the limit as $s \rightarrow 0$, the function $\Phi_{+}$is in $H^{1}\left(\mathbb{R}_{+}\right)$and its value at $x=0$ is equal to $c_{0}$. The same calculus holds for $\Phi_{-}$, hence we constructed an element of $H^{1}(\mathbb{R})$ with the value $c_{0}$ at 0 . The uniqueness comes from the uniqueness of the representation of $L^{2}\left(\mathbb{R}_{+}\right)$through $L$.

As a Corollary, we define and characterize the space of Fourier transforms of elements of $H^{1}\left(\mathbb{R}_{+}\right)$(which are not necessary null at $x=0$ ): 
COROllary 2.1. - The space $L^{1}$, subset of $L$, equal to

$$
L^{1}=\{\phi \in L, \exists c \in \mathbb{C}, z \mapsto z \phi(z)+c \in L\}
$$

is isomorphic to the space $H^{1}\left(\mathbb{R}_{+}\right)$of distributions $f \in L^{2}\left(\mathbb{R}_{+}\right)$such that the distribution $\phi \in C_{0}^{\infty}\left(\mathbb{R}_{+}^{*}\right) \mapsto-\left\langle f, \partial_{x} \phi\right\rangle$ belongs to $L^{2}(\mathbb{R})$.

\subsection{Expression of the boundary operators using the Mellin transform}

Let $\tau>0$. We introduce, for each $\xi \in \mathbb{R}$, the complex number $\xi_{\alpha}$ of positive imaginary part defined through

$$
\xi_{\alpha}=\left(\tau^{2} e^{-2 i \alpha}-\xi^{2}\right)^{1 / 2}
$$

Definition 2.1. - The four operators $I_{\alpha}^{0, \tau}, I_{\alpha}^{1, \tau} T_{\alpha}^{0, \tau}$ and $T_{\alpha}^{1, \tau}$ used for the doublelayer operators are defined through

$$
\begin{aligned}
I_{\alpha}^{0, \tau}(\phi)(z) & =\frac{1}{2 \pi} \int_{-\infty}^{+\infty} \frac{\mathrm{d} \xi}{z+i \xi} \frac{\tau^{2} e^{-2 i \alpha}}{\xi_{\alpha}} \phi(-i \xi) \\
I_{\alpha}^{1, \tau}(\phi)(z) & =\frac{1}{2 \pi} \int_{-\infty}^{+\infty} \frac{\mathrm{d} \xi}{z+i \xi} \frac{\xi}{\xi_{\alpha}} \phi(-i \xi) \\
T_{\alpha}^{0, \tau}(\phi)(z) & =\frac{1}{2 \pi} \int_{-\infty}^{+\infty} \frac{\mathrm{d} \xi}{z+i\left(\xi \cos \gamma+\xi_{\alpha} \sin \gamma\right)} \frac{\tau^{2} e^{-2 i \alpha}}{\xi_{\alpha}} \phi(-i \xi) \\
T_{\alpha}^{1, \tau}(\phi)(z) & =\frac{1}{2 \pi} \int_{-\infty}^{+\infty} \frac{\mathrm{d} \xi}{z+i\left(\xi \cos \gamma+\xi_{\alpha} \sin \gamma\right)}\left[\cos \gamma \frac{\xi}{\xi_{\alpha}}+\sin \gamma\right] \phi(-i \xi) .
\end{aligned}
$$

LEMma 2.5. - These operators can also be written

$$
\begin{aligned}
& I_{\alpha}^{0, \tau}(\phi)(z)=-\frac{1}{2 \pi} \int_{-\infty}^{+\infty} \int_{0}^{\infty} e^{t(z+i \xi)} \frac{\tau^{2} e^{-2 i \alpha}}{\xi_{\alpha}} \phi(-i \xi) \mathrm{d} \xi \mathrm{d} t \\
& I_{\alpha}^{1, \tau}(\phi)(z)=-\frac{1}{2 \pi} \int_{-\infty}^{+\infty} \int_{0}^{\infty} e^{t(z+i \xi)} \frac{\xi}{\xi_{\alpha}} \phi(-i \xi) \mathrm{d} \xi \mathrm{d} t \\
& T_{\alpha}^{0, \tau}(\phi)(z)=-\frac{1}{2 \pi} \int_{-\infty}^{+\infty} \int_{0}^{\infty} e^{t\left(z+i\left(\xi \cos \gamma+\xi_{\alpha} \sin \gamma\right)\right)} \frac{\tau^{2} e^{-2 i \alpha}}{\xi_{\alpha}} \phi(-i \xi) \mathrm{d} \xi \mathrm{d} t \\
& T_{\alpha}^{1, \tau}(\phi)(z)=-\frac{1}{2 \pi} \int_{-\infty}^{+\infty} \int_{0}^{\infty} e^{t\left(z+i\left(\xi \cos \gamma+\xi_{\alpha} \sin \gamma\right)\right)}\left[\cos \gamma \frac{\xi}{\xi_{\alpha}}+\sin \gamma\right] \phi(-i \xi) \mathrm{d} \xi \mathrm{d} t .
\end{aligned}
$$

The proof of Lemma 2.5 is based on the equalities, valid when $\Re z \leqslant 0$ because $\sin \gamma>0$ and $\Im \eta_{1}^{\alpha}>0$ :

$$
\begin{aligned}
\int_{0}^{+\infty} e^{t(z+i \xi)} \mathrm{d} t & =-\frac{1}{z+i \xi} \\
\int_{0}^{+\infty} \mathrm{d} t e^{t\left(z+i\left(\xi \cos \gamma+\eta_{1}^{\alpha} \sin \gamma\right)\right)} & =-\frac{1}{z+i\left(\xi_{1} \cos \gamma+\eta_{1}^{\alpha} \sin \gamma\right)}
\end{aligned}
$$


We give the action of the operators $I_{\alpha}^{0, \tau}, I_{\alpha}^{1, \tau}, T_{\alpha}^{0, \tau}, T_{\alpha}^{1, \tau}$ on the imaginary axis.

Let $\Phi_{+} \in H^{1}\left(\mathbb{R}_{+}\right), \Phi_{-} \in H^{1}\left(\mathbb{R}_{+}\right)$, such that $\Phi_{+}(0)=\Phi_{-}(0)$. This means that the distribution $\Phi(x)=\Phi_{+}(x) 1_{x>0}+\Phi_{-}(-x) 1_{x<0}$ is element of $H^{1}(\mathbb{R})$.

We associate to $\Phi$ the element of $L \times L \times L \times L$ equal to $\left(\phi_{+}, \widetilde{\phi}_{+}, \phi_{-}, \widetilde{\phi}_{-}\right)$, where

$$
\begin{aligned}
\phi_{+}(-i \xi)=\widehat{\Phi}_{+}\left(\frac{\xi}{\cos \gamma_{+}}\right), & \phi_{-}(-i \xi)=\widehat{\Phi}_{-}\left(\frac{\xi}{\cos \gamma_{-}}\right), \\
\widetilde{\phi}_{+}(-i \xi)=\mathcal{F}\left(\partial_{x} \Phi_{+}\right)\left(\frac{\xi}{\cos \gamma_{+}}\right), & \widetilde{\phi}_{-}(-i \xi)=\mathcal{F}\left(\partial_{x} \Phi_{-}\right)\left(\frac{\xi}{\cos \gamma_{-}}\right) .
\end{aligned}
$$

Note that the only difference with the transform introduced in the previous paragraph is the coefficient $1 / \cos \gamma_{ \pm}$.

LEMMA 2.6. - We have the equalities:

$$
\begin{aligned}
& \mathcal{F}\left.1_{x>0} \partial_{+}\left(\Delta+\tau^{2} e^{-2 i \alpha}\right)^{-1}\left(\Phi_{+} \delta_{+}^{\prime}+\Phi_{-} \delta_{-}^{\prime}\right)\right]\left(\Sigma / \cos \gamma_{+}\right)= \\
& \frac{1}{2}\left[-i I_{\alpha}^{0, \tau}\left(\phi_{+}\right)+\cos \gamma_{+} I_{\alpha}^{1, \tau}\left(\widetilde{\phi}_{+}\right)\right](-i \Sigma)+\frac{1}{2} \cos \gamma_{+}\left[\frac{\cos \gamma}{\cos \gamma_{-}} i T_{\alpha}^{0, \tau}\left(\phi_{-}\right)-T_{\alpha}^{1, \tau}\left(\widetilde{\phi}_{-}\right)\right](-i \Sigma) \\
& \mathcal{F}\left[1_{x>0} \partial_{-}\left(\Delta+\tau^{2} e^{-2 i \alpha}\right)^{-1}\left(\Phi_{+} \delta_{+}^{\prime}+\Phi_{-} \delta_{-}^{\prime}\right)\right]\left(\Sigma / \cos \gamma_{-}\right)= \\
& \frac{1}{2}\left[-i I_{\alpha}^{0, \tau}\left(\phi_{-}\right)+\cos \gamma_{-} I_{\alpha}^{1, \tau}\left(\widetilde{\phi}_{-}\right)\right](-i \Sigma)+\frac{1}{2} \cos \gamma_{-}\left[\frac{\cos \gamma}{\cos \gamma_{+}} i T_{\alpha}^{0, \tau}\left(\phi_{+}\right)-T_{\alpha}^{1, \tau}\left(\widetilde{\phi}_{+}\right)\right](-i \Sigma) .
\end{aligned}
$$

Proof. - From the expressions of Proposition 6.2 (rewritten in Lemma 2.3), we notice that

$$
\begin{array}{r}
r_{+, 0}^{+, \tau e^{-i \alpha}}\left(\Phi_{+}\right)\left(x, \tau e^{-i \alpha}\right)=\frac{i}{4 \pi \cos \gamma_{+}} \int \mathrm{d} x e^{i \frac{x \xi}{\cos \gamma_{+}}} \frac{\tau^{2} e^{-2 i \alpha}}{\xi_{\alpha}} \widehat{\Phi}_{+}\left(\frac{\xi}{\cos \gamma_{+}}\right) \\
-\frac{1}{4 \pi} \int \mathrm{d} x e^{i \frac{x \xi}{\cos \gamma_{+}}} \frac{\xi}{\xi_{\alpha}} \widehat{\widetilde{\Phi}}_{+}\left(\frac{\xi}{\cos \gamma_{+}}\right)
\end{array}
$$

We consider the Fourier transform of this expression in $\Sigma / \cos \gamma_{+}$, we use the change of variable $t=x / \cos \gamma_{+}$and we make use of the first relation of (34) to get

$$
\begin{aligned}
\mathcal{F}\left(1_{x>0} r_{+, 0}^{+, \tau e^{-i \alpha}}\left(\Phi_{+}\right)\right)\left(\Sigma / \cos \gamma_{+}\right)=-\frac{i}{4 \pi} \int \frac{\mathrm{d} \xi}{-i \Sigma+i \xi} \frac{\tau^{2} e^{-2 i \alpha}}{\xi_{\alpha}} \widehat{\Phi}_{+}\left(\frac{\xi}{\cos \gamma_{+}}\right) \\
+\frac{1}{4 \pi} \int \frac{\mathrm{d} \xi}{-i \Sigma+i \xi} \frac{\xi}{\xi_{\alpha}} \mathcal{F}\left(\widetilde{\Phi}_{+}\right)\left(\frac{\xi}{\cos \gamma_{+}}\right)
\end{aligned}
$$

The definition of $\widetilde{\phi}_{+}$and of $\phi_{+}$lead to the relation

$$
\mathcal{F}\left(1_{x>0} r_{+}^{+, \tau e^{-i \alpha}}\left(\Phi_{+}\right)\right)\left(\Sigma / \cos \gamma_{+}\right)=\left[\frac{1}{2} \cos \gamma_{+} I_{\alpha}^{1, \tau}\left(\widetilde{\phi}_{+}\right)-\frac{i}{2} I_{\alpha}^{0, \tau}\left(\phi_{+}\right)\right](-i \Sigma) .
$$

Along with the definition of $r_{+}^{+, \tau e^{-i \alpha}}$, this gives the relations of the lemma for the pseudodifferential operators. 
We notice that, again with Proposition 6.2

$$
\begin{aligned}
& r_{+}^{-, \tau e^{-i \alpha}}\left(\Phi_{+}\right)\left(x, \tau e^{-i \alpha}\right)=-\frac{i}{4 \pi} \frac{\cos \gamma}{\cos \gamma_{+}} \int \mathrm{d} \xi \frac{\tau^{2} e^{-2 i \alpha}}{\xi_{\alpha}} \widehat{\Phi}_{+}\left(\frac{\xi}{\cos \gamma_{+}}\right) e^{i \frac{x}{\cos \gamma_{-}}\left[\xi \cos \gamma+\xi_{\alpha} \sin \gamma\right]} \\
&+\frac{1}{4 \pi} \int \mathrm{d} \xi\left[\sin \gamma+\cos \gamma \frac{\xi}{\xi_{\alpha}}\right] \mathcal{F}\left(\widetilde{\Phi}_{+}\right)\left(\frac{\xi}{\cos \gamma_{+}}\right) e^{i \frac{x}{\cos \gamma_{-}}\left[\xi \cos \gamma+\xi_{\alpha} \sin \gamma\right]}
\end{aligned}
$$

The use of the second relation of Laplace yields easily, again with $t=x / \cos \gamma_{-}$and with the introduction of $\phi_{+}$and $\widetilde{\phi}_{+}$, the expression

$$
\begin{aligned}
\mathcal{F}\left(1_{x>0} r_{+}^{-, \tau e^{-i \alpha}}\left(\Phi_{+}\right)\right)\left(\Sigma / \cos \gamma_{-}\right) \\
=\frac{i}{4 \pi} \cos \gamma \frac{\cos \gamma_{-}}{\cos \gamma_{+}} \int \mathrm{d} \xi \frac{\tau^{2} e^{-2 i \alpha}}{\xi_{\alpha}} \phi_{+}(-i \xi) \frac{\mathrm{d} \xi}{-i \Sigma+i\left(\xi \cos \gamma+\xi_{\alpha} \sin \gamma\right)} \\
\quad-\frac{1}{4 \pi} \cos \gamma_{-} \int \mathrm{d} \xi\left[\sin \gamma+\cos \gamma \frac{\xi}{\xi_{\alpha}}\right] \widetilde{\phi}_{+}(-i \xi) \frac{\mathrm{d} \xi}{-i \Sigma+i\left(\xi \cos \gamma+\xi_{\alpha} \sin \gamma\right)}
\end{aligned}
$$

We recognize on the right hand side the operators $T_{\alpha}^{0, \tau}$ and $T_{\alpha}^{1, \tau}$, acting on $z=-i \Sigma$ and on the left hand side, the expression of

$$
\mathcal{F}\left(1_{x>0} \partial_{-}\left[\left(\Delta+\tau^{2} e^{-2 i \alpha}\right)^{-1}\left(\Phi_{+} \delta_{+}^{\prime}\right)\right]\right)\left(\frac{\Sigma}{\cos \gamma_{-}}\right) .
$$

The equality corresponding in Lemma 2.6 follows.

Note that the relation $\widetilde{\phi}_{ \pm}(z)=-\Phi(0)-\frac{z \phi_{ \pm}(z)}{\cos \gamma_{ \pm}}$leads to the relations

$$
\begin{aligned}
\mathcal{F}\left[1_{x>0} \partial_{+}\left(\Delta+\tau^{2} e^{-2 i \alpha}\right)^{-1}\left(\Phi_{+} \delta_{+}^{\prime}+\Phi_{-} \delta_{-}^{\prime}\right)\right] & \left(\frac{\xi}{\cos \gamma_{+}}\right) \\
= & \frac{1}{2}\left[P^{r}\left(\phi_{+}\right)-\frac{\cos \gamma_{+}}{\cos \gamma_{-}} P^{s}\left(\phi_{-}\right)\right](-i \xi) \\
\mathcal{F}\left[1_{x>0} \partial_{-}\left(\Delta+\tau^{2} e^{-2 i \alpha}\right)^{-1}\left(\Phi_{+} \delta_{+}^{\prime}+\Phi_{-} \delta_{-}^{\prime}\right)\right] & \left(\frac{\xi}{\cos \gamma_{-}}\right) \\
= & \frac{1}{2}\left[\frac{\cos \gamma_{-}}{\cos \gamma_{+}} P^{s}\left(\phi_{+}\right)-P^{r}\left(\phi_{-}\right)\right](-i \xi)
\end{aligned}
$$

where a formulation of the operators $P^{r}$ and $P^{s}$ is given by the integrals

$$
\begin{aligned}
& P^{r}(\phi)(z)=\frac{1}{2 \pi} \int_{\mathbb{R}} \frac{i \xi_{\alpha} \phi(-i \xi)}{z+i \xi} \mathrm{d} \xi \\
& P^{s}(\phi)(z)=\frac{1}{2 \pi} \int_{\mathbb{R}} \frac{i\left(\cos \gamma \xi_{\alpha}-\sin \gamma \xi\right) \phi(-i \xi)}{z+i\left(\xi \cos \gamma+\xi_{0} \sin \gamma\right)} \mathrm{d} \xi
\end{aligned}
$$

The operator $\left(\begin{array}{cc}P^{r} & -\frac{\cos \gamma_{+}}{\cos \gamma_{-}} P^{s} \\ \frac{\cos \gamma_{-}}{\cos \gamma_{+}} P^{s} & -P^{r}\end{array}\right)$ is continuous from $\left(L^{1}\right)^{2}$ to $L \times L$. 


\subsection{The fundamental analytic system for the traces}

One of the important steps of this paper is to prove that the operator associated with the straight wedge is invertible. More precisely, we consider the equality of Proposition 1.1 and we write an heuristic analysis:

$$
\left(\Delta-\partial_{t^{2}}^{2}\right) \underline{u}=v_{+} \delta_{+}+v_{-} \delta_{-}+\left.u\right|_{\partial \Omega} \delta_{\partial \Omega}^{\prime},
$$

which leads to the relation $\left(\left(\Delta-\partial_{t^{2}}^{2}\right)_{+}^{-1}\right.$ is the fundamental solution of the d'Alembertian supported in $t \geqslant 0$ )

$$
\underline{u}=u_{i}+\left(\Delta-\partial_{t^{2}}^{2}\right)_{+}^{-1}\left[v_{+} \delta_{+}+v_{-} \delta_{-}+\left.u\right|_{\partial \Omega} \delta_{\partial \Omega}^{\prime}\right]
$$

which implies the two relations

$$
\left\{\begin{array}{l}
\partial_{+} u=\partial_{+} u_{i}+K_{+}^{+}\left(v_{+}\right)+K_{-}^{+}\left(v_{-}\right)+R^{+}\left(\left.u\right|_{\partial \Omega}\right) \\
\partial_{-} u=\partial_{-} u_{i}+K_{+}^{-}\left(v_{+}\right)+K_{-}^{-}\left(v_{-}\right)+R^{-}\left(\left.u\right|_{\partial \Omega}\right)
\end{array}\right.
$$

Hence we need to solve the system

$$
\left\{\begin{array}{l}
v_{+}=\partial_{+} u_{i}+K_{+}^{+}\left(v_{+}\right)+K_{-}^{+}\left(v_{-}\right)+R^{+}\left(\left.u\right|_{\partial \Omega}\right) \\
v_{-}=\partial_{-} u_{i}+K_{+}^{-}\left(v_{+}\right)+K_{-}^{-}\left(v_{-}\right)+R^{-}\left(\left.u\right|_{\partial \Omega}\right) \\
v_{+}-z^{+}\left(x^{\prime}\right) \partial_{t}\left(\left.u\right|_{\Delta_{+}}\right)=0 \\
v_{-}-z^{-}\left(x^{\prime}\right) \partial_{t}\left(\left.u\right|_{\Delta_{-}}\right)=0
\end{array}\right.
$$

Considering the Laplace-Fourier transform in time and $\omega=\tau e^{-i \alpha}$, we obtain, for the straight wedge, the system

$$
\left\{\begin{array}{l}
f_{+}=D_{i}^{+}+k_{+}^{+, \tau e^{-i \alpha}}\left(f_{+}\right)+k_{-}^{+, \tau e^{-i \alpha}}\left(f_{-}\right)+r^{+, \tau e^{-i \alpha}}(g) \\
f_{-}=D_{i}^{-}+k_{+}^{-, \tau e^{-i \alpha}}\left(f_{+}\right)+k_{-}^{-, \tau e^{-i \alpha}}\left(f_{-}\right)+r^{-, \tau e^{-i \alpha}}(g) \\
f_{+}-i \sin \theta_{+} \tau e^{-i \alpha} g_{+}=0 \\
f_{-}-i \sin \theta_{-} \tau e^{-i \alpha} g_{-}=0 .
\end{array}\right.
$$

We finally compute the Fourier transform of the first and third lines of this system at $\Sigma / \cos \gamma_{+}$and of the second and fourth lines at $\Sigma / \cos \gamma_{-}$. With the notations

$$
\left\{\begin{array}{l}
\psi_{+}(-i \theta)=\mathcal{F}\left(f_{+}\right)\left(\frac{\theta}{\cos \gamma_{+}}\right) \\
\psi_{-}(-i \theta)=\mathcal{F}\left(f_{-}\right)\left(\frac{\theta}{\cos \gamma_{-}}\right) \\
\beta_{+}(-i \theta)=\mathcal{F}\left(g_{+}\right)\left(\frac{\theta}{\cos \gamma_{+}}\right) \\
\beta_{-}(-i \theta)=\mathcal{F}\left(g_{-}\right)\left(\frac{\theta}{\cos \gamma_{-}}\right)
\end{array}\right.
$$

and introducing the operators $S_{\alpha}^{+}=2 k_{-}^{+, \tau e^{-i \alpha}}, S_{\alpha}^{-}=2 k_{+}^{-, \tau e^{-i \alpha}}, T_{+, \alpha}^{ \pm}=2 r_{+, 0}^{ \pm, \tau e^{-i \alpha}}$, $T_{-, \alpha}^{ \pm}=2 r_{-, 0}^{ \pm, \tau e^{-i \alpha}}$, we obtain the system

$$
\left\{\begin{array}{lllll}
\psi_{+} & -S_{\alpha}^{+} \psi_{-} & -T_{+, \alpha}^{+} \beta_{+} & -T_{-, \alpha}^{+} \beta_{-} & =\phi_{+} \\
-S_{\alpha}^{-} \psi_{+}+\psi_{-} & -T_{+, \alpha}^{-} \beta_{+} & -T_{-, \alpha}^{-} \beta_{-} & =\phi_{-} \\
\psi_{+} & & -i \sin \theta_{+} \tau e^{-i \alpha} \beta_{+} & =0 \\
& \psi_{-} & & -i \sin \theta_{-} \tau e^{-i \alpha} \beta_{-} & =0 .
\end{array}\right.
$$


We prove in the next paragraph that this system admits, for $\left(\phi_{+}, \phi_{-}\right) \in L \times L$, a solution $\left(\psi_{+}, \psi_{-}\right) \in L \times L$ and $\left(\beta_{+}, \beta_{-}\right) \in\left(L^{1}\right)^{2}$, the space $\left(L^{1}\right)^{2}$ being defined in Lemma 2.4.

\subsection{The invertibility in $L \times L$ of the analytic system}

In this section, we use a solution in $\Omega \times \Omega^{\prime}$ of $\left(\Delta+\tau^{2} e^{-2 i \alpha}\right) U=0$ to solve the previous system. This is to be done constructing a solution which is continuous at the corner (Proposition 2.2) for computing an element of $\left(L^{1}\right)^{2}$, and deducing from this construction the invertibility of a system related to (38) with a jump at the corner $\left(g_{+}(0) \neq g_{-}(0)\right)$ (Corollary 2.2).

Proposition 2.2. - The system (38) admits an unique solution in $L \times L$ such that $\beta_{+}, \beta_{-}$is associated with an element of $H^{1}(\partial \Omega)$.

Proof. - We introduce

$$
\partial_{ \pm}^{1} W= \pm\left.\left(\cos \gamma_{ \pm} \partial_{y}-\sin \gamma_{ \pm} \partial_{x}\right) W\right|_{y-\tan \gamma_{ \pm} x=0^{\mp}, x>0} .
$$

Similarly, we write the two traces on each tangent line that we need:

$$
\begin{cases}\left.W\right|_{D_{+},+}=\left.W\right|_{y-x \tan \gamma_{+}=0^{+}} & \left.W\right|_{D_{+},-}=\left.W\right|_{y-x \tan \gamma_{+}=0^{-}} \\ \left.W\right|_{D_{-},+}=\left.W\right|_{y-x \tan \gamma_{-}=0^{-}} & \left.W\right|_{D_{-},-}=\left.W\right|_{y-x \tan \gamma_{-}=0^{+}}\end{cases}
$$

Let $\mathcal{O}$ be the set $\mathbb{R}^{2}-\left(\left\{y=\tan \gamma_{+} x, x \geqslant 0\right\} \cup\left\{y=\tan \gamma_{-} x, x \geqslant 0\right\}\right)$. Suppose that $U$ is a distribution with the following properties:

- the distribution $U$ is solution of the Helmholtz equation $\left(\Delta+e^{-2 i \alpha} \tau^{2}\right) U=0$ in the wedge $F_{0}$ as well as in the space $\mathbb{R}^{2}-F_{0}$.

- the distributions $\partial_{ \pm} U$ and $\partial_{ \pm}^{1} U$ are in $L^{2}$

- the distributions $\left.U\right|_{D_{ \pm},+}$and $\left.U\right|_{D_{ \pm},-}$are in $H^{1}\left(D_{ \pm}\right)$.

If we denote by $\widetilde{U}$ the distribution defined in $\mathbb{R}^{2}$ equal to $U$ on $\mathcal{O}$, a consequence of the formula (27) and of the regularity at the corner is that:

$$
\begin{aligned}
\left(\Delta+\tau^{2} e^{-2 i \alpha}\right) \widetilde{U}=\left(\partial_{+} U-\right. & \left.\partial_{+}^{1} U\right) \otimes \delta_{+}+\left(\left.U\right|_{D_{+},+}-\left.U\right|_{D_{+},-}\right) \otimes \delta_{+}^{\prime} \\
& +\left(\partial_{-} U-\partial_{-}^{1} U\right) \otimes \delta_{-}+\left(\left.U\right|_{D_{-},+}-\left.U\right|_{D_{-},-}\right) \otimes \delta_{-}^{\prime}
\end{aligned}
$$

We introduce:

$$
\left\{\begin{array}{l}
\Psi_{ \pm}=\partial_{ \pm} U-\partial_{ \pm}^{1} U \\
G_{ \pm}=\left.U\right|_{D_{ \pm},+}-\left.U\right|_{D_{ \pm},-}
\end{array}\right.
$$

For the simplicity of notations, we denote by $\widetilde{G}_{ \pm}$the distribution which is the derivative in $H^{1}\left(D_{ \pm}\right)$of $G_{ \pm}$. The equality (39) rewrites:

$$
\left(\Delta+\tau^{2} e^{-2 i \alpha}\right) \widetilde{U}=\Psi_{+} \delta_{+}+G_{+} \delta_{+}^{\prime}+\Psi_{-} \delta_{-}+G_{-} \delta_{-}^{\prime},
$$


hence

$$
\begin{aligned}
\partial_{ \pm} \tilde{U}(x, \tau) & =k_{+}^{ \pm, \tau e^{-i \alpha}}\left(\Psi_{+}\right)+k_{-}^{ \pm, \tau e^{-i \alpha}}\left(\Psi_{-}\right)+r^{ \pm, \tau e^{-i \alpha}}(G) \\
& =\frac{1}{2} \Psi_{+}+k_{-}^{ \pm, \tau e^{-i \alpha}}\left(\Psi_{-}\right)+r_{+, 0}^{ \pm, \tau e^{-i \alpha}}\left(G_{+}\right)+r_{-, 0}^{ \pm, \tau e^{-i \alpha}}\left(G_{-}\right)
\end{aligned}
$$

We note that

$$
\partial_{ \pm} \widetilde{U}(x, \tau)=\partial_{ \pm} U-\partial_{ \pm}^{1} U+\partial_{ \pm}^{1} U=\Psi_{ \pm}+\partial_{ \pm}^{1} U
$$

This will give a system linking $\Psi_{ \pm}, G_{ \pm}$and $\partial_{ \pm}^{1} U$.

Let us consider the holomorphic functions defined by

$$
\left\{\begin{array}{l}
\psi_{ \pm}(-i \xi)=\mathcal{F}\left(\Psi_{ \pm}\right)\left(\frac{\xi}{\cos \gamma_{ \pm}}\right) \\
\beta_{ \pm}(-i \xi)=\mathcal{F}\left(G_{ \pm}\right)\left(\frac{\xi}{\cos \gamma_{ \pm}}\right) \\
\widetilde{\beta}_{ \pm}=\mathcal{F}\left(\widetilde{G}_{ \pm}\right)\left(\frac{\xi}{\cos \gamma_{ \pm}}\right) .
\end{array}\right.
$$

We obtain $\mathcal{F}\left(\partial_{ \pm} \widetilde{U}\right)\left(\frac{\xi}{\cos \gamma_{ \pm}}\right)=\psi_{ \pm}(-i \xi)+\mathcal{F}\left(\partial_{ \pm}^{1} U\right)\left(\frac{\xi}{\cos \gamma_{ \pm}}\right)$. From Lemma 2.6, $\left(\psi_{+}, \psi_{-}, \beta_{+}, \beta_{-}, \widetilde{\beta}_{+}, \widetilde{\beta}_{-}\right)$satisfies

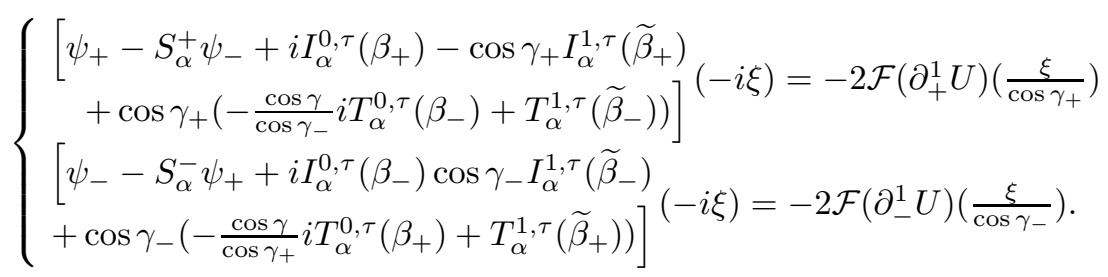

We note that the system (38) is obtained with the boundary condition $\psi_{ \pm}-$ $i \tau e^{-i \alpha} \sin \theta_{ \pm} \beta_{ \pm}=0$ and the source terms such that $\phi_{ \pm}(-i \xi)=\mathcal{F}\left(-2 \partial_{ \pm}^{1} U\right)\left(\frac{\xi}{\cos \gamma_{ \pm}}\right)$.

When $\phi_{ \pm}$are given in $L \times L$, we introduce the distributions $\Phi_{ \pm}$in $L^{2}\left(\mathbb{R}_{+}\right)$such that $\phi_{ \pm}(-i \xi)=\mathcal{F}\left(\Phi_{ \pm}\right)\left(\frac{\xi}{\cos \gamma_{ \pm}}\right)$. We have the relations

$$
\partial_{ \pm}^{1} U=-\frac{1}{2} \Phi_{ \pm}
$$

The boundary conditions yield

$$
\Psi_{ \pm}-i \tau e^{-i \alpha} \sin \theta_{ \pm} G_{ \pm}=0
$$

that is

$$
\left(\partial_{ \pm}-\partial_{ \pm}^{1}\right) U-i \tau e^{-i \alpha} \sin \theta_{ \pm}\left(\left.U\right|_{D_{ \pm},+}-\left.U\right|_{D_{ \pm},-}\right)=0 .
$$

It is thus equivalent to solve (38) and to solve:

- a Neumann boundary problem in the inner wedge:

$$
\left(\Delta+\tau^{2} e^{-2 i \alpha}\right) U=0, \quad \partial_{+}^{1} U=-\frac{1}{2} \Phi_{+}, \quad \partial_{-}^{1} U=-\frac{1}{2} \Phi_{-} .
$$

- following the condition on the traces on the boundary in the outer space associated with the boundary condition (which is the extension of the condition of $[\mathbf{2 0}]$ ), 
we impose a condition on the traces on the boundary in the outer space associated with the boundary condition, namely

$$
\left\{\begin{array}{l}
\partial_{+} U-\left.i e^{-i \alpha} \tau \sin \theta_{+} U\right|_{D_{+},+}=\partial_{+}^{1} U-\left.i e^{-i \alpha} \tau \sin \theta_{+} U\right|_{D_{+},-} \\
\partial_{-} U-\left.i e^{-i \alpha} \tau \sin \theta_{-} U\right|_{D_{-},+}=\partial_{-}^{1} U-\left.i e^{-i \alpha} \tau \sin \theta_{-} U\right|_{D_{-},-}
\end{array}\right.
$$

The system of equations in the open set $\mathcal{O}$ is then

$$
\left\{\begin{array}{l}
\left(\Delta+\tau^{2} e^{-2 i \alpha}\right) U=0 \\
\left(\partial_{+}-\partial_{+}^{1}\right) U-i \tau e^{-i \alpha} \sin \theta_{+}\left(\left.U\right|_{D_{+},+}-\left.U\right|_{D_{+},-}\right)=0 \\
\left(\partial_{-}-\partial_{-}^{1}\right) U-i \tau e^{-i \alpha} \sin \theta_{-}\left(\left.U\right|_{D_{-},+}-\left.U\right|_{D_{-},-}\right)=0 \\
\partial_{+}^{1} U=-\frac{1}{2} \Phi_{+} \\
\partial_{-}^{1} U=-\frac{1}{2} \Phi_{-}
\end{array}\right.
$$

Let $\phi_{ \pm}$be given in $L$. When (42) has a solution $U$ in $\mathcal{O}$, we construct the function $\widetilde{U}$ by (39) and $\psi_{ \pm}$are given by the relations (40). The existence of the solution of (38) is a consequence of the existence of a solution of (42), result given by

LEMma 2.7. - The system (42) is a well posed problem. It admits a solution

$$
U \in C_{\theta}\left(\mathbb{R} / 2 \pi Z, H^{1}\left(\mathbb{R}_{+}\right)\right)
$$

such that

$$
r^{-1} \partial_{\theta} U \in C_{\theta}\left(\left[\gamma_{-}, \gamma_{+}\right], L^{2}\left(\mathbb{R}_{+}\right)\right) \cap C_{\theta}\left(\left[\gamma_{+}, 2 \pi+\gamma_{-}\right], L^{2}\left(\mathbb{R}_{+}\right)\right) .
$$

This solution satisfies $U\left(0, \gamma_{+}\right)=U\left(0, \gamma_{-}\right)$, and thus $V(x)=U\left(x, \gamma_{+}\right) 1_{x \geqslant 0}+$ $U\left(-x, 2 \pi+\gamma_{-}\right) 1_{x \leqslant 0}$ is element of $H^{1}(\mathbb{R})$.

Proof of Lemma 2.7. - From $\phi_{ \pm} \in L$, we obtain $\Phi_{ \pm} \in L^{2}\left(\mathbb{R}_{+}\right)$. Using, for $\omega=$ $\tau e^{-i \alpha} \notin \mathbb{R}^{+}$, the point 2 of Theorem 2 (proven in $[\mathbf{2 0}]$ ), we verify that the problem in the internal wedge $\left(\Delta+\tau^{2} e^{-2 i \alpha}\right) U=0$ in $\gamma_{-} \leqslant \theta \leqslant \gamma_{+}$with the Neumann boundary condition $\partial_{ \pm}^{1} U=-\frac{1}{2} \Phi_{ \pm}$admits a unique solution $U$ such that $r^{-1} U, \partial_{r} U$ and $r^{-1} \partial_{\theta} U$ are in $L^{2}\left(\mathbb{R}_{+}\right)$. This solution $U$ satisfies $U\left(0, \gamma_{+}\right)=U\left(0, \gamma_{-}\right)=$ 0 . We thus compute the traces of $U$ on the internal boundary. The distributions $\chi_{ \pm}=\partial_{ \pm}^{1} U-\left.i e^{-i \alpha} \tau \sin \theta_{ \pm} U\right|_{y-x \tan \gamma_{ \pm}=o^{\mp}}$ are in $L^{2}\left(\mathbb{R}_{+}\right)$. It has been proven in Proposition 2.1 that the problem $\left(\Delta+\tau^{2} e^{-2 i \alpha}\right) U=0$ with the boundary condition $\partial_{ \pm} U-\left.i e^{-i \alpha} \tau \sin \theta_{ \pm} U\right|_{y-x \tan \gamma_{ \pm}=o^{ \pm}}=\chi_{ \pm}$, which satisfies the hypothesis (29) for $\alpha$ small enough, is a well posed problem and admits a solution $\widetilde{U}$ in $H^{1} \cap E$. We deduce that the traces of this solution $\partial_{ \pm} \widetilde{U}$ in the external wedge are in $L^{2}\left(\mathbb{R}_{+}\right)$hence $\psi_{ \pm}$, such that $\psi_{ \pm}(-i \xi)=\widehat{\Psi}_{ \pm}\left(\frac{\xi}{\cos \gamma_{ \pm}}\right)$belongs to $L$. Moreover $\Psi_{ \pm}=\partial_{ \pm} \widetilde{U}-\partial_{ \pm}^{1} U$. An additional but crucial consequence of Proposition 1.1 is that $\left.\widetilde{U}\right|_{\partial \Omega^{0}}$ is in $H^{1}\left(\partial \Omega^{0}\right)$. As $G_{ \pm}=\left.U\right|_{\partial \mathcal{O}_{ \pm},+}-\left.U\right|_{\partial \mathcal{O}_{ \pm},-}$is in $H^{1}(\partial \mathcal{O})$, a consequence of Lemma 2.4 is that the holomorphic functions $\beta_{ \pm}(z)=\int_{0}^{\infty} G_{ \pm}(x) e^{z x} \mathrm{~d} x$ are in $\left(L^{1}\right)^{2}$. Lemma 2.7 is proven.

We obtained $\left(\psi_{+}, \psi_{-}\right) \in L \times L$ and $\left(\beta_{+}, \beta_{-}\right) \in\left(L^{1}\right)^{2}$. The system (38) admits a solution in $L \times L \times\left(L^{1}\right)^{2}$. This ends the proof of Proposition 2.2. 
We prove the

Corollary 2.2. - Let $l_{+} \in L, l_{-} \in L$ and $S_{0}$ a given complex number. We consider the system, for $\phi_{+} \in L^{1}, \phi_{-} \in L^{1}, \psi_{+} \in L, \psi_{-} \in L$ :

$$
\left\{\begin{array}{l}
\psi_{+}+i I_{\alpha}^{0, \tau}\left(\phi_{+}\right)-\cos \gamma_{+} I_{\alpha}^{1, \tau}\left(\widetilde{\phi}_{+}\right)+\cos \gamma_{+}\left[-\frac{\cos \gamma}{\cos \gamma-} i T_{\alpha}^{0, \tau}\left(\phi_{-}\right)+T_{\alpha}^{1, \tau}\left(\widetilde{\phi}_{-}\right)\right]=l_{+} \\
\psi_{-}+i I_{\alpha}^{0, \tau}\left(\phi_{-}\right)-\cos \gamma_{-} I_{\alpha}^{1, \tau}\left(\widetilde{\phi}_{-}\right)+\cos \gamma_{-}\left[-\frac{\cos \gamma}{\cos \gamma_{+}} i T_{\alpha}^{0, \tau}\left(\phi_{+}\right)-T_{\alpha}^{1, \tau}\left(\widetilde{\phi}_{+}\right)\right]=l_{-} \\
\psi_{+}-i \tau e^{-i \alpha} \sin \theta_{+} \phi_{+}=0 \\
\psi_{-}-i \tau e^{-i \alpha} \sin \theta_{-} \phi_{-}=0 \\
\widetilde{\phi}_{+}(z)+\frac{1}{\cos \gamma_{+}} z \phi_{+}(z)=\widetilde{\phi}_{-}(z)+\frac{1}{\cos \gamma_{-}} z \phi_{-}(z)+S_{0}
\end{array}\right.
$$

This system admits an unique solution.

We reduce this system to the system of Proposition 2.2. More precisely, we consider a function $l \in C_{0}^{\infty}(\mathbb{R})$ equal to 1 in a neighborhood of 0 . Let $R$ be a large number. We introduce the distribution which belongs to $L^{2}(\mathcal{O})$, equal to

$$
U_{0}=\left(\Delta+\tau^{2} e^{-2 i \alpha}\right)^{-1}\left[i \tau \sin \theta_{+} e^{-i \alpha} l(x / R) 1_{x \geqslant 0} \delta_{+}+l(x / R) \delta_{+}^{\prime}\right] .
$$

Note that this distribution takes into account a jump at the corner for the trace of $U_{0}$ on the boundary $\partial \mathcal{O}$.

Let $\Phi_{+}$and $\Phi_{-}$be two functions of $H^{1}\left(\mathbb{R}_{+}\right)$such that $\Phi_{-}(0)=\Phi_{+}(0)+S_{0}$. The distribution $\Phi_{-}(-x) 1_{-x \geqslant 0}+\left(\Phi_{+}(x)+S_{0} l(x / R)\right) 1_{x \geqslant 0}$ is an element of $H^{1}(\mathbb{R})$, and the distribution $\Phi_{-} \delta_{-}^{\prime}+\left(\Phi_{+}(x)+S_{0} l(x / R)\right) 1_{x \geqslant 0} \delta_{+}^{\prime}$ is constructed as corresponding to an element of $H^{1}(\partial \Omega)$.

We consider $\Psi_{ \pm} \in L^{2}\left(\mathbb{R}_{+}\right)$, to which we associate $\psi_{ \pm}(z)=\int_{0}^{\infty} e^{x z / \cos \gamma_{ \pm}} \Psi_{ \pm}(x) \mathrm{d} x$. Remember that

$$
\frac{1}{2} S_{\alpha} \psi_{\mp}(-i \xi)=\cos \gamma_{\mp} \mathcal{F}\left[1_{x>0} \partial_{ \pm}\left(\Delta+\tau^{2} e^{-2 i \alpha}\right)^{-1}\left(\Psi_{\mp} \delta_{\mp}\right)\right]\left(\frac{\xi}{\cos \gamma_{ \pm}}\right)
$$

This is an easy extension of the formula (5.7) p. 391 of [20] (according to the new definition of the normal derivative used here). We also proved the equalities of Lemma 2.6 .

Let us introduce the distribution, element of $L^{2}(\mathcal{O})$

$$
U=\left(\Delta+\tau^{2} e^{-2 i \alpha}\right)^{-1}\left[\Psi_{+} \delta_{+}+\Psi_{-} \delta_{-}+\Phi_{+} \delta_{+}^{\prime}+\Phi_{-} \delta_{-}^{\prime}\right] .
$$

We consider the distribution $V=U+S_{0} U_{0}$. We verify

$$
\begin{aligned}
V=\left(\Delta+\tau^{2} e^{-2 i \alpha}\right)^{-1}\left[\left[\Psi_{+}+i \tau \sin \theta_{+} e^{-i \alpha} l(x / R) 1_{x \geqslant 0} S_{0}\right] \delta_{+}+\Psi_{-} \delta_{-}\right. \\
\left.+\left[\Phi_{+}+l(x / R) 1_{x \geqslant 0}\right] \delta_{+}^{\prime}+\Phi_{-} \delta_{-}^{\prime}\right],
\end{aligned}
$$


hence $V \in H^{1}\left(\mathbb{R}^{2}\{y=a x, y=b x, x>0\}\right)$. On this distribution, we apply the jump formula, through

$$
\left\{\begin{array}{l}
\Psi_{+}+i \tau \sin \theta_{+} e^{-i \alpha} l(x / R) 1_{x \geqslant 0} S_{0}=\partial_{+} V-\partial_{+}^{1} V \\
\Psi_{-}=\partial_{-} V-\partial_{-}^{1} V \\
\Phi_{+}+S_{0} l(x / R) 1_{x \geqslant 0}=\left.V\right|_{\Delta_{+},+}-\left.V\right|_{\Delta_{+},-} \\
\Phi_{-}=\left.V\right|_{\Delta_{-},+}-\left.V\right|_{\Delta_{-},-}
\end{array}\right.
$$

The main argument used in the proof of Proposition 2.2 is that, when $\partial_{+}^{1} V$ and $\partial_{-}^{1} V$ are given in $L^{2}\left(\mathbb{R}_{+}\right)$, then the problem associated is solved in $\Omega \cup \Omega^{\prime}$. We use this idea and identify $\partial_{+}^{1} V$ and $\partial_{-}^{1} V$ such that we reduce to the problem of Corollary 2.2 .

Introduce the Fourier transform $\hat{l}$ of $l$. We note that

$$
\begin{aligned}
& \mathcal{F}\left(\Phi_{+}+S_{0} l(x / R) 1_{x \geqslant 0}\right)\left(\frac{\xi}{\cos \gamma_{+}}\right)=\phi_{+}(-i \xi)+R S_{0} \widehat{l}\left(\frac{\xi R}{\cos \gamma_{+}}\right) \\
& \mathcal{F}\left(\partial_{x} \Phi_{+}+\frac{S_{0}}{R} l^{\prime}(x / R)\right) 1_{x \geqslant 0}\left(\frac{\xi}{\cos \gamma_{+}}\right)=\widetilde{\phi}_{+}(-i \xi)-S_{0}+i \frac{\xi R}{\cos \gamma_{+}} \widehat{l}\left(\frac{\xi R}{\cos \gamma_{+}}\right) \\
& \mathcal{F}\left(\Psi_{+}+i \tau e^{-i \alpha} \sin \theta_{+} S_{0} l\left(\frac{x}{R}\right) 1_{x} \geqslant 0\right)\left(\frac{\xi}{\cos \gamma_{+}}\right)=\psi(-i \xi)+i \tau e^{-i \alpha} \sin \theta_{+} S_{0} R \widehat{l}\left(\frac{\xi R}{\cos \gamma_{+}}\right)
\end{aligned}
$$

Introducing (for the simplicity of writing) $l_{1}(z), l_{2}(z), l_{3}(z)$ such that

$$
l_{1}(z)=R \widehat{l}\left(\frac{i z R}{\cos \gamma_{+}}\right), \quad l_{2}(z)=\frac{z l_{1}(z)}{\cos \gamma_{+}}-1 \quad \text { and } \quad l_{3}(z)=i \tau e^{-i \alpha} \sin \theta_{+} l_{1}(z),
$$

we obtain

$$
\begin{aligned}
& \mathcal{F}\left(\partial_{+} V\right)\left(\frac{\xi}{\cos \gamma_{+}}\right)= \\
& \frac{1}{2}\left[\psi_{+}+l_{3}+\cos \gamma_{+} S_{\alpha} \psi_{-}+\left[-i I_{\alpha}^{0, \tau}\left(\phi_{+}+S_{0} l_{1}\right)+\cos \gamma_{+} I_{\alpha}^{1, \tau}\left(\widetilde{\phi}_{+}+S_{0} l_{2}\right)\right]\right. \\
& \left.+\cos \gamma_{+}\left[\frac{\cos \gamma}{\cos \gamma_{-}} i T_{\alpha}^{0, \tau}\left(\phi_{-}\right)-T_{\alpha}^{1, \tau}\left(\widetilde{\phi}_{-}\right)\right]\right](-i \xi), \\
& \mathcal{F}\left(\partial_{-} V\right)\left(\frac{\xi}{\cos \gamma_{-}}\right)=\frac{1}{2}\left[\psi_{-}+\cos \gamma_{-} S_{\alpha}\left(\psi_{+}+S_{0} l_{3}\right)+\left[-i I_{\alpha}^{0, \tau}\left(\phi_{-}\right)+\cos \gamma_{-} I_{\alpha}^{1, \tau}\left(\tilde{\phi}_{-}\right)\right]\right. \\
& \left.+\cos \gamma_{-}\left[\frac{\cos \gamma}{\cos \gamma_{+}} i T_{\alpha}^{0, \tau}\left(\phi_{+}+S_{0} l_{1}\right)-T_{\alpha}^{1, \tau}\left(\widetilde{\phi}_{+}+S_{0} l_{2}\right)\right]\right](-i \xi) .
\end{aligned}
$$

Hence we have the relations

$$
\begin{aligned}
& \psi_{+}-\cos \gamma_{+} S_{\alpha} \psi_{-}+i I_{\alpha}^{0, \tau} \phi_{+}-\cos \gamma_{+} I_{\alpha}^{1, \tau} \widetilde{\phi}_{+}-\cos \gamma_{+}\left[\frac{\cos \gamma}{\cos \gamma_{-}} i T_{\alpha}^{0, \tau} \phi_{-}-T_{\alpha}^{1, \tau} \widetilde{\phi}_{-}\right]=s t_{+} \\
& \psi_{-}-\cos \gamma_{-} S_{\alpha} \psi_{+}+i I_{\alpha}^{0, \tau} \phi_{-}-\cos \gamma_{-} I_{\alpha}^{1, \tau} \widetilde{\phi}_{-}-\cos \gamma_{-}\left[\frac{\cos \gamma}{\cos \gamma_{+}} i T_{\alpha}^{0, \tau} \phi_{+}-T_{\alpha}^{1, \tau} \widetilde{\phi}_{+}\right]=s t_{-}
\end{aligned}
$$

where

$$
\begin{aligned}
& s t_{+}(z)=-2 \mathcal{F}\left(\partial_{+}^{1} V\right)\left(\frac{i z}{\cos \gamma_{+}}\right)+S_{0}\left(-i I_{\alpha}^{0, \tau} l_{1}+\cos \gamma_{+} I_{\alpha}^{1, \tau} l_{2}-l_{3}\right) \\
& s t_{-}(z)=-\mathcal{F}\left(2 \partial_{-}^{1} V\right)\left(\frac{i z}{\cos \gamma_{-}}\right)+S_{0} \cos \gamma_{-}\left[S_{\alpha} l_{3}+\frac{\cos \gamma}{\cos \gamma_{+}} i T_{\alpha}^{0, \tau} l_{1}-T_{\alpha}^{1, \tau} l_{2}\right] .
\end{aligned}
$$


We notice that $l_{1}, l_{2}, l_{3}$ belong to $L$, hence the terms $s t_{+}$and $s t_{-}$belong to $L$ if $\mathcal{F}\left(2 \partial_{ \pm}^{1} V\right)\left(\frac{i z}{\cos \gamma_{ \pm}}\right)$belong to $L$, which is equivalent to $\partial_{ \pm}^{1} V$ belong to $L^{2}\left(\mathbb{R}_{+}\right)$. We denote by $e_{ \pm}$the distributions of $L^{2}\left(\mathbb{R}_{+}\right)$such that

$$
s t_{ \pm}(z)=-2 \mathcal{F}\left(2 \partial_{ \pm}^{1} V\right)\left(\frac{i z}{\cos \gamma_{ \pm}}\right)+S_{0} \mathcal{F}\left(e_{ \pm}\right)\left(\frac{i z}{\cos \gamma_{ \pm}}\right) .
$$

This analysis being done, assume that $l_{+}$and $l_{-}$are given in $L^{2}\left(\mathbb{R}_{+}\right)$. We consider the distributions $\Phi_{ \pm}=l_{ \pm}-S_{0} e_{ \pm}$. They belong to $L^{2}\left(\mathbb{R}_{+}\right)$. From Lemma 2.7, the system (42) admits a solution $V \in C_{\theta}\left(\mathbb{R} / 2 \pi \mathbb{Z}, H^{1}\left(\mathbb{R}_{+}\right)\right)$which is continuous. We compute $\Psi_{+}=\partial_{+} V-\partial_{+}^{1} V-i \tau \sin \theta_{+} e^{-i \alpha} l(x / R) 1_{x \geqslant 0} S_{0}, \Psi_{-}=\partial_{-} V-\partial_{-}^{1} V$, $\Phi_{+}=\left.V\right|_{\Delta_{+},+}-\left.V\right|_{\Delta_{+},-}-S_{0} l(x / R) 1_{x \geqslant 0}, \Phi_{-}=\left.V\right|_{\Delta_{-},+}-\left.V\right|_{\Delta_{-},-}$. These distributions belong respectively to $L^{2}\left(\mathbb{R}_{+}\right), L^{2}\left(\mathbb{R}_{+}\right), H^{1}\left(\mathbb{R}_{+}\right), H^{1}\left(\mathbb{R}_{+}\right)$and, in addition, $\Phi_{+}(0)$ $\Phi_{-}(0)=-S_{0}$. Hence, according to (35), the Mellin transform of $\left(\psi_{+}, \psi_{-}, \Phi_{+}, \Phi_{-}\right)$is in $L \times L \times L^{1} \times L^{1}$ and we have the relation of compatibility

$$
\widetilde{\phi}_{+}(z)+\frac{z}{\cos \gamma_{+}} \phi_{+}(z)=\widetilde{\phi}_{-}(z)+\frac{z}{\cos \gamma_{-}} \phi_{-}(z)+S_{0} .
$$

The proof of Corollary 2.2 is complete. 



\section{CHAPTER 3}

\section{MICROLOCAL PROPAGATION OF SINGULARITIES FOR THE MIXED PROBLEM}

The aim of this Section is to generalize the 2-microlocal regularity result that Gérard and Lebeau obtained for the Dirichlet problem to a mixed boundary condition satisfying the strong Lopatinskii condition. Throughout this Section, we will follow the notations of $[\mathbf{2 0}]$. The proof follows step by step the proof of Gérard and Lebeau, but the result is not a consequence of their result.

We denote, in this section only, the normal coordinate ${ }^{(1)}$ to the face $\widetilde{\Delta}_{+}$of the wedge by $t$, and the "tangential" variables, including the time (denoted by $x_{2}$ ), by $x$. The same presentation may be done for the diffraction by the face -. In the case useful for the propagation in $\mathbb{R}^{2} \times \mathbb{R}_{t}$, we may have $d=2$, and the boundary is of dimension 2. In the semi-geodesic system of coordinates $(t, x)[\mathbf{2 6}]$, the wave operator can be written (up to a conjugation, using the traditional notation $D_{t}=\frac{1}{i} \partial_{t}$ ):

$$
P=D_{t}^{2}+R\left(t, x, D_{x}\right)
$$

where, the boundary $\widetilde{\Delta}_{ \pm}$being analytic, the coefficients of $R$, differential operator, are analytic. We consider in this problem the mixed boundary condition

$$
\partial_{t} u+L u=0
$$

where $L$ is a tangential differential operator of order 1 .

The boundary conditions studied in this paper corresponds to $L=z^{ \pm}\left(x_{1}\right) \partial_{x_{2}}$, $x_{1}$ is the variable on $t=0, x_{2}$ is the time variable, and $t$ is the normal variable to the boundary. The result we prove is true only in the neighborhood of points where the operator $\partial_{t}+L$ is elliptic thus invertible. However, if we return to the boundary conditions used in this paper, we verify that the conditions $\Im z^{+}(0) \neq 0$ and $\Im z^{-}(0) \neq 0$ imply that the operator $\partial_{t}+z^{ \pm} \partial_{x_{2}}$ is always elliptic if $\xi_{2} \neq 0$.

The model case of a constant impedance and a straight boundary. - The model case is the wave equation in the upper half plane. The time variable is $x_{2}$, the variable on the boundary $\Delta=\{t=0\}$ is denoted by $x_{1}$, and the wave operator is

$$
\left(D_{t}\right)^{2}+\left(D_{x_{1}}\right)^{2}-\left(D_{x_{2}}\right)^{2} \text {. }
$$

${ }^{(1)}$ denoted by $n$ in the introduction 
The boundary condition is $\left(\partial_{t}+\alpha \partial_{x_{2}}\right) u\left(0, x_{1}, x_{2}\right)=0, \alpha \in \mathbb{R}$.

Let $u$ be a solution in $t>0$ of the wave equation with the boundary condition. The energy of $u$ is $E(u)\left(x_{2}\right)=\int_{t \geqslant 0}\left[\left(D_{t} u\right)^{2}+\left(D_{x_{1}} u\right)^{2}+\left(D_{x_{2}} u\right)^{2}\right] \mathrm{d} x_{1} \mathrm{~d} t$, and we have $\partial_{x_{2}}(E(u))\left(x_{2}\right)=\alpha \int\left(\partial_{x_{2}} u\left(0, x_{1}, x_{2}\right)\right)^{2} \mathrm{~d} x_{1}$. Hence the energy decreases if and only if $\alpha<0$. The condition $\alpha<0$ leads to a well posed problem.

Now, let us consider an incoming wave $u_{i}\left(x_{1}, x_{2}, t\right)=e^{i\left(\tau t+\xi_{1} x_{1}+\xi_{2} x_{2}\right)}$. We have $u_{i}\left(x_{1}, x_{2}, t\right)=e^{i \xi_{2}\left[x_{2}+\frac{\tau}{\xi_{2}} t+\frac{\xi_{1}}{\xi_{2}} x_{1}\right]}$. Hence the wave front of this plane wave is $x_{2}=$ $-\frac{\tau}{\xi_{2}} t-\frac{\xi_{1}}{\xi_{2}} x_{1}$. This plane wave is incoming when $x_{2}$ increasing implies $t$ decreasing. Hence $\tau / \xi_{2}>0$.

Let $u_{r}$ the reflected wave induced by $u_{i}$. We have $u_{r}\left(x_{1}, x_{2}, t\right)=A e^{-i \tau t+i \xi_{1} x_{1}+i \xi_{2} x_{2}}$ and $u=u_{i}+u_{r}$. Hence $A$ is solution of $\tau-\tau A+\alpha\left(\xi_{2}+A \xi_{2}\right)=0$, hence we have $\left(-\frac{\tau}{\xi_{2}}+\alpha\right) A=\alpha+\tau / \xi_{2}$. For all the points $\rho_{0}=\left(0, x_{2}^{0}, \xi_{1}^{0}, \xi_{2}^{0}\right)$ of $T^{*}(\partial \Omega)$ such that $\tau^{2}+r\left(0,0, x_{2}^{0}, \xi_{1}^{0}, \xi_{2}^{0}\right)=0$ satisfying the inequality $\left|-\frac{\tau}{\xi_{2}^{0}}+\alpha\right| \geqslant \varepsilon_{0}$, we can compute the reflection coefficient and we shall see that the Théorème 3.1 of $[\mathbf{2 0}]$ is true in this case.

We modify the proof of Théorème 3.1 of $[\mathbf{2 0}]$ as follows. As usual, the cotangent bundle to the boundary $T^{*}(\{t=0\})$ is the union of the elliptic set $\mathcal{E}$, the hyperbolic set $\mathcal{H}$, and of the glancing set $\mathcal{G}$. If $r_{0}(x, \xi)$ is the restriction to $t=0$ of the principal symbol $r(t, x, \xi)$ of $R$, we have

$$
\mathcal{E}=\left\{(x, \xi), r_{0}(x, \xi)>0\right\}, \quad \mathcal{H}=\left\{(x, \xi), r_{0}(x, \xi)<0\right\}, \quad \mathcal{G}=\left\{(x, \xi), r_{0}(x, \xi)=0\right\} .
$$

We prove a second microlocalisation theorem along an involutive submanifold of $T^{*}(\{t=0\})$ under the only assumption that this submanifold is transverse to the Hamiltonian field.

We introduce, in a neighborhood of $\rho_{0}=\left(x_{0}, \xi_{0}\right) \in T^{*}(\{t=0\})$, an analytic real function $s(x, \xi)$ such that $s(x, \lambda \xi)=\lambda s(x, \xi), s\left(\rho_{0}\right)=0, d s\left(\rho_{0}\right) \wedge \xi_{0} \mathrm{~d} x \neq 0$, $\left\{r_{0}, s\right\}\left(\rho_{0}\right) \neq 0$. The submanifold $V=s^{-1}(0)$ is an involutive submanifold in $T^{*}(\{t=$ $0\})$, transverse in $\rho_{0}$ to the Hamiltonian vector field $H_{r_{0}}$. We introduce $\operatorname{SS}_{V}^{2,1}(f)$ the second microlocal analytic wave front set of $f$ along $V([\mathbf{2 8}])$. We have

$$
\left(\rho_{0}, \pm 1\right) \notin \mathrm{SS}_{V}^{2,1}(f) \Longrightarrow \mathrm{SS}(f) \cap\{ \pm s>0\} \cap W_{\rho_{0}}=\varnothing .
$$

Notice that \pm 1 indicates the "sense of propagation of $\operatorname{SS}(f)$ along $V$ " in the neighborhood of $\rho_{0}$. The generalized bicharacteristics of $P$ are constructed as usual (see [26] for example). We introduce $\epsilon=\left\{r_{0}, s\right\}\left(\rho_{0}\right) /\left|\left\{r_{0}, s\right\}\left(\rho_{0}\right)\right|$.

Recall the definition of $f V$-outgoing in $\rho_{0}$ (definition $3.4 \mathrm{p} 364$ of [20]):

Definition 3.1. - The distribution $f(t, x)$ in $\{t>0\}$, extendible distribution, solution of $P f=0$, is $V$-outgoing in $\rho_{0}$ when one of the following conditions is fulfilled:

i) $\rho_{0} \in \mathcal{E}$

ii) $\rho_{0} \in \mathcal{H}$ and if $\gamma_{ \pm}$are the two half-bicharacteristic curves of $p$ (principal symbol of $P$ ) passing through $\left(t=0, x_{0}, \pm \sqrt{-r_{0}\left(x_{0}, \xi_{0}\right)}, \xi_{0}\right)$ and contained in $T^{*}(\{t>0\})$, 
we have

$$
\gamma_{+} \cap \mathrm{SS}(f)=\varnothing \quad \text { or } \quad \gamma_{-} \cap \mathrm{SS}(f)=\varnothing .
$$

iii) $\rho_{0} \in \mathcal{G}$ and there exists $\varepsilon>0$ such that the conditions $0 \leqslant t<\varepsilon,\left|\rho-\rho_{0}\right| \leqslant \varepsilon$, $s(\rho)<0$ imply $(\rho, t, \tau) \notin \mathrm{SS}_{b}(f)$.

This definition has to be compared with the definition of [25], where the ray was, at the glancing point, tangent at the second order, and where we chose one of the two characteristics $\gamma_{+}$or $\gamma_{-}$. This definition of microlocally outgoing coincides with the definition given by R. Melrose in [32].

Théorème 3.1 p. 364 of [20] generalizes to the following theorem:

TheOREM 3. - If $f$ is a distribution on $M=\mathbb{R}_{t}^{+} \times \mathbb{R}_{x}$ such that $P f=0$ and $f V$-outgoing, then, for $\rho_{0} \in \mathcal{H} \cup \mathcal{G}$, such that $L\left(\rho_{0}\right)+\left(-r_{0}\left(0, \rho_{0}\right)\right)^{1 / 2} \neq 0$ and $L\left(\rho_{0}\right)-\left(-r_{0}\left(0, \rho_{0}\right)\right)^{1 / 2} \neq 0$, we have

$$
\left(\rho_{0},-1\right) \notin \operatorname{SS}_{V}^{2,1}\left(\left.\left(\partial_{t} f+L f\right)\right|_{t=0}\right) \Longrightarrow\left(\rho_{0},-1\right) \notin \operatorname{SS}_{V}^{2,1}\left(\left.\partial_{t} f\right|_{t=0}\right) .
$$

If $\rho_{0}$ is in $\mathcal{E}$, there exists a pseudodifferential operator of order 0 such that $\rho_{0} \notin \mathrm{SS}\left(\partial_{t} u-\mathcal{C}\left(\partial_{t} u+L u\right)\right)$. For $\rho_{0} \in \mathcal{H}$, we denote by $\rho_{0}^{+}$and $\rho_{0}^{-}$the two points of $\left\{(0, x, \tau, \xi), \tau^{2}+r(0, x, \xi)=0\right\}$ such that the projection $\left(x_{0}, \xi_{0}\right)$ of this point on $T^{*}(\partial \Omega)$ is $\rho_{0}$. These two points are $\left(0, x_{0},\left(-r\left(0, x_{0}, \xi_{0}\right)\right)^{1 / 2}, \xi_{0}\right)$ and $\left(0, x_{0},-\left(-r\left(0, x_{0}, \xi_{0}\right)\right)^{1 / 2}, \xi_{0}\right)$.

As $f$ is $V$-outgoing, $\gamma_{+} \cap \mathrm{SS}(f)=\varnothing$ or $\gamma_{-} \cap \mathrm{SS}(f)=\varnothing$. Assume (for instance) that $\gamma_{+} \cap \mathrm{SS}(f)=\varnothing$. We have thus $\gamma_{-} \subset \mathrm{SS}(f)$. For

$$
\left(-r_{0}\left(\rho_{0}\right)\right)^{1 / 2}+L\left(\rho_{0}\right) \neq 0
$$

there exists a pseudodifferential operator of order 0 , in the neighborhood of $\rho_{0}$, called $\mathcal{C}$, such that

$$
\rho_{0} \notin \mathrm{SS}\left(\partial_{t} u-\mathcal{C}\left(\partial_{t} u+L u\right)\right) .
$$

In a similar fashion, this operator $\mathcal{C}$ is microlocally defined at $\rho_{0}$ for $\gamma_{-} \cap \operatorname{SS}(f)=\varnothing$ and the condition $L\left(\rho_{0}\right)-\left(-r_{0}\left(\rho_{0}\right)\right)^{1 / 2} \neq 0$. This operator is the Calderòn operator (see $[\mathbf{2 6}])$. This operator is defined under the condition :

$$
L\left(\rho_{0}\right) \pm\left(-r_{0}(\rho)\right)^{1 / 2} \neq 0 .
$$

We use then the fact that pseudodifferential operators are 2-microlocal operators to prove Theorem 3. Assume from now that $\rho_{0} \in \mathcal{G}$. There exists a canonical tangential transform $\chi$, from a neighborhood of $\rho_{0}$ in $T^{*}\left(\mathbb{C}^{d}\right)$ to a neighborhood of 0 in $T^{*}\left(\mathbb{C}^{d}\right)$ such that

i) $\chi\left(T^{*}\left(\mathbb{R}^{d}\right)\right)=\Lambda_{\phi_{0}}$, where the phase function $\phi_{0}(z)$ is

$$
\phi_{0}(z)=\frac{1}{2}\left(\Re z_{1}\right)^{2}+\frac{1}{2} \sum_{j=2}^{j=d}\left(\Im z_{j}\right)^{2} .
$$


This implies that

$$
\Lambda_{\phi_{0}}=\left\{\left(z_{1}, z^{\prime},-i \Re z_{1},-\Im z^{\prime}\right)\right\}
$$

ii) the coordinate $z_{1}$ is the "good choice" of symplectic coordinates for $r_{0}$ and $s$, that is for $\epsilon= \pm 1$

$$
\begin{aligned}
\epsilon r_{0} \circ \chi^{-1}(z, \zeta) & =\zeta_{1}+i z_{1} \\
s \circ \chi^{-1}(z, \zeta) & =i \zeta_{1} l_{0}(z, \zeta),\left.\quad l_{0}\right|_{\Lambda_{\phi_{0}}}>0 .
\end{aligned}
$$

iii) $\chi\left(\rho_{0}\right)=0$.

Let $T_{\chi}$ be a Fourier-Bros-Iagolnitzer transform associated with the canonical transform $\chi$ quantized by the analytic phase function $g(z, x)$. The graph of $\chi$ is thus $\left\{\left(x,-g_{x}^{\prime}, z, g_{z}^{\prime}\right)\right\}$, and we have $g\left(0, x_{0}\right)=0$, $\operatorname{det} g_{z x}^{\prime \prime}\left(0, x_{0}\right) \neq 0$.

For $\psi \in C_{0}^{\infty}\left(\mathbb{R}^{d}\right)$, supported in a neighborhood of $x_{0}$, identically equal to 1 near $x_{0}$, we may define

$$
T_{\chi} h(z, \lambda)=\int e^{i \lambda g(z, x)} h(x) \psi(x) \mathrm{d} x .
$$

Let $f$ the function considered in Definition 3.1. We introduce

$$
u(t, z, \lambda)=T_{\chi}(x \mapsto f(t, x))(z, \lambda) .
$$

We consider the algebra of operators in the complex plane used by Gérard and Lebeau, and for the reader's convenience, we reproduce here some of the definitions and results of Section 3.2 of [20].

Let $I=\left[0, t_{0}\right], U$ an open subset in $T^{*}\left(\mathbb{C}^{d}\right), K$ a compact subset of $\mathbb{C} / \mathbb{R}$. We introduce $A^{m}(I, U, K)$ the space of formal series $e=\sum_{n \geqslant 0}(i \lambda)^{-n} e_{n}$ such that $e_{n}(t, \tau, z, \zeta, \lambda)$ is holomorphic for $t \in V$, complex neighborhood of $I,(z, \zeta) \in U$ and such that

$$
\left|e_{n}(t, \tau, z, \zeta, \lambda)\right| \leqslant A B^{n} n !(1+|\tau|)^{m} .
$$

We associate to $e$ the sequence of holomorphic functions

$$
k\left(e, \zeta_{0} ; n, \alpha\right)=\frac{\lambda}{2 \pi} \int_{-\infty}^{+\infty} e^{i \lambda(t-s) \tau} \frac{1}{\alpha !} \partial_{\xi}^{\alpha} e_{n}\left(t, \tau, z, \zeta_{0}, \lambda\right) \mathrm{d} \tau .
$$

If $m \geqslant 0$, there exists a decomposition

$$
e(t, \tau, z, \zeta, \lambda)=\sum_{j=0}^{j=m} e_{n}^{j}(t, z, \zeta, \lambda) \tau^{j}+e_{n}^{-}(t, \tau, z, \zeta, \lambda)
$$

where $e_{n}^{-} \in A^{-1}(I, U, K)$ and each $e_{n}^{j}$ is defined by

$$
e_{n}^{j}(t, z, \zeta, \lambda)=\frac{\partial_{\tau^{j}}^{j} e(t, 0, z, \zeta, \lambda)}{j !}
$$

and $e_{n}^{-}$is the remainder term of the Taylor series, equal to

$$
\tau^{m+1} \int_{0}^{1} \frac{(1-s)^{m}}{m !} \partial_{\tau^{m+1}}^{m+1} e(t, \tau s, z, \zeta, \lambda) \mathrm{d} s .
$$


For $e \in A^{m}(I, U, K), m<0$, we define

$$
k^{ \pm}\left(e, \zeta_{0} ; n, \alpha\right)(t, s, z, \lambda)=\left.k\left(e, \zeta_{0} ; n, \alpha\right)(t, s, z, \lambda)\right|_{ \pm(t-s)>0},
$$

which extends up to $t=s$. We have, for $\int_{0}^{t_{0}} k\left(e, \zeta_{0} ; n, \alpha\right)(t, s, z, \lambda) v(s, z, \lambda) \mathrm{d} s$, the following definition: for $m \geqslant 0$,

$$
\begin{aligned}
& \int_{0}^{t_{0}} k\left(e, \zeta_{0} ; n, \alpha\right)(t, s, z, \lambda) v(s, z, \lambda) \mathrm{d} s=\int_{0}^{t} k^{+}\left(e, \zeta_{0} ; n, \alpha\right)(t, s, z, \lambda) v(s, z, \lambda) \mathrm{d} s \\
+ & \int_{t}^{t_{0}} k^{-}\left(e, \zeta_{0} ; n, \alpha\right)(t, s, z, \lambda) v(s, z, \lambda) \mathrm{d} s+\sum_{j=0}^{j=m}(i \lambda)^{-j} \frac{1}{\alpha !} \partial_{\xi}^{\alpha} e_{n}^{j}\left(t, z, \zeta_{0}, \lambda\right) \partial_{t}^{j} v(t)
\end{aligned}
$$

The definition for $m<0$ is obtained by putting the finite sum as empty. We associate to $e \in A^{m}(I, U, K)$ the differential operator

$$
\begin{aligned}
& \mathrm{Op}(e)\left(\zeta_{0}, C_{1}, C_{2}\right) u(t, z, \lambda)= \\
& \sum_{\substack{n \leqslant \lambda / C_{1} \\
|\alpha| \leqslant \lambda / C_{2}}}(i \lambda)^{-n} \int_{0}^{t_{0}} k\left(e, \zeta_{0} ; n, \alpha\right)(t, s, z, \lambda)\left(\frac{1}{i \lambda} \partial_{z}-\zeta_{0}\right)^{\alpha} u(s, z, \lambda) \mathrm{d} s .
\end{aligned}
$$

We associate also the operator

$$
K^{ \pm}(t, s)\left(\zeta_{0}, C_{1}, C_{2}\right)=\sum_{\substack{n \leqslant \lambda / C_{1} \\|\alpha| \leqslant \lambda / C_{2}}}(i \lambda)^{-n} k^{ \pm}\left(e, \zeta_{0} ; n, \alpha\right)(t, s, z, \lambda)\left(\frac{1}{i \lambda} \partial_{z}-\zeta_{0}\right)^{\alpha}
$$

We note that we have the estimate, owing to the estimates on $e_{n}$, and to the fact that $K$ is a compact of $\mathbb{C}-\mathbb{R}$, which implies that $\operatorname{dist}(K, \mathbb{R})>0$ :

$$
\left|\partial_{s, t}^{\beta} k^{ \pm}\left(e, \zeta_{0} ; n, \alpha\right)(t, s, z, \lambda)\right| \leqslant C_{\beta} \lambda^{|\beta|+1} B^{n} R_{0}^{-|\alpha|} n ! e^{-\frac{\lambda}{2}|t-s| \operatorname{dist}(K, \mathbb{R})} .
$$

These differential operators satisfy the following results

Lemma 3.1. - Let $R_{0}>D>0$ such that

$$
\sup _{z \in \omega}\left\|\frac{2}{i} \frac{\partial \phi}{\partial z}-\zeta_{0}\right\| \leqslant D
$$

If $\omega^{\prime}$ is a subset of $\omega$ such that $\operatorname{dist}\left(\bar{\omega}^{\prime}, C \omega\right) \neq 0$, we introduce $C_{0}=1 / D \operatorname{dist}\left(\bar{\omega}^{\prime}, C \omega\right)$. For $C_{1} \geqslant 2 B / e, C_{2} \geqslant C_{0}, u \in C^{\infty}\left(I, H_{\phi}(\omega)\right)$, we have

$$
\operatorname{Op}(e)\left(\zeta_{0}, C_{1}, C_{2}\right)(u) \in C^{\infty}\left(I, H_{\phi}\left(\omega^{\prime}\right)\right) .
$$

Moreover, if $C_{1}^{\prime} \geqslant 2 B / e, C_{2}^{\prime} \geqslant C_{0}$, there exists $\varepsilon>0$ such that

$$
\operatorname{Op}(e)\left(\zeta_{0}, C_{1}, C_{2}\right)(u)-\mathrm{Op}(e)\left(\zeta_{0}, C_{1}^{\prime}, C_{2}^{\prime}\right)(u) \in C^{\infty}\left(I, H_{\phi-\varepsilon}\left(\omega^{\prime}\right)\right) .
$$

Proof. - See [20], p. 358 (Lemma 3.1). 
LEMma 3.2. - Let $\omega, D, R_{0} \omega^{\prime}$ be as in Lemma 3.1, and $C_{0}$ the constant obtained in Lemma 3.1.

For $\left(\zeta_{0}^{\prime}, R_{0}^{\prime}, D^{\prime}\right)$ such that $\omega \times\left\{\left|\zeta_{0}^{\prime}-\zeta\right| \leqslant R_{0}^{\prime}\right\} \subset U, \sup _{\omega}\left|\frac{2}{i} \partial_{z} \phi-\zeta_{0}^{\prime}\right|<D^{\prime}<R_{0}^{\prime}$, we introduce $C_{0}^{\prime}=1 / D^{\prime} \operatorname{dist}\left(\omega^{\prime}, C \omega\right)$ and for all $C_{1}, C_{1}^{\prime}$ greater than $2 B / e, C_{2} \geqslant C_{0}$, $C_{2}^{\prime} \geqslant C_{0}^{\prime}$, there exists $\varepsilon>0$ such that

$$
\operatorname{Op}(e)\left(\zeta_{0}, C_{1}, C_{2}\right)(u)-\mathrm{Op}(e)\left(\zeta_{0}^{\prime}, C_{1}^{\prime}, C_{2}^{\prime}\right)(u) \in C^{\infty}\left(I, H_{\phi-\varepsilon}\left(\omega^{\prime}\right)\right) .
$$

Proof. — See [20], p. 359 (Lemma 3.2).

Lemma 3.3. - Let $\omega^{\prime} \subset \omega$. There exists $C_{1}^{0}, C_{2}^{0}$, such that, for all $C_{1} \geqslant C_{1}^{0}, C_{2} \geqslant$ $C_{2}^{0}$, there exists $\varepsilon>0$ such that, for all $u \in C^{\infty}\left(I, H_{\phi}(\omega)\right)$,

$\operatorname{Op}(e \circ q)\left(\zeta_{0}, C_{1}, C_{2}\right)(u)-\mathrm{Op}(e)\left(\zeta_{0}, C_{1}, C_{2}\right)\left[\mathrm{Op}(q)\left(\zeta_{0}, C_{1}, C_{2}\right)(u)\right] \in C^{\infty}\left(I, H_{\phi-\varepsilon}\left(\omega^{\prime}\right)\right)$

where the composition calculus is the usual one, $g=e \circ q$ with

$$
g_{n}(t, \tau, z, \zeta, \lambda)=\sum_{n^{\prime}+n^{\prime \prime}+|\alpha|+l=n} \frac{1}{l ! \alpha !} \partial_{\tau}^{l} \partial_{\zeta}^{\alpha} e_{n^{\prime}} \partial_{t}^{l} \partial_{z}^{\alpha} q_{n^{\prime \prime}}(t, z, \zeta, \lambda)
$$

Proof. - See [20], Lemma 3.3 p. 361.

We notice that the differential operator $(i \lambda)^{-1} \partial_{z_{j}}$, whose symbol is $\zeta_{j}$ in the analytic calculus of Sjöstrand, is associated with $e_{0}=\zeta_{j}, e_{n}=0$ for $n>0$ in the definition above. If $L$ is a pseudodifferential operator of order 1 , the symbol in this calculus of the operator $\frac{1}{i \lambda} L$ is the symbol $l(z, \zeta)=L \circ \chi^{-1}(z, \zeta)=L\left(\chi^{-1}(z, \zeta)\right)$ such that $l(\chi(x, \xi))=L(x, \xi)$.

There exists an analytic symbol $q$ defined in a neighborhood of $t \in\left[0, t_{0}\right],\left(z_{0}, \zeta_{0}\right)=$ $(0,0)$, of the form

$$
q(t, z, \zeta, \lambda)=\sum_{n \geqslant 0}(i \lambda)^{-n} q_{n}(t, z, \zeta)
$$

such that $q_{0}(t, \chi(x, \xi))=r(t, x, \xi)$ and that

$$
\lambda^{2} \operatorname{Op}(q)\left(0, C_{1}, C_{2}\right)\left(e^{i \lambda g(z, x)}\right)={ }^{t} R\left(t, x, D_{x}\right)\left(e^{i \lambda g(z, x)}\right)+e^{i \lambda g(z, x)} b(t, x, z, \lambda)
$$

where the symbol $b$ is analytic near $(t, x, 0)$ and is bounded by $e^{-\epsilon^{\prime} \lambda}$ for an $\epsilon^{\prime}>0$. We introduce $\phi_{-}(z)$ the phase function equal to

$$
\phi_{-}(z)= \begin{cases}\frac{1}{2}\left(\Re z_{1}\right)^{2}+\frac{1}{2} \sum_{j=2}^{j=d}\left(\Im z_{j}\right)^{2}, & \text { if } \Re z_{1} \geqslant 0, \\ \frac{1}{2} \sum_{j=2}^{j=d}\left(\Im z_{j}\right)^{2}, & \text { if } \Re z_{1}<0 .\end{cases}
$$

Consider $f$ the function considered in Definition 3.1. The condition iii) of this definition implies that there exists $\varepsilon>0$ such that

$$
\mathrm{SS}(f) \cap\left\{0<t \leqslant \varepsilon / 2,\left|\rho-\rho_{0}\right| \leqslant \varepsilon / 2, s(\rho) \leqslant 0\right\}=\varnothing .
$$


Let $\left.t_{0} \in\right] 0,1[$. As $f$ is an extendible solution of $P f=0$, where $P$ is a partially hypoelliptic operator, there exists $\nu_{0} \in \mathbb{R}$ such that, for all $k \geqslant 0, f \in$ $C^{k}\left(\left[0, t_{0}\right], H^{\nu_{0}-k}(\{|x| \leqslant a / 2\})\right)$.

Hence, by the properties of the Fourier-Bros-Iagolnitzer transform, we may choose a neighborhood $\Omega_{0}$ of $z_{0}=0$ such that $u \in C^{\infty}\left(\left[0, t_{0}\right], H_{\phi_{0}}\left(\Omega_{0}\right)\right)$.

We have $\left(\rho_{0},-1\right) \notin \operatorname{SS}_{V}^{2,1}\left(\partial_{t} f+\left.L f\right|_{t=0}\right)$, which implies that there exists $\Omega_{0}$ small enough such that

$$
\forall z \in \Omega_{0}, \quad\left|\partial_{t} u(0, z, \lambda)+\operatorname{Op}\left(L \circ \chi^{-1}\right)(u(0, z, \lambda))\right| \leqslant A \lambda^{M+1} e^{\lambda \phi_{-}(z)} .
$$

As $u$ is a solution of the problem, there exists $\varepsilon_{0}$ and $\Omega_{1}$ compact subset of $\Omega_{0}$ such that

$$
\mathrm{Op}\left(\tau^{2}+q\right)\left(0, C_{1}, C_{2}\right) u \in C^{\infty}\left(\left[0, t_{0}\right], H_{\phi_{0}-\varepsilon_{0}}\left(\Omega_{1}\right)\right) .
$$

We choose $t_{0}>0$ and we fix $d_{1}>0$ such that

$$
\begin{aligned}
|z| \leqslant 2 d_{1} & \Longrightarrow z \in \Omega_{1} \\
|z| \leqslant 2 d_{1}, \Re z_{1}<0 & \Longrightarrow \exists \varepsilon_{2}>0, u \in C^{\infty}\left(\left[0, t_{0}\right], H_{\phi_{0}-\varepsilon_{2}}\left(\Omega_{0}\right)\right) \\
|z| \leqslant 2 d_{1}, \Re z_{1} \leqslant 0, t_{0}^{\prime}>0 & \Longrightarrow \exists \varepsilon_{3}>0, u \in C^{\infty}\left(\left[t_{0}^{\prime}, t_{0}\right], H_{\phi_{0}-\varepsilon_{3}}\left(\Omega_{0}\right)\right) .
\end{aligned}
$$

Let $\alpha>0$ be given. There exists a function $\theta_{\alpha} \in C^{\infty}\left(\left[-d_{1},+\infty[)\right.\right.$ and three numbers $s_{1}(\alpha), s_{2}(\alpha), s_{3}(\alpha)$ such that $-d_{1}<s_{3}(\alpha)<s_{2}(\alpha)<s_{1}(\alpha)<0$ with the following properties :

$$
\begin{gathered}
\sup \left(0, \frac{s^{2}}{2}-\alpha\right) \leqslant \theta_{\alpha}(s) \leqslant \frac{s^{2}}{2} ; \quad s+\alpha \geqslant \theta_{\alpha}^{\prime}(s) \geqslant s \\
\theta_{\alpha}(s)=\frac{s^{2}}{2}, s \geqslant s_{1}(\alpha) ; \quad \theta_{\alpha}^{\prime}(s)>s,-d_{1} \leqslant s<s_{1}(\alpha) ; \quad \theta_{\alpha}(s)=0, s \in\left[s_{3}(\alpha), s_{2}(\alpha)\right] .
\end{gathered}
$$

Let also introduce a function $\Phi \in C^{\infty}\left(\mathbb{R}, \mathbb{R}_{+}\right)$, such that $\Phi$ is increasing, $\Phi\left(d_{1}\right)>d_{1}$, $\Phi(t)=0$ for $t \leqslant d_{1} / 4$. Using $\Phi, \widetilde{\theta}_{\alpha}(s)=\theta_{\alpha}(s)-s^{2} / 2$ and $\phi_{0}$ we construct $\phi$ (as in $[\mathbf{2 0}])$ for $\left|\Re z_{1}\right| \leqslant d_{1}$ and $\left.\left(\left(\Im z_{1}\right)^{2}+\left|z^{\prime}\right|^{2}\right)\right)^{1 / 2} \leqslant d_{1}$ :

$$
\phi(z)=\phi_{0}(z)+\widetilde{\theta}_{\alpha}\left(\Re z_{1}+\Phi\left(\left(\left(\Im z_{1}\right)^{2}+\left|z^{\prime}\right|^{2}\right)^{1 / 2}\right)\right) .
$$

This is a phase function satisfying :

$$
\phi(z) \leqslant \phi_{0}(z) ; \quad \phi(z)=\phi_{0}(z) \text { on } \Re z_{1}+\Phi(y(z)) \geqslant s_{1}(\alpha) .
$$

Let $y(z)=\left(\left(\Im z_{1}\right)^{2}+\left|z^{\prime}\right|^{2}\right)^{1 / 2}$. We assume that $\phi$ is the phase function associated with the canonical transform, such that

$$
\frac{2}{i} \frac{\partial \phi}{\partial z^{\prime}}=\zeta^{\prime}, \quad \frac{2}{i} \frac{\partial \phi}{\partial z_{1}}=\zeta_{1} .
$$

We deduce from this equality the relation

$$
\zeta_{1}=-i \Re z_{1}+\widetilde{\theta}_{\alpha}^{\prime}\left(\Re z_{1}+\Phi(y(z))\right) \frac{1}{i}\left(1-i \Phi^{\prime}(y(z)) \frac{\Im z_{1}}{y(z)}\right) .
$$

This equality implies $\Im\left(\zeta_{1}+i z_{1}\right)=-\widetilde{\theta}_{\alpha}^{\prime}\left(\Re z_{1}+\Phi(y(z))\right) \leqslant 0$. 
Recall that $\frac{2}{i} \frac{\partial \phi_{0}}{\partial z}=-\Im z^{\prime}$. We introduce, for $0<\delta<1$, the phase function $\phi_{\delta}=\delta \phi+(1-\delta) \phi_{0}$.

We have the following

Lemma 3.4. - Let $|\nabla \Phi|_{\infty}=\sup \left\{\left|\Phi^{\prime}(y)\right|,|y| \leqslant d_{1}\right\}$. There exists $D>0$, independent of $\alpha$, such that, for $0<\delta<1$,

$$
\epsilon \Im q_{0}\left(t, z, \frac{2}{i} \frac{\partial \phi_{\delta}}{\partial z}\right) \leqslant \delta \Im\left(i z_{1}+\zeta_{1}\right)[1-D \delta(1+|\nabla \Phi|) t]
$$

Proof. - Note that

$$
\frac{2}{i} \frac{\partial \phi_{\delta}}{\partial z^{\prime}}-\frac{2}{i} \frac{\partial \phi_{0}}{\partial z^{\prime}}=\delta\left(\frac{2}{i} \frac{\partial \phi}{\partial z^{\prime}}-\frac{\partial \phi_{0}}{\partial z^{\prime}}\right)=\delta\left[\widetilde{\theta}_{\alpha}\left(\Re z_{1}+\Phi(y(z))\right)-\Re z_{1}+\Phi(y(z))\right] \frac{2}{i} \partial_{\bar{z} j} \Phi .
$$

Hence we obtain

which leads to the inequality

$$
\frac{2}{i} \frac{\partial \phi_{\delta}}{\partial z}-\frac{2}{i} \frac{\partial \phi_{0}}{\partial z}=\delta\left[-\Im\left(\zeta_{1}+i z_{1}\right)\right] \frac{2}{i} \partial_{\bar{z} j} \Phi^{\prime}
$$

$$
\left|\frac{2}{i}\left(\partial_{z^{\prime}} \phi_{\delta}-\partial_{z^{\prime}} \phi_{0}\right)\right| \leqslant 2 d_{1} \delta\left\|\Phi^{\prime}\right\|_{\left[-d_{1}, d_{1}\right]}\left|\Im\left(\zeta_{1}+i z_{1}\right)\right| .
$$

We have

$\frac{2}{i}\left(\partial_{z_{1}} \phi_{\delta}-\partial_{z_{1}} \phi_{0}\right)=\delta\left(\frac{2}{i} \partial_{z_{1}} \phi-\frac{2}{i} \partial_{z_{1}} \phi_{0}\right)=\delta\left(-i \Re z_{1}+i \Re z_{1}\right)+\delta\left[-\Im\left(\zeta_{1}+i z_{1}\right)\right] \frac{1}{i}\left(1-i \Phi^{\prime}\right)$.

Hence we obtain

$$
\left|\frac{2}{i}\left(\partial_{z_{1}} \phi_{\delta}-\partial_{z_{1}} \phi_{0}\right)\right| \leqslant \delta\left|\Im\left(\zeta_{1}+i z_{1}\right)\right|(1+|\nabla \Phi|) .
$$

Using the equality $\epsilon r_{0}\left(\chi^{-1}(z, \zeta)\right)=\zeta_{1}+i z_{1}=\epsilon q_{0}(0, z, \zeta)$, we obtain

$$
\epsilon q_{0}\left(0, z, \frac{2}{i} \frac{\partial \phi_{\delta}}{\partial z_{1}}\right)=i z_{1}+\frac{2}{i} \frac{\partial \phi_{\delta}}{\partial z_{1}}=i z_{1}+\delta \zeta_{1}+(1-\delta)\left(-i \Re z_{1}\right)=\delta\left(i z_{1}+\zeta_{1}\right)-\Im z_{1} .
$$

Hence $\epsilon \Im q_{0}\left(0, x, \frac{2}{i} \frac{\partial \phi_{\delta}}{\partial z_{1}}\right)=\delta \Im\left(i z_{1}+\zeta_{1}\right)$.

We note that $\left(z, \frac{2}{i} \frac{\partial \phi_{\delta}}{\partial z}\right)=\left(z, \frac{2}{i} \frac{\partial \phi_{0}}{\partial z}\right)+\left(0, \frac{2}{i} \partial_{z} \phi_{\delta}-\frac{2}{i} \partial_{z} \phi_{0}\right)$, hence there exists a constant $D_{0}$ such that

$$
\begin{aligned}
\left|\chi^{-1}\left(z, \frac{2}{i} \frac{\partial \phi_{\delta}}{\partial z}\right)-\chi^{-1}\left(z, \frac{2}{i} \frac{\partial \phi_{0}}{\partial z}\right)\right| & \leqslant D_{0}\left|\frac{2}{i} \partial_{z} \phi_{\delta}-\frac{2}{i} \partial_{z} \phi_{0}\right| \\
& \leqslant D_{0}\left(1+2 d_{1}\right)(1+|\nabla \Phi|)\left|\Im\left(i z_{1}+\zeta_{1}\right)\right| .
\end{aligned}
$$

As $q_{0}(t, \chi(x, \xi))=r(t, x, \xi)$, we get the equality

$$
q_{0}(t, \chi(x, \xi))=r(0, x, \xi)+t \int_{0}^{1} \partial_{u} r(t u, x, \xi) \mathrm{d} u .
$$

We use the hypothesis that $D_{t}^{2}+r\left(t, x, D_{x}\right)$ is a differential operator of order 2. Hence the principal symbol of $D_{t}^{2}+r\left(t, x, D_{x}\right)$ is $\tau^{2}+\sum_{i, j} a_{i j}(t, x) \xi_{i} \xi_{j}=\tau^{2}+r(t, x, \xi)$, which 
implies that $\partial_{t} r(t, x, \xi)=\sum_{i j} \partial_{t} a_{i j}(t, x) \xi_{i} \xi_{j}$. We introduce $\left(x_{\delta}, \xi_{\delta}\right)=\chi^{-1}\left(z, \frac{2}{i} \frac{\partial \phi_{\delta}}{\partial z}\right)$ and $(x, \xi)=\chi^{-1}\left(z, \frac{2}{i} \frac{\partial \phi_{0}}{\partial z}\right)$. Hence

$$
\begin{aligned}
r\left(t, x_{\delta}, \xi_{\delta}\right) & =r\left(0, x_{\delta}, \xi_{\delta}\right)+t \int_{0}^{1} \partial_{t} r\left(t u, x_{\delta}, \xi_{\delta}\right) \mathrm{d} u \\
& =r\left(0, x_{\delta}, \xi_{\delta}\right)+t \int_{0}^{1}\left[\partial_{t} r\left(t u, x_{\delta}, \xi_{\delta}\right)-\partial_{t} r(t u, x, \xi)\right] \mathrm{d} u+r(t, x, \xi)-r(0, x, \xi) .
\end{aligned}
$$

This yields

$$
\begin{aligned}
\epsilon q_{0}\left(t, z, \frac{2}{i} \frac{\partial \phi_{\delta}}{\partial z}\right) & =\delta\left(i z_{1}+\zeta_{1}\right)-\Im z_{1} \\
& +\epsilon t \int_{0}^{1}\left[\partial_{t} r\left(t u, x_{\delta}, \xi_{\delta}\right)-\partial_{t} r(t u, x, \xi)\right] \mathrm{d} u+\epsilon r(t, x, \xi)-\epsilon r(0, x, \xi) .
\end{aligned}
$$

As $\Lambda_{\phi_{0}}=\left\{\left(z, \frac{2}{i} \frac{\partial \phi_{0}}{\partial z}\right)\right\}=\chi\left(T^{*}\left(\mathbb{R}^{d}\right)\right)$, we get that $(x, \xi) \in T^{*}\left(\mathbb{R}^{d}\right)$ hence $\Im r(t, x, \xi)=$ $\Im r(0, x, \xi)=0$, which imply

$$
\epsilon \Im q_{0}\left(t, z, \frac{2}{i} \frac{\partial \phi_{\delta}}{\partial z}\right)=\delta \Im\left(i z_{1}+\zeta_{1}\right)+\epsilon t \Im\left[\int_{0}^{1}\left[\partial_{t} r\left(t u, x_{\delta}, \xi_{\delta}\right)-\partial_{t} r(t u, x, \xi)\right] \mathrm{d} u\right]
$$

We have

$$
\left|\sum_{i, j} \partial_{t} a_{i j}\left(t u, x_{\delta}\right)\left(\xi_{\delta}\right)_{i}\left(\xi_{\delta}\right)_{j}-\sum_{i, j} \partial_{t} a_{i j}(t u, x) \xi_{i} \xi_{j}\right| \leqslant D_{1}\left|\chi^{-1}\left(z, \frac{2}{i} \frac{\partial \phi_{\delta}}{\partial z}\right)-\chi^{-1}\left(z, \frac{2}{i} \frac{\partial \phi_{0}}{\partial z}\right)\right| .
$$

Thus there exists a constant $D$, independent of $\alpha$, such that

$$
\left|\epsilon \Im q_{0}\left(t, z, \frac{2}{i} \frac{\partial \phi_{\delta}}{\partial z}\right)-\delta \Im\left(i z_{1}+\zeta_{1}\right)\right| \leqslant D \delta(1+|\nabla \Phi|) t\left|\Im\left(i z_{1}+\zeta_{1}\right)\right|
$$

and using the fact that $\Im\left(i z_{1}+\zeta_{1}\right) \leqslant 0$, that

$$
\left.\epsilon \Im q_{0}\left(t, z, \frac{2}{i} \frac{\partial \phi_{\delta}}{\partial z}\right) \leqslant \delta \Im\left(i z_{1}+\zeta_{1}\right)[1-D \delta(1+|\nabla \Phi|) t)\right] .
$$

The lemma 3.4 is proven.

We introduce then $t_{1}$ such that

$$
D t_{1}\left(1+\left\|\Phi^{\prime}\right\|_{\left[-d_{1}, d_{1}\right]}\right) \leqslant \frac{1}{2} .
$$

As we have the conditions (54), and as

$$
\phi(z) \leqslant \phi_{0}(z), \phi(z)=\phi_{0}(z) \text { for } \Re z_{1}+\Phi(y(z)) \geqslant s_{1}(\alpha)
$$

there exists $\alpha_{0}>0$ and $\beta>0$ such that, for all $\left.\alpha \in\right] 0, \alpha_{0}[$, we have

$$
\begin{aligned}
& u \in C^{\infty}\left(\left[t_{1} / 2, t_{1}\right], H_{\phi}\left(\left\{\left|\Re z_{1}\right|<d_{1},|y|<d_{1}\right\}\right)\right) \\
& u \in C^{\infty}\left(\left[0, t_{1}\right], H_{\phi}\left(\left\{\left|\Re z_{1}\right|<d_{1},|y|<d_{1}, \Re z_{1}<-d_{1} / 2\right\}\right)\right) \\
& u \in C^{\infty}\left(\left[0, t_{1}\right], H_{\phi}\left(\left\{\left|\Re z_{1}\right|<d_{1},|y|<d_{1},|y| \geqslant d_{1}-\beta\right\}\right)\right) .
\end{aligned}
$$

We introduce the sets

$$
\Omega_{2}=\left\{\left|\Re z_{1}\right|<d_{1},|y(z)|<d_{1}\right\}, \quad \Omega_{3}=\left\{\left|\Re z_{1}\right|<5 d_{1} / 6,|y|<d_{1}-\beta / 3\right\} .
$$


From $\operatorname{Op}(\widetilde{p})\left(0, C_{1}, C_{2}\right)(u) \in C^{\infty}\left(\left[0, t_{0}\right], H_{\phi_{0}-\varepsilon_{0}}\left(\Omega_{1}\right)\right)$ and from Lemma 3.1, if $C_{1}^{\prime}$ and $C_{2}^{\prime}$ are large enough, there exists $\varepsilon_{1}>0$ such that

$$
\operatorname{Op}(\widetilde{p})\left(0, C_{1}^{\prime}, C_{2}^{\prime}\right)(u) \in C^{\infty}\left(\left[0, t_{1}\right], H_{\phi_{0}-\varepsilon_{1}}\left(\Omega_{3}\right)\right) .
$$

Let now $\alpha \in] 0, \inf \left(\alpha_{0}, \varepsilon_{1} / 2\right)[$. We will prove that, for all $\delta \in[0,1]$,

$$
u \in C^{\infty}\left(\left[0, t_{1}\right], H_{\phi_{\delta}}\left(\Omega_{2}\right)\right) .
$$

First of all, as we have, from $(54) u \in C^{\infty}\left(\left[0, t_{0}\right], H_{\phi_{0}-\varepsilon}\left(\Omega_{0}\right)\right)$ for $|z| \leqslant 2 d_{1}$. There exists $\beta^{\prime}>0$ such that $s_{1}(\alpha)-\beta^{\prime}>s_{2}(\alpha)$, and we have, for $\Re z_{1}+\Phi(y(z)) \geqslant s_{1}(\alpha)$, $\phi(z)=\phi_{0}(z)$, as well as, for every $\epsilon$ small enough, $u \in C^{\infty}\left(\left[0, t_{0}\right], H_{\phi_{0}-\epsilon}\left(\Omega_{z}\right)\right), \Omega_{z}$ being a small neighborhood of $z$, hence if $\phi(z)<\phi_{0}(z)$, we use $\epsilon$ such that $\phi(z)>$ $\phi_{0}(z)-\epsilon$, hence $u \in C^{\infty}\left(\left[0, t_{1}\right], H_{\phi}\left(\Omega_{z}\right)\right)$. Hence

$$
u \in C^{\infty}\left(\left[0, t_{1}\right], H_{\phi}\left(\Omega_{2} \cap\left\{\Re z_{1}>s_{1}(\alpha)-\beta^{\prime}\right\}\right)\right) .
$$

Hence (58) is true for $\delta$ small enough, because, for $\Re z_{1} \leqslant s_{1}(\alpha)-\beta^{\prime}$, we have $\widetilde{\theta}_{\alpha}(s) \leqslant$ $\widetilde{\theta}_{\alpha}\left(s_{1}(\alpha)-\beta^{\prime}\right)<0$. We denote by $\delta_{0}$ the largest value of $\widetilde{\delta}$ such that

$$
\forall \delta<\widetilde{\delta}, \quad u \in C^{\infty}\left(\left[0, t_{1}\right], H_{\phi_{\delta}}\left(\Omega_{2}\right)\right) .
$$

Now, we have $\phi_{\delta}(z)=\phi_{0}(z)+\delta\left(\phi(z)-\phi_{0}(z)\right)$. For $\delta$ small enough, as we have $\phi_{0}(z)-\phi(z) \leqslant \delta_{0}$ (because $-\widetilde{\theta}_{\alpha}$ is bounded between $s^{2} / 2$ and the maximum value on $\left[s_{2}(\alpha), s_{1}(\alpha)-\beta^{\prime}\right]$, hence bounded by $\left.\delta_{0}\right)$ we may choose $\delta$ small enough so that we can take $\varepsilon_{2}=\delta \delta_{0}$. Note that $t_{1}$ plays no role here.

Assume that (58) is true for all $\delta<\delta_{0}$, where $\left.\delta_{0} \in\right] 0,1[$. We consider a point $z^{0}=\left(z_{1}^{0}, y^{0}\right)$ in $\Omega_{2}$, with $-3 d_{1} / 4 \leqslant \Re z_{1}^{0} \leqslant s_{1}(\alpha)-\beta^{\prime} / 2$ and $|y| \leqslant d_{1}-\beta / 2$.

We have, for $\tau \in \mathbb{R}, \epsilon \Im\left(\tau^{2}+q_{0}(t, z, \zeta)\right)=\epsilon \Im q_{0}(t, z, \zeta) \leqslant \frac{1}{2} \Im\left(\zeta_{1}+i z_{1}\right)$. We choose the neighborhood of $\left(z^{0}, \zeta_{0}\right)$. For this point, we have $\Im\left(\zeta_{1}^{0}+i z_{1}^{0}\right)=\left(\Re z_{1}^{0}+\Phi\left(y\left(z^{0}\right)\right)\right)-$ $\theta_{\alpha}^{\prime}\left(\Re z_{1}^{0}+\Phi\left(y\left(z^{0}\right)\right)\right)<0$ because $\Re z_{1}^{0}+\Phi\left(y\left(z^{0}\right)\right) \leqslant s_{1}(\alpha)-\frac{\beta^{\prime}}{2}+\Phi\left(d_{1}-\beta / 2\right)$.

We choose $\beta^{\prime}$ and $\beta$ such that $\Phi\left(d_{1}-\beta / 2\right)<\beta^{\prime} / 2$ and $\left.\beta^{\prime} \in\right] 0, s_{2}(\alpha)-s_{1}(\alpha)[$. Hence we have $\Im\left(\zeta_{1}^{0}+i z_{1}^{0}\right)<0$, and the neighborhood $U$ we choose is such that we have $\Im\left(\zeta_{1}+i z_{1}\right)<\Im\left(\zeta_{1}^{0}+i z_{1}^{0}\right) / 2$. We then obtain $\Im \epsilon\left(\tau^{2}+q_{0}(t, z, \zeta)\right) \leqslant-\left|\Im\left(\zeta_{1}^{0}+i z_{1}^{0}\right)\right| / 4$. The function $\left|\tau^{2}+q_{0}(t, z, \zeta)\right| /\left(1+\tau^{2}\right)$ has for limit 1 in $\pm \infty$, hence is, on the complementary of a compact set in $\tau \in \mathbb{R}$, bounded below by $1 / 2$. On the compact set, it cannot vanish, thus is bounded below by a constant $c_{1}$. We take $c=\min \left(c_{1}, 1 / 2\right)$ to obtain the inequality

$$
\left|\tau^{2}+q_{0}(t, z, \zeta)\right| \geqslant c\left(1+\tau^{2}\right), \quad \forall(z, \zeta) \in U, t \in\left[0, t_{1}\right] \text { and } \tau \in \mathbb{R} .
$$

The roots of $\tau^{2}+q_{0}(t, z, \zeta)$ stay in a compact set $K$ of $\mathbb{C}-\mathbb{R}$. Owing to the definitions, we can consider symbols on $A^{m}\left(\left[0, t_{1}\right], U, K\right)$.

From the usual theory for analytic symbols, $e=\left(\tau^{2}+q\right)^{-1} \in A^{-2}\left(\left[0, t_{1}\right], U, K\right)$. We consider two neighborhoods of $z^{0}$, denoted by $\omega_{1} \subset \omega_{0}$, and $R_{0}>0$ such that $\omega_{0} \times\left\{\left|\zeta^{0}-\zeta\right|<R_{0}\right\} \subset U$, and $\left|\frac{2}{i} \frac{\partial \phi_{\delta_{0}}}{\partial z}-\zeta_{0}\right|<D_{0}<R_{0}$. We may assume (choice of $\left.\theta_{\alpha}\right)$ that $\left|\frac{2}{i} \frac{\partial \phi_{\delta}}{z}\right|<D<R$, where $R$ is a radius of convergence of the series defining 
$q(t, z, \zeta)$ (inequality (46)). We consider, in (57), the constants $C_{1}^{\prime}$ and $C_{2}^{\prime}$ such that $C_{1}^{\prime} \geqslant 2 B / e, C_{2}^{\prime} \geqslant 1 / D \operatorname{dist}\left(\Omega_{3}, C \Omega_{2}\right)$, where $B$ is the constant for $q$ in inequalities of the form (46). Let $C_{2}^{\prime \prime}=C_{2}^{\prime}+\left(D_{0}^{-1}+D^{-1}\right) / \operatorname{dist}\left(\omega_{1}, C \omega_{0}\right)$. From Lemma 3.1, there exists $\varepsilon>0$ such that

$$
\mathrm{Op}\left(\tau^{2}+q(t, z, \zeta)\right)\left(0, C_{1}^{\prime}, C_{2}^{\prime \prime}\right)(u) \in C^{\infty}\left(\left[0, t_{1}\right], H_{\phi_{\delta_{0}}-\varepsilon}\left(\Omega_{3}\right)\right) .
$$

From Lemma 3.2, we deduce immediately that

$$
\operatorname{Op}\left(\tau^{2}+q(t, z, \zeta)\right)\left(\zeta_{0}, C_{1}^{\prime}, C_{2}^{\prime \prime}\right)(u) \in C^{\infty}\left(\left[0, t_{1}\right], H_{\phi_{\delta_{0}}-\varepsilon}\left(\omega_{1}\right)\right) .
$$

This result is still true if $C_{1}^{\prime}, C_{2}^{\prime}$ are replaced by larger constants, by diminishing $\varepsilon$.

The following steps of this proof is the proof of the following Lemmas:

Lemma 3.5. - Let $u \in C^{\infty}\left(I, H_{\phi}(\Omega)\right)$. We have

$$
\begin{aligned}
& \mathrm{Op}(e)\left(\zeta_{0}, C_{1}, C_{2}\right)\left(\frac{1}{i \lambda} \partial_{t}\right)\left[\left(\frac{1}{i \lambda} \partial_{t}\right)^{2} u\left(\frac{1}{i \lambda} \partial_{t}\right)\right]=\operatorname{Op}\left(\tau^{2} e\right)\left(\zeta_{0}, C_{1}, C_{2}\right)(u) \\
& +\lambda^{-2}\left[K^{+}(t, 0) \partial_{t} u(0)-\partial_{s} K^{+}(t, 0) u(0)-K^{-}\left(t, t_{0}\right) \partial_{t} u\left(t_{0}\right)+\partial_{s} K^{-}\left(t, t_{0}\right) u\left(t_{0}\right)\right] .
\end{aligned}
$$

Lemma 3.6. - Introduce the complex number $(q(t, z, \zeta))^{1 / 2}$ such that we have $\Re(q(t, z, \zeta))^{1 / 2}>0$. The operator $K^{+}(0,0)$ is a pseudodifferential operator of order 1 :

$$
K^{+}(0,0)=\sum_{m \geqslant 0}(i \lambda)^{1-m} K_{m}^{1}(0,0)
$$

where $K_{0}^{1}(0,0)$ satisfies, for $v \in H_{\phi}$

$$
\left[\operatorname{Op}\left(K_{0}^{1}(0,0)\right)\left(\zeta_{0}, C_{1}, C_{2}\right)-\operatorname{Op}\left(\frac{1}{2 i(q(0, z, \zeta))^{1 / 2}}\right)\left(\zeta_{0}, C_{1}, C_{2}\right)\right](v) \in H_{\phi-\varepsilon} .
$$

The operator $\partial_{s} K^{+}(0,0)$ is a pseudodifferential operator of order 2 , whose principal symbol is $\lambda^{2} / 2$.

Proof. - We have

$$
\begin{aligned}
e_{0}(t, \tau, z, \zeta) & =\left(\tau^{2}+q(t, z, \zeta)\right)^{-1} \\
& =\frac{1}{2 i(q(t, z, \zeta))^{1 / 2}}\left[\frac{1}{\tau-i(q(t, z, \zeta))^{1 / 2}}-\frac{1}{\tau+i(q(t, z, \zeta))^{1 / 2}}\right] .
\end{aligned}
$$

The relation $e \circ\left(\tau^{2}+q\right)=1$ writes, for $p \geqslant 1$

$$
\sum_{|\alpha|+n=p} \frac{1}{\alpha !} \partial_{\zeta, \tau}^{\alpha} e_{n} \partial_{t, z}^{\alpha} q=0 .
$$

This leads to the choice of each $e_{p}$ for $p \geqslant 1$ because we have

$$
e_{p}(t, \tau, z, \zeta)=-\sum_{\substack{|\alpha|+n=p \\ n \leqslant p-1}} \frac{1}{\alpha !} \partial_{\zeta, \tau}^{\alpha} e_{n} \partial_{t, z}^{\alpha} q .
$$


The relations (60) and (61) show that there exists a sequence of analytic functions $r_{p}(t, z, \zeta)$ and two sequences $A_{p, q}(t, z, \zeta), B_{p, q}(t, z, \zeta)$ such that

$$
\text { (62) } \begin{aligned}
e_{p}(t, \tau, z, \zeta)=r_{p}(t, z, \zeta)\left[\frac{1}{\tau-i(q(t, z, \zeta))^{1 / 2}}-\frac{1}{\tau+i(q(t, z, \zeta))^{1 / 2}}\right] \\
+\sum_{2 \leqslant q \leqslant p} \frac{A_{p, q}}{\left(\tau+i q^{1 / 2}\right)^{p}}+\frac{B_{p, q}}{\left(\tau-i q^{1 / 2}\right)^{p}} .
\end{aligned}
$$

We have thus

$$
\begin{aligned}
\partial_{\tau, \zeta}^{\alpha} e_{p}=\partial_{\tau, \zeta}^{\alpha} r_{p}\left[\frac{1}{\tau-i(q(t, z, \zeta))^{1 / 2}}-\frac{1}{\tau+}+i(q(t, z, \zeta))^{1 / 2}\right] \\
\quad+\sum_{2 \leqslant q \leqslant p+|\alpha|} \frac{A_{p, q}^{\alpha}}{\left(\tau+i q^{1 / 2}\right)^{p}}+\frac{B_{p, q}^{\alpha}}{\left(\tau-i q^{1 / 2}\right)^{p}} .
\end{aligned}
$$

Identifying the term of the form $\left(\tau+i q^{1 / 2}\right)^{-1}$ and the term of the form $\left(\tau-i q^{1 / 2}\right)^{-1}$ (which are the only ones to give a residue), we deduce that

$$
r_{p}+\sum_{|\alpha|+n=p} \frac{1}{\alpha !} \partial_{\tau, \zeta}^{\alpha} r_{n} \partial_{t, z}^{\alpha} q=0
$$

and $r_{0}=\left(2 i q^{1 / 2}\right)^{-1}$.

We compute

$$
k^{+}\left(e, \zeta_{0} ; p, \alpha\right)(t, s, z, \lambda)=\frac{\lambda}{2 \pi} \int_{-\infty}^{+\infty} 1_{t-s>0} e^{i \lambda(t-s) \tau} \frac{1}{\alpha !} \partial_{\zeta}^{\alpha} e_{p}\left(t, \tau, z, \zeta_{0}\right) \mathrm{d} \tau .
$$

The expression of $e_{p}$ given in (62) and a classical formula of residue on the contour $[-R, R] \cup\left\{R e^{i \theta}, 0<\theta<\pi\right\}$ (because $\Re(i(t-s) \tau)<0$ on the curve) around the pole $i(q(t, z, \zeta))^{1 / 2}$ (owing to the fact that, uniformly on $\left[0, t_{1}\right] \times \bar{U}, \Re(q(t, z, \zeta))^{1 / 2}>0$ ) yield:

$$
k^{+}\left(e, \zeta_{0} ; p, \alpha\right)(t, s, z, \lambda)=i \lambda e^{-\lambda(t-s)(q(t, z, \zeta))^{1 / 2}} \frac{1}{\alpha !} \partial_{\zeta}^{\alpha} r_{p}\left(t, z, \zeta_{0}\right) .
$$

The operator $K^{+}(t, t)$ is thus the operator of symbol

$$
i \lambda \sum_{p=0}^{\infty}(i \lambda)^{-p} r_{p}(t, z, \zeta)
$$

because we can take the limit $t-s \rightarrow 0$.

The relation (63) shows that the principal symbol of $K^{+}(t, t)$, which is obtained with $p=0$, is given by

$\sum_{|\alpha| \leqslant \lambda / C} k^{+}\left(e, \zeta_{0} ; 0, \alpha\right)(t, t, z, \lambda)\left(\frac{1}{i \lambda} \partial_{z}-\zeta_{0}\right)^{\alpha}=\frac{\lambda}{2} \sum_{|\alpha| \leqslant \lambda / C} \frac{1}{\alpha !} \partial_{\zeta}^{\alpha}(q(t, z, \zeta))^{-1 / 2}\left(\frac{1}{i \lambda} \partial_{z}-\zeta_{0}\right)^{\alpha}$.

This shows that $K^{+}(t, t)$ is the usual analytic tangential pseudodifferential operator $\frac{\lambda}{2}(q(t, z, \zeta))^{-1 / 2}$ of order 1 , where $\Re(q(0, z, \zeta))^{1 / 2}>0$. 
Similarly, we have

$$
\frac{1}{i \lambda} \partial_{s} k^{+}\left(e, \zeta_{0} ; 0, \alpha\right)=k^{+}\left(-\tau e, \zeta_{0} ; 0, \alpha\right)
$$

and the equality

$$
-\frac{\tau}{\tau^{2}+q(t, z, \zeta)}=-\frac{1}{2}\left[\frac{1}{\tau-i(q(t, z, \zeta))^{1 / 2}}+\frac{1}{\tau+i(q(t, z, \zeta))^{1 / 2}}\right]
$$

leads to the fact that $\partial_{s} k^{+}\left(e, \zeta_{0} ; 0, \alpha\right)$ is the operator of symbol $-\frac{1}{2}(i \lambda)(i \lambda)=\lambda^{2} / 2$. We can also verify that it is $\frac{\lambda}{2(q)^{1 / 2}}\left(-i \lambda\left(i(q)^{1 / 2}\right)\right)$. This ends the proof of Lemma 3.6 .

Proof of Lemma 3.5. - We want to prove

(64) $\operatorname{Op}(e)\left(\zeta_{0}, C_{1}, C_{2}\right)\left[\left(\frac{1}{i \lambda} \partial_{t}\right)^{2} u\right]=\operatorname{Op}\left(\tau^{2} e\right)\left(\zeta_{0}, C_{1}, C_{2}\right)(u)$

$$
+\frac{1}{\lambda^{2}}\left[K^{+}(t, 0) \partial_{t} u(0)-\partial_{s} K^{+}(t, 0) u(0)-K^{-}\left(t, t_{0}\right) \partial_{t} u\left(t_{0}\right)+\partial_{s} K^{-}\left(t, t_{0}\right) u\left(t_{0}\right)\right] .
$$

Note that

$$
\begin{aligned}
& \operatorname{Op}\left(\tau^{2} e\right)\left(\zeta_{0}, C_{1}, C_{2}\right)(u)(t, z, \lambda) \\
= & \sum_{\substack{n \leqslant \lambda / C_{1} \\
|\alpha| \leqslant \lambda / C_{2}}}(i \lambda)^{-n} \int_{0}^{t_{0}} k\left(\tau^{2} e, \zeta_{0} ; n, \alpha\right)(t, s, z, \lambda)\left[e^{i \lambda z \zeta_{0}}\left(\frac{1}{i \lambda} \partial_{z}\right)^{\alpha}\left(e^{-i \lambda z \zeta_{0}} u(s, z, \lambda)\right)\right] \mathrm{d} s .
\end{aligned}
$$

We calculate each term. We have

$$
\begin{aligned}
k\left(\tau^{2} e, \zeta_{0} ; n, \alpha\right)(t, s, z, \lambda) & =\frac{\lambda}{2 \pi} \int_{-\infty}^{+\infty} e^{i \lambda(t-s) \tau} \tau^{2} \frac{1}{\alpha !} \partial_{\zeta}^{\alpha} e_{n}\left(t, \tau, z, \zeta_{0}, \lambda\right) \mathrm{d} \tau \\
& =\left(\frac{1}{i \lambda} \partial_{t}\right)^{2}\left[\frac{\lambda}{2 \pi} \int_{-\infty}^{+\infty} e^{i \lambda(t-s) \tau} \frac{1}{\alpha !} \partial_{\zeta}^{\alpha} e_{n}\left(t, \tau, z, \zeta_{0}, \lambda\right) \mathrm{d} \tau\right] \\
& =\left(\frac{1}{i \lambda} \partial_{t}\right)^{2} k\left(e, \zeta_{0} ; n, \alpha\right)=\left(\frac{1}{i \lambda} \partial_{s}\right)^{2} k\left(e, \zeta_{0} ; n, \alpha\right) .
\end{aligned}
$$

We have the equality

$$
\begin{aligned}
& \int_{0}^{t_{0}} k\left(\tau^{2} e, \zeta_{0} ; n, \alpha\right)(t, s, z, \lambda)\left(e^{i \lambda z \zeta_{0}}\left(\frac{1}{i \lambda} \partial_{z}\right)^{\alpha}\left(e^{-i \lambda z \zeta_{0}} u(s, z, \lambda)\right)\right) d d s \\
& =\int_{0}^{t_{0}}\left(\frac{1}{i \lambda} \partial_{s}\right)^{2}\left[k\left(e, \zeta_{0} ; n, \alpha\right)(t, s, z, \lambda)\right]\left(e^{i \lambda z \zeta_{0}}\left(\frac{1}{i \lambda} \partial_{z}\right)^{\alpha}\left(e^{-i \lambda z \zeta_{0}} u(s, z, \lambda)\right)\right) \mathrm{d} s \\
& =-\int_{0}^{t_{0}}\left(\frac{1}{i \lambda} \partial_{s}\right)\left[k\left(e, \zeta_{0} ; n, \alpha\right)(t, s, z, \lambda)\right]\left(e^{i \lambda z \zeta_{0}}\left(\frac{1}{i \lambda} \partial_{s}\right)\left(\frac{1}{i \lambda} \partial_{z}\right)^{\alpha}\left(e^{-i \lambda z \zeta_{0}} u(s, z, \lambda)\right)\right) \mathrm{d} s \\
& \quad+\left(\frac{1}{i \lambda}\right)^{2} \partial_{s} k^{-}\left(e, \zeta_{0} ; n, \alpha\right)\left(t, t_{0}, z, \lambda\right)\left(e^{i \lambda z \zeta_{0}} \partial_{s}\left(\frac{1}{i \lambda} \partial_{z}\right)^{\alpha}\left(e^{-i \lambda z \zeta_{0}} u(s, z, \lambda)\right)\right) \\
& \quad-\left(\frac{1}{i \lambda}\right)^{2} \partial_{s} k^{+}\left(e, \zeta_{0} ; n, \alpha\right)(t, 0, z, \lambda)\left(e^{i \lambda z \zeta_{0}} \partial_{s}\left(\frac{1}{i \lambda} \partial_{z}\right)^{\alpha}\left(e^{-i \lambda z \zeta_{0}} u(s, z, \lambda)\right)\right)
\end{aligned}
$$




$$
\begin{aligned}
=\int_{0}^{t_{0}} k\left(e, \zeta_{0}\right. & ; n, \alpha)(t, s, z, \lambda)\left[e^{i \lambda z \zeta_{0}}\left(\frac{1}{i \lambda} \partial_{s}\right)^{2}\left(\frac{1}{i \lambda} \partial_{z}\right)^{\alpha}\left(e^{-i \lambda z \zeta_{0}} u(s, z, \lambda)\right)\right] \mathrm{d} s \\
+ & \left(\frac{1}{i \lambda}\right)^{2} \partial_{s} k^{-}\left(e, \zeta_{0} ; n, \alpha\right)\left(t, t_{0}, z, \lambda\right)\left(e^{i \lambda z \zeta_{0}}\left(\frac{1}{i \lambda} \partial_{z}\right)^{\alpha}\left(e^{-i \lambda z \zeta_{0}} u(s, z, \lambda)\right)\right) \\
& -\left(\frac{1}{i \lambda}\right)^{2} \partial_{s} k^{-}\left(e, \zeta_{0} ; n, \alpha\right)(t, 0, z, \lambda)\left(e^{i \lambda z \zeta_{0}}\left(\frac{1}{i \lambda} \partial_{z}\right)^{\alpha}\left(e^{-i \lambda z \zeta_{0}} u(s, z, \lambda)\right)\right) \\
& -\left(\frac{1}{i \lambda}\right)^{2} k^{+}\left(e, \zeta_{0} ; n, \alpha\right)\left(t, t_{0}, z, \lambda\right)\left(e^{i \lambda z \zeta_{0}}\left(\frac{1}{i \lambda} \partial_{z}\right)^{\alpha}\left(e^{-i \lambda z \zeta_{0}} \partial_{s} u(s, z, \lambda)\right)\right) \\
& +\left(\frac{1}{i \lambda}\right)^{2} k^{+}\left(e, \zeta_{0} ; n, \alpha\right)(t, 0, z, \lambda)\left(e^{i \lambda z \zeta_{0}}\left(\frac{1}{i \lambda} \partial_{z}\right)^{\alpha}\left(e^{-i \lambda z \zeta_{0}} \partial_{s} u(s, z, \lambda)\right)\right)
\end{aligned}
$$

Note that, in the above equalities, we considered the integral $\int_{0}^{t_{0}}$. From the definition of the operator (48), it is in reality $\int_{0}^{t} k^{+}+\int_{t}^{t_{0}} k^{-}$, with $k^{+}=k 1_{t \geqslant s}$ and $k^{-}=k 1_{t \leqslant s}$. Hence, in the integration by parts, we see the terms $k^{-}\left(t, t_{0}\right)-$ $k^{-}(t, t)+k^{+}(t, t)-k^{+}(t, 0)$. We use then the relations $k^{+}(t, t)=k^{-}(t, t)$ and $\partial_{s} k^{+}(t, t)=\partial_{s} k^{-}(t, t)$, which proves precisely the equality above. Using Definition (50) of the operator $K^{+}$and of the operator $K^{-}$we obtain the relation (64), which is the relation of Lemma 3.5.

We have thus

$$
\begin{aligned}
{\left[\mathrm{Op}(1)(u)-\mathrm{Op}(e) \operatorname{Op}\left(\tau^{2}+q(t, z, \zeta)\right)\right]\left(\zeta_{0}, C_{1}, C_{2}\right)(u) } \\
=\left[\mathrm{Op}(1)-\mathrm{Op}(e) \operatorname{Op}(q)-\mathrm{Op}(e) \operatorname{Op}\left(\tau^{2}\right)\right]\left(\zeta_{0}, C_{1}, C_{2}\right)(u) .
\end{aligned}
$$

From the relation $\operatorname{Op}\left(\tau^{2}\right)(u)=\left(\frac{1}{i \lambda} \partial_{t}\right)^{2} u(t)$ (case of Definition (49) and the expression (48) for $m=2$ and $e_{0}^{2}=1, e_{0}^{1}=e_{0}^{0}=0$ and $e^{-}=0$ ), and from the previous formula, we get

$$
\begin{aligned}
& {\left[\operatorname{Op}(1)-\operatorname{Op}(e) \operatorname{Op}\left(\tau^{2}+q\right)\right]\left(\zeta_{0}, C_{1}, C_{2}\right)(u)} \\
& =\left[\operatorname{Op}(1)-\operatorname{Op}(e) \operatorname{Op}(q)-\operatorname{Op}\left(\tau^{2} e\right)\right](u) \\
& -\lambda^{-2}\left[K^{+}(t, 0) \partial_{t} u(0)-\partial_{s} K^{+}(t, 0) u(0)-K^{-}\left(t, t_{0}\right) \partial_{t} u\left(t_{0}\right)+K^{-}\left(t, t_{0}\right) u\left(t_{0}\right)\right] \\
& =[\operatorname{Op}(e q)-\operatorname{Op}(e) \operatorname{Op}(q)](u) \\
& -\lambda^{-2}\left[K^{+}(t, 0) \partial_{t} u(0)-\partial_{s} K^{+}(t, 0) u(0)-K^{-}\left(t, t_{0}\right) \partial_{t} u\left(t_{0}\right)+K^{-}\left(t, t_{0}\right) u\left(t_{0}\right)\right]
\end{aligned}
$$

Using Lemma 3.3 of [20] for the composition of such operators, we have

$$
[\mathrm{Op}(e q)-\mathrm{Op}(e) \operatorname{Op}(q)](u) \in C^{\infty}\left(\left[0, t_{1}\right], H_{\phi_{\delta_{0}}-\varepsilon}\left(\omega_{1}\right)\right.
$$

We write the formula (65) for $t_{1}$ instead of $t_{0}$, and we use this result. Hence we get

$$
\begin{aligned}
& \operatorname{Op}(1) u(t)-\operatorname{Op}(e) \operatorname{Op}(\widetilde{p})\left(\zeta_{0}, \widetilde{C}_{1}, \widetilde{C}_{2}\right) u(t) \\
&+\lambda^{-2}\left[K^{+}(t, 0) \partial_{t} u(0)-\partial_{s} K^{+}(t, 0) u(0)-K^{-}\left(t, t_{1}\right) \partial_{t} u\left(t_{1}\right)+\partial_{s} K^{-}\left(t, t_{1}\right) u\left(t_{1}\right)\right] \\
& \in C^{\infty}\left(\left[0, t_{1}\right], H_{\phi_{\delta_{0}}-\varepsilon}\left(\omega_{1}\right)\right)
\end{aligned}
$$


From this equality, by putting $t=0$ and using the inequality (51), we get $K^{-}\left(0, t_{1}\right) \partial_{t} u\left(t_{1}\right) \in H_{\phi_{\delta_{0}}-\varepsilon}, \partial_{s} K^{-}\left(0, t_{1}\right) u\left(t_{1}\right) \in H_{\phi_{\delta_{0}}-\varepsilon}$, thus

$\frac{1}{\lambda^{2}}\left[K^{+}(0,0) \partial_{t} u(0)-\partial_{s} K^{+}(0,0) u(0)\right]+\mathrm{Op}(1) u(0)-\mathrm{Op}(e) \operatorname{Op}\left(\tau^{2}+q\right)(u)(0) \in H_{\phi_{\delta_{0}}-\varepsilon}$.

As $u$ is a solution of $\left(\tau^{2}+q\right)(u)=0$ (by hypothesis), the equality (59) holds. As $\mathrm{Op}(1)=\mathrm{Id}$, this gives

$$
\frac{1}{\lambda^{2}}\left[K^{+}(0,0) \partial_{t} u(0)-\partial_{s} K^{+}(0,0) u(0)\right]+u(0) \in H_{\phi_{\delta_{0}}-\varepsilon},
$$

that is

$$
K^{+}(0,0) \partial_{t} u(0, z, \lambda)=\partial_{s} K^{+}(0,0) u(0, z, \lambda)-\lambda^{2} u(0, z, \lambda)+w(0, z, \lambda)
$$

where $w \in H_{\phi_{\delta_{0}}-\varepsilon}\left(\omega_{1}\right)$.

We introduce $v=\left.\frac{1}{i \lambda}\left(\partial_{t} u+T_{\chi}(L f)\right)\right|_{t=0}$. By the usual pseudodifferential calculus, there exists an operator $\widetilde{L}$, of principal symbol $L\left(\chi^{-1}(z, \zeta)\right)=l(z, \zeta)$, such that $v=\frac{1}{i \lambda} \partial_{t} u(0, z, \lambda)+\widetilde{L}(u(0, z, \lambda))$. We express the relation (67) in terms of $v$. The strong Lopatinskii condition yields $u(0, z, \lambda)=(\widetilde{L})^{-1}\left(v-\frac{1}{i \lambda} \partial_{t} u(0, z, \lambda)\right)$, and the equality (67) leads to the two relations

$$
\begin{gathered}
{\left[K^{+}(0,0)+\left(\frac{1}{i \lambda} \partial_{s} K^{+}(0,0)+i \lambda\right) \circ(\widetilde{L})^{-1}\right]\left(\frac{1}{i \lambda} \partial_{t} u(0, z, \lambda)\right)} \\
=\left(\frac{1}{i \lambda} \partial_{s} K^{+}(0,0)+i \lambda\right)(\widetilde{L})^{-1} v+\frac{1}{i \lambda} w \\
{\left[\frac{1}{i \lambda} \partial_{s} K^{+}(0,0)+i \lambda+K^{+}(0,0) \widetilde{L}\right] u=K^{+}(0,0) v-\frac{1}{i \lambda} w .}
\end{gathered}
$$

The inequality for $v$ in the hypothesis of Theorem 3 implied the relation (52):

$$
|v(z, \lambda)| \leqslant A \lambda^{M} e^{\lambda \phi_{0}(z)}
$$

We use now the following result

LEMMA 3.7. - There exists a neighborhood $\widetilde{U} \subset U$ of $\left(z^{0}, \zeta_{0}\right)$ in which the operator $K=K^{+}(0,0)+\left(\frac{1}{i \lambda} \partial_{s} K^{+}(0,0)+i \lambda\right)(\widetilde{L})^{-1}$ is elliptic.

Proof. - We check that, owing to Lemma 3.6, the symbol of $K$ in the analytic calculus is

$$
\frac{\lambda}{2} \frac{1}{(q(0, z, \zeta))^{1 / 2}}+\frac{i \lambda}{2} \frac{1}{l(z, \zeta)}=\frac{\lambda}{2(q(0, z, \zeta))^{1 / 2} l(z, \zeta)}\left[l(z, \zeta)+i(q(0, z, \zeta))^{1 / 2}\right] .
$$

For $(z, \zeta)=\left(z^{0}, \zeta_{0}\right)$, we have $|q(0, z, \zeta)| \geqslant \widetilde{c}$, and the operator $(q(0, z, \zeta))^{1 / 2}$ is elliptic on $U$. In a same way, $\widetilde{l}(z, \zeta)$ is elliptic on $U$. The ellipticity of $K$ on $U$ is thus equivalent to the ellipticity of $l(z, \zeta)+i(q(0, z, \zeta))^{1 / 2}$ in $U$.

For $z$ in a neighborhood of the glancing point $(0,0)$, we know that $q(0, z, \zeta)$ is small, and going back to the beginning, as $|\Re l(0,0)|>0$, we can choose a neighborhood of the point $(0,0)$ such that $|q(0, z, \zeta)| \leqslant|\Re l(0,0)| / 2$, which imply that the condition $l(z, \zeta)+i(q(0, z, \zeta))^{1 / 2} \neq 0$ is fulfilled. Hence the operator is invertible. The boundary 
operator $\partial_{t}+L$ is elliptic in the neighborhood of a glancing point as soon as $L$ is not 0 at the glancing point.

Note that this is a consequence of the fact that, for $\Re z>0$, the operator $\tau+\xi_{2} z$ is elliptic for $\tau=0$, corresponding to glancing points. Lemma 3.7 is proven.

Lemma 3.7 allows us to deduce the regularity of $(i \lambda)^{-1} \partial_{t} u(0, z, \lambda)$ in terms of the regularity of $v(z, \lambda)$.

From the inequality

$$
|v(z, \lambda)| \leqslant A \lambda^{M} e^{\lambda \phi_{-}(z)}
$$

for $z \in \Omega_{0}$ small, we deduce that $u(0, z, \lambda)$ and $\partial_{t} u(0, z, \lambda)$ satisfy the inequalities, owing to Lemma 3.7 and to the ellipticity of $L$ :

$$
\begin{aligned}
\left|\frac{1}{i \lambda} \partial_{t} u(0, z, \lambda)\right| & \leqslant A^{\prime} \lambda^{M} e^{\lambda \phi_{-}(z)} \\
|u(0, z, \lambda)| & \leqslant A^{\prime \prime} \lambda^{M+1} e^{\lambda \phi_{-}(z)} .
\end{aligned}
$$

Hence $\partial_{t} u(0)$ and $u(0)$ are in $H_{\phi_{\delta_{0}}-\varepsilon}\left(\omega_{3}\right)$ where $\omega_{3} \subset \omega_{2}$. As $\operatorname{Op}(1)(u)=u$, and $u \in C^{\infty}\left(\left[t_{1} / 2, t_{1}\right], H_{\phi}\left(\omega_{2}\right)\right)$, we deduce that $u \in C^{\infty}\left(\left[0, t_{1}\right], H_{\phi_{\delta_{0}}-\varepsilon}\left(\omega_{3}\right)\right)$ because $\phi \geqslant$ $\phi_{\delta_{0}}-\varepsilon$ for $\varepsilon$ small enough.

Hence there exists $\delta_{0}^{\prime}>\delta_{0}$ such that $u \in C^{\infty}\left(\left[0, t_{1}\right], H_{\phi_{\delta_{0}^{\prime}}}\left(\omega_{3}\right)\right)$, which is contradictory with the hypothesis that $\delta_{0}$ is the largest $\delta$ for which it is possible.

Hence we have

$$
\forall \delta \in] 0,1\left[, \quad u \in C^{\infty}\left(\left[0, t_{1}\right], H_{\phi_{\delta}}\left(\Omega_{2}\right)\right) .\right.
$$

The case of $\delta_{0}=1$ is achieved by the fact that $\widetilde{w} \in H_{\phi_{1}-\varepsilon}$ and that the relations on $(i \lambda)^{-1} \partial_{t} u(0)$ and $u(0)$ are still valid, and as $\phi_{1} \geqslant \phi_{-}$, we get, for $z=\left(z_{1}, z^{\prime}\right)$, $\Re z_{1} \in\left[s_{3}(\alpha), s_{2}(\alpha)\right],|y(z)| \leqslant d_{1} / 4$

$$
\begin{gathered}
\left|\partial_{t} u(0, z, \lambda)\right| \leqslant A^{\prime} \lambda^{M+1} e^{\lambda \phi(z)} \\
|u(0, z, \lambda)| \leqslant A^{\prime \prime} \lambda^{M} e^{\lambda \phi(z)} .
\end{gathered}
$$

The result of Theorem 3 is proven in the neighborhood of the elliptic and hyperbolic points, hence, if $z^{0}=\left(z_{1}^{0},\left(z^{\prime}\right)^{0}\right), \Im z_{1}^{0} \neq 0$, as we get $\epsilon r_{0}\left(\chi^{-1}(z, \zeta)\right)=-\Im z_{1}$ for $(z, \zeta) \in \Lambda_{\phi_{0}}$, we deduce that such points are either elliptic or hyperbolic, hence the theorem is true and the inequalities (69) are valid for all $z \in V_{0}$, neighborhood of $z_{0}$.

$$
\begin{gathered}
\left|\partial_{t} u(0, z, \lambda)\right| \leqslant C^{\prime} \lambda^{M+1} e^{\lambda \phi_{-}(z)} \\
|u(0, z, \lambda)| \leqslant C^{\prime \prime} \lambda^{M} e^{\lambda \phi_{-}(z)} .
\end{gathered}
$$

Finally, for $|y(z)| \leqslant d_{1} / 4$ and $\Re z_{1} \in\left[s_{3}(\alpha), s_{2}(\alpha)\right]$, we have $\phi(z)=\phi_{-}(z)=\frac{1}{2}\left(\Im z^{\prime}\right)^{2}$. From (68) and (69), by the maximum principle, if we choose $\alpha$ small enough such that the region $\left[s_{3}(\alpha), 0\right]$ is large, we get (69) for $z$ in a neighborhood of 0 .

Hence $\left(\rho_{0},-1\right) \notin \operatorname{SS}_{V}^{2,1}\left(\left.\partial_{t} f\right|_{t=0}\right)$ and $\left(\rho_{0},-1\right) \notin \operatorname{SS}_{V}^{2,1}\left(\left.f\right|_{t=0}\right)$. The Theorem 3 is proven. 
We deduce from that theorem that, by considering the waves $u_{ \pm}$constructed in the Introduction, we have the same regularity result as in Proposition 3.1 of [20]. Let $\tilde{f}_{ \pm}=v_{ \pm}-1_{x>0} \partial_{ \pm} u_{ \pm}$. The following Proposition gives the analytic wave front set of $\tilde{f}_{ \pm}$.

Proposition 3.1. - For all $\varepsilon_{0}>0$, there exists $\varepsilon_{1}>0$ such that

$$
\begin{aligned}
& \mathrm{SS}\left(\tilde{f}_{ \pm}\right) \cap\left\{x>0, t \geqslant \varepsilon_{1}\right\} \\
& \quad \subset\left\{\left|\xi+\frac{\tau}{\cos \gamma_{ \pm}}\right|<\varepsilon_{0}\right\} \cup\left\{\left|\xi+\frac{\tau}{\cos \gamma_{ \pm}} \cos \theta_{ \pm}\right|<\varepsilon_{0}\right\} \cup\left\{\left|\xi-\frac{\tau}{\cos \gamma_{ \pm}} \cos \theta_{ \pm}\right|<\varepsilon_{0}\right\}
\end{aligned}
$$

and a point $t \geqslant \varepsilon_{1},(0, t, \xi, \tau)$ is not in $S S_{V_{ \pm}}^{2,1}\left(\widetilde{f}_{ \pm}\right)$when

$$
\left|\xi+\frac{\tau}{\cos \gamma_{ \pm}}\right| \geqslant \varepsilon_{0}, \quad\left|\xi+\frac{\tau}{\cos \gamma_{ \pm}} \cos \theta_{ \pm}\right| \geqslant \varepsilon_{0}, \quad\left|\xi-\frac{\tau}{\cos \gamma_{ \pm}} \cos \theta_{ \pm}\right| \geqslant \varepsilon_{0} .
$$

In the particular case $\Im \sin \theta_{+} \Im \sin \theta_{-} \neq 0$ (which means that the boundary condition is absorbing), these conditions reduce to

$$
\operatorname{SS}\left(\widetilde{f}_{ \pm}\right) \cap\left\{x>0, t \geqslant \varepsilon_{1}\right\} \subset\left\{\left|\xi+\tau / \cos \gamma_{ \pm}\right|<\varepsilon_{0}\right\}
$$

and a point $(0, t, \xi, \tau), t \geqslant \varepsilon_{1}$ is not in $S S_{V_{ \pm}}^{2,1}\left(\widetilde{f}_{ \pm}\right)$when $\left|\xi+\tau / \cos \gamma_{ \pm}\right| \geqslant \varepsilon_{0}$.

We denote by $L_{ \pm}$the operator in $\Omega_{ \pm}$defined by

$$
L_{+} \phi(x, y, t)=\left(1+\left(a^{\prime}(x)\right)^{2}\right)^{-1 / 2}\left(\partial_{y} \phi-a^{\prime}(x) \partial_{x} \phi\right)-z^{+}(x) \partial_{t} \phi
$$

Notice that $\left.\widetilde{f}_{ \pm}\right|_{x>0}=\left.\partial_{ \pm}\left(u-u_{ \pm}\right)\right|_{x>0}$. We have

$$
\begin{gathered}
\left.\left(u-u_{ \pm}\right)\right|_{t<0}=\left.u_{r, \pm}\right|_{t<0}, \\
\left.L_{ \pm}\left(u-u_{ \pm}\right)\right|_{\partial \Omega_{ \pm} \cap x>0}=0 .
\end{gathered}
$$

We introduce the involutive submanifold $V_{ \pm}=T^{*}\left(\Delta_{ \pm} \times \mathbb{R}\right) \cap\{x=0\}$, associated with $s(x)=x$. Similarly, we introduce the involutive submanifold $-V_{ \pm}=T^{*}\left(\Delta_{ \pm} \times \mathbb{R}\right) \cap$ $\{x=0\}$, associated with $s(x)=-x$ (we changed the orientation on the cotangent space). A solution of $\left(\Delta-\partial_{t^{2}}^{2}\right) g=0$ is $V_{ \pm}$-incoming if and only if $g$ is $-V_{ \pm}$-outgoing (following Definition 3.1).

We intend to show that $u-u_{ \pm}$is $V_{ \pm}$-incoming. If this is true, then Theorem 3 proves that

$$
\left(\rho_{0},-1\right) \notin \operatorname{SS}_{V_{ \pm}}^{2,1}\left(\partial_{ \pm}\left(u-u_{ \pm}\right)-\left.z^{ \pm}(x)\left(u-u_{ \pm}\right)\right|_{\partial \Omega_{ \pm}}\right)
$$

and the condition

$$
\exists \varepsilon^{\prime \prime}>0, \quad\left|\left(L\left(\rho_{0}\right)\right)^{2}-r_{0}\left(0, \rho_{0}\right)\right| \geqslant \varepsilon^{\prime \prime}
$$

implies

$$
\left(\rho_{0},-1\right) \notin \operatorname{SS}_{V_{ \pm}}^{2,1}\left(\partial_{ \pm}\left(u-u_{ \pm}\right)\right) \cap \operatorname{SS}_{V_{ \pm}}^{2,1}\left(u-\left.u_{ \pm}\right|_{\partial \Omega_{ \pm}}\right) .
$$

It is important to notice that (72) is automatically fulfilled if $\Im z^{ \pm}(0) \neq 0$, because $\rho$ is real. This means that the impedance boundary condition correspond to an absorbing material. It means also that the Brewster angles are complex. 
The Proposition 3.1 is a consequence of Lemma 3.8 .

LEMMA 3.8. - If $\rho=(x, t, \widetilde{\xi}, \tau), 0 \leqslant x \leqslant c t,\left|\widetilde{\xi}+\tau / \cos \gamma_{ \pm}\right| \geqslant \varepsilon_{0}$, the relations (70), (71) imply

$$
u-u_{ \pm} \text {is } V_{ \pm} \text {-incoming at } \rho .
$$

Proof of Lemma 3.8. - The argument of Gérard and Lebeau is valid, owing to an analytic theorem of propagation of singularities for a mixed boundary condition, in the neighborhood of points where this mixed boundary condition is elliptic, that is where the Calderòn operator exists. This result is a generalization of the usual theorem of propagation of singularities for Dirichlet boundary conditions of Sjöstrand [38].

If $\rho \in \mathcal{E}$, the elliptic regularity shows that, owing to the boundary condition

$$
\partial_{ \pm}\left(u-u_{ \pm}\right)-\left.z^{ \pm}\left(u-u_{ \pm}\right)\right|_{\{x>0\} \cap \partial \Omega_{ \pm}}=0
$$

and the representation of the Calderòn operator, the result of Proposition 3.1 is true.

If $\rho \notin \mathcal{E}$, we consider the half bicharacteristic of $\partial_{t^{2}}^{2}-\Delta$ starting at $\rho$, included in $\Omega_{ \pm}$, in the direction of $t<0^{(2)}$.

- Let us assume first that $\left|\widetilde{\xi}-\tau / \cos \gamma_{ \pm}\right| \geqslant \varepsilon_{0}^{\prime}$, where $\varepsilon_{0}^{\prime}$ is to be given. There exists $\varepsilon_{1}$ such that, for $t \leqslant \varepsilon_{1}, \gamma$ does not cross the "real" boundary $\Delta_{ \pm}$before reaching $t<0$. The half-bicharacteristic $\gamma$ is constructed such that the distance to $\Delta_{ \pm}$is decreasing along $\gamma$ in the neighborhood of $\Delta_{ \pm}$.

But the choice of the phase function $\theta_{ \pm}$implies that, on the bicharacteristics included in $\operatorname{SS}\left(u_{r, \pm}\right) \cap \Omega_{ \pm}$, the distance to $\Delta_{ \pm}$is increasing along the bicharacteristic parametrized by $t$. This shows that $u_{r, \pm}$ is regular along $\gamma$.

- If $x=0$, this means exactly (see Definition 3.1) that $u-u_{ \pm}$is $V_{ \pm}$-incoming.

- If $x>0$, we know that $u-\left.u_{ \pm}\right|_{t<0}=\left.u_{r, \pm}\right|_{t<0}$. By construction, the halfbicharacteristic $\gamma$ enters the region $t<0$ while staying in $D_{ \pm}$. Hence there exists $\epsilon_{1}>0$ such that $\gamma \cup\left\{t>-\epsilon_{1}\right\} \subset D_{ \pm}$, and thus on this part of the bicharacteristic there is no interaction with the boundary. Hence as $u_{r, \pm}$ is regular for $-\epsilon_{1}<t<0$, from the

${ }^{(2)}$ To give a precise idea to the reader, we consider the (elementary) case where $\widetilde{\Delta}_{+}=\{y=0\}$, $D_{+}=\{y>0\}$, the normal variable to the boundary is $y=n$, and the bicharacteristic strips starting from a point $\left(t, x, 0, \tau_{0}, \xi_{0}, \eta_{0}\right)$ which is hyperbolic, that is $\eta_{0}= \pm\left(\tau_{0}^{2}-\xi_{0}^{2}\right)^{1 / 2}$, are

$$
x(s)=x+2 \xi_{0} s, \quad n(s)=2 \eta_{0} s, \quad t(s)=t-2 \tau_{0} s .
$$

Hence the half bicharacteristic strips starting at $\left(t, x, \tau_{0}, \xi_{0}\right)$ included in $n>0$ are

$$
x(s)=x+2 \xi_{0} s, \quad n(s)= \pm 2\left(\tau_{0}^{2}-\xi_{0}^{2}\right)^{1 / 2} s, \quad t(s)=t-2 \tau_{0} s, \quad \pm s>0 .
$$

This bicharacteristic is directed towards $t<0$ when $-2 \tau_{0} s<0$. Hence, for $\tau_{0}>0$, the half bicharacteristic included in $n>0$ directed towards $t<0$ is

$$
x(s)=x+2 \xi_{0} s, \quad n(s)=2\left(\tau_{0}^{2}-\xi_{0}^{2}\right)^{1 / 2} s, \quad t(s)=t-2 \tau_{0} s, \quad s>0
$$

and for $\tau_{0}<0$, the half bicharacteristic included in $n>0$ directed towards $t<0$ is

$$
x(s)=x+2 \xi_{0} s, \quad n(s)=-2\left(\tau_{0}^{2}-\xi_{0}^{2}\right)^{1 / 2} s, \quad t(s)=t-2 \tau_{0} s, \quad s<0 .
$$


propagation of singularities theorem in an open set, we obtain that $\gamma \cap \operatorname{SS}\left(u-u_{ \pm}\right)=\varnothing$. This means that $u-u_{ \pm}$is $V_{ \pm}$-incoming.

- Consider the second case $\left|\widetilde{\xi}-\tau / \cos \gamma_{ \pm}\right| \leqslant \varepsilon_{0}^{\prime}$. For $\varepsilon_{0}^{\prime}$ small enough, it is geometric to see that every half-ray $\gamma_{1}$ issued from $\rho$ (there is no uniqueness because of the glancing points) in the direction of $t<0$ is locally contained in $x>0$. There is thus a point of $\gamma_{1}$ in $\{t<0\} \cap\{x>0\}$ such that $u_{r, \pm}$ is regular.

- When the point is hyperbolic, that is $\widetilde{\xi} \neq \tau / \cos \gamma_{ \pm}$, if $\varepsilon_{0}^{\prime}$ is chosen small enough, we know that the boundary operator $L_{ \pm}$is elliptic at $\rho$. From the conditions (70), (71) and the theorem of reflection of singularities with a mixed boundary condition which is elliptic (which is the case when $\left|\widetilde{\xi}-\tau / \cos \gamma_{ \pm}\right|$is small), we obtain that $\mathrm{SS}_{b}\left(u-u_{ \pm}\right) \cap \gamma_{1}=\varnothing$.

- For $x=0, u-u_{ \pm}$is $V_{ \pm}$-incoming if $\rho$ is hyperbolic.

- For $x>0$, we also get that $u-u_{ \pm}$is $V_{ \pm}$-incoming.

- When the point is glancing, that is $\widetilde{\xi}=\tau / \cos \gamma_{ \pm}$, this means that

$$
\rho=\left(0, t, \tau, \tau / \cos \gamma_{ \pm}\right) \text {. }
$$

We verify that the half rays $\gamma^{*}$ starting from $\rho$ stay in $x>0$ before entering $t<0$ (that is because $\gamma_{+}<\pi / 2$ and $\gamma_{-}>-\pi / 2$ ). This means that there exists a point of $\{t<0\} \cap\{x>0\}$ where $u_{r, \pm}$ is regular. Thus the same argument of propagation of singularities for the mixed boundary problem (the boundary condition is elliptic because we are at the glancing point) applied to this new point show that $u-u_{ \pm}$is $V_{ \pm}$-incoming.

Finally, there is no other assumption needed to prove that the wave $u-u_{ \pm}$is $V_{ \pm}$-incoming, hence Lemma 3.8 is proven.

Note that is is not surprising that the points in the neighborhood of $\widetilde{\xi}=-\tau / \cos \gamma_{ \pm}$ are not incoming, because it is near the tangent direction which excludes a propagation in the good region.

Let us end the proof of Proposition 3.1. It is enough to apply Theorem 3 to the wave $u-u_{ \pm}$, excluding the points of the form $\left|\left(L_{ \pm}(\rho)\right)^{2}-r_{0}(0, \rho)\right| \leqslant \varepsilon_{0}^{\prime}$, using Lemma 3.8 . 



\section{CHAPTER 4}

\section{REDUCED SYSTEM ON THE BOUNDARY}

As in the section 5 of [20], we prove that the principal symbol of the system calculating the normal derivatives on each face is the symbol associated with the same system corresponding to the tangent plane wedge and the boundary conditions where the coefficient of $\left.\partial_{t} u\right|_{\Delta_{ \pm}}$is $z^{ \pm}(0)$. This idea has already been used by J.M. Bernard who proved (annex of [5] pp. 327-329) that for a convex boundary (for which is known the source on the boundary), the boundary condition on the plane tangent "face" of the diedra (given in (2) p. 322) and the boundary condition deduced on the solution near the curved boundary (given by the expression A.9 p. 329) have the same leading term. This was done using Maliuzhinets integral ([31]).

In a first part of this section, we shall introduce the unknowns and give their microlocal regularity. In a second part, after introducing the F.B.I. transform in time and the F.B.I. transform in $x$, we shall give the explicit calculation of the symbols.

Let $e$ be a strictly positive number and $E_{e}$ the space of tempered distributions $u(t, x)$, supported in $t \geqslant 0$, such that the partial Fourier transform in time of $u$, $\mathcal{F} u(k, x)$, is in $L^{2}\left(\mathbb{R}^{2}\right)$ for $\Im k<0$ and its norm is bounded by $A(1+|k|)^{B}|\Im k|^{-e}$. This space of tempered distributions has been introduced in Definition 2.1 of [20]. The partial FBI in time of such functions is defined by

$$
T_{0} u(\tau, x, \lambda)=\left(\frac{\lambda}{2 \pi}\right)^{1 / 2} \int_{\Im k=-a} e^{i \lambda k \tau-\lambda k^{2} / 2} \mathcal{F} u(\lambda k, x) \mathrm{d} k .
$$

There is an inversion formula for this transformation, given by [20] (2.12).

Let $W$ be an open subset of $\mathbb{C}, \phi \in C^{0}(W, \mathbb{R})$.

Definition 4.1. - We denote by $H_{\phi}\left(W, L^{2}\right)$ the Sjöstrand space of functions $f(w, x, \lambda)$, holomorphic in $w \in W, L^{2}(\mathbb{R})$ in $x$, defined for $\lambda \geqslant 1$, such that for all $K$ compact subset of $W$, there exists $A, B>0$ such that

$$
\sup _{w \in K}\left\|e^{-\lambda \phi(w)} f(w, \cdot \lambda)\right\|_{L^{2}(\mathbb{R})} \leqslant A \lambda^{B} .
$$


For $\kappa>0$, we define

$$
W_{\kappa}=\{W \in \mathbb{C},|\Re W|<\kappa,|1+\Im W|<\kappa\} .
$$

Introduce $t_{0}>\kappa$. The function $\phi_{0}(w)=\frac{1}{2}(\Im w)^{2}$ is the usual weight function of Sjöstrand (see Sjöstrand [38]). The following Lemma gives the regularity of $T_{0} u$ for a function supported in $t \geqslant t_{0}$ :

Lemma 4.1. - Let $\phi(w)=\phi_{0}(w)-\frac{1}{2}\left(t_{0}-\kappa\right)^{2}$. For $u \in E_{e}$, such that $\operatorname{supp} u$ is contained in $\left\{t \geqslant t_{0}\right\}$, we have

$$
T_{0} u \in H_{\phi}\left(W_{\kappa}, L^{2}\right) .
$$

If $K_{k}$ is a family of operators bounded on $L^{2}\left(\mathbb{R}^{2}\right)$ and satisfying the inequality

$$
\left\|K_{k}\right\| \leqslant A_{1}(1+|k|)^{B_{1}}|\Im k|^{-e}
$$

for $\Im k<0$ and the family is holomorphic in $k$, $\Im k<0$, then $K_{k}$ defines an operator from $E_{e_{1}}$ into $E_{e_{1}+e}$ through

$$
\mathcal{F}(K u)(k, x)=K_{k}(\mathcal{F} u)(k, x) .
$$

We can compute $T_{0} K u$ with Proposition 2.2 of $[\mathbf{2 0}]$ p. 350, that we recall below:

Proposition 4.1. - For $u \in E_{e^{\prime}}$ and $K$ from $E_{e^{\prime}}$ to $E_{e+e^{\prime}}$, we have the identity

$$
T_{0} K u-\frac{\lambda}{2 \pi} \int_{t_{-}}^{t_{+}} \mathrm{d} t \int_{\tau_{-}}^{\tau_{+}} d \tau e^{i \lambda \tau(w-t)-\lambda \tau^{2}} K_{\lambda \tau} T_{0} u(t-i \tau, \cdot, \lambda) \in H_{\phi}\left(W, L^{2}\right)
$$

where $t_{ \pm}= \pm 2 \kappa, \tau_{ \pm}=1 \pm 2 \kappa-i / \lambda$, and $\phi(w)=\phi_{0}(w)-\kappa^{2} / 4$.

The relation proven in Proposition 1.1 allows us to write a first system between the trace on the boundary and the traces of the normal derivatives on each piece of the boundary.

\subsection{The fundamental identity}

Let us introduce localizing functions in time $\phi_{1}, \phi_{2}$ and in space $\psi$. We fix a $t_{0}>0$ small. The functions $\phi_{1}, \phi_{2}$ are in $C_{0}^{\infty}\left(\mathbb{R}_{t}\right)$ and $\psi \in C_{0}^{\infty}(\mathbb{R})$ (they are defined as in p. 351 of $[\mathbf{2 0}])$ :

- On a neighborhood of $\left[0, t_{0}\right], \phi_{1} \equiv 1$ and $\phi_{1} \equiv 0$ for $t \geqslant 2 t_{0}$,

- On a neighborhood of $\left[0, t_{0} / 2\right], \phi_{2} \equiv 1$ and $\phi_{2} \equiv 0$ for $t \geqslant t_{0}$,

- For $-3 c t_{0} \leqslant x \leqslant 3 c t_{0}, c$ introduced above in Lemma $1.1, \psi \equiv 1$, and $\psi \equiv 0$ for $|x| \geqslant 4 c t_{0}$.

In particular, $\phi_{1} \phi_{2}=\phi_{2}$ and $(1-\psi) \phi_{1} \times 1_{0 \leqslant x \leqslant c t}=0$. Recall that $u$ is the solution of (6) and $\underline{u}=1_{\Omega} u$.

From Proposition 1.1, we have

$$
\left(\Delta-\partial_{t^{2}}^{2}\right) \underline{u}=\left(v_{+} \delta_{+}+v_{-} \delta_{-}\right)+\left(\left.u\right|_{\partial \Omega} \delta_{\partial \Omega}^{\prime}\right)=G
$$


with $\left.u\right|_{t<0}=\left.u_{i}\right|_{t<0}$. As $\left(\Delta-\partial_{t^{2}}^{2}\right)_{+}^{-1}$ is the fundamental solution of $\left(\Delta-\partial_{t^{2}}^{2}\right)$ supported in $t \geqslant 0$, we obtain the equality (77) comparing the solution in $t<0$ and in $t>0$ :

$$
\underline{u}=u_{i}+\left(\Delta-\partial_{t^{2}}^{2}\right)_{+}^{-1}\left[v_{+} \delta_{+}\right]+\left(\Delta-\partial_{t^{2}}^{2}\right)_{+}^{-1}\left[v_{-} \delta_{-}\right]+\left(\Delta-\partial_{t^{2}}^{2}\right)_{+}^{-1}\left[\left.u\right|_{\partial \Omega} \delta_{\partial \Omega}^{\prime}\right] .
$$

From this relation, we get

$$
\begin{aligned}
\phi_{2} \underline{u}=\phi_{2} u_{i}+\phi_{2}\left(\Delta-\partial_{t^{2}}^{2}\right)_{+}^{-1}\left[\phi_{1} v_{+} \delta_{+}\right] & +\phi_{2}\left(\Delta-\partial_{t^{2}}^{2}\right)_{+}^{-1}\left[\phi_{1} v_{-} \delta_{-}\right] \\
& +\phi_{2}\left(\Delta-\partial_{t^{2}}^{2}\right)_{+}^{-1}\left[\left.\phi_{1} u\right|_{\partial \Omega} \delta_{\partial \Omega}^{\prime}\right]+S_{1}(x, y, t) .
\end{aligned}
$$

with

$$
S_{1}(x, y, t)=\phi_{2}\left(\Delta-\partial_{t^{2}}^{2}\right)_{+}^{-1}\left(\left(1-\phi_{1}\right) G\right) \text {. }
$$

The support of $G$ is contained in $t \geqslant 0$, hence the support of $\left(1-\phi_{1}\right) G$ is contained in $t \geqslant t_{0}+\delta_{0}, \delta_{0}>0$. Hence, as $\left(\Delta-\partial_{t^{2}}^{2}\right)_{+}^{-1}$ is the outgoing solution, the support of $\left(\Delta-\partial_{t^{2}}^{2}\right)_{+}^{-1}\left(1-\phi_{1}\right) G$ is contained in $t \geqslant t_{0}+\delta_{0}$, where $\phi_{2} \equiv 0$. Thus $S_{1} \equiv 0$.

We consider the normal derivative of this relation, and we take the partial Fourier transform in time, which is valid because $\phi_{1} v_{ \pm} \in L^{2}\left(\mathbb{R}^{2}\right)$ supported in $t \geqslant 0$, hence the partial Fourier transform in time of $\phi_{1} v_{ \pm}$exist and is holomorphic in $\varsigma k<0$. We check that

$$
\left\|\mathcal{F}\left(\phi_{1} v_{ \pm}\right)(\cdot, k)\right\|_{L^{2}(\mathbb{R})} \leqslant C t_{0}^{1 / 2}\left\|v_{ \pm} \phi_{1}\right\|_{L^{2}\left(\mathbb{R}_{+} \times \mathbb{R}_{+}\right)},
$$

hence $\phi_{1} v_{ \pm} \in E_{0}$. From the boundary equality, $\left.\phi_{1} u\right|_{\partial \Omega} \in E_{0}$. Hence we have the equality, after Fourier transform in time (denoted by $\widehat{u}$ or by $\mathcal{F}(u)$ ), for $k \in \mathbb{C}^{-}$:

$$
\begin{aligned}
\widehat{\phi}_{2}(k) \star \underline{\widehat{u}} & =\widehat{\phi}_{2}(k) \star \widehat{u}_{i}+\widehat{\phi}_{2}(k) \star\left(\Delta+k^{2}\right)^{-1}\left[\mathcal{F}\left(\phi_{1} v_{+}\right) \delta_{+}\right] \\
& +\widehat{\phi}_{2}(k) \star\left(\Delta+k^{2}\right)^{-1}\left[\mathcal{F}\left(\phi_{1} v_{-}\right) \delta_{-}\right]+\widehat{\phi}_{2}(k) \star\left(\Delta+k^{2}\right)^{-1}\left[\mathcal{F}\left(\left.\phi_{1} u\right|_{\partial \Omega}\right) \delta_{\partial \mathcal{O}}^{\prime}\right] .
\end{aligned}
$$

Recall that the operators $K_{ \pm}^{+, k}, K_{ \pm}^{-, k}, R^{+, k}$ and $R^{-, k}$ are defined in Definition 1.1. Hence we get

$$
\begin{aligned}
\widehat{\phi}_{2}(k) \star \psi(x) \mathcal{F}\left(\partial_{+} \underline{u}\right)=\widehat{\phi}_{2}(k) & \star K_{+}^{+, k}\left[\mathcal{F}\left(\phi_{1} v_{+}\right)\right]+\widehat{\phi}_{2}(k) \star K_{-}^{+, k}\left[\mathcal{F}\left(\phi_{1} v_{-}\right)\right] \\
& +\widehat{\phi}_{2}(k) \star R^{+, k}\left[\mathcal{F}\left(\left.\phi_{1} u\right|_{\partial \Omega}\right)\right]+\widehat{\phi}_{2}(k) \star \psi(x) \partial_{+} \widehat{u}_{i} . \\
\widehat{\phi}_{2}(k) \star \psi(x) \mathcal{F}\left(\partial_{-} \underline{u}\right)=\widehat{\phi}_{2}(k) \star & K_{+}^{-, k}\left[\mathcal{F}\left(\phi_{1} v_{+}\right)\right]+\widehat{\phi}_{2}(k) \star K_{-}^{-, k}\left[\mathcal{F}\left(\phi_{1} v_{-}\right)\right] \\
& +\widehat{\phi}_{2}(k) \star R^{-, k}\left[\mathcal{F}\left(\left.\phi_{1} u\right|_{\partial \Omega}\right)\right]+\widehat{\phi}_{2}(k) \star \psi(x) \partial_{-} \widehat{u}_{i} .
\end{aligned}
$$

We state in the next section the results on the operators $R^{ \pm, k}$ and $K_{ \pm}^{ \pm, k}, K_{ \pm}^{\mp, k}$. The regularity results allow us, along with (76) to write the system deduced from the two equalities with the operators $R^{ \pm}$and $K_{ \pm}^{ \pm}, K_{ \pm}^{\mp}$. 


\subsection{Expression of the Neumann to Neumann operators}

In this Section, we calculate using a residue theorem the operators $K_{ \pm}^{+, k}, K_{ \pm}^{-, k}$, $R_{ \pm}^{+, k}, R_{ \pm}^{-, k}$ defined in Definition 1.1. From the simple example of the function equal to 1 on $[0,1]$, and the distribution $\partial_{y}\left[\left(\Delta+k^{2}\right)^{-1}\left(1_{[0,1]} \otimes \delta_{x=0}^{\prime}\right)\right]$, which is equal to two Dirac distributions concentrated at 0 and at 1 , we check that the operators $R_{+}^{ \pm, k}$ and $R_{-}^{ \pm, k}$ send functions of $H^{1}\left(\mathbb{R}_{+}\right)$to distributions of $H^{-1-\varepsilon}(\mathbb{R})$. However, we prove that these operators send $H_{0}^{1}\left(\mathbb{R}_{+}\right)$on $L^{2}\left(\mathbb{R}_{+}\right)$and that the operators $R^{+, k}$ and $R^{-, k}$ live from $H^{1}(\mathbb{R})$ to $L^{2}\left(\mathbb{R}_{+}\right)$. We need in what follows some definitions:

$$
\left\{\begin{array}{l}
\mu_{+}\left(x, x^{\prime}\right)\left(x-x^{\prime}\right)=a(x)-a\left(x^{\prime}\right) \\
\mu_{-}\left(x, x^{\prime}\right)\left(x-x^{\prime}\right)=b(x)-b\left(x^{\prime}\right) \\
\xi_{1}^{ \pm}=\xi+\mu_{ \pm} \eta \\
\eta_{1}^{ \pm}=-\mu_{ \pm} \xi+\eta \\
\xi_{ \pm}\left(x, x^{\prime}, k, \xi_{1}^{ \pm}\right)=\left[k^{2}\left(1+\mu_{ \pm}^{2}\right)-\left(\xi_{1}^{ \pm}\right)^{2}\right]^{1 / 2} \\
\xi_{0}=\left[k^{2}-\xi^{2}\right]^{1 / 2}
\end{array}\right.
$$

the functions and dual variables allowing us to study the behavior of the faces of the wedge, where $\Im \xi_{+}>0, \Im \xi_{0}>0, \Im \xi_{-}>0$. We have $\eta=\frac{\mu_{ \pm} \xi_{1}^{ \pm}+\eta_{1}^{ \pm}}{1+\mu_{ \pm}^{2}}, \xi=\frac{\xi_{1}^{ \pm}-\mu_{ \pm} \eta_{1}^{ \pm}}{1+\mu_{ \pm}^{2}}$. We note that

$\left(1+\mu_{ \pm}^{2}\right)\left(k^{2}-\xi^{2}-\eta^{2}\right)=\left(\xi_{1}^{ \pm}\right)^{2}+\left(\eta_{1}^{ \pm}\right)^{2}-k^{2}\left(1+\mu_{ \pm}^{2}\right), \quad\left(1+\mu_{ \pm}^{2}\right) \mathrm{d} \xi \wedge \mathrm{d} \eta=\mathrm{d} \xi_{1}^{ \pm} \wedge \mathrm{d} \eta_{1}^{ \pm}$, hence

$$
\frac{\mathrm{d} \xi \mathrm{d} \eta}{k^{2}-\xi^{2}-\eta^{2}}=\frac{\mathrm{d} \xi_{1}^{ \pm} \mathrm{d} \eta_{1}^{ \pm}}{\left(\xi_{ \pm}\right)^{2}-\left(\eta_{1}^{ \pm}\right)^{2}} .
$$

The operators $K$ have been calculated in $[\mathbf{2 0}]$ and we only reproduce the results in the

Lemma 4.2. - Let $\sigma^{+}, \sigma^{-}, s^{+,-}, s^{-,+}$the symbols equal to

$$
\left\{\begin{array}{l}
\sigma^{+}\left(x, x^{\prime}, \xi_{1}^{+}, k\right)=\frac{\mu_{+}-a^{\prime}(x)}{1+\mu_{+}^{2}}\left(\frac{\xi_{1}^{+}}{\xi_{+}}-\mu_{+}\right) \\
\sigma^{-}\left(x, x^{\prime}, \xi_{1}^{-}, k\right)=\frac{b^{\prime}(x)-\mu_{-}}{1+\mu_{-}^{2}}\left(\frac{\xi_{1}^{-}}{\xi_{-}}+\mu_{-}\right) \\
s^{+,-}(x, \xi, k)=1-a^{\prime}(x) \frac{\xi}{\xi_{0}} \\
s^{-,+}(x, \xi, k)=1+b^{\prime}(x) \frac{\xi}{\xi_{0}} .
\end{array}\right.
$$

Let

$$
\begin{aligned}
& \psi_{+,-}\left(x, x^{\prime}, \xi, k\right)=\left(x-x^{\prime}\right) \xi+\left(a(x)-b\left(x^{\prime}\right)\right) \xi_{0} \\
& \psi_{-,+}\left(x, x^{\prime}, \xi, k\right)=\left(x-x^{\prime}\right) \xi+\left(a\left(x^{\prime}\right)-b(x)\right) \xi_{0} .
\end{aligned}
$$


We have, for $f \in L^{2}\left(\mathbb{R}_{+}\right)$

(81) $K_{+}^{+, k}(f)(x, k)=\frac{1}{4 \pi} \int_{\mathbb{R}_{+} \times \mathbb{R}} \psi(x) \psi\left(x^{\prime}\right) f\left(x^{\prime}\right) e^{i\left(x-x^{\prime}\right) \xi_{1}^{+}}$

$$
\times \frac{\left(1+\left(a^{\prime}\left(x^{\prime}\right)\right)^{2}\right)^{1 / 2}}{\left(1+\left(a^{\prime}(x)\right)^{2}\right)^{1 / 2}}\left[1+\sigma^{+}\left(x, x^{\prime}, \xi_{1}^{+}, k\right)\right] \mathrm{d} x^{\prime} \mathrm{d} \xi_{1}^{+}
$$

(82) $\quad K_{-}^{-, k}(f)(x, k)=\frac{1}{4 \pi} \int_{\mathbb{R}_{+} \times \mathbb{R}} \psi(x) \psi\left(x^{\prime}\right) f\left(x^{\prime}\right) e^{i\left(x-x^{\prime}\right) \xi_{1}^{-}}$

$$
\times \frac{\left(1+\left(b^{\prime}\left(x^{\prime}\right)\right)^{2}\right)^{1 / 2}}{\left(1+\left(b^{\prime}(x)\right)^{2}\right)^{1 / 2}}\left[1+\sigma^{-}\left(x, x^{\prime}, \xi_{1}^{-}, k\right)\right] \mathrm{d} x^{\prime} \mathrm{d} \xi_{1}^{-}
$$

$$
\begin{aligned}
1_{x \geqslant 0} K_{+}^{-, k}(f)(x, k)=\frac{1}{4 \pi} \int_{\mathbb{R}^{2}} \psi(x) \psi\left(x^{\prime}\right) f( & \left.x^{\prime}\right) 1_{x \geqslant 0} 1_{x^{\prime} \geqslant 0} e^{i \psi_{-,+}\left(x, x^{\prime}, \xi, k\right)} \\
& \times \frac{\left(1+\left(a^{\prime}\left(x^{\prime}\right)\right)^{2}\right)^{1 / 2}}{\left(1+\left(b^{\prime}(x)\right)^{2}\right)^{1 / 2}} s^{-,+}(x, \xi, k) \mathrm{d} x^{\prime} \mathrm{d} \xi
\end{aligned}
$$

$$
\begin{aligned}
1_{x \geqslant 0} K_{-}^{+, k}(f)(x, k)=\frac{1}{4 \pi} \int_{\mathbb{R}^{2}} \psi(x) \psi\left(x^{\prime}\right) f( & \left.x^{\prime}\right) \\
& 1_{x \geqslant 0} 1_{x^{\prime} \geqslant 0} e^{i \psi_{+,-}\left(x, x^{\prime}, \xi, k\right)} \\
& \times \frac{\left(1+\left(b^{\prime}\left(x^{\prime}\right)\right)^{2}\right)^{1 / 2}}{\left(1+\left(a^{\prime}(x)\right)^{2}\right)^{1 / 2}} s^{+,-}(x, \xi, k) \mathrm{d} x^{\prime} \mathrm{d} \xi
\end{aligned}
$$

Equivalently,

$$
\begin{array}{rl}
\text { (83) } K_{+}^{-, k}(f)=-\frac{1}{4 \pi} \int_{\mathbb{R}_{+} \times \mathbb{R}} & f\left(x^{\prime}\right) \psi\left(x^{\prime}\right) \psi\left(x^{\prime}\right) e^{i\left(x-x^{\prime}\right) \xi_{1}^{+}+i\left(b\left(x^{\prime}\right)-a\left(x^{\prime}\right)\right) \frac{\xi_{1}^{+}-\mu_{+} \xi_{+}}{1+\mu_{+}^{2}}} \mathrm{~d} x^{\prime} \mathrm{d} \xi_{1}^{+} \\
& \times \frac{\left(1+\left(a^{\prime}\left(x^{\prime}\right)\right)^{2}\right)^{1 / 2}}{\left(1+\left(b^{\prime}(x)\right)^{2}\right)^{1 / 2}}\left[\frac{\mu_{+}-b^{\prime}(x)}{1+\mu_{+}^{2}} \frac{\xi_{1}^{+}}{\xi_{+}}-\frac{1+b^{\prime}(x) \mu_{+}}{1+\mu_{+}^{2}}\right] \\
(84) \quad K_{-}^{+, k}(f)=\frac{1}{4 \pi} \int_{\mathbb{R}_{+} \times \mathbb{R}} f\left(x^{\prime}\right) \psi\left(x^{\prime}\right) \psi\left(x^{\prime}\right) e^{i\left(x-x^{\prime}\right) \xi_{1}^{-}+i\left(a\left(x^{\prime}\right)-b\left(x^{\prime}\right)\right) \frac{\xi_{1}^{-}+\mu_{-} \xi_{-}}{1+\mu_{-}^{2}}} \mathrm{~d} x^{\prime} \mathrm{d} \xi_{1}^{-} \\
\times \frac{\left(1+\left(b^{\prime}\left(x^{\prime}\right)\right)^{2}\right)^{1 / 2}}{\left(1+\left(a^{\prime}(x)\right)^{2}\right)^{1 / 2}}\left[\frac{\mu_{-}-a^{\prime}(x)}{1+\mu_{-}^{2}} \frac{\xi_{1}^{-}}{\xi_{-}}-\frac{1+a^{\prime}(x) \mu_{-}}{1+\mu_{-}^{2}}\right]
\end{array}
$$

\subsection{The Dirichlet to Neumann operators}

The results of what follows are similar to (2.27), (2.28) and the system of equations given from $(2.29)$ to $(2.42)$ of $[\mathbf{2 0}]$. We prove in this Section the two 
Proposition 4.2. - There exist distributions $S_{+}^{+}, S_{-}^{-}, S_{-}^{+}$and $S_{+}^{-}$of $H^{-1}(\mathbb{R})$ such that, for $f \in H^{1}\left(\mathbb{R}_{+}\right)$

$$
\begin{aligned}
& R_{ \pm}^{ \pm, k} f=R_{ \pm, 0}^{ \pm, k} f+f(0) S_{ \pm}^{ \pm} \\
& R_{\mp}^{ \pm, k} f=R_{\mp, 0}^{ \pm, k} f+f(0) S_{\mp}^{ \pm} .
\end{aligned}
$$

The operators $R_{ \pm, 0}^{ \pm, k}$ are operators from $H^{1}\left(\mathbb{R}_{+}\right)$to $L^{2}(\mathbb{R})$ and $R_{\mp, 0}^{ \pm, k}$ are operators from $H^{1}\left(\mathbb{R}_{+}\right)$to $L^{2}\left(\mathbb{R}_{+}\right)$whose expression is given in Proposition 4.3 below.

We introduce some new notations for the symbols: (85)

$$
\left\{\begin{array}{l}
L_{1}^{+}\left(x, x^{\prime}\right)=\frac{1}{\left(1+\left(a^{\prime}(x)\right)^{2}\right)^{1 / 2}}\left[\frac{\mu_{+}-a^{\prime}(x)}{1+\mu_{+}^{2}} \frac{\mu_{+}-a^{\prime}\left(x^{\prime}\right)}{1+\mu_{+}^{2}}-\frac{1+\mu_{+} a^{\prime}(x)}{1+\mu_{+}^{2}} \frac{1+\mu_{+} a^{\prime}\left(x^{\prime}\right)}{1+\mu_{+}^{2}}\right] \\
L_{2}^{+}\left(x, x^{\prime}\right)=\frac{1}{\left(1+\left(a^{\prime}(x)\right)^{2}\right)^{1 / 2}}\left[\frac{\mu_{+}-a^{\prime}(x)}{1+\mu_{+}^{2}} \frac{1+\mu_{+} a^{\prime}\left(x^{\prime}\right)}{1+\mu_{+}^{2}}+\frac{\mu_{+}-a^{\prime}\left(x^{\prime}\right)}{1+\mu_{+}^{2}} \frac{1+\mu_{+}^{\prime}(x)}{1+\mu_{+}^{2}}\right] \\
L_{3}^{+}\left(x, x^{\prime}\right)=\frac{1}{\left(1+\left(a^{\prime}(x)\right)^{2}\right)^{1 / 2}}\left[\frac{\left(1+\mu_{+} a^{\prime}(x)\right)\left(1+\mu_{+} a^{\prime}\left(x^{\prime}\right)\right)}{1+\mu_{+}^{2}}\right] \\
L_{1}^{-}\left(x, x^{\prime}\right)=\frac{1}{\left(1+\left(b^{\prime}(x)\right)^{2}\right)^{1 / 2}}\left[\frac{\mu_{-}-b^{\prime}(x)}{1+\mu_{-}^{2}} \frac{\mu_{-}-b^{\prime}\left(x^{\prime}\right)}{1+\mu_{-}^{2}}-\frac{1+\mu_{-} b^{\prime}(x)}{1+\mu_{-}^{2}} \frac{1+\mu_{-} b^{\prime}\left(x^{\prime}\right)}{1+\mu_{-}^{2}}\right] \\
L_{2}^{-}\left(x, x^{\prime}\right)=-\frac{1}{\left(1+\left(b^{\prime}(x)\right)^{2}\right)^{1 / 2}}\left[\frac{\mu-b^{\prime}(x)}{1+\mu_{-}^{2}} \frac{1+\mu_{-}^{\prime}\left(x^{\prime}\right)}{1+\mu_{-}^{2}}+\frac{\mu_{-}-b^{\prime}\left(x^{\prime}\right)}{1+\mu_{-}^{2}} \frac{1+\mu_{-}^{\prime}(x)}{1+\mu_{-}^{2}}\right] \\
\left.L_{3}^{-}\left(x, x^{\prime}\right)=\frac{\left(1+\mu_{-}^{\prime}(x)\right)\left(1+\mu_{-} b^{\prime}\left(x^{\prime}\right)\right)}{1+\mu_{-}^{2}}\right] .
\end{array}\right.
$$

and we introduce the symbols used here

$$
\begin{aligned}
& \left\{\begin{array}{l}
q_{1}^{+}\left(x, x^{\prime}, \xi_{1}^{+}, k\right)=\psi(x) \psi\left(x^{\prime}\right)\left[L_{2}^{+}\left(x, x^{\prime}\right)+L_{1}^{+}\left(x, x^{\prime}\right) \frac{\xi_{1}^{+}}{\xi_{+}}\right] \\
\sigma_{2}^{+}\left(x, x^{\prime}, \xi_{1}^{+}, k\right)=\psi(x) L_{3}^{+}\left(x, x^{\prime}\right) \psi\left(x^{\prime}\right) \frac{k^{2}}{\xi_{+}}+\frac{1}{i} \partial_{x^{\prime}} q_{1}^{+}\left(x, x^{\prime}, \xi_{1}^{+}, k\right) \\
q_{1}^{-}\left(x, x^{\prime}, \xi_{1}^{-}, k\right)=\psi(x) \psi\left(x^{\prime}\right)\left[L_{2}^{-}\left(x, x^{\prime}\right)+L_{1}^{-}\left(x, x^{\prime}\right) \frac{\xi_{1}^{-}}{\xi_{-}}\right] \\
\sigma_{2}^{-}\left(x, x^{\prime}, \xi_{1}^{-}, k\right)=\psi(x) L_{3}^{-}\left(x, x^{\prime}\right) \psi\left(x^{\prime}\right) \frac{k^{2}}{\xi_{-}}+\frac{1}{i} \partial_{x^{\prime}} q_{1}^{-}\left(x, x^{\prime}, \xi_{1}^{-}, k\right)
\end{array}\right. \\
& \left\{\begin{array}{l}
r_{1}^{+,-}(x, \xi, k)=\frac{1}{\left(1+\left(a^{\prime}(x)\right)^{2}\right)^{1 / 2}}\left(a^{\prime}(x)+\frac{\xi}{\xi_{0}}\right) \\
r_{2}^{+,-}\left(x, x^{\prime}, \xi, k\right)=\frac{k^{2}}{\xi_{0}} \frac{1+a^{\prime}(x) b^{\prime}\left(x^{\prime}\right)}{\left(1+\left(a^{\prime}(x)\right)^{2}\right)^{1 / 2}} \\
r_{1}^{-,+}(x, \xi, k)=\frac{1}{\left(1+\left(b^{\prime}(x)\right)^{2}\right)^{1 / 2}}\left(\frac{\xi}{\xi_{0}}-b^{\prime}(x)\right) \\
r_{2}^{-,+}\left(x, x^{\prime}, \xi, k\right)=\frac{k^{2}}{\xi_{0}} \frac{1+b^{\prime}(x) a^{\prime}\left(x^{\prime}\right)}{\left(1+\left(b^{\prime}(x)\right)^{2}\right)^{1 / 2}}
\end{array}\right.
\end{aligned}
$$


Proposition 4.3. - The operators $R_{ \pm, 0}^{ \pm, k}$ and $R_{\mp, 0}^{ \pm, k}$ defined on $H^{1}\left(\mathbb{R}_{+}\right)$are defined through the equalities

$$
\begin{aligned}
& R_{ \pm, 0}^{ \pm, k}(f)=\frac{i}{4 \pi} \int_{\mathbb{R}_{+} \times \mathbb{R}} \sigma_{2}^{ \pm}\left(x, x^{\prime}, \xi_{1}^{ \pm}, k\right) e^{i\left(x-x^{\prime}\right) \xi_{1}^{ \pm}} f\left(x^{\prime}\right) \mathrm{d} x^{\prime} \mathrm{d} \xi_{1}^{ \pm} \\
& +\frac{1}{4 \pi} \int_{\mathbb{R}_{+} \times \mathbb{R}_{1}} q_{1}^{ \pm}\left(x, x^{\prime}, \xi_{1}^{ \pm}, k\right) e^{i\left(x-x^{\prime}\right) \xi_{1}^{ \pm}} \partial_{x^{\prime}} f \mathrm{~d} x^{\prime} \mathrm{d} \xi_{1}^{ \pm} \\
& 1_{x \geqslant 0} R_{\mp, 0}^{ \pm, k}(f)=-\frac{i}{4 \pi} \int_{\mathbb{R}_{+} \times \mathbb{R}} e^{i \psi_{ \pm, \mp} \psi(x) \psi\left(x^{\prime}\right) r_{2}^{ \pm, \mp}\left(x, x^{\prime}, \xi, k\right) 1_{x \geqslant 0} 1_{x^{\prime} \geqslant 0} f\left(x^{\prime}\right) \mathrm{d} x^{\prime} \mathrm{d} \xi} \\
& +\frac{1}{4 \pi} \int_{\mathbb{R}_{+} \times \mathbb{R}} e^{i \psi_{ \pm, \mp} \psi(x) r_{1}^{ \pm, \mp}(x, \xi, k) 1_{x \geqslant 0} 1_{x^{\prime} \geqslant 0} \partial_{x^{\prime}}(\psi f)\left(x^{\prime}\right) \mathrm{d} x^{\prime} \mathrm{d} \xi}
\end{aligned}
$$

These operators are operators from $H^{1}\left(\mathbb{R}_{+}\right)$to $L^{2}(\mathbb{R})$ satisfying the relation (75) for $e=3$. We have, on $H_{0}^{1}\left(\mathbb{R}_{+}\right)$, the equality $R_{\mp, 0}^{ \pm, k}=R_{0, \mp}^{ \pm, k}=R_{\mp}^{ \pm, k}$. The same result holds for the operators $R^{+}$and $R^{-}$.

The main result we deduce from this relation is

THEOREM 4. - The operators $1_{x \geqslant 0} R^{+}$and $1_{x \geqslant 0} R^{-}$are well defined on $H^{1}(\mathbb{R})$. If $f \in H^{1}(\mathbb{R})$ is associated to $f_{+} \in H^{1}\left(\mathbb{R}_{+}\right)$and to $f_{-}$in $H^{1}\left(\mathbb{R}_{+}\right)$(the first one being $f 1_{x \geqslant 0}$ and the second one being $f(-x) 1_{x \geqslant 0}$ ) and to $f(0)$ (which is bounded by $\left.\|f\|_{H^{1}(\mathbb{R})}\right)$, they are respectively given by

$$
\begin{aligned}
& 1_{x \geqslant 0} R^{+}(f)=1_{x \geqslant 0} R_{+, 0}^{+}\left(f_{+}\right)+1_{x \geqslant 0} R_{-, 0}^{+}\left(f_{-}\right)+f(0) T^{+} \\
& 1_{x \geqslant 0} R^{-}(f)=1_{x \geqslant 0} R_{+, 0}^{-}\left(f_{+}\right)+1_{x \geqslant 0} R_{-, 0}^{-}\left(f_{-}\right)+f(0) T^{-} .
\end{aligned}
$$

The distributions $T^{+}$and $T^{-}$are the restriction to $\mathbb{R}^{+}$of the distributions of $L^{2}(\mathbb{R})$ given by

$$
\left\langle\widetilde{T}^{ \pm}, l\right\rangle=\frac{i}{4 \pi} \iint T_{ \pm}(x) l(x) \frac{k^{2}}{\xi_{ \pm}^{3}}\left[\left(1+\mu_{ \pm}^{2}\right)+3 i \frac{\xi_{1}^{ \pm}}{\xi_{ \pm}^{2}} \mu_{ \pm} \partial_{x} \mu_{ \pm}\right] e^{i x \xi_{1}^{ \pm}} \mathrm{d} x \mathrm{~d} \xi_{1}^{ \pm}
$$

We begin with the proof of this theorem. The expression of the distributions $S_{+}^{+}$ and $S_{-}^{+}$, given in (151), (153) lead to the expression (154) for the distribution $S_{+}^{+}+S_{-}^{+}$. The theorem 4 is a consequence of the results of regularity on $R_{ \pm, 0}^{ \pm}$and on $R_{\mp, 0}^{ \pm}$of Proposition 4.3 as well as the regularity of the distribution $T^{+}$. For $T^{+}$, we notice that the functions

$$
g_{1}\left(\xi_{1}^{ \pm}\right)=\frac{k^{2}}{\xi_{ \pm}^{3}}, \quad g_{2}\left(\xi_{1}^{ \pm}\right)=3 i \frac{\xi_{1}^{ \pm}}{\xi_{ \pm}^{2}}
$$

belong to $L^{2}\left(\mathbb{R}_{\xi_{1}^{ \pm}}\right)$, and that

$$
\int\left(1+\left|\xi_{1}^{ \pm}\right|^{2}\right)^{s}\left|g_{j}\left(\xi_{1}^{ \pm}\right)\right|^{2} \mathrm{~d} \xi_{1}^{ \pm}<+\infty \text { for } 2 s-6<-1 .
$$

hence its inverse Fourier transform is in $H^{\frac{5}{2}-\varepsilon}(\mathbb{R})$, which imply that $T^{+}$is in $H^{\frac{5}{2}-\varepsilon}(\mathbb{R})$. 
The expression of the distribution $T^{+}$is also easily computed using (151) and (153). Note that, using integration by parts and the relation $\partial_{\xi_{1}^{+}} \xi_{+}=-\xi_{1}^{+} / \xi_{+}$and $\partial_{x} \xi_{+}=k^{2} \mu_{+} \partial_{x} \mu_{+} / \xi_{+}$, we have

$$
\left\langle T^{+}, l\right\rangle=\frac{i k^{2}}{4 \pi} \int_{\mathbb{R}^{2}} T_{+}(x) l(x) e^{i x \xi_{1}^{+}}\left[\frac{1+\mu_{+}^{2}}{\xi_{+}^{3}}+3 i \frac{\xi_{1}^{+} \mu_{+} \partial_{x} \mu_{+}}{\xi_{+}^{5}}\right] \mathrm{d} x \mathrm{~d} \xi_{1}^{+} .
$$

This relation will be used for the asymptotic expansion of the corner term. The proof of Proposition 4.2 and of Proposition 4.3 is the aim of Sections 4.2 to 4.4. We use the regularity result of Proposition 4.3 to write, from the equalities (78) and (79) the system deduced from the jump formula.

We showed in Proposition 1.1 that $\left.u\right|_{\partial \mathcal{O}} \in H^{1}\left(\mathbb{R}_{t} \times \partial \mathcal{O}\right)$. Hence $\left.u\right|_{\partial \mathcal{O}} \circ j^{-1}$ is a distribution of $H^{1}\left(\mathbb{R}^{2}\right)$ supported in $t \geqslant 0$. The support of the distribution $\left.u\right|_{\partial \mathcal{O}}$ is included in $0 \leqslant x \leqslant c t$, hence the support of $\left.\phi_{1} u\right|_{\partial \mathcal{O}}$ is included in $(t, x) \in\left[0,2 t_{0}\right] \times$ $\left[0,2 c t_{0}\right]$. The partial Fourier transform in time of $\left.\phi_{1} u\right|_{\partial \mathcal{O}}$ is thus bounded in $H^{1}(\mathbb{R})$, independently of $k$. This imply

$$
\left.\phi_{1} u\right|_{\partial \mathcal{O}} \in E_{0}, \quad\left\|\mathcal{F}\left(\left.\phi_{1} u\right|_{\partial \mathcal{O}}\right)\right\|_{H^{1}(\mathbb{R})} \leqslant A^{\prime} .
$$

The Proposition 1.1 shows that $v_{ \pm} \in L^{2}\left([0, T] \times \mathbb{R}_{+}\right)$, which gives

$$
\phi_{1} v_{ \pm} \in E_{0} .
$$

We thus deduce, using the fact that $R^{+, k}$ and $R^{-, k}$ are bounded from $H^{1}(\mathbb{R})$ to $L^{2}\left(\mathbb{R}_{+}\right)$and satisfy the inequality (75) for $e=3$ that we can apply $R^{ \pm, k}$ to $\left.\phi_{1} u\right|_{\partial \mathcal{O}}$ and that $R^{ \pm, k}\left(\left.\phi_{1} u\right|_{\partial \mathcal{O}}\right)$ is bounded in $L^{2}\left(\mathbb{R}_{+}\right)$and satisfy

$$
\left\|R^{ \pm, k}\left(\left.\phi_{1} u\right|_{\partial \mathcal{O}}\right)\right\|_{L^{2}\left(\mathbb{R}_{+}\right)} \leqslant C \frac{(1+|k|)^{A}}{|\Im k|^{3}}\left\|\mathcal{F}\left(\left.\phi_{1} u\right|_{\partial \mathcal{O}}\right)\right\|_{H^{1}(\mathbb{R})} .
$$

The inversion formula can be performed on $R^{ \pm, k}\left(\left.\phi_{1} u\right|_{\partial \mathcal{O}}\right)(x, k)$, which defines $R^{ \pm}\left(\left.\phi_{1} u\right|_{\partial \mathcal{O}}\right) \in E_{3}$. Finally

$$
\phi_{2} R^{ \pm}\left(\left.\phi_{1} u\right|_{\partial \mathcal{O}}\right) \in E_{3}, \quad \phi_{2} K_{+}^{ \pm}\left(\phi_{1} v_{+}\right) \in E_{1}, \quad \phi_{2} K_{-}^{ \pm}\left(\left.\phi_{1} u\right|_{\partial \mathcal{O}}\right) \in E_{1} .
$$

The system of equations (78) and (79) lead to

$$
\left\{\begin{array}{l}
\psi \phi_{2} v_{+}=\phi_{2} \psi \partial_{+} u_{i}+\phi_{2} K_{+}^{+}\left(\phi_{1} v_{+}\right)+\phi_{2} K_{-}^{+}\left(\phi_{1} v_{-}\right)+\phi_{2} R^{+}\left(\left.\phi_{1} u\right|_{\mathcal{O}}\right) \\
\psi \phi_{2} v_{-}=\phi_{2} \psi \partial_{-} u_{i}+\phi_{2} K_{+}^{-}\left(\phi_{1} v_{+}\right)+\phi_{2} K_{-}^{-}\left(\phi_{1} v_{-}\right)+\phi_{2} R^{-}\left(\left.\phi_{1} u\right|_{\mathcal{O}}\right)
\end{array}\right.
$$

We consider the same localizing function as Gérard and Lebeau [20] (the function $\theta(x)$, which is $C^{\infty}, 0$ for $x \leqslant 1,1$ for $x \geqslant 2$ ) and we show that, by multiplying (88) by $\theta(x / \varepsilon)$, we obtain, in $L^{2}\left(\mathbb{R}_{+}\right)$, the equality

$$
\left\{\begin{array}{c}
\psi \phi_{2} v_{+}=1_{x \geqslant 0} \phi_{2} \psi \partial_{+} u_{i}+\phi_{2} 1_{x \geqslant 0} K_{+}^{+}\left(\phi_{1} v_{+}\right)+\phi_{2} 1_{x \geqslant 0} K_{-}^{+}\left(\phi_{1} v_{-}\right) \\
+\phi_{2} 1_{x \geqslant 0} R^{+}\left(\left.\phi_{1} u\right|_{\mathcal{O}}\right) \\
\psi \phi_{2} v_{-}=1_{x \geqslant 0} \phi_{2} \psi \partial_{-} u_{i}+\phi_{2} 1_{x \geqslant 0} K_{+}^{-}\left(\phi_{1} v_{+}\right)+\phi_{2} 1_{x \geqslant 0} K_{-}^{-}\left(\phi_{1} v_{-}\right) \\
+\phi_{2} 1_{x \geqslant 0} R^{-}\left(\left.\phi_{1} u\right|_{\mathcal{O}}\right) .
\end{array}\right.
$$


We have computed another expression of the operators $1_{x \geqslant 0} K_{\mp}^{ \pm, k}$ and $R_{\mp}^{ \pm, k}$. We introduce the operators $Q^{ \pm}$and $S^{ \pm, \mp}$ (which are not exactly the same as the operators introduced in $[\mathbf{2 0}]$ by the relations $(2.32)$ p. 353 but differ only slightly, according to the definition of the trace and the normal derivative we chose) through

$$
\left\{\begin{array}{l}
K_{ \pm}^{ \pm}=\left(\frac{1}{2} \psi(x) \otimes \psi\left(x^{\prime}\right)+\frac{1}{2} Q^{ \pm}\right) \\
1_{x \geqslant 0} K_{\mp}^{ \pm}=\frac{1}{2} S^{ \pm, \mp} .
\end{array}\right.
$$

The Dirichlet to Neumann operators can be decomposed using the result of Proposition 4.3 and Proposition 4.2. We have in particular, for $\Phi \in H^{1}\left(\mathbb{R}^{2} \times \mathbb{R}\right)$, the existence of $\Phi^{ \pm}=\psi\left(\left(1_{x>0} \Phi \circ j^{-1}\right) \circ j_{ \pm}\right)$and $\widetilde{\Phi}^{ \pm}=\psi\left(\left(1_{x>0} \partial_{x} \Phi \circ j^{-1}\right) \circ j_{ \pm}\right)$.

We introduce the operators $I^{ \pm, 0}, I^{ \pm, 1}, R^{ \pm, \mp, 0}, R^{ \pm, \mp, 1}$ on $E_{e}$ such that we have the equalities, for $\Phi \in H^{1}(\partial \Omega)$ :

$$
\begin{aligned}
& 2 R^{+}(\Phi)=2 T^{+}(x) \Phi(0)+I^{+, 0}\left(\Phi^{+}\right)+I^{+, 1}\left(\widetilde{\Phi}^{+}\right)+R^{+,-, 0}\left(\Phi^{-}\right)+R^{+,-, 1}\left(\widetilde{\Phi}^{-}\right) \\
& 2 R^{-}(\Phi)=2 T^{-}(x) \Phi(0)+I^{-, 0}\left(\Phi^{-}\right)+I^{-, 1}\left(\widetilde{\Phi}^{-}\right)+R^{-,+, 0}\left(\Phi^{+}\right)+R^{-,+, 1}\left(\widetilde{\Phi}^{+}\right) .
\end{aligned}
$$

Comparing with the relations of Proposition 4.3, we have

$$
\begin{aligned}
& I^{+, 0}(f)(x, k)=\frac{1}{2 \pi} \int_{\mathbb{R}_{+} \times \mathbb{R}} i \sigma_{2}^{+}\left(x, x^{\prime}, \xi_{1}^{+}, k\right) e^{i\left(x-x^{\prime}\right) \xi_{1}^{+}} f\left(x^{\prime}, k\right) \mathrm{d} x^{\prime} \mathrm{d} \xi_{1}^{+} \\
& I^{+, 1}(\widetilde{f})(x, k)=\frac{1}{2 \pi} \int_{\mathbb{R}_{+} \times \mathbb{R}} q_{1}^{+}\left(x, x^{\prime}, \xi_{1}^{+}, k\right) e^{i\left(x-x^{\prime}\right) \xi_{1}^{+}} \widetilde{f}\left(x^{\prime}, k\right) \mathrm{d} x^{\prime} \mathrm{d} \xi_{1}^{+} \\
& R^{+,-, 0}(f)(x, k)=\frac{1}{2 \pi} \int_{\mathbb{R}_{+} \times \mathbb{R}} e^{i \psi_{+,-}\left(x, x^{\prime}, \xi, k\right)} r^{+,-, 0}\left(x, x^{\prime}, \xi, k\right) f\left(x^{\prime}, k\right) \mathrm{d} x^{\prime} \mathrm{d} \xi \\
& R^{+,-, 1}(\widetilde{f})(x, k)=\frac{1}{2 \pi} \int_{\mathbb{R}_{+} \times \mathbb{R}} e^{i \psi_{+,-}\left(x, x^{\prime}, \xi, k\right)} r^{+,-, 0}\left(x, x^{\prime}, \xi, k\right) \widetilde{f}\left(x^{\prime}, k\right) \mathrm{d} x^{\prime} \mathrm{d} \xi
\end{aligned}
$$

with $r^{+,-, 0}\left(x, x^{\prime}, \xi, k\right)=-i \psi(x) \psi\left(x^{\prime}\right) r_{2}^{+,-}\left(x, x^{\prime}, \xi, k\right)+r_{1}^{+,-}(x, \xi, k) \psi(x) \partial_{x^{\prime}} \psi\left(x^{\prime}\right)$ and $r^{+,-, 0}\left(x, x^{\prime}, \xi, k\right)=\psi(x) \psi\left(x^{\prime}\right) r_{1}^{+,-}(x, \xi, k) 1_{x \geqslant 0} 1_{x^{\prime} \geqslant 0}$ and similar relations lead to $I^{-, 0}$, $I^{-, 1}, R^{-,+, 0}, R^{-,+, 1}$. The system of equations on

$$
\psi \phi_{1} v_{+}, \psi \phi_{1} v_{-}, \quad \psi \phi_{1} u(x, a(x), t)=\left.\psi \phi_{1} u\right|_{\mathcal{O}_{+}}, \quad \psi \phi_{1} u(x, b(x), t)=\left.\psi \phi_{1} u\right|_{\mathcal{O}_{-}}
$$

and the derivatives

$$
\psi \phi_{1}\left(\partial_{x} u+a^{\prime}(x) \partial_{y} u\right)=\left.\psi \phi_{1} \partial_{\tan } u\right|_{\mathcal{O}_{+}}, \quad \psi \phi_{1}\left(\partial_{x} u+b^{\prime}(x) \partial_{y} u\right)=\left.\psi \phi_{1} \partial_{\tan } u\right|_{\mathcal{O}_{-}}
$$


is deduced from the system (89) and the identities, valid for $f \in L^{2}$ supported in $0 \leqslant x \leqslant c t$ (using $\phi_{1} \phi_{2}=\phi_{2}$ ). This system writes

$$
\left\{\begin{array}{l}
\left(1-1_{x \geqslant 0} Q^{+}\right)\left(\psi \phi_{1} v_{+}\right) \\
-I^{+, 0}\left(\left.\psi \phi_{1} u\right|_{\partial \mathcal{O}_{+}}\right)-I^{+, 1}\left(\left.\psi \phi_{1} \partial_{t a n} u\right|_{\partial \mathcal{O}_{+}}\right) \\
-S^{+,-}\left(\psi \phi_{1} v_{-}\right) \\
-R^{+,-, 0}\left(\left.\psi \phi_{1} u\right|_{\partial \mathcal{O}_{-}}\right)-R^{+,-, 1}\left(\left.\psi \phi_{1} \partial_{t a n} u\right|_{\partial \mathcal{O}_{-}}\right) \\
-2 T^{+} u(0,0, t) \\
\left(1-1_{x \geqslant 0} Q^{-}\right)\left(\psi \phi_{2} v_{-}\right) \\
-I^{-, 0}\left(\left.\psi \phi_{1} u\right|_{\partial \mathcal{O}_{-}}\right)-I^{-, 1}\left(\left.\psi \phi_{1} \partial_{\tan } u\right|_{\partial \mathcal{O}_{-}}\right) \\
-S^{-,+}\left(\psi \phi_{1} v_{+}\right) \\
-R^{-,+, 0}\left(\left.\psi \phi_{1} u\right|_{\partial \mathcal{O}_{+}}\right)-r_{+} \\
-2 T^{-} u(0,0, t)
\end{array}\right.
$$

where

$$
\left\{\begin{array}{l}
r_{+}=\left(1-\phi_{2}\right)\left[1_{x \geqslant 0} Q^{+}\left(\psi \phi_{1} v_{+}\right)+S^{+,-}\left(\psi \phi_{1} v_{-}\right)+2 R^{+}\left(\left.\psi \phi_{1} u\right|_{\partial \mathcal{O}}\right)-\psi \phi_{1} v_{+}\right] \\
r_{-}=\left(1-\phi_{2}\right)\left[1_{x \geqslant 0} Q^{-}\left(\psi \phi_{1} v_{-}\right)+S^{-,+}\left(\psi \phi_{1} v_{+}\right)+2 R^{-}\left(\left.\psi \phi_{1} u\right|_{\partial \mathcal{O}}\right)-\psi \phi_{1} v_{-}\right]
\end{array}\right.
$$

The terms $r_{ \pm}$are element of $E_{3}$, they are compactly supported in $x$ (because of $\psi$ in the operators and in the source term), supported in $t \geqslant t_{0} / 2$ (because $\phi_{2} \equiv 1$ on $\left.\left[0, t_{0} / 2\right]\right)$. The system $(93)$ is the system we base ourselves on. If we define the operator $\Upsilon$ from $E_{0}$ to $E_{0}$ through

$$
\Upsilon^{k}(\mathcal{F}(u(\cdot, k))(x, k)=i k \mathcal{F} u(x, k)
$$

that is $\Upsilon^{k}=i k \operatorname{Id}_{L^{2}}$, the system we study for computing the solution is (93) to which we add the two boundary relations. We use then that the support of $\partial_{t} \phi_{1}$ is included in $\left[t_{0}, 2 t_{0}\right]$ and that $1-\phi_{2}=1$ for $t \geqslant t_{0}$ to conclude that $\left.\left(1-\phi_{2}\right) \partial_{t} \phi_{1} z^{ \pm}(x) \psi u\right|_{\partial \mathcal{O}_{ \pm}}$ has the same properties as the terms $r_{ \pm}$. The final system (obtained with the system (93) and the impedance boundary conditions) is the following:

$$
\left\{\begin{array}{r}
\left(1-1_{x \geqslant 0} Q^{+}\right)\left(\psi \phi_{1} v_{+}\right)-I^{+, 0}\left(\left.\psi \phi_{1} u\right|_{\partial \mathcal{O}_{+}}\right)-I^{+, 1}\left(\left.\psi \phi_{1} \partial_{\tan } u\right|_{\partial \mathcal{O}_{+}}\right) \\
-S^{+,-}\left(\psi \phi_{1} v_{-}\right)-R^{+,-, 0}\left(\left.\psi \phi_{1} u\right|_{\partial \mathcal{O}_{-}}\right)-R^{+,-, 1}\left(\left.\psi \phi_{1} \partial_{\tan } u\right|_{\partial \mathcal{O}_{-}}\right) \\
-2 T^{+} u(0,0, t) \\
=2 \phi_{2} \psi \partial_{+} u_{i}+r_{+} \\
\left(1-1_{x \geqslant 0} Q^{-}\right)\left(\psi \phi_{2} v_{-}\right)-I^{-, 0}\left(\left.\psi \phi_{1} u\right|_{\partial \mathcal{O}_{-}}\right)-I^{-, 1}\left(\left.\psi \phi_{1} \partial_{\tan } u\right|_{\partial \mathcal{O}_{-}}\right) \\
-S^{-,+}\left(\psi \phi_{1} v_{+}\right)-R^{-,+, 0}\left(\left.\psi \phi_{1} u\right|_{\partial \mathcal{O}_{+}}\right)-R^{-,+, 1}\left(\left.\psi \phi_{1} \partial_{\tan } u\right|_{\partial \mathcal{O}_{+}}\right) \\
-2 T^{-} u(0,0, t) \\
=2 \phi_{2} \psi \partial_{-} u_{i}+r_{-} \\
\psi \phi_{1} v_{+}-\Upsilon\left(\left.z^{+}(x) \psi \phi_{1} u\right|_{\partial \mathcal{O}_{+}}\right)=-\left.z^{+}(x) \psi u\right|_{\partial \mathcal{O}_{+}}\left(1-\phi_{2}\right) \partial_{t} \phi_{1} \\
\psi \phi_{1} v_{-}-\Upsilon\left(\left.z^{-}(x) \psi \phi_{1} u\right|_{\partial \mathcal{O}_{-}}\right)=-\left.z^{-}(x) \psi u\right|_{\partial \mathcal{O}_{-}}\left(1-\phi_{2}\right) \partial_{t} \phi_{1}
\end{array}\right.
$$

We seek a solution of $(94),\left(\psi \phi_{1} v_{+}, \psi \phi_{1} v_{-},\left.\psi \phi_{1} u\right|_{\partial \mathcal{O}_{+}},\left.\psi \phi_{1} u\right|_{\partial \mathcal{O}_{-}}\right) \in L^{2}\left(\mathbb{R}^{2}\right) \times L^{2}\left(\mathbb{R}^{2}\right) \times$ $\left(H^{1}\left(\mathbb{R}_{+} \times \mathbb{R}_{t}\right) \times H^{1}\left(\mathbb{R}_{+} \times \mathbb{R}_{t}\right)\right)_{\text {comp }}$ supported in $t \geqslant 0$. 
This system will be modified in Section 5.4 to include the terms generated by $u_{e}$, the classical reflected wave written in the Introduction.

\subsection{Regularity of the operators $R_{+, 0}^{ \pm, k}$ and $R_{-, 0}^{ \pm}$}

We generalize the results of Gérard and Lebeau, stated in Lemma 2.3 of [20], to the operators $R_{+}^{ \pm, k}$ and $R_{-}^{ \pm, k}$. This proof relies on Lemma 2.4 and Lemma 2.5 of [20] which give the $L^{2}$ regularity of compactly supported symbols. Of course, we study the regularity of the operators $R_{+, 0}^{+, k}$ (and the similar ones written from the relations of Lemma 6.5). These operators satisfy

Proposition 4.4. - The operators $R_{ \pm, 0}^{ \pm, k}$ and $R_{\mp, 0}^{ \pm, k}$ are bounded from $H^{1}\left(\mathbb{R}_{+}\right)$to $L^{2}(\mathbb{R})$ of norm in $\mathcal{C}\left(H^{1}\left(\mathbb{R}_{+}\right), L^{2}(\mathbb{R})\right)$ satisfying the inequality (75) for $e=3$.

We associate to these operators, according to Definition (76), the operators from $E_{e}$ to $E_{e+3}$. The operators $R^{+, k}$ and $R^{-, k}$ are bounded in $\mathcal{C}\left(H^{1}(\mathbb{R}), L^{2}(\mathbb{R})\right)$ and satisfy the inequality (75) for $e=3$.

We have

Corollary 4.1. - Note that a slight change of the definition may show that the operators are bounded from $E_{e}$ to $E_{e+1}$ because we can perform the estimates before the integration by parts in $x^{\prime}$.

We state the two results of Gérard and Lebeau which will be used here.

LEMMA 4.3 (Lemma 2.4 of $[\mathbf{2 0}])$. - If $q\left(x, x^{\prime}, \xi\right)$ is a symbol, compactly supported in $\left(x, x^{\prime}\right)$, such that

$$
\left\|\partial_{x}^{l} \partial_{x^{\prime}}^{l^{\prime}} q\left(x, x^{\prime}, \xi\right)\right\|_{\infty} \leqslant C_{l, l^{\prime}}
$$

then the pseudodifferential operator of symbol $q\left(x, x^{\prime}, \xi\right)$ is continuous on $L^{2}(\mathbb{R})$, and we have

$$
\|\mathrm{Op}(q) f\|_{L^{2}(\mathbb{R})} \leqslant c_{0}\left(\max _{l \leqslant 2, l^{\prime} \leqslant 2} C_{l, l^{\prime}}\right)\|f\|_{L^{2}(\mathbb{R})}
$$

Its proof comes from the fact that, on the support of the symbol, we can expand $f$ as a Fourier series which is in $l^{2}$. We evaluate the action of $\operatorname{Op}(q)$ on the Fourier series, and the condition $l \leqslant 2, l^{\prime} \leqslant 2$ comes from the fact that $\sum \frac{1}{1+n^{2}}<+\infty$.

LEMMA 4.4 (Lemma 2.5 of $[\mathbf{2 0}])$. - Let $q_{\varepsilon}(x, y, \xi)$ be a compactly supported symbol in $(x, y)$ such that there exists a constant $c_{1}$ and constants $C_{l}$ such that, for all $y \geqslant 0$, for $l \geqslant 0$

$$
\sup _{x}\left|\partial_{x}^{l} q_{\varepsilon}\right| \leqslant C_{l} e^{-c_{1}(y+\varepsilon)|\xi|} .
$$

The operator

$$
Q_{\varepsilon}(f)(x)=\int e^{i(x-y) \xi} q_{\varepsilon}(x, y, \xi) f(y) \mathrm{d} y \mathrm{~d} \xi
$$


is bounded from $L^{2}\left(\mathbb{R}_{+}\right)$to $L^{2}(\mathbb{R})$ and its norm is estimated, uniformly in $\varepsilon$, by $c_{1}, C_{l}, l \leqslant 2$.

Proof of Proposition 4.4. - We consider the equalities (150) (for the case of the pseudodifferential operator) and (152) (for the case of the singular face-to-face operator) proven above. We check that, for $t_{0}$ given, $\left(x, x^{\prime}\right) \in \operatorname{supp} \psi, 1+\left(\mu_{+}\left(x, x^{\prime}\right)\right)^{2}$ is bounded. Hence there exists $D_{0}>0$ such that, for $\left|\xi_{1}^{+}\right| \geqslant D_{0}(1+|k|)$, the real part of $\left(\xi_{1}^{+}\right)^{2}-k^{2}\left(1+\mu_{+}^{2}\right)$ is positive, greater than $\frac{1}{2}\left(\xi_{1}^{+}\right)^{2}$, the real part of the square root of this complex number (of positive real part) is greater than $\frac{1}{2}\left|\xi_{1}^{+}\right|$. This writes $\Im \xi_{+} \geqslant \frac{1}{2}\left|\xi_{1}^{+}\right|$for $\left|\xi_{1}^{+}\right| \geqslant D_{0}(1+|k|)$, hence $\xi_{1}^{+} / \xi_{+}$is bounded by $1 / 2$ and $\left|\xi_{+}^{-1}\right| \leqslant 2 /\left|\xi_{1}^{+}\right| \leqslant 2 / D_{0}(1+|k|)$.

We split the integration in $\xi_{1}^{ \pm}$in two parts, the part where $\left|\xi_{1}^{ \pm}\right| \leqslant D_{0}(1+|k|)$ in which we use $L^{\infty}$ estimates on the compactly supported symbol, and the part where $\left|\xi_{1}^{ \pm}\right| \geqslant D_{0}(1+|k|)$, where we use the preceding lemmas. ${ }^{(1)}$

Let us consider the other terms. The symbol $\sigma_{2}^{+} 1_{\left|\xi_{1}^{+}\right| \geqslant D_{0}(1+|k|)}, \sigma_{2}^{-} 1_{\left|\xi_{1}^{+}\right| \geqslant D_{0}(1+|k|)}$, $q_{1}^{+} 1_{\left|\xi_{1}^{+}\right| \geqslant D_{0}(1+|k|)}, \quad q_{1}^{-} 1_{\left|\xi_{1}^{+}\right| \geqslant D_{0}(1+|k|)}$ satisfy the assumptions of Lemma 4.3, while the symbols $e^{i \phi_{\varepsilon}^{+}} q_{\varepsilon}^{+} 1_{\left|\xi_{1}^{+}\right| \geqslant D_{0}(1+|k|)}, \quad e^{i \phi_{\varepsilon}^{+}} r_{\varepsilon}^{+} 1_{\left|\xi_{1}^{+}\right| \geqslant D_{0}(1+|k|)}, \quad e^{i \phi_{\varepsilon}^{-}} q_{\varepsilon}^{-} 1_{\left|\xi_{1}^{+}\right| \geqslant D_{0}(1+|k|)}$, $e^{i \phi_{\varepsilon}^{-}} r_{\varepsilon}^{-} 1_{\left|\xi_{1}^{+}\right| \geqslant D_{0}(1+|k|)}$ satisfy the assumptions of Lemma 4.4. For example, we use the fact that $\partial_{x^{\prime}}\left(\xi_{+}\right)^{-1}=-\frac{k^{2} \mu_{+} \partial_{x^{\prime}} \mu_{+}}{\xi_{+}^{3}}$ to obtain, for $\left|\xi_{1}^{+}\right| \geqslant D_{0}(1+|k|)$

$$
\left\|\partial_{x^{\prime}}\left(\xi_{+}\right)^{-1}\right\| \leqslant \frac{M(1+|k|)^{2}}{\frac{1}{8}\left|\xi_{1}^{+}\right|^{3}} \leqslant \frac{8 M}{D_{0}^{3}(1+|k|)}
$$

hence

$$
\left\|\partial_{x^{\prime}} \frac{\xi_{1}^{+}}{\xi_{+}}\right\| \leqslant \frac{M(1+|k|)^{2}}{\frac{1}{8}\left|\xi_{1}^{+}\right|^{3}} \leqslant \frac{8 M}{D_{0}^{3}} .
$$

From Lemma 4.3, we deduce that there exists $C>0$ such that

$$
\begin{gathered}
\left\|-\frac{1}{4 \pi} \int_{\mathbb{R}^{2}} 1_{x^{\prime} \geqslant 0} e^{i\left(x-x^{\prime}\right) \xi_{1}^{+}} q_{1}^{+} 1_{\left|\xi_{1}^{+}\right| \geqslant D_{0}(1+|k|)} \partial_{x^{\prime}} f \mathrm{~d} x^{\prime} \mathrm{d} \xi_{1}^{+}\right\|_{L^{2}(\mathbb{R})} \leqslant C\left\|\partial_{x^{\prime}} f 1_{x^{\prime} \geqslant 0}\right\|_{L^{2}(\mathbb{R})}, \\
\left\|-\frac{1}{4 \pi} \int_{\mathbb{R}^{2}} e^{i\left(x-x^{\prime}\right) \xi_{1}^{+}} 1_{x^{\prime} \geqslant 0} \sigma_{2}^{+} 1_{\left|\xi_{1}^{+}\right| \geqslant D_{0}(1+|k|)} f \mathrm{~d} x^{\prime} \mathrm{d} \xi_{1}^{+}\right\|_{L^{2}(\mathbb{R})} \leqslant C\left\|f 1_{x^{\prime} \geqslant 0}\right\|_{L^{2}(\mathbb{R})} .
\end{gathered}
$$

(1) The result obtained is not optimal in $\Im k$; we may have the optimal result, based on the inequalities

$$
\begin{gathered}
\left\|1_{\left\|\xi_{1}^{+}\right\| \leqslant D_{0}(1+|k|)} L_{1}^{+}\left(x, x^{\prime}\right) \frac{\left(\xi_{1}^{+}\right)^{2}}{\xi_{+}}+L_{2}^{+}\left(x, x^{\prime}\right) \xi_{1}^{+}+L_{3}^{+}\left(x, x^{\prime}\right) \frac{k^{2}}{\xi_{+}}\right\|_{\infty} \leqslant D_{1} \frac{(1+|k|)^{2}}{|\Im k|} \\
\left\|1_{\left\|\xi_{1}^{+}\right\| \leqslant D_{0}(1+|k|)} e^{i \phi_{\varepsilon}^{+}} M_{1}^{+}\left(x, x^{\prime}\right) \xi_{+}+M_{2}^{+}\left(x, x^{\prime}\right) \xi_{1}^{+}+M_{3}^{+}\left(x, x^{\prime}\right) \frac{k^{2}}{\xi_{+}}\right\|_{\infty} \leqslant D_{2} \frac{(1+|k|)^{2}}{|\Im k|}
\end{gathered}
$$

and the fact that the support of the integral is the support in $x, x^{\prime}$ times the support $1_{\left|\xi_{1}^{+}\right| \leqslant D_{0}(1+|k|)}$, hence the integral with a symbol $t$ truncated in $\xi_{1}^{+}$satisfies (continuous injection)

$$
\left\|\iint \mathrm{d} x^{\prime} \mathrm{d} \xi_{1}^{+} t\left(x, x^{\prime}, \xi_{1}^{+}\right) f\left(x^{\prime}\right) e^{i\left(x-x^{\prime}\right) \xi_{1}^{+}}\right\|_{L^{2}(\mathbb{R})} \leqslant\left(2 D_{0} R(1+|k|)\right)\|t\|_{\infty}\|f\|_{L^{2}(\mathbb{R})}
$$

and we perform the inverse integration by parts to introduce these symbols. 
Along with the inequalities

$$
\begin{gathered}
\left\|-\frac{1}{4 \pi} \int_{\mathbb{R}^{2}} 1_{x^{\prime} \geqslant 0} e^{i\left(x-x^{\prime}\right) \xi_{1}^{+}} q_{1}^{+} 1_{\left|\xi_{1}^{+}\right| \leqslant D_{0}(1+|k|)} \partial_{x^{\prime}} f \mathrm{~d} x^{\prime} \mathrm{d} \xi_{1}^{+}\right\|_{L^{2}(\mathbb{R})} \\
\leqslant \frac{C(1+|k|)^{3}}{|\Im k|^{3}}\left\|\partial_{x^{\prime}} f 1_{x^{\prime} \geqslant 0}\right\|_{L^{2}(\mathbb{R})}, \\
\left\|-\frac{1}{4 \pi} \int_{\mathbb{R}^{2}} e^{i\left(x-x^{\prime}\right) \xi_{1}^{+}} 1_{x^{\prime} \geqslant 0} \sigma_{2}^{+} 1_{\left|\xi_{1}^{+}\right| \leqslant D_{0}(1+|k|)} f \mathrm{~d} x^{\prime} \mathrm{d} \xi_{1}^{+}\right\|_{L^{2}(\mathbb{R})} \\
\leqslant \frac{C(1+|k|)^{3}}{|\Im k|^{3}}\left\|f 1_{x^{\prime} \geqslant 0}\right\|_{L^{2}(\mathbb{R})},
\end{gathered}
$$

we deduce that $R_{+, 0}^{+, k}$ is bounded from $H^{1}\left(\mathbb{R}_{+}\right)$to $L^{2}(\mathbb{R})$ and that there exists $D_{*}>0$ such that

$$
\left\|R_{+, 0}^{+, k}\right\|_{H^{1}\left(\mathbb{R}_{+}\right), L^{2}\left(\mathbb{R}_{+}\right)} \leqslant D_{*} \frac{(1+|k|)^{3}}{|\Im k|^{3}} .
$$

We thus define an operator $R_{+, 0}^{+}$from $E_{e}$ to $E_{e+3}$ through the equality

$$
\mathcal{F}\left[R_{+, 0}^{+}(u)\right](k, x)=R_{+, 0}^{+, k}[\mathcal{F}(u)](k, \cdot)(x) .
$$

The case of the operators from a face to another face is similar. Denote the minimum of $\frac{1}{1+\mu_{+}^{2}}$ on the support of $\psi \otimes \psi$ by $\delta_{1}$, introduce the minimum $\delta_{2}>0$ of $\int_{0}^{1}\left(a^{\prime}\left(u x^{\prime}\right)-b^{\prime}\left(u x^{\prime}\right)\right) \mathrm{d} u$. Consider the constant $c_{0}=\frac{1}{4} \delta_{1} \min \left(1, \delta_{2}\right)$. We have

$$
\begin{aligned}
& \Im \phi_{\varepsilon}^{+}\left(x, x^{\prime}, \xi_{1}^{+}, k\right)=\frac{\left(a\left(x^{\prime}\right)-b\left(x^{\prime}\right)+\varepsilon\right)}{1+\mu_{+}^{2}} \Im \xi_{+} \geqslant c_{0}\left(x^{\prime}+\varepsilon\right)\left|\xi_{1}^{+}\right|, \\
& \forall n, \exists D_{n}, \quad\left|\partial_{x}^{n}\left(\phi_{\varepsilon}^{+}-x \cdot \xi_{1}^{+}\right)\right| \leqslant D_{n}\left|\xi_{1}^{+}\right|\left(x^{\prime}+\varepsilon\right) .
\end{aligned}
$$

The second relation comes from $\partial_{x} \xi_{+}=\mu_{+} \partial_{x^{\prime}} \mu_{+} k^{2} / \xi_{+}$and successive derivatives of $\xi_{+}$. This imply that, for all $n$ and for $x^{\prime} \geqslant 0$,

$$
\left\|\partial_{x}^{n}\left(e^{i\left(\phi_{\varepsilon}^{+}-\left(x-x^{\prime}\right) \cdot \xi_{1}^{+}\right)} \psi\left(x^{\prime}\right) \psi(x) r_{j}^{\varepsilon}\right) 1_{\left|\xi_{1}^{+}\right| \geqslant D_{0}(1+|k|)}\right\|_{L^{\infty}(x, k)} \leqslant D_{n} e^{-c_{0}\left(x^{\prime}+\varepsilon\right)\left|\xi_{1}^{+}\right| / 2}
$$

Hence there exists a constant $D$, depending on $c_{0}, D_{0}, D_{1}, D_{2}$, such that, if the limit of $R_{+, 0}^{-, k, \varepsilon}$ is denoted by $R_{+, 0}^{-, k}$,

$$
\left\|R_{+, 0}^{-, k}(f)\right\|_{L^{2}(\mathbb{R})} \leqslant D \frac{(1+|k|)^{3}}{|\Im k|^{3}}\left[\left\|f 1_{x^{\prime} \geqslant 0}\right\|_{L^{2}\left(\mathbb{R}_{+}\right)}+\left\|\partial_{x^{\prime}} f 1_{x^{\prime} \geqslant 0}\right\|_{L^{2}\left(\mathbb{R}_{+}\right)}\right] .
$$

The proof of the inequalities for $R_{-, 0}^{+, k}$ and $R_{-, 0}^{-, k}$ is left to the reader.

The last thing to be checked is the behavior of the corner terms in $R^{+, k}$ and in $R^{-, k}$. As the result will be used later, we intend to study it carefully.

Recall that the expression of the kernels of the operators $R_{ \pm}^{ \pm, k}$ are written in Lemma 6.5. The result on $R_{\mp}^{ \pm, k}$ is a consequence of the calculus of this section, which shows 
that the operator

$$
\begin{aligned}
& -\frac{i}{4 \pi} \int_{\mathbb{R}_{+} \times L} e^{i \psi_{ \pm, \mp}} r_{2}^{ \pm, \mp}\left(x, x^{\prime}, \xi, k\right) \psi(x) \psi\left(x^{\prime}\right) 1_{x \geqslant 0} 1_{x^{\prime} \geqslant 0} f\left(x^{\prime}\right) \mathrm{d} x^{\prime} \mathrm{d} \xi \\
& +\frac{1}{4 \pi} \int_{\mathbb{R}_{+} \times L} e^{i \psi_{ \pm, \mp}} \psi(x) r_{1}^{ \pm, \mp}(x, \xi, k) 1_{x \geqslant 0} 1_{x^{\prime} \geqslant 0} \partial_{x^{\prime}}\left(\psi\left(x^{\prime}\right) f\left(x^{\prime}\right)\right) \mathrm{d} x^{\prime} \mathrm{d} \xi
\end{aligned}
$$

is continuous from $H^{1}\left(\mathbb{R}_{+}\right)$to $L^{2}(\mathbb{R})$ when $L=\left\{\xi \in \mathbb{R},|\xi| \geqslant C_{0}(1+|k|)\right\}$ and that, before the integration by parts, the symbol obtained is bounded by $\frac{(1+|k|)^{2}}{|\Im k|}$. Hence the expression of $R_{\mp}^{ \pm, k}$ written in Lemma 6.5 and in Proposition 4.3 have the same regularity and they have the same value for $f \in C_{0}^{\infty}\left(\mathbb{R}_{+}\right)$, and moreover the same value for $f(0)$. Hence they are equal.

We introduce the operators on which we will perform the asymptotic expansion, for $f \in H^{1}\left(\mathbb{R}^{2}\right), \tilde{f} \in L^{2}\left(\mathbb{R}^{2}\right)$

$$
\begin{aligned}
& R_{ \pm, 0}^{ \pm, 1, k}(\widetilde{f})(x, k)=\frac{1}{4 \pi} \int_{\mathbb{R}_{+} \times \mathbb{R}} q_{1}^{ \pm}\left(x, x^{\prime}, \xi_{1}^{ \pm}, k\right) \widetilde{f} \mathrm{~d} x^{\prime} \mathrm{d} \xi_{1}^{ \pm} \\
& S_{ \pm, 0}^{ \pm, 2, k}(f)(x, k)=\frac{i}{4 \pi} \int_{\mathbb{R}_{+} \times \mathbb{R}} \psi(x) \psi\left(x^{\prime}\right) L_{3}^{ \pm}\left(x, x^{\prime}\right) \frac{k^{2}}{\xi_{ \pm}} e^{i\left(x-x^{\prime}\right) \xi_{1}^{ \pm}} f\left(x^{\prime}\right) \mathrm{d} x^{\prime} \mathrm{d} \xi_{1}^{ \pm} \\
& S_{ \pm, 0}^{ \pm, 1, k}(f)(x, k)=\frac{1}{4 \pi} \int_{\mathbb{R}_{+} \times \mathbb{R}} \partial_{x^{\prime}} q_{1}^{ \pm}\left(x, x^{\prime}, \xi_{1}^{ \pm}, k\right) e^{i\left(x-x^{\prime}\right) \xi_{1}^{ \pm}} f\left(x^{\prime}\right) \mathrm{d} x^{\prime} \mathrm{d} \xi_{1}^{ \pm}
\end{aligned}
$$

such that

$$
R_{ \pm, 0}^{ \pm, k}(f)=R_{ \pm, 0}^{ \pm, 1, k}\left(\partial_{x} f 1_{x \geqslant 0}\right)+S_{ \pm, 0}^{ \pm, 2, k}\left(f 1_{x \geqslant 0}\right)+S_{ \pm, 0}^{ \pm, 1, k}\left(f 1_{x \geqslant 0}\right)
$$

Introduce

$$
\begin{array}{r}
R_{\mp}^{ \pm, 1, k}(\widetilde{f})(x, k)=\frac{1}{2 \pi} \int_{\mathbb{R}^{2}} e^{i \psi_{ \pm, \mp}\left(x, x^{\prime}, \xi, k\right)} 1_{x \geqslant 0, x^{\prime} \geqslant 0} \psi(x) \psi\left(x^{\prime}\right) r_{1}^{ \pm, \mp}(x, \xi, k) \\
\cdot \widetilde{f}\left(x^{\prime}\right) \mathrm{d} x^{\prime} \mathrm{d} \xi \\
S_{\mp}^{ \pm, 2, k}(f)(x, k)=-\frac{i}{2 \pi} \int_{\mathbb{R}^{2}} e^{i \psi_{ \pm, \mp}\left(x, x^{\prime}, \xi, k\right)} 1_{x \geqslant 0, x^{\prime} \geqslant 0} \psi(x) \psi\left(x^{\prime}\right) r_{2}^{ \pm, \mp}\left(x, x^{\prime} \xi, k\right) \\
\cdot f\left(x^{\prime}\right) \mathrm{d} x^{\prime} \mathrm{d} \xi \\
S_{\mp}^{ \pm, 1, k}(f)(x, k)=\frac{1}{2 \pi} \int_{\mathbb{R}^{2}} e^{i \psi_{ \pm, \mp}\left(x, x^{\prime}, \xi, k\right)} 1_{x \geqslant 0, x^{\prime} \geqslant 0} \psi(x) \partial_{x^{\prime}} \psi\left(x^{\prime}\right) r_{1}^{ \pm, \mp}(x, \xi, k) \\
\cdot f\left(x^{\prime}\right) \mathrm{d} x^{\prime} \mathrm{d} \xi .
\end{array}
$$

We have

$$
R_{\mp, 0}^{ \pm, k}(f)=R_{\mp, 0}^{ \pm, 1, k}\left(\partial_{x} f 1_{x \geqslant 0}\right)+S_{\mp, 0}^{ \pm, 2, k}\left(f 1_{x \geqslant 0}\right)+S_{\mp, 0}^{ \pm, 1, k}\left(f 1_{x \geqslant 0}\right)
$$

which is a consequence of the equality (92).

All the operators involved have bounded symbols when $\xi$ is large. We note that the operators introduced above in the relations (92) from Proposition 4.3 and written in system (93), that is $I^{+, 0}, I^{+, 1}, R^{+,-, 0}, R^{+,-, 1}$, are easily related with the operators 
introduced above through the equalities

$$
\begin{aligned}
\mathcal{F}\left(I^{+, 0}(f)\right)(x, k) & =2\left(S_{+}^{+, 2, k}+S_{+}^{+, 1, k}\right)(\mathcal{F}(f))(x) \\
\mathcal{F}\left(I^{+, 1}(f)\right)(x, k) & =2 R_{+}^{+, 1, k}(\mathcal{F}(f))(x) \\
\mathcal{F}\left(R^{+,-, 0}(f)\right)(x, k) & =2\left(S_{-}^{+, 2, k}+S_{-}^{+, 1, k}\right)(\mathcal{F}(f))(x, k) \\
\mathcal{F}\left(R^{+,-, 1}(f)\right)(x, k) & =2 R_{-}^{+, 1, k}(\mathcal{F}(f))(x) .
\end{aligned}
$$

Similar equalities hold for the operators corresponding to - upper indices.

The operators $S_{ \pm, 0}^{ \pm, 2, k}$ and $S_{ \pm, 0}^{ \pm, 1, k}$ are not put together in this equality because they differ through their order in $k$; in particular the symbol of the operator $S_{ \pm, 0}^{ \pm, 2, k}$ behaves, for $\xi=\lambda \zeta$ and for $k=\lambda \widetilde{k}$ as $\lambda$, while the symbols of $S_{ \pm, 0}^{ \pm, 1, k}$ are of order $\lambda^{0}$.

We end this Section with a Lemma allowing us to perform the asymptotic expansion of the operators $R_{ \pm}^{ \pm, k}$ and $R_{\mp}^{ \pm, k}$. This asymptotic expansion is complex valued, hence we have to consider $x, x^{\prime}, \xi, k$ in $\mathbb{C}$ instead of $\mathbb{R}$. More precisely, we allow $x, x^{\prime}$ to be "not far" from the real axis, $\xi$ to be complex. We have

Lemma 4.5. - Let $\mathcal{C}_{1}$ be the complex contour defined in Section 6.3, depending on the parameters $a^{\prime}, a^{\prime \prime}$. There exists a constant $D_{1}$ such that, for $a^{\prime} \ll t_{0} \ll 1 / D_{1}$, the symbols $q_{1}^{+}\left(x, x^{\prime}, \xi_{1}^{+}, k\right), \sigma_{2}^{+}\left(x, x^{\prime}, \xi_{1}^{+}, k\right)$ are holomorphic in $\left(x, x^{\prime}, k\right)$ for $\xi_{1}^{+} \in \mathcal{C}_{1}$, $\max \left(|\Re x|,\left|\Re x^{\prime}\right|\right) \leqslant 5 c t_{0}, \max \left(|\Im x|,\left|\Im x^{\prime}\right|\right) \leqslant 2 a^{\prime} / D_{1},|k-1| \leqslant 4 a^{\prime} / D_{1}$ and satisfy

$$
\left|q_{1}^{+}\left(x, x^{\prime}, \xi_{1}^{+}, k\right)\right| \leqslant \frac{D_{1}}{a^{\prime}}, \quad\left|\sigma_{2}^{+}\left(x, x^{\prime}, \xi_{1}^{+}, k\right)\right| \leqslant \frac{D_{1}}{\left(a^{\prime}\right)^{3}} .
$$

A similar result is available for the complex contour dilated by the coefficient $\frac{\cos \gamma_{+}}{\cos \gamma_{-}}$ in the $\Im \xi$ direction and for the symbols $q_{1}^{-}$and $\sigma_{2}^{-}$.

Proof. - This Lemma is a consequence of $\xi_{+}=\left(\left(1+\mu_{+}^{2}\right) k^{2}-\left(\xi_{1}^{+}\right)^{2}\right)^{1 / 2}$, for which we verify that, for $k=1$ and $x=x^{\prime}=0, \xi_{1}^{+}$must be different from $1 / \cos \gamma_{+}$, hence, for $\xi_{1}^{+} \in \mathcal{C}_{1}$, we verify that there exists $\widetilde{c}>0$ such that

$$
\left|\left(\xi_{1}^{+}\right)^{2}-\frac{1}{\cos ^{2} \gamma_{+}}\right| \geqslant \widetilde{c} a^{\prime}
$$

If $D_{1}$ is large enough, for $\xi_{1}^{+} \in \mathcal{C}_{1}, \max _{\sim}\left(|\Re x|,\left|\Re x^{\prime}\right|\right) \leqslant 5 c t_{0}, \max \left(|\Im x|,\left|\Im x^{\prime}\right|\right) \leqslant 2 a^{\prime} / D_{1}$, $|k-1| \leqslant 4 a^{\prime} / D_{1}$, we obtain $\left|\xi_{+}\right| \geqslant \widetilde{d} a^{\prime}$.

As the operator $q_{1}^{+}$is of the form $\psi(x) \psi\left(x^{\prime}\right)\left[L_{2}\left(x, x^{\prime}\right)+L_{1}\left(x, x^{\prime}\right) \xi_{1}^{+} / \xi_{+}\right]$, we get the result for the symbol $q_{1}^{+}$. The result on the symbol $\sigma_{2}^{+}$is a consequence of

$$
\begin{aligned}
\partial_{x^{\prime}} q_{1}^{+}=\partial_{x^{\prime}}\left(\psi(x) \psi\left(x^{\prime}\right) L_{2}\left(x, x^{\prime}\right)\right)+\partial_{x^{\prime}}\left(\psi(x) \psi\left(x^{\prime}\right) L_{1}\left(x, x^{\prime}\right)\right) \frac{\xi_{1}^{+}}{\xi_{+}} & \\
& -\psi(x) \psi\left(x^{\prime}\right) L_{1}^{+}\left(x, x^{\prime}\right) \frac{\xi_{1}^{+} k^{2} \mu_{+} \partial_{x^{\prime}} \mu_{+}}{\xi_{+}^{3}} .
\end{aligned}
$$


We verify that, for $|\xi| \geqslant C_{0}(1+|k|)$

$$
\begin{array}{r}
\left|e^{i \psi_{+,-}}\left(a^{\prime}(x)+\frac{\xi}{\xi_{0}}\right) \psi(x) \psi\left(x^{\prime}\right) 1_{x \geqslant 0} 1_{x^{\prime} \geqslant 0}\right| \leqslant e^{-c_{0}\left(x+x^{\prime}\right)|\xi|} \\
\left|e^{i \psi_{+,-}} \frac{k^{2}}{\xi_{0}} \psi(x) \psi\left(x^{\prime}\right) 1_{x \geqslant 0} 1_{x^{\prime} \geqslant 0}\right| \leqslant e^{-c_{0}\left(x+x^{\prime}\right)|\xi|} .
\end{array}
$$

As we have

$$
\int_{0}^{\infty} d y \int \mathrm{d} \xi e^{-c_{0}(x+y)}|\xi| f(y) \mathrm{d} \xi=\frac{2}{c_{0}} \int_{0}^{\infty} \frac{f(y) d y}{x+y}
$$

which is bounded from $L^{2}\left(\mathbb{R}_{+}\right)$to $L^{2}\left(\mathbb{R}_{+}\right)$, we show that the operators $\widetilde{R}_{ \pm}^{ \pm, k}$ are bounded from $H^{1}\left(\mathbb{R}_{+}\right)$to $L^{2}\left(\mathbb{R}_{+}\right)$(the estimate on $|\xi| \leqslant C_{0}(1+|k|)$ is a $L^{\infty}$ estimate).

\subsection{Asymptotic expansions of the operators $K_{ \pm}^{+, k}$ and $R_{ \pm, 0}^{+, k}$}

This section summarizes the formalism of Section 3 and Section 4 of [20], in which the authors microlocalize the problem.

We notice that the main result of this section, proven in Proposition 4.5 and in Proposition 4.6, leads to

\section{COROLlary 4.2}

- The asymptotic expansion of the operators $S_{+}^{+, 2, k}, S_{+}^{+, 1, k}, R_{+}^{+, 1, k}, Q^{+}$follow the same pattern and lead to similar symbols in the pseudodifferential calculus of Gérard and Lebeau. The integrals obtained can be deformed on the contour $C(+)$ defined in Section 6.3.

- The asymptotic expansion of the operators $S_{-}^{+, 2, k}, S_{-}^{+, 1, k}, R_{-}^{+, 1, k}$ and $S^{+,-}$follow the same pattern and lead to similar symbols in the pseudodifferential calculus of Gérard and Lebeau. The integrals obtained can be deformed on the contour $\mathcal{C}(l, b)$ defined also in Section 6.3.

Hence we may be able to use again the same techniques as in [20].

We define the pseudodifferential operators used in this section. The operator $T_{0}$ which is the Fourier-Bros-Iagolnitzer (FBI) transform in time has been introduced by (73). We introduce the partial FBI transform in $x$, the set $W_{\kappa}$ was defined in (74). In what follows, we consider $A, \varepsilon$ such that $A \sin \varepsilon>4$ and we denote by $U=\omega(\varepsilon, A)=\{z \in \mathbb{C}, \arg z \in] \pi / 2+\varepsilon, 3 \pi / 2-\varepsilon[,|z| \geqslant A\}$. Let $\omega$ be a $\varepsilon$-sector, that is $U \cup \omega^{\prime}$, where $A$ is given and $\omega^{\prime}$ a bounded open set. For $\omega$ of this form, we denote by $H_{\omega}$ the space of functions $g(z, \lambda)$ on $\omega$ associated with the norm

$$
\|g(\cdot, \lambda)\|_{\omega}=\sup _{\theta}\left(\int_{A}^{\infty}\left|g\left(\rho e^{i \theta}, \lambda\right)\right|^{2} d \rho\right)^{1 / 2}+\|g\|_{\infty, \omega^{\prime}}
$$

such that $\exists C_{0}>0,\|g(\cdot, \lambda)\|_{\omega} \leqslant C_{0} \lambda^{C_{0}}$. We say that $g \in H_{\omega}$ is negligible if there exists $C_{1}>0$ such that $\|g(\cdot, \lambda)\|_{\omega} \leqslant C_{1} e^{-\lambda / C_{1}}$. 
We introduce $X$ open set in $\mathbb{C}^{2}$. Recall that $\phi_{0}(w)=\frac{1}{2}(\Im w)^{2}$. The space $H\left(\phi_{0}, X, \omega\right)$ is the space of functions $g(t, k, z, \lambda)$, holomorphic for $(t, k) \in X$, living in $H_{\omega}$, such that

$$
\exists C_{2}>0, \forall(t, k) \in X, \quad\|g(t, k, \cdot, \lambda)\|_{\omega} \leqslant C_{2} e^{\lambda C_{2}} e^{\lambda \phi_{0}(t-i k)} .
$$

We say that $g$ is negligible in $H\left(\phi_{0}, X, \omega\right)$ if there exists $C_{3}>0$ such that

$$
\forall(t, k) \in X, \quad\|g(t, k, \cdot, \lambda)\|_{\omega} \leqslant C_{3} e^{-\lambda / C_{3}} e^{\lambda \phi_{0}(t-i k)} .
$$

We define a family of sectors $\omega_{\sigma}$ such that $d\left(\omega_{\sigma}, \omega_{\sigma^{\prime}}^{c}\right)=d_{1}\left(\sigma^{\prime}-\sigma\right)\left(\sigma^{\prime}>\sigma\right)$ and we simplify the notation with

$$
H_{\sigma}=H_{\omega_{\sigma}} .
$$

For the purpose of this paper, we define also

$$
H_{\sigma}^{ \pm}=H_{\omega_{\sigma}^{ \pm}}
$$

where $\omega_{\sigma}^{ \pm}=\frac{1}{\cos \gamma_{ \pm}} \omega_{\sigma}$. We introduce the FBI transform in $x$ as in Section 4.1 of [20]. In particular, the FBI transform in $x$, denoted by $T_{1}$, is described through (4.1) to (4.5) of $[\mathbf{2 0}]$ p. 372 , and a Gaussian localization operator $J_{\rho}$ is defined through (4.16) of $[\mathbf{2 0}]$. We reproduce these definitions here.

Let $\psi_{2} \in C_{0}^{\infty}$, equal to 1 on $\operatorname{supp}(\psi)$, equal to 0 for $|x| \geqslant 5 c t_{0}$. For $g \in L_{\text {loc }}^{2}(\mathbb{R})$, $\operatorname{supp}(g) \subset \mathbb{R}_{+}, z \in \mathbb{C}$, we define

$$
T_{1} g(z, \lambda)=\int_{0}^{\infty} e^{i \lambda x z-\lambda x^{2} / 2} \psi_{2}(x) g(x) \mathrm{d} x .
$$

For $h(z, \lambda)$ holomorphic in $z$ and $\rho>0$, we introduce

$$
\begin{aligned}
J_{\rho}(h)(z, \lambda) & =\left(\frac{\lambda}{2 \pi}\right)^{1 / 2} \int_{-\rho}^{+\rho} h(z+x, \lambda) e^{-\lambda x^{2} / 2} \mathrm{~d} x, \\
J_{\rho}^{-1}(h)(z, \lambda) & =\left(\frac{\lambda}{2 \pi}\right)^{1 / 2} \int_{-\rho}^{\rho} e^{-\lambda x^{2} / 2} h(z+i x) \mathrm{d} x .
\end{aligned}
$$

Properties of these operators $T_{0}, T_{1}, J_{\rho}, J_{\rho}^{-1}$ are the following (from p. 374-375 of $[20]):$

- For $\omega^{\prime} \subset_{<} \omega$ (that is $\omega$ is a $\varepsilon$-sector and $\omega^{\prime}$ is a $\varepsilon^{\prime}$-sector, with $\varepsilon^{\prime}>\varepsilon$ ), there exists $\rho_{0}$ such that, for $\left.\left.\rho \in\right] 0, \rho_{0}\right], J_{\rho}$ and $J_{\rho}^{-1}$ send $H_{\omega}$ into $H_{\omega^{\prime}}$, and these operators are independent of $\rho$, up to a negligible operator $R$ satisfying

$$
\exists C, \forall g \in H_{\omega}, \quad\|R(g)\|_{\omega^{\prime}} \leqslant C e^{-\lambda / C}\|g\|_{\omega} .
$$

- There exist two negligible operators $R_{\rho}^{1,2}$ such that

$$
J_{\rho} \circ J_{\rho}^{-1}=\mathrm{Id}+R_{\rho}^{1}, \quad J_{\rho}^{-1} \circ J_{\rho}=\mathrm{Id}+R_{\rho}^{2} .
$$

- Moreover, $J_{\rho}$ is a quantization of the canonical transform $(x, \xi) \mapsto(x-i \xi, \xi)$. 
Let $f(t, x) \in L^{2}, \operatorname{supp} f \subset\left\{0 \leqslant x \leqslant c t, 0 \leqslant t \leqslant 2 t_{0}\right\}$. If we introduce $F(w, z, \lambda)=$ $\left(T_{0} \otimes T_{1}\right)(f)(w, z, \lambda)$, we obtain, as a consequence of

$$
\left(\frac{\lambda}{2 \pi}\right)^{1 / 2} \int_{\Im k=-a} e^{-\frac{\lambda}{2}(k-i(w-t))^{2}} \mathrm{~d} k=1
$$

that

$$
F(w, z, \lambda)=\int_{-\infty}^{+\infty} e^{\lambda\left(x z-x^{2} / 2-(w-t)^{2} / 2\right)} f(x, t) \mathrm{d} x \mathrm{~d} t .
$$

The value at $x=0$ is microlocalized through the

Lemma 4.6. - Let $f(t, x) \in L^{2}\left(\mathbb{R}^{2}\right)$, $\operatorname{supp} f \subset\left\{0 \leqslant x \leqslant c t, 0 \leqslant t \leqslant 2 t_{0}\right\}$ and $1_{x>0} \partial_{x} f(t, x) \in L^{2}\left(\mathbb{R}^{2}\right)$. We introduce $\widetilde{F}(w, z, \lambda)=\left(T_{0} \otimes T_{1}\right)\left(1_{x>0} \partial_{x} f\right)$. We have the identity

$$
\widetilde{F}(w, z, \lambda)=-T_{0}(f(0, \cdot))(w, \lambda)+\left(\partial_{z}-\lambda z\right) F(w, z, \lambda)+r(w, z, \lambda)
$$

where $r(w, z, \lambda)$ is bounded by $\frac{C}{z} e^{-\lambda / C}$.

This writes also

$$
-\frac{1}{\lambda} T_{0}(f(0, \cdot))(w, \lambda)=\frac{1}{\lambda} \widetilde{F}(w, z, \lambda)+\left(\frac{1}{\lambda} \partial_{z}-z\right) F(w, z, \lambda)+\frac{1}{\lambda} r(w, z, \lambda)
$$

We deduce from this relation

$$
J_{\rho}\left(\frac{1}{\lambda} \widetilde{F}\right)(z, w, \lambda)=-z J_{\rho}(F)(w, z, \lambda)-\frac{1}{\lambda} T_{0}(f(0, \cdot))(w, \lambda)+\frac{e^{-\lambda \rho^{2} / 2}}{(2 \pi \lambda)^{1 / 2}} \widetilde{r}(w, z, \lambda, \rho)
$$

where the term $\widetilde{r}$ is bounded.

The equality (104) implies that

$$
\begin{aligned}
\widetilde{F}(w, z, \lambda)= & \int_{-\infty}^{+\infty} \mathrm{d} t \int_{0}^{\infty} \mathrm{d} x e^{\left(\lambda x z-\lambda x^{2}\right) / 2} \psi_{2}(x) e^{-\lambda(w-t)^{2} / 2} \partial_{x} f(x, t) \\
= & \int_{-\infty}^{+\infty} \mathrm{d} t \int_{0}^{\infty} \mathrm{d} x \partial_{x}\left[f(x, t) e^{\left(\lambda x z-\lambda x^{2}\right) / 2} \psi_{2}(x)\right] e^{-\lambda(w-t)^{2} / 2} \\
& \quad-\int_{-\infty}^{+\infty} \mathrm{d} t \int_{0}^{\infty} \mathrm{d} x f(x, t) \partial_{x}\left[e^{\left(\lambda x z-\lambda x^{2}\right) / 2} \psi_{2}(x)\right] e^{-\lambda(w-t)^{2} / 2} \\
= & -\int_{-\infty}^{+\infty} \mathrm{d} t f(0, t) e^{-\lambda(w-t)^{2} / 2} \\
& \quad-\int_{-\infty}^{+\infty} \mathrm{d} t \int_{0}^{\infty} \mathrm{d} x f(x, t)(\lambda z-\lambda x) e^{\left(\lambda x z-\lambda x^{2}\right) / 2} \psi_{2}(x) e^{-\lambda(w-t)^{2} / 2} \\
& \quad-\int_{-\infty}^{+\infty} \mathrm{d} t \int_{0}^{\infty} \mathrm{d} x f(x, t) \partial_{x} \psi_{2}(x) e^{\left(\lambda x z-\lambda x^{2}\right) / 2} e^{-\lambda(w-t)^{2} / 2} .
\end{aligned}
$$

From the equality (103), we get that

$$
\left(\frac{\lambda}{2 \pi}\right)^{1 / 2} \int_{\Im k=-a} e^{-\lambda k^{2} / 2-i k \lambda(t-w)} \mathrm{d} k=e^{-\lambda(w-t)^{2} / 2},
$$


which imply that the first term above is

$$
\begin{aligned}
-\int_{-\infty}^{+\infty} \mathrm{d} t f(0, t) e^{-\lambda(w-t)^{2} / 2} & =-\left(\frac{\lambda}{2 \pi}\right)^{1 / 2} \int_{\Im k=-a} e^{-\lambda k^{2} / 2+i k \lambda w} \int_{-\infty}^{+\infty} \mathrm{d} t e^{-i k \lambda t} f(0, t) \mathrm{d} k \\
& =-T_{0}(f(0, t))(w, \lambda) .
\end{aligned}
$$

On the other hand, we notice that

$$
\partial_{z} F(z, w, \lambda)=\int_{-\infty}^{+\infty} \mathrm{d} t \int_{0}^{\infty} \mathrm{d} x \lambda x e^{\lambda x z-\lambda x^{2} / 2} \psi_{2}(x) e^{-\lambda(w-t)^{2} / 2} f(x, t) .
$$

We deduce then the identity (105). Hence we have the equality

$$
\begin{aligned}
& J_{\rho}\left(\frac{1}{\lambda}\left(T_{0} \otimes T_{1}\right)\left(\partial_{x} f\right)\right)(z, w, \lambda)= \\
& \quad-z J_{\rho}\left(T_{0} \otimes T_{1}\right)(f)(w, z, \lambda)-\left(\frac{1}{\sqrt{2 \pi}} \int_{-\rho \sqrt{\lambda}}^{\rho \sqrt{\lambda}} e^{-u^{2} / 2} d u\right) \frac{1}{\lambda} T_{0}(f(0, \cdot))(w, \lambda) \\
& \quad+\frac{e^{-\lambda \rho^{2} / 2}}{\sqrt{2 \pi \lambda}}\left[\left(T_{0} \otimes T_{1}\right)(f)(w, z+\rho, \lambda)-\left(T_{0} \otimes T_{1}\right)(f)(w, z-\rho, \lambda)\right]
\end{aligned}
$$

which imply the equality (107), where the term $\widetilde{r}$ is equal to

$$
\begin{aligned}
\widetilde{r}(w, z, \lambda, \rho)=\left(T_{0} \otimes T_{1}\right)(f)(w, z+\rho, \lambda) & -\left(T_{0} \otimes T_{1}\right)(f)(w, z-\rho, \lambda) \\
& +2 \rho \int_{1}^{\infty} e^{-\frac{\rho \lambda^{2}}{2} \frac{u^{2}-1}{2}} \mathrm{~d} u \frac{1}{\lambda} T_{0}(f(0, \cdot))(w, \lambda) .
\end{aligned}
$$

Let $f(t, x) \in H^{1}(\mathbb{R} \times \mathbb{R})$, supported in $\left\{-c t \leqslant x \leqslant c t, t \leqslant 3 t_{0}\right\}$. Note that the two distributions $f(t, x) 1_{x \geqslant 0}$ and $\partial_{x} f(t, x) 1_{x>0}$ are element of $L^{2}\left(\mathbb{R}^{2}\right)$, supported in $\left\{0 \leqslant x \leqslant c t, t \leqslant 3 t_{0}\right\}$. We define

$$
\begin{aligned}
& F(w, z, \lambda)=\left(T_{0} \otimes T_{1}\right)\left(f(t, x) 1_{x \geqslant 0}\right)(w, z, \lambda), \\
& \widetilde{F}(w, z, \lambda)=\left(T_{0} \otimes T_{1}\right)\left(\partial_{x} f(t, x) 1_{x \geqslant 0}\right)(w, z, \lambda) .
\end{aligned}
$$

It is a consequence of Proposition 3.1 that, if $f(t, x)$ is the trace of $u-u_{ \pm}$on the boundary, considered as Lipschitz equivalent to $\mathbb{R}$, the part $\mathbb{R}_{+}$being associated with $\Delta_{+}$and the part $\mathbb{R}_{-}$being associated with $\Delta_{-}$,

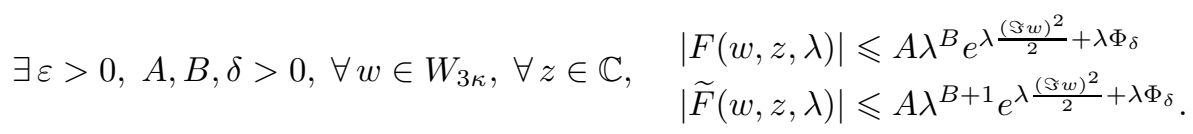

We note that, as $\psi$ is flat in the neighborhood of 0 , the symbol $\sigma_{2}^{+}$can be replaced by the symbol $L_{3}^{+}\left(x, x^{\prime}\right)\left(k^{2} / \xi_{+}\right)+i^{-1} \partial_{x^{\prime}}\left(L_{2}^{+}\left(x, x^{\prime}\right)+L_{1}^{+}\left(x, x^{\prime}\right) \xi_{1}^{+} / \xi_{+}\right)$, and the symbol $q_{1}^{+}$by the symbol $L_{2}^{+}\left(x, x^{\prime}\right)+L_{1}^{+}\left(x, x^{\prime}\right) \xi_{1}^{+} / \xi_{+}$. We introduce the symbols $s_{2}^{+}\left(x, x^{\prime}, \xi_{1}^{+}, k\right)=L_{3}^{+}\left(x, x^{\prime}\right) k^{2} / \xi_{+}$and $s_{1}^{+}\left(x, x^{\prime}, \xi_{1}^{+}, k\right)=\partial_{x^{\prime}}\left(L_{2}^{+}\left(x, x^{\prime}\right)+\right.$ $\left.L_{1}^{+}\left(x, x^{\prime}\right) \xi_{1}^{+} / \xi_{+}\right)$. We use the asymptotic expansion of Gérard and Lebeau (Appendix B, (B.1) to (B.22) of [20]) noticing that $s_{2}^{+}\left(x, x^{\prime}, \lambda \xi_{1}^{+}, \lambda k\right)=\lambda s_{2}^{+}\left(x, x^{\prime}, \xi_{1}^{+}, k\right)$ and $s_{1}^{+}\left(x, x^{\prime}, \lambda \xi_{1}^{+}, \lambda k\right)=s_{1}^{+}\left(x, x^{\prime}, \xi_{1}^{+}, k\right)$ as well as $q_{1}^{+}\left(x, x^{\prime}, \lambda \xi_{1}^{+}, \lambda k\right)=q_{1}^{+}\left(x, x^{\prime}, \xi_{1}^{+}, k\right)$. 
We define the elementary symbols

$$
\begin{aligned}
s_{2, n, m}^{+}(k, \xi) & =\frac{\partial_{x}^{n} \partial_{x^{\prime}}^{m} s_{2}^{+}}{n ! m !}(0,0, \xi, k) \\
q_{1, n, m}^{+}(k, \xi) & =\frac{\partial_{x}^{n} \partial_{x^{\prime}}^{m} q_{1}^{+}}{n ! m !}(0,0, \xi, k) . \\
s_{1, n, m}^{+}(k, \xi) & =\frac{\partial_{x}^{n} \partial_{x^{\prime}}^{m} s_{1}^{+}}{n ! m !}(0,0, \xi, k)
\end{aligned}
$$

Applying Proposition 4.1, which is Proposition 2.2 of [20], we compute $\left(T_{0} \otimes\right.$ $\left.T_{1}\right)\left(R_{+, 0}^{+}\right)(f)$ using the relation, for $f \in E_{e^{\prime}}$,

$$
T_{0} R_{+}^{+} f-\frac{\lambda}{2 \pi} \int_{t_{-}}^{t_{+}} \mathrm{d} t \int_{\tau_{-}}^{\tau_{+}} \mathrm{d} \tau e^{i \lambda \tau(w-t)-\lambda \tau^{2}} I(t, \tau, z, \lambda) \in H_{\phi}\left(W, L^{2}\right) .
$$

We recall some definitions and results of $[\mathbf{2 0}]$ on the Sjöstrand spaces used here, namely Definition 2.2 and Lemma 2.2 and Proposition 2.2 p. 350 of [20].

Recall also that if $R \in H_{\phi_{0}}\left(W, L^{2}\right)$, then $T_{1} R \in H\left(\phi_{0}, X_{a^{\prime} / D_{1}}, U\right)$ because $T_{1}$ sends continuously $L^{2}$ into $H_{U}$ (the Sjöstrand spaces are defined in Definition 4.1 and following equalities). As

$$
T_{1} \otimes T_{0}\left(1_{x>0} R_{+, 0}^{+}(f)\right)=\frac{\lambda}{2 \pi} \int_{t_{-}}^{t_{+}} \mathrm{d} t \int_{\tau_{-}}^{\tau_{+}} \mathrm{d} \tau e^{i \lambda \tau(w-t)-\lambda \tau^{2}} I(t, \tau, z, \lambda)+T_{1} R,
$$

the remainder term is negligible. The application of Proposition 4.5 below yields the asymptotic expansion of $I$. The same pattern applies to all the terms of the system (94).

Proposition 4.5. - There exists $t_{0}^{1}$, and, for $\left.t_{0} \in\right] 0, t_{0}^{1}[$ given, three constants $a^{\prime}, \rho, C_{2}^{0}$ such that

i) $I(t, k, z, \lambda)=T_{1}\left(1_{x>0} R_{+, 0}^{+, \lambda k} T_{0} f\right)(t-i k, z, \lambda) \in H\left(\phi_{0}, X_{a^{\prime} / D_{1}}, U\right)$

ii) For $C_{1} \geqslant e D_{1} / a^{\prime}, C_{2} \geqslant C_{2}^{0}$, the function

$$
\begin{aligned}
r(t, k, z, \lambda) & =T_{1}\left(1_{x>0} R_{+, 0}^{+, \lambda k} T_{0} f\right)(t-i k, z, \lambda) \\
& -\frac{i \lambda}{2} J_{\rho}^{-1}\left(\sum_{n \leqslant \lambda / C_{1}, m \leqslant \lambda / C_{2}}\left(\frac{1}{\lambda} \partial_{z}\right)^{n} \frac{1}{2 \pi} \int_{\mathcal{C}_{1}} \frac{\mathrm{d} \xi}{z+i \xi} s_{2, n, m}^{+}(k, \xi)\left(\frac{1}{\lambda} \partial_{z}\right)^{m} J_{\rho} F(-i \xi)\right) \\
& -\frac{1}{2} J_{\rho}^{-1}\left(\sum_{n \leqslant \lambda / C_{1}, m \leqslant \lambda / C_{2}}\left(\frac{1}{\lambda} \partial_{z}\right)^{n} \frac{1}{2 \pi} \int_{\mathcal{C}_{1}} \frac{\mathrm{d} \xi}{z+i \xi} s_{1, n, m}^{+}(k, \xi)\left(\frac{1}{\lambda} \partial_{z}\right)^{m} J_{\rho} F(-i \xi)\right) \\
& -\frac{1}{2} J_{\rho}^{-1}\left(\sum_{n \leqslant \lambda / C_{1}, m \leqslant \lambda / C_{2}}\left(\frac{1}{\lambda} \partial_{z}\right)^{n} \frac{1}{2 \pi} \int_{\mathcal{C}_{1}} \frac{\mathrm{d} \xi}{z+i \xi} q_{1, n, m}^{+}(k, \xi)\left(\frac{1}{\lambda} \partial_{z}\right)^{m} J_{\rho} \widetilde{F}(-i \xi)\right)
\end{aligned}
$$

is negligible in $H\left(\phi_{0}, Z_{a^{\prime} / D_{1}}, U\right)$.

These integrals can be deformed on the contour $C(+)$ defined in Section 6.3. Note that $\widetilde{F}$ is of "weight" $\lambda$ hence contributions of $s_{+}^{2}$ and of $q_{1}^{+}$are of "weight" $\lambda$. 
Note that, for $f \in E_{e}$, verifying $\partial_{x} f 1_{x \geqslant 0} \in E_{e}$, we have the identity $T_{0}\left(\partial_{x} f 1_{x \geqslant 0}\right)=$ $1_{x \geqslant 0} \partial_{x}\left(T_{0}(f)\right)$, because the integration is only in the time variable. As

$$
\begin{aligned}
\frac{1}{4 \pi} \iint \mathrm{d} \xi_{1}^{+} \mathrm{d} x^{\prime} s_{2}^{+}\left(x, x^{\prime}, \xi_{1}^{+}, \lambda k\right) & T_{0} f\left(t-i k, x^{\prime}, \lambda\right) \\
= & \frac{\lambda^{2}}{4 \pi} \iint d \zeta_{1}^{+} \mathrm{d} x^{\prime} s_{2}^{+}\left(x, x^{\prime}, \zeta_{1}^{+}, k\right) T_{0} f\left(t-i k, x^{\prime}, \lambda\right)
\end{aligned}
$$

because $s_{2}^{+}\left(x, x^{\prime}, \lambda \zeta_{1}^{+}, \lambda k\right)=\lambda s_{2}^{+}\left(x, x^{\prime}, \zeta_{1}^{+}, k\right)$,

$$
\begin{aligned}
\frac{1}{4 \pi} \iint \mathrm{d} \xi_{1}^{+} \mathrm{d} x^{\prime} s_{1}^{+}\left(x, x^{\prime}, \xi_{1}^{+}, \lambda k\right) & T_{0} f\left(t-i k, x^{\prime}, \lambda\right) \\
= & \frac{\lambda}{4 \pi} \iint d \zeta_{1}^{+} \mathrm{d} x^{\prime} s_{1}^{+}\left(x, x^{\prime}, \zeta_{1}^{+}, k\right) T_{0} f\left(t-i k, x^{\prime}, \lambda\right)
\end{aligned}
$$

because $s_{1}^{+}\left(x, x^{\prime}, \lambda \zeta_{1}^{+}, \lambda k\right)=s_{1}^{+}\left(x, x^{\prime}, \zeta_{1}^{+}, k\right)$ and

$$
\begin{aligned}
R_{+, 0}^{+, 1, \lambda k}\left(T_{0} f\right)(t-i k, x, \lambda) & =\frac{1}{4 \pi} \iint \mathrm{d} \xi_{1}^{+} \mathrm{d} x^{\prime} q_{1}^{+}\left(x, x^{\prime}, \xi_{1}^{+}, \lambda k\right) 1_{x^{\prime} \geqslant 0} \partial_{x^{\prime}} T_{0} f\left(t-i k, x^{\prime}, \lambda\right) \\
& =\frac{\lambda}{4 \pi} \iint d \zeta_{1}^{+} \mathrm{d} x^{\prime} q_{1}^{+}\left(x, x^{\prime}, \zeta_{1}^{+}, k\right) T_{0}\left(1_{x^{\prime} \geqslant 0} \partial_{x^{\prime}} f\right)\left(t-i k, x^{\prime}, \lambda\right)
\end{aligned}
$$

because $q_{1}^{+}\left(x, x^{\prime}, \lambda \zeta_{1}^{+}, \lambda k\right)=q_{1}^{+}\left(x, x^{\prime}, \zeta_{1}^{+}, k\right)$, we get three different terms to evaluate. The proof of this Proposition, identical to the proof of Proposition 4.1 of [20], is given in Section 6.3 of the Appendix

The three sequences of operators $s_{2, n, m}^{+}(k, \xi), s_{1, n, m}^{+}(k, \xi)$ and $q_{1, n, m}^{+}(k, \xi)$ lead to the construction of the three sequences:

$$
\begin{aligned}
& q_{1, n}^{+}(k, \lambda)(f)=\sum_{j+m=n} \partial_{z}^{j}\left(\frac{1}{2 \pi} \int_{\mathcal{C}(+)} \frac{\mathrm{d} \xi}{z+i \xi} q_{1, n m}^{+}\left(\partial_{x^{\prime}} f 1_{x^{\prime} \geqslant 0}\right)(k, \xi,-i \xi)\right), \\
& s_{2, n}^{+}(k, \lambda)(f)=\sum_{j+m=n} \partial_{z}^{j}\left(\frac{1}{2 \pi} \int_{\mathcal{C}(+)} \frac{\mathrm{d} \xi}{z+i \xi} s_{2, n m}^{+}(f)(k, \xi,-i \xi)\right), \\
& s_{1, n}^{+}(k, \lambda)(f)=\sum_{j+m=n} \partial_{z}^{j}\left(\frac{1}{2 \pi} \int_{\mathcal{C}(+)} \frac{\mathrm{d} \xi}{z+i \xi} s_{1, n m}^{+}(f)(k, \xi,-i \xi)\right) .
\end{aligned}
$$

The three symbols $s_{2}^{+}(k, \lambda), s_{1}^{+}(k, \lambda)$ and $q_{1}^{+}(k, \lambda)$ are valued in the chain of spaces $H_{\sigma}^{+}$.

The case of the operators $R_{-, 0}^{+, k}$ is similar to the case of the operators $S^{ \pm, \mp}$ of $[\mathbf{2 0}]$. We introduce now the two phase functions

$$
\phi_{+}(x)=i\left(a(x)-a^{\prime}(0) x\right) \xi_{0}-\frac{x^{2}}{2}, \quad \phi_{-}(x)=-i\left(b\left(x^{\prime}\right)-b^{\prime}(0) x^{\prime}\right) \xi_{0}-\frac{\left(x^{\prime}\right)^{2}}{2} .
$$


Let

$$
\begin{aligned}
& r_{1, n, m}^{-,+}(f)(k, \xi, z)= \\
& \left.\frac{k^{2}}{\xi_{0}} \frac{1}{n !} \frac{1}{m !} \partial_{x}^{n} \partial_{x^{\prime}}^{m}\left[e^{\lambda \phi_{+}(x)+\lambda \phi_{-}\left(x^{\prime}\right)}\left(\frac{1+a^{\prime}(x) b^{\prime}\left(x^{\prime}\right)}{\left(1+\left(a^{\prime}(x)\right)^{2}\right)^{1 / 2}}\right) \partial_{z}^{m} f\left(x^{\prime}+z\right)\right]\right|_{x=0, x^{\prime}=0}, \\
& r_{2, n, m}^{-,+}(f)(k, \xi, z)= \\
& \left.\frac{1}{n !} \frac{1}{m !} \partial_{x}^{n} \partial_{x^{\prime}}^{m}\left[e^{\lambda \phi_{+}(x)+\lambda \phi_{-}\left(x^{\prime}\right)}\left(\frac{a^{\prime}(x)+\xi / \xi_{0}}{\left(1+\left(a^{\prime}(x)\right)^{2}\right)^{1 / 2}}\right) \partial_{z}^{m} f\left(x^{\prime}+z\right)\right]\right|_{x=0, x^{\prime}=0 .} .
\end{aligned}
$$

Let $\widetilde{F}\left(x^{\prime}, z, \lambda, t-i k\right)=F\left(t-i k, x^{\prime}+z, \lambda\right)$. We have the

Proposition 4.6. - If $t_{0}^{1}$ and $t_{0}$ are given as in Proposition 4.5, there exists $l, \kappa_{0}, C_{1}^{0}, C_{2}^{0}$ such that, for $\left.\left.\kappa \in\right] 0, \kappa_{0}\right], C_{1} \geqslant C_{1}^{0}, C_{2} \geqslant C_{2}^{0}$

i) the function $T_{1}\left(R_{-}^{+, \lambda k} T_{0} f\right)(t-i k, z, \lambda)$ is in $H\left(\phi_{0}, X_{\kappa}, U\right)$,

ii) $T_{1}\left(R_{-}^{+, \lambda k} T_{0} f\right)(t-i k, z, \lambda)$

$$
\begin{aligned}
& +\frac{\lambda}{2} \sum_{\substack{n \leqslant \lambda / C_{1} \\
m \leqslant \lambda / C_{2}}}\left(\frac{1}{\lambda} \partial_{z}\right)^{n}\left(\frac{1}{2 \pi} \int_{\mathcal{C}(l, b)} \frac{\mathrm{d} \xi}{z+i\left(\xi+a^{\prime}(0) \xi_{0}\right)} r_{1, n, m}^{-,+}(F)\left(k, \xi,-i\left(\xi+b^{\prime}(0) \xi_{0}\right)\right)\right) \\
& -\frac{i}{2} \sum_{\substack{n \leqslant \lambda / C_{1} \\
m \leqslant \lambda / C_{2}}}\left(\frac{1}{\lambda} \partial_{z}\right)^{n}\left(\frac{1}{2 \pi} \int_{\mathcal{C}(l, b)} \frac{\mathrm{d} \xi}{z+i\left(\xi+a^{\prime}(0) \xi_{0}\right)} r_{2, n, m}^{-,+}(\widetilde{F})\left(k, \xi,-i\left(\xi+b^{\prime}(0) \xi_{0}\right)\right)\right)
\end{aligned}
$$

is negligible in $H\left(\phi_{0}, X_{\kappa}, U\right)$. The contour used here is shown in Section 6.3.

This is the extension of proposition 4.2 of $[\mathbf{2 0}]$ for the operator $S^{-,+}$. We check that the function $\partial_{x^{\prime}} \psi\left(x^{\prime}\right)$ in the calculus instead of $\psi\left(x^{\prime}\right)$ leads to a symbol which is equal to 0 , hence only the symbol $\frac{1+a^{\prime}(x) b^{\prime}\left(x^{\prime}\right)}{\left(1+\left(a^{\prime}(x)\right)^{2}\right)^{1 / 2}}\left(a^{\prime}(x)+\xi / \xi_{0}\right)$ contributes to the symbol after the asymptotic expansion. We have the equalities $r_{1}^{ \pm, \mp}\left(x, x^{\prime}, \lambda \zeta, \lambda k\right)=$ $r_{1}^{ \pm, \mp}\left(x, x^{\prime}, \zeta, k\right)$ and $r_{2}^{ \pm, \mp}\left(x, x^{\prime}, \lambda \zeta, \lambda k\right)=r_{1}^{ \pm, \mp}\left(x, x^{\prime}, \zeta, k\right)$.

These two sequences of operators lead to the construction of the two sequences:

$$
\begin{aligned}
& r_{1, n}^{-,+}(k, \lambda)(f)= \\
& \quad-\sum_{j+m=n} \partial_{z}^{j}\left(\frac{1}{2 \pi} \int_{\mathcal{C}(l, b)} \frac{\mathrm{d} \xi}{z+i\left(\xi+a^{\prime}(0) \xi_{0}\right)} r_{1, n m}^{-,+}(f)\left(k, \xi,-i\left(\xi+b^{\prime}(0) \xi_{0}\right)\right)\right) \\
& r_{2, n}^{-,+}(k, \lambda)(f)= \\
& \quad-\sum_{j+m=n} \partial_{z}^{j}\left(\frac{1}{2 \pi} \int_{\mathcal{C}(l, b)} \frac{\mathrm{d} \xi}{z+i\left(\xi+a^{\prime}(0) \xi_{0}\right)} r_{2, n m}^{-,+}\left(\partial_{x^{\prime}} f\right)\left(k, \xi,-i\left(\xi+b^{\prime}(0) \xi_{0}\right)\right)\right) .
\end{aligned}
$$

Notice that the symbols $r_{j, n}^{-,+}(k, \lambda)$ operate from $H_{0}^{-}$to $H_{U}$. The symbol $r^{-,+}(k, \lambda)$ is an analytic symbol from the bounded operators of $H_{0}^{-}$into $H_{1}^{+}$. We prove this 
Proposition in Section 6.3, and it is a reproduction of the proof of Gérard and Lebeau $[20]$.

The same results apply for the operators with the "minus" index.

Note that we have to write the asymptotic expansion of the operators $S^{ \pm, \mp}$ and $Q^{ \pm}$. The asymptotic expansion of these operators takes into account the term $\left(\frac{\left(1+\left(a^{\prime}\left(x^{\prime}\right)\right)^{2}\right.}{\left.1+\left(a^{\prime}(x)\right)^{2}\right)}\right)^{1 / 2}$ and the term $\left(\frac{\left(1+\left(a^{\prime}\left(x^{\prime}\right)\right)^{2}\right.}{\left.1+\left(b^{\prime}(x)\right)^{2}\right)}\right)^{1 / 2}$ we added in the symbol to $Q^{+}$and to $S^{+,-}$. Hence we obtain a slight modification of the expressions of the asymptotic expansion (4.34) of [20] into

$$
\begin{gathered}
T_{1}\left(1_{x>0} Q^{+, \lambda k} T_{0} f\right)(t-i \tau, z, \lambda) \simeq J_{\rho}^{-1}\left(\sum_{m \leqslant \lambda / C_{1}, n \leqslant \lambda / C_{2}}\left(\frac{1}{\lambda} \partial_{z}\right)^{n} \frac{1}{2 \pi} \int_{\mathcal{C}_{1}} \frac{\mathrm{d} \xi_{1}^{+}}{z+i \xi_{1}^{+}}\right. \\
\left.. \frac{\partial_{x}^{n} \partial_{x}^{\prime m}}{n ! m !}\left[\left(\frac{1+\left(a^{\prime}\left(x^{\prime}\right)\right)^{2}}{1+\left(a^{\prime}(x)\right)^{2}}\right)^{1 / 2}\left(1+\sigma^{+}\left(x, x^{\prime}, \xi_{1}^{+}, k\right)\right)-1\right]\left(\frac{1}{\lambda} \partial_{z}\right)^{m} J_{\rho} F\left(-i \xi_{1}^{+}\right)\right)
\end{gathered}
$$

(and the term of order 0 obtained for $m=n=0$ is still 0 ) and we modify the relation (4.49) of $[\mathbf{2 0}]$ into

$$
\begin{gathered}
T_{1}\left(1_{x>0} S^{+,-, \lambda k} T_{0} f\right)(t-i \tau, z, \lambda) \simeq-\sum_{m \leqslant \lambda / C_{1}, n \leqslant \lambda / C_{2}}\left(\frac{1}{\lambda} \partial_{z}\right)^{n} \frac{1}{2 \pi} \int_{C(l, b)} \frac{\mathrm{d} \xi_{1}^{+}}{z+i\left(\xi+a \xi_{0}\right)} \\
\cdot \frac{\partial_{x}^{n} \partial_{x}^{\prime m}}{n ! m !}\left[e^{\lambda \phi_{+}+\lambda \phi_{-}}\left(\frac{1+\left(b^{\prime}\left(x^{\prime}\right)\right)^{2}}{1+\left(a^{\prime}(x)\right)^{2}}\right)^{1 / 2}\left(1-a^{\prime}(x) \xi / \xi_{0}\right)\right]\left(\frac{1}{\lambda} \partial_{z}\right)^{m} F\left(-i\left(\xi+b \xi_{0}\right)\right)
\end{gathered}
$$

We end the asymptotic expansion by computing the expansion of the source terms associated with the values at $t=0$ of the trace $g(x, t)$ on the boundary, which belongs to $H^{1}\left(\mathbb{R}^{2}\right)$. Note that this imply that $g(0, t)$ is bounded by the derivative of $g$, hence we need to introduce $G=\left(T_{0} \otimes T_{1}\right)(g) 1_{x \geqslant 0}$ and $\widetilde{G}=\left(T_{0} \otimes T_{1}\right)\left(\partial_{x} g 1_{x \geqslant 0}\right)$. We intend to compute

$$
\left(T_{0} \otimes T_{1}\right)\left(\mathcal{F}^{-1}\left(T^{+} \widehat{f}(0, k)\right)(x, t)\right) .
$$

We use again Proposition 4.1 (Proposition 2.2 of $[\mathbf{2 0}]$ ), because the distribution $T^{+}$is regular in $k$ in the sense of the spaces $E_{3}$, to obtain (here we gave the explicit notation of $T^{+}$depending on $k: T^{+, k}$ )

$$
T_{0}\left(\mathcal{F}^{-1}\left(T^{+} \widehat{g}(0, k)\right)=\frac{\lambda}{2 \pi} \int_{t_{-}}^{t_{+}} \int_{\tau_{-}}^{\tau_{+}} \mathrm{d} t \mathrm{~d} \tau e^{i \lambda \tau(w-t)-\lambda \tau^{2}} T^{+, \lambda \tau}(x) T_{0} g(t-i \tau, 0, \lambda)+R\right.
$$

the remainder term is in $H_{\phi}\left(W, L^{2}\right)$.

Recall that

$$
\begin{aligned}
& \left\langle T^{+, \lambda \tau}, l\right\rangle= \\
& \frac{i}{4 \pi} \int_{\mathbb{R}^{2}} e^{i x \cdot \xi_{1}^{+}} \frac{T_{+}(x) l(x) \lambda^{2} \tau^{2}}{\left(\lambda^{2} \tau^{2}\left(1+\mu_{+}^{2}\right)-\left(\xi_{1}^{+}\right)^{2}\right)^{3 / 2}}\left[1+\mu_{+}^{2}+\frac{3 \mu_{+} \partial_{x} \mu_{+} \xi_{1}^{+}}{\lambda^{2} \tau^{2}\left(1+\mu_{+}^{2}\right)-\left(\xi_{1}^{+}\right)^{2}}\right] \mathrm{d} x \mathrm{~d} \xi_{1}^{+} .
\end{aligned}
$$


We write

$$
t\left(x, \xi_{1}^{+}, \lambda\right)=\frac{T_{+}(x) \tau^{2}}{\left(\tau^{2}\left(1+\mu_{+}^{2}\right)-\left(\xi_{1}^{+}\right)^{2}\right)^{3 / 2}}\left[1+\mu_{+}^{2}+\frac{3}{\lambda} \mu_{+} \partial_{x} \mu_{+} \frac{\xi_{1}^{+}}{\tau^{2}\left(1+\mu_{+}^{2}\right)-\left(\xi_{1}^{+}\right)^{2}}\right] .
$$

We consider now the application of $T_{1}$. It concerns only the $x$ variable, hence we compute

$$
S(t, \tau, z, \lambda)=T_{1}\left(T^{+, \lambda \tau}\right)(z, \lambda) T_{0} g(t-i \tau, 0, \lambda) .
$$

Proposition 4.7. - We have the equality

$$
\left(T_{0} \otimes T_{1}\right)\left(\mathcal{F}^{-1}\left(T^{+} \widehat{f}(0, k)\right)(x, t)\right)=\frac{\lambda}{2 \pi} \int_{t_{-}}^{t_{+}} \int_{\tau_{-}}^{\tau_{+}} \mathrm{d} t \mathrm{~d} \tau e^{i \lambda \tau(w-t)-\lambda \tau^{2}} S(t, \tau, z, \lambda)
$$

where $S$ has the following asymptotic expansion

$$
S(t, \tau, z, \lambda) \simeq \frac{1}{\lambda} T_{0} g(t-i \tau, 0, \lambda) \frac{i}{2} J_{\rho}^{-1}\left[\sum_{n \leqslant \lambda / C_{1}} \frac{1}{p !}\left(\frac{1}{\lambda} \partial_{z}\right)^{p} \frac{1}{2 \pi} \int_{\mathcal{C}(+)} \frac{t_{p}\left(\xi_{1}^{+}, \lambda\right) \mathrm{d} \xi_{1}^{+}}{z+i \xi_{1}^{+}}\right],
$$

where $t_{p}\left(\xi_{1}^{+}, \lambda\right)=\left(\frac{\partial}{\partial_{x}}\right)^{p} t\left(0, \xi_{1}^{+}, \lambda\right)$.

We also have

$$
\left(T_{0} \otimes T_{1}\right)\left(z(x) 1_{x \geqslant 0} \partial_{t} g(t, x)\right) \simeq \frac{\lambda}{2 \pi} \int_{t_{-}}^{t_{+}} \int_{\tau_{-}}^{\tau_{+}} \mathrm{d} t \mathrm{~d} \tau e^{i \lambda \tau(w-t)-\lambda \tau^{2}} Z(t, \tau, z, \lambda)
$$

with

(111) $Z(t, \tau, z, \lambda) \simeq i \tau \lambda J_{\rho}^{-1}\left[\sum_{n \leqslant \lambda / C_{1}}\left(\frac{1}{\lambda} \partial_{z}\right)^{n} \frac{1}{2 \pi} \int_{\mathbb{R}} \frac{\mathrm{d} \xi}{z+i \xi} \frac{\partial_{x}^{n} z(0)}{n !} J_{\rho} G(t-i \tau,-i \xi, \lambda)\right]$.

Apply the definition of $T^{+, k}$ to $l(x)=e^{i \lambda x z-\lambda x^{2} / 2} \psi_{2}(x) 1_{x} \geqslant 0$, which belongs to $L^{2}$ (it is necessary to notice that because the expression of $T^{+}$is defined for functions of $C_{0}^{\infty}(\mathbb{R})$ but can be extended to such functions). Hence we have, changing $\xi_{1}^{+}$into $\lambda \xi_{1}^{+}$

$$
T_{1}\left(T^{+, \lambda \tau}\right)(z, \lambda)=\frac{i}{4 \pi} \lambda \int_{\mathbb{R}} \mathrm{d} \xi_{1}^{+} \int_{0}^{\infty} e^{\lambda x z+i \lambda x \xi_{1}^{+}-\lambda x^{2} / 2} \lambda^{-1} t\left(x, \xi_{1}^{+}, \lambda\right) \psi_{2}(x) \mathrm{d} x
$$

The Taylor expansion of $t$ writes

$$
t\left(x, \xi_{1}^{+}\right)=\sum_{p=0}^{p=N}\left(\frac{\partial}{\partial x}\right)^{p} t\left(0, \xi_{1}^{+}\right) \frac{x^{p}}{p !}+\frac{x^{N+1}}{N !} t_{N}\left(x, \xi_{1}^{+}\right)
$$

and

$$
S_{N}(z, \lambda)=\frac{i}{4 \pi} \int_{\mathbb{R}} \mathrm{d} \xi_{1}^{+} \int_{0}^{\infty} e^{\lambda x\left(z+i \xi_{1}^{+}\right)-\lambda \frac{x^{2}}{2}-\nu\left(\xi_{1}^{+}\right)^{2}} \frac{x^{N+1}}{N !} t_{N}\left(x, \xi_{1}^{+}\right)
$$

is negligible in $H\left(\phi_{0}, X_{\kappa}, U\right)$ when $\nu \rightarrow 0_{+}$and $N=O(\lambda)$, because the symbol $B_{+}(x) / \xi_{+}^{3}$ is of the same type as $\sigma$. The only thing left in the calculus is

$$
\frac{i}{4 \pi} \int_{\mathbb{R}} \mathrm{d} \xi_{1}^{+} \frac{1}{p !}\left(\frac{\partial}{\partial x}\right)^{p} t\left(0, \xi_{1}^{+}\right) \int_{0}^{\infty} x^{p} e^{\lambda x\left(z+i \xi_{1}^{+}\right)-\lambda x^{2} / 2} \mathrm{~d} x .
$$


We use the relation

$$
\lambda \int_{0}^{\infty} e^{\lambda x\left(z+i \xi_{1}^{+}\right)-\lambda x^{2} / 2} x^{p} \psi(x) \mathrm{d} x=\left(\frac{1}{\lambda} \partial_{z}\right)^{p}\left[J_{\rho}^{-1}\left(\frac{1}{z+i \xi_{1}^{+}}\right)+r\left(z+i \xi_{1}^{+}, \lambda\right)\right]
$$

which is the result of Lemme 4.3 of $[\mathbf{2 0}]$, hence, as $\left[J_{\rho}, \partial_{z}\right]=0$, we have, using the appendix B of $[\mathbf{2 0}]$,

$$
\begin{aligned}
\frac{i}{4 \pi} \int_{\mathbb{R}} \mathrm{d} \xi_{1}^{+} \frac{1}{p !}\left(\frac{\partial}{\partial x}\right)^{p} t\left(0, \xi_{1}^{+}\right) \int_{0}^{\infty} x^{p} e^{\lambda x\left(z+i \xi_{1}^{+}\right)-\lambda x^{2} / 2} \mathrm{~d} x \\
\quad=\frac{i}{4 \pi \lambda} \int_{C(+)} \mathrm{d} \xi_{1}^{+} \frac{1}{p !}\left(\frac{\partial}{\partial x}\right)^{p} t\left(0, \xi_{1}^{+}\right)\left(\frac{1}{\lambda} \partial_{z}\right)^{p}\left[J_{\rho}^{-1}\left(\frac{1}{z+i \xi_{1}^{+}}\right)+r\left(z+i \xi_{1}^{+}, \lambda\right)\right]
\end{aligned}
$$

We replace $T_{0} g(t-i \tau, 0, \lambda)$ by the relation obtained in Lemma 4.6 through the equality (107), which imply

$$
-\frac{1}{\lambda} T_{1}\left(T^{+, \lambda \tau}\right) T_{0} g(t-i \tau, 0, \lambda) \simeq T_{1}\left(T^{+, \lambda \tau}\right)\left[J_{\rho}\left(\frac{1}{\lambda} \widetilde{F}\right)(z, w, \lambda)+z J_{\rho}(F)(w, z, \lambda)\right] .
$$

Hence we have the asymptotic expansion of the contribution of the corner.

The second item of the proposition comes easily from Proposition 4.1 of [20] using the representation

$$
z(x) \partial_{t} g(x, t)=\int_{\mathbb{R} \times \mathbb{R}_{+}} e^{i\left(x-x^{\prime}\right) \xi} z(x) \partial_{t} g\left(t, x^{\prime}\right) \mathrm{d} x^{\prime} \mathrm{d} \xi
$$

and the partial Fourier transform in time of $\partial_{t} u(t, x)$ which leads to a term of the form $\lambda i \tau \widehat{g}(\lambda \tau, x)$.

In the end of this Section, we write the leading order term of all the operators we computed (and symbols we computed) coming from the system (94).

Introduce

$$
X=\left(\begin{array}{l}
\left(T_{0} \otimes T_{1}\right)\left(\psi \phi_{1} v_{+}\right) \\
\left(T_{0} \otimes T_{1}\right)\left(\left.\psi \phi_{1} u\right|_{\partial \mathcal{O}_{+}}\right) \\
\left(T_{0} \otimes T_{1}\right)\left(\left.\psi \phi_{1} u\right|_{\partial \mathcal{O}_{-}}\right) \\
\left(T_{0} \otimes T_{1}\right)\left(\psi \phi_{1} v_{-}\right) \\
\left(T_{0} \otimes T_{1}\right)\left(\left.\psi \phi_{1} \partial_{\tan } u\right|_{\partial \mathcal{O}_{+}}\right) \\
\left(T_{0} \otimes T_{1}\right)\left(\left.\psi \phi_{1} \partial_{\tan } u\right|_{\partial \mathcal{O}_{-}}\right) \\
T_{0}\left(\phi_{1} u(0,0, t)\right)
\end{array}\right) \quad \text { and } \quad L=\left(\begin{array}{l}
2 \phi_{2} \psi \partial_{+} u_{i}+r_{+} \\
2 \phi_{2} \psi \partial_{-} u_{i}+r_{-} \\
-\left.z^{+}(x) \psi u\right|_{\partial O_{+}}\left(1-\phi_{2}\right) \partial_{t} \phi_{1} \\
-\left.z^{-}(x) \psi u\right|_{\partial O_{-}}\left(1-\phi_{2}\right) \partial_{t} \phi_{1}
\end{array}\right)
$$

Let $B$ be the operator which symbol is

$$
\begin{aligned}
& \text { (112) } \mathrm{op}(b)= \\
& \left(\begin{array}{ccccccc}
\mathrm{Id}-\mathrm{op}\left(q^{+}\right) & -\mathrm{op}\left(i^{+, 0}\right) & -\mathrm{op}\left(i^{+, 1}\right) & -\mathrm{op}\left(s^{+,-}\right) & -\mathrm{op}\left(r^{+,-, 0}\right) & -\mathrm{op}\left(r^{+,-, 1}\right) & -2 t_{+} \\
-\mathrm{op}\left(s^{-,+}\right) & -\mathrm{op}\left(r^{-,+, 0}\right) & -\mathrm{op}\left(r^{-,+, 1}\right) & \mathrm{Id}-\mathrm{op}\left(q^{-}\right) & -\mathrm{op}\left(i^{-, 0}\right) & -\mathrm{op}\left(i^{-, 1}\right) & -2 t_{-} \\
\mathrm{Id} & i \lambda \mathrm{op}\left(z^{+}\right) & 0 & 0 & 0 & 0 & 0 \\
0 & 0 & 0 & \mathrm{Id} & i \lambda \mathrm{op}\left(z^{-}\right) & 0 & 0
\end{array}\right)
\end{aligned}
$$


where the definition of $\operatorname{op}\left(z^{ \pm}\right)$is straightforward owing to (111). The principal symbol of $b$ is given by the leading order term of each symbol, taking into account the behavior in $\lambda$. We obtain:

$$
b_{0}=\left(\begin{array}{ccccccc}
1 & -i \lambda s_{2,0,0}^{+} & -q_{1,0,0}^{+} & -s^{+} & -\lambda r_{1,0,0}^{+,-} & i r_{2,0,0}^{+,-} & 0 \\
-s^{-} & -\lambda r_{1,0,0}^{-,+} & i r_{2,0,0}^{-},+ & 1 & -i \lambda s_{2,0,0}^{-} & -q_{1,0,0}^{-} & 0 \\
1 & i \tau \lambda z^{+}(0) & 0 & 0 & 0 & 0 & 0 \\
0 & 0 & 0 & 1 & i \tau \lambda z^{-}(0) & 0 & 0
\end{array}\right)
$$

We notice that, in this symbol, we mixed some terms with $\lambda$ and other terms without $\lambda$, because, when we use the two last lines of the equality into the two first lines, the terms 1 and $-s^{+}$transform into $-i \tau \lambda z^{+}(0)$ and into $i \tau \lambda z^{-}(0) s^{+}$.

We also notice that it is equivalent to replace the two terms $t^{+}$and $t^{-}$by 0 , because in the final system, all terms will be of order $\lambda$ except for these two terms.

Proposition 4.8. - The system (94) imply the equality $B X=L$, where $B$ is the matricial operator of symbol op $(b)$ given in (112).

The operators op $\left(q^{+}\right), \operatorname{op}\left(q^{-}\right), \operatorname{op}\left(i^{+, 0}\right)$ op $\left(i^{+, 1}\right), \operatorname{op}\left(s^{+,-}\right), \operatorname{op}\left(r^{+,-, 0}\right), \operatorname{op}\left(r^{+,-, 1}\right)$, $\mathrm{op}\left(s^{-,+}\right), \operatorname{op}\left(r^{-,+, 0}\right), \mathrm{op}\left(r^{-,+, 1}\right), \mathrm{op}\left(i^{-, 0}\right), \mathrm{op}\left(i^{-, 1}\right)$ written in this system admit an asymptotic expansion.

The expression of the principal symbol is given in (118).

We remark that the second, third and last column of this system can be associated with an operator acting on the trace $\left.u\right|_{\partial \mathcal{O}_{+}}$and the fifth and sixth columns are the representation of an operator acting on $\left.u\right|_{\partial \mathcal{O}_{-}}$.

Corollary 4.3. - For the straight wedge, the total symbols obtained are exactly the symbols with $, 0,0$. The matrix (113) is the matrix of the system associated with the straight wedge.

4.5.1. Calculus of $u_{0}$ - - We compute now the distribution $u_{0}(x, y, t)$ introduced in (19). We consider its partial Fourier transform in time $\widehat{u}_{0}(x, y, \tau)$, and we have:

$$
\begin{aligned}
\widehat{u}_{0}(x, y, \tau)=\frac{\sigma_{+}(0,0, \tau)-\sigma_{-}(0,0, \tau)}{4 \pi^{2}} \\
\cdot \int_{\mathbb{R}^{2}} \mathrm{~d} \xi \mathrm{d} \eta \int_{0}^{+\infty} \mathrm{d} x^{\prime} \frac{e^{i\left(x-x^{\prime}\right) \cdot \xi+i\left(y-a\left(x^{\prime}\right)\right) \cdot \eta}}{k^{2}-\xi^{2}-\eta^{2}} \theta\left(x^{\prime} / R\right)\left(\eta-a^{\prime}\left(x^{\prime}\right) \xi\right) .
\end{aligned}
$$

We notice that the calculus of this function is similar to the calculus done for $R_{+}^{+, k}$ and of $R_{+}^{-, k}$. We use exactly the same residue theorem as before in both contributions (the one in $y>a(x), y>0$ and the one in $y<b(x), y<0$ for $x>0$ ), as well as in the contribution in $x<0$. The symbol obtained each time using the residue theorem is equivalent to a constant when $\xi_{1}^{+}$or $\xi$ goes to $\pm \infty$, hence the symbol obtained does 
not belong to $L^{2}$. We note that, integrating by parts in $x^{\prime}$ the expression

$$
\int_{\mathbb{R}} \mathrm{d} \xi \int_{0}^{+\infty} s_{0}\left(x, x^{\prime}, \xi, k\right) \theta\left(x^{\prime} / R\right) e^{i \phi\left(x, y, x^{\prime}, \xi, k\right)} \mathrm{d} x^{\prime}
$$

we get, with the equality

$$
s_{0}\left(x, x^{\prime}, \xi, k\right)=\partial_{x^{\prime}} \phi\left(x, y, x^{\prime}, \xi, k\right) s_{-1}\left(x, y, x^{\prime}, \xi, k\right)+s_{-1}^{1}\left(x, y, x^{\prime}, \xi, k\right),
$$

where both symbols behave as $1 /(1+|\xi|)$ when $\xi$ goes to $\pm \infty$

$$
\begin{gathered}
\int_{\mathbb{R}} \mathrm{d} \xi \int_{0}^{+\infty} s_{0}\left(x, x^{\prime}, \xi, k\right) \theta\left(x^{\prime} / R\right) e^{i \phi\left(x, y, x^{\prime}, \xi, k\right)} \mathrm{d} x^{\prime}= \\
\int_{\mathbb{R}} \mathrm{d} \xi \int_{0}^{+\infty} s_{-1}^{1}\left(x, y, x^{\prime}, \xi, k\right) \theta\left(x^{\prime} / R\right) e^{i \phi\left(x, y, x^{\prime}, \xi, k\right)} \mathrm{d} x^{\prime} \\
+i \int_{\mathbb{R}} \mathrm{d} \xi \int_{0}^{+\infty} \partial_{x^{\prime}}\left(s_{-1} \theta\left(x^{\prime} / R\right)\right) e^{i \phi\left(x, x^{\prime}, \xi, k\right)} \mathrm{d} x^{\prime}+i \int_{\mathbb{R}} \mathrm{d} \xi s_{1}(x, y, 0, \xi, k) e^{i \phi(x, y, 0, \xi, k)} .
\end{gathered}
$$

The last contribution is the less regular one, it is in $L_{\text {loc }}^{2}$. The two other contributions are in $H_{\mathrm{loc}}^{1}$. Hence we identified the leading order term of $u_{0}$.

\subsection{System of equations and microlocalisation of the sources}

For our purpose in this section, we introduce the new unknowns that will be used for the microlocal system. It is necessary to use these unknowns, so that we may be able to remove the behavior of the classical reflected wave and the results of Section 2 of outgoing waves. Recall that we defined $u_{r, \pm}$ in the introduction by (9) and (10) and $u_{ \pm}=u_{i}-u_{r, \pm}$.

Let us denote by $\widetilde{f}_{ \pm}=v_{ \pm}-1_{x>0} \partial_{ \pm} u_{ \pm}$and by $\widetilde{g}_{ \pm}=\left.\left(u-u_{ \pm}\right)\right|_{\partial \mathcal{O}_{ \pm}}$. Note that $\widetilde{g}_{+}\left(0_{+}, t\right) \neq \widetilde{g}_{-}\left(0_{+}, t\right)$. Note also that $\left.u_{ \pm}\right|_{\widetilde{\Delta}_{ \pm}} \in H^{1}\left(\mathbb{R}^{2}\right)$, hence the traces $\widetilde{g}_{+}$and $\widetilde{g}_{-}$ are both in $H^{1}\left(\mathbb{R}_{+}^{*} \times \mathbb{R}\right)$. We also get $\widetilde{f}_{ \pm} \in L^{2}\left(\mathbb{R}_{+} \times \mathbb{R}\right)$.

We introduce $f_{ \pm}=\phi_{1} \widetilde{f}_{ \pm}, g_{ \pm}=\phi_{1} \widetilde{g}_{ \pm}$. We write the new unknowns for the microlocalized system

$$
\begin{aligned}
& F_{ \pm}=\left(T_{0} \otimes T_{1}\right)\left(f_{ \pm}\right) \\
& G_{ \pm}=\left(T_{0} \otimes T_{1}\right)\left(g_{ \pm}\right) \\
& \widetilde{G}_{ \pm}=\left(T_{0} \otimes T_{1}\right)\left(\phi_{1}\left(1_{\Delta_{ \pm}} \partial_{x}\left(\left.u\right|_{\partial \mathcal{O}}\right)-\partial_{\tan }\left(u_{ \pm}\right) 1_{\Delta_{ \pm}}\right)\right)
\end{aligned}
$$

(which means, for the last waves, that we took the derivative of a function in $H^{1}(V)$, $V$ being regular or not regular, which is in $L^{2}(V)$, and that we took afterwards the restriction to a part of $V$ ).

It is clear that $\phi_{1} v_{ \pm}=\phi_{1} \widetilde{f}_{ \pm}+\phi_{1} 1_{x>0} \partial_{ \pm} u_{ \pm}$and that $\left.\phi_{1} u\right|_{\partial \mathcal{O}}=\left[\phi_{1} \widetilde{g}_{ \pm}+\phi_{1} u_{ \pm}\right]$, where the bracket [ ] means that the corresponding distribution is the superposition of the + part on $\Delta_{+}$and of the - part on $\Delta_{-}$. 
The system (94) writes, using the new unknowns and the representation of the operators

$$
\left\{\begin{array}{l}
f_{+}-1_{x \geqslant 0} Q^{+}\left(f_{+}\right)-S^{+,-}\left(f_{-}\right)-2 T^{+} u(0,0, t) \\
-I^{+, 0}\left(g_{+}\right)-I^{+, 1}\left(\widetilde{g}_{+}\right)-R^{+,-, 0}\left(g_{-}\right)-R^{+,-1}\left(\widetilde{g}_{-}\right) \\
-S^{-,+}\left(f_{+}\right)+f_{-}-1_{x \geqslant 0} Q^{-}\left(f_{-}\right)-2 T^{-} u(0,0, t) \\
-R^{-,+, 0}\left(g_{+}\right)-R^{-,+, 1}\left(\widetilde{g}_{+}\right)-I^{-, 0}\left(g_{-}\right)-I^{-, 1}\left(\widetilde{g}_{-}\right) \\
f_{+}-\Upsilon\left(z^{+}(x) g_{+}\right)=-\left.z^{+}(x) \psi u\right|_{\partial \mathcal{O}_{+}}\left(1-\phi_{2}\right) \partial_{t} \phi_{1} \\
f_{-}-\Upsilon\left(z^{-}(x) g_{-}\right)=-\left.z^{-}(x) \psi u\right|_{\partial \mathcal{O}_{-}}\left(1-\phi_{2}\right) \partial_{t} \phi_{1} .
\end{array}\right.
$$

The modification is generated by the source terms, and we have

$$
\left\{\begin{array}{rl} 
& 2 \phi_{2} \psi \partial_{+} u_{i}-\psi \phi_{1} \partial_{+} u_{+}+1_{x \geqslant 0} Q^{+}\left(\psi \phi_{1} \partial_{+} u_{+}\right)+S^{+,-}\left(\psi \phi_{1} \partial_{-} u_{-}\right) \\
h_{+}= & +I^{+, 0}\left(\left.\psi \phi_{1} u_{+}\right|_{\partial \mathcal{O}_{+}}\right)+I^{+, 1}\left(\left.\psi \phi_{1} \partial_{\tan } u_{+}\right|_{\partial \mathcal{O}_{+}}\right) \\
& +R^{+,-, 0}\left(\left.\psi \phi_{1} u_{-}\right|_{\partial \mathcal{O}_{-}}\right)+R^{+,-, 1}\left(\left.\psi \phi_{1} \partial_{\tan } u_{-}\right|_{\partial \mathcal{O}_{-}}\right) \\
& 2 \phi_{2} \psi \partial_{-} u_{i}+S^{-,+}\left(\psi \phi_{1} \partial_{+} u_{+}\right)-\psi \phi_{1} \partial_{-} u_{-}-1_{x \geqslant 0} Q^{-}\left(\psi \phi_{2} \partial_{-} u_{-}\right) \\
h_{-}= & +R^{-,+, 0}\left(\left.\psi \phi_{1} u_{+}\right|_{\partial \mathcal{O}_{+}}\right)+R^{-,+, 1}\left(\left.\psi \phi_{1} \partial_{\tan } u_{+}\right|_{\partial \mathcal{O}_{+}}\right) \\
& +I^{-, 0}\left(\psi \phi_{1} u_{-} \mid \partial_{\mathcal{O}_{-}}\right)+I^{-, 1}\left(\psi \phi_{1} \partial_{\tan } u_{-} \mid \partial \mathcal{O}_{-}\right)
\end{array} .\right.
$$

The system of equations (93) becomes (as (3.109)):

(114)

$$
\left\{\begin{array}{l}
f_{+}-1_{x \geqslant 0} Q^{+}\left(f_{+}\right)-S^{+,-}\left(f_{-}\right)-2 T^{+}\left[g_{+}(0, t)\right] \\
-I^{+, 0}\left(g_{+}\right)-I^{+, 1}\left(\widetilde{g}_{+}\right)-R^{+,-, 0}\left(g_{-}\right)-R^{+,-, 1}\left(\widetilde{g}_{-}\right)
\end{array}=h_{+}+2 T^{+}\left[u_{+}(0,0, t)\right]+\widetilde{r}_{+}\right.
$$

with the relations:

$$
\begin{aligned}
& \widetilde{r}_{+}=\left(1-g_{2}\right) 2 \phi_{2} \psi \partial_{+} u_{i}-\left(1-g_{2}\right)\left[1_{x \geqslant 0} Q^{+}\left(g_{+}\right)+S^{+,-}\left(g_{-}\right)+R^{+}(g)\right] \\
& \widetilde{r}_{-}=-\left(1-g_{2}\right)\left[S^{-,+}\left(g_{+}\right)+1_{x \geqslant 0} Q^{-}\left(g_{-}\right)+R^{-}(g)\right] .
\end{aligned}
$$

Note finally that the compatibility condition between the traces $g_{+}$and $g_{-}$is a consequence of $u(x, a(x), t)-u(x, b(x), t)$ goes to 0 when $x$ goes to 0 and writes

$$
g_{+}(0, t)-g_{-}(0, t)=u_{-}(0,0, t)-u_{+}(0,0, t)
$$

The asymptotic expansion of the right hand of the system (114) is given, as in the section 4.5 of $[\mathbf{2 0}]$, by the expansion of $\left(T_{0} \times T_{1}\right)\left(h_{ \pm}\right)$. There exists six symbols, of 
the same order as $\sigma_{i}$, such that

$$
\begin{aligned}
\partial_{+} u_{+}(x, t) & =\frac{1}{2 \pi} \int_{\mathbb{R}} i k p_{+}(x, k) e^{i k\left(t-\theta_{a}(x)\right)} \mathrm{d} k \\
u_{+}(x, a(x), t) & =\frac{1}{2 \pi} \int_{\mathbb{R}} q_{+}(x, k) e^{i k\left(t-\theta_{a}(x)\right)} \mathrm{d} k \\
\partial_{x}\left[u_{+}(x, a(x), t)\right] & =\frac{1}{2 \pi} \int_{\mathbb{R}} i k \widetilde{q}_{+}(x, k) e^{i k\left(t-\theta_{a}(x)\right)} \mathrm{d} k \\
\partial_{-} u_{-}(x, t) & =\frac{1}{2 \pi} \int_{\mathbb{R}} i k p_{-}(x, k) e^{i k\left(t-\theta_{b}(x)\right)} \mathrm{d} k \\
u_{-}(x, b(x), t) & =\frac{1}{2 \pi} \int_{\mathbb{R}} q_{-}(x, k) e^{i k\left(t-\theta_{b}(x)\right)} \mathrm{d} k \\
\partial_{x}\left[u_{-}(x, b(x), t)\right] & =\frac{1}{2 \pi} \int_{\mathbb{R}} i k \widetilde{q}_{-}(x, k) e^{i k\left(t-\theta_{b}(x)\right)} \mathrm{d} k .
\end{aligned}
$$

We have the relations

$$
\left\{\begin{array}{l}
p_{+}(x, k)=z^{+}(x)\left[\sigma_{z}^{+}-\sigma_{i}\right](x, a(x), k) \\
p_{-}(x, k)=z^{-}(x)\left[\sigma_{z}^{-}-\sigma_{i}\right](x, b(x), k) \\
q_{+}(x, k)=\left[\sigma_{z}^{+}-\sigma_{i}\right](x, a(x), k) \\
q_{-}(x, k)=\left[\sigma_{z}^{-}-\sigma_{i}\right](x, b(x), k) \\
\widetilde{q}_{+}(x, k)=-\partial_{x} \theta_{a}(x) q_{+}(x, k)+\frac{1}{i k} \partial_{x} q_{+}(x, k) \\
\widetilde{q}_{-}(x, k)=-\partial_{x} \theta_{b}(x) q_{-}(x, k)+\frac{1}{i k} \partial_{x} q_{-}(x, k)
\end{array}\right.
$$

We write

$$
\begin{aligned}
& p_{+}(x, k)=p_{+}(x) \sigma_{i}(x, a(x), k)\left(1+O\left(k^{-1}\right)\right) \\
& q_{+}(x, k)=q_{+}(x) \sigma_{i}(x, a(x), k)\left(1+O\left(k^{-1}\right)\right) \\
& \widetilde{q}_{+}(x, k)=\widetilde{q}_{+}(x) \sigma_{i}(x, a(x), k)\left(1+O\left(k^{-1}\right)\right) \\
& p_{-}(x, k)=p_{-}(x) \sigma_{i}(x, b(x), k)\left(1+O\left(k^{-1}\right)\right) \\
& q_{-}(x, k)=q_{-}(x) \sigma_{i}(x, b(x), k)\left(1+O\left(k^{-1}\right)\right) \\
& \widetilde{q}_{-}(x, k)=\widetilde{q}_{-}(x) \sigma_{i}(x, b(x), k)\left(1+O\left(k^{-1}\right)\right),
\end{aligned}
$$

where

$$
\left\{\begin{array}{l}
p_{ \pm}(x)=\frac{2 z^{ \pm}(x) \partial_{ \pm} \theta_{i}(x)}{z^{ \pm}(x)-\partial_{ \pm} \theta_{i}(x)} \\
q_{ \pm}(x)=\frac{2 \partial_{ \pm} \theta_{i}(x)}{z^{ \pm}-\partial_{ \pm} \theta_{i}(x)} \\
\widetilde{q}_{+}(x)=-q_{+}(x) \partial_{x} \theta_{a}(x), \widetilde{q}_{-}(x)=-q_{-}(x) \partial_{x} \theta_{b}(x) .
\end{array}\right.
$$

Note that

$$
\widetilde{q}_{ \pm}(0)= \pm \frac{2 \sin \gamma_{ \pm}}{\sin \theta_{ \pm} \pm \sin \gamma_{ \pm}}, q_{ \pm}(0)=-\widetilde{q}_{ \pm}(0), p_{ \pm}(0)=-\sin \theta_{ \pm} \widetilde{q}_{ \pm}(0) .
$$


We introduce $T_{0}\left(\left.\psi \phi_{1} u_{+}\right|_{\partial \mathcal{O}_{+}}\right)(x, \tau)=T_{0}\left(\left.\psi(x) \phi_{1}(\cdot) 1_{x \geqslant 0} u_{+}\right|_{\partial \mathcal{O}_{+}}(x, \cdot)\right)(\tau)$ for example. Making use of Proposition 4.1 we get

$$
\begin{aligned}
\left(T_{0} \otimes T_{1}\right)\left(I^{+, 0}\left(\left.\psi \phi_{1} u_{+}\right|_{\partial \mathcal{O}_{+}}\right)\right)(w, z, \lambda) & \simeq \frac{\lambda}{2 \pi} \int_{t_{-}}^{t_{+}} \mathrm{d} t \int_{\tau_{-}}^{\tau_{+}} \mathrm{d} \tau e^{i \lambda \tau(w-t)-\lambda \tau^{2}} \\
& T_{1}\left(I^{+, 0, \lambda \tau}\left(T_{0}\left(\left.\psi \phi_{1} u_{+}\right|_{\partial \mathcal{O}_{+}}\right)(\cdot, \lambda \tau)\right)\right)(t-i \tau, \lambda)
\end{aligned}
$$

Making use of the definition of $T_{0}$, we obtain

$$
\begin{aligned}
& T_{1}\left(I^{+, 0, \lambda \tau}\left(T_{0}\left(\left.\psi \phi_{1} u_{+}\right|_{\partial \mathcal{O}_{+}}\right)(\cdot, \lambda \tau)\right)\right)(t-i \tau, \lambda)= \\
& \left(\frac{\lambda}{2 \pi}\right)^{1 / 2} \int_{\Im k=-a} e^{i k \lambda(t-i \tau)-\lambda k^{2} / 2} T_{1}\left[I^{+, 0, \lambda \tau}\left[\mathcal{F}\left(\left.\psi \phi_{1}(\cdot) 1_{x \geqslant 0} u_{+}(x, \cdot)\right|_{\partial \mathcal{O}_{+}}\right)\right](x, \lambda k)\right] \mathrm{d} k .
\end{aligned}
$$

Hence, for the calculus of this terms, is left the calculus of

$$
T_{1}\left[I^{+, 0, \lambda \tau}\left(\mathcal{F}\left(\psi \phi_{1} 1_{x \geqslant 0} u_{+} \mid \partial \mathcal{O}_{+}\right)\right)(x, \lambda k)\right]
$$

which is a function of $\tau, k, z, \lambda$.

We get rid of the localization $\phi_{1}$ because, owing to the finite speed of propagation, $\psi \phi_{1} 1_{x \geqslant 0} u_{+}=\psi 1_{x \geqslant 0} u_{+}$. Hence we compute

$$
T_{1}\left[I^{+, 0, \lambda \tau}\left(\psi(\cdot) q_{+}(\cdot, \lambda k) e^{-i \lambda k \theta_{a}(\cdot)}\right)\right](z, \lambda) .
$$

The same method applies to all the terms of the source term $h_{+}$. We write

$$
\left(T_{0} \otimes T_{1}\right)\left(h_{+}\right)(w, z, \lambda)=\left(T_{0} \otimes T_{1}\right)\left(2 \psi \partial_{+} u_{i}\right)+l_{2}^{+}(w, z, \lambda)+l_{3}^{+}(w, z, \lambda)+o_{+}(w, z, \lambda)
$$

where

$$
\begin{aligned}
& l_{2}^{+}(w, z, \lambda)=\left(T_{0} \otimes T_{1}\right)\left[1_{x \geqslant 0} Q^{+}\left(\psi \phi_{1} \partial_{+} u_{+}\right)-\psi \phi_{1} \partial_{+} u_{+}\right] \\
& +\left(T_{0} \otimes T_{1}\right)\left[I^{+, 0}\left(\left.\psi \phi_{1} u_{+}\right|_{\partial \mathcal{O}_{+}}\right)\right]+\left(T_{0} \otimes T_{1}\right)\left[I^{+, 1}\left(\psi \phi_{1} \partial_{x}\left(u_{+} \mid \partial \mathcal{O}_{+}\right)\right)\right], \\
& l_{3}^{+}(w, z, \lambda)=\left(T_{0} \otimes T_{1}\right)\left[1_{x \geqslant 0} S^{+,-}\left(\psi \phi_{1} \partial_{-} u_{-}\right)\right] \\
& +\left(T_{0} \otimes T_{1}\right)\left[R^{+,-, 0}\left(\psi \phi_{1} u_{-} \mid \partial_{\mathcal{O}_{-}}\right)\right]+\left(T_{0} \otimes T_{1}\right)\left[R^{+,-, 1}\left(\psi \phi_{1} \partial_{x}\left(\left.u_{-}\right|_{\partial \mathcal{O}_{-}}\right)\right)\right],
\end{aligned}
$$

and the term $\left(T_{0} \otimes T_{1}\right)\left(2 \psi \partial_{+} u_{i}\right)$ has been computed in [20].

We write each term by using Proposition 4.1, hence

$$
\begin{aligned}
& l_{2}^{+}(w, z, \lambda) \simeq \frac{\lambda}{2 \pi} \int_{t_{-}}^{t_{+}} \mathrm{d} t \int_{\tau_{-}}^{\tau_{+}} \mathrm{d} \tau e^{i \lambda \tau(w-t)-\lambda \tau^{2}} \Lambda_{2}^{+}(t, \tau, z, \lambda), \\
& l_{3}^{+}(w, z, \lambda) \simeq \frac{\lambda}{2 \pi} \int_{t_{-}}^{t_{+}} \mathrm{d} t \int_{\tau_{-}}^{\tau_{+}} \mathrm{d} \tau e^{i \lambda \tau(w-t)-\lambda \tau^{2}} \Lambda_{3}^{+}(t, \tau, z, \lambda)
\end{aligned}
$$


(with the obvious definition of $\Lambda_{2}^{+}$and $\Lambda_{3}^{+}$). Then there exists two symbols $\lambda_{2}^{+}(t, \tau, k, z, \lambda)$ and $\lambda_{3}^{+}(t, \tau, k, z, \lambda)$ such that

$$
\begin{aligned}
\Lambda_{2}^{+}(t, \tau, z, \lambda) & =\left(\frac{\lambda}{2 \pi}\right)^{1 / 2} \int_{\Im k=-a} e^{i \lambda k(t-i \tau)-\lambda k^{2} / 2} \lambda_{2}^{+}(t, \tau, k, z, \lambda) \mathrm{d} k \\
& \simeq\left(\frac{\lambda}{2 \pi}\right)^{1 / 2} \int_{1-4 \kappa}^{1+4 \kappa} e^{i \lambda k(t-i \tau)-\lambda k^{2} / 2} \lambda_{2}^{+}(t, \tau, k, z, \lambda) \mathrm{d} k \\
\Lambda_{3}^{+}(t, \tau, z, \lambda) & =\left(\frac{\lambda}{2 \pi}\right)^{1 / 2} \int_{\Im k=-a} e^{i \lambda k(t-i \tau)-\lambda k^{2} / 2} \lambda_{3}^{+}(t, \tau, k, z, \lambda) \mathrm{d} k \\
& \simeq\left(\frac{\lambda}{2 \pi}\right)^{1 / 2} \int_{1-4 \kappa}^{1+4 \kappa} e^{i \lambda k(t-i \tau)-\lambda k^{2} / 2} \lambda_{3}^{+}(t, \tau, k, z, \lambda) \mathrm{d} k .
\end{aligned}
$$

We concentrate on the calculus of $\lambda_{2}^{+}$and of $\lambda_{3}^{+}$. The pseudodifferential calculus of [20] p 382-387 is still valid. The analogous of Proposition 4.3 of [20] holds to compute the symbol of $h_{+}$. Note that the total phase function is

$$
\phi(w, t, \tau, k)=\tau(w-t)+i \tau^{2}+k(t-i \tau)+i \frac{k^{2}}{2}
$$

which critical point is $(t, \tau, k)$ is $(0, i w, i w)$, of associated critical value $\phi(w, 0, i w, i w)$ $=i w^{2} / 2$. We then introduce $\phi_{1}(w)=-\Im\left(i w^{2} / 2\right)=\frac{1}{2}\left((\Im w)^{2}-(\Re w)^{2}\right)$. We get $l_{2}^{+}(w, z, \lambda) \in H_{\phi_{1}}\left(W_{\kappa}, H_{1}^{+}\right)$, as well as $l_{3}^{+}(w, z, \lambda)$. The sign $\simeq$ used here means that the difference can be neglected in $H_{\phi_{0}}\left(W_{\kappa}, H_{U}\right)$. We have

Proposition 4.9. - There exists $t_{0}^{1}>0$ such that, for $\left.\left.t_{0} \in\right] 0, t_{0}^{1}\right]$, there exists $\kappa, a^{\prime}, l, C_{0}^{1}, C_{0}^{2}, \rho$ such that, for $C^{1} \geqslant C_{0}^{1}, C^{2} \geqslant C_{0}^{2}$, we have

$$
\begin{array}{r}
\lambda_{2}^{+}(t, \tau, k, z, \lambda)=\frac{1}{2 \pi} \int_{C(+)} \mu_{2}^{+}\left(t, \tau, k, \xi_{1}^{+}, z, \lambda\right) \mathrm{d} \xi_{1}^{+} \\
\lambda_{3}^{+}(t, \tau, k, z, \lambda)=-\frac{1}{2 \pi} \int_{C\left(l, \tan \gamma_{-}\right)} \mu_{3}^{+}(t, \tau, k, \xi, z, \lambda) \mathrm{d} \xi
\end{array}
$$

where $\mu_{2}^{+}$and $\mu_{3}^{+}$admit the following expansions:

$$
\begin{aligned}
& \mu_{2}^{+} \simeq \lambda J_{\rho}^{-1} \sum_{n \leqslant \lambda / C_{1}, m \leqslant \lambda / C_{2}}\left(\frac{1}{\lambda} \partial_{z}\right)^{n}\left[\frac{1}{z+i \xi_{1}^{+}} \frac{\lambda^{-m-1}}{\left(i\left(\xi_{1}^{+}+k\right)\right)^{m+1}}\right. \\
&\left.\left.\frac{1}{n !} \partial_{x}^{n} \partial_{x^{\prime}}^{m}\left(\widetilde{\mu}_{2}^{+}\left(x, x^{\prime}, \xi_{1}^{+}, \tau, k, \lambda\right) e^{-i \lambda k\left(\theta_{a}\left(x^{\prime}\right)-x^{\prime}\right)}\right)\right|_{x=x^{\prime}=0}\right] \\
& \mu_{3}^{+} \simeq \lambda J_{\rho}^{-1} \sum_{n \leqslant \lambda / C_{1}, m \leqslant \lambda / C_{2}}\left(\frac{1}{\lambda} \partial_{z}\right)^{n}\left[\frac{1}{z+i\left(\xi+\tan \gamma_{+} \xi_{0}\right)} \frac{\lambda^{-m-1}}{\left(i\left(\xi+\tan \gamma_{-} \xi_{0}+k\right)\right)^{m+1}}\right. \\
&\left.\frac{1}{n !} \partial_{x}^{n} \partial_{x^{\prime}}^{m}\left(e^{i \lambda \phi_{+}+i \lambda \phi_{-}} \widetilde{\mu}_{3}^{+}\left(x, x^{\prime}, \xi, \tau, k, \lambda\right) e^{-i \lambda k\left(\theta_{b}\left(x^{\prime}\right)-x^{\prime}\right)}\right)\right]
\end{aligned}
$$


with the symbols

$$
\begin{aligned}
& \widetilde{\mu}_{2}^{+}\left(x, x^{\prime}, \xi_{1}^{+}, \tau, k, \lambda\right)= \\
& {\left[\left(\frac{1+\left(a^{\prime}\left(x^{\prime}\right)\right)^{2}}{1+\left(a^{\prime}(x)\right)^{2}}\right)^{1 / 2}\left[1+\sigma^{+}\left(x, x^{\prime}, \xi_{1}^{+}, \tau\right)\right]-1\right] i k p_{+}\left(x^{\prime}, \lambda k\right)-i k p_{+}\left(x^{\prime}, \lambda k\right)} \\
& +i\left[L_{3}^{+}\left(x, x^{\prime}\right) \frac{\tau^{2}}{\xi_{+}}+\frac{1}{i \lambda} \partial_{x^{\prime}}\left(L_{2}^{+}\left(x, x^{\prime}\right)+L_{1}^{+}\left(x, x^{\prime}\right) \frac{\xi_{1}^{+}}{\xi_{+}}\right)\right] q_{+}\left(x^{\prime}, \lambda k\right) \\
& +\left[L_{2}^{+}\left(x, x^{\prime}\right)+L_{1}^{+}\left(x, x^{\prime}\right) \frac{\xi_{1}^{+}}{\xi_{+}}\right] i k \widetilde{q}_{+}\left(x^{\prime}, \lambda k\right)
\end{aligned}
$$

and

$$
\begin{aligned}
& \widetilde{\mu}_{3}^{+}\left(x, x^{\prime}, \xi, \tau, k, \lambda\right)= \\
&\left(\frac{1+\left(b^{\prime}\left(x^{\prime}\right)\right)^{2}}{1+\left(a^{\prime}(x)\right)^{2}}\right)^{1 / 2}\left(1-a^{\prime}(x) \xi / \xi_{0}\right) i k p_{-}\left(x^{\prime}, \lambda k\right)+i \frac{k^{2}}{\xi_{0}} \frac{1+a^{\prime}(x) b^{\prime}\left(x^{\prime}\right)}{\left(1+\left(a^{\prime}(x)\right)^{2}\right)^{1 / 2}} q_{-}\left(x^{\prime}, \lambda k\right) \\
&-\frac{1}{\left(1+\left(a^{\prime}(x)\right)^{2}\right)^{1 / 2}}\left(a^{\prime}(x)+\xi / \xi_{0}\right) i k \widetilde{q}_{-}\left(x^{\prime}, \lambda k\right) .
\end{aligned}
$$

The proof of this Proposition is a straightforward transcription of the proof of Proposition 4.3 of $[\mathbf{2 0}]$ and we do not reproduce it here.

Note that we have

$$
\begin{aligned}
\frac{\tilde{\mu}_{2}^{+}\left(0,0, \xi_{1}^{+}, \tau, k\right)}{q_{+}(0)} & =-i k z^{+}(0)+\frac{i}{\cos \gamma_{+}} \frac{\tau^{2}}{\xi_{+}}+\cos \gamma_{+} i k \frac{\xi_{1}^{+}}{\xi_{+}} \\
\frac{\widetilde{\mu}_{3}^{+}(0,0, \xi, \tau, k, \lambda)}{q_{-}(0)} & =\frac{i k z^{-}(0)}{\cos \gamma_{-}}\left(\cos \gamma_{+}-\sin \gamma_{+} \frac{\xi}{\xi_{0}}\right)+i \frac{\tau^{2}}{\xi_{0}} \frac{\cos \gamma}{\cos \gamma_{-}}+i k\left(\sin \gamma_{+}+\cos \gamma_{+} \frac{\xi}{\xi_{0}}\right) .
\end{aligned}
$$

We check that, when $k=\tau$ we have

$$
\begin{gathered}
\frac{\tilde{\mu}_{2}^{+}\left(0,0, \xi_{1}^{+}, k, k\right)}{q_{+}(0)}=-i k z^{+}(0)+i \cos \gamma_{+} \xi_{+}+\cos \gamma_{+} i\left(k+\xi_{1}^{+}\right) \frac{\xi_{1}^{+}}{\xi_{+}}, \\
\frac{\widetilde{\mu}_{3}^{+}(0,0, \xi, k, k, \lambda)}{q_{-}(0)}=i \frac{k z^{-}(0)+\cos \gamma_{-} \xi_{0}-\sin \gamma_{-} \xi}{\cos \gamma_{-}}\left(\cos \gamma_{+}-\sin \gamma_{+} \frac{\xi}{\xi_{0}}\right) \\
+i \cos \gamma_{-}\left(\cos \gamma_{+} \frac{\xi}{\xi_{0}}+\sin \gamma_{+}\right)\left(\xi+\tan \gamma_{-} \xi_{0}+k\right) .
\end{gathered}
$$

The symbols associated with the expansion of $h_{-}$are given in a similar way by:

$$
\begin{aligned}
& \widetilde{\mu}_{2}^{-}\left(x, x^{\prime}, \xi, \tau, k\right)=\left(\frac{1+\left(a^{\prime}\left(x^{\prime}\right)\right)^{2}}{1+\left(b^{\prime}(x)\right)^{2}}\right)^{1 / 2}\left(1+b^{\prime}(x) \xi / \xi_{0}\right) i k p_{+}\left(x^{\prime}, \lambda k\right) \\
& \quad+i \frac{\tau^{2}}{\xi_{0}} \frac{1+a^{\prime}\left(x^{\prime}\right) b^{\prime}(x)}{\left(1+\left(b^{\prime}(x)\right)^{2}\right)^{1 / 2}} q_{+}\left(x^{\prime}, \lambda k\right)-\frac{1}{\left(1+\left(b^{\prime}(x)\right)^{2}\right)^{1 / 2}}\left(b^{\prime}(x)-\xi / \xi_{0}\right) i k \widetilde{q}_{+}\left(x^{\prime}, \lambda k\right) .
\end{aligned}
$$




$$
\begin{aligned}
& \widetilde{\mu}_{3}^{-}\left(x, x^{\prime}, \xi_{1}^{-}, \tau, k, \lambda\right)= \\
& {\left[\left(\frac{1+\left(b^{\prime}\left(x^{\prime}\right)\right)^{2}}{1+\left(b^{\prime}(x)\right)^{2}}\right)^{1 / 2}\left[1+\sigma^{-}\left(x, x^{\prime}, \xi_{1}^{-}, \tau\right)\right]-1\right] i k p_{-}\left(x^{\prime}, \lambda k\right)-i k p_{-}\left(x^{\prime}, \lambda k\right)} \\
& +i\left[L_{3}^{-}\left(x, x^{\prime}\right) \frac{\tau^{2}}{\xi_{-}}+\frac{1}{i \lambda} \partial_{x^{\prime}}\left(L_{2}^{-}\left(x, x^{\prime}\right)+L_{1}^{-}\left(x, x^{\prime}\right) \frac{\xi_{1}^{-}}{\xi_{-}}\right)\right] q_{-}\left(x^{\prime}, \lambda k\right) \\
& +\left[L_{2}^{-}\left(x, x^{\prime}\right)+L_{1}^{-}\left(x, x^{\prime}\right) \frac{\xi_{1}^{-}}{\xi_{-}}\right] i k \widetilde{q}_{-}\left(x^{\prime}, \lambda k\right) .
\end{aligned}
$$

We get

$$
\begin{aligned}
& \frac{\tilde{\mu}_{2}^{-}(0,0, \xi, \tau, k)}{q_{+}(0)}=i \frac{k z^{+}(0)}{\cos \gamma_{+}}\left(\cos \gamma_{-}+\sin \gamma_{-} \frac{\xi}{\xi_{0}}\right)+i \frac{\tau^{2}}{\xi_{0}} \frac{\cos \gamma}{\cos \gamma_{+}} \\
& +\left(\sin \gamma_{-}-\cos \gamma_{-} \frac{\xi}{\xi_{0}}\right) i k \\
& \frac{\tilde{\mu}_{3}^{-}\left(0,0, \xi_{1}^{-}, \tau, k\right)}{q_{-}(0)}=-i k z^{-}(0)+\frac{i \tau^{2}}{\cos \gamma_{-} \xi_{-}}+\cos \gamma_{-} \frac{i k \xi_{1}^{-}}{\xi_{-}} \text {. }
\end{aligned}
$$

For $\tau=k$, we have

$$
\begin{aligned}
& \frac{\widetilde{\mu}_{2}^{-}(0,0, \xi, k, k)}{q_{+}(0)}=i \frac{k z^{+}(0)+\cos \gamma_{+} \xi_{0}+\sin \gamma_{+} \xi}{\cos \gamma_{+}}\left(\cos \gamma_{-}+\sin \gamma_{-} \frac{\xi}{\xi_{0}}\right) \\
& +i\left(\frac{\xi}{\xi_{0}} \cos \gamma_{-}-\sin \gamma_{-}\right)\left[\xi-\xi_{0} \tan \gamma_{+}-i k\right] \\
& \frac{\widetilde{\mu}_{3}^{-}\left(0,0, \xi_{1}^{-}, k, k\right)}{q_{-}(0)}=-i k z^{-}(0)+i \cos \gamma_{-} \xi_{-}+i \frac{k \cos \gamma_{-}}{\xi_{-}}\left(k+\xi_{1}^{-}\right) \text {. }
\end{aligned}
$$

The calculus of $l_{2}^{+}(w, z, \lambda)$ and of $l_{3}^{+}(w, z, \lambda)$ is a consequence of successive applications of the stationary phase theorem. From

$$
\begin{aligned}
\Lambda_{2}^{+}(t, \tau, z, \lambda) & \simeq\left(\frac{\lambda}{2 \pi}\right)^{1 / 2} \int_{1-4 \kappa}^{1+4 \kappa} e^{i \lambda k(t-i \tau)-\lambda k^{2} / 2} \lambda_{2}^{+}(t, \tau, k, z, \lambda) \mathrm{d} k \\
& \simeq\left(\frac{\lambda}{2 \pi}\right)^{1 / 2} \int_{1-4 \kappa}^{1+4 \kappa} e^{-\lambda(t-i \tau)^{2} / 2} e^{-\lambda(k-i(t-i \tau))^{2} / 2} \lambda_{2}^{+}(t, \tau, k, z, \lambda) \mathrm{d} k
\end{aligned}
$$

we obtain

$$
\Lambda_{2}^{+}(t, \tau, z, \lambda) \simeq e^{-\lambda t^{2} / 2} e^{\lambda\left(\frac{\tau^{2}}{2}+i t \tau\right)} \sum_{p \leqslant \lambda / C_{1}} \frac{1}{p ! \lambda^{p}} \frac{\partial^{2} p}{\partial k^{2 p}} \lambda_{2}^{+}(t, \tau, \tau+i t, z, \lambda) .
$$

As we have

$$
l_{2}^{+}(w, z, \lambda) \simeq \frac{\lambda}{2 \pi} \int_{t_{-}}^{t_{+}} \mathrm{d} t \int_{\tau_{-}}^{\tau_{+}} \mathrm{d} \tau e^{i \lambda \tau(w-t)-\lambda \tau^{2}+\lambda \frac{\tau^{2}}{2}+i t \tau \lambda-\lambda \frac{t^{2}}{2}} \Lambda_{2}^{+}(t, \tau, z, \lambda),
$$


we obtain

$$
\begin{aligned}
& l_{2}^{+}(w, z, \lambda) \simeq e^{-\lambda w^{2} / 2} \frac{\lambda}{2 \pi} \int_{t_{-}}^{t_{+}} \mathrm{d} t e^{-\lambda t^{2} / 2} \int_{\tau_{-}}^{\tau_{+}} \mathrm{d} \tau e^{-\lambda(\tau-i w)^{2} / 2} \Lambda_{2}^{+}(t, \tau, z, \lambda) \\
& \simeq \sum_{p \leqslant \lambda / C_{1}} \frac{1}{p ! \lambda^{p}} e^{-\lambda w^{2} / 2} \frac{\lambda}{2 \pi} \int_{t_{-}}^{t_{+}} \mathrm{d} t e^{-\lambda t^{2} / 2} \\
& \cdot \int_{\tau_{-}}^{\tau_{+}} \mathrm{d} \tau e^{-\lambda(\tau-i w)^{2} / 2} \frac{\partial^{2 p}}{\partial k^{2 p}} \lambda_{2}^{+}(t, \tau, \tau+i t, z, \lambda) .
\end{aligned}
$$

The usual stationary phase theorem at $\tau=i w$ and at $t=0$ yields

$$
\begin{aligned}
l_{2}^{+}(w, z, \lambda) \simeq \sum_{\substack{p \leqslant \lambda / C_{1} \\
q \leqslant \lambda / C_{2} \\
r \leqslant \lambda / C_{3}}} \frac{1}{p ! q ! r ! \lambda^{p+q+r}} e^{-\lambda w^{2} / 2} \\
\cdot \frac{\partial^{2 q}}{\partial t^{2 q}} \frac{\partial^{2 r}}{\partial \tau^{2 r}}\left[\frac{\partial^{2} p}{\partial k^{2 p}} \lambda_{2}^{+}(t, \tau, \tau+i t, z, \lambda)\right]_{t=0, \tau=i w}
\end{aligned}
$$

We deduce from this asymptotic expansion that the leading order term of $l_{2}^{+}$is given by

$$
\lambda J_{\rho}^{-1} \int_{C(+)} \frac{\mathrm{d} \xi_{1}^{+}}{z+i \xi_{1}^{+}} \frac{1}{i \lambda\left(\xi_{1}^{+}+i w\right)} \widetilde{\mu}_{2}^{+}\left(0,0, \xi_{1}^{+}, i w, i w, \lambda\right) e^{-\lambda w^{2} / 2}
$$

and the leading order term of $l_{3}^{+}$is given by

$$
\begin{aligned}
\lambda \int_{C\left(l, \tan \gamma_{-}\right)} \frac{\mathrm{d} \xi}{z+i\left(\xi+\tan \gamma_{+} \xi_{0}\right)} \frac{1}{i \lambda\left(\xi+\tan \gamma_{-} \xi_{0}\right)+i w} \\
\cdot \widetilde{\mu}_{3}^{+}(0,0, \xi, i w, i w, \lambda) e^{-\lambda w^{2} / 2} .
\end{aligned}
$$

Is left in this calculus the calculus of the term induced by $\partial_{+} u_{i}$. It is a consequence of the expressions (4.69) and (4.70) of [20] that

$$
\begin{gathered}
\left(T_{0} \otimes T_{1}\right)\left(2 \partial_{+} u_{i}\right)(w, z, \lambda) \simeq 2\left(\frac{\lambda}{2 \pi}\right)^{1 / 2} \int_{1-2 \kappa}^{1+2 \kappa} d k e^{i \lambda w k-\lambda k^{2} / 2}\left[\sum_{n \leqslant \lambda / C} \frac{1}{\lambda^{n+1}}\left(\frac{1}{i(k+i z)}\right)^{n+1}\right. \\
\left.\left.\cdot \partial_{x}^{n}\left(e^{-\lambda \frac{x^{2}}{2}-i \lambda k \widetilde{\theta}_{a}(x)}\left[-i k \lambda \partial_{+} \theta_{i}(x) \sigma_{i}(x, a(x), \lambda k)+\partial_{+} \sigma_{i}(x, a(x), k \lambda)\right]\right)\right|_{x=0}\right] .
\end{gathered}
$$

The stationary phase theorem applied to this integral leads to a critical point at $k=$ $i w$, a critical value equal to $e^{-\lambda w^{2} / 2}$, hence the leading order term of $\left(T_{0} \otimes T_{1}\right)\left(2 \partial_{+} u_{i}\right)$ is $\frac{2 \lambda \sin \gamma_{+} w}{w+z} \sigma_{i}(0,0, i \lambda w)$. We deduce from these relations the leading order term of 
the source term $L_{+}$:

$$
\begin{aligned}
& \frac{e^{\lambda w^{2} / 2}}{\lambda} L_{+}^{0}(w, z, \lambda)=\frac{2 w \partial_{+} \sin \gamma_{+}(0)}{i w+z} \sigma_{i}(0,0, i \lambda w) \\
&+\frac{1}{2 \pi} \int_{C(+)} \frac{\mathrm{d} \xi_{1}^{+}}{z+i \xi_{1}^{+}} \frac{1}{i \lambda\left(\xi_{1}^{+}+i w\right)} \widetilde{\mu}_{2}^{+}\left(0,0, \xi_{1}^{+}, i w, i w, \lambda\right) \\
&+\frac{1}{2 \pi} \int_{C\left(l, \tan \gamma_{-}\right)} \frac{1}{z+i\left(\xi+\tan \gamma_{+} \xi_{0}\right)} \frac{1}{i \lambda\left(\xi+\tan \gamma_{-} \xi_{0}+i w\right)} \widetilde{\mu}_{3}^{+}(0,0, \xi, i w, i w, \lambda) .
\end{aligned}
$$

Let us introduce the symbol $S I_{+}(w, z)$ such that

$$
L_{+}^{0}(w, z, \lambda)=\lambda J_{\rho}\left(S I_{+}\right) \sigma_{i}(0,0, i \lambda w) e^{-\lambda w^{2} / 2} .
$$

We have

$$
\begin{aligned}
& \frac{1}{2} S I_{+}(w, z)= \\
& \quad \frac{\sin \gamma_{+} w}{w+z}-\frac{\sin \gamma_{+}}{\sin \theta_{+}+\sin \gamma_{+}}\left[\frac{1}{2 \pi} \int_{C(+)} \frac{\left(w \sin \theta_{+}-i \frac{w^{2}}{\cos \gamma_{+} \xi_{+}}-w \cos \gamma_{+} \frac{\xi_{1}^{+}}{\xi_{+}}\right) \mathrm{d} \xi_{1}^{+}}{i \lambda\left(\xi_{1}^{+}+i w\right)\left(z+i \xi_{1}^{+}\right)}\right] \\
& -\frac{\tan \gamma_{-}}{\sin \theta_{-}-\sin \gamma_{-}} . \\
& {\left[\frac{1}{2 \pi} \int_{C\left(l, \tan \gamma_{-}\right)} \frac{w \sin \theta_{-}\left(\cos \gamma_{+}+\sin \gamma_{+} \frac{\xi}{\xi_{0}}\right)+i \frac{w^{2}}{\xi_{0}} \cos \gamma+w \cos \gamma_{-}\left(\sin \gamma_{+}+\cos \gamma_{+} \frac{\xi}{\xi_{0}}\right)}{i \lambda\left(\xi+\tan \gamma_{-} \xi_{0}+i w\right)\left(z+i\left(\xi+\tan \gamma_{+} \xi_{0}\right)\right)} \mathrm{d} \xi\right] .}
\end{aligned}
$$

It is important to note that we cannot use the relations (116) to simplify the expression of $S I_{+}(w, z)$, because if we apply the residue theorem around $k=-\xi_{1}^{+}$, we get an integral which does not converge when $\left|\xi_{1}^{+}\right| \rightarrow \infty$. It is the same reason that compelled us to write the boundary operator $R_{+}^{+}$using the symbols $\frac{k^{2}}{\xi_{+}}$and $\frac{\xi_{1}^{+}}{\xi_{+}}$instead of $\xi_{+}$.

The expression of $S I_{-}(w, z)$, defined through

$$
L_{-}^{0}(w, z, \lambda)=\lambda J_{\rho}\left(S I_{-}\right)(w, z) \sigma_{i}(0,0, i w \lambda) e^{-\lambda w^{2} / 2},
$$

is given by

$$
\begin{aligned}
& \quad \frac{1}{2} S I_{-}(w, z)= \\
& -\frac{\sin \gamma_{-} w}{z+w}+\frac{\sin \gamma_{-}}{\sin \theta_{-}-\sin \gamma_{-}}\left[\frac{1}{2 \pi} \int_{C(-)} \frac{w \sin \theta_{-}-i \frac{w^{2}}{\cos \gamma_{-}}-w \cos \gamma_{-} \frac{\xi_{1}^{-}}{\xi}}{z+i \xi_{1}^{-}} \frac{\mathrm{d} \xi_{1}^{-}}{i \lambda\left(i w+\xi_{1}^{-}\right)}\right] \\
& \quad-\frac{\tan \gamma_{+}}{\sin \theta_{+}+\sin \gamma_{+}} \cdot \\
& {\left[\frac{1}{2 \pi} \int_{C\left(l, \tan \gamma_{+}\right)} \frac{w \sin \theta_{+}\left(\cos \gamma_{-}+\sin \gamma_{-} \frac{\xi}{\xi_{0}}\right)+i \frac{w^{2}}{\xi_{0}} \cos \gamma+w \cos \gamma_{+}\left(\sin \gamma_{-}-\cos \gamma_{-} \frac{\xi}{\xi_{0}}\right)}{i \lambda\left(\xi-\tan \gamma_{+} \xi_{0}+i w\right)\left(z+i\left(\xi-\tan \gamma_{-} \xi_{0}\right)\right.} \mathrm{d} \xi\right]}
\end{aligned}
$$

These functions will be used when writing the system in the holomorphic domain. For the integral on $C(+)$ or on $C(-)$, we can respectively change $\xi_{1}^{ \pm}$to $\frac{\xi}{\cos \gamma_{ \pm}}$, which 
imply $\xi_{ \pm}=\frac{\xi_{0}}{\cos \gamma_{ \pm}}$. We check that the symbol associated with $\frac{1}{2 \pi} \int_{C(-)}$ changes to

$$
\frac{2 \sin \gamma_{-}}{\sin \theta_{-}-\sin \gamma_{-}} \frac{1}{2 \pi} \int_{C} \frac{w \sin \theta_{-}-i \frac{w^{2}}{\xi_{0}}-w \cos \gamma_{-} \frac{\xi}{\xi_{0}}}{\cos \gamma_{-} z+i \xi} \frac{\cos \gamma_{-} \mathrm{d} \xi}{i \lambda\left(\xi+i w \cos \gamma_{-}\right)}
$$

Note that

$$
\xi_{0}-i \sin \gamma_{-} w=\frac{\cos ^{2} \gamma_{-}(z-w)(z+w)-\left(\xi-i \cos \gamma_{-} z\right)\left(\xi+i \cos \gamma_{-} z\right)}{\xi_{0}+i \sin \gamma_{-} w}
$$

and that

$$
\begin{aligned}
& w \sin \theta_{-}-i \frac{w^{2}}{\xi_{0}}-w \cos \gamma_{-} \frac{\xi}{\xi_{0}}= \\
& w\left(\sin \theta_{-}-\sin \gamma_{-}\right)-\sin \gamma_{-} \frac{w}{\xi_{0}}\left(\xi_{0}-i \sin \gamma_{-} w\right)-\frac{w}{\xi_{0}}\left(\xi+i \cos \gamma_{-} w\right) .
\end{aligned}
$$

Hence

$$
\begin{aligned}
& \frac{\left(w \sin \theta_{-}-i \frac{w^{2}}{\xi_{0}}-w \cos \gamma_{-} \frac{\xi}{\xi_{0}}\right) 2 \sin \gamma_{-} w}{\sin \theta_{-}-\sin \gamma_{-}}= \\
& 2 \sin \gamma_{-} w+\frac{2 \sin \gamma_{-} w}{\sin \theta_{-}-\sin \gamma_{-}}\left[-\sin \gamma_{-} \frac{w}{\xi_{0}}\left(\xi_{0}-i \sin \gamma_{-} w\right)-\frac{w}{\xi_{0}}\left(\xi+i \cos \gamma_{-} w\right)\right],
\end{aligned}
$$

the terms with $\xi+i \cos \gamma_{-} w$ canceling in the integral, hence is left the calculus for the residue at $z \cos \gamma_{-}+i \xi=(z+w) \cos \gamma_{-}+i\left(\xi+i \cos \gamma_{-} w\right)$.

The reflected solution $u_{r, \pm}$ is computed using the boundary condition, hence there is a jump between $u_{+}(0,0, t)$ and $u_{-}(0,0, t)$ when $z^{+}(0) \neq z^{-}(0)$. It is thus necessary to check that the integral on $C(-)$ cancels with the source term $\left(T_{0} \otimes T_{1}\right)\left(2 \partial_{-} u_{i}\right)$, which is the case.

\subsection{Inversion of the principal symbol}

The inversion of the principal symbol of the system (114) and the compatibility condition (115) is done in this paper following the proof of Croisille and Lebeau [16]. We may not use the argument proposed by Gérard and Lebeau (Section 5 of [20]) mainly because the system is not of the form $\mathrm{Id}-A$, which was studied using a partial Neumann series (called a Bremmer coupling series by M. Van de Hoop [22]).

We apply $\left(T_{0} \otimes T_{1}\right)$ to the system (93). We use Proposition 4.5 and Proposition 4.6 for the microlocalisation of the left hand side of the system, and Proposition 4.9 for the microlocalisation of the right hand side. We notice that

$\left(T_{0} \otimes T_{1}\right)\left(h_{+}\right)(w, z, \lambda)=\left(T_{0} \otimes T_{1}\right)\left(2 \psi \partial_{+} u_{i}\right)+l_{2}^{+}(w, z, \lambda)+l_{3}^{+}(w, z, \lambda)+o^{+}(w, z, \lambda)$

$\left(T_{0} \otimes T_{1}\right)\left(h_{-}\right)(w, z, \lambda)=\left(T_{0} \otimes T_{1}\right)\left(2 \psi \partial_{-} u_{i}\right)+l_{2}^{-}(w, z, \lambda)+l_{3}^{-}(w, z, \lambda)+o^{-}(w, z, \lambda)$.

with $l_{2}^{ \pm} \in H_{\phi_{1}}\left(W_{\kappa}, H_{1}^{ \pm}\right), l_{3}^{ \pm} \in H_{\phi_{1}}\left(W_{\kappa}, H_{1}^{ \pm}\right)$. We seek a solution such that $F_{ \pm}, G_{ \pm}, \widetilde{G}_{ \pm} \in H_{\phi_{0}}\left(W_{\kappa}, H_{1}^{ \pm}\right)$. Remember that $s_{ \pm}(w, z, \lambda)=\left(T_{0} \otimes T_{1}\right)\left(2 \psi \partial_{ \pm} u_{i}\right)$ is also known. 
Recall that the spaces $H_{\sigma}, H_{\sigma}^{ \pm}$are defined in (100). If $\rho$ is small enough, we verify that $J_{\rho} F_{ \pm} \in H_{\phi_{0}}\left(W_{\kappa}, H_{2 / 3}^{ \pm}\right), J_{\rho} G_{ \pm} \in H_{\phi_{0}}\left(W_{\kappa}, H_{2 / 3}^{ \pm}\right), J_{\rho} \widetilde{G}_{ \pm} \in H_{\phi_{0}}\left(W_{\kappa}, H_{2 / 3}^{ \pm}\right)$and that

$$
J_{\rho}\left(l_{2}^{ \pm}+l_{3}^{ \pm}+s_{ \pm}+o^{ \pm}(w, z, \lambda)\right) \in H_{\phi_{1}}\left(W_{\kappa}, H_{2 / 3}^{ \pm}\right) .
$$

Moreover, the operators op $\left(q^{ \pm}\right), \operatorname{op}\left(i^{ \pm, 0}\right), \operatorname{op}\left(i^{ \pm, 1}\right)$ send $H_{\sigma}^{ \pm}$into $H_{\sigma}^{ \pm}$(for $\sigma=1 / 3$ or for $\sigma=2 / 3$ for example $\left.{ }^{(2)}\right)$. The operators $\operatorname{op}\left(s^{ \pm, \mp}\right), \operatorname{op}\left(r^{ \pm, \mp, 0}\right)$ and $\operatorname{op}\left(r^{ \pm, \mp, 0}\right)$ are regularizing; they send $H_{\frac{1}{3}}^{\mp}$ onto $H_{2 / 3}^{ \pm}$. Let us introduce the operators $M_{ \pm}$such that

$$
M_{ \pm} f(z)=f\left(\cos \gamma_{ \pm} z\right)
$$

The operators $M_{ \pm}$send exactly $H_{\sigma}$ to $H_{\sigma}^{ \pm}$. We consider the operators, acting from $H_{\sigma}$ to $H_{\sigma^{\prime}}$, through the relations

$$
\begin{aligned}
I^{0}(k)(g)(z) & =\frac{1}{2 \pi} \int_{\mathcal{C}} \frac{k^{2}}{\xi_{0}} \frac{\mathrm{d} \xi}{z+i \xi} g(-i \xi) \\
I^{1}(k)(g)(z) & =\frac{1}{2 \pi} \int_{\mathcal{C}} \frac{\xi}{\xi_{0}} \frac{\mathrm{d} \xi}{z+i \xi} g(-i \xi) \\
T^{0}(k)(g)(z) & =\frac{1}{2 \pi} \int_{\Gamma} \frac{\mathrm{d} \xi}{z+i\left(\xi \cos \gamma+\xi_{0} \sin \gamma\right)} \frac{k^{2}}{\xi_{0}} g(-i \xi) \mathrm{d} \xi \\
T^{1}(k)(g)(z) & =\frac{1}{2 \pi} \int_{\Gamma} \frac{\mathrm{d} \xi}{z+i\left(\xi \cos \gamma+\xi_{0} \sin \gamma\right)}\left(\cos \gamma \frac{\xi}{\xi_{0}}+\sin \gamma\right) g(-i \xi) \mathrm{d} \xi \\
S(k)(g)(z) & =\frac{1}{2 \pi} \int_{\Gamma} \frac{\mathrm{d} \xi}{z+i\left(\xi \cos \gamma+\xi_{0} \sin \gamma\right)}\left(\cos \gamma-\sin \gamma \frac{\xi}{\xi_{0}}\right) g(-i \xi) \mathrm{d} \xi
\end{aligned}
$$

Hence the principal symbol of the operator of the system deduced from (112) by considering only the equations on the quantities $v_{ \pm},\left.u\right|_{\partial \mathcal{O}_{ \pm}},\left.\partial_{\tan } u\right|_{\partial \mathcal{O}_{ \pm}}$and forgetting the contribution of the corner is

$$
B(k)=\widetilde{M}\left(\begin{array}{llllll}
I & S(k)-I^{0}(k) & -I^{1}(k) & -T^{0}(k) & -T^{1}(k) \\
-S(k) & I & -T^{0}(k) & -T^{1}(k) & -I^{0}(k) & -I^{1}(k) \\
I & 0 & -i k \sin \theta_{+} & 0 & 0 & 0 \\
0 & I & 0 & -i k \sin \theta_{-} & 0
\end{array}\right) M
$$

where $\widetilde{M}=\left(M_{+} M_{-} M_{+} M_{-}\right)$and

$$
M=\left(\begin{array}{c}
M_{+}^{-1} \\
M_{-}^{-1} \\
M_{+}^{-1} \\
M_{+}^{-1} \\
M_{-}^{-1} \\
M_{-}^{-1}
\end{array}\right) .
$$

${ }^{(2)} \mathrm{A}$ remark that will be interesting is that a certain combination of these operators will be invertible in $H_{\sigma}^{ \pm}$for every $\sigma$. 
The principal term of this system was obtained by considering the unknowns

$$
\begin{aligned}
& \left(T_{0} \otimes T_{1}\right)\left(\psi \phi_{1} v_{+}\right), \quad\left(T_{0} \otimes T_{1}\right)\left(\psi \phi_{1} v_{-}\right), \quad \lambda\left(T_{0} \otimes T_{1}\right)\left(\left.\psi \phi_{1} u\right|_{\partial \mathcal{O}_{+}}\right), \\
& \lambda\left(T_{0} \otimes T_{1}\right)\left(\left.\psi \phi_{1} u\right|_{\partial \mathcal{O}_{-}}\right), \quad\left(T_{0} \otimes T_{1}\right)\left(\left.\psi \phi_{1} \partial_{\tan } u\right|_{\partial \mathcal{O}_{+}}\right), \quad\left(T_{0} \otimes T_{1}\right)\left(\left.\psi \phi_{1} \partial_{\tan } u\right|_{\partial \mathcal{O}_{-}}\right) .
\end{aligned}
$$

and this scaling of selected unknowns by $\lambda$ leads to a well posed problem. We invert the system of symbol equal to the principal symbol (118) and of right hand side equal to $L_{+}, L_{-}, 0,0$ up to regularizing terms. This system is thus to be inverted from $\left(H_{\sigma^{\prime}}^{+} \times H_{\sigma^{\prime}}^{+, 1} \times H_{\sigma^{\prime}}^{+}\right) \oplus\left(H_{\sigma^{\prime}}^{-} \times H_{\sigma^{\prime}}^{-, 1} \times H_{\sigma^{\prime}}^{-}\right)$to $\left(H_{\sigma}^{+} \times H_{\sigma}^{+, 1} \times H_{\sigma}^{+}\right) \oplus\left(H_{\sigma}^{-} \times H_{\sigma}^{-, 1} \times H_{\sigma}^{-}\right)$ (with $\sigma^{\prime}>\sigma$ ). For $k$ in a neighborhood of 1 , we consider $k=1$ and we denote by $\xi_{0}=\left(1-\xi^{2}\right)^{1 / 2}$. The choice of the square root is given by the analytic continuation in $\mathbb{C}-\left[1,+\infty\right.$ [ of the square root of $1-i \epsilon-\xi^{2}$ of positive imaginary part. We prove in this Section the proposition:

Proposition 4.10. - The system $B(1) F=L$ admits an unique solution in $H_{0}^{+} \times$ $H_{0}^{+}$.

The main difference here with the method introduced in [16] is that the problem considered is not the problem with straight boundaries, hence the spaces $H_{\sigma}$ are not the simple ones considered in [16], because we had to take into account the control in $W_{\kappa}$ of the position of the branching points $\left(1+\mu_{+}^{2}\right)^{1 / 2}$ and $\left(1+\mu_{-}^{2}\right)^{1 / 2}$, while in [16] these branching points are always at $1 / \cos \gamma_{ \pm}$.

We consider from now on the solution of the "principal system" $B(1) F_{0}=L$. Our aim is to show that this system is invertible in $\left(H_{0}^{+} \times H_{0}^{+, 1} \times H_{0}^{+}\right) \oplus\left(H_{0}^{-} \times H_{0}^{-, 1} \times H_{0}^{-}\right)$. The result for $k \neq 1$ and for the general system follows from the general results on elliptic systems of the Section A of [20]. Notice that we removed from the solution the incoming part of the diffracted wave, hence the + components of $F_{0}$ are $V_{+}$outgoing and the - components of $F_{0}$ are $V_{-}$-outgoing. Hence, $L_{+}(z)$ and $L_{-}(z)$ are holomorphic in $\mathbb{C}-\left[i, i \infty\left[\right.\right.$. Introduce $R_{\alpha} \phi(z)=\phi\left(e^{i \alpha} z\right)$. The modified sources $R_{\alpha} L_{+}$and $R_{\alpha} L_{-}$are holomorphic in $\Re z<0$. They belong to $L$. Let us introduce the operator $B^{\alpha}$, which is the operator associated with $I_{\alpha}^{0,1}, I_{\alpha}^{1,1}, S_{\alpha}, T_{\alpha}^{0,1}, T_{\alpha}^{1,1}$ defined in Definition 2.1. Introduce the unknowns in $\left(L \times L^{1}\right)^{2}$ that we denote by $\left(F_{+}, F_{-}, G_{+}, \widetilde{G}_{+}, G_{-}, \widetilde{G}_{-}\right)$. Let $\alpha_{0}$ be such that $z^{ \pm}(0) e^{i \alpha_{0}}$ satisfies (4). For all $\alpha \in$ $\left[0, \alpha_{0}\right], z^{ \pm}(0) e^{i \alpha}$ satisfies (4). Note that the choice of $\alpha_{0}=\pi / 2$ is not possible as in [16] but the argument of [16] is valid.

From Corollary 2.2, the problem

$$
\left\{\begin{array}{l}
B^{\alpha}(1) F=R_{\alpha} L \\
J_{\rho}\left(\widetilde{G}_{+}(w, z, \lambda)-\widetilde{G}_{-}(w, z, \lambda)\right)+\lambda z J_{\rho}\left(G_{+}(w, z, \lambda)-G_{-}(w, z, \lambda)\right) \\
\quad=T_{0}\left(u_{+}(0,0, t)-u_{-}(0,0, t)\right)(w, \lambda)
\end{array}\right.
$$

admits an unique solution. 
In this system, we took into account the condition at the corner, which was removed from the operator $B(k)$. However, the system $B(1) F=L$ has, regardless of the corner condition, a unique solution.

We denote by $F_{\alpha} \circ R_{\alpha}$ the solution of the problem (119) for $\alpha \leqslant \alpha_{0}$. We have

LEMma 4.7. - The set of functions $F_{\alpha}$ defines without ambiguity an holomorphic set of functions in

$$
\mathcal{U}_{\alpha_{0}}=\cup_{0<\alpha \leqslant \alpha_{0}}\left\{z, \Re\left(z e^{i \alpha}\right)<0\right\} .
$$

Proof. - Let $\left.\alpha \in] 0, \alpha_{0}\right]$. The solution $F_{\alpha} \circ R_{\alpha}=\left[z \mapsto F_{\alpha}\left(e^{i \alpha} z\right)\right]$ belongs to $\left(L \times L^{1}\right)^{2}$. We have, by construction

$$
\begin{gathered}
F_{+, \alpha}\left(z e^{i \alpha}\right)-I_{\alpha}^{0,1} G_{+, \alpha}-I_{\alpha}^{1,1} \widetilde{G}_{+, \alpha} \\
=L_{+}\left(e^{i \alpha} z\right)+S_{\alpha}(1) F_{-, \alpha}\left(z e^{i \alpha}\right)+R_{\alpha}^{0,1} G_{-, \alpha}\left(e^{i \alpha} z\right)+R_{\alpha}^{1,1} \widetilde{G}_{-, \alpha}\left(e^{i \alpha} z\right)=Z_{+}(z), \\
F_{+, \alpha}\left(z e^{i \alpha}\right)=i \sin \theta_{+} e^{-i \alpha} G_{+, \alpha}\left(z e^{i \alpha}\right) .
\end{gathered}
$$

The functions $L_{+}$and $L_{-}$are holomorphic in $\mathbb{C}-\left[0, i \infty\left[\right.\right.$, hence $z \mapsto L_{ \pm}\left(e^{i\left(\alpha+\alpha^{\prime}\right)} z\right)$ is holomorphic in $\Re z<0$. The operators $S(1)$ and $R^{0,1}, R^{1,1}$ are regularizing (send functions of $L$ to functions holomorphic in a larger domain equal to the union of a bounded set of $\mathbb{C}$ and of $\left.\left\{|z| \geqslant A, \operatorname{Arg} z \in\left[\frac{\pi}{2}-\varepsilon_{0}, \frac{3 \pi}{2}+\varepsilon_{0}\right]\right\}\right)$, hence $Z_{+}\left(z e^{i \alpha^{\prime}}\right)$ is in $L$ for $\alpha^{\prime}$ small. It is then enough to show that the operator on $L^{1}$ defined by $\left[i \sin \theta_{+} e^{-i \alpha}-I_{\alpha}^{0,1}\right] G_{+, \alpha}-I_{\alpha}^{1,1} \widetilde{G}_{+, \alpha}$ is characterized by an Hilbert projector, as p. 48 of $[\mathbf{1 6}]$.

We check, because of the relation, valid for $G_{+, \alpha}$ being the Laplace transform of a function of $H_{0}^{1}\left(\mathbb{R}_{+}\right)$, that $\widetilde{G}_{+, \alpha}(-i \xi)=i \xi G_{+, \alpha}(-i \xi)$. This imply the relation

$$
\begin{aligned}
I_{\alpha}^{0,1}\left(G_{+, \alpha}\right)(z)+I_{\alpha}^{1,1}\left(\widetilde{G}_{+, \alpha}\right)(z) & =\frac{1}{2 \pi} \int_{\mathbb{R}} \frac{\mathrm{d} \xi}{z+i \xi}\left(\frac{i e^{-2 i \alpha} G_{+, \alpha}(-i \xi)}{\xi_{\alpha}}-\widetilde{G}_{+, \alpha}(-i \xi) \frac{\xi}{\xi_{\alpha}}\right) \\
& =\frac{1}{2 \pi} \int_{\mathbb{R}} \frac{\mathrm{d} \xi}{z+i \xi} \frac{i\left(e^{-2 i \alpha}-\xi^{2}\right)}{\xi_{\alpha}} G_{+, \alpha}(-i \xi) \\
& =\frac{1}{2 \pi} \int_{\mathbb{R}} \frac{\mathrm{d} \xi}{z+i \xi} i \xi_{\alpha} G_{+, \alpha}(-i \xi)
\end{aligned}
$$

In this integral, we use the fact that $G_{+, \alpha}$ is in $L^{1}$, hence the behavior at infinity of the symbol is correct. Hence we modify slightly the integral around 0 , such that we have

$I_{\alpha}^{0,1}\left(G_{+, \alpha}\right)+I_{\alpha}^{1,1}\left(\widetilde{G}_{+, \alpha}\right)=\frac{1}{2 \pi} \int_{D_{\varepsilon}} \frac{\mathrm{d} \xi}{z+i \xi} \frac{\xi_{\alpha}}{\xi} \widetilde{G}_{+, \alpha}(-i \xi)+\frac{1}{2 \pi} \int_{B_{\varepsilon}} \frac{\mathrm{d} \xi}{z+i \xi} i \xi_{\alpha} G_{+, \alpha}(-i \xi)$,

where $\varepsilon>0, D_{\varepsilon}$ is $\left.]-\infty,-\varepsilon\right] \cup\left[-\varepsilon e^{i \theta}, 0 \leqslant \theta \leqslant \pi\right] \cup[\varepsilon,+\infty[$ and

$$
B_{\varepsilon}=[-\varepsilon, \varepsilon] \cup\left[\varepsilon e^{-i \theta}, 0 \leqslant \theta \leqslant \pi\right] .
$$

The contribution of $B_{\varepsilon}$ gives the behavior at 0 when $\varepsilon \rightarrow 0$. 
We notice that $F_{+\alpha}=i e^{-i \alpha} \sin \theta_{+} G_{+, \alpha}$. Moreover, as $\xi_{\alpha}=a_{\alpha}(\xi)+i b_{\alpha}(\xi)$, with $b_{\alpha}(\xi)>0$. As $b_{\alpha}(\xi) a_{\alpha}(\xi)=-\sin \alpha \cos \alpha$, if $\alpha$ is small, $a_{\alpha}(\xi)<0$. The Lopatinskii condition $\Re \sin \theta_{+}>0$ imply that $\sin \theta_{+} e^{-i \alpha}-\xi_{\alpha}$ is never 0 , and its real part is bounded below by $\Re\left(\sin \theta_{+} e^{-i \alpha}\right)$. Hence, for $\alpha$ small, it is strictly positive.

The symbol of the Hilbert projector is non zero because $\sin \theta_{ \pm} e^{-i \alpha}$ satisfies the Lopatinskii condition. Hence the regularity of $Z_{ \pm}\left(e^{i \alpha^{\prime}} z\right)$ imply the regularity of $G_{ \pm, \alpha}\left(e^{i \alpha^{\prime}} z\right), \widetilde{G}_{ \pm, \alpha}\left(e^{i \alpha^{\prime}} z\right), F_{ \pm, \alpha}\left(e^{i \alpha^{\prime}} z\right)$.

We know that $G_{+, \alpha}$ is holomorphic in $\Re\left(z e^{-i \alpha-i \alpha^{\prime}}\right)<0$ for $\left|\alpha^{\prime}\right| \leqslant \varepsilon$. Hence, deforming the contour of integration $I_{\alpha}^{0,1}$ onto $\mathbb{R} e^{i \alpha^{\prime}}$, we get that

$$
\begin{aligned}
I_{\alpha}^{0,1}\left(G_{+, \alpha}\right)(z) & =\frac{1}{2 \pi} \int_{\mathbb{R}} \frac{d \zeta}{e^{-i \alpha^{\prime}} z+i \zeta} \frac{e^{-2 i \alpha-i \alpha^{\prime}}}{\zeta_{\alpha+\alpha^{\prime}}} G_{+, \alpha}\left(e^{i \alpha^{\prime}}(-i \zeta)\right) \\
& =\frac{1}{2 \pi} e^{i \alpha^{\prime}} \int_{\mathbb{R}} \frac{e^{-2 i\left(\alpha+\alpha^{\prime}\right)}}{\zeta_{\alpha+\alpha^{\prime}}} \frac{d \zeta}{e^{-i \alpha^{\prime}} z+i \zeta} G_{+, \alpha}\left(e^{i \alpha^{\prime}}(-i \zeta)\right) .
\end{aligned}
$$

This is the relation $I_{\alpha}^{0,1}\left(G_{+, \alpha}\right)\left(z e^{i \alpha^{\prime}}\right)=e^{i \alpha^{\prime}} I_{\alpha+\alpha^{\prime}}^{0,1}\left(R_{\alpha^{\prime}} G_{+, \alpha}\right)(z)$. The other operators modify in a similar way. Hence we have

$$
B_{\alpha+\alpha^{\prime}}(1)\left(R_{\alpha^{\prime}} F_{\alpha}\right)\left(z e^{i \alpha^{\prime}}\right)=R_{\alpha^{\prime}} L_{\alpha}=L_{\alpha+\alpha^{\prime}}
$$

which means that $R_{\alpha^{\prime}} F_{\alpha}=F_{\alpha+\alpha^{\prime}}$. We then showed that the solution of the problem for $\alpha+\alpha^{\prime}$ was the extension of the problem for $\alpha$ to the set $\Re\left(z e^{i \alpha+\alpha^{\prime}}\right)<0$, which proves that we can superpose the solutions. The Lemma 4.7 is proven. 


\section{CHAPTER 5}

\section{CALCULUS OF THE DIFFRACTION COEFFICIENT}

The calculus of the diffraction coefficient $D\left(\theta, \gamma_{+}, \gamma_{-}, \theta_{+}, \theta_{-}\right)$of Theorem 1 is the aim of this Section. This coefficient is obtained by comparing two coefficients, the first one is the total diffraction coefficient (Section 5.2), and the second one is the calculus of the diffraction coefficient included in $u_{e}(x, y, t)$ (calculated in Section 5.3).

This Section begins with a Proposition, used for computing the wave obtained by the diffraction of a source on a half line. This result is used for the computation of $\widehat{u}_{e}$, which is a sum of two terms of the form $\left(\Delta+\tau^{2}\right)^{-1}\left(f^{ \pm}(x, \tau) 1_{x>0} \otimes \delta_{ \pm}+g^{ \pm}(x, \tau) 1_{x>0} \otimes\right.$ $\left.\delta_{ \pm}^{\prime}\right)$

\subsection{Diffraction by a curved antenna}

Let $f_{+}(x, t)$ and $g_{+}(x, t)$ two distributions supported in the set $t \geqslant 0,0 \leqslant x^{\prime}$, $\left(x^{\prime}\right)^{2}+\left(a\left(x^{\prime}\right)\right)^{2} \leqslant t^{2}$. They are typically traces of distributions supported in $\left(x^{\prime}\right)^{2}+$ $\left(y^{\prime}\right)^{2} \leqslant t^{2}$. Assume, moreover, that $\phi(t) f_{+}$is in $L^{2}\left(\mathbb{R}_{+} \times \mathbb{R}\right)$ and that $\phi(t) g_{+}$is in $H^{1}\left(\mathbb{R}_{+} \times \mathbb{R}\right)$.

Let $S_{+}(x, y, t)$ be the forward outgoing solution generated by $f_{+} \delta_{+}+g_{+} \delta_{+}^{\prime}$ :

$$
S_{+}(x, y, t)=\left(\Delta-\partial_{t^{2}}^{2}\right)_{+}^{-1}\left[f_{+} \delta_{+}+g_{+} \delta_{+}^{\prime}\right] .
$$

Let us introduce

$$
w_{0}(x, y, \tau)=-\frac{i}{4 \pi} \int_{\mathbb{R}} \frac{\mathrm{d} \xi}{\xi_{0}} \frac{\xi}{\xi_{0}} e^{i x \xi+i y \xi_{0}}
$$

for $(x, y)$ such that $(x, y, t) \in \Omega^{\varepsilon}, t \leqslant \varepsilon_{1}, y>0$. We denote by $S G_{0}(x, y, t)$ the inverse Fourier transform in time at $(x, y, t) \in \Omega^{\varepsilon}$ of $\widehat{g}_{+}(0, \tau) w_{0}(x, y, \tau)$.

Proposition 5.1. - We have the following results.

- The distribution $S_{+}(x, y, t)-\left(\Delta-\partial_{t^{2}}^{2}\right)_{+}^{-1}\left[\psi_{l_{1}} f_{+} \delta_{+}+\psi_{l_{1}} g_{+} \delta_{+}^{\prime}\right]$ is analytic in $\Omega^{\varepsilon} \cap$ $\left\{t<\varepsilon_{1}\right\}$.

- The distribution $\left(\Delta-\partial_{t^{2}}^{2}\right)_{+}^{-1}\left[\psi_{l_{1}} f_{+} \delta_{+}+\psi_{l_{1}} g_{+} \delta_{+}^{\prime}\right]-S G_{0}(x, y, t)$ is supported in $t \geqslant 0$, and, for $\Im \tau<0$, its partial Fourier transform in time is of the form $S T_{+}(x, y, \tau)$ 
equal to

$$
\begin{aligned}
-\frac{i}{4 \pi} \int \mathrm{d} \xi \frac{e^{i\left(x-x^{\prime}\right) \xi-i\left(y-a\left(x^{\prime}\right)\right) \xi_{0}}}{\xi_{0}} \int_{0}^{l_{1}} \psi_{l_{1}}\left(x^{\prime}\right) \mathrm{d} x^{\prime}\left[\widehat{f}_{+}\left(x^{\prime}, \tau\right)\left(1+\left(a^{\prime}\left(x^{\prime}\right)\right)^{2}\right)^{1 / 2}\right. \\
\left.+i \widehat{g}_{+}\left(x^{\prime}, \tau\right) \frac{\tau^{2}}{\xi_{0}}-\partial_{x^{\prime}} \widehat{g}_{+}\left(x^{\prime}, \tau\right) \frac{\xi}{\xi_{0}}-\widehat{g}_{+}\left(x^{\prime}, \tau\right) \frac{\xi}{\xi_{0}} \partial_{x^{\prime}} \psi_{l_{1}}\left(x^{\prime}\right)\right]
\end{aligned}
$$

- Introduce

$$
\begin{aligned}
& A T_{+}(x, y, \lambda, \tau)=\frac{1}{\cos \gamma_{+}} \mathcal{F} f_{+}\left(-\lambda \tau \frac{x+\tan \gamma_{+} y}{r}, \lambda \tau\right) \\
& -\tau \frac{r}{y} \lambda \mathcal{F} g_{+}\left(-\lambda \tau \frac{x+\tan \gamma_{+} y}{r}, \lambda \tau\right)-\frac{x}{y} \mathcal{F}\left(g_{+}^{\prime}\right)\left(-\lambda \tau \frac{x+\tan \gamma_{+} y}{r}, \lambda \tau\right) .
\end{aligned}
$$

There exists a constant $C>0$ such that

$$
\left|S T_{+}(x, y, \lambda \tau)-e^{-i \lambda \tau r} \frac{e^{3 i \pi} / 4}{\sqrt{8 \pi \lambda \tau}} r^{-1 / 2} A T_{+}(x, y, \lambda, \tau)\right| \leqslant \lambda^{-1} C e^{\lambda \Im \tau r} .
$$

- For $w \in \mathbb{C}$ such that $-\Re w \gg 0$, we have

$$
\begin{aligned}
& T_{0}\left(\Delta-\partial_{t^{2}}^{2}\right)^{-1}\left[1_{x^{\prime}>0} \psi f\right.\left.\otimes \delta_{+}+\psi g 1_{x^{\prime}>0} \otimes \delta_{+}^{\prime}\right](w, x, y, \lambda) \simeq \\
&\left.e^{\lambda \tau^{2} / 2} \frac{e^{3 i \pi / 4}}{(8 \pi \lambda \tau)^{1 / 2}} \widehat{S}\left(\lambda \tau \frac{x+a y}{r},-\lambda \tau \frac{x}{r},-\lambda \tau \frac{y}{r}, \lambda \tau\right)\right|_{\tau=i(w-r)}
\end{aligned}
$$

Proof. - The support in time of $S_{+}$is contained in $t \geqslant 0$, because the fundamental outgoing solution is supported in $t \geqslant 0$. Hence the partial Fourier transform in time $\widehat{S}_{+}(x, y, \tau)$ is holomorphic in $\Im \tau<0$. We have, denoting by $\sigma_{+}\left(x^{\prime}, \tau, \xi, \eta\right)=$ $\widehat{f}_{+}\left(x^{\prime}, \tau\right)\left(1+\left(a^{\prime}\left(x^{\prime}\right)\right)^{2}\right)^{1 / 2}+i \widehat{g}_{+}\left(x^{\prime}, \tau\right)\left(\eta-a^{\prime}\left(x^{\prime}\right) \xi\right)$

$$
\widehat{S}_{+}(x, y, \tau)=\frac{1}{(2 \pi)^{2}} \int_{\mathbb{R}^{2} \times \mathbb{R}_{+}} \mathrm{d} \xi \mathrm{d} \eta \mathrm{d} x^{\prime} \frac{e^{i\left(x-x^{\prime}\right) \xi+i\left(y-a\left(x^{\prime}\right)\right) \eta}}{\tau^{2}-\xi^{2}-\eta^{2}} \sigma_{+}\left(x^{\prime}, \tau, \xi, \eta\right)
$$

We introduce the notation $\alpha=\frac{y-a\left(x^{\prime}\right)}{\left|y-a\left(x^{\prime}\right)\right|}$ when $y \neq a(x)$. We assume $\Im \tau<0$ and, for $\xi \in \mathbb{R}$, we introduce as before $\xi_{0}=\left(\tau^{2}-\xi^{2}\right)^{1 / 2}, \Im \xi_{0}>0$. As in Section 4.2, the residue theorem selects the contribution of the pole in $\eta$ located in the region $\Im\left(\eta\left(y-a\left(x^{\prime}\right)\right)>0\right.$, hence we have $\eta=\alpha \xi_{0}$ and the residue is $2 \pi i \alpha$. Hence

$$
\widehat{S}_{+}(x, y, \tau)=-\frac{i}{4 \pi} \int_{\mathbb{R}^{\prime} \mathbb{R}_{+}} \mathrm{d} \xi \mathrm{d} x^{\prime} \frac{e^{i\left(x-x^{\prime}\right) \xi+i\left|y-a\left(x^{\prime}\right)\right| \xi_{0}}}{\xi_{0}} \sigma_{+}\left(x^{\prime}, \tau, \xi, \xi_{0}\right)
$$

Introduce $l_{1}>0$ and $\psi_{l_{1}}(x)$ a $C_{0}^{\infty}(\mathbb{R})$ function, such that $\psi_{l_{1}} \equiv 1$ on $\left[-l_{1} / 2, l_{1} / 2\right]$, $\psi_{l_{1}} \equiv 0$ on $|x| \geqslant l_{1}$. From Lemma 4.1 , the contribution of

$$
r(x, y, t)=S_{+}(x, y, t)-\left(\Delta-\partial_{t^{2}}^{2}\right)_{+}^{-1}\left[\psi_{l_{1}} f_{+} \delta_{+}+\psi_{l_{1}} g_{+} \delta_{+}^{\prime}\right]
$$

is negligible for the contribution on the front $t=r$. Let $y>0$ be given. There exists $l_{1}^{0}$ such that, for $l_{1} \leqslant l_{1}^{0}$ and $x^{\prime} \in \operatorname{supp} \psi_{l_{1}} \cap \mathbb{R}_{+}, y \geqslant a\left(x^{\prime}\right)$. Hence $\alpha=1$ for $y, x^{\prime}$. We 
reduce to the evaluation of

$$
\widehat{S}_{+}(x, y, \tau)-\widehat{r}(x, y, \tau)=-\frac{i}{4 \pi} \int_{\mathbb{R} \times \mathbb{R}_{+}} \mathrm{d} \xi \frac{e^{i\left(x-x^{\prime}\right) \xi+i\left(y-a\left(x^{\prime}\right)\right) \xi_{0}}}{\xi_{0}} \psi_{l_{1}}\left(x^{\prime}\right) \sigma_{+}\left(x^{\prime} \tau, \xi, \xi_{0}\right)
$$

The method of the Section 4.2 applies to the calculation of this integral using symbols bounded in $L^{2}\left(\mathbb{R}_{\xi}\right)$. We shall use the two different equalities. Two ideas may apply, because the form of the denominator has to be chosen in the integral, as we see here.

$$
\begin{aligned}
& \left(\xi_{0}-a^{\prime}\left(x^{\prime}\right) \xi\right)^{2}+\left(\xi+a^{\prime}\left(x^{\prime}\right) \xi_{0}\right)^{2}=\tau^{2}\left(1+\left(a^{\prime}\left(x^{\prime}\right)\right)^{2}\right) \\
& i\left(\xi_{0}-a^{\prime}\left(x^{\prime}\right) \xi\right) \xi_{0}=i \tau^{2}-i \xi\left(\xi+a^{\prime}\left(x^{\prime}\right) \xi_{0}\right) .
\end{aligned}
$$

and this yields two different forms of $\left.\widehat{S}_{+}(x, y, \tau)-\widehat{r}\right](x, y, \tau)$ as follows. Making use of the first equality, we get

$$
\begin{array}{r}
\widehat{S}_{+}(x, y, \tau)-\widehat{r}(x, y, \tau)=-\frac{i}{4 \pi} \int_{\mathbb{R} \times \mathbb{R}_{+}} \mathrm{d} \xi \mathrm{d} x^{\prime} \frac{e^{i\left(x-x^{\prime}\right) \xi+i\left(y-a\left(x^{\prime}\right)\right) \xi_{0}}}{\xi_{0}} \psi_{l_{1}}\left(x^{\prime}\right) \\
\times\left(\widehat{f}_{+}\left(x^{\prime}, \tau\right)\left(1+\left(a^{\prime}\left(x^{\prime}\right)\right)^{2}\right)^{1 / 2}+i \widehat{g}_{+}\left(x^{\prime}, \tau\right) \frac{\tau^{2}\left(1+\left(a^{\prime}\left(x^{\prime}\right)\right)^{2}\right)}{\xi_{0}-a^{\prime}\left(x^{\prime}\right) \xi}\right) \\
-\frac{i}{4 \pi} \int_{\mathbb{R}^{\prime} \times \mathbb{R}_{+}} \mathrm{d} \xi \mathrm{d} x^{\prime} \frac{\partial_{x^{\prime}}\left[e^{i\left(x-x^{\prime}\right) \xi+i\left(y-a\left(x^{\prime}\right)\right) \xi_{0}}\right]}{\xi_{0}} \psi_{l_{1}}\left(x^{\prime}\right) \widehat{g}_{+}\left(x^{\prime}, \tau\right) \frac{\xi+a^{\prime}\left(x^{\prime}\right) \xi_{0}}{\xi_{0}-a^{\prime}\left(x^{\prime}\right) \xi} .
\end{array}
$$

From the second equality, we obtain

$$
\begin{aligned}
& \widehat{S}_{+}(x, y, \tau)-\widehat{r}(x, y, \tau)=-\frac{i}{4 \pi} \int_{\mathbb{R} \times \mathbb{R}_{+}} \mathrm{d} \xi \mathrm{d} x^{\prime} \frac{e^{i\left(x-x^{\prime}\right) \xi+i\left(y-a\left(x^{\prime}\right)\right) \xi_{0}}}{\xi_{0}} \psi_{l_{1}}\left(x^{\prime}\right) \\
& \times\left(\widehat{f}_{+}\left(x^{\prime}, \tau\right)\left(1+\left(a^{\prime}\left(x^{\prime}\right)\right)^{2}\right)^{1 / 2}+i \widehat{g}_{+}\left(x^{\prime}, \tau\right) \frac{\tau^{2}}{\xi_{0}}\right) \\
& +\frac{i}{4 \pi} \int_{\mathbb{R} \times \mathbb{R}_{+}} \mathrm{d} \xi \mathrm{d} x^{\prime} \frac{\partial_{x^{\prime}}\left[e^{i\left(x-x^{\prime}\right) \xi+i\left(y-a\left(x^{\prime}\right)\right) \xi_{0}}\right]}{\xi_{0}} \psi_{l_{1}}\left(x^{\prime}\right) \widehat{g}_{+}\left(x^{\prime}, \tau\right) \frac{\xi}{\xi_{0}} .
\end{aligned}
$$

Introduce

$$
\begin{aligned}
& H_{+}\left(x^{\prime}, \xi, \tau\right)= \\
& \quad \psi_{l_{1}}\left(x^{\prime}\right)\left(\widehat{f}_{+}\left(x^{\prime}, \tau\right)\left(1+\left(a^{\prime}\left(x^{\prime}\right)\right)^{2}\right)^{1 / 2}+\widehat{g}_{+}\left(x^{\prime}, \tau\right) \frac{i \tau^{2}}{\xi_{0}}\right)-\partial_{x^{\prime}}\left(\psi_{l_{1}}\left(x^{\prime}\right) \widehat{g}_{+}\left(x^{\prime}, \tau\right)\right) \frac{\xi}{\xi_{0}} .
\end{aligned}
$$

We perform an integration by parts in $x^{\prime}$ on the last term of (121), which gives

$$
\begin{array}{r}
\widehat{S}_{+}(x, y, \tau)-\widehat{r}(x, y, \tau)=-\frac{i}{4 \pi} \int_{\mathbb{R} \times \mathbb{R}_{+}} \mathrm{d} \xi \mathrm{d} x^{\prime} \frac{e^{i\left(x-x^{\prime}\right) \xi+i\left(y-a\left(x^{\prime}\right)\right) \xi_{0}}}{\xi_{0}} H_{+}\left(x^{\prime}, \xi, \tau\right) \\
-\frac{i}{4 \pi} \int_{\mathbb{R}} \mathrm{d} \xi \frac{e^{i x \xi+y \xi_{0}}}{\xi_{0}} \widehat{g}_{+}(0, \tau) \frac{\xi}{\xi_{0}} .
\end{array}
$$

Note that the term involving $\widehat{g}_{+}(0, \tau)$ in $(120)$ is

$$
\left(\frac{i}{4 \pi} \int \frac{\mathrm{d} \xi}{\xi_{0}} e^{i x \xi+i y \xi_{0}} \frac{\xi+\tan \gamma_{+} \xi_{0}}{\xi_{0}-\tan \gamma_{+} \xi}\right) \widehat{g}_{+}(0, \tau)
$$


which has the same behavior as $-\frac{i}{4 \pi} \int \mathrm{d} \xi \frac{e^{i x \xi+y \xi_{0}}}{\xi_{0}} \widehat{g}_{+}(0, \tau) \frac{\xi}{\xi_{0}}$ when $\xi \rightarrow \pm \infty$. This term cancels with the same term arising in $\widehat{S}_{-}(x, y, \tau)$ when $\widehat{g}_{-}(0, \tau)=\widehat{g}_{+}(0, \tau)$. We get

$$
\begin{array}{r}
\widehat{S}_{+}(x, y, \tau)-\widehat{r}(x, y, \tau)=-\frac{i}{4 \pi} \int \frac{\mathrm{d} \xi}{\xi_{0}} \int_{0}^{\infty} \mathrm{d} x^{\prime} e^{i x \xi+i y \xi_{0}} e^{-i x^{\prime} \xi-i a\left(x^{\prime}\right) \xi_{0}} H_{+}\left(x^{\prime}, \xi, \tau\right) \\
-\frac{i}{4 \pi} \int \mathrm{d} \xi \frac{e^{i x \xi+y \xi_{0}}}{\xi_{0}} \widehat{g}_{+}(0, \tau) \frac{\xi}{\xi_{0}}
\end{array}
$$

and we denote by

$$
J_{+}(\xi, \tau)=\int_{0}^{\infty} e^{-i x^{\prime} \xi-i a\left(x^{\prime}\right) \xi_{0}} H_{+}\left(x^{\prime}, \xi, \tau\right) \mathrm{d} x^{\prime} .
$$

Define $\phi(\xi)=x \xi+y \xi_{0}$. We verify that $\phi^{\prime}(\xi)=x-y \xi / \xi_{0}$ and $\phi^{\prime \prime}(\xi)=-y \tau^{2} / \xi_{0}^{3}$. The phase $\phi$ admits a critical complex point at $\xi / \xi_{0}=x / y$, that is, using $\Im \tau<0$ and $\Im \xi_{0}>0, \xi=-\tau x / r$, which comes from $\xi_{0}=-\tau y / r$. The contour of integration on $\xi$ is $\mathbb{R}$. We deform this contour on $\Sigma=\{\Im \xi=-\Im \tau x / r\}$. As $y>0$, we verify that we do not cross the branching points when deforming the contour because $|x| / r<1$. For $l_{2}>0$ given, we divide $\Sigma$ into $\Sigma_{l_{2}}=\Sigma \cap\{|\xi+\tau x / r|\}$ and $\Sigma \backslash \Sigma_{l_{2}}$. For $\xi \in \Sigma_{l_{2}}$, we have $\xi_{0}=-\tau y / r+O\left(l_{2}\right)$, which implies that there exists a constant $r_{0}>0$ such that

$$
\forall \xi \in \Sigma-\Sigma_{l_{2}}, \quad|\Im \phi(\xi)+r \Im \tau|>r_{0} l_{2} .
$$

This constant $r_{0}$ is graphically estimated. In fact, $\tau^{2}-\xi^{2}=\tau^{2} \frac{y^{2}}{r^{2}}-u^{2}+2 \frac{x}{r} \tau u$, which describes a parabola, and the zone $|u| \geqslant l_{2}$ excludes on this parabola a neighborhood of $\tau^{2} y^{2} / r^{2}$ of size $r_{0}^{\prime} l_{2}$ (we are not at the minimum of the parabola), hence on the square root of this curve we exclude a neighborhood of size $r_{0} l_{2}$ (same reason).

We then link the constants $l_{1}$ used for the definition of $\widehat{S}_{+}-\widehat{r}$ and this constant $l_{2}$ such that $r_{0} l_{2} \gg l_{1}$. As there exists $C$ such that

$$
\max \left(\int_{0}^{+\infty}\left|\widehat{f}_{+}\left(x^{\prime}, \lambda \tau\right)\right|^{2} \mathrm{~d} x^{\prime}, \int_{0}^{+\infty} \lambda^{2}\left|\widehat{g}_{+}\left(x^{\prime}, \lambda \tau\right)\right|^{2} \mathrm{~d} x^{\prime}, \int_{0}^{+\infty}\left|\partial_{x^{\prime}} \widehat{g}_{+}\left(x^{\prime}, \lambda \tau\right)\right|^{2} \mathrm{~d} x^{\prime}\right) \leqslant C,
$$

the contribution of $\Sigma \backslash \Sigma_{l_{2}}$ in $\widehat{S}_{+}-\widehat{r}$ is exponentially decreasing compared with $e^{-\lambda \Im \tau r}$. We reduce then to the study of the integral on $\Sigma_{l_{2}}$. We have immediately, for $\xi \in \Sigma_{l_{2}}$,

$$
\xi+\tan \gamma_{+} \xi_{0}=-\tau\left(\frac{x}{r}+\tan \gamma_{+} \frac{y}{r}\right)+O\left(l_{2}\right) .
$$

We assume

$$
(x, y, t) \in \Omega^{\varepsilon} .
$$

We write

$$
\widehat{S}_{+}(x, y, \lambda \tau)-\widehat{r}_{+}(x, y, \lambda \tau)=\int \frac{\mathrm{d} \xi}{\xi_{0}} e^{i \lambda\left(x \xi+y \xi_{0}\right)} J_{+}(\lambda \xi, \lambda \tau)
$$


We study $J_{+}(\lambda \xi, \lambda \tau)$. We have

$$
\begin{aligned}
J_{+}(\lambda \xi, \lambda \tau) & =\int_{0}^{\infty} e^{-i \lambda x^{\prime} \xi-i \lambda a\left(x^{\prime}\right) \xi_{0}} H_{+}\left(x^{\prime}, \lambda \xi, \lambda \tau\right) \mathrm{d} x^{\prime} \\
& =\int_{0}^{\infty} e^{-i \lambda x^{\prime} \xi-i \lambda \tan \gamma_{+} \xi_{0}-i \lambda\left(a\left(x^{\prime}\right)-a^{\prime}(0) x^{\prime}\right) \xi_{0}} H_{+}\left(x^{\prime}, \lambda \xi, \lambda \tau\right) \mathrm{d} x^{\prime} .
\end{aligned}
$$

We then check that $\frac{\cos \left(\theta-\gamma_{+}\right)}{\cos \gamma_{+}} \leqslant \frac{\cos \varepsilon}{\cos \gamma_{+}}$. We denote by $D_{0}$ a constant such that

$$
\forall \xi \in \Sigma_{l_{2}}, \quad\left|\xi+\tan \gamma_{+} \xi_{0}+\tau\left(\frac{x}{r}+\frac{y}{r} \tan \gamma_{+}\right)\right| \leqslant D_{0} l_{2} .
$$

For $l_{2} \leqslant \frac{1}{D_{0}}\left(\frac{\cos \varepsilon / 3-\cos \varepsilon}{\cos \gamma+}\right)(-\Im \tau)$ we have

$$
\forall \xi \in \Sigma_{l_{2}}, \quad \Im\left(\xi+\tan \gamma_{+} \xi_{0}\right)<-\frac{\Im \tau \cos \varepsilon / 3}{\cos \gamma_{+}} .
$$

We choose $s$ such that $\sup _{\xi \in \Sigma_{l_{2}}} \Im\left(\xi+\tan \gamma_{+} \xi_{0}\right)<s<-\frac{\Im \tau \cos \varepsilon / 3}{\cos \gamma_{+}}$. We use

LEMmA 5.1. - For all $c_{0}>\cos \gamma_{+}$, there exists $l_{1}>0$ such that the Fourier transforms $\mathcal{F}\left(\psi_{l_{1}} f_{+}\right)\left(\lambda \tau, \lambda \xi^{\prime}\right), \mathcal{F}\left(\psi_{l_{1}} g_{+}\right)\left(\lambda \tau, \lambda \xi^{\prime}\right), \mathcal{F}\left(\psi_{l_{1}} \partial_{x^{\prime}} g_{+}\right)\left(\lambda \tau, \lambda \xi^{\prime}\right)$ can be extended holomorphically to the domain $\Im \xi^{\prime}<-\Im \tau / c_{0}$, where the following estimates hold

$$
\begin{gathered}
\sup _{\lambda} \sup _{s<-\Im \tau / c_{0}} \int_{\mathbb{R}_{\xi^{\prime}}-i s}\left|\mathcal{F}\left(\psi_{l_{1}} f_{+}\right)\left(\lambda \tau, \lambda \xi^{\prime}\right)\right|^{2} \mathrm{~d} \xi^{\prime}<+\infty, \\
\sup _{\lambda} \sup _{s<-\Im \tau / c_{0}} \int_{\mathbb{R}_{\xi^{\prime}}-i s}\left|\lambda^{2} \mathcal{F}\left(\psi_{l_{1}} g_{+}\right)\left(\lambda \tau, \lambda \xi^{\prime}\right)\right|^{2} \mathrm{~d} \xi^{\prime}<+\infty, \\
\sup _{\lambda} \sup _{s<-\Im \tau / c_{0}} \int_{\mathbb{R}_{\xi^{\prime}-i s}}\left|\mathcal{F}\left(\partial_{x^{\prime}}\left(\psi_{l_{1}} g_{+}\right)\right)\left(\lambda \tau, \lambda \xi^{\prime}\right)\right|^{2} \mathrm{~d} \xi^{\prime}<+\infty .
\end{gathered}
$$

Proof. - This behavior and these estimates comes from the fact that $f_{+}\left(x^{\prime}, t\right)=0$ for $\left(x^{\prime}\right)^{2}+\left(a\left(x^{\prime}\right)\right)^{2}>t^{2}$ because of the finite speed of propagation. As we have $a\left(x^{\prime}\right)=\left(\tan \gamma_{+}+O\left(l_{1}\right)\right) x^{\prime}$ for $x^{\prime} \leqslant l_{1}$, we get that the support of $f_{+}\left(x^{\prime}, t\right)$ is contained in $\left(x^{\prime}\right)^{2}\left(1+\tan \gamma_{+}^{2}\right)+O\left(l_{1}\right) \leqslant t$, which is $\left(\frac{x^{\prime}}{\cos \gamma_{+}}\right)^{2}\left(1+O\left(l_{1}\right)\right) \leqslant t^{2}$. As $c_{0}>\cos \gamma_{+}$, there exists $l_{1}^{1}$ such that $\frac{1+O\left(l_{1}\right)}{\cos ^{2} \gamma_{+}}<1 / c_{0}^{2}$ for $l_{1} \leqslant l_{1}^{1}$, which imply that the support of $f_{+}$is contained in $x^{\prime} \leqslant c_{0} t$. The Fourier transform of a function whose support

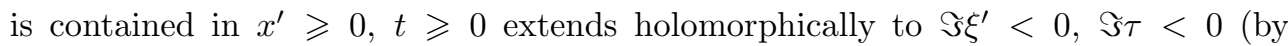
the Paley-Wiener theorem), and the Fourier transform of a function supported for $x^{\prime}-c_{0} t \leqslant 0$ extends holomorphically to $\Im \xi^{\prime}<-\Im \tau / c_{0}$. We apply the Paley-Wiener theorem on the distribution $L f_{+}(u, t)=f_{+}\left(c_{0} t-u, t\right)$, supported in $u \geqslant 0$, which Fourier transform in $u, t$ writes $\mathcal{F} L f_{+}(U, \tau)=\mathcal{F} f_{+}\left(-U, \tau+c_{0} U\right)$. The distribution $\mathcal{F} f_{+}$belongs to $L^{2}(U, \tau)$ on each line $\Im U<0$. 
Return to the proof of Proposition 5.1. Using the inverse Fourier transform of $H_{+}\left(x^{\prime}, \lambda \xi, \lambda \tau\right)$, we have the equality

$$
\begin{aligned}
H_{+}\left(x^{\prime}, \lambda \xi, \lambda \tau\right)=\frac{\lambda}{2 \pi} \int_{\mathbb{R}_{\xi^{\prime}}-i s} \mathrm{~d} \xi^{\prime} e^{i \lambda x^{\prime}\left(\xi^{\prime}-\xi-\tan \gamma_{+} \xi_{0}\right)} e^{-i \lambda\left(a\left(x^{\prime}\right)-a^{\prime}(0) x^{\prime}\right) \xi_{0}} \\
\cdot\left[\mathcal{F}\left(\left(1+\left(a^{\prime}\left(x^{\prime}\right)\right)^{2}\right)^{1 / 2} \psi_{l_{1}} \widehat{f}_{+}\right)\left(\lambda \xi^{\prime}, \lambda \tau\right)+\mathcal{F}\left(\psi_{l_{1}}\left(x^{\prime}\right) \widehat{g}_{+}\right)\left(\lambda \xi^{\prime}, \lambda \tau\right) \frac{i \tau^{2}}{\xi_{0}}\right. \\
\left.\quad-\mathcal{F}\left(\partial_{x^{\prime}}\left(\psi_{l_{1}}\left(x^{\prime}\right) \widehat{g}_{+}\left(x^{\prime}, \tau\right)\right)\right)\left(\lambda \xi^{\prime}, \lambda \tau\right) \frac{\xi}{\xi_{0}}\right] .
\end{aligned}
$$

We have thus

$$
\begin{aligned}
& J_{+}(\lambda \xi, \lambda \tau)= \\
& \quad \frac{\lambda}{2 \pi} \int_{0}^{l_{1}} \mathrm{~d} x^{\prime} \int_{\mathbb{R}_{\xi^{\prime}}-i s} \mathrm{~d} \xi^{\prime} e^{i \lambda x^{\prime}\left(\xi^{\prime}-\xi-\tan \gamma_{+} \xi_{0}\right)-i \lambda\left(a\left(x^{\prime}\right)-a^{\prime}(0) x^{\prime}\right)} \mathcal{F} H_{+}\left(\lambda \xi^{\prime}, \lambda \xi, \lambda \tau\right) .
\end{aligned}
$$

We expand $e^{-i \lambda\left(a\left(x^{\prime}\right)-\tan \gamma_{+} x^{\prime}\right) \xi_{0}}$ in the neighborhood of $x^{\prime}=0$. We have

$$
\begin{aligned}
J_{+}(\lambda \xi, \lambda \tau) \simeq \frac{\lambda}{2 \pi} \int_{0}^{l_{1}} & \int_{\mathbb{R}-i s} \mathrm{~d} t e^{i x^{\prime}\left(t-\xi-\tan \gamma_{+} \xi_{0}\right)} \\
& \cdot \sum_{m \leqslant \lambda / C} \frac{\left(x^{\prime}\right)^{m}}{m !} \mathcal{F} H_{+}(\lambda t, \lambda \xi, \lambda \tau)\left(\frac{\partial_{x^{\prime}}}{\lambda}\right)^{m}\left[e^{-i \lambda\left(a\left(x^{\prime}\right)-\tan \gamma_{+} x^{\prime}\right) \xi_{0}}\right] .
\end{aligned}
$$

Hence, using

$$
\frac{\lambda}{2 \pi} \int_{\mathbb{R}-i s} e^{i x^{\prime}\left(t-\xi-\tan \gamma_{+} \xi_{0}\right)} \frac{\left(x^{\prime}\right)^{m}}{m !} \mathcal{F} H_{+}(\lambda t, \lambda \xi, \lambda \tau)=i^{m} \frac{\partial_{t}^{m} H_{+}}{m !}\left(\lambda\left(\xi+\tan \gamma_{+} \xi_{0}\right), \lambda \xi, \lambda \tau\right)
$$

we obtain

$$
J_{+}(\lambda \xi, \lambda \tau) \simeq \sum_{m \leqslant \frac{\lambda}{C}} \frac{i^{m}}{m !}\left(\frac{\partial_{x^{\prime}}}{\lambda}\right)^{m}\left[e^{-i \lambda\left(a\left(x^{\prime}\right)-\tan \gamma_{+} x^{\prime}\right) \xi_{0}}\right] \partial_{t}^{m} \mathcal{F} H_{+}\left(\lambda\left(\xi+\tan \gamma_{+} \xi_{0}\right), \lambda \xi, \lambda \tau\right) .
$$

We apply the stationary phase lemma in the relation

$$
\widehat{S}_{+}(x, y, \lambda \tau)-\widehat{r}(x, y, \lambda \tau)=-\frac{i}{4 \pi} \int_{\Sigma_{l_{2}}} \frac{\mathrm{d} \xi}{\xi_{0}} e^{i \lambda\left(x \xi+y \xi_{0}\right)} J_{+}(\lambda \xi, \lambda \tau)+r_{1}(x, y, \lambda \tau) .
$$

The critical point of $x \xi+y \xi_{0}$ is $\xi_{c}=-\tau x / r$, with $\phi^{\prime \prime}\left(\xi_{c}\right)=\frac{r}{\tau} \frac{r^{2}}{y^{2}}, \phi\left(\xi_{c}\right)=-\tau r$, $\xi_{0}^{c}=-\tau y / r$, which gives

$$
\begin{aligned}
\widehat{S}_{+}(x, y, \lambda \tau) & \simeq-\frac{i}{4 \pi}\left(\frac{2 \pi}{r^{2}} \lambda \tau y^{2}\right)^{1 / 2} e^{i \pi / 4} e^{-i \tau r} \sum_{m \leqslant \frac{\lambda}{C}, p \leqslant \frac{\lambda}{C}} \alpha_{p, m}(x, y, \tau) \lambda^{-p} \tau^{-p} \lambda^{-m} \\
& \simeq e^{-i \tau r} e^{3 i \pi / 4} \frac{1}{\sqrt{8 \pi \lambda \tau r}} \sum_{m, p \leqslant \frac{\lambda}{C}} \alpha_{m, p}(x, y, \tau) \lambda^{-m-p} \tau^{-p} .
\end{aligned}
$$


We identify

$$
\begin{aligned}
\alpha_{0,0}(x, y, \tau) & =J_{+}\left(-\lambda \tau \frac{x}{r}, \lambda \tau\right) \\
& \simeq \mathcal{F} H_{+}\left(-\lambda \tau\left(\frac{x}{r}+\frac{y}{r} \tan \gamma_{+}\right),-\tau \frac{x}{r}, \lambda\right) \\
\simeq & \frac{1}{\cos \gamma_{+}} \mathcal{F} f_{+}\left(-\lambda \tau \frac{\cos \left(\theta-\gamma_{+}\right)}{\cos \gamma_{+}}, \lambda \tau\right) \\
& -i \lambda \tau \frac{r}{y} \mathcal{F} g_{+}\left(-\lambda \tau \frac{\cos \left(\theta-\gamma_{+}\right)}{\cos \gamma_{+}}, \lambda \tau\right) \\
& \quad-\frac{x}{y} \mathcal{F}\left(g_{+}^{\prime}\right)\left(-\lambda \tau \frac{\cos \left(\theta-\gamma_{+}\right)}{\cos \gamma_{+}}, \lambda \tau\right) .
\end{aligned}
$$

This ends the proof of Proposition 5.1, which is used to compute the contribution of the source on each half-boundary, et precisely from the point $(0,0)$.

In the following Section, we use the result of J.M. Bernard and other authors to identify the total diffraction coefficient.

\subsection{Calculus of the total diffraction coefficient}

In the case of the Dirichlet boundary condition the problem of the diffraction of an incident wave by a straight wedge was solved explicitly by Garnir [19]. This was possible because the Green function of this problem could be computed by a change of variables. However, in the case of a mixed boundary condition, such an explicit form of the Green function is not known. Many authors, following Maliuzinetz [30], have given a form of the diffraction coefficient, among them J.M. Bernard [5], D. Bouche [9] and we present this method here. Let $u_{i}$ be a plane incident wave

$$
u_{i}(x, y, t)=\int_{-\infty}^{+\infty} e^{i \tau(t-x)} \sigma_{0}(\tau) \mathrm{d} \tau
$$

They obtained the

Proposition 5.2. - The diffracted wave generated by a straight wedge with the boundary condition $\partial_{n} u-\sin \theta_{ \pm} u=0$ on each face admits an asymptotic expansion, and we have

$$
\begin{aligned}
u_{d}(x, y, t)= & \int_{-\infty}^{+\infty} \sigma_{r}^{+}(\tau) e^{i \tau\left(t-x \cos 2 \gamma_{+}-y \sin 2 \gamma_{+}\right)} \mathrm{d} \tau \\
& +\int_{-\infty}^{+\infty} \sigma_{r}^{-}(\tau) e^{i \tau\left(t-x \cos 2 \gamma_{-}-y \sin 2 \gamma_{-}\right)} \mathrm{d} \tau+\int_{-\infty}^{+\infty} \sigma_{d}(r, \tau) e^{i \tau(t-r)} \mathrm{d} \tau
\end{aligned}
$$

We have

$$
\sigma_{d}(r, \tau)=\sigma_{0}^{0}(\tau) \frac{D\left(\theta, \gamma_{+}, \gamma_{-}, \theta_{+}, \theta_{-}\right) e^{i \pi / 4}}{(2 \pi \tau r)^{1 / 2}}
$$

The diffraction coefficient is computed through a special function $\Psi$ that is given below in (124). 
This method is valid for the Helmholtz equation, and we denote also by "wave" the Fourier transform of the waves we studied.

The incident wave considered by Bernard [5] is $u^{i}(r, \theta, k)=u_{0} e^{i k r \cos \left(\theta-\theta_{0}\right)}$, and we represent the solution of the diffraction problem in $]-\gamma / 2, \gamma / 2[$ as

$$
u(r, \theta, k)=\frac{1}{2 \pi i} \int_{\mathcal{C}} f(\alpha+\theta) e^{i k r \cos \alpha} \mathrm{d} \alpha .
$$

Under the assumption $\theta_{0}=0$, we check that

$$
\frac{1}{2 \pi} \int_{-\infty}^{+\infty} u_{i}(r, \theta, k)\left(1+|k|^{2}\right)^{-2} e^{-i k t} \mathrm{~d} k
$$

is the conjugate of an analytic conormal wave $u_{i}(x, y, t)$, where the function $\theta_{i}(x, y)$ is $x$ and the symbol is $\sigma_{i}(x, y, k)=u_{0} /\left(1+|k|^{2}\right)^{2}$, which satisfies the hypotheses on $\sigma_{i}$ and $\theta_{i}$. If we obtain the function $f$ solution of the problem stated by J. M. Bernard, the linearity of the Helmholtz operator shall lead to a solution of the problem (6) with such an $u_{i}$. The purpose of this paragraph is to give the construction of $f$ associated with an incident plane wave. The function $f$ is assumed to verify the two relations

i) $f(\alpha)-u_{0} /\left(\alpha-\theta_{0}\right)$ is regular for $|\Re \alpha| \leqslant \gamma / 2$

ii) $|f(\alpha)-f( \pm i \infty)| \leqslant e^{-c|\Im \alpha|}$ for $\Im \alpha \rightarrow \pm \infty$.

If the solution $u$ of the diffraction problem satisfies $i)$ and ii), the function $f$ verifies the functional equality:

$$
\left(\sin \alpha \pm \sin \theta_{ \pm}\right) f(\alpha \pm \gamma / 2)-\left(-\sin \alpha \pm \sin \theta_{ \pm}\right) f(-\alpha \pm \gamma / 2)=0 .
$$

We need only to check that i) and ii) are satisfied for the solution of the problem we study. Maliuzinetz [31] gives an inversion formula for $f$ in terms of $u$. A solution in terms of special functions has been found by Maliuzhinets in his $\mathrm{PhD}$ thesis and can be found in [30]. Simple expressions of the special function involved were given by Senior and Volakis [36] and Molinet [33], and the following formula is found in Bouche and Molinet $[\mathbf{9}]$. A review of the history of the results on the diffraction by a wedge is given in $[\mathbf{6}]$. We outline the expression below.

Let $\gamma=\gamma_{+}-\gamma_{-}$. We introduce a function $\Psi_{\gamma}$ :

$$
\Psi_{\gamma}(u)=\left(\cos \frac{\pi u}{4 \pi-2 \gamma}\right)^{1 / 2} \exp \left(\frac{2 \pi-\gamma}{\pi^{2}} \int_{0}^{1} \frac{\ln \left(1+t^{2} \tan ^{2} \frac{\pi u}{4 \pi-2 \gamma}\right)}{\left(1-t^{2}\right) \cosh \left(\frac{4 \pi-2 \gamma}{\pi} \operatorname{argth} t\right)} \mathrm{d} t\right) .
$$

We consider the function (expressed both with the angle of the wedge we consider $\gamma$ and the exterior angle to the wedge $\widetilde{\gamma}=2 \pi-\gamma=n \pi)$ :

$$
\begin{aligned}
& \Psi(\alpha)=\Psi_{\gamma}\left(\alpha+\frac{\tilde{\gamma}}{2}+\frac{\pi}{2}-\theta^{+}\right) \Psi_{\gamma}\left(\alpha-\frac{\tilde{\gamma}}{2}-\frac{\pi}{2}+\theta^{-}\right) \Psi_{\gamma}\left(\alpha-\frac{\tilde{\gamma}}{2}+\frac{\pi}{2}-\theta^{-}\right) \Psi_{\gamma}\left(\alpha+\frac{\tilde{\gamma}}{2}-\frac{\pi}{2}+\theta^{+}\right), \\
& \Psi(\alpha)=\Psi_{\gamma}\left(\alpha+\frac{\gamma}{2}-\frac{3 \pi}{2}+\theta^{+}\right) \Psi_{\gamma}\left(\alpha+\frac{\gamma}{2}-\frac{\pi}{2}-\theta^{-}\right) \Psi_{\gamma}\left(\alpha-\frac{\gamma}{2}+\frac{3 \pi}{2}-\theta^{+}\right) \Psi_{\gamma}\left(\alpha-\frac{\gamma}{2}+\frac{\pi}{2}+\theta^{-}\right) .
\end{aligned}
$$


We verify that the angles introduced in $[\mathbf{9}]$ are

$$
\left\{\begin{array}{l}
\Phi^{\prime}-\frac{n \pi}{2}=\frac{\gamma}{2}-\gamma_{+} \\
\Phi=\theta-\gamma_{-} \\
\Phi-\frac{\pi n}{2}+\pi=\theta-\gamma_{-}+\frac{\gamma}{2} \\
\Phi-\frac{\pi n}{2}-\pi=\theta-\gamma_{-}+\frac{\gamma}{2}-2 \pi
\end{array}\right.
$$

which leads to the relations

$$
\begin{aligned}
& \Psi\left(\Phi-\frac{n \pi}{2}-\pi\right)=\Psi_{\gamma}\left(\theta-\gamma_{-}-\theta_{+}-\frac{\pi}{2}\right) \Psi_{\gamma}\left(\theta-\gamma_{+}-\theta_{+}-\frac{7 \pi}{2}\right) \\
& \quad \times \Psi_{\gamma}\left(\theta-\gamma_{-}+\theta_{-}-\frac{3 \pi}{2}\right) \Psi_{\gamma}\left(\theta-\gamma_{+}+\theta_{-}-\frac{5 \pi}{2}\right) \\
& \Psi\left(\Phi-\frac{n \pi}{2}+\pi\right)=\Psi_{\gamma}\left(\theta-\gamma_{-}-\theta_{+}+\frac{3 \pi}{2}\right) \Psi_{\gamma}\left(\theta-\gamma_{+}-\theta_{+}-\frac{3 \pi}{2}\right) \\
& \times \Psi_{\gamma}\left(\theta-\gamma_{-}+\theta_{-}+\frac{\pi}{2}\right) \Psi_{\gamma}\left(\theta-\gamma_{+}+\theta_{-}-\frac{\pi}{2}\right) .
\end{aligned}
$$

The diffraction coefficient is then

$$
\frac{D\left(\theta, \gamma_{+}, \gamma_{-}, \theta_{+}, \theta_{-}\right) e^{i \pi / 4}}{\sqrt{2 \pi k r}}
$$

with

$$
\begin{aligned}
& D\left(\theta, \gamma_{+}, \gamma_{-}, \theta_{+}, \theta_{-}\right) \Psi\left(\frac{\gamma}{2}-\gamma_{+}\right)= \\
& \quad \frac{\Psi\left(\theta-\frac{\gamma_{+}+\gamma_{-}}{2}-2 \pi\right)}{\cos \left(\frac{\pi^{2}+\pi\left(\theta-\gamma_{+}\right)}{2 \pi-\gamma}\right)-\cos \left(\frac{\pi^{2}-\pi \gamma_{+}}{2 \pi-\gamma}\right)}-\frac{\Psi\left(\theta-\frac{\gamma_{+}+\gamma_{-}}{2}\right)}{\cos \left(\frac{\pi^{2}-\pi\left(\theta-\gamma_{+}\right)}{2 \pi-\gamma}\right)-\cos \left(\frac{\pi^{2}-\pi \gamma_{+}}{2 \pi-\gamma}\right)} .
\end{aligned}
$$

This function depends on the angles $\theta-\gamma_{+}$and $\theta-\gamma_{-}$. We check that $\Psi\left(\theta-\gamma_{+}\right)$is equal to $\Psi\left(\theta-\gamma_{-}\right)$when we exchange the Brewster angles of the faces + and - . We notice that the special function which gives the diffraction coefficient is solution of the functional relation (123). Note that finding a solution of the functional relation (123) is closely related with the inversion of $B(k)$.

From Maliuzhinets, we check that the diffraction coefficient has poles. We get (from $[\mathbf{3 0}]$ ) the relation (where $\widetilde{\gamma}=2 \pi-\gamma$ denotes the exterior angle of the wedge)

$$
\Psi_{\gamma}(z \pm(\widetilde{\gamma}+3 \pi / 2))= \pm \sin \frac{\pi(\pi \pm z)}{2 \widetilde{\gamma}} \frac{1}{\sin (\pi z / 2 \widetilde{\gamma})} \Psi_{\gamma}(\widetilde{\gamma}-\pi / 2 \pm z)
$$

Hence the poles of $\Psi_{\gamma}$ are $z= \pm(\widetilde{\gamma}+3 \pi / 2)$. 
We thus deduce that the poles of $\Psi$ are given by the list

$$
\left\{\begin{array}{l}
\alpha=\theta_{+}+\pi+\widetilde{\gamma} / 2 \\
\alpha=\theta_{+}-2 \pi-3 \widetilde{\gamma} / 2 \\
\alpha=-\theta_{-}+2 \pi+3 \widetilde{\gamma} / 2 \\
\alpha=-\theta_{-}-\pi-\widetilde{\gamma} / 2 \\
\alpha=\theta_{-}+\pi+3 \widetilde{\gamma} / 2 \\
\alpha=\theta_{-}-2 \pi-\widetilde{\gamma} / 2 \\
\alpha=-\theta_{+}+2 \pi+\widetilde{\gamma} / 2 \\
\alpha=-\theta_{+}-\pi-3 \widetilde{\gamma} / 2 .
\end{array}\right.
$$

As we consider $\Psi$ at the points $\Phi-\widetilde{\gamma} / 2 \pm \pi$, the list of poles of the diffraction coefficient is given by the following angles

$$
\left\{\begin{array}{l}
\Phi= \pm \pi+\widetilde{\gamma}+\theta_{+}+\pi \\
\Phi= \pm \pi-\widetilde{\gamma}+\theta_{+}-2 \pi \\
\Phi= \pm \pi+2 \widetilde{\gamma}-\theta_{-}+\pi \\
\Phi= \pm \pi-\theta_{-}-\pi \\
\Phi= \pm \pi+2 \widetilde{\gamma}+\theta_{-}+\pi \\
\Phi= \pm \pi+\theta_{-}-2 \pi \\
\Phi= \pm \pi+2 \widetilde{\gamma}-\theta_{+}+2 \pi \\
\Phi= \pm \pi-\theta_{+}-\pi-\widetilde{\gamma}
\end{array}\right.
$$

We recover $\theta$ by $\Phi=\theta-\gamma_{-}$and $\Phi+\widetilde{\gamma}=2 \pi+\theta-\gamma_{+}$.

In particular, for admissible angles and assuming that the angle $\widetilde{\gamma} \in] \pi, 2 \pi[$, we obtain $\Phi=\theta_{-}-\pi$ or $\Phi=-\theta_{-}$and $\Phi+\widetilde{\gamma}=\theta_{+}-\pi$ and $\Phi+\widetilde{\gamma}=-\theta_{+}$. These angles are complex when $\Im z^{+}(0) \neq 0$ or $\Im z^{-}(0) \neq 0$.

Corollary 5.1. - When $\Im z^{+}(0)=0$ or $\Im z^{-}(0)=0$, we obtain the singular directions of Proposition 3.1.

\subsection{Calculus of $u_{e}$}

The aim of this section is to compute the principal symbol of $u_{e}$, which is obtained by

$$
\left(\Delta-\partial_{t^{2}}^{2}\right)^{-1}\left(\partial_{+} u_{+} 1_{x^{\prime} \geqslant 0} \otimes \delta_{+}+\partial_{-} u_{-} 1_{x^{\prime} \geqslant 0} \otimes \delta_{-}+u_{+} 1_{x^{\prime} \geqslant 0} \otimes \delta_{+}^{\prime}+u_{-} 1_{x^{\prime} \geqslant 0} \otimes \delta_{-}^{\prime}\right) .
$$

We write

$$
u_{e}(x, y, t)=u_{e}^{+}(x, y, t)+u_{e}^{-}(x, y, t)
$$

with

$$
u_{e}^{+}(x, y, t)=\left(\Delta-\partial_{t^{2}}^{2}\right)^{-1}\left[1_{x>0} \partial_{+} u_{+}\left(x^{\prime}, t^{\prime}\right) \otimes \delta_{+}+u_{+}\left(x^{\prime}, a\left(x^{\prime}\right), t^{\prime}\right) \otimes \delta_{+}^{\prime}\right] .
$$

We prove in this section the 
LEMMA 5.2. - There exists symbols $\sigma_{ \pm}(x, y, \tau), p_{ \pm}(x, \tau), q_{ \pm}(x, \tau), \widetilde{q}_{ \pm}(x, \tau)$, satisfying the inequality (2), such that

$$
\begin{aligned}
u_{ \pm}(x, y, t) & =\frac{1}{2 \pi} \int \sigma_{ \pm}(x, y, \tau) e^{i \tau\left(t-\theta_{ \pm}(x, y)\right)} \mathrm{d} \tau \\
\partial_{+} u_{+}(x, t) & =\frac{1}{2 \pi} \int i \tau p_{+}(x, \tau) e^{i \tau\left(t-\theta_{a}(x)\right)} \mathrm{d} \tau \\
u_{+}(x, a(x), t) & =\frac{1}{2 \pi} \int q_{+}(x, \tau) e^{i \tau\left(t-\theta_{a}(x)\right)} \mathrm{d} \tau \\
\partial_{x}\left[u_{+}(x, a(x), t)\right] & =\frac{1}{2 \pi} \int i \tau \widetilde{q}_{+}(x, \tau) e^{i \tau\left(t-\theta_{a}(x)\right)} \mathrm{d} \tau \\
\partial_{-} u_{-}(x, t) & =\frac{1}{2 \pi} \int i \tau p_{-}(x, \tau) e^{i \tau\left(t-\theta_{b}(x)\right)} \mathrm{d} \tau \\
u_{-}(x, b(x), t) & =\frac{1}{2 \pi} \int q_{-}(x, \tau) e^{i \tau\left(t-\theta_{b}(x)\right)} \mathrm{d} \tau \\
\partial_{x}\left[u_{-}(x, b(x), t)\right] & =\frac{1}{2 \pi} \int i \tau \widetilde{q}_{-}(x, \tau) e^{i \tau\left(t-\theta_{b}(x)\right)} \mathrm{d} \tau
\end{aligned}
$$

Note that $u_{ \pm}(0,0, t)=\frac{1}{2 \pi} \int \sigma_{ \pm}(0,0, \tau) e^{i t \tau} \mathrm{d} \tau$.

This is a consequence of the relations (1), (11), (16). We obtain

$$
\begin{aligned}
\sigma_{ \pm}(x, y, \tau) & =\sigma_{i}(x, y, \tau)-\sigma_{ \pm}^{z}(x, y, \tau) \\
p_{+}(x, \tau) & =\frac{\partial_{+} \sigma_{+}}{i \tau}-\partial_{+} \theta_{+} \sigma_{+}(x, a(x), \tau) \\
p_{-}(x, \tau) & =\frac{\partial_{-} \sigma_{-}}{i \tau}-\partial_{-} \theta_{-} \sigma_{-}(x, a(x), \tau) \\
q_{+}(x, \tau) & =\sigma_{+}(x, a(x), \tau) \\
q_{-}(x, \tau) & =\sigma_{-}(x, b(x), \tau) \\
\widetilde{q}_{+}(x, \tau) & =-\frac{\partial_{x} \theta_{+}+a^{\prime}(x) \partial_{y} \theta_{+}}{\left(1+\left(a^{\prime}(x)\right)^{2}\right)^{1 / 2}} \sigma_{+}(x, a(x), \tau)+\frac{1}{i \tau} \frac{\partial_{x} \sigma_{+}+a^{\prime}(x) \partial_{y} \sigma_{+}}{\left(1+\left(a^{\prime}(x)\right)^{2}\right)^{1 / 2}} \\
& =\frac{a^{\prime}(x)}{\left(1+\left(a^{\prime}(x)\right)^{2}\right)^{1 / 2}} p_{+}(x, \tau)+\left(1+\left(a^{\prime}(x)\right)^{2}\right)^{1 / 2}\left[\frac{\partial_{x} \sigma_{+}}{i \tau}-\partial_{x} \theta_{+} q_{+}(x, \tau)\right] \\
\widetilde{q}_{-}(x, \tau) & =-\frac{\partial_{x} \theta_{-}+b^{\prime}(x) \partial_{y} \theta_{-}}{\left(1+\left(b^{\prime}(x)\right)^{2}\right)^{1 / 2}} \sigma_{-}(x, b(x), \tau)+\frac{1}{i \tau} \frac{\partial_{x} \sigma_{-}+b^{\prime}(x) \partial_{y} \sigma_{-}}{\left(1+\left(b^{\prime}(x)\right)^{2}\right)^{1 / 2}}
\end{aligned}
$$

For example, we get, for $p_{+}(x, \tau)$

$$
p_{+}(x, \tau)=\frac{-2 \partial_{+} \theta_{i} z^{+}(x)}{z^{+}(x)-\partial_{+} \theta_{i}(x)} \sigma_{i}(x, a(x), \tau)+\frac{1}{i \tau}\left[\partial_{+} \sigma_{i}-\partial_{+} \sigma_{+}^{z}\right](x, \tau) .
$$

Similarly,

$$
u_{+}(x, a(x), t)=\frac{1}{2 \pi} \int_{\mathbb{R}} \mathrm{d} \tau e^{i \tau\left(t-\theta_{i}(x, a(x))\right)}\left(\sigma_{i}-\sigma_{+}^{z}\right)(x, a(x), \tau) \mathrm{d} \tau .
$$


Let us assume now

$$
\begin{aligned}
& u_{e}(x, y, t)=u_{e,+}(x, t)+u_{e,-}(x, y, t)+u_{e, d}(x, y, t) \\
& =\frac{1}{2 \pi} \int_{-\infty}^{+\infty} e^{i \tau\left(t-\theta_{+}(x, y)\right)} \sigma_{e,+}(x, y, \tau) \mathrm{d} \tau+\frac{1}{2 \pi} \int_{-\infty}^{+\infty} e^{i \tau\left(t-\theta_{-}(x, y)\right)} \sigma_{e,-}(x, y, \tau) \mathrm{d} \tau \\
& +\frac{1}{2 \pi} \int_{-\infty}^{+\infty} e^{i \tau(t-r)} \sigma_{e, d}(x, y, \tau) \mathrm{d} \tau
\end{aligned}
$$

As $\left(x_{0}, y_{0}\right) \in \Omega^{\varepsilon}$ satisfies $\theta_{ \pm}\left(x_{0}, y_{0}\right) \neq r_{0}$, there exists a neighborhood $W_{+}$of $-i+\theta_{+}\left(x_{0}, y_{0}\right)$, a neighborhood $W_{-}$of $-i+\theta_{-}\left(x_{0}, y_{0}\right)$, and a neighborhood $W$ of $-i+r_{0}$ such that $W \cap W_{+}=W \cap W_{-}=\varnothing$. We thus consider $w \in W$. The relation $T_{0} v(w, x, y, \lambda)=\left(\frac{\lambda}{2 \pi}\right)^{1 / 2} \int_{-\infty-i a_{0}}^{\infty-i a_{0}} e^{i \lambda \tau w-\lambda \tau^{2} / 2} \widehat{v}(x, y, \lambda \tau) \mathrm{d} \tau$ will allow us to compute the symbol associated with $u_{e}$. Owing to the fact that the Fourier transform in time of a wave conormal analytic is known, we get

$$
\begin{aligned}
& T_{0} u_{e, \pm}(w, x, y, \lambda)=e^{-\frac{\lambda}{2}\left(w-\theta_{ \pm}(x, y)\right)^{2}} \sum_{n \leqslant \lambda / C} \frac{1}{(2 \lambda)^{n} n !} \frac{\partial^{2 n} \sigma_{e, \pm}}{\partial \tau^{2 n}}\left(x, y, i \lambda\left(w-\theta_{ \pm}(x, y)\right)\right), \\
& T_{0} u_{e, d}(w, x, y, \lambda)=e^{-\frac{\lambda}{2}(w-r)^{2}} \sum_{n \leqslant \lambda / C} \frac{1}{(2 \lambda)^{n} n !} \frac{\partial^{2 n} \sigma_{e, d}}{\partial \tau^{2 n}}(x, y, i \lambda(w-r)) .
\end{aligned}
$$

The parametrix of the wave operator in the neighborhood of a point $\left(x_{0}, a\left(x_{0}\right)\right), x_{0}>0$ allow us to compute the solution on $\Sigma_{r,+}$, and it coincides with $u_{+}$. This will give the asymptotic behavior of the term obtained when the source term of the forward wave equation is $(1-\psi) S$. To apply Proposition 5.1, we compute the Fourier transform of $\psi S$ using the phase function $-i \tau \theta_{+}\left(x^{\prime}, a\left(x^{\prime}\right)\right)$. We have

LEMMA 5.3. - The Fourier transform in $x^{\prime}$ of the symbol

$$
S\left(x^{\prime}, \xi, \xi_{0}, \tau\right)=i \tau l\left(x^{\prime}, \xi, \xi_{0}, \tau\right) \psi\left(x^{\prime}\right) 1_{x^{\prime} \geqslant 0} e^{-i \tau \theta_{+}\left(x^{\prime}, a\left(x^{\prime}\right)\right)}
$$

is defined and admits, for $\xi^{\prime} \neq-\tau$, an asymptotic expansion of the form

$$
\sum \frac{S_{p}\left(\xi^{\prime}, \xi, \xi_{0}, \tau\right)}{\left(\xi^{\prime}+\tau\right)^{p+1}}
$$

where $S_{p}$ is a polynomial function of order at most $p$ in $\xi^{\prime}$ and such that

$$
S_{0}\left(\xi^{\prime}, \xi, \xi_{0}, \tau\right)=i \tau l\left(0, \xi, \xi_{0}, \tau\right) .
$$

In particular, if we consider

$$
S\left(x^{\prime}, \xi, \xi_{0}, \tau\right)=\partial_{+} u_{+}\left(x^{\prime}, \tau\right)+i u_{+}\left(x^{\prime}, a\left(x^{\prime}\right), \tau\right) \frac{\xi-a^{\prime}\left(x^{\prime}\right) \xi_{0}}{\left(1+\left(a^{\prime}\left(x^{\prime}\right)\right)^{2}\right)^{1 / 2}},
$$

we have

$$
S_{0}\left(\xi^{\prime}, \xi, \xi_{0}, \tau\right)=\frac{2 i \tau \sin \gamma_{+} \sigma_{i}(0,0, \tau)}{\sin \theta_{+}+\sin \gamma_{+}}\left[\sin \theta_{+}+\cos \gamma_{+} \frac{\xi}{\tau}-\sin \gamma_{+} \frac{\xi_{0}}{\tau}\right]
$$


We deduce the

Proposition 5.3. - We have

$$
\begin{aligned}
& D\left(\theta, \gamma_{+}, \gamma_{-}, \theta_{+}, \theta_{-}\right)= \\
& D_{+}\left(\theta, \gamma_{+}, \gamma_{-}, \theta_{+}, \theta_{-}\right)+D_{-}\left(\theta, \gamma_{+}, \gamma_{-}, \theta_{+}, \theta_{-}\right)+D_{d}\left(\theta, \gamma_{+}, \gamma_{-}, \theta_{+}, \theta_{-}\right),
\end{aligned}
$$

the two diffraction coefficients $D_{+}$and $D_{-}$(computed by the diffraction of the two faces) are

$$
\begin{aligned}
& D_{+}\left(\theta, \gamma_{+}, \gamma_{-}, \theta_{+}, \theta_{-}\right)=-\frac{\sin \gamma_{+} \cos \gamma_{+}}{\cos \gamma_{+}-\cos \left(\theta-\gamma_{+}\right)}\left(\sin \theta_{+}-\cos \left(\theta-\gamma_{+}\right)\right) \\
& D_{-}\left(\theta, \gamma_{+}, \gamma_{-}, \theta_{+}, \theta_{-}\right)=-\frac{\sin \gamma_{-} \cos \gamma_{-}}{\cos \gamma_{-}-\cos \left(\theta-\gamma_{-}\right)}\left(\sin \theta_{-}-\cos \left(\theta-\gamma_{-}\right)\right)
\end{aligned}
$$

We have

$$
D_{d}\left(\theta, \gamma_{+}, \gamma_{-}, \theta_{+}, \theta_{-}\right)=\frac{i}{2} e^{-k / 2} \frac{\left[F_{+}\left(-i, i \frac{\cos \left(\theta-\gamma_{+}\right)}{\cos \gamma_{+}}, k\right)+F_{-}\left(-i, i \frac{\cos \left(\theta-\gamma_{-}\right)}{\cos \gamma_{-}}, k\right)\right]}{\sigma_{i}(0,0, k)}
$$

Corollary 5.2. - The diffraction coefficient $D_{d}=D-D_{+}-D_{-}$is not equal to 0 . The diffraction by the wedge is thus not the superposition of the diffraction by the two antennas. There is a contribution, not equal to 0 , from the point $(0,0)$.

Proof of the Lemma and of the Corollary. - It is easy to check that there exists $l\left(x^{\prime}, \xi, \xi_{0}, \tau\right)$ such that

$$
\psi S\left(x^{\prime}, \xi, \xi_{0}, \tau\right)=i \tau l\left(x^{\prime}, \xi, \xi_{0}, \tau\right) \psi\left(x^{\prime}\right) e^{-i \tau \theta_{+}\left(x^{\prime}, a\left(x^{\prime}\right)\right)} .
$$

The term $l$ is equal to

$$
l\left(x^{\prime}, \xi, \xi_{0}, \tau\right)=s_{+}\left(x^{\prime}, \tau\right)+\left(\sigma_{i}-\sigma_{z}^{+}\right)\left(x^{\prime}, a\left(x^{\prime}\right), \tau\right) \frac{\xi-a^{\prime}\left(x^{\prime}\right) \xi_{0}}{\tau\left(1+\left(a^{\prime}\left(x^{\prime}\right)\right)^{2}\right)^{1 / 2}} .
$$

We check that

$$
l\left(0, \xi, \xi_{0}, \tau\right)=-\left(1+O\left(\tau^{-1}\right)\right) \frac{2 \partial_{+} \theta_{i}(0) \sigma_{i}(0,0, \tau)}{z^{+}(0)-\partial_{+} \theta_{i}(0)}\left[z^{+}(0)+\cos \gamma_{+} \frac{\xi}{\tau}-\sin \gamma_{+} \frac{\xi_{0}}{\tau}\right]
$$

Denote by $M$ the operator

$$
M F\left(x^{\prime}\right)=\frac{\partial}{\partial x^{\prime}}\left[\frac{F\left(x^{\prime}\right) \psi\left(x^{\prime}\right)}{\xi^{\prime}+\tau\left[\partial_{x} \theta_{+}+a^{\prime}\left(x^{\prime}\right) \partial_{y} \theta_{+}\right]\left(x^{\prime}, a\left(x^{\prime}\right)\right)}\right] .
$$

The Fourier transform $\mathcal{F}\left(\xi^{\prime}, \xi, \xi_{0}, \tau\right)$ of $S$ is

$$
\mathcal{F}\left(\psi S 1_{x^{\prime} \geqslant 0}\right)\left(\xi^{\prime}, \xi, \xi_{0}, \tau\right)=\int_{0}^{l_{1}} F\left(x^{\prime}\right) \psi\left(x^{\prime}\right) e^{-i \tau \theta_{+}\left(x^{\prime}, a\left(x^{\prime}\right)\right)-i x^{\prime} \xi^{\prime}} \mathrm{d} x^{\prime} .
$$


Usual integration by parts leads to the formula, owing to $\psi^{(j)}\left(l_{1}\right)=0$ for all $j \geqslant 0$

$$
\begin{aligned}
\mathcal{F}\left(\psi S 1_{x^{\prime} \geqslant 0}\right)\left(\xi^{\prime}, \xi, \xi_{0}, \tau\right) & = \\
& \sum_{p=0}^{p=N}(-i)^{p} \frac{M^{p} F(0)}{\xi^{\prime}+\tau}+(-i)^{N} \int_{0}^{l_{1}} M^{N} F\left(x^{\prime}\right) e^{-i \tau \theta_{+}\left(x^{\prime}, a\left(x^{\prime}\right)\right)-i x^{\prime} \xi^{\prime}} \mathrm{d} x^{\prime} .
\end{aligned}
$$

We note that $\frac{M^{p} F(0)}{\xi^{\prime}+\tau}$ is a classical symbol in the variables $\left(\xi^{\prime}, \tau\right)$, of order $-p-1$. We thus gave an asymptotic expansion of the symbol of $\mathcal{F}\left(\psi S 1_{x^{\prime}} \geqslant 0\right)$. The leading order term of the asymptotic expansion of the symbol of $\mathcal{F}\left(\psi S 1_{x \geqslant 0}\right)$ is given by

$$
S_{0}\left(\xi^{\prime}, \xi, \xi_{0}, \tau\right)=\frac{i \tau \sigma_{+}(0,0, \tau)}{\xi^{\prime}+\tau}\left[\cos \gamma_{+} \frac{\xi}{\tau}-\sin \gamma_{+} \frac{\xi_{0}}{\tau}+\sin \gamma_{+}\right]
$$

We obtained the leading order term of the symbol as

$$
\frac{2 i \tau \sin \gamma_{+} \sigma_{i}(0,0, \tau)}{\left(\xi^{\prime}+\tau\right)\left(\sin \theta_{+}+\sin \gamma_{+}\right)}\left[\sin \theta_{+}+\cos \gamma_{+} \frac{\xi}{\tau}-\sin \gamma_{+} \frac{\xi_{0}}{\tau}\right]
$$

From Proposition 5.1, we get

$$
\xi^{\prime}=-\tau \frac{x+a y}{r}=-\tau \frac{\cos \left(\theta-\gamma_{+}\right)}{\cos \gamma_{+}}, \quad \xi=-\tau \cos \theta, \quad \xi_{0}=-\tau \sin \theta .
$$

The result of Proposition 5.3 follows.

\subsection{Proof of Theorem 1}

We consider the system of unknowns written in Section 4 in (93) and (94). It has been shown that $u_{ \pm} \in H_{\text {loc }}^{1}\left(\mathbb{R}^{2} \times \mathbb{R}_{t}\right)$ and that $\widetilde{f}_{ \pm} \in L_{\text {loc }}^{2}\left(\Delta_{ \pm}\right)$with support in $\{0 \leqslant x \leqslant C t\}$.

We introduce $L_{ \pm}=J_{\rho}\left(2 \phi_{2} \psi \partial_{ \pm} u_{i}+l_{2}^{ \pm}+l_{3}^{ \pm}+o_{ \pm}+\left(T_{0} \otimes T_{1}\right)\left(\widetilde{r}_{ \pm}\right)\right)$. We notice that $f_{ \pm}-z^{ \pm}(x) \partial_{t} g_{ \pm}(x)=z^{ \pm}(x)\left[u-u_{ \pm}\right](x) \phi_{1}^{\prime}(t)$, hence it is supported outside $t=0$. We introduce $R E_{ \pm}=o_{ \pm}^{\prime}+J_{\rho}\left(T_{0} \otimes T_{1}\right)\left(z^{ \pm}(x)\left[u-u_{ \pm}\right](x) \phi_{1}^{\prime}(t)\right)$, the terms $o_{ \pm}^{\prime}$ are the difference $J_{\rho}\left[\left(T_{0} \otimes T_{1}\right)\left(z^{ \pm} \partial_{t} g_{ \pm}\right)\right]-i \lambda$ op $\left(z^{ \pm}\right)\left(T_{0} \otimes T_{1}\right) g_{ \pm}$. The system (94) of Proposition 4.8 yields

$$
J_{\rho} \mathrm{op}(b) J_{\rho}^{-1}\left(\begin{array}{c}
J_{\rho} F_{+} \\
J_{\rho} F_{-} \\
J_{\rho} G_{+} \\
J_{\rho} G_{-} \\
J_{\rho} \widetilde{G}_{+} \\
J_{\rho} \widetilde{G}_{-}
\end{array}\right)=\left(\begin{array}{c}
L_{+} \\
L_{-} \\
R E_{+} \\
R E_{-}
\end{array}\right)
$$

Note that

$$
\left(T_{0} \otimes T_{1}\right)\left(h_{+}\right)=\left(T_{0} \otimes T_{1}\right)\left(2 \psi \phi_{1} \partial_{+} u_{i}\right)+l_{2}^{+}+l_{3}^{+}+\sum_{n \leqslant \lambda / C} \frac{r_{n}(w, \lambda)}{(z+w)^{n+1}}+r
$$

where $r$ is negligible in $H_{\phi_{0}}\left(W_{\kappa}, H_{U}\right.$ ) (Sjöstrand space defined by Definition 2.2. of [20] p. 350) and where $r_{n}$ satisfies the inequality

$$
\left|r_{n}(w, \lambda)\right| \leqslant D e^{\lambda \phi_{1}(w)}[\theta(C)]^{n}
$$


with $\theta(C) \rightarrow 0$ if $C \rightarrow \infty$.

The elements $L_{ \pm}$belong to $H_{\phi_{1}}\left(W_{\kappa}, H_{2 / 3}^{ \pm}\right)$. The unknowns $J_{\rho} F_{ \pm}, J_{\rho} G_{ \pm}, J_{\rho} \widetilde{G}_{ \pm}$are in $H_{\phi_{0}}\left(W_{\kappa}, H_{2 / 3}^{ \pm}\right)$. The symbols $\widetilde{s}^{ \pm, \mp}=J_{\rho} s^{ \pm, \mp} J_{\rho}^{-1}, \widetilde{r}^{ \pm, \mp, 0}=J_{\rho} r^{ \pm, \mp, 0} J_{\rho}^{-1}, \widetilde{r}^{ \pm, \mp, 1}=$ $J_{\rho} r^{ \pm, \mp, 1} J_{\rho}^{-1}$ are defined from $H_{1 / 3}^{\mp}$ to $H_{2 / 3}^{ \pm}$. The symbols $\widetilde{q}^{ \pm}=J_{\rho} q^{ \pm} J_{\rho}^{-1}, \tilde{i}^{ \pm, 1}=$ $J_{\rho} i^{ \pm, 1} J_{\rho}^{-1}, \widetilde{i}^{ \pm, 0}=J_{\rho} i^{ \pm, 0} J_{\rho}^{-1}$ are defined from $H_{1 / 3}^{\mp}$ to $H_{1 / 3}^{ \pm}$. This system is thus in the chain of spaces $H_{\sigma}^{+} \otimes H_{\sigma}^{-}, \sigma \in[1 / 3,2 / 3]$.

The principal symbol of $L_{ \pm}$, denoted by $L_{ \pm}^{0}$, is given by

$$
L_{ \pm}^{0}(w, z, \lambda)=\lambda w J_{\rho}\left(S I_{ \pm}\right) \sigma_{i}(0,0, i \lambda w) e^{-\frac{\lambda}{2} w^{2}}
$$

The theorem 1 is a consequence of the invertibility of the system corresponding to the principal term of the operator $J_{\rho} \mathrm{op}(b) J_{\rho}^{-1}$, which is $B(1)$. Moreover, as we have the equality (118), the principal symbol to be studied is

$$
A(1)=\left(\begin{array}{ccccccc}
I & -S(1) & -I^{0}(1) & -I^{1}(1) & -T^{0}(1) & -T^{1}(1) & 0 \\
-S(1) & I & -T^{0}(1) & -T^{1}(1) & -I^{0}(1) & -I^{1}(1) & 0 \\
I & 0 & -i \sin \theta_{+} & 0 & 0 & 0 & 0 \\
0 & I & 0 & 0 & -i \sin \theta_{-} & 0 & 0 \\
0 & 0 & z & 1 & 0 & 0 & -1 \\
0 & 0 & 0 & 0 & z & 1 & -1
\end{array}\right),
$$

where the last column takes into account the term $u_{+}(0,0, t)-u_{-}(0,0, t)$ and the two last lines express the relation between the Laplace transform of $f$ and of $\partial_{x} f$, for $f \in H^{1}\left(\mathbb{R}_{+}\right)$. This construction of $A(1)$ from $B$ is natural owing to the definition of $G$ and $\widetilde{G}$ but has to be taken explicitly into account. As the operator $A(1)$ is invertible in the space $\left(H_{0}^{+} \times H_{0}^{-}\right)^{3}$ (by rearranging the unknowns), we obtain the relation giving $F_{ \pm}, G_{ \pm}, \widetilde{G}_{ \pm}$:

$$
\left(\begin{array}{c}
F_{+} \\
F_{-} \\
G_{+} \\
G_{-} \\
-z G_{+}(z)-G_{+}(0) \\
-z G_{-}(z)-G_{-}(0)
\end{array}\right)=M(A(1))^{-1}\left(\begin{array}{c}
S I_{+} \\
S I_{-} \\
0 \\
0 \\
T_{0}\left[u_{+}(0,0, \cdot)-u_{-}(0,0, \cdot)\right]
\end{array}\right) .
$$

The invertibility of $A(1)$ in $H_{0}^{+} \times H_{0}^{-}$is proven in Proposition 4.10. The symbol $A(1)$ depends only on the constants $\theta_{+}, \theta_{-}, \gamma_{+}, \gamma_{-}$. Hence we get the solution of the system

$$
A(1)\left(\begin{array}{c}
\mathcal{K}_{+} \\
\mathcal{K}_{-} \\
K_{+} \\
\widetilde{K}_{+} \\
K_{-} \\
\widetilde{K}_{-}
\end{array}\right)(z)=\left(\begin{array}{c}
S I_{+} \\
S I_{-} \\
0 \\
0 \\
0 \\
0
\end{array}\right)(-i, z)
$$


equal to

$$
\left(\begin{array}{l}
i \sin \theta_{+} K_{+}^{\theta_{+}, \theta_{-}, \gamma_{+}, \gamma_{-}}(-i, z) \\
K_{+}^{\theta_{+}, \theta_{-}, \gamma_{+}, \gamma_{-}}(-i, z) \\
-z K_{+}^{\theta_{+}, \theta_{-}, \gamma_{+}, \gamma_{-}}(-i, z) \\
i \sin \theta_{-} K_{-}^{\theta_{+}, \theta_{-}, \gamma_{+}, \gamma_{-}}(-i, z) \\
K_{-}^{\theta_{+}, \theta_{-}, \gamma_{+}, \gamma_{-}}(-i, z) \\
-z K_{-}^{\theta_{+}, \theta_{-}, \gamma_{+}, \gamma_{-}}(-i, z)
\end{array}\right)
$$

We denote by $r=\sqrt{x^{2}+y^{2}}$ and $\theta$ the usual polar coordinates. The wave diffracted by the curved wedge with boundary condition is

$$
u_{d}(x, y, t)=\int e^{i k(t-r)} \sigma_{d}(x, y, k) \frac{d k}{2 \pi}
$$

where the first term of the symbol $\sigma_{d}$ is $\sigma_{d}^{0}$ with:

$$
\begin{aligned}
\sigma_{d}^{0}(x, y, k)=i \frac{\sigma_{i}(0,0, k)}{(k r)^{1 / 2}}\left[\left(\sin \theta_{+}-\sin \left(\theta-\gamma_{+}\right)\right) K_{+}\left(\cos \left(\theta-\gamma_{+}\right)\right)\right. \\
\left.\quad+\left(\sin \theta_{-}-\sin \left(\theta-\gamma_{-}\right)\right) K_{-}\left(\cos \left(\theta-\gamma_{-}\right)\right)\right]
\end{aligned}
$$

The end of the computation of the principal symbol of the diffracted solution follows exactly the same pattern as in Section 6 of [20]. In particular, the regularity results are the same.

We need to compute $g_{+}$and $g_{-}$, defined, as in (6.11), by:

$$
g_{ \pm}=\left[-\Delta+\partial_{t^{2}}^{2}\right]^{-1}\left(\tilde{f}_{ \pm} 1_{x>0} \otimes \delta_{+}\right)
$$

where the source $\widetilde{f}_{ \pm}$is computed using the derivative in time and the normal derivative. The solution $u_{d}$ will be $g_{+}+g_{-}$. We have:

$$
g_{+}(x, y, \tau)=\left(-\Delta-\tau^{2}\right)^{-1}\left(f_{+}\left(x^{\prime}, \tau\right) 1_{x^{\prime} \geqslant 0} \otimes \delta_{+}+\frac{f_{+}\left(x^{\prime}, \tau\right)}{i \tau z^{+}\left(x^{\prime}\right)} 1_{x^{\prime} \geqslant 0} \otimes \delta_{+}^{\prime}\right) .
$$

Introducing

$$
S_{+}\left(x^{\prime}, \xi, \xi_{0}, \tau\right)=f_{+}\left(x^{\prime}, \tau\right)\left(1+\frac{\xi-a^{\prime}\left(x^{\prime}\right) \xi_{0}}{\tau z^{+}\left(x^{\prime}\right)\left(1+\left(a^{\prime}\left(x^{\prime}\right)\right)^{2}\right)^{1 / 2}}\right),
$$

we find, using $f_{+}\left(x^{\prime}, \tau\right)=\frac{1}{2 \pi} \int_{\mathbb{R}} e^{i x^{\prime} \cdot \xi^{\prime}} \sigma_{+}\left(\xi^{\prime}, \tau\right) \mathrm{d} \xi^{\prime}$ that, owing to Lemma 5.3

$$
\mathcal{F}(\psi S)\left(\xi^{\prime}, \xi, \xi_{0}, \tau\right)=\frac{\sigma_{+}\left(\xi^{\prime}, \tau\right)}{\tau-\xi^{\prime}}\left(1+\frac{\cos \gamma_{+}}{\sin \theta_{+}} \frac{\xi}{\tau}-\frac{\sin \gamma_{+}}{\sin \theta_{+}} \frac{\xi_{0}}{\tau}\right) .
$$

We use Proposition 5.1 proven in Section 5 to obtain that

$$
\begin{aligned}
\left(\Delta-\partial_{t^{2}}^{2}\right)^{-1}\left[1_{x^{\prime} \geqslant 0} f\left(x^{\prime}, t\right) \otimes \delta_{+}+\frac{f\left(x^{\prime}, t\right) 1_{x^{\prime} \geqslant 0}}{i \tau z^{+}\left(x^{\prime}\right)} \otimes \delta_{+}^{\prime}\right](x, y, \lambda \tau) \simeq \\
e^{-i \tau r} \frac{e^{3 i \pi / 4}}{\cos \gamma_{+} \sqrt{8 \pi \tau r}} \frac{\sigma_{+}\left(\tau, \tau \frac{x+a y}{r}\right)}{1-\frac{x+a y}{r}}\left(1+\frac{-\cos \gamma_{+} x+\sin \gamma_{+} y}{\sin \theta_{+} r}\right)+\sigma_{s}
\end{aligned}
$$

where the term $\sigma_{s}$ is a remainder term of order $\lambda^{-1}$. 
We recall that the holomorphic function $F_{+}$is known from $f_{+}$through

$$
\begin{aligned}
F_{+}(w, z, \lambda) & =\int_{-\infty}^{+\infty} \int_{0}^{\infty} e^{i \lambda\left(x z-x^{2} / 2-(w-t)^{2} / 2\right)} f_{+}(x, t) \mathrm{d} x \mathrm{~d} t \\
& =\left(\frac{\lambda}{2 \pi}\right)^{2} \int_{\mathbb{R}^{3} \times \mathbb{R}_{+}} e^{\lambda\left(x z-x^{2} / 2-(w-t)^{2} / 2+i \tau t+i \xi^{\prime} x\right)} \sigma_{+}\left(\lambda \xi^{\prime}, \lambda \tau\right) \mathrm{d} x \mathrm{~d} t \mathrm{~d} \xi^{\prime} \mathrm{d} \tau
\end{aligned}
$$

The critical point in $t$ is $t=w-i \tau$, which implies, after the application of the stationary phase theorem in $x^{\prime}, \tau, \xi^{\prime}$ for $-\Re w$ and $-\Re z$ large

$$
\begin{aligned}
F_{+}(w, z, \lambda) & =\left(\frac{\lambda}{2 \pi}\right)^{3 / 2} \int_{\mathbb{R}^{3}} 1_{x \geqslant 0} e^{\lambda\left(x z-x^{2} / 2+i \xi^{\prime} x\right)} e^{\lambda \tau^{2} / 2+i \lambda \tau w} \sigma_{+}\left(\lambda \xi^{\prime}, \lambda \tau\right) \mathrm{d} x \mathrm{~d} \xi^{\prime} \mathrm{d} \tau \\
& =e^{-\lambda w^{2} / 2} \sum_{m, n \leqslant \lambda / C} \frac{1}{2^{n} \lambda^{n+m} n ! m !} \frac{\partial^{2 n} \partial^{2 m} \sigma_{+}}{\partial^{2 n} \tau \partial^{2 m} \xi^{\prime}}(i \lambda w, i \lambda z)\left(i \partial_{x}\right)^{m}\left(e^{-\lambda x^{2} / 2}\right) .
\end{aligned}
$$

We thus obtain $\sigma_{+}(i \lambda z, i \lambda w)$ in terms of $F_{+}(w, z, \lambda)$ for $-\Re w>D,-\Re z>D$. Using the maximum principle, we can propagate this equality to $w=-i \tau, z=i \tau \frac{x+a y}{r}$, and we obtain

$$
\sigma_{+}\left(-\lambda \tau \frac{x+a y}{r}, \lambda \tau\right) \simeq F_{+}\left(-i \tau, i \tau \frac{x+a y}{r}, \lambda\right) e^{-\lambda \tau^{2} / 2}
$$

Finally, by the maximum principle again, we can see that this equality is true for $\tau \in \mathbb{R}_{+}$, hence for $\tau=1$ and $\lambda=k>0$ we obtain

$$
\sigma_{+}\left(-k \frac{x+a y}{r}, k\right) \simeq F_{+}\left(-i, i \frac{x+a y}{r}, k\right) e^{-k / 2} .
$$

We obtain the symbol of $\sigma_{+}$once we have the symbol of $F_{+}$(given by (129)). Note that $T_{0} g_{+}(w, x, y, \tau)$ is given by

$$
T_{0} g_{+}(w, x, y, \lambda)=\left(\frac{\lambda}{2 \pi}\right)^{1 / 2} \int_{-\infty-i a}^{+\infty-i a} e^{i \lambda \tau w-\lambda \tau^{2} / 2+i \lambda \tau r}\left[e^{i \lambda \tau r} \widehat{g}_{+}(x, y, \lambda \tau)\right] \mathrm{d} \tau
$$

with $e^{i \tau r} \widehat{g}_{+}(x, y, \tau)$ is in $L^{2}$ in the variable $\tau$. The stationary phase theorem in the variable $\tau$ (which is correct for $-\Re w \gg 0$ ) whose critical point is $\tau_{c}=i(w-r)$ yields

$$
\begin{aligned}
& T_{0} g_{+}(w, x, y, \lambda) \simeq \frac{e^{-\lambda(w-r)^{2} / 2} e^{3 i \pi / 4}}{\sqrt{8 \pi r \lambda i(w-r)}} \sigma_{+}\left(i \lambda(w-r),-i \lambda(w-r) \frac{x+a y}{r}\right) . \\
& T_{0} g_{-}(w, x, y, \lambda) \simeq \frac{e^{-\lambda(w-r)^{2} / 2} e^{3 i \pi / 4}}{\sqrt{8 \pi r \lambda i(w-r)}} \sigma_{-}\left(i \lambda(w-r),-i \lambda(w-r) \frac{x+b y}{r}\right) .
\end{aligned}
$$

Note that, if $u_{d}(x, y, t)=(2 \pi)^{-1} \int_{\mathbb{R}} \sigma_{d}(x, y, \tau) e^{i \tau(t-r)} \mathrm{d} \tau$, then

$$
T_{0} u_{d}(w, x, y, \lambda)=e^{-\frac{\lambda}{2}(w-r)^{2}} \sum_{n \leqslant \lambda / C} \frac{1}{(2 \lambda)^{n} n !} \frac{\partial^{2 n} \sigma_{d}}{\partial_{\tau^{2 n}}}(x, y, i \lambda(w-r)) .
$$

As $u_{d}=g_{+}+g_{-}+v_{r}$, where $\operatorname{SS}\left(v_{r}\right) \cap \Sigma_{d}=\varnothing$, equalities (132), (133) and (134) yield, for $k \in \mathbb{R}_{+}$:

$$
\sigma_{d}(x, y, k)=\frac{e^{3 i \pi / 4}}{\sqrt{8 \pi k r}}\left[\sigma_{+}\left(-k \frac{x+a y}{r}, k\right)+\sigma_{-}\left(-k \frac{x+b y}{r}, k\right)\right]
$$


Equality (131) gives then

Hence

$$
\begin{aligned}
& \sigma_{+}\left(-k \frac{x+a y}{r}, k\right) \simeq e^{-k / 2} F_{+}\left(-i, i \frac{x+a y}{r}, k\right) \\
& \sigma_{-}\left(-k \frac{x+b y}{r}, k\right) \simeq e^{-k / 2} F_{-}\left(-i, i \frac{x+b y}{r}, k\right) .
\end{aligned}
$$

$$
\sigma_{d}(x, y, k)=\frac{e^{3 i \pi / 4}}{\sqrt{8 \pi k r}} e^{-k / 2}\left[F_{+}\left(-i, i \frac{x+a y}{r}, k\right)+F_{-}\left(-i, i \frac{x+b y}{r}, k\right)\right] .
$$

We have to identify $F_{+}$and $F_{-}$. These functions are the scattering functions associated with the normal derivative on each face, while the solution of the global system involves both the normal derivative on each face and the trace on each face. But, again from the system above, the normal derivative on the face + is equal to the trace on the face + multiplied by the coefficient $i \sin \theta_{ \pm}$, hence we get all the terms needed. We identified the diffraction term of Proposition 5.3. This ends the proof the diffraction relation of Theorem 1 .

The last item to prove is the result on $u_{d}+u_{0}$. In fact, the inversion of the system (94) leads to the expression of $f_{+}, f_{-}, g_{+}, g_{-}$, and we get a jump condition, owing to $u\left(0_{-}, b\left(0_{-}\right), t\right)=u\left(0_{+}, a\left(0_{+}\right), t\right)$ and $g_{+}(x, t)=u(x, a(x), t)-u_{+}(x, a(x), t), g_{-}(x, t)=$ $u(x, b(x), t)-u_{-}(x, b(x), t)$, hence $g_{+}(0, t)-g_{-}(0, t)=u_{-}(0,0, t)-u_{+}(0,0, t)$. We check that

$$
\begin{aligned}
g_{+} \delta_{+}^{\prime}+g_{-} \delta_{-}^{\prime}+\left(u_{+}(0,0, t)-\right. & \left.u_{-}(0,0, t)\right) \theta(x / R) \delta_{+}^{\prime}= \\
& \left(g_{+}+\left(u_{+}(0,0, t)-u_{-}(0,0, t)\right) \theta(x / R)\right) \delta_{+}^{\prime}+g_{-} \delta_{-}^{\prime},
\end{aligned}
$$

and that $\lim _{x \rightarrow 0_{+}}\left(g_{+}+\left(u_{+}(0,0, t)-u_{-}(0,0, t)\right) \theta(x / R)\right)=g_{+}(0, t)+u_{+}(0,0, t)-$ $u_{-}(0,0, t)=g_{-}(0, t)$. The distribution

$$
g_{+} \delta_{+}^{\prime}+g_{-} \delta_{-}^{\prime}+\left(u_{+}(0,0, t)-u_{-}(0,0, t)\right) \theta(x / R) \delta_{+}^{\prime}
$$

writes $h \otimes \delta_{\partial \Omega}^{\prime}$ with $h \in H^{1}(\partial \Omega)$, hence from Proposition 5.1 shows that $u_{d}+u_{0} \in$ $H_{\mathrm{loc}}^{3 / 2}\left(\bar{\Omega}^{\varepsilon}\right)$. The proof of Theorem 1 is now complete. 


\section{CHAPTER 6}

\section{APPENDIX}

\subsection{Properties of the Helmholtz equations around corners}

\subsubsection{Single-layer and double-layer distributions for the Helmholtz equa-} tion in $\mathbb{R}^{2}$. - In this Section, we recall the already known results for the distributions of single and double layer for the Helmholtz problem in an open set with boundary. This framework has been introduced to describe the influence of an electric current and of a magnetic current on a boundary.

Recall that Gérard and Lebeau [20] only used the single-layer boundary distributions. In this Memoire, it is not enough to consider these distributions; we have also to consider the double-layer distributions in order to take into account the jump of $u$ across the boundary. The approach here is entirely developed in the book of Colton and Kress [14].

We begin by recalling that, in $\mathbb{R}^{2}$, the fundamental solution of the Laplace operator, which is the distribution $\Phi_{0}(x, y)$ verifying $\Delta_{y} \Phi_{0}(x, y)=\delta_{y=x}$ is $\Phi_{0}(x, y)=$ $-\frac{1}{2 \pi} \log |x-y|, x \neq y$. If $J_{0}$ is the solution (usual Bessel function of order 0 ) which is finite for $t \rightarrow 0$ of the differential equation $t f^{\prime \prime}(t)+f^{\prime}(t)+t f(t)=0$, and $Y_{0}$ is the linearly independent solution of this same equation known as the Neumann function of order 0 , we introduce the Hankel function of order 0 of the first kind $H_{0}^{(1)}=J_{0}+i Y_{0}$. The fundamental outgoing solution of the Helmholtz equation in $\mathbb{R}^{2}$ is then $\frac{i}{2} H_{0}^{(1)}(\tau|x-y|)=\Phi_{\tau}(x, y)$.

Introduce a bounded set $D$ whose boundary $\partial D$ is of class $C^{2}$. The outgoing unit normal vector $n(x)$ is of class $C^{1}$ and is defined on any point of $\partial D$. We can apply Theorem 2.4 of $[\mathbf{1 4}]$ hence, for $u_{\tau}$ a solution in $C^{2}\left(\mathbb{R}^{2}-\bar{D}\right) \cap C\left(\mathbb{R}^{2}-D\right)$ of $\left(\Delta+\tau^{2}\right) u=0$ in $\mathbb{R}^{2}-\bar{D}$, which admits a normal derivative $\partial_{n} u$ on the boundary (defined as the uniform limit on $\partial D$, for $h \rightarrow 0_{+}$of $n(x) \cdot \nabla u(x+h n(x))$ ), we have

$$
u_{\tau}(x)=\int_{\partial D}\left[u_{\tau}(y) \partial_{n_{y}} \Phi_{\tau}(x, y)-\partial_{n} u_{\tau}(y) \Phi_{\tau}(x, y)\right] \mathrm{d} s(y)
$$


From this representation, we introduce the single layer and double layer potentials, for $\phi$ a function of $L^{1}(\partial D)$ :

$$
\begin{aligned}
& u_{s}^{\tau}(\phi)(x)=\int_{\partial D} \phi(y) \Phi_{\tau}(x, y) \mathrm{d} s(y), \\
& u_{d}^{\tau}(\phi)(x)=\int_{\partial D} \phi(y) \partial_{n_{y}} \Phi_{\tau}(x, y) \mathrm{d} s(y) .
\end{aligned}
$$

Assume $\phi \in C^{0}(\partial D)$. The function $u_{s}^{\tau}(\phi)$ is continuous in $\mathbb{R}^{2}$. If we define, for $x \in \partial D$, the distributions

$$
\begin{gathered}
\partial_{n} u_{s}^{\tau}(\phi)(x)=\lim _{h \rightarrow 0_{+}} n(x) \nabla u_{s}^{\tau}(\phi)(x+h n(x)) \\
\partial_{n}^{\prime} u_{s}^{\tau}(\phi)(x)=\lim _{h \rightarrow 0_{+}} n(x) \nabla u_{s}^{\tau}(\phi)(x-h n(x)) \\
u_{d,+}^{\tau}(\phi)(x)=\lim _{h \rightarrow 0_{+}} u_{d}^{\tau}(\phi)(x+h n(x)) \\
u_{d,-}^{\tau}(\phi)(x)=\lim _{h \rightarrow 0_{+}} u_{d}^{\tau}(\phi)(x-h n(x))
\end{gathered}
$$

we have, on the boundary

$$
\begin{gathered}
u_{s}^{\tau}(\phi)=\int_{\partial D} \phi(y) \Phi_{\tau}(x, y) \mathrm{d} s(y) \\
\partial_{n} u_{s}^{\tau}(x)=\int_{\partial D} \phi(y) \partial_{n_{y}} \Phi_{\tau}(x, y) \mathrm{d} s(y)-\frac{1}{2} \phi(x) \\
\partial_{n}^{\prime} u_{s}^{\tau}(x)=\int_{\partial D} \phi(y) \partial_{n_{y}} \Phi_{\tau}(x, y) \mathrm{d} s(y)+\frac{1}{2} \phi(x) \\
u_{d,+}^{\tau}(\phi)(x)=\int_{\partial D} \phi(y) \partial_{n_{y}} \Phi_{\tau}(x, y) \mathrm{d} s(y)+\frac{1}{2} \phi(y) \\
u_{d,-}^{\tau}(\phi)(x)=\int_{\partial D} \phi(y) \partial_{n_{y}} \Phi_{\tau}(x, y) \mathrm{d} s(y)-\frac{1}{2} \phi(y) .
\end{gathered}
$$

The integrals written here are improper integrals. There are properties of Holder continuity for these integrals (Theorem 3.3 of [14]).

We define the operators

$$
\begin{aligned}
& S^{\tau} \phi(x)=2 \int_{\partial D} \Phi_{\tau}(x, y) \phi(y) \mathrm{d} s(y) \\
& K^{\tau} \phi(x)=2 \int_{\partial D} \partial_{n_{y}} \Phi_{\tau}(x, y) \phi(y) \mathrm{d} s(y) \\
& K^{\prime}, \tau \phi(x)=2 \int_{\partial D} \partial_{n_{x}} \Phi_{\tau}(x, y) \phi(y) \mathrm{d} s(y) \\
& T^{\tau} \phi(x)=2 \partial_{n_{x}} \int_{\partial D} \partial_{n_{y}} \Phi_{\tau}(x, y) \phi(y) \mathrm{d} s(y) .
\end{aligned}
$$

The operator $S^{\tau}$ is bounded from $L^{2}(\partial D)$ to $H^{1}(\partial D)$. If the boundary $\partial D$ is more regular than $C^{2}$, that is $C^{2, \alpha}$, the operators $K^{\tau}, K^{\prime}, \tau$ are also bounded from $L^{2}(\partial D)$ into $H^{1}(\partial D)$, and $T$ is bounded from $H^{1}(\partial D)$ to $L^{2}(\partial D)$. 
Introduce

$$
u^{\tau}(x)=\int_{\partial D}\left[\partial_{n_{y}} \Phi_{\tau}(x, y)-i \zeta \Phi_{\tau}(x, y)\right] \phi(y) \mathrm{d} s(y)
$$

The potential $u^{\tau}$ solves the problem

$$
\left\{\begin{array}{l}
\left(\Delta+\tau^{2}\right) u^{\tau}=0 \text { in } \mathbb{R}^{2}-D \\
\left.u\right|_{\partial D}=f \in C^{0}(\partial D)
\end{array}\right.
$$

if $\phi$ is solution of the impedance layer potential problem ( $\zeta$ is a positive constant, called the impedance of the problem)

$$
\phi+K \phi-i \zeta S \phi=2 f .
$$

This is a problem of the kind $(I+A) \phi=2 f, A$ is a compact operator from $C(\partial D)$ to $C(\partial D)$ under the previous hypotheses.

In [14], they construct the layer potential problem for an open set with a corner, which angle is $\gamma, 0<\gamma<2 \pi$. We restrict ourselves to $\gamma \in] 0, \pi[$. The boundary is thus the reunion of piecewise analytic parts, and is $C^{2}$ except at $S$. If $x_{0}$ is the position of $S$, we reduce the problem to

$$
u(x)=\int_{\partial D}\left[\left[\partial_{n_{y}} \Phi_{\tau}(x, y)-i \zeta \Phi_{\tau}(x, y)\right] \phi(y)-\partial_{n_{y}} \Phi_{0}(x, y) \phi\left(x_{0}\right)\right] \mathrm{d} s(y) .
$$

The equation (137) is then

$$
\begin{array}{r}
\phi(x)-\phi\left(x_{0}\right)+2 \int_{\partial D}\left[\partial_{n_{y}} \Phi_{\tau}(x, y)-i \zeta \Phi_{\tau}(x, y)\right] \phi(y) \mathrm{d} s(y) \\
-2 \int_{\partial D} \partial_{n_{y}} \Phi_{0}(x, y) \phi\left(x_{0}\right) d y=2 f
\end{array}
$$

We introduce the operator

$$
K_{0} \phi(x)=2 \int_{\partial D}\left[\partial_{n_{y}} \Phi_{0}(x, y)\left[\phi(y)-\phi\left(x_{0}\right)\right] \mathrm{d} s(y)\right.
$$

and its truncated form $K_{0, r} \phi=\psi K_{0}(\psi \phi)$, where $\psi$ is a cutoff function null in $\left|x-x_{0}\right| \geqslant$ $r$, identically equal to 1 in the neighborhood of 0 . Colton and Kress prove that there exists a $r$ such that $\left\|K_{0, r}\right\|_{\infty, 0}<1$. The singularity of $\Phi_{\tau}$ and of $\Phi_{0}$ in $\log |x-y|$ induce a singularity for the normal derivative in $1 /|x-y|$. This singularity cancels in the difference $\partial_{n_{y}} \Phi_{\tau}-\partial_{n_{y}} \Phi_{0}$. Hence for $x \notin \partial D$, the relations

$$
\begin{array}{r}
\int_{\partial D}\left(\partial_{n_{y}} \Phi_{\tau}-\partial_{n_{y}} \Phi^{0}\right)(x, y) \mathrm{d} s(y)=0 \\
\int_{\partial D} \Phi_{\tau}(x, y) \mathrm{d} s(y)=0
\end{array}
$$

lead to the equation replacing (138) for $\widetilde{\phi}(x)=\phi(x)-\phi\left(x_{0}\right)$ :

$\widetilde{\phi}(x)+K_{0} \phi(x)+2 \int_{\partial D} \partial_{n_{y}} \Phi_{\tau}-\partial_{n_{y}} \Phi_{0} \widetilde{\phi}(y) \mathrm{d} s(y)-2 i \zeta \int_{\partial D} \Phi_{\tau}(x, y) \widetilde{\phi}(y) \mathrm{d} s(y)=2 f(x)$. 
Hence, with continuous data $f$, there exists a potential solution of (137). This solution is continuous, but its derivative has singularities at the corner (as we may see in Grisvard, [21], Dauge [2]...).

6.1.2. Existence and uniqueness of some variational problems. - In this part of the Annex, we deal with the problems which are related to the existence of a solution of the wave equation with the mixed boundary condition in a domain with corners. We begin with the construction of the normal derivative. We introduce $\mathcal{O}^{R}$ the bounded open subset of $\mathbb{R}^{2}$ equal to $\left(\mathbb{R}^{2}-F\right) \cap\{|x| \leqslant R\}$. It is clear that $\mathcal{O}^{R}$ admits a Lipschitz boundary, piecewise analytic (we may note that $\partial \mathcal{O}^{R}=$ $C(R) \cup \Delta_{+}^{R} \cup \Delta_{-}^{R} \cup\left\{O, A_{+}, A_{-}\right\}$, where $A_{ \pm}$is the intersection of $\Delta_{ \pm}$and of the circle of radius $R$, and $C(R)$ is the part of the circle of radius $R$ linking $A_{+}$and $A_{-}$in $\left.\mathbb{R}^{2}-F\right)$. Hence, from Theorem 1.5.1.3 of [21], the mapping $\left.u \rightarrow u\right|_{\partial \mathcal{O}}$ is well defined as an operator from $H^{1}\left(\mathcal{O}^{R}\right)$ onto $H^{1 / 2}\left(\partial \mathcal{O}^{R}\right)=H^{1 / 2}(C(R)) \otimes H^{1 / 2}\left(\Delta_{+}^{R}\right) \otimes H^{1 / 2}\left(\Delta_{-}^{R}\right)$. Moreover, the mapping $\left.u \rightarrow \partial_{n} u\right|_{\partial \mathcal{O}^{R}}$ is well defined for $u \in H^{2}\left(\mathcal{O}^{R}\right)$. The half Green's formula is true for a Lipschitz boundary (Lemma 1.5.3.7 of [21]):

For $u \in H^{2}\left(\mathcal{O}^{R}\right)$ and $v \in H^{1}\left(\mathcal{O}^{R}\right)$, we have

$$
\int_{\mathcal{O}^{R}}(\Delta u) v \mathrm{~d} x+\int_{\mathcal{O}^{R}} \nabla u \cdot \nabla v \mathrm{~d} x=\left.\int_{\partial \mathcal{O}^{R}} \partial_{n} u v\right|_{\partial \mathcal{O}^{R}} \mathrm{~d} \sigma .
$$

We know that the space $C_{0}^{\infty}\left(\overline{\mathcal{O}}^{R}\right)$ is dense in $E(\Delta)=\left\{u \in H^{1}\left(\mathcal{O}^{R}\right), \Delta u \in L^{2}\left(\mathcal{O}^{R}\right)\right\}$ (Lemma 1.5.3.9 of [21]). Hence the relation for $u \in H^{2}\left(\mathcal{O}^{R}\right)$ and $v \in H^{1}\left(\mathcal{O}^{R}\right)$ extends (because $H^{2}\left(\mathcal{O}^{R}\right)$ is dense in $E\left(\Delta, \mathcal{O}^{R}\right)$ ) for $u \in E(\Delta)$ and $v \in H^{1}\left(\mathcal{O}^{R}\right)$ and defines the normal derivative, for $u \in E\left(\Delta, \mathcal{O}^{R}\right)$, in the dual space of $H^{1 / 2}\left(\partial \mathcal{O}^{R}\right)$ which is $H^{-1 / 2}\left(\mathcal{O}^{R}\right)$ through the equality (139). Here we made use of the fact that the right continuous inverse of the mapping $\left.u \rightarrow u\right|_{\partial \mathcal{O}^{R}}$ exists (which indicates that, for every function $\psi$ in $H^{1 / 2}\left(\partial \mathcal{O}^{R}\right)$, there exists a function $u$ of $H^{1}\left(\mathcal{O}^{R}\right)$ such that $\left.\left.u\right|_{\partial \mathcal{O}^{R}}=\psi\right)$.

The extension to the non bounded set $\mathcal{O}=\mathbb{R}^{2}-F$ is straightforward.

For $u \in C_{0}^{\infty}(\overline{\mathcal{O}})$, the mapping $\left.u \mapsto u\right|_{\Delta_{+} \cup \Delta_{-}}$is continuous and extends to $u \in$ $H^{1}(\mathcal{O})$, and has the same properties. There is no difference for the proof of the Green's formula for this unbounded domain. As $C_{0}^{\infty}(] 0,+\infty[)$ is (up to the diffeomorphism) dense in $H^{1 / 2}\left(\Delta_{ \pm}\right)$we may define by duality $H^{-1 / 2}(\partial \mathcal{O})$. Hence we have

LEMmA 6.1. - The open set $\Theta$ on which we consider the result is either $\mathcal{O}$ or $\mathcal{O}^{R}$ or $F$. For every $u \in E(\Delta, \Theta)$ and $v \in H^{1}(\Theta)$, the normal derivative is defined through the relation (139):

$$
\int_{\Theta} \Delta u v \mathrm{~d} x+\int_{\Theta} \nabla u \nabla v \mathrm{~d} x=\left\langle\partial_{n} u,\left.v\right|_{\Theta}\right\rangle
$$

where $\langle$,$\rangle denotes the duality between H^{1 / 2}(\Theta)$ and $H^{-1 / 2}(\Theta)$. 
The normal derivative being thus defined, we introduce the problem for $u \in H^{1}(\mathcal{O})$,

$$
\left\{\begin{array}{l}
\left(\Delta+\omega^{2}\right) u(x, y, \omega)=f \text { in } \mathcal{O} \\
\partial_{n} u(x, \omega)+\left.i \omega z u\right|_{\partial \mathcal{O}}=g \text { on } \partial \mathcal{O}
\end{array}\right.
$$

The last line is exactly (remember that $\partial_{ \pm} u=-\left.\partial_{n} u\right|_{\Delta_{ \pm}}$)

$$
\partial_{+} u-i \omega z^{+}(x) u(x, a(x), \omega)=g_{+}(x, \omega), \quad \partial_{-} u-i \omega z(x) u(x, b(x), \omega)=g_{-}(x, \omega) .
$$

We seek a solution of (140) for $f \in L^{2}(\mathcal{O}), g \in H^{-1 / 2}(\partial \mathcal{O})$. Hence $\Delta u=f-\omega^{2} u \in$ $L^{2}(\mathcal{O})$ and we may apply Lemma 6.1 , the normal derivative is well defined.

LEMMA 6.2. - Under the condition

$$
\Im \omega<0, \quad \Re\left(z^{+}(x)\right)>0, \quad \Re\left(z^{-}(x)\right)>0, \quad z^{ \pm} \text {bounded }
$$

the problem (140) has a unique solution in $H^{1}(\mathcal{O})$.

Let $\phi \in C_{0}^{\infty}(\overline{\mathcal{O}})$. We have, if $u$ is a solution

$$
\omega^{2} \int_{\mathcal{O}} u \bar{\phi} \mathrm{d} x-\int_{\mathcal{O}} \nabla u \nabla \bar{\phi} \mathrm{d} x+\int_{\partial \mathcal{O}} \partial_{n} u \bar{\phi} \mathrm{d} \sigma=\int_{\mathcal{O}} f \bar{\phi} \mathrm{d} x
$$

or, using the boundary condition

$$
\omega^{2} \int_{\mathcal{O}} u \bar{\phi} \mathrm{d} x-\int_{\mathcal{O}} \nabla u \nabla \bar{\phi} \mathrm{d} x+\int_{\partial \mathcal{O}}(g-i \omega z u) \bar{\phi} \mathrm{d} \sigma=\int_{\mathcal{O}} f \bar{\phi} \mathrm{d} x
$$

Introduce, for $(u, v) \in H^{1}(\mathcal{O})$

$$
\begin{gathered}
-a(u, v)=\omega^{2} \int_{\mathcal{O}} u \bar{v} \mathrm{~d} x-\int_{\mathcal{O}} \nabla u \nabla \bar{v} \mathrm{~d} x-i \omega \int_{\partial \mathcal{O}} z u \bar{v} \mathrm{~d} \sigma \\
L(v)=\int_{\mathcal{O}} f \bar{v} \mathrm{~d} x-\left\langle g,\left.v\right|_{\partial \mathcal{O}}\right\rangle .
\end{gathered}
$$

These two forms are continuous respectively on $\left(H^{1}(\mathcal{O})\right)^{2}$ and on $H^{1}(\mathcal{O})$. We have

$$
\Re(-i \bar{\omega} a(u, u))=(-\Im \omega)\left[|\omega|^{2} \int_{\mathcal{O}}|u|^{2} \mathrm{~d} x+\int_{\mathcal{O}}|\nabla u|^{2} \mathrm{~d} x\right]+|\omega|^{2} \int_{\partial \mathcal{O}} \Re z|u|^{2} \mathrm{~d} \sigma .
$$

We assume that (141) is fulfilled. We introduce the Hilbert space $V$, which is the completed of $C_{0}^{\infty}(\overline{\mathcal{O}})$ for the norm

$$
\left\|\left|u\left\|\left.\right|^{2}=\right\| u\left\|_{H^{1}(\mathcal{O})}^{2}+\right\| u\right|_{\partial \mathcal{O}}\right\|_{L^{2}(\partial \mathcal{O})}^{2} .
$$

This space is a Hilbert space, and the trace of $u \in V$ on $\partial \mathcal{O}$ is uniquely defined. This space is a subspace of $H^{1}(\mathcal{O})$, and the definition of the trace coincides.

From the equality above, if we denote by

$$
c=\min \left(-\Im \omega|\omega|^{2},-\Im \omega,|\omega|^{2} \min \Re z^{+},|\omega|^{2} \min \Re z^{-}\right)>0,
$$

we obtain $\Re(-i \bar{\omega} a(u, u)) \geqslant c\|u\|_{H^{1}(\mathcal{O})} \geqslant 0$, hence, with $|\omega| \cdot|a(u, u)| \geqslant \Re(-i \bar{\omega} a(u, u))$, and we deduce that the sesquilinear form $a(u, u)$ is coercitive. Hence the problem

$$
a(u, v)=L(v), \quad \forall v \in H^{1}(\mathcal{O})
$$


has a unique solution in $H^{1}(\mathcal{O})$ through the Lax-Milgram lemma. The lemma 6.2 is proven.

- We assume $\omega=-i p, p>0$. We have

$$
\Re a(u, u)=p^{2} \int_{\mathcal{O}}|u|^{2} \mathrm{~d} x+\int_{\mathcal{O}}|\nabla u|^{2} \mathrm{~d} x+p \int_{\partial \mathcal{O}} \Re z|u|^{2} \mathrm{~d} \sigma
$$

The sesquilinear form $a(u, v)$ is coercive in the ordinary sense and the problem $(142)$ has a unique solution in $H^{1}(\mathcal{O})$. This is extended to the Neumann boundary condition.

We end this section with the Hille-Yosida theorem for the diffraction problem. We prove

Lemma 6.3. - Under the assumption $\left.u_{i}\right|_{t=-\delta} \in H^{1}(\mathcal{O}),\left.\partial_{t} u_{i}\right|_{t=-\delta} \in H^{1}(\mathcal{O})$, $\left.\partial_{t^{2}}^{2} u_{i}\right|_{t=-\delta} \in L^{2}(\mathcal{O})$, the problem

$$
\left\{\begin{array}{l}
\left.\left(\Delta-\partial_{t^{2}}^{2}\right) u=0 \text { in } \mathcal{O} \times\right] 0,+\infty[ \\
\left.u\right|_{t=-\delta}=\left.u_{i}\right|_{t=-\delta} \\
\left.\partial_{n} u+z \partial_{t} u=0 \text { on } \partial \mathcal{O} \times\right] 0,+\infty[
\end{array}\right.
$$

admits an unique solution which is in the domain $D(A)$, of class $C^{1}$ in time.

Let

$$
A=\left(\begin{array}{cccc}
0 & 0 & -1 & 0 \\
0 & 0 & 0 & -1 \\
-\Delta & 0 & 0 & 0 \\
0 & -\Delta & 0 & 0
\end{array}\right)
$$

The wave equation with the boundary condition $\partial_{n} u+\left.z \partial_{t} u\right|_{\partial \Omega}=0$ is equivalent, for $u$ regular enough, to (we write $u=u_{1}+i u_{2}$ )

$$
\frac{d}{d t}\left(\begin{array}{c}
u_{1} \\
u_{2} \\
\partial_{t} u_{1} \\
\partial_{t} u_{2}
\end{array}\right)+A\left(\begin{array}{c}
u_{1} \\
u_{2} \\
\partial_{t} u_{1} \\
\partial_{t} u_{2}
\end{array}\right)=\left(\begin{array}{l}
0 \\
0 \\
0 \\
0
\end{array}\right)
$$

with

$$
D(A)=\left\{\begin{array}{c}
\left(\begin{array}{c}
x_{0} \\
x_{1} \\
y_{0} \\
y_{1}
\end{array}\right) \in\left(H^{1}(\mathcal{O})\right)^{2} \times L^{2}(\mathcal{O})^{2}, A\left(\begin{array}{c}
x_{0} \\
x_{1} \\
y_{0} \\
y_{1}
\end{array}\right) \in\left(H^{1}(\mathcal{O})\right)^{2} \times L^{2}(\mathcal{O})^{2}, \\
\partial_{n} x_{0}+\left.\Re z y_{0}\right|_{\partial \mathcal{O}}-\left.\Im z y_{1}\right|_{\partial \mathcal{O}}=0, \\
\partial_{n} x_{1}+\left.\Re z y_{1}\right|_{\partial \mathcal{O}}+\left.\Im z y_{0}\right|_{\partial \mathcal{O}}=0
\end{array}\right\} .
$$

From Lemma 6.1, the boundary condition is well defined in the domain $D(A)$, because $x_{0}$ and $x_{1}$ are in $E(\Delta, \mathcal{O})$ hence we can consider their normal derivative. As $y_{0}$ and $y_{1}$ are in $H^{1}$, we can consider the trace on $\partial \mathcal{O}$. Using Lemma 6.1, we have, with $X=\left(\begin{array}{l}x_{0} \\ x_{1} \\ y_{0} \\ y_{1}\end{array}\right)$

$$
\begin{gathered}
((A+I) X, X)_{\left(H^{1}(\mathcal{O})\right)^{2} \times L^{2}(\mathcal{O})^{2}}=\left(x_{0}, x_{0}\right)_{H^{1}(\mathcal{O})}+\left(y_{0}, y_{0}\right)_{H^{1}(\mathcal{O})}+\left(y_{0}, y_{0}\right)_{L^{2}(\mathcal{O})} \\
+\left(y_{1}, y_{1}\right)_{L^{2}(\mathcal{O})}+\left(x_{0},-y_{0}\right)_{H^{1}(\mathcal{O})}+\left(x_{1},-y_{1}\right)_{H^{1}(\mathcal{O})}+\left(y_{0},-\Delta x_{0}\right)_{L^{2}(\mathcal{O})}+\left(y_{1},-\Delta x_{1}\right)_{L^{2}(\mathcal{O})}
\end{gathered}
$$


Making use of Lemma 6.1, we have

$$
\begin{aligned}
& ((A+I) X, X)_{\left(H^{1}(\mathcal{O})\right)^{2} \times L^{2}(\mathcal{O})^{2}}=\left(x_{0}, x_{0}\right)_{H^{1}(\mathcal{O})}+\left(x_{1}, x_{1}\right)_{H^{1}(\mathcal{O})}+\left(y_{0}, y_{0}\right)_{L^{2}(\mathcal{O})} \\
& \quad+\left(y_{1}, y_{1}\right)_{L^{2}(\mathcal{O})}-\left(x_{0}, y_{0}\right)_{L^{2}(\mathcal{O})}-\left(x_{1}, y_{1}\right)_{L^{2}(\mathcal{O})}-\left\langle\left. y_{0}\right|_{\partial \mathcal{O}}, \partial_{n} x_{0}\right\rangle-\left\langle\left. y_{1}\right|_{\partial \mathcal{O}}, \partial_{n} x_{1}\right\rangle \\
& \quad((A+I) X, X)_{\left(H^{1}(\mathcal{O})\right)^{2} \times L^{2}(\mathcal{O})^{2}}=\left(x_{0}, x_{0}\right)_{H^{1}(\mathcal{O})}+\left(x_{1}, x_{1}\right)_{H^{1}(\mathcal{O})}+\left(y_{0}, y_{0}\right)_{L^{2}(\mathcal{O})} \\
& \quad+\left(y_{1}, y_{1}\right)_{L^{2}(\mathcal{O})}-\left(x_{0}, y_{0}\right)_{L^{2}(\mathcal{O})}-\left(x_{1}, y_{1}\right)_{L^{2}(\mathcal{O})}-\left\langle\left. y_{0}\right|_{\partial \mathcal{O}}, \partial_{n} x_{0}\right\rangle-\left\langle\left. y_{1}\right|_{\partial \mathcal{O}}, \partial_{n} x_{1}\right\rangle
\end{aligned}
$$

We consider first the boundary terms. We obtain

$$
\begin{aligned}
-\left\langle\left. y_{0}\right|_{\partial \mathcal{O}}, \partial_{n} x_{0}\right\rangle & -\left\langle\left. y_{1}\right|_{\partial \mathcal{O}}, x_{1}\right\rangle \\
& =\left\langle\left. y_{0}\right|_{\partial \mathcal{O}},\left.\Re z y_{0}\right|_{\partial \mathcal{O}}-\left.\Im z y_{1}\right|_{\partial \mathcal{O}}\right\rangle+\left\langle\left. y_{1}\right|_{\partial \mathcal{O}},\left.\Re z y_{1}\right|_{\partial \mathcal{O}}+\left.\Im z y_{0}\right|_{\partial \mathcal{O}}\right\rangle \\
& =\left\langle\left. y_{0}\right|_{\partial \mathcal{O}},\left.\Re z y_{0}\right|_{\partial \mathcal{O}}\right\rangle+\left\langle\left. y_{1}\right|_{\partial \mathcal{O}},\left.\Re z y_{1}\right|_{\partial \mathcal{O}}\right\rangle .
\end{aligned}
$$

As $\left(x_{0}-\frac{1}{2} y_{0}, x_{0}-\frac{1}{2} y_{0}\right)_{L^{2}(\mathcal{O})}=\left(x_{0}, x_{0}\right)_{L^{2}(\mathcal{O})}-\left(x_{0}, y_{0}\right)_{L^{2}(\mathcal{O})}+\frac{1}{4}\left(y_{0}, y_{0}\right)_{L^{2}(\mathcal{O})}$, we obtain that, under the condition $\Re z \geqslant 0$, that $A+I$ is accretive.

Let us consider now the system $(A+2 I) X=F$, where $F=\left(\begin{array}{l}f_{0} \\ f_{1} \\ g_{0} \\ g_{1}\end{array}\right)$, with $f_{i} \in$ $H^{1}(\mathcal{O})$ and $g_{i} \in H^{2}(\mathcal{O})$. This system, for $X$ in $D(A)$, is equivalent to the system (140) with $u=x_{0}+i x_{1}, f=-2\left(f_{0}+i f_{1}\right), g=g_{0}+i g_{1}+\left(z_{1}+i z_{2}\right)\left(f_{0}+i f_{1}\right)$, $\omega=-2 i, y_{0}=2 x_{0}-f_{0}, y_{1}=2 x_{1}-f_{1}$. As the problem (140) has a unique solution in $H^{1}(\mathcal{O})$ satisfying $\partial_{n} u \in L^{2}(\partial \mathcal{O}), \Delta u \in L^{2}(\mathcal{O})$ we get a solution $x_{0}, x_{1}$ in $H^{1}(\mathcal{O})$, $\Delta x_{0}, \Delta x_{1} \in L^{2}(\mathcal{O}), y_{0}=2 x_{0}-f_{0} \in H^{1}(\mathcal{O}), y_{1} \in H^{1}(\mathcal{O})$, thus the solution is in the domain $D(A)=D(A+I)$. Hence the operator $A+I$ is accretive and maximal, thus, from the Hille-Yosida theorem [10], the problem of Lemma 6.3 with $u_{i}$ as in the hypothesis and $u_{i}(x, y, t)$ supported in $t<\theta_{i}(x, y)$ (hence $u_{i}(x, y,-\delta)$ is uniformly zero on $\partial \mathcal{O}$, which implies that $u_{i}(x, y,-\delta)$ is in $\left.D(A+I)\right)$ admits an unique solution in $C^{2}(] 0, \infty\left[, H^{1} \times L^{2}\right) \cap C^{1}(] 0,+\infty[, D(A))$.

\subsection{Calculus of the distributions on the boundary}

6.2.1. Calculus of the Dirichlet to Neumann operators. - In this section, we express the operators $R_{+}^{ \pm, k}$ and $R_{-}^{ \pm, k}$ with the same expression as (81), (83), (84), (82). We denote by $\partial_{+} f$ the limit for $\varepsilon \rightarrow 0$ of $D_{+} f(x, a(x)+\varepsilon)$ (rigorous way of defining the trace on the boundary, according to $[\mathbf{1 4}])$. We compute the wave $u_{+}(x, y, k)$ (respectively $u_{-}$) associated with a distribution $f(x)$ on the face $\Delta_{+}$(resp. $\Delta_{-}$), for $(x, y)$ outside $\Delta_{+}\left(\right.$resp. $\left.\Delta_{-}\right)$, that is

$$
\begin{gathered}
u_{+}(x, y, k)=\left(\Delta+k^{2}\right)^{-1}\left(f \otimes \delta_{+}^{\prime}\right) \\
u_{-}(x, y, k)=\left(\Delta+k^{2}\right)^{-1}\left(f \otimes \delta_{-}^{\prime}\right) .
\end{gathered}
$$

We have 
Lemma 6.4. - The following relations are true:

$$
\begin{aligned}
&\left(1+\left(a^{\prime}(x)\right)^{2}\right)^{1 / 2} \partial_{+} u_{+}(x, k)=\lim _{\varepsilon \rightarrow 0_{+}} \frac{1}{4 \pi} \int_{\mathbb{R} \times \mathbb{R}_{+}} i f\left(x^{\prime}\right) e^{i\left(x-x^{\prime}\right) \xi_{1}^{+}+i \varepsilon \frac{\mu_{+} \xi_{1}^{+}+\xi_{+}}{1+\mu_{+}^{2}}} \mathrm{~d} x^{\prime} \mathrm{d} \xi_{1}^{+} \\
& \times\left[\frac{\mu_{+}-a^{\prime}(x)}{1+\mu_{+}^{2}} \xi_{1}^{+}+\frac{1+\mu_{+} a^{\prime}\left(x^{\prime}\right)}{1+\mu_{+}^{2}} \xi_{+}\right]\left[\frac{\mu_{+}-a^{\prime}\left(x^{\prime}\right)}{1+\mu_{+}^{2}} \frac{\xi_{1}^{+}}{\xi_{+}}+\frac{1+\mu_{+} a^{\prime}\left(x^{\prime}\right)}{1+\mu_{+}^{2}}\right] \\
&\left(1+\left(a^{\prime}(x)\right)^{2}\right)^{1 / 2} \partial_{+} u_{-}(x, k)= \\
&-\lim _{\varepsilon \rightarrow 0_{+}} \frac{1}{4 \pi} \int_{\mathbb{R} \times \mathbb{R}_{+}} i f\left(x^{\prime}\right) e^{i\left(x-x^{\prime}\right) \xi_{1}^{+}+i\left(a\left(x^{\prime}\right)-b\left(x^{\prime}\right)+\varepsilon\right) \frac{\mu_{+} \xi_{1}^{+}+\xi_{+}}{1+\mu_{+}^{2}}} \mathrm{~d} x^{\prime} \mathrm{d} \xi_{1}^{+} \\
& \times\left[\frac{\mu_{+}-a^{\prime}(x)}{1+\mu_{+}^{2}} \xi_{1}^{+}+\frac{1+\mu_{+} a^{\prime}(x)}{1+\mu_{+}^{2}} \xi_{+}\right]\left[\frac{1+b^{\prime}\left(x^{\prime}\right) \mu_{+}}{1+\mu_{+}^{2}}+\frac{\mu_{+}-b^{\prime}\left(x^{\prime}\right)}{1+\mu_{+}^{2}} \frac{\xi_{1}^{+}}{\xi_{+}}\right] .
\end{aligned}
$$

Proof. - For $y-a(x)>0$, using the Cauchy formula

$$
\begin{gathered}
u_{+}(x, y, k)=\frac{1}{4 \pi} \int_{\mathbb{R} \times \mathbb{R}_{+}} e^{i\left(x-x^{\prime}\right) \xi_{1}^{+}+i(y-a(x)) \frac{\mu_{+} \xi_{1}^{+}+\xi_{+}}{1+\mu_{+}^{2}}} \\
\times\left[\frac{\mu_{+}-a^{\prime}\left(x^{\prime}\right)}{1+\mu_{+}^{2}} \frac{\xi_{1}^{+}}{\xi_{+}}+\frac{1+\mu_{+} a^{\prime}\left(x^{\prime}\right)}{1+\mu_{+}^{2}}\right] f\left(x^{\prime}\right) \mathrm{d} x^{\prime} \mathrm{d} \xi_{1}^{+} \\
u_{-}(x, y, k)=-\frac{1}{4 \pi} \int_{\mathbb{R} \times \mathbb{R}_{+}} e^{i\left(x-x^{\prime}\right) \xi_{1}^{+}+i\left(a\left(x^{\prime}\right)-b\left(x^{\prime}\right)+(y-a(x))\right) \frac{\mu_{+} \xi_{1}^{+}+\xi_{+}}{1+\mu_{+}^{2}}} \\
\times\left[\frac{1+b^{\prime}\left(x^{\prime}\right) \mu_{+}}{1+\mu_{+}^{2}}+\frac{\mu_{+}-b^{\prime}\left(x^{\prime}\right)}{1+\mu_{+}^{2}} \frac{\xi_{1}^{+}}{\xi_{+}}\right] f\left(x^{\prime}\right) \mathrm{d} x^{\prime} \mathrm{d} \xi_{1}^{+} .
\end{gathered}
$$

As the support of $f$ is included in $[0,+\infty[$ and $y-a(x)=\varepsilon>0$, both relations define $C^{\infty}$ functions through the Paley-Wiener-Schwartz theorem. We thus:

- compute, using (143) and (144), the derivative $D_{+} u_{+}$and $D_{+} u_{-}$,

- show that this derivative has the limit corresponding to the usual one for $\varepsilon \rightarrow 0$, $\varepsilon>0$.

We have

$$
\begin{array}{r}
D_{+} u_{+}(x, y, k)=\frac{1}{4 \pi} \int_{\mathbb{R} \times \mathbb{R}_{+}} e^{i\left(x-x^{\prime}\right) \xi_{1}^{+}+i(y-a(x)) \frac{\mu_{+} \xi_{1}^{+}+\xi_{+}}{1+\mu_{+}^{2}}} \frac{f\left(x^{\prime}\right) \mathrm{d} x^{\prime} \mathrm{d} \xi_{1}^{+}}{\left(1+\left(a^{\prime}(x)\right)^{2}\right)^{1 / 2}} \\
{\left[i\left[\frac{\mu_{+} \xi_{1}^{+}+\xi_{+}}{1+\mu_{+}^{2}}-a^{\prime}(x)\left(\xi_{1}^{+}-a^{\prime}(x) \frac{\mu_{+} \xi_{1}^{+}+\xi_{+}}{1+\mu_{+}^{2}}+(y-a(x)) \partial_{x}\left(\frac{\mu_{+} \xi_{1}^{+}+\xi_{+}}{1+\mu_{+}^{2}}\right)\right)\right]\right.} \\
\times\left[\frac{\mu_{+}-a^{\prime}\left(x^{\prime}\right)}{1+\mu_{+}^{2}} \frac{\xi_{1}^{+}}{\xi_{+}}+\frac{1+\mu_{+} a^{\prime}\left(x^{\prime}\right)}{1+\mu_{+}^{2}}\right] \\
\left.-a^{\prime}(x) \partial_{x}\left(\frac{\mu_{+}-a^{\prime}\left(x^{\prime}\right)}{1+\mu_{+}^{2}} \frac{\xi_{1}^{+}}{\xi_{+}}+\frac{1+\mu_{+} a^{\prime}\left(x^{\prime}\right)}{1+\mu_{+}^{2}}\right)\right]
\end{array}
$$


hence

$$
\begin{gathered}
D_{+} u_{+}(x, y, k)=\frac{1}{4 \pi} \int_{\mathbb{R} \times \mathbb{R}_{+}} e^{i\left(x-x^{\prime}\right) \xi_{1}^{+}+i(y-a(x)) \frac{\mu_{+} \xi_{1}^{+}+\xi_{+}}{1+\mu_{+}^{2}}} \frac{f\left(x^{\prime}\right) \mathrm{d} x^{\prime} \mathrm{d} \xi_{1}^{+}}{\left(1+\left(a^{\prime}(x)\right)^{2}\right)^{1 / 2}} \\
{\left[\left[i\left(\frac{\mu_{+}-a^{\prime}(x)}{1+\mu_{+}^{2}} \xi_{1}^{+}+\frac{1+\mu_{+} a^{\prime}(x)}{1+\mu_{+}^{2}} \xi_{+}\right)\left(\frac{\mu_{+}-a^{\prime}\left(x^{\prime}\right)}{1+\mu_{+}^{2}} \frac{\xi_{1}^{+}}{\xi_{+}}+\frac{1+\mu_{+} a^{\prime}\left(x^{\prime}\right)}{1+\mu_{+}^{2}}\right)\right.\right.} \\
\left.+i a^{\prime}(x)\left(\left(a^{\prime}(x)-\mu_{+}\right) \frac{\mu_{+} \xi_{1}^{+}+\xi_{+}}{1+\mu_{+}^{2}}-(y-a(x)) \partial_{x}\left(\frac{\mu_{+} \xi_{1}^{+}+\xi_{+}}{1+\mu_{+}^{2}}\right)\right)\right] \\
\times\left[\frac{\mu_{+}-a^{\prime}\left(x^{\prime}\right)}{1+\mu_{+}^{2}} \frac{\xi_{1}^{+}}{\xi_{+}}+\frac{1+\mu_{+} a^{\prime}\left(x^{\prime}\right)}{1+\mu_{+}^{2}}\right] \\
\left.-a^{\prime}(x) \partial_{x}\left(\frac{\mu_{+}-a^{\prime}\left(x^{\prime}\right)}{1+\mu_{+}^{2}} \frac{\xi_{1}^{+}}{\xi_{+}}+\frac{1+\mu_{+} a^{\prime}\left(x^{\prime}\right)}{1+\mu_{+}^{2}}\right)\right] .
\end{gathered}
$$

We concentrate on the last terms. We use the relations

$$
\begin{aligned}
a^{\prime}(x)-\mu_{+} & =\left(x-x^{\prime}\right) \partial_{x} \mu_{+}, \\
\partial_{x}\left(\frac{\mu_{+} \xi_{1}^{+}+\xi_{+}}{1+\mu_{+}^{2}}\right) & =-\partial_{x} \mu_{+} \frac{\mu_{+} \xi_{1}^{+}+\xi_{+}}{1+\mu_{+}^{2}} \partial_{\xi_{1}^{+}}\left(\frac{\mu_{+} \xi_{1}^{+}+\xi_{+}}{1+\mu_{+}^{2}}\right)
\end{aligned}
$$

to obtain

$$
\begin{gathered}
D_{+} u_{+}(x, y, k)=\frac{1}{4 \pi} \int_{\mathbb{R} \times \mathbb{R}_{+}} e^{i\left(x-x^{\prime}\right) \xi_{1}^{+}+i(y-a(x)) \frac{\mu_{+} \xi_{1}^{+}+\xi_{+}}{1+\mu_{+}^{+}}} \frac{f\left(x^{\prime}\right) \mathrm{d} x^{\prime} \mathrm{d} \xi_{1}^{+}}{\left(1+\left(a^{\prime}(x)\right)^{2}\right)^{1 / 2}} \\
{\left[\left[i\left(\frac{\mu_{+}-a^{\prime}(x)}{1+\mu_{+}^{2}} \xi_{1}^{+}+\frac{1+\mu_{+} a^{\prime}(x)}{1+\mu_{+}^{2}} \xi_{+}\right)\left(\frac{\mu_{+}-a^{\prime}\left(x^{\prime}\right)}{1+\mu_{+}^{2}} \frac{\xi_{1}^{+}}{\xi_{+}}+\frac{1+\mu_{+} a^{\prime}\left(x^{\prime}\right)}{1+\mu_{+}^{2}}\right)\right.\right.} \\
\left.+i a^{\prime}(x)\left(\partial_{x} \mu_{+}\left(x-x^{\prime}\right) \frac{\mu_{+} \xi_{1}^{+}+\xi_{+}}{1+\mu_{+}^{2}}+\partial_{x} \mu_{+}(y-a(x)) \partial_{\xi_{1}^{+}}\left(\frac{\mu_{+} \xi_{1}^{+}+\xi_{+}}{1+\mu_{+}^{2}}\right) \frac{\mu_{+} \xi_{1}^{+}+\xi_{+}}{1+\mu_{+}^{2}}\right)\right] \\
\times\left(\frac{\mu_{+}-a^{\prime}\left(x^{\prime}\right)}{1+\mu_{+}^{2}} \frac{\xi_{1}^{+}}{\xi_{+}}+\frac{1+\mu_{+} a^{\prime}\left(x^{\prime}\right)}{1+\mu_{+}^{2}}\right) \\
\left.-a^{\prime}(x) \partial_{x}\left(\frac{\mu_{+}-a^{\prime}\left(x^{\prime}\right)}{1+\mu_{+}^{2}} \frac{\xi_{1}^{+}}{\xi_{+}}+\frac{1+\mu_{+} a^{\prime}\left(x^{\prime}\right)}{1+\mu_{+}^{2}}\right)\right] \\
D_{+} u_{+}(x, y, k)=\frac{1}{4 \pi} \int_{\mathbb{R} \times \mathbb{R}_{+}} e^{i\left(x-x^{\prime}\right) \xi_{1}^{+}+i(y-a(x)) \frac{\mu_{+} \xi_{1}^{+} \xi_{+}}{1+\mu_{+}^{2}} \frac{f\left(x^{\prime}\right) \mathrm{d} x^{\prime} \mathrm{d} \xi_{1}^{+}}{\left(1+\left(a^{\prime}(x)\right)^{2}\right)^{1 / 2}}} \\
{\left[\left[i\left(\frac{\mu_{+}-a^{\prime}\left(x^{\prime}\right)}{1+\mu_{+}^{2}} \xi_{1}^{+}+\frac{1+\mu_{+} a^{\prime}\left(x^{\prime}\right)}{1+\mu_{+}^{2}} \xi_{+}\right)\left(\frac{\mu_{+}-a^{\prime}\left(x^{\prime}\right)}{1+\mu_{+}^{2}} \frac{\xi_{1}^{+}}{\xi_{+}}+\frac{1+\mu_{+} a^{\prime}\left(x^{\prime}\right)}{1+\mu_{+}^{2}}\right)\right.\right.} \\
\left.+i a^{\prime}(x) \partial_{x} \mu_{+} \partial_{\xi_{1}^{+}}\left(\left(x-x^{\prime}\right) \xi_{1}^{+}+(y-a(x)) \frac{\mu_{+} \xi_{1}^{+}+\xi_{+}}{1+\mu_{+}^{2}}\right) \frac{\mu_{+} \xi_{1}^{+}+\xi_{+}}{1+\mu_{+}^{2}}\right] \\
\times\left[\frac{\mu_{+}-a^{\prime}\left(x^{\prime}\right)}{1+\mu_{+}^{2}} \frac{\xi_{1}^{+}}{\xi_{+}}+\frac{1+\mu_{+} a^{\prime}\left(x^{\prime}\right)}{1+\mu_{+}^{2}}\right] \\
\left.-a^{\prime}(x) \partial_{x}\left(\frac{\mu_{+}-a^{\prime}\left(x^{\prime}\right)}{1+\mu_{+}^{2}} \frac{\xi_{1}^{+}}{\xi_{+}}+\frac{1+\mu_{+} a^{\prime}\left(x^{\prime}\right)}{1+\mu_{+}^{2}}\right)\right]
\end{gathered}
$$


We finally check that (using $\partial_{x} \xi_{+}^{-1}=-\frac{k^{2}}{\xi_{+}^{3}} \mu_{+} \partial_{x} \mu_{+}$)

$$
\begin{aligned}
& \partial_{x}\left[\frac{1+\lambda \mu_{+}}{1+\mu_{+}^{2}}+\frac{\mu_{+}-\lambda}{1+\mu_{+}^{2}} \frac{\xi_{1}^{+}}{\xi_{+}}\right]= \\
& \partial_{x} \mu_{+}\left[\frac{\lambda\left(1-\mu_{+}^{2}\right)-2 \mu_{+}}{\left(1+\mu_{+}^{2}\right)^{2}}+\frac{1-\mu_{+}+2 \mu_{+} \lambda}{\left(1+\mu_{+}^{2}\right)^{2}} \frac{\xi_{1}^{+}}{\xi_{+}}-\frac{\mu_{+}\left(\mu_{+}-\lambda\right)}{1+\mu_{+}^{2}} \frac{k^{2} \xi_{1}^{+}}{\xi_{+}^{3}}\right]
\end{aligned}
$$

and (using $\partial_{\xi_{1}^{+}} \xi_{+}=-\xi_{1}^{+} / \xi_{+}$and $\partial_{\xi_{1}^{+}}\left(\xi_{+}^{-1}\right)=\xi_{1}^{+} / \xi_{+}^{3}$ ) we have

$$
\begin{aligned}
\partial_{\xi_{1}^{+}}\left[\frac{\mu_{+} \xi_{1}^{+}+\xi_{+}}{1+\mu_{+}^{2}}\right. & \left.\left(\frac{1+\lambda \mu_{+}}{1+\mu_{+}^{2}}+\frac{\mu_{+}-\lambda}{1+\mu_{+}^{2}} \frac{\xi_{1}^{+}}{\xi_{+}}\right)\right]= \\
- & {\left[\frac{\lambda\left(1-\mu_{+}^{2}\right)-2 \mu_{+}}{\left(1+\mu_{+}^{2}\right)^{2}}+\frac{1-\mu_{+}^{2}+2 \mu_{+} \lambda}{\left(1+\mu_{+}^{2}\right)^{2}}-\frac{\mu_{+}\left(\mu_{+}-\lambda\right) k^{2} \xi_{1}^{+}}{\xi_{+}^{3}}\right] }
\end{aligned}
$$

which leads to

(148) $\partial_{x}\left[\frac{1+\lambda \mu_{+}}{1+\mu_{+}^{2}}+\frac{\mu_{+}-\lambda}{1+\mu_{+}^{2}} \frac{\xi_{1}^{+}}{\xi_{+}}\right]=-\partial_{x} \mu_{+} \partial_{\xi_{1}^{+}}\left[\frac{\mu_{+} \xi_{1}^{+}+\xi_{+}}{1+\mu_{+}^{2}}\left(\frac{1+\lambda \mu_{+}}{1+\mu_{+}^{2}}+\frac{\mu_{+}-\lambda}{1+\mu_{+}^{2}} \frac{\xi_{1}^{+}}{\xi_{+}}\right)\right]$

We finally get

$$
\begin{aligned}
& D_{+} u_{+}(x, y, k)=\frac{1}{4 \pi} \int_{\mathbb{R} \times \mathbb{R}_{+}} e^{i\left(x-x^{\prime}\right) \xi_{1}^{+}+i(y-a(x)) \frac{\mu_{+} \xi_{1}^{+}+\xi_{+}}{1+\mu_{+}^{2}}} \frac{f\left(x^{\prime}\right) \mathrm{d} x^{\prime} \mathrm{d} \xi_{1}^{+}}{\left(1+\left(a^{\prime}(x)\right)^{2}\right)^{1 / 2}} \\
& {\left[i\left(\frac{\mu_{+}-a^{\prime}(x)}{1+\mu_{+}^{2}} \xi_{1}^{+}+\frac{1+\mu_{+} a^{\prime}(x)}{1+\mu_{+}^{2}} \xi_{+}\right)\left(\frac{\mu_{+}-a^{\prime}\left(x^{\prime}\right)}{1+\mu_{+}^{2}} \frac{\xi_{1}^{+}}{\xi_{+}}+\frac{1+\mu_{+} a^{\prime}\left(x^{\prime}\right)}{1+\mu_{+}^{2}}\right)+a^{\prime}(x) \partial_{x} \mu_{+}\right.} \\
& \left.\cdot \partial_{\xi_{1}^{+}}\left[e^{i\left(x-x^{\prime}\right) \xi_{1}^{+}+i(y-a(x)) \frac{\mu_{+} \xi_{1}^{+}+\xi_{+}}{1+\mu_{+}^{2}}} \frac{\mu_{+} \xi_{1}^{+}+\xi_{+}}{1+\mu_{+}^{2}}\left(\frac{\mu_{+}-a^{\prime}\left(x^{\prime}\right)}{1+\mu_{+}^{2}} \frac{\xi_{1}^{+}}{\xi_{+}}+\frac{1+\mu_{+} a^{\prime}\left(x^{\prime}\right)}{1+\mu_{+}^{2}}\right)\right]\right]
\end{aligned}
$$

which imply the result.

In a similar fashion,

$$
\begin{gathered}
D_{+} u_{-}(x, y, k)=-\frac{1}{4 \pi} \int_{\mathbb{R} \times \mathbb{R}_{+}} e^{i\left(x-x^{\prime}\right) \xi_{1}^{+}+i\left(a\left(x^{\prime}\right)-b\left(x^{\prime}\right)+(y-a(x))\right) \frac{\mu_{+} \xi_{1}^{+}+\xi_{+}}{1+\mu_{+}^{2}}} \\
\times\left[i \left(\frac{\mu_{+} \xi_{1}^{+}+\xi_{+}}{1+\mu_{+}^{2}}-a^{\prime}(x)\left(\xi_{1}^{+}+\left(a\left(x^{\prime}\right)-b\left(x^{\prime}\right)+y-a(x)\right) \partial_{x}\left(\frac{\mu_{+} \xi_{1}^{+}+\xi_{+}}{1+\mu_{+}^{2}}\right)\right.\right.\right. \\
\left.\left.-a^{\prime}(x) \frac{\mu_{+} \xi_{1}^{+}+\xi_{+}}{1+\mu_{+}^{2}}\right)\right) \times\left(\frac{1+b^{\prime}\left(x^{\prime}\right) \mu_{+}}{1+\mu_{+}^{2}}+\frac{\mu_{+}-b^{\prime}\left(x^{\prime}\right)}{1+\mu_{+}^{2}} \frac{\xi_{1}^{+}}{\xi_{+}}\right) \\
\left.-a^{\prime}(x) \partial_{x}\left(\frac{1+b^{\prime}\left(x^{\prime}\right) \mu_{+}}{1+\mu_{+}^{2}}+\frac{\mu_{+}-b^{\prime}\left(x^{\prime}\right)}{1+\mu_{+}^{2}} \frac{\xi_{1}^{+}}{\xi_{+}}\right)\right] \frac{f\left(x^{\prime}\right) \mathrm{d} x^{\prime} \mathrm{d} \xi_{1}^{+}}{\left(1+\left(b^{\prime}(x)\right)^{2}\right)^{1 / 2}}
\end{gathered}
$$


hence

$$
\begin{gathered}
D_{+} u_{-}(x, y, k)=-\frac{1}{4 \pi} \int_{\mathbb{R} \times \mathbb{R}_{+}} e^{i\left(x-x^{\prime}\right) \xi_{1}^{+}+i\left(a\left(x^{\prime}\right)-b\left(x^{\prime}\right)+(y-a(x))\right) \frac{\mu_{+} \xi_{1}^{+}+\xi_{+}}{1+\mu_{+}^{2}}} \\
\times\left[i \left[\left(\frac{\mu_{+} \xi_{1}^{+}+\xi_{+}}{1+\mu_{+}^{2}}-a^{\prime}(x) \frac{\xi_{1}^{+}-\mu_{+} \xi_{+}}{1+\mu_{+}^{2}}\right)\right.\right. \\
\left.+i a^{\prime}(x)\left[\left(a^{\prime}(x)-\mu_{+}\right) \frac{\mu_{+} \xi_{1}^{+}+\xi_{+}}{1+\mu_{+}^{2}}-\left(a\left(x^{\prime}\right)-b\left(x^{\prime}\right)+y-a(x)\right) \partial_{x}\left(\frac{\mu_{+} \xi_{1}^{+}+\xi_{+}}{1+\mu_{+}^{2}}\right)\right]\right] \\
\quad \times\left(\frac{1+b^{\prime}\left(x^{\prime}\right) \mu_{+}}{1+\mu_{+}^{2}}+\frac{\mu_{+}-b^{\prime}\left(x^{\prime}\right)}{1+\mu_{+}^{2}} \frac{\xi_{1}^{+}}{\xi_{+}}\right) \\
\left.-a^{\prime}(x) \partial_{x}\left(\frac{1+b^{\prime}\left(x^{\prime}\right) \mu_{+}}{1+\mu_{+}^{2}}+\frac{\mu_{+}-b^{\prime}\left(x^{\prime}\right)}{1+\mu_{+}^{2}} \frac{\xi_{1}^{+}}{\xi_{+}}\right)\right] \frac{f\left(x^{\prime}\right) \mathrm{d} x^{\prime} \mathrm{d} \xi_{1}^{+}}{\left(1+\left(b^{\prime}(x)\right)^{2}\right)^{1 / 2}}
\end{gathered}
$$

We use the relation (145) to have

$$
\begin{aligned}
& \left(a^{\prime}(x)-\mu_{+}\right) \frac{\mu_{+} \xi_{1}^{+}+\xi_{+}}{1+\mu_{+}^{2}}-\left(a\left(x^{\prime}\right)-b\left(x^{\prime}\right)+y-a(x)\right) \partial_{x}\left(\frac{\mu_{+} \xi_{1}^{+}+\xi_{+}}{1+\mu_{+}^{2}}\right) \\
& \quad=\frac{\mu_{+} \xi_{1}^{+}+\xi_{+}}{1+\mu_{+}^{2}} \partial_{x} \mu_{+}\left[\left(x-x^{\prime}\right)+\left(a\left(x^{\prime}\right)-b\left(x^{\prime}\right)+y-a(x)\right) \partial_{\xi_{1}^{+}}\left(\frac{\mu_{+} \xi_{1}^{+}+\xi_{+}}{1+\mu_{+}^{2}}\right)\right]
\end{aligned}
$$

which leads, with (148), to

$$
\begin{aligned}
& -a^{\prime}(x) \partial_{x}\left[\frac{1+b^{\prime}\left(x^{\prime}\right) \mu_{+}}{1+\mu_{+}^{2}}+\frac{\mu_{+}-b^{\prime}\left(x^{\prime}\right)}{1+\mu_{+}^{2}} \frac{\xi_{1}^{+}}{\xi_{+}}\right] \\
& =a^{\prime}(x) \partial_{x} \mu_{+}\left[\frac{\mu_{+} \xi_{1}^{+}+\xi_{+}}{1+\mu_{+}^{2}}\left(\frac{1+b^{\prime}\left(x^{\prime}\right) \mu_{+}}{1+\mu_{+}^{2}}+\frac{\mu_{+}-b^{\prime}\left(x^{\prime}\right)}{1+\mu_{+}^{2}} \frac{\xi_{1}^{+}}{\xi_{+}}\right)\right] .
\end{aligned}
$$

We have then

$$
\begin{gathered}
D_{+} u_{-}(x, a(x)+\varepsilon, k)=-\frac{i}{4 \pi} \int_{\mathbb{R} \times \mathbb{R}_{+}} e^{\left.i\left(x-x^{\prime}\right) \xi_{1}^{+}+i\left(a\left(x^{\prime}\right)-b\left(x^{\prime}\right)+\varepsilon\right)\right) \frac{\mu_{+} \xi_{1}^{+}+\xi_{+}}{1+\mu_{+}^{2}}} \\
\times\left[\left(\frac{\mu_{+} \xi_{1}^{+}+\xi_{+}}{1+\mu_{+}^{2}}-a^{\prime}(x) \frac{\xi_{1}^{+}-\mu_{+} \xi_{+}}{1+\mu_{+}^{2}}\right)\left(\frac{1+b^{\prime}\left(x^{\prime}\right) \mu_{+}}{1+\mu_{+}^{2}}+\frac{\mu_{+}-b^{\prime}\left(x^{\prime}\right)}{1+\mu_{+}^{2}} \frac{\xi_{1}^{+}}{\xi_{+}}\right)+\right. \\
\left.\frac{1}{i} a^{\prime}(x) \partial_{x} \mu_{+} \partial_{\xi_{1}^{+}}\left[e^{\left.i\left(x-x^{\prime}\right) \xi_{1}^{+}+i\left(a\left(x^{\prime}\right)-b\left(x^{\prime}\right)+\varepsilon\right)\right) \frac{\mu_{+} \xi_{1}^{+}+\xi_{+}}{1+\mu_{+}^{2}}}\left(\frac{\mu_{+} \xi_{1}^{+}+\xi_{+}}{1+\mu_{+}^{2}}-a^{\prime}(x) \frac{\xi_{1}^{+}-\mu_{+} \xi_{+}}{1+\mu_{+}^{2}}\right)\right]\right] \\
\cdot \frac{f\left(x^{\prime}\right) \mathrm{d} x^{\prime} \mathrm{d} \xi_{1}^{+}}{\left(1+\left(b^{\prime}(x)\right)^{2}\right)^{1 / 2}} .
\end{gathered}
$$

The proof of Lemma 6.4 is complete.

6.2.2. Transformation of the operators $R_{+}^{ \pm, k}$ and $R_{-}^{ \pm, k}$ - We notice that the symbols of the operators of Lemma 6.4 are bounded, for $\xi_{1}^{ \pm}$large, by $\left|\xi_{1}^{ \pm}\right|$, which 
proves that they are operators of order 1 . However, let

$$
\widetilde{f} \in H^{1}(\{y=a(x), x \geqslant 0\} \cap\{y-b(x), x \geqslant 0\}) .
$$

To this distribution is canonically associated a distribution $f$ of $H^{1}(\mathbb{R})$, and two distributions $f_{+}$and $f_{-}$of $H^{1}\left(\mathbb{R}_{+}^{*}\right)$, which are $f_{+}(x)=f(x) 1_{x \geqslant 0}=\tilde{f}(x, a(x), k)$ and $\tilde{f}_{-}(x)=f(-x) 1_{x \leqslant 0}=\tilde{f}(x, b(x))$. The distributions $f_{+} 1_{x \geqslant 0}$ and $f_{-} 1_{x \geqslant 0}$ are not element of $H^{1}(\mathbb{R})$, because of the value $f_{+}(0)$ and of the value $f_{-}(0)$. Note that ${ }^{(1)}$ $H^{1}(\{y=a(x), x \geqslant 0\} \cap\{y-b(x), x \geqslant 0\})$ is associated with

$$
\left\{\left(f_{+}, f_{+}\right) \in L^{2}\left(\mathbb{R}_{+}\right) \times L^{2}\left(\mathbb{R}_{+}\right),\left(f_{+}, f_{-}\right) \in H^{1}\left(\mathbb{R}_{+}^{*}\right) \times H^{1}\left(\mathbb{R}_{+}^{*}\right), f_{+}(0)=f_{-}(0)\right\} .
$$

The contribution of the distribution on the boundary and the contribution of the corner have thus to be explicitly written. Moreover, we must check that the distribution constructed with $\tilde{f} \in H^{1}(\partial \Omega)$, which is $\partial_{+}\left(\left(\Delta+k^{2}\right)^{-1}\left(\widetilde{f} \otimes \delta_{\partial \Omega}^{\prime}\right)\right)$, is element of $L^{2}(\Omega)$.

Notice that

$$
\left(x-x^{\prime}\right) \xi+\left(y-a\left(x^{\prime}\right)\right) \eta=\left(x-x^{\prime}\right) \xi_{-}^{1}+\left(b\left(x^{\prime}\right)-a\left(x^{\prime}\right)\right) \eta+(y-b(x)) \eta
$$

and

$$
\left(x-x^{\prime}\right) \xi+\left(y-b\left(x^{\prime}\right)\right) \eta=\left(x-x^{\prime}\right) \xi_{+}^{1}+\left(a\left(x^{\prime}\right)-b\left(x^{\prime}\right)\right) \eta+(y-a(x)) \eta .
$$

We introduce the two phase functions

$$
\begin{aligned}
& \phi_{\varepsilon}^{+}\left(x, x^{\prime}, \xi_{+}^{1}, k\right)=\left(x-x^{\prime}\right) \xi_{1}^{+}+\left(a\left(x^{\prime}\right)-b\left(x^{\prime}\right)+\varepsilon\right) \frac{\mu_{+} \xi_{1}^{+}+\xi_{+}}{1+\mu_{+}^{2}} \\
& \phi_{\varepsilon}^{-}\left(x, x^{\prime}, \xi_{-}^{1}, k\right)=\left(x-x^{\prime}\right) \xi_{1}^{-}+\left(b\left(x^{\prime}\right)-a\left(x^{\prime}\right)-\varepsilon\right) \frac{\mu_{-} \xi_{1}^{-}-\xi_{-}}{1+\mu_{-}^{2}}
\end{aligned}
$$

We prove

LEMMA 6.5. - There exists symbols $q_{1}^{ \pm}, \sigma_{2}^{ \pm}, q_{\varepsilon}^{ \pm}, r_{\varepsilon}^{ \pm}$, bounded for $\left|\xi_{1}^{ \pm}\right|$large, given by the relations (86) and distributions $S_{+}^{ \pm}$and $S_{-}^{ \pm}$, which are in $H^{-1}(\mathbb{R})$, given by (151) and (153) such that the action of the operators $R_{+}^{ \pm, k}$ and $R_{-}^{ \pm, k}$ is given by the following relations:

$$
\begin{aligned}
R_{+}^{+, k}(f)(x, k)=f(0) S_{+}^{+}+ & \frac{i}{4 \pi} \int_{\mathbb{R}_{+} \times \mathbb{R}} \sigma_{2}^{+}\left(x, x^{\prime}, \xi_{1}^{+}, k\right) e^{i\left(x-x^{\prime}\right) \xi_{1}^{+}} f\left(x^{\prime}\right) \mathrm{d} x^{\prime} \mathrm{d} \xi_{1}^{+} \\
& +\frac{1}{4 \pi} \int_{\mathbb{R}_{+} \times \mathbb{R}} q_{1}^{+}\left(x, x^{\prime}, \xi_{1}^{+}, k\right) e^{i\left(x-x^{\prime}\right) \xi_{1}^{+}} \partial_{x^{\prime}} f\left(x^{\prime}\right) \mathrm{d} x^{\prime} \mathrm{d} \xi_{1}^{+}
\end{aligned}
$$

$\overline{{ }^{(1)} \text { The values of }} f_{+}$and of $f_{-}$are well defined because the distributions are element of $L^{2}\left(\mathbb{R}_{+}\right) \cap$ $H^{1}\left(\mathbb{R}_{+}^{*}\right)$, hence the trace of these functions at the end is well defined. 


$$
\begin{aligned}
& R_{-}^{-, k}(f)(x, k)=f(0) S_{-}^{-}+ \frac{i}{4 \pi} \int_{\mathbb{R}_{+} \times \mathbb{R}} \sigma_{2}^{-}\left(x, x^{\prime}, \xi_{1}^{-}, k\right) e^{i\left(x-x^{\prime}\right) \xi_{1}^{-}} f\left(x^{\prime}\right) \mathrm{d} x^{\prime} \mathrm{d} \xi_{1}^{-} \\
&+\frac{1}{4 \pi} \int_{\mathbb{R}_{+} \times \mathbb{R}} q_{1}^{-}\left(x, x^{\prime}, \xi_{1}^{-}, k\right) e^{i\left(x-x^{\prime}\right) \xi_{1}^{-}} \partial_{x^{\prime}} f\left(x^{\prime}\right) \mathrm{d} x^{\prime} \mathrm{d} \xi_{1}^{-} \\
& R_{-}^{+, k}(f)(x, k)=f(0) S_{-}^{+}+\frac{1}{4 \pi} \lim _{\varepsilon \rightarrow 0_{+}, \mathcal{D}^{\prime}} \int_{\mathbb{R}_{+} \times \mathbb{R}_{\varepsilon}} q_{\varepsilon}^{+}\left(x, x^{\prime}, \xi_{1}^{+}, k\right) e^{i \phi_{\varepsilon}^{+}} \partial_{x^{\prime}} f\left(x^{\prime}\right) \mathrm{d} x^{\prime} \mathrm{d} \xi_{1}^{+} \\
&-\frac{i}{4 \pi} \lim _{\varepsilon \rightarrow 0_{+}, \mathcal{D}^{\prime}} \int_{\mathbb{R}_{+} \times \mathbb{R}} r_{\varepsilon}^{+}\left(x, x^{\prime}, \xi_{1}^{+}, k\right) e^{i \phi_{\varepsilon}^{+}} f\left(x^{\prime}\right) \mathrm{d} x^{\prime} \mathrm{d} \xi_{1}^{+} \\
& R_{+}^{-, k}(f)(x, k)=f(0) S_{+}^{-}+\frac{1}{4 \pi} \lim _{\varepsilon \rightarrow 0_{+}, \mathcal{D}^{\prime}} \int_{\mathbb{R}_{+} \times \mathbb{R}_{\varepsilon}} q_{\varepsilon}^{-}\left(x, x^{\prime}, \xi_{1}^{-}, k\right) e^{i \phi_{\varepsilon}^{-}} \partial_{x^{\prime}} f\left(x^{\prime}\right) \mathrm{d} x^{\prime} \mathrm{d} \xi_{1}^{-} \\
&-\frac{i}{4 \pi} \lim _{\varepsilon \rightarrow 0_{+}, \mathcal{D}^{\prime}} \int_{\mathbb{R}_{+} \times \mathbb{R}} r_{\varepsilon}^{-}\left(x, x^{\prime}, \xi_{1}^{-}, k\right) e^{i \phi_{\varepsilon}^{-}} f\left(x^{\prime}\right) \mathrm{d} x^{\prime} \mathrm{d} \xi_{1}^{-}
\end{aligned}
$$

This induces a definition of regular operators associated with the double layer distributions:

DEFinition 6.1. - The regular part of the Dirichlet to Neumann operators is given by

$$
\begin{gathered}
R_{ \pm, 0}^{ \pm, k}(f)(x, k)=\frac{i}{4 \pi} \int_{\mathbb{R}_{+} \times \mathbb{R}} \sigma_{2}^{ \pm}\left(x, x^{\prime}, \xi_{1}^{ \pm}, k\right) e^{i\left(x-x^{\prime}\right) \xi_{1}^{ \pm}} f\left(x^{\prime}\right) \mathrm{d} x^{\prime} \mathrm{d} \xi_{1}^{ \pm} \\
\frac{1}{4 \pi} \int_{\mathbb{R}_{+} \times \mathbb{R}} q_{1}^{ \pm}\left(x, x^{\prime}, \xi_{1}^{ \pm}, k\right) e^{i\left(x-x^{\prime}\right) \xi_{1}^{ \pm}} \partial_{x^{\prime}} f\left(x^{\prime}\right) \mathrm{d} x^{\prime} \mathrm{d} \xi_{1}^{ \pm} \\
R_{\mp, 0}^{ \pm, k, \varepsilon}(f)(x, k)=\frac{1}{4 \pi} \int_{\mathbb{R}_{+} \times \mathbb{R}} q_{\varepsilon}^{ \pm}\left(x, x^{\prime}, \xi_{1}^{ \pm}, k\right) e^{i \phi_{\varepsilon}^{ \pm}} \partial_{x^{\prime}} f\left(x^{\prime}\right) \mathrm{d} x^{\prime} \mathrm{d} \xi_{1}^{ \pm} \\
-\frac{i}{4 \pi} \int_{\mathbb{R}_{+} \times \mathbb{R}} r_{\varepsilon}^{ \pm}\left(x, x^{\prime}, \xi_{1}^{ \pm}, k\right) e^{i \phi_{\varepsilon}^{ \pm}} f\left(x^{\prime}\right) \mathrm{d} x^{\prime} \mathrm{d} \xi_{1}^{ \pm}
\end{gathered}
$$

Proof of Lemma 6.5. - Introduce the following functions of $\left(x, x^{\prime}\right)$ (additional to the functions defined in (85)):

$$
\begin{aligned}
& M_{1}^{+}\left(x, x^{\prime}\right)=\frac{1}{\left(1+\left(a^{\prime}(x)\right)^{2}\right)^{1 / 2}}\left[\frac{1+\mu_{+} a^{\prime}(x)}{1+\mu_{+}^{2}} \frac{1+\mu_{+} b^{\prime}\left(x^{\prime}\right)}{1+\mu_{+}^{2}}-\frac{\mu_{+}-a^{\prime}(x)}{1+\mu_{+}^{2}} \frac{\mu_{+}-b^{\prime}\left(x^{\prime}\right)}{1+\mu_{+}^{2}}\right] \\
& M_{2}^{+}\left(x, x^{\prime}\right)=\frac{1}{\left(1+\left(a^{\prime}(x)\right)^{2}\right)^{1 / 2}}\left[\frac{\mu_{+}-a^{\prime}(x)}{1+\mu_{+}^{2}} \frac{1+\mu_{+} b^{\prime}\left(x^{\prime}\right)}{1+\mu_{+}^{2}}+\frac{\mu_{+}-b^{\prime}\left(x^{\prime}\right)}{1+\mu_{+}^{2}} \frac{1+\mu_{+} a^{\prime}(x)}{1+\mu_{+}^{2}}\right] \\
& M_{3}^{+}\left(x, x^{\prime}\right)=\frac{1}{\left(1+\left(a^{\prime}(x)\right)^{2}\right)^{1 / 2}}\left[\frac{\left(\mu_{+}-a^{\prime}(x)\right)\left(\mu_{+}-b^{\prime}\left(x^{\prime}\right)\right)}{1+\mu_{+}^{2}}\right] .
\end{aligned}
$$




$$
\begin{aligned}
& M_{1}^{-}\left(x, x^{\prime}\right)=\frac{1}{\left(1+\left(b^{\prime}(x)\right)^{2}\right)^{1 / 2}}\left[\frac{1+\mu_{-} b^{\prime}(x)}{1+\mu_{-}^{2}} \frac{1+\mu_{-} a^{\prime}\left(x^{\prime}\right)}{1+\mu_{-}^{2}}-\frac{\mu_{-}-a^{\prime}\left(x^{\prime}\right)}{1+\mu_{-}^{2}} \frac{\mu_{-}-b^{\prime}(x)}{1+\mu_{-}^{2}}\right] \\
& M_{2}^{-}\left(x, x^{\prime}\right)=-\frac{1}{\left(1+\left(b^{\prime}(x)\right)^{2}\right)^{1 / 2}}\left[\frac{\mu_{-}-a^{\prime}\left(x^{\prime}\right)}{1+\mu_{-}^{2}} \frac{1+\mu_{-}(x)}{1+\mu_{-}^{2}}+\frac{\mu_{-}-b^{\prime}(x)}{1+\mu_{-}^{2}} \frac{1+\mu_{-} a^{\prime}\left(x^{\prime}\right)}{1+\mu_{-}^{2}}\right] \\
& M_{3}^{-}\left(x, x^{\prime}\right)=\frac{1}{\left(1+\left(b^{\prime}(x)\right)^{2}\right)^{1 / 2}}\left[\frac{\left(\mu_{-}-a^{\prime}\left(x^{\prime}\right)\right)\left(\mu_{-}-b^{\prime}(x)\right)}{1+\mu_{-}^{2}}\right] .
\end{aligned}
$$

We note that the symbols involved in the expressions of $\left(1+\left(a^{\prime}(x)\right)^{2}\right)^{1 / 2} \partial_{+} u_{+}$and of $\left(1+\left(a^{\prime}(x)\right)^{2}\right)^{1 / 2} \partial_{+} u_{-}$obtained below in Lemma 6.4 are exactly

$$
\frac{i}{4 \pi} \frac{L_{1}^{+}\left(\xi_{1}^{+}\right)^{2}+L_{2}^{+} \xi_{1}^{+} \xi_{+}+L_{3}^{+} k^{2}}{\xi_{+}} \text {and }-\frac{i}{4 \pi} \frac{M_{1}^{+} \xi_{+}^{2}+M_{2}^{+} \xi_{1}^{+} \xi_{+}+M_{3}^{+} k^{2}}{\xi_{+}} .
$$

We verify the relations

$$
\left\{\begin{array}{l}
A_{+}^{+, 2}\left(x, x^{\prime}, \frac{\xi_{1}^{+}-\mu_{+} \xi_{+}}{1+\mu_{+}^{2}}, \frac{\mu_{+} \xi_{1}^{+}+\xi_{+}}{1+\mu_{+}^{2}}\right)=-L_{1}^{+}\left(x, x^{\prime}\right)\left(\xi_{1}^{+}\right)^{2}-L_{2}^{+}\left(x, x^{\prime}\right) \xi_{1}^{+} \xi_{+}-L_{3}^{+}\left(x, x^{\prime}\right) k^{2} \\
A_{-}^{-, 2}\left(x, x^{\prime}, \frac{\xi_{1}^{-}+\mu_{-} \xi_{-}}{1+\mu_{-}^{2}}, \frac{\mu_{-} \xi_{1}^{-}-\xi_{-}}{1+\mu_{-}^{2}}\right)=-L_{1}^{-}\left(x, x^{\prime}\right)\left(\xi_{1}^{-}\right)^{2}-L_{2}^{-}\left(x, x^{\prime}\right) \xi_{1}^{-} \xi_{-}-L_{3}^{-}\left(x, x^{\prime}\right) k^{2} \\
A_{-}^{+, 2}\left(x, x^{\prime}, \frac{\xi_{1}^{+}-\mu_{+} \xi_{+}}{1+\mu_{+}^{2}}, \frac{\mu_{+} \xi_{1}^{+}+\xi_{+}}{1+\mu_{+}^{2}}\right)=M_{1}^{+}\left(x, x^{\prime}\right) \xi_{+}^{2}+M_{2}^{+}\left(x, x^{\prime}\right) \xi_{+} \xi_{1}^{+}+M_{3}^{+}\left(x, x^{\prime}\right) k^{2} \\
A_{+}^{-, 2}\left(x, x^{\prime}, \frac{\xi_{1}^{+}+\mu_{-} \xi_{-}}{1+\mu_{-}^{2}}, \frac{\mu_{-} \xi_{1}^{-}-\xi_{-}}{1+\mu_{-}^{2}}\right)=M_{1}^{-}\left(x, x^{\prime}\right) \xi_{-}^{2}+M_{2}^{-}\left(x, x^{\prime}\right) \xi_{1}^{-} \xi_{-}+M_{3}^{-}\left(x, x^{\prime}\right) k^{2}
\end{array}\right.
$$

Note that $\partial_{x^{\prime}} \xi_{+}=\frac{k^{2}}{\xi_{+}} \mu_{+} \partial_{x^{\prime}} \mu_{+}$and $\partial_{x^{\prime}} \xi_{-}=\frac{k^{2}}{\xi_{-}} \mu_{-} \partial_{x^{\prime}} \mu_{-}$. We introduce

$$
\left\{\begin{array}{l}
m_{\varepsilon}^{+}\left(x, x^{\prime}\right)=-1+\partial_{x^{\prime}}\left(\frac{\mu_{+}\left(a\left(x^{\prime}\right)-b\left(x^{\prime}\right)+\varepsilon\right)}{1+\mu_{+}^{2}}\right) \\
n_{\varepsilon}^{+}\left(x, x^{\prime}\right)=\partial_{x^{\prime}}\left(\frac{a\left(x^{\prime}\right)-b\left(x^{\prime}\right)+\varepsilon}{1+\mu_{+}^{2}}\right) \\
m_{\varepsilon}^{-}\left(x, x^{\prime}\right)=-1+\partial_{x^{\prime}}\left(\frac{-\mu_{-}\left(a\left(x^{\prime}\right)-b\left(x^{\prime}\right)+\varepsilon\right)}{1+\mu_{-}^{2}}\right) \\
n_{\varepsilon}^{-}\left(x, x^{\prime}\right)=\partial_{x^{\prime}}\left(\frac{a\left(x^{\prime}\right)-b\left(x^{\prime}\right)+\varepsilon}{1+\mu_{-}^{2}}\right) \\
t_{\varepsilon}^{+}\left(x, x^{\prime}\right)=-1+\varepsilon \partial_{x^{\prime}}\left(\frac{\mu_{+}^{2}}{1+\mu_{+}^{2}}\right) \\
s_{\varepsilon}^{+}\left(x, x^{\prime}\right)=\varepsilon \partial_{x^{\prime}}\left(\frac{1}{1+\mu_{+}^{2}}\right) \\
t_{\varepsilon}^{-}\left(x, x^{\prime}\right)=-1-\varepsilon \partial_{x^{\prime}}\left(\frac{\mu_{-}}{1+\mu_{-}^{2}}\right) \\
s_{\varepsilon}^{-}\left(x, x^{\prime}\right)=\varepsilon \partial_{x^{\prime}}\left(\frac{1}{1+\mu_{-}^{2}}\right)
\end{array}\right.
$$

We have

$$
\begin{aligned}
& \partial_{x^{\prime}} \phi_{\varepsilon}^{+}\left(x, x^{\prime}, \xi_{1}^{+}, k\right)=\xi_{1}^{+} m_{\varepsilon}^{+}\left(x, x^{\prime}\right)+\xi_{+} n_{\varepsilon}^{+}\left(x, x^{\prime}\right)+\left(a\left(x^{\prime}\right)-b\left(x^{\prime}\right)+\varepsilon\right) \mu_{+} \partial_{x^{\prime}} \mu_{+} \frac{k^{2}}{\xi_{+}} \\
& \partial_{x^{\prime}} \phi_{\varepsilon}^{-}\left(x, x^{\prime}, \xi_{1}^{-}, k\right)=\xi_{1}^{-} m_{\varepsilon}^{-}\left(x, x^{\prime}\right)+\xi_{-} n_{\varepsilon}^{-}\left(x, x^{\prime}\right)+\left(a\left(x^{\prime}\right)-b\left(x^{\prime}\right)+\varepsilon\right) \mu_{-} \partial_{x^{\prime}} \mu_{-} \frac{k^{2}}{\xi_{-}}
\end{aligned}
$$


We denote by $\alpha_{\varepsilon}^{ \pm}$and $\beta_{\varepsilon}^{ \pm}$the functions such that there exists functions $\gamma_{\varepsilon}^{ \pm}$and $\delta_{\varepsilon}^{ \pm}$ satisfying

$$
\begin{aligned}
& A_{-}^{+, 2}\left(x, x^{\prime}, \frac{\xi_{1}^{+}-\mu_{+} \xi_{+}}{1+\mu_{+}^{2}}, \frac{\mu_{+} \xi_{1}^{+}+\xi_{+}}{1+\mu_{+}^{2}}\right)=\left[\alpha_{\varepsilon}^{+} \xi_{1}^{+}+\beta_{\varepsilon}^{+} \xi_{+}\right] \partial_{x^{\prime}} \phi_{\varepsilon}^{+}+\gamma_{\varepsilon}^{+} k^{2} \frac{\xi_{1}^{+}}{\xi_{+}}+\delta_{\varepsilon}^{+} k^{2} \\
& A_{+}^{-, 2}\left(x, x^{\prime}, \frac{\xi_{1}^{-}+\mu_{-} \xi_{-}}{1+\mu_{-}^{2}}, \frac{\mu_{-} \xi_{1}^{-}-\xi_{-}}{1+\mu_{-}^{2}}\right)=\left[\alpha_{\varepsilon}^{-} \xi_{1}^{-}+\beta_{\varepsilon}^{-} \xi_{-}\right] \partial_{x^{\prime}} \phi_{\varepsilon}^{-}+\gamma_{\varepsilon}^{-} k^{2} \frac{\xi_{1}^{-}}{\xi_{-}}+\delta_{\varepsilon}^{-} k^{2} .
\end{aligned}
$$

In a similar fashion, we denote by $A_{\varepsilon}^{ \pm}, B_{\varepsilon}^{ \pm}, \Gamma_{\varepsilon}^{ \pm}, \Delta_{\varepsilon}^{ \pm}$the functions such that

$$
\begin{aligned}
& -A_{+}^{+, 2}\left(x, x^{\prime}, \frac{\xi_{1}^{+}-\mu_{+} \xi_{+}}{1+\mu_{+}^{2}}, \frac{\mu_{+} \xi_{1}^{+}+\xi_{+}}{1+\mu_{+}^{2}}\right) \\
& =\left[A_{\varepsilon}^{+} \xi_{1}^{+}+B_{\varepsilon}^{+} \xi_{+}\right] \partial_{x^{\prime}}\left(\left(x-x^{\prime}\right) \xi_{1}^{+}+\varepsilon \frac{\mu_{+} \xi_{1}^{+}+\xi_{+}}{1+\mu_{+}^{2}}\right)+\Gamma_{\varepsilon}^{+} k^{2} \frac{\xi_{1}^{+}}{\xi_{+}}+\Delta_{\varepsilon}^{+} k^{2} \\
& -A_{-}^{-, 2}\left(x, x^{\prime}, \frac{\xi_{1}^{-}+\mu_{-} \xi_{-}}{1+\mu_{-}^{2}}, \frac{\mu_{-} \xi_{1}^{-}-\xi_{-}}{1+\mu_{-}^{2}}\right) \\
& \quad=\left[A_{\varepsilon}^{-} \xi_{1}^{-}+B_{\varepsilon}^{-} \xi_{-}\right] \partial_{x^{\prime}}\left(\left(x-x^{\prime}\right) \xi_{1}^{-}+\varepsilon \frac{-\mu_{-} \xi_{1}^{-}+\xi_{-}}{1+\mu_{-}^{2}}\right)+\Gamma_{\varepsilon}^{-} k^{2} \frac{\xi_{1}^{-}}{\xi_{-}}+\Delta_{\varepsilon}^{-} k^{2} .
\end{aligned}
$$

These functions are given by the relations

$$
\left\{\begin{aligned}
\alpha_{\varepsilon}^{ \pm} & =\frac{-m_{\varepsilon}^{ \pm} M_{1}^{ \pm}+n_{\varepsilon}^{ \pm} M_{2}^{ \pm}}{\left(m_{\varepsilon}^{ \pm}\right)^{2}+\left(n_{\varepsilon}^{ \pm}\right)^{2}} \\
\beta_{\varepsilon}^{ \pm} & =\frac{n_{\varepsilon}^{ \pm} M_{1}^{ \pm}+m_{\varepsilon}^{ \pm} M_{2}^{ \pm}}{\left(m_{\varepsilon}^{ \pm}\right)^{2}+\left(n_{\varepsilon}^{ \pm}\right)^{2}} \\
\gamma_{\varepsilon}^{ \pm}\left(x, x^{\prime}\right) & =-\alpha_{\varepsilon}^{ \pm}\left(x, x^{\prime}\right) \mu_{ \pm} \partial_{x^{\prime}} \mu_{ \pm}\left(a\left(x^{\prime}\right)-b\left(x^{\prime}\right)+\varepsilon\right) \\
\delta_{\varepsilon}^{ \pm}\left(x, x^{\prime}\right) & =M_{3}^{ \pm}\left(x, x^{\prime}\right)-\left(1+\mu_{ \pm}^{2}\right) m_{\varepsilon}^{ \pm} \alpha_{\varepsilon}^{ \pm}-\beta_{\varepsilon}^{ \pm} \mu_{ \pm} \partial_{x^{\prime}} \mu_{ \pm}\left(a\left(x^{\prime}\right)-b\left(x^{\prime}\right)+\varepsilon\right) \\
A_{\varepsilon}^{+} & =\frac{t_{\varepsilon}^{ \pm} L_{1}^{ \pm}+s_{\varepsilon}^{ \pm} L_{2}^{ \pm}}{\left(t_{\varepsilon}^{ \pm}\right)^{2}+\left(s_{\varepsilon}^{ \pm}\right)^{2}} \\
B_{\varepsilon}^{ \pm} & =\frac{-s_{\varepsilon}^{ \pm} L_{1}^{ \pm}+t_{\varepsilon}^{ \pm} L_{2}^{ \pm}}{\left(t_{\varepsilon}^{ \pm}\right)^{2}+\left(s_{\varepsilon}^{ \pm}\right)^{2}} \\
\Gamma_{\varepsilon}^{ \pm}\left(x, x^{\prime}\right) & =-A_{\varepsilon}^{ \pm}\left(x, x^{\prime}\right) \mu_{ \pm} \partial_{x^{\prime}} \mu_{ \pm}\left(a\left(x^{\prime}\right)-b\left(x^{\prime}\right)+\varepsilon\right) \\
\Delta_{\varepsilon}^{ \pm}\left(x, x^{\prime}\right) & =L_{3}^{ \pm}\left(x, x^{\prime}\right)-\left(1+\mu_{ \pm}^{2}\right) s_{\varepsilon}^{ \pm} B_{\varepsilon}^{ \pm}-B_{\varepsilon}^{ \pm} \mu_{ \pm} \partial_{x^{\prime}} \mu_{ \pm}\left(a\left(x^{\prime}\right)-b\left(x^{\prime}\right)+\varepsilon\right) .
\end{aligned}\right.
$$


We consider the expressions of Lemma 6.4. We have

$$
\begin{aligned}
& R_{+}^{+, k}(f)(x, k)=\lim _{\varepsilon \rightarrow 0_{+}} \frac{1}{4 \pi} \int_{\mathbb{R} \times \mathbb{R}_{+}} e^{i\left(x-x^{\prime}\right) \xi_{1}^{+}+i \varepsilon \frac{\mu_{+} \xi_{1}^{+}+\xi_{+}}{1+\mu_{+}^{2}}} i f\left(x^{\prime}\right) \mathrm{d} x^{\prime} \mathrm{d} \xi_{1}^{+} \\
& \times\left[\frac{\mu_{+}-a^{\prime}(x)}{1+\mu_{+}^{2}} \xi_{1}^{+}+\frac{1+\mu_{+} a^{\prime}\left(x^{\prime}\right)}{1+\mu_{+}^{2}} \xi_{+}\right]\left[\frac{\mu_{+}-a^{\prime}\left(x^{\prime}\right)}{1+\mu_{+}^{2}} \frac{\xi_{1}^{+}}{\xi_{+}}+\frac{1+\mu_{+} a^{\prime}\left(x^{\prime}\right)}{1+\mu_{+}^{2}}\right] \\
& =\lim _{\varepsilon \rightarrow 0_{+}} \frac{1}{4 \pi} \int_{\mathbb{R} \times \mathbb{R}_{+}} e^{i\left(x-x^{\prime}\right) \xi_{1}^{+}+i \varepsilon \frac{\mu_{+} \xi_{1}^{+}+\xi_{+}}{1+\mu_{+}^{2}}} \text { if }\left(x^{\prime}\right) \frac{\left[L_{1}^{+}\left(\xi_{1}^{+}\right)^{2}+L_{2}^{+} \xi_{1}^{+} \xi_{+}+L_{3}^{+} k^{2}\right]}{\xi_{+}} \mathrm{d} x^{\prime} \mathrm{d} \xi_{1}^{+} .
\end{aligned}
$$

We use the relation

$$
\begin{aligned}
L_{1}^{+}\left(\xi_{1}^{+}\right)^{2}+ & L_{2}^{+} \xi_{1}^{+} \xi_{+}+L_{3}^{+} k^{2}= \\
& {\left[A_{\varepsilon}^{+} \xi_{1}^{+}+B_{\varepsilon}^{+} \xi_{+}\right] \partial_{x^{\prime}}\left(\left(x-x^{\prime}\right) \xi_{1}^{+}+\varepsilon \frac{\mu_{+} \xi_{1}^{+}+\xi_{+}}{1+\mu_{+}^{2}}\right)+\Gamma_{\varepsilon}^{+} \frac{k^{2} \xi_{1}^{+}}{\xi_{+}}+\Delta_{\varepsilon}^{+} k^{2} }
\end{aligned}
$$

hence

$$
\begin{aligned}
& R_{+}^{+, k}(f)(x, k)=\lim _{\varepsilon \rightarrow 0_{+}} \frac{1}{4 \pi} \int_{\mathbb{R} \times \mathbb{R}_{+}} e^{i\left(x-x^{\prime}\right) \xi_{1}^{+}+i \varepsilon \frac{\mu_{+} \xi_{1}^{+}+\xi_{+}}{1+\mu_{+}^{2}}}\left[i \partial_{x^{\prime}}\left(\left(x-x^{\prime}\right) \xi_{1}^{+}+\varepsilon \frac{\mu_{+} \xi_{1}^{+}+\xi_{+}}{1+\mu_{+}^{2}}\right)\right. \\
&\left.\times\left[A_{\varepsilon}^{+} \frac{\xi_{1}^{+}}{\xi_{+}}+B_{\varepsilon}^{+}\right]+i\left[\Gamma_{\varepsilon}^{+} \frac{k^{2} \xi_{1}^{+}}{\xi_{+}^{2}}+\Delta_{\varepsilon}^{+} \frac{k^{2}}{\xi_{+}}\right]\right] f\left(x^{\prime}\right) \mathrm{d} x^{\prime} \mathrm{d} \xi_{1}^{+} \\
&= \lim _{\varepsilon \rightarrow 0_{+}} \frac{1}{4 \pi} \int_{\mathbb{R} \times \mathbb{R}_{+}}\left[A_{\varepsilon}^{+} \frac{\xi_{1}^{+}}{\xi_{+}}+B_{\varepsilon}^{+}\right] f\left(x^{\prime}\right) \partial_{x^{\prime}}\left(e^{i\left(x-x^{\prime}\right) \xi_{1}^{+}+i \varepsilon \frac{\mu_{+} \xi_{1}^{+}+\xi_{+}}{1+\mu_{+}^{+}}}\right) \mathrm{d} x^{\prime} \mathrm{d} \xi_{1}^{+} \\
&+\lim _{\varepsilon \rightarrow 0_{+}} \frac{1}{4 \pi} \int_{\mathbb{R} \times \mathbb{R}_{+}} e^{i\left(x-x^{\prime}\right) \xi_{1}^{+}+i \varepsilon \frac{\mu_{+} \xi_{1}^{+}+\xi_{+}}{1+\mu_{+}^{2}}} i\left[\Gamma_{\varepsilon}^{+} \frac{k^{2} \xi_{1}^{+}}{\xi_{+}^{2}}+\Delta_{\varepsilon}^{+} \frac{k^{2}}{\xi_{+}}\right] f\left(x^{\prime}\right) \mathrm{d} x^{\prime} \mathrm{d} \xi_{1}^{+} .
\end{aligned}
$$

We use an integration by parts in the first term. We have

$$
\begin{aligned}
\int_{\mathbb{R}_{+}}\left[A_{\varepsilon}^{+} \frac{\xi_{1}^{+}}{\xi_{+}}+B_{\varepsilon}^{+}\right] f\left(x^{\prime}\right) \frac{1}{i} \partial_{x^{\prime}}\left(e^{i\left(x-x^{\prime}\right) \xi_{1}^{+}+i \varepsilon \frac{\mu_{+} \xi_{1}^{+}+\xi_{+}}{1+\mu_{+}^{2}}}\right) \mathrm{d} x^{\prime}= \\
-\int_{\mathbb{R}_{+}} \partial_{x^{\prime}}\left(\left[A_{\varepsilon}^{+} \frac{\xi_{1}^{+}}{\xi_{+}}+B_{\varepsilon}^{+}\right] f\left(x^{\prime}\right)\right) e^{i\left(x-x^{\prime}\right) \xi_{1}^{+}+i \varepsilon \frac{\mu_{+} \xi_{1}^{+}+\xi_{+}}{1+\mu_{+}^{2}}} \mathrm{~d} x^{\prime} \\
-f(0)\left[A_{\varepsilon}^{+} \frac{\xi_{1}^{+}}{\xi_{+}}+B_{\varepsilon}^{+}\right](x, 0) e^{i x \xi_{1}^{+}+i \varepsilon \frac{\mu_{+} \xi_{1}^{+}+\xi_{+}}{1+\mu_{+}^{2}}}
\end{aligned}
$$


This leads to

$$
\begin{aligned}
R_{+}^{+, k}(f)(x, k) & = \\
-\lim _{\varepsilon \rightarrow 0_{+}} & \frac{1}{4 \pi} \int_{\mathbb{R} \times \mathbb{R}_{+}} \partial_{x^{\prime}}\left(\left[A_{\varepsilon}^{+} \frac{\xi_{1}^{+}}{\xi_{+}}+B_{\varepsilon}^{+}\right] f\left(x^{\prime}\right)\right) e^{i\left(x-x^{\prime}\right) \xi_{1}^{+}+i \varepsilon \frac{\mu_{+} \xi_{1}^{+}+\xi_{+}}{1+\mu_{+}^{2}}} \mathrm{~d} x^{\prime} \mathrm{d} \xi_{1}^{+} \\
& -f(0) \lim _{\varepsilon \rightarrow 0_{+}} \frac{1}{4 \pi} \int_{\mathbb{R}}\left(A_{\varepsilon}^{+} \frac{\xi_{1}^{+}}{\xi_{+}}+B_{\varepsilon}^{+}\right) e^{i x \xi_{1}^{+}+i \varepsilon \frac{\mu_{+} \xi_{1}^{+}+\xi_{+}}{1+\mu_{+}^{2}}} \mathrm{~d} \xi_{1}^{+} \\
& +\lim _{\varepsilon \rightarrow 0_{+}} \frac{i}{4 \pi} \int_{\mathbb{R} \times \mathbb{R}_{+}} e^{i\left(x-x^{\prime}\right) \xi_{1}^{+}+i \varepsilon \frac{\mu_{+} \xi_{1}^{+}+\xi_{+}}{1+\mu_{+}^{2}}}\left[\Gamma_{\varepsilon}^{+} \frac{k^{2} \xi_{1}^{+}}{\xi_{+}^{2}}+\Delta_{\varepsilon}^{+} \frac{k^{2}}{\xi_{+}}\right] f\left(x^{\prime}\right) \mathrm{d} x^{\prime} \mathrm{d} \xi_{1}^{+} .
\end{aligned}
$$

Let us study the corner term. We introduce the sequence of functions

$$
S_{+}^{+, \varepsilon}(x)=-\frac{1}{4 \pi} \int_{\mathbb{R}}\left(A_{\varepsilon}^{+} \frac{\xi_{1}^{+}}{\xi_{+}}+B_{\varepsilon}^{+}\right) e^{i x \xi_{1}^{+}+i \varepsilon \frac{\mu_{+} \xi_{1}^{+}+\xi_{+}}{1+\mu_{+}^{2}}} \mathrm{~d} \xi_{1}^{+} .
$$

We have

$$
\left\langle S_{+}^{+, \varepsilon}, l\right\rangle=-\frac{1}{4 \pi} \iint l(x)\left(A_{\varepsilon}^{+} \frac{\xi_{1}^{+}}{\xi_{+}}+B_{\varepsilon}^{+}\right) e^{i x \xi_{1}^{+}+i \varepsilon \frac{\mu_{+} \xi_{1}^{+}+\xi_{+}}{1+\mu_{+}^{2}}} \mathrm{~d} \xi_{1}^{+} \mathrm{d} x .
$$

Note that

$$
\begin{aligned}
A_{\varepsilon}^{+} \xi_{1}^{+}+B_{\varepsilon}^{+} \xi_{+}= & \frac{A_{\varepsilon}^{+}(x, 0)}{1+\varepsilon \partial_{x}\left(\frac{\mu_{+}}{1+\mu_{+}^{2}}\right)} \partial_{x}\left(x \xi_{1}^{+}+\varepsilon \frac{\mu_{+} \xi_{1}^{+}+\xi_{+}}{1+\mu_{+}^{2}}\right) \\
& +\left(B_{\varepsilon}^{+}-\varepsilon A_{\varepsilon}^{+} \frac{\partial_{x}\left(\frac{1}{1+\mu_{+}^{2}}\right)}{1+\varepsilon \partial_{x}\left(\frac{\mu_{+}}{1+\mu_{+}^{2}}\right)}\right) \xi_{+}-\varepsilon \frac{A_{\varepsilon}^{+}(x, 0)}{1+\varepsilon \partial_{x}\left(\frac{\mu_{+}}{1+\mu_{+}^{2}}\right)} \frac{k^{2} \mu_{+} \partial_{x} \mu_{+}}{\xi_{+}}
\end{aligned}
$$

which imply the equality

$$
\begin{aligned}
\left\langle S_{+}^{+, \varepsilon}, l\right\rangle=-\frac{i}{4 \pi} & \iint \partial_{x}\left(\frac{l(x) A_{\varepsilon}^{+}(x, 0)}{\xi_{+}\left(1+\varepsilon \partial_{x}\left(\frac{\mu_{+}}{1+\mu_{+}^{2}}\right)\right)}\right) e^{i x \xi_{1}^{+}+i \varepsilon \frac{\mu_{+} \xi_{1}^{+}+\xi_{+}}{1+\mu_{+}^{2}}} \mathrm{~d} x \mathrm{~d} \xi_{1}^{+} \\
& -\frac{1}{4 \pi} \iint l(x)\left(B_{\varepsilon}^{+}-\varepsilon A_{\varepsilon}^{+} \frac{\partial_{x}\left(\frac{1}{1+\mu_{+}^{2}}\right)}{1+\varepsilon \partial_{x}\left(\frac{\mu_{+}}{1+\mu_{+}^{2}}\right)}\right) e^{i x \xi_{1}^{+}+i \varepsilon \frac{\mu_{+} \xi_{1}^{+}+\xi_{+}}{1+\mu_{+}^{2}}} \mathrm{~d} x \mathrm{~d} \xi_{1}^{+} \\
& +\frac{\varepsilon}{4 \pi} \iint l(x) \frac{A_{\varepsilon}^{+}(x, 0)}{1+\varepsilon \partial_{x}\left(\frac{\mu_{+}}{1+\mu_{+}^{2}}\right)} \frac{k^{2} \mu_{+} \partial_{x} \mu_{+}}{\xi_{+}^{2}} e^{i x \xi_{1}^{+}+i \varepsilon \frac{\mu_{+} \xi_{1}^{+}+\xi_{+}}{1+\mu_{+}^{2}}} \mathrm{~d} x \mathrm{~d} \xi_{1}^{+}
\end{aligned}
$$

The limit of such integrals exist when $\varepsilon \rightarrow 0$. We verify that $A_{0}^{+}(x, 0)=-L_{1}^{+}(x, 0)$ and $B_{0}^{+}(x, 0)=-L_{2}^{+}(x, 0)$. We have thus

$$
\left\langle S_{+}^{+}, l\right\rangle=\frac{i}{4 \pi} \iint e^{i x \xi_{1}^{+}} \partial_{x}\left(\frac{l(x) L_{1}^{+}(x, 0)}{\xi_{+}}\right) \mathrm{d} x \mathrm{~d} \xi_{1}^{+}+\frac{1}{4 \pi} \iint e^{i x \xi_{1}^{+}} l(x) L_{2}^{+}(x, 0) \mathrm{d} x \mathrm{~d} \xi_{1}^{+}
$$


which proves that

$$
\left\langle S_{+}^{+}, l\right\rangle=\frac{i}{4 \pi} \iint e^{i x \xi_{1}^{+}} \partial_{x}\left(\frac{l(x) L_{1}^{+}(x, 0)}{\xi_{+}}\right) \mathrm{d} x \mathrm{~d} \xi_{1}^{+}+\frac{1}{2} L_{2}^{+}(0,0) l(0) .
$$

As $L_{2}^{+}(0,0)=0$, the distribution $S_{+}^{+}$is element of $H^{-1}(\mathbb{R})$ and is equal to

$$
\left\langle S_{+}^{+}, l\right\rangle=\frac{i}{4 \pi} \iint e^{i x \xi_{1}^{+}} \partial_{x}\left(\frac{l(x) L_{1}^{+}(x, 0)}{\xi_{+}}\right) \mathrm{d} x \mathrm{~d} \xi_{1}^{+} .
$$

In a similar fashion, the symbol associated with the operator $R_{-}^{+, k}$ is

$$
\xi_{+}^{-1}\left[M_{1}^{+} \xi_{+}^{2}+M_{2}^{+} \xi_{1}^{+} \xi_{+}+M_{3}^{+} k^{2}\right]=\partial_{x^{\prime}} \phi_{\varepsilon}^{+}\left[\alpha_{\varepsilon} \frac{\xi_{1}^{+}}{\xi_{+}}+\beta_{\varepsilon}^{+}\right]+\gamma_{\varepsilon}^{+} \frac{k^{2} \xi_{1}^{+}}{\xi_{+}^{2}}+\delta_{\varepsilon}^{+} \frac{k^{2}}{\xi_{+}}
$$

and the phase function is $\phi_{\varepsilon}^{+}$. We have similarly

$$
\begin{gathered}
\int_{0}^{\infty} \partial_{x^{\prime}} \phi_{\varepsilon}^{+} e^{i \phi_{\varepsilon}^{+}}\left[\alpha_{\varepsilon}^{+} \frac{\xi_{1}^{+}}{\xi_{+}}+\beta_{\varepsilon}^{+}\right] f\left(x^{\prime}\right) \psi\left(x^{\prime}\right) \mathrm{d} x^{\prime}= \\
i f(0) e^{i \phi_{\varepsilon}^{+}\left(x, 0, \xi_{1}^{+}, k\right)}\left[\alpha_{\varepsilon}^{+}(x, 0) \frac{\xi_{1}^{+}}{\xi_{+}}+\beta_{\varepsilon}^{+}(x, 0)\right]+i \int_{0}^{\infty} e^{i \phi_{\varepsilon}^{+}} \partial_{x^{\prime}}\left(f\left(x^{\prime}\right) \psi\left(x^{\prime}\right)\left[\alpha_{\varepsilon}^{+} \frac{\xi_{1}^{+}}{\xi_{+}}+\beta_{\varepsilon}^{+}\right]\right)
\end{gathered}
$$

hence

$$
\begin{aligned}
& -\frac{i}{4 \pi} \int_{0}^{+\infty} e^{i \phi_{\varepsilon}^{+}\left(x, x^{\prime}, \xi_{1}^{+}, k\right)} f\left(x^{\prime}\right) \psi\left(x^{\prime}\right) \frac{A_{+}^{-, 2}\left(x, x^{\prime}, \frac{\xi_{1}^{+}-\mu_{+} \xi_{+}}{1+\mu_{+}^{2}}, \frac{\mu_{+} \xi_{1}^{+}+\xi_{+}}{1+\mu_{+}^{2}}\right)}{\xi_{+}} \mathrm{d} x^{\prime} \\
& =\frac{1}{4 \pi} f(0) e^{i \phi_{\varepsilon}^{+}\left(x, 0, \xi_{1}^{+}, k\right)}\left[\alpha_{\varepsilon}^{+}(x, 0) \frac{\xi_{1}^{+}}{\xi_{+}}+\beta_{\varepsilon}^{+}\right] \\
& \quad-\frac{i}{4 \pi} \int_{0}^{+\infty} e^{i \phi_{\varepsilon}^{+}\left(x, x^{\prime}, \xi_{1}^{+}, k\right)} f\left(x^{\prime}\right) \psi\left(x^{\prime}\right)\left[\gamma^{\varepsilon}\left(x, x^{\prime}\right) \frac{k^{2} \xi_{1}^{+}}{\xi_{+}^{2}}+\delta^{\varepsilon} \frac{k^{2}}{\xi_{+}}\right] \mathrm{d} x^{\prime} \\
& \quad+\frac{1}{4 \pi} \int_{0}^{+\infty} e^{i \phi_{\varepsilon}^{+}\left(x, x^{\prime}, \xi_{1}^{+}, k\right)} \partial_{x^{\prime}}\left[f\left(x^{\prime}\right) \psi\left(x^{\prime}\right)\left(\alpha_{\varepsilon}^{+}\left(x, x^{\prime}\right) \frac{\xi_{1}^{+}}{\xi_{+}}+\beta_{\varepsilon}^{+}\right)\right] \mathrm{d} x^{\prime} .
\end{aligned}
$$

We obtain

$$
\begin{gathered}
R_{-}^{+, k}(f)=-\lim _{\varepsilon \rightarrow 0_{+}} \frac{i}{4 \pi} \int_{\mathbb{R} \times \mathbb{R}_{+}} e^{i \phi_{\varepsilon}^{+}\left(x, x^{\prime}, \xi_{1}^{+}, k\right)} f\left(x^{\prime}\right) \psi\left(x^{\prime}\right)\left[\gamma^{\varepsilon}\left(x, x^{\prime}\right) \frac{k^{2} \xi_{1}^{+}}{\xi_{+}^{2}}+\delta^{\varepsilon} \frac{k^{2}}{\xi_{+}}\right] \mathrm{d} x^{\prime} \\
+\lim _{\varepsilon \rightarrow 0_{+}} \frac{1}{4 \pi} \int_{\mathbb{R} \times \mathbb{R}_{+}} e^{i \phi_{\varepsilon}^{+}\left(x, x^{\prime}, \xi_{1}^{+}, k\right)} \partial_{x^{\prime}}\left[f\left(x^{\prime}\right) \psi\left(x^{\prime}\right)\left(\alpha_{\varepsilon}^{+}\left(x, x^{\prime}\right) \frac{\xi_{1}^{+}}{\xi_{+}}+\beta_{\varepsilon}^{+}\right)\right] \mathrm{d} x^{\prime}+S_{-}^{+} f(0)
\end{gathered}
$$

where the distribution at the corner is the limit of $S_{-}^{+, \varepsilon}$ equal to

$$
S_{-}^{+, \varepsilon}(x)=\frac{1}{4 \pi} \int_{\mathbb{R}}\left[\alpha_{\varepsilon}^{+}(x, 0) \frac{\xi_{1}^{+}}{\xi_{+}}+\beta_{\varepsilon}^{+}(x, 0)\right] e^{i \phi_{\varepsilon}^{+}\left(x, 0, \xi_{1}^{+}, k\right)} \mathrm{d} \xi_{1}^{+} .
$$

Noting that

$$
\partial_{x} \phi_{+}^{\varepsilon}\left(x, 0, \xi_{1}^{+}, k\right)=\xi_{1}^{+}+\varepsilon \partial_{x}\left(\mu_{+}(x, 0)\right)\left[\frac{1-\mu_{+}^{2}}{\left(1+\mu_{+}^{2}\right)^{2}} \xi_{1}^{+}+\frac{-2 \mu_{+}}{\left(1+\mu_{+}^{2}\right)^{2}} \xi_{+}+\frac{k^{2} \mu_{+}}{\left(1+\mu_{+}^{2}\right) \xi_{+}}\right],
$$


which imply the relation

$$
\alpha_{\varepsilon}^{+} \xi_{1}^{+}+\beta_{\varepsilon}^{+} \xi_{+}=\widetilde{\alpha}_{\varepsilon}^{+} \partial_{x}\left(\phi_{\varepsilon}^{+}\left(x, 0, \xi_{1}^{+}, k\right)\right)+\widetilde{\beta}_{\varepsilon}^{+} \xi_{+}+\varepsilon \widetilde{\gamma}_{\varepsilon}^{+} \frac{k^{2}}{\xi_{+}},
$$

we obtain

$$
\left\langle S_{-}^{+}, l\right\rangle=\frac{1}{2} \widetilde{\beta}_{0}^{+}(0) l(0)+\frac{i}{4 \pi} \int_{\mathbb{R}^{2}} \partial_{x}\left[\frac{\widetilde{\alpha}_{0}^{+} l(x)}{\xi_{+}}\right] e^{i x \xi_{1}^{+}} \mathrm{d} x \mathrm{~d} \xi_{1}^{+}
$$

and this distribution is in $H^{-1}$, according to $\widetilde{\alpha}_{0}^{+}(0)=\alpha_{0}^{+}(0,0) \neq 0$.

We have the relations $\widetilde{\alpha}_{0}^{+}(x)=\alpha_{0}^{+}(x, 0)$ and $\widetilde{\beta}_{0}^{+}(x)=\beta_{0}^{+}(x, 0)$ (because the phase is $\phi_{0}^{+}\left(x, 0, \xi_{1}^{+}, k\right)=x \xi_{1}^{+}$and $\left.m_{0}^{+}=\frac{\mu_{+}(a-b)-1-\mu_{+}^{2}}{1+\mu_{+}^{2}}, n_{0}^{+}=\frac{a-b}{1+\mu_{+}^{2}}\right)$. Hence $\alpha_{0}^{+}(0,0)=$ $\left(1+a^{2}\right)^{-1 / 2}$, which imply the existence of a function $T_{+}$such that $\alpha_{0}^{+}(x, 0)+L_{1}^{+}(x, 0)=$ $x T_{+}(x)$.

We obtain thus the relation

$$
\left\langle S_{+}^{+}+S_{-}^{+}, l\right\rangle=\frac{i}{4 \pi} \int_{\mathbb{R}^{2}} e^{i x \xi_{1}^{+}} \partial_{x}\left(\frac{x l(x) T_{+}(x)}{\xi_{+}}\right) \mathrm{d} x \mathrm{~d} \xi_{1}^{+} .
$$

Simple integrations by parts lead to

$$
\begin{aligned}
& \left\langle S_{+}^{+}+S_{-}^{+}, l\right\rangle \\
& \quad=\frac{i}{4 \pi} \int_{\mathbb{R}^{2}} l(x) e^{i x \xi_{1}^{+}}\left[T_{+}(x) \frac{\left(\xi_{1}^{+}\right)^{2}}{\xi_{+}^{3}}-i \partial_{x}\left(\frac{T_{+}(x)}{\xi_{+}^{2}}\right) \xi_{1}^{+}+\partial_{x}\left(\frac{x T_{+}(x)}{\xi_{+}}\right)\right] \mathrm{d} x \mathrm{~d} \xi_{1}^{+} .
\end{aligned}
$$

The two integrations by parts defined here lead to the expression of the symbols used in Lemma 6.5 (using the symbols given in (86)):

$$
\left\{\begin{aligned}
& q_{\varepsilon}^{+}\left(x, x^{\prime}, \xi_{1}^{+}, k\right)=\psi(x) \psi\left(x^{\prime}\right)\left[\alpha_{\varepsilon}^{+}\left(x, x^{\prime}\right) \frac{\xi_{1}^{+}}{\xi_{+}}+\beta_{\varepsilon}^{+}\right] \\
& q_{\varepsilon}^{-}\left(x, x^{\prime}, \xi_{1}^{-}, k\right)=\psi(x) \psi\left(x^{\prime}\right)\left[\alpha_{\varepsilon}^{-}\left(x, x^{\prime}\right) \frac{\xi_{1}^{-}}{\xi_{-}}+\beta_{\varepsilon}^{-}\right] \\
& r_{\varepsilon}^{+}\left(x, x^{\prime}, \xi_{1}^{+}, k\right)=\psi\left(x^{\prime}\right) \psi(x)\left[\gamma_{\varepsilon}^{+}\left(x, x^{\prime}\right) \frac{k^{2} \xi_{1}^{+}}{\xi_{+}^{2}}+\delta_{\varepsilon}^{+}\left(x, x^{\prime}\right) \frac{k^{2}}{\xi_{+}}\right] \\
& \quad+i \partial_{x^{\prime}} q_{\varepsilon}^{+}\left(x, x^{\prime}, \xi_{1}^{+}, k\right) \\
& r_{\varepsilon}^{-}\left(x, x^{\prime}, \xi_{1}^{-}, k\right)=\psi\left(x^{\prime}\right) \psi(x)\left[\gamma_{\varepsilon}^{-}\left(x, x^{\prime}\right) \frac{k^{2} \xi_{1}^{-}}{\xi_{-}^{2}}+\right.\left.\delta_{\varepsilon}^{-}\left(x, x^{\prime}\right) \frac{k^{2}}{\xi_{-}}\right] \\
&+i \partial_{x^{\prime}} q_{\varepsilon}^{-}\left(x, x^{\prime}, \xi_{1}^{-}, k\right) .
\end{aligned}\right.
$$

6.2.3. Another representation of $R_{\mp}^{ \pm, k}$. - The expression of $R_{-}^{+, k}$ involves the phase function with $\left(a\left(x^{\prime}\right)-b\left(x^{\prime}\right)\right)$. In order to be exactly in the framework of Gérard and Lebeau, we need to find another expression of this operator involving the complex number $\xi_{0}=\left(k^{2}-\xi^{2}\right)^{1 / 2}$ with positive imaginary part. Recall that the phase functions $\psi_{+,-}$and $\psi_{-},+$have been introduced in Lemme 4.2. We prove the 
Proposition 6.1. - There exists $\phi>0, \widetilde{\phi}>0$ and two contours $\Gamma_{\phi}, \Gamma_{\widetilde{\phi}}$ described in Figure 6.1 such that

$$
\begin{aligned}
& 1_{x \geqslant 0} R_{-}^{+, k}(f)(x, k)= \\
& -\frac{i}{4 \pi} \int_{\mathbb{R} \times \Gamma_{\phi}} \frac{e^{i \psi_{+,-}\left(x, x^{\prime}, \xi, k\right)}}{\left(1+\left(a^{\prime}(x)\right)^{2}\right)^{1 / 2}} \frac{k^{2}\left(1+a^{\prime}(x) b^{\prime}\left(x^{\prime}\right)\right)}{\xi_{0}} \psi(x) 1_{x \geqslant 0} \psi\left(x^{\prime}\right) 1_{x^{\prime} \geqslant 0} f\left(x^{\prime}\right) \mathrm{d} x^{\prime} \mathrm{d} \xi \\
& +\frac{1}{4 \pi} \int_{\mathbb{R} \times \Gamma_{\phi}} \frac{e^{i \psi_{+,-}\left(x, x^{\prime}, \xi, k\right)}}{\left(1+\left(a^{\prime}(x)\right)^{2}\right)^{1 / 2}}\left(a^{\prime}(x)+\frac{\xi}{\xi_{0}}\right) \psi(x) 1_{x \geqslant 0} 1_{x^{\prime} \geqslant 0} \partial_{x^{\prime}}\left(\psi\left(x^{\prime}\right) f\left(x^{\prime}\right)\right) \mathrm{d} x^{\prime} \mathrm{d} \xi \\
& +\psi(x) 1_{x \geqslant 0} \frac{f(0)}{4 \pi} \int_{\Gamma_{\phi}}\left(a^{\prime}(x)+\frac{\xi}{\xi_{0}}\right) \frac{e^{i x \xi+i a(x) \xi_{0}}}{\left(1+\left(a^{\prime}(x)\right)^{2}\right)^{1 / 2}} \mathrm{~d} \xi . \\
& 1_{x \geqslant 0} R_{+}^{-, k}(f)(x, k)= \\
& -\frac{i}{4 \pi} \int_{\mathbb{R} \times \Gamma_{\tilde{\phi}}} \frac{e^{i \psi_{-,+}\left(x, x^{\prime}, \xi, k\right)}}{\left(1+\left(b^{\prime}(x)\right)^{2}\right)^{1 / 2}} \frac{k^{2}\left(1+a^{\prime}\left(x^{\prime}\right) b^{\prime}(x)\right)}{\xi_{0}} \psi(x) 1_{x \geqslant 0} \psi\left(x^{\prime}\right) 1_{x^{\prime} \geqslant 0} f\left(x^{\prime}\right) \mathrm{d} x^{\prime} \mathrm{d} \xi \\
& +\frac{1}{4 \pi} \int_{\mathbb{R} \times \Gamma_{\tilde{\phi}}} \frac{e^{i \psi_{-,+}\left(x, x^{\prime}, \xi, k\right)}}{\left(1+\left(b^{\prime}(x)\right)^{2}\right)^{1 / 2}}\left(b^{\prime}(x)-\frac{\xi}{\xi_{0}}\right) \psi(x) 1_{x \geqslant 0} 1_{x^{\prime} \geqslant 0} \partial_{x^{\prime}}\left(\psi\left(x^{\prime}\right) f\left(x^{\prime}\right)\right) \mathrm{d} x^{\prime} \mathrm{d} \xi \\
& +\psi(x) 1_{x \geqslant 0} \frac{f(0)}{4 \pi} \int_{\Gamma_{\widetilde{\phi}}}\left(b^{\prime}(x)-\frac{\xi}{\xi_{0}}\right) \frac{e^{i x \xi-i b(x) \xi_{0}}}{\left(1+\left(b^{\prime}(x)\right)^{2}\right)^{1 / 2}} \mathrm{~d} \xi .
\end{aligned}
$$

Proof. — From the relation

$$
u_{-}(x, y, k)=-\frac{1}{(2 \pi)^{2}} \int \frac{e^{i\left(x-x^{\prime}\right) \xi+i\left(y-b\left(x^{\prime}\right)\right) \eta}}{\xi_{0}^{2}-\eta^{2}} f\left(x^{\prime}\right) i\left[\eta-b^{\prime}\left(x^{\prime}\right) \xi\right] \mathrm{d} x^{\prime} \mathrm{d} \xi \mathrm{d} \eta
$$

we use the residue theorem to get, in $y>0$ (because $b\left(x^{\prime}\right) \leqslant 0$ for $x^{\prime} \geqslant 0$ )

$$
u_{-}(x, y, k)=-\frac{1}{4 \pi} \int_{\mathbb{R}_{+} \times \mathbb{R}} e^{i\left(x-x^{\prime}\right) \xi+i\left(y-b\left(x^{\prime}\right)\right) \xi_{0}}\left[1-b^{\prime}\left(x^{\prime}\right) \frac{\xi}{\xi_{0}}\right] f\left(x^{\prime}\right) \mathrm{d} x^{\prime} \mathrm{d} \xi .
$$

We want to extend this integral to negative values of $y$, because we are not able to compute the trace on $y=a(x)$ and then restrict to $x \geqslant 0$ without having informations for this integral on $y-a(x)>0,-\delta_{0} \leqslant x \leqslant \delta_{0}$. This is done by a deformation of the integration contour in the variable $\xi$, which is $\mathbb{R}$ and avoids the branching points $\pm k$, in the complex plane. This deformation is to be done in $\Im \xi<0$, because we want to compensate the term $\Re\left(i y \Im \xi_{0}\right)=-y \Im \xi_{0}$, which is positive when $y<0$.

Assume that, for large values of $\xi$, the integral is to be deformed on $\Im \xi=$ $-\tan \phi|\Re \xi|, \phi \in] 0, \pi / 2[$. We note that

$$
\Re\left(i\left(x-x^{\prime}\right) \xi+i\left(y-b\left(x^{\prime}\right) \xi_{0}\right)=-\left(x-x^{\prime}\right) \Im \xi-\left(y-b\left(x^{\prime}\right)\right) \Im \xi_{0} .\right.
$$

It is simple to see that $\xi_{0}^{2}=-\xi^{2}\left(1-k^{2} / \xi^{2}\right)$, hence we have, on the branch $\xi=$ $a(-\cos \phi-i \sin \phi), a>0$ (which is the negative branch), $\xi_{0}=a(i \cos \phi-\sin \phi)+$ 
$O\left(a^{-1}\right)$, and on the branch $\xi=a(\cos \phi-i \sin \phi), a>0$ (positive branch), we have $\xi_{0}=a(i \cos \phi+\sin \phi)+O\left(a^{-1}\right)$. Hence, for $x^{\prime} \geqslant 0$,

$$
\begin{aligned}
\Re\left(i\left(x-x^{\prime}\right) \xi+i\left(y-b\left(x^{\prime}\right) \xi_{0}\right)\right. & =a\left[\left(x-x^{\prime}\right) \sin \phi-\left(y-b\left(x^{\prime}\right)\right) \cos \phi\right]+O\left(a^{-1}\right) \\
& \leqslant a[x \sin \phi-y \cos \phi]+O\left(a^{-1}\right) .
\end{aligned}
$$

We construct then such a contour $\Gamma_{\phi}$, which is the interval $[-2 \Re k, 2 \Re k]$, two vertical lines linking the real axis at $\pm 2 \Re k$ to the points $-2 i|\Re k| \tan \phi$, and the two straight lines $\Im \xi=-\tan \phi|\Re \xi|$ (shown in Figure 6.1 below):

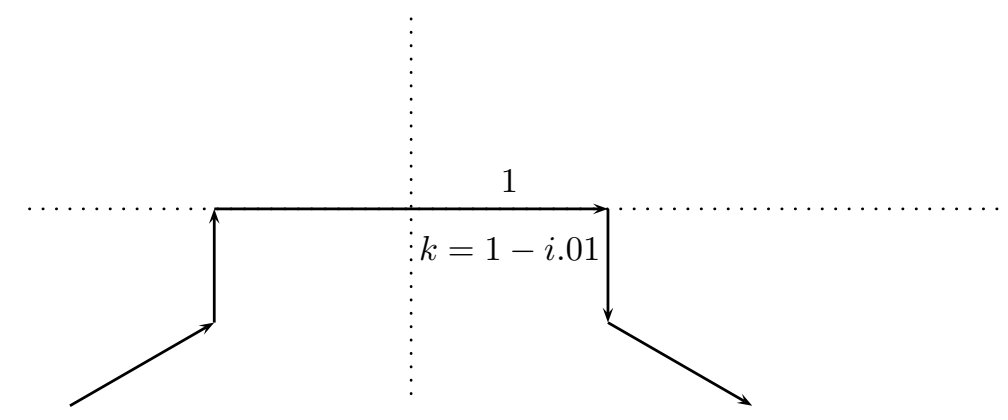

Figure 6.1. Contour $\Gamma_{\pi / 3}$

We consider $x \in \operatorname{supp} \psi$, hence $5 c t_{0} \geqslant x \geqslant-5 c t_{0}$, and thus, for $t_{0}$ small enough, there exists $\phi_{0}>0$ such that $\max \frac{a(x)}{x}=\tan \phi_{0}$. We then consider $\varepsilon_{0}>0$, and we introduce $\phi=\phi_{0}+\varepsilon_{0}$. We have:

$$
u_{-}(x, y, k)=\frac{1}{4 \pi} \int_{\mathbb{R}_{+} \times \Gamma_{\phi}} e^{i\left(x-x^{\prime}\right) \xi+i\left(y-b\left(x^{\prime}\right) \xi_{0}\right.}\left(1-b^{\prime}\left(x^{\prime}\right) \frac{\xi}{\xi_{0}}\right) f\left(x^{\prime}\right) \mathrm{d} x^{\prime} \mathrm{d} \xi .
$$

This expression extends holomorphically in $y-\tan \phi x>0$, owing to (156). The curve $y=a(x),-5 c t_{0} \leqslant x \leqslant 5 c t_{0}$ is included in this region, hence we can take the limits and all kind of derivatives in the above equality.

We obtain

$$
\begin{aligned}
& R_{-}^{+, k}(f)(x, k)=-\frac{i}{4 \pi} \int_{\mathbb{R}_{+} \times \Gamma_{\phi}} e^{i\left(x-x^{\prime}\right) \xi+i\left(a(x)-b\left(x^{\prime}\right)\right) \xi_{0}} \psi(x) \psi\left(x^{\prime}\right) f\left(x^{\prime}\right) \mathrm{d} x^{\prime} \mathrm{d} \xi \\
& \frac{\left[\xi_{0}-a^{\prime}(x) \xi\right]\left[\xi_{0}-b^{\prime}\left(x^{\prime}\right) \xi\right]}{\left(1+\left(a^{\prime}(x)\right)^{2}\right)^{1 / 2} \xi_{0}} .
\end{aligned}
$$

As we have the identity

$$
\left[\xi_{0}-a^{\prime}(x) \xi\right]\left[\xi_{0}-b^{\prime}\left(x^{\prime}\right) \xi\right]=\left(1+a^{\prime}(x) b^{\prime}\left(x^{\prime}\right)\right) k^{2}-\left[\xi+b^{\prime}\left(x^{\prime}\right) \xi_{0}\right]\left[\xi+a^{\prime}(x) \xi_{0}\right],
$$


we recognize $-\partial_{x^{\prime}} \psi_{+,-}=\xi+b^{\prime}\left(x^{\prime}\right) \xi_{0}$. Hence, in $\mathcal{D}^{\prime}$,

$$
\begin{gathered}
R_{-}^{+, k}(f)(x, k)=-\frac{i}{4 \pi} \int_{\mathbb{R}_{+} \times \Gamma_{\phi}} \frac{e^{i \psi_{+,-}}}{\left(1+\left(a^{\prime}(x)\right)^{2}\right)^{1 / 2}} \frac{k^{2}}{\xi_{0}}\left(1+a^{\prime}(x) b^{\prime}\left(x^{\prime}\right)\right) \psi(x) \psi\left(x^{\prime}\right) f\left(x^{\prime}\right) \mathrm{d} x^{\prime} \mathrm{d} \xi \\
+\frac{1}{4 \pi} \int_{\mathbb{R}_{+} \times \Gamma_{\phi}} \frac{e^{i \psi_{+,-}}}{\left(1+\left(a^{\prime}(x)\right)^{2}\right)^{1 / 2}}\left[a^{\prime}(x)+\frac{\xi}{\xi_{0}}\right] \psi(x) \partial_{x^{\prime}}\left(\psi\left(x^{\prime}\right) f\left(x^{\prime}\right)\right) \mathrm{d} x^{\prime} \mathrm{d} \xi \\
+\psi(x) \frac{f(0)}{4 \pi} \int_{\Gamma_{\phi}} \mathrm{d} \xi \frac{e^{i x \xi+i a(x) \xi_{0}}}{\left(1+\left(a^{\prime}(x)\right)^{2}\right)^{1 / 2}}\left[a^{\prime}(x)+\frac{\xi}{\xi_{0}}\right] .
\end{gathered}
$$

In this equality, the limit of the last term when we have $\phi=0$ is to be considered as a distribution. We introduce the operator $\widetilde{R}_{-, 0}^{+, k}(f)$ defined by

$$
\begin{aligned}
& \widetilde{R}_{-, 0}^{+, k}(f)(x, k)= \\
& -\frac{i}{4 \pi} 1_{x} \geqslant 0 \int_{\mathbb{R}_{+} \times \mathbb{R}} \frac{e^{i \psi_{+,-}}}{\left(1+\left(a^{\prime}(x)\right)^{2}\right)^{1 / 2}} \frac{k^{2}}{\xi_{0}}\left(1+a^{\prime}(x) b^{\prime}\left(x^{\prime}\right)\right) \psi(x) \psi\left(x^{\prime}\right) f\left(x^{\prime}\right) \mathrm{d} x^{\prime} \mathrm{d} \xi \\
& \quad+\frac{1}{4 \pi} \int_{\mathbb{R}_{+} \times \mathbb{R}} \frac{e^{i \psi_{+,-}}}{\left(1+\left(a^{\prime}(x)\right)^{2}\right)^{1 / 2}}\left[a^{\prime}(x)+\frac{\xi}{\xi_{0}}\right] \psi(x) \partial_{x^{\prime}}\left(\psi\left(x^{\prime}\right) f\left(x^{\prime}\right)\right) \mathrm{d} x^{\prime} \mathrm{d} \xi .
\end{aligned}
$$

A similar representation is available for the operator $R_{+}^{-, k}$. We leave the details to the reader; in particular the integral in $\eta$ is to be closed in $\Im \eta<0$. We find (the contour $\Gamma_{\widetilde{\phi}}$ being constructed with $\left.\widetilde{\phi}_{0}=\max -b(x) / x\right)$

$$
\begin{array}{r}
R_{+}^{-, k}(f)(x, k)=-\frac{i}{4 \pi} \int_{\mathbb{R}_{+} \times \Gamma_{\tilde{\phi}}} \psi(x) \psi\left(x^{\prime}\right) f\left(x^{\prime}\right) e^{i\left(x-x^{\prime}\right) \xi+i\left(a\left(x^{\prime}\right)-b(x)\right) \xi_{0}} \mathrm{~d} x^{\prime} \mathrm{d} \xi \\
\frac{\left[\xi_{0}+b^{\prime}(x) \xi\right]\left[\xi_{0}+a^{\prime}(x) \xi\right]}{\xi_{0}\left(1+\left(b^{\prime}(x)\right)^{2}\right)^{1 / 2}} .
\end{array}
$$

Making use of the identity

$$
\left[\xi_{0}+b^{\prime}(x) \xi\right]\left[\xi_{0}+a^{\prime}(x) \xi\right]=k^{2}\left(1+a^{\prime}\left(x^{\prime}\right) b^{\prime}(x)\right)-\left[\xi-a^{\prime}\left(x^{\prime}\right) \xi_{0}\right]\left[\xi-b^{\prime}(x) \xi_{0}\right]
$$

we obtain similarly

$$
\begin{aligned}
& R_{+}^{-, k}(f)(x, k)=-\frac{i}{4 \pi} \int_{\mathbb{R}_{+} \times \Gamma_{\tilde{\phi}}} f\left(x^{\prime}\right) \psi\left(x^{\prime}\right) \psi(x) e^{i \psi_{-,+}} \frac{k^{2}}{\xi_{0}} \frac{1+a^{\prime}\left(x^{\prime}\right) b^{\prime}(x)}{\left(1+\left(b^{\prime}(x)\right)^{2}\right)^{1 / 2}} \mathrm{~d} x^{\prime} \mathrm{d} \xi \\
& +\frac{1}{4 \pi} \int_{\mathbb{R}_{+} \times \Gamma_{\tilde{\Phi}}} e^{i \psi_{-,+}}\left[\frac{\xi}{\xi_{0}}-b^{\prime}(x)\right] \psi(x) \frac{\partial_{x^{\prime}}(f \psi)}{\left(1+\left(b^{\prime}(x)\right)^{2}\right)^{1 / 2}} \mathrm{~d} x^{\prime} \mathrm{d} \xi \\
& +\frac{f(0)}{4 \pi} \int_{\Gamma_{\tilde{\phi}}} e^{i x \xi-i b(x) \xi_{0}}\left[\frac{\xi}{\xi_{0}}-b^{\prime}(x)\right] \mathrm{d} \xi .
\end{aligned}
$$


The operator $R_{+, 0}^{-, k}$ defined by

$$
\begin{array}{r}
\widetilde{R}_{+, 0}^{-, k}(f)(x, k)=-\frac{i}{4 \pi} 1_{x} \geqslant 0 \int_{\mathbb{R}_{+} \times \mathbb{R}} f\left(x^{\prime}\right) \psi\left(x^{\prime}\right) \psi(x) e^{i \psi_{-,+}} \frac{k^{2}}{\xi_{0}} \frac{1+a^{\prime}\left(x^{\prime}\right) b^{\prime}(x)}{\left(1+\left(b^{\prime}(x)\right)^{2}\right)^{1 / 2}} \mathrm{~d} x^{\prime} \mathrm{d} \xi \\
+\frac{1}{4 \pi} \int_{\mathbb{R}_{+} \times \mathbb{R}} e^{i \psi_{-,+}}\left[\frac{\xi}{\xi_{0}}-b^{\prime}(x)\right] \psi(x) \frac{\partial_{x^{\prime}}(f \psi)}{\left(1+\left(b^{\prime}(x)\right)^{2}\right)^{1 / 2}} \mathrm{~d} x^{\prime} \mathrm{d} \xi
\end{array}
$$

is also introduced.

The symbols used here were introduced in (87) above and they are the symbols involved in this new representation of $R_{\mp, 0}^{ \pm, k}$. For $f \in C_{0}^{\infty}\left(\mathbb{R}_{+}^{*}\right)$, for which $f(0)=0$, we get explicitly from the above calculation (we have the right to consider the deformation of the contour $\Gamma_{\phi}$ or $\Gamma_{\tilde{\phi}}$ on $\mathbb{R}$ because $f \in C_{0}^{\infty}$ and we use the dominated convergence theorem).

6.2.4. Expression of the operators for straight boundaries. - From the expressions above, we deduce the expression of all the operators involved in the calculus when $a(x)=a x, a=\tan \gamma_{+}$and $b(x)=b x=\tan \gamma_{-} x$. We have (the operators are denoted with small letters instead of capital letters in the general case)

Proposition 6.2. - The boundary operators for the straight case are given by

$$
\begin{aligned}
& r_{+}^{+, k}(f)(x, k)=\frac{i}{4 \pi \cos \gamma_{+}} \iint e^{i\left(x-x^{\prime}\right) \frac{\xi}{\cos \gamma+} \frac{k^{2}}{\xi_{0}}} f\left(x^{\prime}\right) \mathrm{d} x^{\prime} \mathrm{d} \xi
\end{aligned}
$$

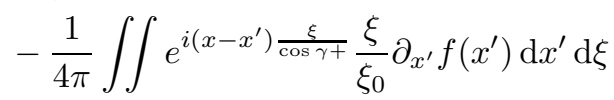

$$
\begin{aligned}
& r_{-}^{-, k}(f)(x, k)=\frac{i}{4 \pi \cos \gamma_{-}} \iint e^{i\left(x-x^{\prime}\right) \frac{\xi}{\cos \gamma_{-}}} \frac{k^{2}}{\xi_{0}} f\left(x^{\prime}\right) \mathrm{d} x^{\prime} \mathrm{d} \xi \\
& -\frac{1}{4 \pi} \iint e^{i\left(x-x^{\prime}\right) \frac{\xi}{\cos \gamma-}} \frac{\xi}{\xi_{0}} \partial_{x^{\prime}} f\left(x^{\prime}\right) \mathrm{d} x^{\prime} \mathrm{d} \xi \\
& r_{-}^{+, k}(f)(x, k)=-\frac{i}{4 \pi} \frac{\cos \gamma}{\cos \gamma_{-}} \iint e^{i \frac{x}{\cos \gamma_{+}}\left[\xi \cos \gamma+\xi_{0} \sin \gamma\right]-i \frac{x^{\prime} \xi}{\cos \gamma_{-}}} \frac{k^{2}}{\xi_{0}} f\left(x^{\prime}\right) \mathrm{d} x^{\prime} \mathrm{d} \xi \\
& +\frac{1}{4 \pi} \iint e^{i \frac{x}{\cos \gamma_{+}}\left[\xi \cos \gamma+\xi_{0} \sin \gamma\right]-i \frac{x^{\prime} \xi}{\cos \gamma-}}\left[\sin \gamma+\cos \gamma \frac{\xi}{\xi_{0}}\right] \partial_{x^{\prime}} f\left(x^{\prime}\right) \mathrm{d} x^{\prime} \mathrm{d} \xi \\
& +\frac{f(0)}{4 \pi} \int e^{i \frac{x}{\cos \gamma_{+}}\left[\xi \cos \gamma+\xi_{0} \sin \gamma\right]}\left[\sin \gamma+\cos \gamma \frac{\xi}{\xi_{0}}\right] \mathrm{d} \xi
\end{aligned}
$$




$$
\begin{array}{r}
r_{+}^{-, k}(f)(x, k)=-\frac{i}{4 \pi} \frac{\cos \gamma}{\cos \gamma_{+}} \iint e^{i \frac{x}{\cos \gamma_{-}}\left[\xi \cos \gamma+\xi_{0} \sin \gamma\right]-i \frac{x^{\prime} \xi}{\cos \gamma+}} \frac{k^{2}}{\xi_{0}} f\left(x^{\prime}\right) \mathrm{d} x^{\prime} \mathrm{d} \xi \\
+\frac{1}{4 \pi} \iint e^{i \frac{x}{\cos \gamma-}\left[\xi \cos \gamma+\xi_{0} \sin \gamma\right]-i \frac{x^{\prime} \xi}{\cos \gamma+}}\left[\sin \gamma+\cos \gamma \frac{\xi}{\xi_{0}}\right] \partial_{x^{\prime}} f\left(x^{\prime}\right) \mathrm{d} x^{\prime} \mathrm{d} \xi \\
+\frac{f(0)}{4 \pi} \int e^{i \frac{x}{\cos \gamma_{-}}\left[\xi \cos \gamma+\xi_{0} \sin \gamma\right]}\left[\sin \gamma+\cos \gamma \frac{\xi}{\xi_{0}}\right] \mathrm{d} \xi
\end{array}
$$

Proof. - We begin with the equalities coming from (149) and (86) written in the straight case, the operators being given by Lemma 6.5 . We have

$$
\begin{aligned}
r_{+}^{+, k}(f)(x, k)=\frac{i}{4 \pi} \iint e^{i\left(x-x^{\prime}\right) \xi_{1}^{+}} \frac{k^{2}}{\xi_{+} \cos \gamma_{+}} f\left(x^{\prime}\right) \mathrm{d} x^{\prime} \mathrm{d} \xi_{1}^{+} & -\frac{1}{4 \pi} \iint e^{i\left(x-x^{\prime}\right) \xi_{1}^{+}} \frac{\xi_{1}^{+}}{\xi_{+}} f\left(x^{\prime}\right) \mathrm{d} x^{\prime} \cos \gamma_{+} \mathrm{d} \xi_{1}^{+}, \\
r_{-}^{-, k}(f)(x, k)=\frac{i}{4 \pi} \iint e^{i\left(x-x^{\prime}\right) \xi_{1}^{-}} & \frac{k^{2}}{\xi_{-} \cos \gamma_{-}} f\left(x^{\prime}\right) \mathrm{d} x^{\prime} \mathrm{d} \xi_{1}^{-} \\
& -\frac{1}{4 \pi} \iint e^{i\left(x-x^{\prime}\right) \xi_{1}^{-}} \frac{\xi_{1}^{-}}{\xi_{-}} \partial_{x^{\prime}} f\left(x^{\prime}\right) \mathrm{d} x^{\prime} \cos \gamma_{-} \mathrm{d} \xi_{1}^{-} .
\end{aligned}
$$

The change of variable $\xi=\xi_{1}^{ \pm} \cos \gamma_{ \pm}$, which imply $\xi_{0}=\xi_{ \pm} \cos \gamma_{ \pm}$, yields

$$
u_{-}(x, y, k)=-\frac{1}{4 \pi^{2}} \int \frac{e^{i(x \xi+y \eta)-i x^{\prime}(\xi+b \eta)}}{k^{2}-\xi^{2}-\eta^{2}} i[\eta-b \xi] f\left(x^{\prime}\right) \mathrm{d} x^{\prime} \mathrm{d} \xi \mathrm{d} \eta .
$$

We introduce the change of variables $\xi+b \eta=\widetilde{\xi}, \eta-b \xi=\widetilde{\eta}$, hence

$$
u_{-}(x, y, k)=-\frac{1}{4 \pi^{2}} \int \frac{e^{i \frac{x(\widetilde{\xi}-b \tilde{\eta})+y(b \tilde{\xi}+\widetilde{\eta})}{1+b^{2}}-i x^{\prime} \widetilde{\xi}}}{k^{2}\left(1+b^{2}\right)-(\widetilde{\xi})^{2}-(\widetilde{\eta})^{2}} i \widetilde{\eta} f\left(x^{\prime}\right) \mathrm{d} x^{\prime} d \widetilde{\xi} d \widetilde{\eta} .
$$

Note that the phase function is

$$
(y-b x) \widetilde{\eta}+(x+b y) \widetilde{\xi}
$$

hence when we consider $y=a x$ and $x \geqslant 0$, the good deformation of the contour is in $\Im \widetilde{\eta}>0$, which imposes that $\widetilde{\eta}=\widetilde{\xi}_{0}$. We obtain, in $y-a x>0, x \geqslant 0$ :

$$
u_{-}(x, y, k)=-\frac{1}{4 \pi} \int e^{i \frac{x+b y}{1+b^{2}} \widetilde{\xi}+\frac{y-b x}{1+b^{2}} \widetilde{F}_{0}-i x^{\prime} \widetilde{\xi}} f\left(x^{\prime}\right) \mathrm{d} x^{\prime} d \widetilde{\xi} .
$$

We deduce that, formally

$$
\partial_{+} u_{-}(x, k)=-\frac{i \cos \gamma_{+}}{4 \pi} \int e^{i x \frac{\cos \gamma_{-}}{\cos \gamma_{+}}\left[\cos \gamma \widetilde{\xi}+\sin \gamma \widetilde{\xi}_{0}\right]-i x^{\prime} \widetilde{\xi}}\left[\cos \gamma \widetilde{\xi}_{0}-\sin \gamma \widetilde{\xi}\right] f\left(x^{\prime}\right) \mathrm{d} x^{\prime} d \widetilde{\xi}
$$


Note that $\widetilde{\xi}_{0}=\frac{k^{2}}{\widetilde{\xi}_{0} \cos ^{2} \gamma_{-}}-\widetilde{\xi} \frac{\widetilde{\xi}}{\widetilde{\xi}_{0}}$, hence we have

$$
\begin{gathered}
\partial_{+} u_{-}(x, k)=-\frac{\cos \gamma_{-}}{4 \pi} \int e^{i x \frac{\cos \gamma_{-}}{\cos \gamma_{+}}\left[\cos \gamma \widetilde{\xi}+\sin \gamma \widetilde{\xi_{0}}\right]-i x^{\prime} \widetilde{\xi}}\left[\cos \gamma \frac{\widetilde{\xi}}{\widetilde{\xi}_{0}}+\sin \gamma\right](-i \widetilde{\xi}) f\left(x^{\prime}\right) \mathrm{d} x^{\prime} d \widetilde{\xi} \\
-\frac{i}{4 \pi} \frac{\cos \gamma}{\cos \gamma_{-}} \int e^{i x \frac{\cos \gamma_{-}}{\cos \gamma_{+}}\left[\cos \gamma \widetilde{\xi}+\sin \gamma \widetilde{\xi}_{0}\right]-i x^{\prime} \widetilde{\xi}} \frac{k^{2}}{\widetilde{\xi}_{0}} f\left(x^{\prime}\right) \mathrm{d} x^{\prime} d \widetilde{\xi}
\end{gathered}
$$

After the integration by parts in the first term, we get

$$
\begin{aligned}
\partial_{+} u_{-}(x, k)= & \frac{\cos \gamma-}{4 \pi} \int e^{i x \frac{\cos \gamma_{-}}{\cos \gamma_{+}}\left[\cos \gamma \widetilde{\xi}+\sin \gamma \widetilde{\xi_{0}}\right]-i x^{\prime} \tilde{\xi}}\left[\cos \gamma \frac{\widetilde{\xi}}{\widetilde{\xi}_{0}}+\sin \gamma\right] \partial_{x^{\prime}} f\left(x^{\prime}\right) \mathrm{d} x^{\prime} d \widetilde{\xi} \\
+ & \frac{f(0) \cos \gamma_{-}}{4 \pi} \int e^{i x \frac{\cos \gamma_{-}}{\cos \gamma_{+}}\left[\cos \gamma \widetilde{\xi}+\sin \gamma \widetilde{\xi}_{0}\right]}\left[\cos \gamma \frac{\widetilde{\xi}}{\widetilde{\xi}_{0}}+\sin \gamma\right] \mathrm{d} \widetilde{\xi} \\
& -\frac{i}{4 \pi} \frac{\cos \gamma}{\cos \gamma_{-}} \int e^{i x \frac{\cos \gamma_{-}}{\cos \gamma_{+}}\left[\cos \gamma \widetilde{\xi}+\sin \gamma \widetilde{\xi}_{0}\right]-i x^{\prime} \widetilde{\xi}} \frac{k^{2}}{\widetilde{\xi}_{0}} f\left(x^{\prime}\right) \mathrm{d} x^{\prime} d \widetilde{\xi}
\end{aligned}
$$

We revert to more usual coordinates with $\widetilde{\xi}=\frac{\xi}{\cos \gamma_{-}}, \widetilde{\xi}_{0}=\frac{\xi_{0}}{\cos \gamma_{-}}$, and we have

$$
\begin{aligned}
\partial_{+} u_{-}(x, k) & =\frac{1}{4 \pi} \int e^{i \frac{x}{\cos \gamma_{+}}\left[\cos \gamma \xi+\sin \gamma \xi_{0}\right]-i \frac{x^{\prime} \xi}{\cos \gamma_{-}}}\left[\cos \gamma \frac{\xi}{\xi_{0}}+\sin \gamma\right] \partial_{x^{\prime}} f\left(x^{\prime}\right) \mathrm{d} x^{\prime} \mathrm{d} \xi \\
+ & \frac{f(0) \cos \gamma_{-}}{4 \pi} \int e^{i x \frac{\cos \gamma-}{\cos \gamma_{+}}\left[\cos \gamma \widetilde{\xi}+\sin \gamma \widetilde{\xi_{0}}\right]}\left[\cos \gamma \frac{\widetilde{\xi}}{\widetilde{\xi}_{0}}+\sin \gamma\right] \mathrm{d} \widetilde{\xi} \\
- & \frac{\cos \gamma}{\cos \gamma_{-}} \frac{i}{4 \pi} \int e^{i \frac{x}{\cos \gamma_{+}}\left[\cos \gamma \xi+\sin \gamma \xi_{0}\right]-i \frac{x^{\prime} \xi}{\cos \gamma_{-}}} \frac{k^{2}}{\xi_{0}} f\left(x^{\prime}\right) \mathrm{d} x^{\prime} \mathrm{d} \xi
\end{aligned}
$$

The method for the term $\partial_{-} u_{+}$is the same, based on the change of variables $\widetilde{\xi}=\xi+a \eta$, $\widetilde{\eta}=-a \xi+\eta$.

\subsection{Asymptotic expansions of the DTN operators (from Gérard and Lebeau)}

In this Section, we reproduce the proofs of the asymptotic expansions of the operators of the type $K_{+}^{+}$and $K_{-}^{+}$as these expansions were done in the Appendix B of [20]. This is done here for the reader's convenience so that the text is quite self-contained. We recall that the $\varepsilon$-sectors were defined in Section 4.5. We introduce the sequence $\omega_{\sigma}$ of $\delta_{\sigma}$-sectors used in $[20]$ p. 375 , that is a sequence such that $\left(0<\delta_{1}<\delta_{0}\right)$

$$
\begin{gathered}
\delta_{\sigma}=(1-\sigma) \delta_{0}+\sigma \delta_{1} \\
\sigma<\sigma^{\prime} \Longrightarrow \bar{\omega}_{\sigma} \subset \omega_{\sigma^{\prime}} \\
\sigma<\sigma^{\prime} \Longrightarrow d\left(\omega_{\sigma}, \mathcal{C} \omega_{\sigma^{\prime}}\right)=d_{1}\left(\sigma^{\prime}-\sigma\right)
\end{gathered}
$$

We assume the existence of three constants $c_{2}, \varepsilon_{2}, d_{2}$ such that $d\left(i, \omega_{\sigma}\right) \geqslant \varepsilon_{2}$, $d\left(i y, \mathcal{C} \omega_{\sigma}\right) \geqslant c_{2}$ for the points $y \in \mathbb{R} \cap[-5,5] \cap\left[1-2 \varepsilon_{2}, 1+2 \varepsilon_{2}\right]$, and $\omega_{\sigma} \subset\left\{\Re z \leqslant d_{2}\right\}$. 
We introduce the weight function $\Phi_{\delta}$ (as in $[\mathbf{2 0}]$ p $373(4.7)$ ):

$$
\left\{\begin{array}{l}
\Phi_{\delta}(u)=0, \quad u \leqslant 0 \\
\Phi_{\delta}(u)=\frac{u^{2}}{2}, \quad 0 \leqslant u \delta \\
\Phi_{\delta}(u)=\frac{u^{2}}{2}-\frac{1}{2\left(1+c^{2}\right)}(u-\delta)^{2}, \quad u \geqslant \delta
\end{array}\right.
$$

the constant $c$ being the constant obtained in Lemma 1.1. We introduce the weight function $\Phi_{\delta}^{ \pm}$through

$$
\Phi_{\delta}^{ \pm}(z)=0, \Re z \in[0, d], \quad\left|\Im z-\frac{1}{\cos \gamma_{ \pm}}\right| \geqslant \varepsilon_{0}, \quad|z| \leqslant \frac{10}{\cos \gamma_{ \pm}}
$$

and $\Phi_{\delta}^{ \pm}(z)=\Phi_{\delta}(\Re z)$ otherwise. Note that, if

$$
\Phi(\Re w, \Re z)=\sup _{0 \leqslant x \leqslant c t}\left[-\frac{1}{2} \Re(w-t)^{2}+x \Re z-\frac{x^{2}}{2}\right]-\frac{1}{2}(\Im w)^{2}
$$

we have $\Phi_{\delta}(u)=\Phi\left(\frac{\delta}{c}, u\right)$. Then (this is Lemme 4.1 of [20]), for $\delta=3 \kappa c$ :

For $w \in \mathbb{C}, \Re w \leqslant 3 \kappa, x \in \mathbb{R}$, we have:

$$
\begin{gathered}
\left\|\left(\frac{1}{\lambda} \partial_{z}\right)^{k}\left[\left(T_{0} \otimes T_{1}\right)(f)(w, x-i \alpha, \lambda)\right]\right\|_{L^{2}(\mathbb{R}-i \alpha)} \leqslant D\left(2 c t_{0}\right)^{k}\|f\|_{L^{2}\left(\mathbb{R}_{+}\right)} e^{\lambda \frac{(\Im w)^{2}}{2}+\lambda \Phi_{\delta}(x)} \\
\left\|\left(\frac{1}{\lambda} \partial_{z}\right)^{k}\left[\left(T_{0} \otimes T_{1}\right)(f)(w, x-i \alpha, \lambda)\right]\right\|_{L^{\infty}(\mathbb{R}-i \alpha)} \leqslant D\left(2 c t_{0}\right)^{k}\|f\|_{L^{2}\left(\mathbb{R}_{+}\right)} e^{\lambda \frac{(\Im w)^{2}}{2}+\lambda \Phi_{\delta}(x)} .
\end{gathered}
$$

We introduce again $W_{\kappa}=\{w \in \mathbb{C},|\Re w|<\kappa,|1+\Im w|<\kappa\}$. From Proposition 3.1, there exists three constants $A, B, d$ (with $d \leqslant c t_{0}$ ) such that, for $f \in H^{1}\left(\mathbb{R}_{+}\right)$, with the two possibilities $f=u-u_{+}$, or $f=u-u_{-}$, which allows us to use Proposition 3.1

Lemma 6.6. - For $\delta \leqslant \varepsilon_{1} / 3 c, z \in \mathbb{C},|\Re w| \leqslant \delta / c,|1+\Im w| \leqslant \delta / c$, we have

$$
\begin{gathered}
\left|\left(T_{0} \otimes T_{1}\right)(f)(w, z, \lambda)\right| \leqslant A \lambda^{B} e^{\frac{\lambda}{2}(\Im w)^{2}+\lambda \Phi_{\delta}^{ \pm}(z)}, \\
\left|\left(T_{0} \otimes T_{1}\right)\left(\partial_{x} f\right)(w, z, \lambda)\right| \leqslant A \lambda^{B+1} e^{\frac{\lambda}{2}(\Im w)^{2}+\lambda \Phi_{\delta}^{ \pm}(z) .}
\end{gathered}
$$

This lemma, (Lemma 4.2 of [20]), leads to the definition of the constant $d$, used in the assumption on $\varepsilon_{2}$ and $d_{2}$ :

$$
\varepsilon_{0} \cos \gamma_{ \pm}<\varepsilon_{2}, \quad d_{2} \cos \gamma_{ \pm}<d
$$

6.3.1. Expansion of pseudodifferential operators. - Introduce

$$
\begin{aligned}
I(t, k, z, \lambda)= & T_{1}\left(1_{x>0} R_{+, 0}^{+, \lambda k}\left(T_{0} f\right)\right)(t-i k, z, \lambda) \\
= & T_{1}\left(1_{x>0} R_{+, 0}^{+, 1, \lambda k}\left(1_{x^{\prime}} \geqslant 0 \partial_{x^{\prime}} T_{0} f\right)\right)(t-i k, z, \lambda) \\
& \quad+T_{1}\left(1_{x>0} S_{+, 0}^{+, 1, \lambda k}\left(T_{0} f\right)\right)(t-i k, z, \lambda)+T_{1}\left(1_{x>0} R_{+, 0}^{+, 2, \lambda k}\left(T_{0} f\right)\right)
\end{aligned}
$$


The equality similar to (B.1) p. 400 of $[\mathbf{2 0}]$ is then

$$
\begin{aligned}
I^{\nu}=\int_{0}^{\infty} e^{\lambda x z-\lambda x^{2} / 2} \times\left[\psi_{2}(x) \frac{\lambda}{4 \pi} \int_{\mathbb{R}} \mathrm{d} x^{\prime} \int_{\mathcal{C}_{1}} d \zeta_{1}^{+} e^{i \lambda\left(x-x^{\prime}\right) \zeta_{1}^{+}-\nu\left(\zeta_{1}^{+}\right)^{2}}\right. \\
\left.\times q_{1}^{+}\left(x, x^{\prime}, \zeta_{1}^{+}, k\right)\left(T_{0}\left(1_{x^{\prime} \geqslant 0} \partial_{x^{\prime}} f\right)\left(t-i k, x^{\prime}, \lambda\right)\right) \mathrm{d} x\right] \\
+\int_{0}^{\infty} e^{\lambda x z-\lambda x^{2} / 2} \times\left[\psi_{2}(x) \frac{i \lambda^{2}}{4 \pi} \int_{\mathbb{R}} \mathrm{d} x^{\prime} \int_{\mathcal{C}_{1}} d \zeta_{1}^{+} e^{i \lambda\left(x-x^{\prime}\right) \zeta_{1}^{+}-\nu\left(\zeta_{1}^{+}\right)^{2}}\right. \\
\left.\quad \times s_{2}^{+}\left(x, x^{\prime}, \zeta_{1}^{+}, k\right)\left(T_{0}\left(1_{x^{\prime} \geqslant 0} f\right)\left(t-i k, x^{\prime}, \lambda\right)\right) \mathrm{d} x\right] \\
+\int_{0}^{\infty} e^{\lambda x z-\lambda x^{2} / 2} \times\left[\psi_{2}(x) \frac{\lambda}{4 \pi} \int_{\mathbb{R}} \mathrm{d} x^{\prime} \int_{\mathcal{C}_{1}} d \zeta_{1}^{+} e^{i \lambda\left(x-x^{\prime}\right) \zeta_{1}^{+}-\nu\left(\zeta_{1}^{+}\right)^{2}}\right. \\
\left.\quad \times s_{1}^{+}\left(x, x^{\prime}, \zeta_{1}^{+}, k\right)\left(T_{0}\left(1_{x^{\prime} \geqslant 0} f\right)\left(t-i k, x^{\prime}, \lambda\right)\right) \mathrm{d} x\right]
\end{aligned}
$$

We have, in $\Im k<0, I=\lim _{\nu \rightarrow 0_{+}} I^{\nu}=I_{1}+I_{2}+I_{3}=\lim _{\nu \rightarrow 0_{+}} I_{1}^{\nu}+\lim _{\nu \rightarrow 0_{+}} I_{2}^{\nu}+$ $\lim _{\nu \rightarrow 0_{+}} I_{3}^{\nu}$. We reproduce the proof of Proposition 4.1 of [20] (appendix B, (B.1) to (B.27)) for the integral defining $I_{1}^{\nu}$.

Let us write the Taylor expansion of $q_{1}^{+}\left(x, x^{\prime}, \zeta_{1}^{+}, k\right)$ in the variable $x$ in the neighborhood of $x=0$. We have

$$
\begin{aligned}
& q_{1}^{+}\left(x, x^{\prime}, \zeta_{1}^{+}, k\right)= \\
& \quad \sum_{n \leqslant N} \partial_{x^{n}}^{n} q_{1}^{+}\left(0, x^{\prime}, \zeta_{1}^{+}, k\right) \frac{x^{n}}{n !}+\frac{x^{N+1}}{(N+1) !} \int_{0}^{1} \partial_{x^{N+1}}^{N+1} q_{1}^{+}\left(s x, x^{\prime}, \zeta_{1}^{+}, k\right)(1-s)^{N} d s .
\end{aligned}
$$

Hence, if we denote by

$$
\begin{aligned}
& I_{1, R, N}^{\nu}=\int_{0}^{\infty} e^{\lambda x z-\lambda x^{2} / 2} \times\left[\psi_{2}(x) \frac{\lambda}{4 \pi} \int_{\mathbb{R}} \mathrm{d} x^{\prime} \int_{\mathcal{C}_{\infty}} d \zeta_{1}^{+} e^{i \lambda\left(x-x^{\prime}\right) \zeta_{1}^{+}-\nu\left(\zeta_{1}^{+}\right)^{2}}\right. \\
\times & {\left.\left[\frac{x^{N+1}}{(N+1) !} \int_{0}^{1} \partial_{x^{N+1}}^{N+1} q_{1}^{+}\left(s x, x^{\prime}, \zeta_{1}^{+}, k\right)(1-s)^{N} d s\right]\left(T_{0}\left(1_{x^{\prime} \geqslant 0} \partial_{x^{\prime}} f\right)\left(t-i k, x^{\prime}, \lambda\right)\right) \mathrm{d} x\right] }
\end{aligned}
$$

as well as

$$
\begin{aligned}
I_{1, n}^{\nu}=\int_{0}^{\infty} e^{\lambda x z-\lambda x^{2} / 2} \times[ & \frac{x^{n}}{n !} \psi_{2}(x) \frac{\lambda}{4 \pi} \int_{\mathbb{R}} \mathrm{d} x^{\prime} \int_{\mathcal{C}_{\infty}} d \zeta_{1}^{+} e^{i \lambda\left(x-x^{\prime}\right) \zeta_{1}^{+}-\nu\left(\zeta_{1}^{+}\right)^{2}} \\
& \left.\times \partial_{x^{n}}^{n} q_{1}^{+}\left(0, x^{\prime}, \zeta_{1}^{+}, k\right)\left(T_{0}\left(1_{x^{\prime} \geqslant 0} \partial_{x^{\prime}} f\right)\left(t-i k, x^{\prime}, \lambda\right)\right) \mathrm{d} x\right]
\end{aligned}
$$

such that, of course, $I_{1}^{\nu}=\sum_{n \leqslant N} \frac{1}{n !} I_{1, n}^{\nu}+I_{1, R, N}^{\nu}$, we have, using an integration by parts in $x$ three times and the compactly supported $C^{\infty}$ function $\psi_{2}(x)$, the relation, 
valid for $N \geqslant 3$ (to cancel the terms coming at $x=0$ from the integration by parts)

$$
\begin{gathered}
\quad I_{1, R, N}^{\nu}=\int_{0}^{\infty} \frac{e^{\lambda x z+i \lambda x \zeta_{1}^{+}}}{\left(z+i \zeta_{1}^{+}\right)^{3}} \times\left(-\frac{1}{\lambda} \partial_{x}\right)^{3}\left[\psi_{2}(x) \frac{\lambda}{4 \pi} \int_{\mathbb{R}} \mathrm{d} x^{\prime} \int_{\mathcal{C}_{\infty}} d \zeta_{1}^{+} e^{-i \lambda x^{\prime} \zeta_{1}^{+}-\lambda x^{2} / 2-\nu\left(\zeta_{1}^{+}\right)^{2}}\right. \\
\left.\times\left[\frac{x^{N+1}}{(N+1) !} \int_{0}^{1} \partial_{x^{N+1}}^{N+1} q_{1}^{+}\left(s x, x^{\prime}, \zeta_{1}^{+}, k\right)(1-s)^{N} d s\right]\left(T_{0}\left(1_{x^{\prime} \geqslant 0} \partial_{x^{\prime}} f\right)\left(t-i k, x^{\prime}, \lambda\right)\right) \mathrm{d} x\right] .
\end{gathered}
$$

Letting $\nu$ go to $0_{+}$, we get the limit because now it is absolutely converging as an integral in $\zeta_{1}^{+}$. More precisely, for $z \in U$ and $\zeta_{1}^{+} \in \mathcal{C}_{1}$, we have the relation $\Re z-\Im \zeta_{1}^{+} \leqslant$ $-A \sin \varepsilon+a^{\prime \prime} \leqslant-2$, and we use the holomorphy result of Lemma 4.5. This leads to the estimate, using for $z \in U$

$$
\begin{gathered}
\int_{\mathcal{C}_{1}} \frac{d \zeta_{1}^{+}}{\left|z+i \zeta_{1}^{+}\right|^{3}} \leqslant \frac{D}{(1+|z|)^{2}} \\
\left|\lim _{\nu \rightarrow 0_{+}} I_{1, R, N}^{\nu}\right| \leqslant D \lambda^{D}\left(D_{1} / a^{\prime}\right)^{N+4} \frac{N !}{\lambda^{N}} \frac{e^{4 \lambda c t_{0} a^{\prime}}}{1+|z|^{2}}\left\|\left(T_{0}\left(1_{x^{\prime} \geqslant 0} \partial_{x^{\prime}} f\right)(t-i k, \cdot, \lambda)\right)\right\|_{L^{2}\left(\mathbb{R}_{+}\right)} .
\end{gathered}
$$

Hence, for $N=\left[\lambda a^{\prime} / e D_{1}\right]$, we get $\left(D_{1} N / a^{\prime} \lambda\right)^{N} \leqslant e^{-N} \leqslant e e^{-\lambda a^{\prime} / e D_{1}}$. Hence

$$
\begin{aligned}
& \left|\lim _{\nu \rightarrow 0_{+}} I_{1, R, N}^{\nu}\right| \leqslant \\
& \quad D \lambda^{D}\left(D_{1} / a^{\prime}\right)^{4} \frac{N !}{N^{N}} \frac{1}{1+|z|^{2}} e^{-\lambda a^{\prime}\left(\frac{1}{e D_{1}}-4 c t_{0}\right)}\left\|\left(T_{0}\left(1_{x^{\prime} \geqslant 0} \partial_{x^{\prime}} f\right)(t-i k, \cdot, \lambda)\right)\right\|_{L^{2}\left(\mathbb{R}_{+}\right) .}
\end{aligned}
$$

For $t_{0}$ small enough, we have $1 / e D_{1}>4 c t_{0}$, hence $\lim _{\nu \rightarrow 0_{+}} I_{1, R, N}^{\nu}$ is negligible in $H\left(\phi_{0}, X_{\kappa}, U\right)$.

We now perform the asymptotic expansion on each term $I_{1, n}^{\nu}$. For this purpose, we introduce

$$
\begin{aligned}
& J_{1, n}\left(\zeta_{1}^{+}, t, k, \lambda\right) \\
& \quad=\int_{\mathbb{R}} \psi\left(x^{\prime}\right) \mathrm{d} x^{\prime} e^{-i \lambda x^{\prime} \zeta_{1}^{+}} \times \partial_{x^{n}}^{n} q_{1}^{+}\left(0, x^{\prime}, \zeta_{1}^{+}, k\right)\left(T_{0}\left(1_{x^{\prime} \geqslant 0} \partial_{x^{\prime}} f\right)\left(t-i k, x^{\prime}, \lambda\right)\right) \mathrm{d} x
\end{aligned}
$$

such that

$$
I_{1, n}^{\nu}=\int_{0}^{\infty} \mathrm{d} x \int_{\mathcal{C}_{1}} d \zeta_{1}^{+} e^{\lambda x z-\lambda \frac{x^{2}}{2}+i \lambda x \zeta_{1}^{+}} x^{n} \psi(x) \frac{\lambda}{4 \pi} e^{-\nu\left(\zeta_{1}^{+}\right)^{2}} J_{1, n}\left(\zeta_{1}^{+}, t, k, \lambda\right) .
$$

We use Lemme B.1 of [20], which writes

We assume that $J_{1, n}^{1}\left(\zeta_{1}^{+}, t, k, \lambda\right)$ is a family of holomorphic functions for $(t, k) \in$ $X_{\kappa}$, which is $L^{2}$ in the variable $\zeta_{1}^{+} \in \mathcal{C}_{1}$, satisfying the estimate, for at least $C<0$ :

$$
\left\|J_{1, n}^{1}(\cdot, t, k, \lambda)\right\|_{L^{2}\left(\mathcal{C}_{1}\right)} \leqslant C e^{-\lambda / C}\left(D_{1} / a^{\prime}\right)^{n+1} e^{\lambda \phi_{0}(t-i k)} .
$$

Then

$$
\sum_{n \leqslant \lambda a^{\prime} / e D_{1}} \int_{0}^{\infty} \mathrm{d} x \int_{\mathcal{C}_{1}} d \zeta_{1}^{+} e^{\lambda x z-\lambda \frac{x^{2}}{2}+i \lambda x \zeta_{1}^{+}} x^{n} \psi(x) \frac{\lambda}{2 \pi} e^{-\nu\left(\zeta_{1}^{+}\right)^{2}} J_{1, n}\left(\zeta_{1}^{+}, t, k, \lambda\right)
$$

is uniformly in $\nu$ negligible in $H\left(\phi_{0}, X_{\kappa}, U\right)$. 
Recall that the FBI transform $T_{1}$ has an inversion formula

$$
\psi_{2} g(x)=\frac{\lambda}{2 \pi} \int e^{i \lambda x \alpha-\lambda x^{2} / 2} T_{1} g(x-i \alpha, \lambda) \mathrm{d} \alpha
$$

which imply

$$
e^{-i \lambda x, \zeta_{1}^{+}} \psi_{2} g(x)=\frac{\lambda}{2 \pi} \int e^{i \lambda x \alpha-\lambda x^{2} / 2} T_{1} g\left(x-i \zeta_{1}^{+}-i \alpha, \lambda\right) \mathrm{d} \alpha .
$$

We apply this formula to $g=T_{0} f_{+}\left(t-i k, x^{\prime}, \lambda\right)$ which yields

$$
\begin{aligned}
J_{1, n}\left(\zeta_{1}^{+}, t, k, \lambda\right)=\frac{\lambda}{4 \pi} \int_{\mathbb{R}^{2}} & \psi\left(x^{\prime}\right) \mathrm{d} x^{\prime} e^{i \lambda \alpha-\lambda\left(x^{\prime}\right)^{2} / 2} \partial_{x^{n}}^{n} q_{1}^{+}\left(0, x^{\prime}, \zeta_{1}^{+}, k\right) \\
& \times\left(T_{0} \otimes T_{1}\left(1_{x^{\prime} \geqslant 0} \partial_{x^{\prime}} f\right)\left(t-i k, x^{\prime}-i \zeta_{1}^{+}-i \alpha, \lambda\right)\right) \mathrm{d} x \mathrm{~d} \alpha .
\end{aligned}
$$

Using an integration by parts for the formula of $J_{n, 1}$, we obtain, with the help of $\left(1-\frac{1}{\lambda^{2}} \partial_{\left(x^{\prime}\right)^{2}}^{2}\right)\left(e^{i \lambda \alpha x^{\prime}}\right)=\left(1+\alpha^{2}\right) e^{i \lambda \alpha x^{\prime}}$, the identity (which defines $\left.j_{1, n}\left(\zeta_{1}^{+} t, k, \lambda, x^{\prime}, \alpha\right)\right)$

$$
\begin{aligned}
J_{1, n}\left(\zeta_{1}^{+}, t, k, \lambda\right)= & \int_{\mathbb{R}^{2}} \mathrm{~d} x^{\prime} \mathrm{d} \alpha j_{1, n} \\
= & \frac{\lambda}{2 \pi} \int_{\mathbb{R}^{2}} \psi\left(x^{\prime}\right) \mathrm{d} x^{\prime} \frac{e^{i \lambda \alpha}}{1+\alpha^{2}}\left(1-\frac{1}{\lambda^{2}} \partial_{\left(x^{\prime}\right)^{2}}^{2}\right)\left[e^{-\lambda\left(x^{\prime}\right)^{2} / 2} \partial_{x^{n}}^{n} q_{1}^{+}\left(0, x^{\prime}, \zeta_{1}^{+}, k\right)\right. \\
& \left.\left(T_{0} \otimes T_{1}\right)\left(\left(1_{x^{\prime} \geqslant 0} \partial_{x^{\prime}} f\right)\left(t-i k, x^{\prime}-i \zeta_{1}^{+}-i \alpha, \lambda\right)\right)\right] \mathrm{d} x \mathrm{~d} \alpha .
\end{aligned}
$$

We split $\mathcal{C}_{1}$ in two parts, called $\mathcal{C}_{1}^{-}$and $\mathcal{C}_{1}^{+}$, with

$$
\mathcal{C}_{1}^{-}=\left\{\zeta_{1}^{+} \in \mathcal{C}_{1}, \quad\left|\Re \zeta_{1}^{+}-\frac{1}{\cos \gamma_{+}}\right| \geqslant 1+D_{1} t_{0}\right\}
$$

For $\zeta_{1}^{+} \in \mathcal{C}_{1}^{-}$and $x^{\prime}$ in $\operatorname{supp} \psi$, we have $\Re\left(x^{\prime}-i \zeta_{1}^{+}-i \alpha\right) \leqslant 4 c t_{0}-a^{\prime \prime} \leqslant-1$, owing to the form of the contour $\mathcal{C}_{1}$, given in 6.3.2. Hence

$$
\left\|j_{1, n}\right\|_{L^{2}\left(\mathcal{C}_{1}^{-}\right)} \leqslant D \lambda^{D} e^{-\lambda\left(x^{\prime}\right)^{2} / 2}\left(D_{1} / a^{\prime}\right)^{n+1} e^{\lambda \phi_{0}(t-i k)} \frac{\left\|f_{+}\right\|_{L^{2}\left(\mathbb{R}^{2}\right)}}{\alpha^{2}+1}
$$

owing to Lemma 4.1 of [20] which gives an estimate of $\left(\frac{1}{\lambda} \partial_{z}\right)^{k}\left(T_{0} \otimes T_{1}\right) f(w, x-i \alpha, \lambda)$ for $f \in L^{2}\left(\mathbb{R}^{2}\right)$, supported in $0 \leqslant x \leqslant c t, 0 \leqslant t \leqslant 2 t_{0}$.

Introduce a function $g, C_{0}^{\infty}(]-2 c t_{0}, 2 c t_{0}[), 0 \leqslant g \leqslant 1, g=1$ on $\left[-c t_{0}, c t_{0}\right]$ and

$$
\Sigma=\left\{\left(\alpha, x^{\prime}\right), x^{\prime}=y^{\prime}+i g\left(y^{\prime}\right) \frac{\alpha}{\left(1+\alpha^{2}\right)^{1 / 2}} \frac{a^{\prime}}{D_{1}}, y^{\prime} \in \operatorname{supp} \psi\right\} .
$$

We have the inequality, replacing $x^{\prime}$ by $y^{\prime}+i g\left(y^{\prime}\right) \frac{\alpha}{\left(1+\alpha^{2}\right)^{1 / 2}} \frac{a^{\prime}}{D_{1}}$ in $i \lambda \alpha x^{\prime}-\lambda \frac{\left(x^{\prime}\right)^{2}}{2}$ and using $\frac{g\left(y^{\prime}\right)}{\left(1+\alpha^{2}\right)^{1 / 2}} \frac{a^{\prime}}{D_{1}} \leqslant 1$ :

$$
\left\|j_{1, n}\right\|_{L^{2}\left(\mathcal{C}_{1}^{-}\right)} \leqslant D \lambda^{D} e^{-\left(\frac{\lambda}{2}\right)\left[\left(y^{\prime}\right)^{2}+\left(\frac{a^{\prime}}{D_{1}} \frac{\alpha^{2}}{1+\alpha^{2}}\left(g\left(y^{\prime}\right)^{2}\right)\right]\right.}\left(D_{1} / a^{\prime}\right)^{n+1} e^{\lambda \phi_{0}(t-i k)} \frac{\left\|f_{+}\right\|_{L^{2}\left(\mathbb{R}^{2}\right)}}{\alpha^{2}+1}
$$


for $(t, k) \in X_{\kappa},\left(\alpha, x^{\prime}\right) \in \Sigma$. We split $\Sigma$ into the part contained in $\left\{\left|y^{\prime}\right|+|\alpha| \leqslant r\right\}$, called $\Sigma_{0}$ and $\Sigma_{1}$, we get

$$
J_{1, n}\left(\zeta_{1}^{+}, t, k, \lambda\right)=\int_{\Sigma_{1}} \mathrm{~d} x^{\prime} \mathrm{d} \alpha j_{1, n}+\int_{\Sigma_{0}} \mathrm{~d} x^{\prime} \mathrm{d} \alpha j_{1, n} .
$$

The family of functions $J_{1, n}^{1}\left(\zeta_{1}^{+}, t, k, \lambda\right)=\int_{\Sigma_{1}} \mathrm{~d} x^{\prime} \mathrm{d} \alpha j_{1, n}$ satisfies the hypothesis of Lemme B.1 above, hence

$$
\begin{aligned}
& \int_{0}^{\infty} \mathrm{d} x x^{n} \int_{\mathcal{C}_{1}} d \zeta_{1}^{+} e^{\lambda x z-\lambda \frac{x^{2}}{2}+i \lambda x \zeta_{1}^{+}} \frac{\lambda}{4 \pi} e^{-\nu\left(\zeta_{1}^{+}\right)^{2}} J_{1, n}\left(\zeta_{1}^{+}, t, k, \lambda\right) \\
&-\int_{0}^{\infty} \mathrm{d} x x^{n} \int_{\mathcal{C}_{1}^{-}} d \zeta_{1}^{+} e^{\lambda x z-\lambda \frac{x^{2}}{2}+i \lambda x \zeta_{1}^{+}} \frac{\lambda}{4 \pi} e^{-\nu\left(\zeta_{1}^{+}\right)^{2}} \int_{\Sigma_{0}} \mathrm{~d} x^{\prime} \mathrm{d} \alpha j_{1, n} \\
& \quad-\int_{0}^{\infty} \mathrm{d} x x^{n} \int_{\mathcal{C}_{1}^{+}} d \zeta_{1}^{+} e^{\lambda x z-\lambda \frac{x^{2}}{2}+i \lambda x \zeta_{1}^{+}} \frac{\lambda}{4 \pi} e^{-\nu\left(\zeta_{1}^{+}\right)^{2}} J_{1, n}\left(\zeta_{1}^{+}, t, k, \lambda\right)
\end{aligned}
$$

is negligible in $H\left(\phi_{0}, X_{\kappa}, U\right)$. We reduced the problem to these two integrals.

For the integral on $\mathcal{C}_{1}^{+}$, we use $\Re\left(x^{\prime}-i \zeta_{1}^{+}-i \alpha\right) \leqslant x^{\prime}+a^{\prime}$, hence we get

$$
\left\|j_{1, n}\right\|_{L^{2}\left(\mathcal{C}_{1}^{+}\right)} \leqslant D \lambda^{D}\left(D_{1} / a^{\prime}\right)^{n+1} e^{-\lambda \frac{\left(x^{\prime}\right)^{2}}{2}+\lambda \phi_{0}(t-i k)+\lambda \Phi_{\delta}\left(x^{\prime}+a^{\prime}\right)} \frac{\left\|f_{+}\right\|_{L^{2}\left(\mathbb{R}^{2}\right)}}{\alpha^{2}+1},
$$

From this inequality, if we deform the integral on $\mathbb{R}^{2}$

$$
\begin{aligned}
& J_{1, n}\left(\zeta_{1}^{+}, t, k, \lambda\right)=\int_{\mathbb{R}^{2}} \mathrm{~d} x^{\prime} \mathrm{d} \alpha j_{1, n} \\
& =\frac{\lambda}{4 \pi} \int_{\mathbb{R}^{2}} \psi\left(x^{\prime}\right) \mathrm{d} x^{\prime} \frac{e^{i \lambda \alpha x^{\prime}}}{1+\alpha^{2}}\left(1-\frac{1}{\lambda^{2}} \partial_{\left(x^{\prime}\right)^{2}}^{2}\right)\left[e^{-\lambda \frac{\left(x^{\prime}\right)^{2}}{2}} \partial_{x^{n}}^{n} q_{1}^{+}\left(0, x^{\prime}, \zeta_{1}^{+}, k\right)\right. \\
& \left.\left(T_{0} \otimes T_{1}\left(1_{x^{\prime} \geqslant 0} \partial_{x^{\prime}} f\right)\left(t-i k, x^{\prime}-i \zeta_{1}^{+}-i \alpha, \lambda\right)\right)\right] \mathrm{d} x \mathrm{~d} \alpha
\end{aligned}
$$

on the same $\Sigma$ for $\zeta_{1}^{+} \in \mathcal{C}_{1}^{+}$, we get, using the inequality

$$
\left\|j_{1, n}\right\|_{L^{2}\left(\mathcal{C}_{1}^{+}\right)} \leqslant D \lambda^{D}\left(D_{1} / a^{\prime}\right)^{n+1} e^{\lambda \phi_{0}(t-i k)+\lambda G} \frac{\left\|f_{+}\right\|_{L^{2}\left(\mathbb{R}^{2}\right)}}{\alpha^{2}+1}
$$

where

$G \leqslant-g\left(y^{\prime}\right) \frac{\alpha^{2}}{\left(1+\alpha^{2}\right)^{1 / 2}} \frac{a^{\prime}}{2 D_{1}}-\frac{\left(y^{\prime}\right)^{2}}{2}+\Phi_{\delta}^{+}\left(y^{\prime}+\Im \zeta_{1}^{+}+i\left(\alpha\left(\frac{g\left(y^{\prime}\right)}{\left(1+\alpha^{2}\right)^{1 / 2}} \frac{a^{\prime}}{D_{1}}-1\right)-\Re \zeta_{1}^{+}\right)\right)$.

We use the result of $[\mathbf{2 0}]$ p. 403, that is $G<0$. This is true because $\sup \left(a^{\prime} \delta\right)=$ $\sup \left(a^{\prime}, 3 \kappa c\right)=\sup \left(a^{\prime}, 3 a^{\prime} c / D_{1}\right) \ll t_{0}$. Hence the decomposition $\Sigma=\Sigma_{0} \cup \Sigma_{1}$ gives 
again the relation

$$
\begin{aligned}
& J_{1, n}\left(\zeta_{1}^{+}, t, k, \lambda\right)- \int_{\Sigma_{0}} \mathrm{~d} x^{\prime} \mathrm{d} \alpha j_{1, n} \\
&=\frac{\lambda}{4 \pi} \int_{\mathbb{R}^{2}} \psi\left(x^{\prime}\right) \mathrm{d} x^{\prime} \frac{e^{i \lambda \alpha x^{\prime}}}{1+\alpha^{2}}\left(1-\frac{1}{\lambda^{2}} \partial_{\left(x^{\prime}\right)^{2}}^{2}\right)\left[e^{-\lambda \frac{\left(x^{\prime}\right)^{2}}{2}} \partial_{x^{n}}^{n} q_{1}^{+}\left(0, x^{\prime}, \zeta_{1}^{+}, k\right)\right. \\
&\left.\quad\left(T_{0} \otimes T_{1}\right)\left(\left(1_{x^{\prime}} \geqslant 0 \partial_{x^{\prime}} f\right)\left(t-i k, x^{\prime}-i \zeta_{1}^{+}-i \alpha, \lambda\right)\right)\right] \mathrm{d} x \mathrm{~d} \alpha
\end{aligned}
$$

is negligible in $H\left(\phi_{0}, X_{\kappa}, U\right)$.

Moreover, integrating by parts in the variable $x^{\prime}$, the contribution of $\partial \Sigma_{0}$ yields a term negligible in $H\left(\phi_{0}, X_{\kappa}, U\right)$, hence we get

$$
\begin{aligned}
& J_{1, n}\left(\zeta_{1}^{+}, t, k, \lambda\right)-\int_{\Sigma_{0}} \mathrm{~d} x^{\prime} \mathrm{d} \alpha j_{1, n}=\frac{\lambda}{4 \pi} \int_{\mathbb{R}^{2}} \psi\left(x^{\prime}\right) \mathrm{d} x^{\prime} e^{i \lambda \alpha x^{\prime}-\lambda\left(x^{\prime}\right)^{2} / 2} \\
& \left.\partial_{x^{n}}^{n} q_{1}^{+}\left(0, x^{\prime}, \zeta_{1}^{+}, k\right)\left(T_{0} \otimes T_{1}\right)\left(1_{x^{\prime} \geqslant 0} \partial_{x^{\prime}} f\right)\left(t-i k, x^{\prime}-i \zeta_{1}^{+}-i \alpha, \lambda\right)\right) \mathrm{d} x \mathrm{~d} \alpha .
\end{aligned}
$$

Note that all this part has been done to prove that we could reduce to the integral on a compact subset, which is $\Sigma_{0}$. Note also that this is valid as well for $\zeta_{1}^{+} \in \mathcal{C}_{1}^{+}$and $\zeta_{1}^{+} \in \mathcal{C}_{1}^{-}$.

We use now the assumption $a^{\prime} \ll d$. This proves that, for $\left(x^{\prime}, \alpha\right) \in \Sigma_{0}$ and $\zeta_{1}^{+} \in \mathcal{C}_{1}$, we have

$$
\begin{aligned}
& \left|\left(T_{0} \otimes T_{1}\right)\left(1_{x^{\prime} \geqslant 0} \partial_{x^{\prime}} f\right)\left(t-i k, x^{\prime}-i \zeta_{1}^{+}-i \alpha, \lambda\right)\right| \leqslant D \lambda^{d} e^{\lambda \phi_{0}(t-i k)}, \\
& \left|\partial_{z}^{m}\left(T_{0} \otimes T_{1}\left(1_{x^{\prime} \geqslant 0} \partial_{x^{\prime}} f\right)\left(t-i k, x^{\prime}-i \zeta_{1}^{+}-i \alpha, \lambda\right)\right)\right| \leqslant D \lambda^{d} e^{\lambda \phi_{0}(t-i k)} m ! D^{m+1} .
\end{aligned}
$$

We write the Taylor expansion on $x^{\prime}=0$ of $\partial_{x^{n}}^{n} q_{1}^{+}\left(0, x^{\prime}, \zeta_{1}^{+}, k\right)$ :

$$
\begin{aligned}
\partial_{x^{n}}^{n} q_{1}^{+}\left(0, x^{\prime}, \zeta_{1}^{+}, k\right)=\sum_{m \leqslant M} \frac{\partial_{x^{n}\left(x^{\prime}\right)^{m}}^{n+m} q_{1}^{+}\left(0,0, \zeta_{1}^{+}, k\right)}{m !}\left(x^{\prime}\right)^{m} & \\
& \quad+\frac{\left(x^{\prime}\right)^{M+1}}{(M+1) !} \int_{0}^{1} \partial_{x^{n}\left(x^{\prime}\right)^{m}}^{n+m} q_{1}^{+}\left(0, s x^{\prime}, \zeta_{1}^{+}, k\right)(1-s)^{M} \mathrm{~d} s
\end{aligned}
$$

for $M=E\left(\lambda / C_{2}\right)$.

We plug the remainder term in the expression of $J_{1, n}$. It gives a term which is negligible in $H\left(\phi_{0}, X_{\kappa}, U\right)$. Moreover, integrating by parts in $\alpha$ leads to

$$
\begin{gathered}
J_{1, n}\left(\zeta_{1}^{+}, t, k, \lambda\right)+\frac{\partial_{x^{n}\left(x^{\prime}\right) m}^{n+m} q_{1}^{+}\left(0,0, \zeta_{1}^{+}, k\right)}{m !} \frac{\lambda}{2 \pi} \int_{\Sigma_{0}} \psi\left(x^{\prime}\right) \mathrm{d} x^{\prime} e^{i \lambda \alpha x^{\prime}-\lambda\left(x^{\prime}\right)^{2} / 2} \\
\left(\frac{1}{\lambda} \partial_{z}\right)^{m}\left(T_{0} \otimes T_{1}\left(1_{x^{\prime} \geqslant 0} \partial_{x^{\prime}} f\right)\left(t-i k, x^{\prime}-i \zeta_{1}^{+}-i \alpha, \lambda\right)\right) \mathrm{d} x \mathrm{~d} \alpha+B_{1}+J_{1, M}^{2},
\end{gathered}
$$

where $B_{1}$ is the sum of the terms on $\partial \Sigma_{0}$ are negligible and $J_{1, M}^{2}$ is the remainder term, including the remainder term of the Taylor expansion in $x^{\prime}$ and all the other 
terms (in particular on $\Sigma_{1}$ for $\zeta_{1}^{+} \in \mathcal{C}_{1}^{ \pm}$). The term $B_{1}$ is bounded by

$$
D \lambda^{D} e^{\lambda \phi_{0}(t-i k)}\left(D / a^{\prime}\right)^{n+1} \sum_{m \leqslant M}\left(D / a^{\prime}\right)^{m+1} \lambda^{-m} e^{-\frac{r^{2} a^{\prime} \lambda}{D D_{2}}} D^{m} m !
$$

and the remainder term $J_{1, M}^{2}$ is bounded by

$$
D \lambda^{D} e^{\lambda \phi_{0}(t-i k)}\left(D / a^{\prime}\right)^{n+1}\left(D / a^{\prime}\right)^{M+1} \lambda^{-M / 2}(M / 2) !
$$

Both terms are negligible in $H\left(\phi_{0}, X_{\kappa}, U\right)$, as soon as $C_{2} \geqslant C_{2}^{0}$, where $C_{2}^{0}$ can be computed in terms of $a^{\prime}$. Hence we can replace the integral on $\Sigma_{0}$ by an integral on any contour localized in the neighborhood of $(0,0)$ this contour being a "good contour" in the sense of Sjöstrand for the phase function $\alpha x^{\prime}+i\left(x^{\prime}\right)^{2} / 2$.

We note now that, if we denote by $H\left(t-i k,-i \zeta_{1}^{+}, \lambda\right)$ the holomorphic function

$$
\begin{array}{r}
\frac{\lambda}{2 \pi} \int_{\Sigma_{0}} \psi\left(x^{\prime}\right) \mathrm{d} x^{\prime} e^{i \lambda \alpha x^{\prime}-\lambda\left(x^{\prime}\right)^{2} / 2}\left(T_{0} \otimes T_{1}\left(1_{x^{\prime} \geqslant 0} \partial_{x^{\prime}} f\right)\left(t-i k, x^{\prime}-i \zeta_{1}^{+}-i \alpha, \lambda\right)\right) \mathrm{d} x \mathrm{~d} \alpha \\
-J_{\rho}\left(T_{0} \otimes T_{1}\left(1_{x^{\prime} \geqslant 0} \partial_{x^{\prime}} f\right)\left(t-i k,-i \zeta_{1}^{+}, \lambda\right)\right)
\end{array}
$$

we have

$$
\left\|\partial_{z}^{m} H(t-i k, . \lambda)\right\|_{L^{2}\left(\mathcal{C}_{1}\right)} \leqslant C e^{-\lambda / C} D^{m} m ! e^{\lambda \phi_{0}(t-i k)} .
$$

We have also the relation

$$
\lambda \int_{0}^{\infty} x^{n} \psi(x) e^{\lambda x\left(z+i \zeta_{1}^{+}\right)} e^{-\lambda x^{2} / 2}=\left(\frac{1}{\lambda} \partial_{z}\right)^{n}\left[J_{\rho}^{-1}\left(\frac{1}{z+i \zeta_{1}^{+}}\right)+r\left(z+i \zeta_{1}^{+}, \lambda\right)\right]
$$

where, for $z \in U, \zeta_{1}^{+} \in \mathcal{C}_{1},\left|\partial_{z}^{j} r\right| \leqslant C e^{-\lambda / C} j ! /|z|$.

Replacing in the equality

$$
\begin{aligned}
I_{1, n}^{\nu}=\int_{0}^{\infty} e^{\lambda x z-\lambda x^{2} / 2} \times[ & \frac{x^{n}}{n !} \psi_{2}(x) \frac{\lambda}{4 \pi} \int_{\mathbb{R}} \mathrm{d} x^{\prime} \int_{\mathcal{C}_{\infty}} d \zeta_{1}^{+} e^{i \lambda\left(x-x^{\prime}\right) \zeta_{1}^{+}-\nu\left(\zeta_{1}^{+}\right)^{2}} \\
& \left.\times \partial_{x^{n}}^{n} q_{1}^{+}\left(0, x^{\prime}, \zeta_{1}^{+}, k\right)\left(T_{0}\left(1_{x^{\prime} \geqslant 0} \partial_{x^{\prime}} f\right)\left(t-i k, x^{\prime}, \lambda\right)\right) \mathrm{d} x\right],
\end{aligned}
$$

the relation (159) giving the asymptotic expansion of $J_{1, n}$, and integrating in $x$ using (161), we get, as $\left[J_{\rho}^{-1}, \partial_{z}\right]=0$ (which allows us to exchange the derivation and $J_{\rho}^{-1}$ in $(161)$ ) and $\left[J_{\rho}, \partial_{z}\right]=0$ (which enables us to transform the following relation, written using (160)):

$$
\begin{aligned}
& \frac{\partial_{x^{n}\left(x^{\prime}\right)^{m}}^{n+m} q_{1}^{+}\left(0,0, \zeta_{1}^{+}, k\right)}{m !} \frac{\lambda}{2 \pi} \int_{\Sigma_{0}} \psi\left(x^{\prime}\right) \mathrm{d} x^{\prime} e^{i \lambda \alpha x^{\prime}-\lambda\left(x^{\prime}\right)^{2} / 2} \\
& \left.\left(\frac{1}{\lambda} \partial_{z}\right)^{m}\left(T_{0} \otimes T_{1}\left(1_{x^{\prime} \geqslant 0} \partial_{x^{\prime}} f\right)\left(t-i k, x^{\prime}-i \zeta_{1}^{+}-i \alpha, \lambda\right)\right)\right] \mathrm{d} x \mathrm{~d} \alpha \\
& \left.\simeq \frac{\partial_{x^{n}\left(x^{\prime}\right)^{m}}^{n+m} q_{1}^{+}\left(0,0, \zeta_{1}^{+}, k\right)}{m !} J_{\rho}\left(\frac{1}{\lambda} \partial_{z}\right)^{m}\left(T_{0} \otimes T_{1}\left(1_{x^{\prime} \geqslant 0} \partial_{x^{\prime}} f\right)\left(t-i k,-i \zeta_{1}^{+}, \lambda\right)\right)\right] .
\end{aligned}
$$

We have only to let $\nu$ go to $0_{+}$. The remainder terms are negligible in $H\left(\phi_{0}, X_{\kappa}, U\right)$. This is valid when $C_{1}^{0}$ is replaced by $C_{1} \geqslant C_{1}^{0}=d e D_{1} / a^{\prime}$. This ends the proof of the 
asymptotic expansion of $I_{1}$. The asymptotic expansion of $I_{2}$ and $I_{3}$ follow the same pattern. We have, when $f$ is constructed from $u-u_{+}$on $\Delta_{+}$:

$$
I_{1}-i \frac{\lambda}{2} J_{\rho}^{-1}\left(\sum_{\substack{n \leqslant \lambda / C_{1} \\ m \leqslant \lambda / C_{2}}}\left(\frac{1}{\lambda} \partial_{z}\right)^{n} \frac{1}{2 \pi} \int_{\mathcal{C}_{1}} \frac{\mathrm{d} \xi}{z+i \xi} s_{2, n, m}^{+}(k, \xi)\left(\frac{1}{\lambda} \partial_{z}\right)^{m} J_{\rho} F(-i \xi)\right)
$$

is negligible in $H\left(\phi_{0}, Z_{a^{\prime} / D_{1}}, U\right)$ and

$$
I_{3}-\frac{1}{2} J_{\rho}^{-1}\left(\sum_{\substack{n \leqslant \lambda / C_{1} \\ m \leqslant \lambda / C_{2}}}\left(\frac{1}{\lambda} \partial_{z}\right)^{n} \frac{1}{2 \pi} \int_{\mathcal{C}_{1}} \frac{\mathrm{d} \xi}{z+i \xi} s_{1, n, m}^{+}(k, \xi)\left(\frac{1}{\lambda} \partial_{z}\right)^{m} J_{\rho} F(-i \xi)\right)
$$

is negligible in $H\left(\phi_{0}, Z_{a^{\prime} / D_{1}}, U\right)$. We have also for the terms with $\widetilde{F}$, owing to $\widetilde{F}(w, z, \lambda)=\left(T_{0} \otimes T_{1}\right)\left(1_{x \geqslant 0} \partial_{x} f\right)(w, z, \lambda)=T_{1}\left(1_{x \geqslant 0} \partial_{x}\left(T_{0} f\right)(w, x, \lambda)\right)(w, z, \lambda)$,

$$
I_{2}-\frac{1}{2} J_{\rho}^{-1}\left(\sum_{\substack{n \leqslant \lambda / C_{1} \\ m \leqslant \lambda / C_{2}}}\left(\frac{1}{\lambda} \partial_{z}\right)^{n} \frac{1}{2 \pi} \int_{\mathcal{C}_{1}} \frac{\mathrm{d} \xi}{z+i \xi} q_{1, n, m}^{+}(k, \xi)\left(\frac{1}{\lambda} \partial_{z}\right)^{m} J_{\rho} \widetilde{F}(-i \xi)\right)
$$

is negligible in $H\left(\phi_{0}, Z_{a^{\prime} / D_{1}}, U\right)$. Proposition 4.5 is proven.

Let us consider now the singular terms, used in Proposition 4.6. We prove Proposition 4.6, following step by step the proof of [20]: (B.28) to (B.113). Some steps may be omitted here, the interested reader must refer to $[\mathbf{2 0}]$ for the completion of some expansions.

We prove the result on the term

$$
T_{1}\left(R_{-}^{+, \lambda k, 2}\left(T_{0} f\right)\right)
$$

where the operator $R_{-}^{+, \lambda k, 2}$ is given by the limit, when $\nu \rightarrow 0_{+}$, of the integral:

$$
\int_{-\infty}^{+\infty} \mathrm{d} \xi e^{-\nu \xi^{2}} r^{2}(t, z, \xi, \lambda) j(t, \tau, \xi, \lambda)
$$

with the relation

$$
\begin{gathered}
r^{2}(t, z, \xi, \lambda)=\int_{0}^{\infty} \mathrm{d} x \psi(x) \frac{a^{\prime}(x)+\xi / \xi_{0}}{\left(1+\left(a^{\prime}(x)\right)^{2}\right)^{1 / 2}} e^{\lambda\left(x(z+i \xi)+i a(x) \xi_{0}-x^{2} / 2\right)} \\
j(t, \tau, \xi, \lambda)=\frac{\lambda}{2 \pi} \int_{0}^{\infty} \mathrm{d} x^{\prime} \psi\left(x^{\prime}\right) e^{-i \lambda x^{\prime} \xi-i \lambda b\left(x^{\prime}\right) \xi_{0}} T_{0}\left(1_{x^{\prime} \geqslant 0} \partial_{x^{\prime}} f\left(t, x^{\prime}\right)\left(t-i k, x^{\prime}, \lambda\right) .\right.
\end{gathered}
$$

Recall that $\xi_{0}=\left(k^{2}-\xi^{2}\right)^{1 / 2}, \Im k<0, \Im \xi_{0}>0$. We consider the contour $\mathcal{C}\left(l_{1}\right)$. If $\kappa \ll l_{1} \ll 1$, for $\xi \in \mathcal{C}\left(l_{1}\right)$, the function $k \rightarrow \xi_{0}$ can be extended to an holomorphic function on $|k-1|<3 \kappa$, and there exists $D_{2}, D_{3}, D_{4}, D_{5}, D_{6}$ positive constants, 
independent of $l_{1}$ and $\kappa$, such that

$$
\begin{aligned}
& \Im \xi_{0} \geqslant-D_{2} l_{1} \\
& D_{3}(1+|\Im k|) \geqslant\left|\xi_{0}\right| \geqslant\left(l_{1}^{2}+\kappa\right) / D_{3} \\
& \Im\left(\xi+\tan \gamma_{-} \xi_{0}\right) \geqslant D_{4} \kappa \\
& \Im\left(\xi+\tan \gamma_{-} \xi_{0}\right) \geqslant-l_{1} / D_{5} \Longrightarrow\left|\xi-\cos \gamma_{-}\right| \leqslant \sqrt{l_{1}} \\
& \left|\xi+\tan \gamma_{-} \xi_{0}-\cos \gamma_{-}\right| \leqslant D_{6} l_{1} .
\end{aligned}
$$

The inequalities on $\Im\left(\xi+\tan \gamma_{-} \xi_{0}\right)$ are obtained when considering the contour $\left\{\xi+\tan \gamma_{-} \xi_{0}, \xi \in \mathcal{C}\left(l_{1}\right)\right\}$ shown in (172).

When we deform (162) on $\mathcal{C}\left(l_{1}\right)$, we show that this integral is defined for $(t, k) \in X_{\kappa}$ and $z \in U$. We also easily check the inequalities, obtained for $t_{0}$ small enough,

$$
\frac{\tan \gamma_{+}}{2} x \leqslant a(x) \leqslant \frac{3 \tan \gamma_{+}}{2} \text { and } \frac{3 \tan \gamma_{-}}{2} x^{\prime} \leqslant b\left(x^{\prime}\right) \leqslant \frac{\tan \gamma_{-}}{2} x^{\prime}
$$

for $\left(x, x^{\prime}\right) \in(\operatorname{supp} \psi)^{2}$. Hence we obtain

$$
\Re\left(z+i\left(\xi+\frac{a(x)}{x} \xi_{0}\right)\right) \leqslant-4+D l_{1} \leqslant-1, \quad \Re\left(-i\left(\xi+\frac{b\left(x^{\prime}\right)}{x^{\prime}} \xi_{0}\right)\right) \leqslant D l_{1} .
$$

This imply the inequality

$$
|j(t, k, \xi, \lambda)| \leqslant D \lambda^{d} e^{\lambda D l_{1} t_{0}}\left\|1_{x^{\prime} \geqslant 0} \partial_{x^{\prime}} f\left(t, x^{\prime}\right)\right\|_{L^{2}\left(\mathbb{R}_{+}\right)} e^{\lambda \phi_{0}(t-i k)} .
$$

Let us write the Taylor expansion of the symbol contained in $k$, using the notation:

$$
\begin{gathered}
\sigma_{n}(y, \xi, k \lambda)=\left(\partial_{y}\right)^{n} \frac{a^{\prime}(y)+\xi / \xi_{0}}{\left(1+\left(a^{\prime}(y)\right)^{2}\right)^{1 / 2}} e^{i \lambda(a(y)-\tan \gamma+y) \xi_{0}-y^{2} / 2} . \\
\frac{a^{\prime}(x)+\xi / \xi_{0}}{\left(1+\left(a^{\prime}(x)\right)^{2}\right)^{1 / 2}} e^{i \lambda\left(a(x)-\tan \gamma_{+} x\right) \xi_{0}-x^{2} / 2} \\
=\sum_{n \leqslant N} \frac{x^{n}}{n !} \sigma_{n}(0, \xi, k, \lambda)+\int_{0}^{x} \frac{(x-y)^{N}}{n !} \sigma_{N+1}(y, \xi, k, \lambda) \mathrm{d} y .
\end{gathered}
$$

Note that, for $y \in \operatorname{supp} \psi$, we have, if $a$ is an holomorphic function on $\{z, d(z, \operatorname{supp} \psi) \leqslant \rho\}, \rho$ small enough, the inequality

$$
\begin{aligned}
& \Re\left[i\left(a\left(y+\rho e^{i \theta}\right)-\left(y+\rho e^{i \theta}\right) \tan \gamma_{+}\right) \xi_{0}-\frac{\left(y+\rho e^{i \theta}\right)^{2}}{2}\right] \\
& \leqslant \Re\left(i\left(a(y)-y \tan \gamma_{+}\right) \xi_{0}\right)-\frac{y^{2}}{2}+D\left(y \rho+\rho^{2}\right)\left(1+\left|\xi_{0}\right|\right) .
\end{aligned}
$$

Let $\zeta=z+i \xi+i \tan \gamma_{+} \xi_{0}$. We get the inequality

$$
\begin{aligned}
& \left|\int_{0}^{\infty} \mathrm{d} x \psi(x) e^{\lambda x\left(z+i \xi+\tan \gamma+\xi_{0}\right)} \int_{0}^{x} \frac{(x-y)^{N}}{n !} \sigma_{N+1}(y, \xi, k, \lambda) \mathrm{d} y\right| \\
& \quad \leqslant \int_{0}^{\infty} \int_{0}^{x} \mathrm{~d} x \mathrm{~d} y \psi(x)(x-y)^{N} e^{\lambda(x-y) \Re \zeta+\lambda \phi(y)}(N+1) \rho^{-N-1} e^{\lambda D \rho^{2}\left(1+\left|\xi_{0}\right|\right)},
\end{aligned}
$$


where

$$
\begin{aligned}
\phi(y) & =\Re\left(y\left(z+i \xi+i \frac{a(y)}{y} \xi_{0}\right)\right)+D y \rho\left(1+\left|\xi_{0}\right|\right) \\
& \leqslant y \Re\left(z+D_{3} \rho+i \xi+i\left(\frac{a(y)}{y}+D_{3} \rho\right) \xi_{0}\right) .
\end{aligned}
$$

We have $\phi(y) \leqslant 0$ if $\rho \ll 1$. Hence, the change $x-y=u$ in the integral obtained in the right hand side of the equality above yields

$$
\begin{aligned}
\int_{0}^{\infty} \int_{0}^{x} \mathrm{~d} x \mathrm{~d} y \psi(x)(x-y)^{N} e^{\lambda(x-y) \Re \zeta+\lambda \phi(y)} & \frac{(N+1)}{\rho^{N+1}} e^{\lambda D \rho^{2}\left(1+\left|\xi_{0}\right|\right)} \\
& \leqslant D \frac{(N+1)}{\rho^{N+1}} e^{\lambda D \rho_{2}\left(1+\left|\xi_{0}\right|\right)} \int_{0}^{\infty} d u u^{N} e^{\lambda u \Re \zeta} \\
& \leqslant \frac{D(N+1) !}{(-\lambda \rho \Re \zeta)^{N+1}} e^{\lambda D \rho^{2}\left(1+\left|\xi_{0}\right|\right)} .
\end{aligned}
$$

For the choice of $N+1=\left[\lambda / C_{0}\right]$ with $C_{0} \gg 1$ and $\rho^{2}=(N+1) / \lambda\left(1+\left|\xi_{0}\right|\right)$, we obtain the inequality

$$
\begin{aligned}
& \int_{0}^{\infty} \int_{0}^{x} \mathrm{~d} x \mathrm{~d} y \psi(x)(x-y)^{N} e^{\lambda(x-y) \Re \zeta+\lambda \phi(y)} \frac{(N+1)}{\rho^{N+1}} e^{\lambda D \rho^{2}\left(1+\left|\xi_{0}\right|\right)} \\
& \leqslant\left(\frac{D(N+1)}{\lambda}\right)^{N+1 / 2}\left(\frac{1+\left|\xi_{0}\right|}{|\Re z|+\Im \xi_{0}}\right)^{N+1 / 2} .
\end{aligned}
$$

We fix $C_{0} \geqslant e D$, hence, using the second inequality of (163), we have

$$
\begin{aligned}
\int_{0}^{\infty} \int_{0}^{x} \mathrm{~d} x \mathrm{~d} y \psi(x)(x-y)^{N} e^{\lambda(x-y) \Re \zeta+\lambda \phi(y)} & \frac{(N+1)}{\rho^{N+1}} e^{\lambda D \rho^{2}\left(1+\left|\xi_{0}\right|\right)} \\
& \leqslant D e^{-\lambda / 2 C_{0}}\left(|\Re z|+\Im \xi_{0}\right)^{-(N+1) / 2} .
\end{aligned}
$$

When $l_{1} t_{0} \ll \frac{1}{C_{0}}$, using the inequality (164) we deduce that

$$
\int_{-\infty}^{+\infty} \mathrm{d} \xi e^{-\nu \xi^{2}} \int_{0}^{\infty} \mathrm{d} x \psi(x) e^{\lambda x\left(z+i \xi+\tan \gamma_{+} \xi_{0}\right)} \int_{0}^{x} \frac{(x-y)^{N}}{n !} \sigma_{N+1}(y, \xi, k, \lambda) d y j(t, \tau, \xi, \lambda)
$$

is negligible in $H\left(\phi_{0}, X_{\kappa}, U\right)$, which allows us to consider only the Taylor expansion up to the order $N$, with $N=\left[\lambda / C_{0}\right]-1$.

A similar calculus yields

$$
\left|\int_{0}^{\infty} \mathrm{d} x \psi(x) e^{\lambda x\left(z+i \xi+\tan \gamma+\xi_{0}\right)} \frac{x^{n}}{n !} \sigma_{n}(0, \xi, k, \lambda)\right| \leqslant D e^{-n / 2}\left(|\Re z|+\Im \xi_{0}\right)^{-(n+1) / 2} .
$$

The asymptotic expansion relies on the expansion of $j$. Let

$$
\mathcal{C}\left(l_{1}\right) \cap\left\{\Im\left(\xi+\tan \gamma_{-} \xi_{0}\right) \leqslant-l_{1} / D_{5}\right\}=\mathcal{C}\left(l_{1}\right)^{-},
$$

and $\mathcal{C}\left(l_{1}\right)=\mathcal{C}\left(l_{1}\right)^{-} \cup \mathcal{C}\left(l_{1}\right)^{+}$. The aim of what follows is to prove: 
(1) for $\xi \in \mathcal{C}\left(l_{1}\right)^{-}$,

$$
\begin{aligned}
j(t, k, \xi, \lambda) \simeq \frac{\lambda}{2 \pi} \sum_{m \leqslant \lambda / C_{0}^{\prime}} \frac{\lambda^{-m}}{m !}\left(\partial_{x^{\prime}}\right)^{m}\left(e^{-\lambda \frac{\left(x^{\prime}\right)^{2}}{2}-i \lambda\left(b\left(x^{\prime}\right)-\tan \gamma_{-} x^{\prime}\right) \xi_{0}}\right) \\
\quad \times\left(\partial_{z}\right)^{m}\left(T_{0} \otimes T_{1}\right)\left(\partial_{x^{\prime}} f\right)\left(t-i k,-i\left(\xi+\tan \gamma_{-} \xi_{0}\right), \lambda\right),
\end{aligned}
$$

which is achieved by using the inversion formula for the F.B.I. transform $T_{1}$, and deforming the integral in $\left(\alpha, x^{\prime}\right) \in \mathbb{R} \times \mathbb{R}_{+}$obtained on the contour $\Gamma\left(l_{2}\right) \times \mathbb{R}_{+}=$ $\left\{\beta+i l_{2}\left(1+\left|\Im \xi_{0}\right|\right)\right\} \times \mathbb{R}_{+}$. with $t_{0}+l_{2} \ll l_{1} \ll 1$,

(2) for $\xi \in \mathcal{C}\left(l_{1}\right)^{+}$we deform the integral obtained after using the inversion formula on $\Sigma=\left\{\left(\alpha, x^{\prime}\right), x^{\prime}=y^{\prime}+i g\left(y^{\prime}\right) \frac{\alpha}{\left(1+\alpha^{2}\right)^{1 / 2}} l_{3}, \quad\left(\alpha, y^{\prime}\right) \in \mathbb{R}^{2}\right\}$ where $l_{3}$ is such that $0<l_{3} \ll 1$, and we prove that $j(t, k, \xi, \lambda)$ is estimated by the integral on $\Sigma_{0}$, the remainder term being $O\left(e^{\lambda\left(\phi_{0}(t-i k)-1 / C(r)\right)}\right)$. We use the stationary phase method to obtain the asymptotic expansion of this term.

Proof of (1). - We check that, for $\alpha \in \Gamma\left(l_{2}\right)$, we have

$$
\Re\left(x^{\prime}-i\left(\xi+\tan \gamma_{-} \xi_{0}\right)-i \alpha\right) \leqslant\left\{\begin{array}{l}
4 c t_{0}-\frac{l_{1}}{D_{5}}+3 l_{2}, \quad\left|\Im \xi_{0}\right| \leqslant 2 \\
4 c t_{0} 2\left(\tan \gamma_{-}-l_{2}\right)+l_{2}, \quad\left|\Im \xi_{0}\right| \leqslant 2 .
\end{array}\right.
$$

This is a consequence of $\xi \in \mathbb{R},|\xi| \geqslant 1+l_{1}, \Im \xi_{0}>0$. If we choose $t_{0}+l_{2} \ll l_{1} \ll 1$, we obtain that the leading term is $-l_{1} / D_{5}$, hence

$$
\Re\left(x^{\prime}-i\left(\xi+\tan \gamma_{-} \xi_{0}\right)-i \alpha\right) \leqslant-\frac{l_{1}}{D_{5}^{\prime}}
$$

where the constant $D_{5}^{\prime}$ is not too different from $D_{5}$. This implies

$$
\begin{aligned}
\| \partial_{z}^{m}\left(T_{0} \otimes T_{1}\right)\left(\partial_{x^{\prime}} f\right)\left(t-i k, x^{\prime}-i\left(\xi+\tan \gamma_{-} \xi_{0}\right)\right. & -i \alpha, \lambda) \|_{L^{2}\left(\Gamma_{l_{2}}\right)} \\
& \leqslant\left(D_{5}^{\prime} / l_{1}\right)^{m+1} m ! e^{\lambda \phi_{0}(t-i k)} .
\end{aligned}
$$

As we have the inequality, for $t_{0} \ll l_{2}$ :

$$
\begin{aligned}
\Re\left(i x^{\prime} \alpha-i\left(b\left(x^{\prime}\right)-\tan \gamma_{-} x^{\prime}\right) \xi_{0}-\left(x^{\prime}\right)^{2} / 2\right) & \leqslant-x^{\prime} l_{2}\left(1+\left|\Im \xi_{0}\right|\right)+D_{5}^{\prime}\left(x^{\prime}\right)^{2}\left|\Im \xi_{0}\right| \\
& \leqslant-x^{\prime} l_{2} / 2
\end{aligned}
$$

we write, for $\alpha \in \Gamma\left(l_{2}\right)$ :

$$
\begin{aligned}
& \int_{0}^{\infty} e^{i \lambda x^{\prime} \alpha-\lambda \frac{\left(x^{\prime}\right)^{2}}{2}-i \lambda\left(b\left(x^{\prime}\right)-\tan \gamma_{-} x^{\prime}\right) \xi_{0}} \psi\left(x^{\prime}\right) \\
& \left(T_{0} \otimes T_{1}\right)\left(\partial_{x^{\prime}} f\right)\left(t-i k, x^{\prime}-i\left(\xi+\tan \gamma_{-} \xi_{0}\right)-i \alpha, \lambda\right) \mathrm{d} x^{\prime} \\
& =\sum_{m \leqslant M} \int_{0}^{\infty} \mathrm{d} x^{\prime} e^{i \lambda x^{\prime} \alpha} \frac{\left(x^{\prime}\right)^{m}}{m !} \partial_{x^{\prime}}^{m}\left[e^{-\lambda \frac{\left(x^{\prime}\right)^{2}}{2}-i \lambda\left(b\left(x^{\prime}\right)-\tan \gamma-x^{\prime}\right) \xi_{0}} \psi\left(x^{\prime}\right)\right. \\
& \left.\quad \times\left(T_{0} \otimes T_{1}\right)\left(\partial_{x^{\prime}} f\right)\left(t-i k, x^{\prime}-i\left(\xi+\tan \gamma_{-} \xi_{0}\right)-i \alpha, \lambda\right)\right]+R_{M}(t, k, \xi, \alpha, \lambda),
\end{aligned}
$$


where $R_{M}$ is given by the integral Taylor formula. We perform an integration by parts in $R_{M}$ in the variable $x^{\prime}$, such that the coefficient becomes $-1 / i \lambda \alpha$. Hence, if $\rho$ (which is such that $a$ is holomorphic on $\{z, d(z, \operatorname{supp} \psi) \leqslant \rho\}$ ) verifies $\rho \ll l_{2}$, we get, as

$$
\left(\int_{\Gamma\left(l_{2}\right)} \frac{\mathrm{d} \alpha}{|\alpha|^{2}}\right)^{1 / 2} \leqslant \frac{D}{\left(l_{2}\left(1+\left|\Im \xi_{0}\right|\right)\right)^{1 / 2}}
$$

we obtain

$$
\int_{\Gamma\left(l_{2}\right)}\left|R_{M}(t, k, \xi, \alpha, \lambda)\right| \mathrm{d} \alpha \leqslant \frac{D(M+1) !}{(\lambda \rho)^{M+1}\left(l_{2}\left(1+\left|\Im \xi_{0}\right|\right)\right)^{M+1 / 2}} e^{\lambda D \rho^{2}\left(1+\left|\xi_{0}\right|\right)+\lambda \phi_{0}(t-i k)} .
$$

We choose $M+1=\left[\lambda / C_{0}^{\prime}\right]$, with $C_{0}^{\prime} \gg l_{2}^{-2}$, and $\rho=\left(\frac{M+1}{\lambda\left(1+\left|\xi_{0}\right|\right)}\right)^{1 / 2}$. This imply

$$
\int_{\Gamma\left(l_{2}\right)}\left|R_{M}\right| \mathrm{d} \alpha \leqslant D e^{-\lambda / 2 C_{0}^{\prime}}\left(1+\left|\Im \xi_{0}\right|\right)^{-M / 2}
$$

Hence the contribution of $R_{M}$ in (162) is negligible, thanks to (165).

Let us study now each term of the Taylor series. We use the relation

$$
\int_{0}^{\infty} \mathrm{d} x^{\prime} e^{i \lambda x^{\prime} \alpha} \psi\left(x^{\prime}\right) \frac{\left(x^{\prime}\right)^{m}}{m !}=\left(-\frac{1}{i \lambda \alpha}\right)^{m+1}+r_{m}(\alpha)
$$

where, using again an integration by parts in $x^{\prime}$ and $\psi(0)=1$

$$
\begin{aligned}
\left|r_{m}(\alpha)\right| & =\mid \frac{1}{\lambda \alpha} \int_{0}^{\infty} \partial_{x^{\prime}}\left(e^{i \lambda x^{\prime} \alpha}\left(1-\psi\left(x^{\prime}\right)\right) \frac{\left(x^{\prime}\right)^{m}}{m !} \mathrm{d} x^{\prime} \mid\right. \\
& \leqslant \frac{1}{\lambda|\alpha|} e^{-2 l_{2} c t_{0}\left(1+\left|\Im \xi_{0}\right|\right) \lambda}\left(\frac{D}{\lambda l_{2}\left(1+\left|\Im \xi_{0}\right|\right)}\right)^{m-1}
\end{aligned}
$$

because, on $\operatorname{supp}(1-\psi)$, we have $x^{\prime} \geqslant 3 c t_{0}$.

Note now that, when $\rho \ll l_{1}$,

$$
\begin{aligned}
& \|\left(\partial_{x^{\prime}}\right)^{m}\left(e^{-\lambda \frac{\left(x^{\prime}\right)^{2}}{2}-i \lambda\left(b\left(x^{\prime}\right)-\tan \gamma_{-} x^{\prime}\right) \xi_{0}}\right. \\
&\left.\left(T_{0} \otimes T_{1}\right)\left(\partial_{x^{\prime}} f\right)\left(t-i k, x^{\prime}-i\left(\xi+\tan \gamma_{-} \xi_{0}\right)-i \alpha, \lambda\right)\right)\left.\right|_{x^{\prime}=0} \|_{L^{2}\left(\Gamma\left(l_{2}\right)\right)} \\
& \leqslant \frac{m !}{\rho^{m}} e^{\lambda \phi_{0}(t-i k)+\lambda D \rho^{2}\left(1+\left|\xi_{0}\right|\right)},
\end{aligned}
$$

hence, choosing $\rho=\left(\frac{m}{\lambda\left(1+\left|\xi_{0}\right|\right)}\right)^{1 / 2} \ll l_{2}$, we obtain, using $C_{0}^{\prime} \ll l_{2}^{-2}$ :

$$
\begin{aligned}
\sum_{m \leqslant \lambda / C_{0}^{\prime}} \int_{\Gamma\left(l_{2}\right)} \mathrm{d} \alpha\left\|r_{m}(\alpha)\right\|_{L^{2}\left(\Gamma\left(l_{2}\right)\right)} \|\left(\partial_{x^{\prime}}\right)^{m}\left(e^{-\lambda \frac{\left(x^{\prime}\right)^{2}}{2}-i \lambda\left(b\left(x^{\prime}\right)-\tan \gamma_{-} x^{\prime}\right) \xi_{0}}\right. \\
\left.\cdot\left[\left(T_{0} \otimes T_{1}\right)\left(\partial_{x^{\prime}} f\right)\left(t-i k, x^{\prime}-i\left(\xi+\tan \gamma_{-} \xi_{0}\right)-i \alpha, \lambda\right)\right]\right)\left.\right|_{x^{\prime}=0} \|_{L^{2}\left(\Gamma\left(l_{2}\right)\right)} \\
\leqslant D_{10} e^{-\lambda / D_{10}} e^{\lambda \phi_{0}(t-i k)}
\end{aligned}
$$


From (165), this term is negligible, hence

$$
\begin{aligned}
j(t, k, \xi, \lambda) \simeq \sum_{m \leqslant \frac{\lambda}{C_{0}^{\prime}}}\left(\frac{\lambda}{2 \pi}\right)^{2} \int_{\Gamma\left(l_{2}\right)}\left(-\frac{1}{i \lambda \alpha}\right)^{m+1}\left(\partial_{x^{\prime}}\right)^{m}\left(e^{-\lambda \frac{\left(x^{\prime}\right)^{2}}{2}-i \lambda\left(b\left(x^{\prime}\right)-\tan \gamma_{-} x^{\prime}\right) \xi_{0}}\right. \\
\left.\cdot\left[\left(T_{0} \otimes T_{1}\right)\left(\partial_{x^{\prime}} f\right)\left(t-i k, x^{\prime}-i\left(\xi+\tan \gamma_{-} \xi_{0}\right)-i \alpha, \lambda\right)\right]\right)\left.\right|_{x^{\prime}=0} \mathrm{~d} \alpha
\end{aligned}
$$

The residue theorem allows us to use the pole $\alpha=0$, for which we compute (owing to the term $\alpha^{-m-1}$ ) the $m$-th derivative in $\alpha$ of

$$
\begin{aligned}
&\left(\partial_{x^{\prime}}\right)^{m}\left(e^{-\lambda \frac{\left(x^{\prime}\right)^{2}}{2}-i \lambda\left(b\left(x^{\prime}\right)-\tan \gamma_{-} x^{\prime}\right) \xi_{0}}\right. \\
&\left.\cdot\left[\left(T_{0} \otimes T_{1}\right)\left(\partial_{x^{\prime}} f\right)\left(t-i k, x^{\prime}-i\left(\xi+\tan \gamma_{-} \xi_{0}\right)-i \alpha, \lambda\right)\right]\right)\left.\right|_{x^{\prime}=0}
\end{aligned}
$$

This term is exactly

$$
\begin{aligned}
&(-i)^{m}\left(\partial_{x^{\prime}}\right)^{m}\left(e^{-\lambda \frac{\left(x^{\prime}\right)^{2}}{2}-i \lambda\left(b\left(x^{\prime}\right)-\tan \gamma_{-} x^{\prime}\right) \xi_{0}}\right. \\
&\left.\cdot\left(\partial_{z}\right)^{m}\left[\left(T_{0} \otimes T_{1}\right)\left(\partial_{x^{\prime}} f\right)\left(t-i k, x^{\prime}-i\left(\xi+\tan \gamma_{-} \xi_{0}\right), \lambda\right)\right]\right)\left.\right|_{x^{\prime}=0},
\end{aligned}
$$

hence, as the residue is $-2 \pi i$, we get

$$
\begin{aligned}
j(168), k, \xi, \lambda) \simeq & \frac{\lambda}{2 \pi} \sum_{m \leqslant \lambda / C_{0}^{\prime}} \lambda^{-m} \partial_{x^{\prime}}^{m}\left(e^{-\lambda\left(\frac{\left(x^{\prime}\right)^{2}}{2}+i\left(b\left(x^{\prime}\right)-\tan \gamma_{-} x^{\prime}\right) \xi_{0}\right)}\right. \\
& \left.\cdot\left(\partial_{z}\right)^{m}\left[\left(T_{0} \otimes T_{1}\right)\left(\partial_{x^{\prime}} f\right)\left(t-i k, x^{\prime}-i\left(\xi+\tan \gamma_{-} \xi_{0}\right), \lambda\right)\right]\right)\left.\right|_{x^{\prime}=0} .
\end{aligned}
$$

This ends the proof of the item 1 in the program.

Proof of Point (2). - We notice that $T_{0}\left(\partial_{x^{\prime}} f\right)$ is equal to 0 if $x^{\prime}<0$ (functions supported for $\left.x^{\prime} \geqslant 0\right)$. Hence, for $\xi \in \mathcal{C}\left(l_{1}\right)^{+}$, we have

$$
j(t, k, \xi, \lambda)=\frac{\lambda}{2 \pi} \iint_{\mathbb{R}^{2}} \sigma\left(t, k, \xi, \lambda ; x^{\prime}, \alpha\right) \mathrm{d} x^{\prime} \mathrm{d} \alpha
$$

where

$$
\begin{aligned}
\sigma\left(t, k, \xi, \lambda ; x^{\prime}, \alpha\right)=\frac{\lambda}{2 \pi} \frac{e^{i \lambda x^{\prime} \alpha}}{1+\alpha^{2}}\left(1-\frac{1}{\lambda^{2}} \partial_{x^{\prime}}^{2}\right) e^{-\lambda\left(\frac{\left(x^{\prime}\right)^{2}}{2}+i\left(b\left(x^{\prime}\right)-\tan \gamma_{-} x^{\prime}\right) \xi_{0}\right)} \psi\left(x^{\prime}\right) \\
\cdot\left(T_{0} \otimes T_{1}\right)\left(\partial_{x^{\prime}} f\right)\left(t-i k, x^{\prime}-i\left(\xi+\tan \gamma_{-} \xi_{0}\right)-i \alpha, \lambda\right)
\end{aligned}
$$

We use the same proof as the proof for the pseudodifferential terms, starting at the contour $\Sigma$ (157). We use the result of [20], pp. 410-412, to obtain

$$
\left|\sigma\left(t, k, \xi, \lambda ; x^{\prime}, \alpha\right)\right| \leqslant D \lambda^{D} \frac{\left\|\partial_{x^{\prime}} f\right\|_{L^{2}}}{1+\alpha^{2}} e^{\lambda \phi_{0}(t-i k)} e^{\lambda G} .
$$

The aim of the proof is to obtain $G<0$. This is done in (B.79) to (B.92) of [20].

Finally, the conditions we obtained on the constants are

$$
\kappa \ll l_{3}, \quad \kappa \ll \delta_{1}<d, \quad l_{4} \leqslant D\left(l_{1}^{1 / 2}+l_{3}^{1 / 2}\right), \quad \delta_{1}^{2} l_{4} \ll l_{3}
$$


which is all right (and all the conditions we came across are satisfied) if

$$
\kappa \ll l_{3}, \quad \kappa \ll t_{0} \ll l_{2} \ll l_{1}, \quad \kappa \ll \min \left(l_{3}^{1 / 2}, d\right) .
$$

The proof of point 2 was sketched.

Now, we replace $\sigma$ by the original symbol $\sigma_{0}$ by integrating by parts, the boundary terms being negligible, and we have

$$
\begin{aligned}
\sigma_{0}\left(t, k, \xi, \lambda ; x^{\prime}, \alpha\right)= & \frac{\lambda}{2 \pi} e^{i \lambda x^{\prime} \alpha} e^{-\lambda\left(\frac{\left(x^{\prime}\right)^{2}}{2}+i\left(b\left(x^{\prime}\right)-\tan \gamma_{-} x^{\prime}\right) \xi_{0}\right)} \\
& \cdot \psi\left(x^{\prime}\right)\left(T_{0} \otimes T_{1}\right)\left(\partial_{x^{\prime}} f\right)\left(t-i k, x^{\prime}-i\left(\xi+\tan \gamma_{-} \xi_{0}\right)-i \alpha, \lambda\right) .
\end{aligned}
$$

Recall the inequality $(\kappa \ll d)$, which is a consequence of Lemma 6.6:

$$
\left|\partial_{z}^{m}\left(T_{0} \otimes T_{1}\right)\left(\partial_{x^{\prime}} f\right)\left(t-i k, x^{\prime}-i\left(\xi+\tan \gamma_{-} \xi_{0}\right)-i \alpha, \lambda\right)\right| \leqslant D^{m+1} m ! e^{\lambda \phi_{0}(t-i k)} .
$$

We use the stationary phase lemma on the phase function $\phi\left(x^{\prime}, z\right)=x^{\prime} z-\frac{\left(x^{\prime}\right)^{2}}{2}-$ $i \xi_{0} b^{\prime \prime}(0)\left(x^{\prime}\right)^{2} / 2$ using the symbol

$$
\begin{aligned}
& v\left(t, k, \xi, \lambda ; x^{\prime}, z\right)=e^{-i \lambda \xi_{0}\left(b\left(x^{\prime}\right)-\tan \gamma_{-} x^{\prime}-b^{\prime \prime}(0) \frac{\left(x^{\prime}\right)^{2}}{2}\right)} \\
& \cdot\left(T_{0} \otimes T_{1}\right)\left(\partial_{x^{\prime}} f\right)\left(t-i k, x^{\prime}-i\left(\xi+\tan \gamma_{-} \xi_{0}\right)+z, \lambda\right) .
\end{aligned}
$$

The critical point is $x^{\prime}=z=0$, the "laplacian operator" is

and the stationary phase expansion yields

$$
\frac{\partial^{2}}{\partial x^{\prime} \partial z}-\frac{1}{2}\left(1+i b^{\prime \prime}(0) \xi_{0}\right) \frac{\partial^{2}}{\partial z^{2}}
$$

$$
\begin{array}{r}
\left.\frac{\lambda}{2 \pi} \sum_{m \leqslant \lambda / C_{0}^{\prime \prime}} \frac{1}{m !} \lambda^{-m}\left[\frac{\partial^{2}}{\partial x^{\prime} \partial z}-\frac{1}{2}\left(1+i b^{\prime \prime}(0) \xi_{0}\right) \frac{\partial^{2}}{\partial z^{2}}\right]^{m} v\left(t, k, \xi, \lambda ; x^{\prime}, z\right)\right|_{x^{\prime}=z=0} \\
+O\left(e^{\lambda \phi_{0}(t-i k)-\lambda / D}\right) .
\end{array}
$$

The expansion obtained in (168) for $\xi \in \mathcal{C}\left(l_{1}\right)^{-}$can be rewritten in a straightforward manner:

(171) $j(t, k, \xi, \lambda) \simeq \frac{\lambda}{2 \pi} \sum_{m \leqslant \lambda / C_{0}^{\prime}} \frac{1}{m !} \lambda^{-m}\left(\partial_{x}^{\prime} \partial_{z}\right)^{m}\left(e^{-\lambda \frac{\left(x^{\prime}\right)^{2}}{2}\left(1+b^{\prime \prime}(0) \xi_{0}\right)} v\left(t, k, \xi, \lambda ; x^{\prime}, z\right)\right)$

Hence (170) and (171) are quite similar. We intend to show now that both expansions are equivalent (their difference is negligible). The asymptotic expansion is thus valid for $\xi \in \mathcal{C}\left(l_{1}\right)^{-} \cup \mathcal{C}\left(l_{1}\right)^{+}$.

The end of the proof of Proposition 4.6 is thus a consequence of the precise calculus of the expansion of the stationary phase theorem using the operator

$$
\frac{\partial^{2}}{\partial x^{\prime} \partial z}-\frac{1}{2}\left(1+i b^{\prime \prime}(0) \xi_{0}\right) \frac{\partial^{2}}{\partial z^{2}} \text {. }
$$


More precisely, considering the same formal computation as [20], we introduce the polynomials in $X$ :

$$
\begin{aligned}
& P_{C}(X)=\sum_{m \leqslant \lambda / C} \frac{1}{m !} \lambda^{-m}\left[\frac{\partial^{2}}{\partial x^{\prime} \partial z}+X \frac{\partial^{2}}{\partial z^{2}}\right]^{m} v \\
& Q_{C}(X)=\sum_{m \leqslant \lambda / C} \frac{1}{m !} \lambda^{-m}\left[\frac{\partial^{2}}{\partial x^{\prime} \partial z}\right]^{m}\left[e^{i \lambda X\left(x^{\prime}\right)^{2}} v\right]
\end{aligned}
$$

which are both polynomials of degree less that $\lambda / C$, and we have, for $\rho=\rho(m)=$ $(m / \lambda)^{1 / 3}$, the inequality

$$
\left|P_{C}(A)-Q_{C}(A)\right| \leqslant \sum_{\lambda / 2 C \leqslant m \leqslant \lambda / C}\left(\frac{4(1+|A|) e^{D}}{C^{1 / 3}}\right)^{m} \leqslant e^{-\frac{\lambda}{C}+\phi_{0}(t-i k)}
$$

which is true as soon as $C \geqslant 64(1+|A|)^{3} e^{3 D+3}$. Hence the result on the expansions.

The last step is to evaluate the term $k$ in the integral (162). We obtain the expansion as in the pseudodifferential case, using

$$
\int_{0}^{\infty} e^{i \lambda \zeta} \psi(x) \frac{x^{n}}{n !} \mathrm{d} x=\left(-\frac{1}{\lambda \zeta}\right)^{n+1}+r_{n}(\zeta) .
$$

The key inequality is obtained considering $\rho=\left(n / \lambda\left(1+\left|\xi_{0}\right|\right)\right)^{1 / 2}$ :

$$
\left|\sum_{n \leqslant \lambda / C_{0}} r_{n}(\zeta) \sigma_{n}(0, \xi, k, \lambda)\right| \leqslant D e^{-\lambda / D} .
$$

Hence the expansion of Proposition 4.6 follows, using $\zeta=\xi+\tan \gamma_{+} \xi_{0}$.

6.3.2. Contours in the complex plane. - The modified sector $\omega_{\sigma}^{ \pm}$is $\frac{1}{\cos \gamma_{ \pm}} \omega_{\sigma}$. Let

$$
V_{\rho}=\left\{z \in \mathbb{C},\left|\Im z+\frac{1}{\cos \gamma_{+}}\right| \leqslant \frac{1}{\rho \cos \gamma_{+}},|\Re z| \leqslant \frac{c_{2}}{\rho \cos \gamma_{+}}\right\} .
$$

For example, we may see $V_{4}$ for $c_{2}=2$ and $\gamma_{+}=\pi / 3$ :

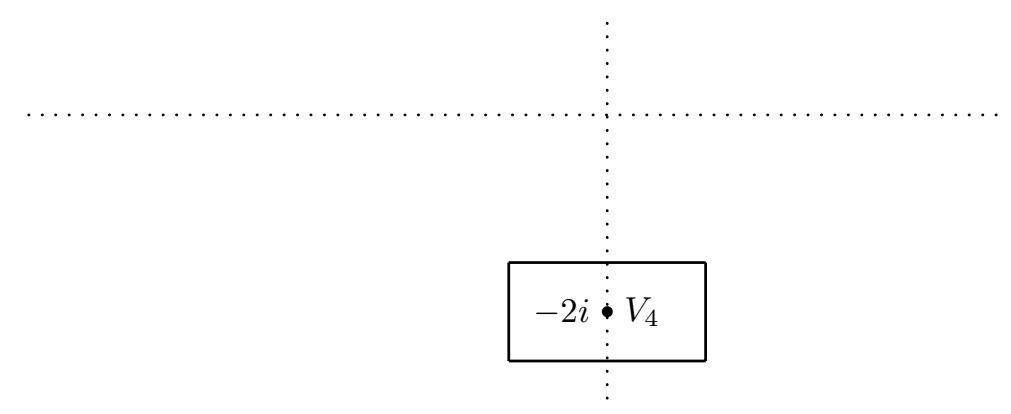

Figure 6.2. Contour $V_{\rho}$

The contour $\mathcal{C}_{1}$ used in Proposition 4.5 is defined in the following figure: 


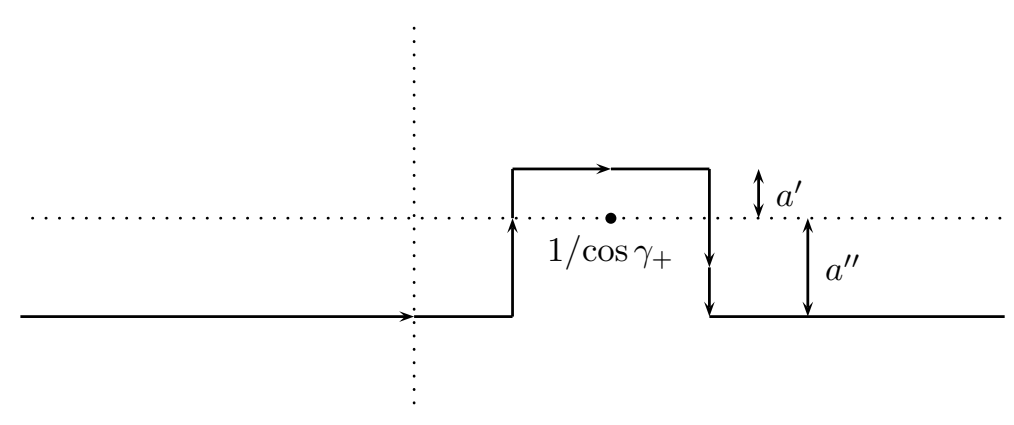

Figure 6.3. Contour $C_{1}$

It is

$$
\begin{array}{r}
\left.-\infty-i a^{\prime \prime}, \frac{1}{\cos \gamma_{+}}-l-i a^{\prime \prime}\right] \cup\left[\frac{1}{\cos \gamma_{+}}-l-i a^{\prime \prime}, \frac{1}{\cos \gamma_{+}}-l+i a^{\prime}\right] \\
\cup\left[\frac{1}{\cos \gamma_{+}}-l+i a^{\prime}, \frac{1}{\cos \gamma_{+}}+l+i a^{\prime}\right] \cup\left[\frac{1}{\cos \gamma_{+}}+l+i a^{\prime}, \frac{1}{\cos \gamma_{+}}+l-i a^{\prime \prime}\right] \\
\cup\left[\frac{1}{\cos \gamma_{+}}+l-i a^{\prime \prime}+\infty-i a^{\prime \prime}[\right.
\end{array}
$$

It is deformed on $i \partial \omega_{\sigma}^{+}$for all $\sigma$, and finally it can be deformed on the contour $\mathcal{C}(+)$ of the figure below, around $i V_{6}$ for $z \notin V_{4}$, because the branching points of the symbol of $I^{+}$or of $Q^{+}$satisfy $-i \xi \in V_{8}$. Thus the integral used in Proposition 4.5 can be deformed on the contour $\mathcal{C}(+)$ below:

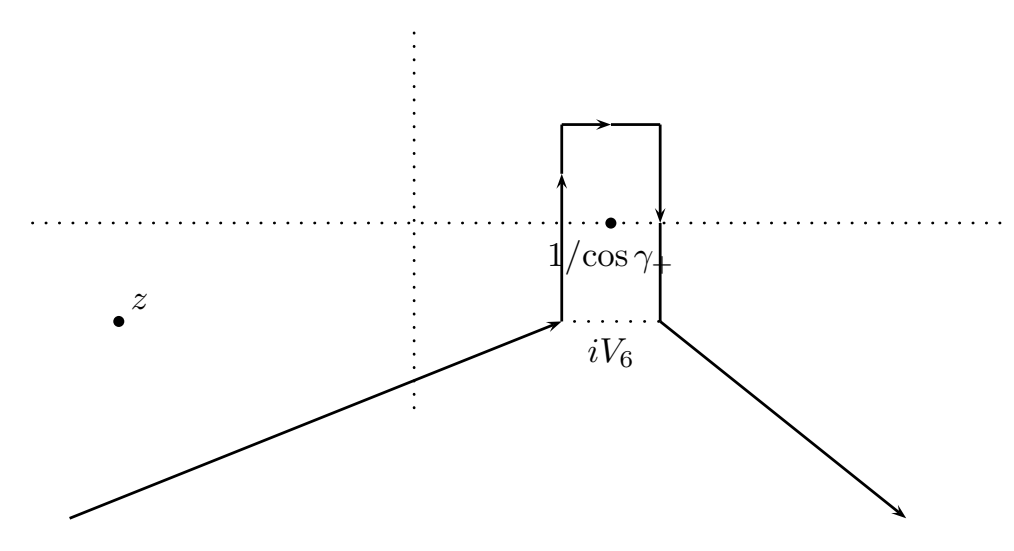

Figure 6.4. Contour $C(+)$ 
We introduce the contour

$$
\begin{aligned}
\left.C=]-\infty,-\frac{5}{4}\right] \cup\left[-\frac{5}{4},-\frac{5}{4}-i \frac{c_{2}}{4}\right] \cup\left[-\frac{5}{4}-i \frac{c_{2}}{4},-\frac{3}{4}-i \frac{c_{2}}{4}\right] \\
\cup\left[-\frac{3}{4}-i \frac{c_{2}}{4},-\frac{3}{4}\right] \cup\left[-\frac{3}{4}, \frac{3}{4}\right] \cup\left[\frac{3}{4}, \frac{3}{4}+i \frac{c_{2}}{4}\right] \\
\cup\left[\frac{3}{4}+i \frac{c_{2}}{4}, \frac{5}{4}+i \frac{c_{2}}{4}\right] \cup\left[\frac{5}{4}+i \frac{c_{2}}{4}, \frac{5}{4}\right] \cup\left[\frac{5}{4},+\infty[.\right.
\end{aligned}
$$

Figure 6.5. Contour $C$

We notice that $\cos \gamma_{+} \mathcal{C}(+)$ can be deformed on $C$, as well as the contour $\cos \gamma_{-} \mathcal{C}(-)$, $\mathcal{C}(-)$ being obtained as the contour on which we could deform for the calculus of $Q^{-}$, $I_{-}^{0, k}, I_{-}^{1, k}$. Let $\mathcal{C}\left(l, \tan \gamma_{ \pm}\right)$be the contours

(172) $\left.\left.\mathcal{C}\left(l, \tan \gamma_{ \pm}\right)=\right]-\infty,-1-l\right] \cup[-1-l,-1-l-i l] \cup\left[-1-l-i l, \cos \gamma_{ \pm}-i l\right]$ $\cup\left[\cos \gamma_{ \pm}-i l, \cos \gamma_{ \pm}+i l\right] \cup\left[\cos \gamma_{ \pm}+i l, 1+l+i l\right] \cup[1+l+i l, 1+l] \cup[1+l,+\infty[$. shown below:

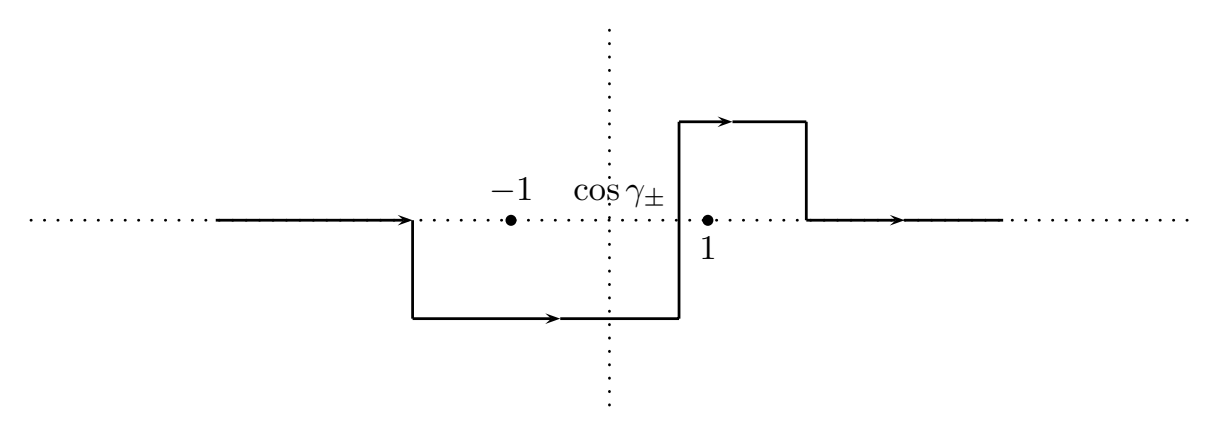

Figure 6.6. Contour $C\left(l, \tan \gamma_{ \pm}\right)$

Let finally $\Gamma$ be the contour introduced in [20] by (4.54), that we reproduce here Let $\tau$ be a complex number such that $|\tau-1| \leqslant \delta / c$. We define the two contours $\mathcal{C}\left(\tan \gamma_{-}\right)$and $\mathcal{C}\left(-\tan \gamma_{+}\right)$, through:

$$
\begin{aligned}
\mathcal{C}\left(\tan \gamma_{-}\right) & =\left\{\zeta \cos \gamma_{-}-\left(\tau^{2}-\zeta^{2}\right)^{1 / 2} \sin \gamma_{-}, \zeta \in \Gamma\right\} \\
\mathcal{C}\left(-\tan \gamma_{+}\right) & =\left\{\zeta \cos \gamma_{+}+\left(\tau^{2}-\zeta^{2}\right)^{1 / 2} \sin \gamma_{+}, \zeta \in \Gamma\right\}
\end{aligned}
$$




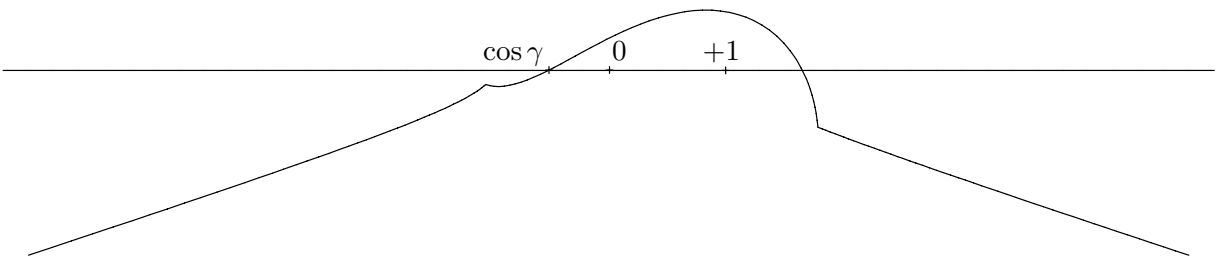

Figure 6.7. Contour $\Gamma$

The integrals of $s^{+,-}, R^{0,+,-}, R^{1,+,-}$ and $s^{-,+}, R^{0,-,+}, R^{1,-,+}$ can be deformed on $\mathcal{C}\left(\mp \tan \gamma_{ \pm}\right)$, which proves that the operators $S^{ \pm}$and $R^{0, \pm}, R^{1, \pm}$ are represented by the integrals on $\Gamma$ written in Definition 4.8. 



\section{BIBLIOGRAPHY}

[1] F. Assous, P. Ciarlet \& E. Sonnendrucker - Résolution des équations de Maxwell dans un domaine avec un coin rentrant, C. R. Acad. Sci. Paris Sér. I Math. 323 (1996), p. 203-208.

[2] M. Azaiez, M. Dauge \& Y. Maday - Méthodes spectrales, in École des ondes: Méthodes numériques d'ordre élevé pour les ondes en régime transitoire, Collection didactique de l'INRIA, janvier 1994.

[3] J.-M. BERnARD - Diffraction by a metallic wedge covered with a dielectric material, Wave Motion 9 (1987), p. 543-561.

[4] perfectly conducting wedge, Ann. Telecom. 45 (1990), p. 30-39.

[5] Diffraction par un dièdre à faces courbes non parfaitement conducteur, Rev. Tech. Thomson-CSF 23 (1991), no. 2, p. 321-330.

[6] Sur les solutions explicites des problèmes de diffraction par un dièdre, Rev. Tech. Thomson-CSF 25 (1993), no. 4.

[7] _ Diffraction by a cone, Note CEA, 1998.

[8] J.-M. Bernard \& G. Pelosi - Diffraction par un dièdre avec variation d'impédance dépendant de la distance à l'arête, Ann. Telecom. 47 (1992), no. 910, p. 421-423.

[9] D. Bouche \& F. Molinet - Méthodes asymptotiques en électromagnétisme, Mathématiques et Applications, vol. 16, Springer Verlag, 1994.

[10] H. BRezis - Analyse fonctionnelle : Théorie et applications, Mathématiques appliquées pour la maîtrise, Masson, 1992.

[11] N. BurQ - Pôles de diffusion engendrés par un coin, Astérisque, vol. 242, Société Mathématique de France, 1997. 
[12] M. Cessenat - Résolution des problèmes de Helmholtz par séparation des variables en coordonnées polaires, C. R. Acad. Sci. Paris Sér. I Math. 309 (1990), p. 105-109.

[13] _ Mathematical methods in electromagnetism. Linear theory and applications, Series on Advances in Mathematics to Applied Sciences, vol. 41, World Scientific, Singapure, 1996.

[14] D. Colton \& R. KRess - Inverse Acoustic and Electromagnetic Scattering Theory, Applied Maths Sciences, vol. 93, Springer, 1992.

[15] M. Costabel \& M. Dauge - General edge asymptotics of solutions of secondorder elliptic boundary value problems I and II, Proc. Edinburgh Math. Soc. (2) 123 A (1993), p. 157-184 and 109-155.

[16] J.-P. Croisille \& G. Lebeau - Diffraction by an immersed elastic wedge: theory and numerical computation, Lecture Notes in Mathematics, vol. 1723, Springer, 1999.

[17] G. Eskin - Mixed initial-boundary value problems for second order hyperbolic equations, Comm. Partial Differential Equations 12 (1987), no. 5, p. 503-587.

[18] Partial Differential Equations 17 (1992), no. 1 and 2, p. 99-160.

[19] H. GARNiR - La fonction de Green pour l'opérateur métaharmonique dans un angle ou un dièdre, Bull. Soc. Roy. Sci. Liège (1952), p. 119-140, 207-231, 328344 .

[20] P. Gérard \& G. Lebeau - Diffusion d'une onde par un coin, J. Amer. Math. Soc. 6 (1993), no. 2.

[21] P. GRISVARD - Elliptic problems in nonsmooth domains, Monograph and Studies in Mathematics, vol. 24, Pitman, New York, 1985.

[22] M. V. DE Hoop - Generalisation of the Bremmer coupling series, J. Math. Phys. 37 (1996), no. 7, p. 3246-3282.

[23] L. Kaminetzky \& J. B. Keller - Diffraction coefficients for high order edges and vertices, SIAM J. Appl. Math. 22 (1972), no. 1, p. 109-134.

[24] V. A. Kondrat'EV - Boundary problems for elliptic equations in domains with conical or angular points, Trans. Moscow Math. Soc. 16 (1967), p. 227-313.

[25] O. LAfitTe - The kernel of the Neumann operator for a strictly diffractive problem, Comm. Partial Differential Equations 20 (1995), no. 3-4, p. 419-483.

[26] _ Opérateurs intégraux de Fourier et asymptotiques pour les ondes, Notes de cours de DEA, Université de Paris Nord, 1995-1996. 
[27] The impedance boundary condition on a thin layer of material over a perfectly conducting body, SIAM J. Appl. Math. 59 (1999), no. 3, p. 1028-1052.

[28] G. LeBEAU - Seconde microlocalisation sur les sous-variétés isotropes, Ann. Inst. Fourier (Grenoble) 35 (1985), no. 2, p. 145-216.

[29] _ Propagation des ondes dans les dièdres, Mém. Soc. math. France (N.S.), vol. 60, Société Mathématique de France, 1995.

[30] G. D. Malyuzhinets - Excitation, reflection and emission of surface waves from a wedge with given face impedances, Soviet Phys. Dokl. 3 (1958), p. 752-755.

[31] _ Inversion formula for the Sommerfeld integral, Dokl. Akad. Nauk SSSR 118 (1958), p. 1099-1102.

[32] R. B. Melrose - Geometric Scattering Lectures, Stanford Lectures, Cambridge University Press, Cambridge, 1995.

[33] F. Molinet - Geometrical theory of diffraction, IEEE APS 1, 2 (1987 and 1997), p. 6-17 and p. 5-16.

[34] H. Poincaré - Sur la polarisation par diffraction, Acta Math. 16 (1892), p. 297339.

[35] Sur la polarisation par diffraction (deuxième partie), Acta Math. 20 (1896), p. 313-356.

[36] T. B. A. Senior \& J. Volakis - A simple expression for a function occuring in diffraction theory, IEEE APS 33, no. 11, p. 678-680.

[37] J. Simon - Sobolev, Besov and Nikolskii fractional spaces: imbeddings and comparisons for vector valued spaces on an interval, unpublished.

[38] J. SJÖSTRAND - Propagation of analytic singularities for second order Dirichlet problems, Comm. Partial Differential Equations 5 (1980), no. 1, p. 41-94.

[39] _ Singularités analytiques microlocales, Astérisque, vol. 95, Société Mathématique de France, 1982.

[40] A. Sommerfeld - Mathematische Theorie der Diffraction, Matematische Annalen 47 (1897), p. 317-1897.

[41] C. H. WiLCOX - Scattering Theory for Diffraction Gratings, Appl. Mathematical Sciences, vol. 46, Springer Verlag, 1984. 